\title{
A twist in NMR relaxation experiments: Application to the study of protein motions
}

\author{
Dissertation \\ for the award of the degree \\ "Doctor rerum naturalium" (Dr.rer.nat.) \\ of the Georg-August-Universität Göttingen
}

within the doctoral program "Physics of Biological and Complex Systems" of the Georg-August-University School of Science (GAUSS)

submitted by

Sebastian Frischkorn

from Bremerhaven, Germany

Göttingen, 2019 


\section{Thesis Committee}

Prof. Dr. Christian Griesinger

NMR-based Structural Biology

Max Planck Institute for Biophysical Chemistry, Göttingen, Germany

Prof. Dr. Marina Bennati

Electron-Spin Resonance Spectroscopy

Max Planck Institute for Biophysical Chemistry, Göttingen, Germany

Prof. Dr. Bert de Groot

Computational Biomolecular Dynamics

Max Planck Institute for Biophysical Chemistry, Göttingen, Germany

\section{Members of the Examination Board}

Reviewer: $\quad$ Prof. Dr. Christian Griesinger NMR-based Structural Biology

Max Planck Institute for Biophysical Chemistry, Göttingen, Germany

Second Reviewer: $\quad$ Prof. Dr. Marina Bennati

Electron-Spin Resonance Spectroscopy

Max Planck Institute for Biophysical Chemistry, Göttingen, Germany

\section{Further Members of the Examination Board}

Prof. Dr. Bert de Groot

Computational Biomolecular Dynamics

Max Planck Institute for Biophysical Chemistry, Göttingen, Germany

Prof. Dr. Jörg Enderlein

Single Molecule Spectroscopy and Imaging for Biophysics and Complex Systems

Third Institute of Physics Georg August University, Göttingen Germany

Prof. Dr. Martin Suhm

Vibrational Spectroscopy and Intermolecular Interactions

Institute for Physical Chemistry, Göttingen, Germany

Prof. Dr. Simone Techert

Structural Dynamics of Chemical Systems

Institute for X-Ray Physics, Göttingen, Germany

Date of the oral examination: 04.06 .2019 


\section{Affidavit}

I hereby affirm that this thesis entitled "A twist in NMR relaxation experiments: Application to the study of protein motions" represents my own original work, except where acknowledged by general and specific references.

Place, Date

Signature 


\section{Acknowledgment}

First of all, I would like to express my gratitude to my supervisor Prof. Dr. Christian Griesinger for giving me the opportunity to work on these interesting and challenging projects, as well as for mentoring and guidance throughout these years.

I am also very grateful to Prof. Dr. Donghan Lee for sharing his knowledge with me as well as mentoring me despite the long distance via many skype meetings.

I would like to thank Prof. Dr. Marina Bennati and Prof. Dr. Bert de Groot for being part of my thesis committee and their feedback during our meetings. Furthermore, I would like to thank all members of the examination board for the evaluation of my dissertation.

I greatly thank Dr. Pablo Trigo Mourino and Supriya Pratihar for their support, comments and discussions as well as the great office atmosphere and coffee breaks that helped me to keep the wheels turning.

Furthermore, I would like to thank all present and past "cubic" members for the nice working atmosphere and all the unforgettable memories I could collect during this time.

Dr. Ching-Yu Chou for the collaboration as well as all the travels to Göttingen for troubleshooting the shuttle setup.

Dr. Stefan Becker, Melanie Wegstroth and Claudia Schwiegk for preparing the samples, as without them this work would not have been possible.

I would like to thank Dr. Jithender Reddy Gurrala, who taught me a lot about pulse programming and helped me with discussions and suggestions.

Dr. Dirk Bockelmann for listening to all my IT related problems as well as handling my plenty python packages and special software requests.

My board game group for the necessary distraction, "Saturday canteen" meetings and making life in Göttingen so enjoyable.

I would like to thank my family for their love and support. Without them all of this would not have been possible.

Finally Christin, for being always there for me, supporting me and so much more than I can say. You kept me motivated and without you I would have never achieved all of this. 


\section{Abstract}

Understanding biological processes requires knowledge about the structure as well as dynamics of the involved molecules. Proteins are complex structures, which experience time dependent changes due to atomic motions. The large number of atoms that compose proteins also cause a large variety of motions that can be observed in these macromolecules. These motions range from femtoseconds to seconds and induce changes in the structure, which might be pivotal for the functionality of a protein. Nuclear magnetic resonances (NMR) spectroscopy provides a powerful tool to study protein motions, since it enables the study of protein dynamics under physiological conditions and offers experiments that cover large parts of the protein motion time scale.

The first part of this thesis focuses on the study of protein motions that occur in the microsecond to millisecond range. This regime of motions is targeted by so-called relaxation dispersion (RD) experiments. Here, the extreme CPMG (E-CPMG) experiment is presented, which combines the time scales of the two conventional RD experiments (CPMG and $R_{1 \rho}$ ) and allows a more accurate determination of fast kinetic processes by CPMG type experiments. Application of E-CPMG to study the folding/unfolding process of gpW protein in solution is presented. Previous studies have identified 68 residue gpW as an ultra-fast downhill folding protein that forms a $\alpha+\beta$ topology, which in solution stays in equilibrium with an unfolded $\beta$-hairpin conformation. Here, we show that the $\alpha$-helices in gpW are also involved in this conformational exchange with a similar time scale as the $\beta$-hairpin. Furthermore, it is shown that residues in the $\alpha$-helices are involved also in another much faster folding process. The RD profiles of these residues can only be described by a three-site exchange model and for the first time two distinct exchange processes are detected in a single RD experiment. Complementary E-CPMG experiments of methyl side-chains as additional probe for structural changes, showed similar exchange kinetics as they were previously observed for the backbone of gpW $\beta$-hairpin region. A temperature dependent study of the slow exchange process lead to similar results for the activation energy reported by the different probes. These results indicate that global changes in the structure are involved in the formation of the $\beta$-hairpin region and also support the hypothesis of a hydrophobic collapse that assists its formation. Temperature dependent data of the folding kinetics reveal that the $\beta$-hairpin folding process of $\mathrm{gpW}$ in solution can be explained by a two-state model with an energy barrier much larger than expected for a downhill folding protein. Thus, it can be assumed that the energy landscape of gpW in solution is more complex than it is described for a downhill folding protein. 
The second part of this thesis is dedicated to the study of protein motions that occur in the sub- $\tau_{\mathrm{c}}$ regime by shuttle relaxometry. Shuttle relaxometry provides an alternative to the commonly used model free analysis for the study of local protein motions. The model free analysis is limited to high magnetic field strengths to ensure a high resolution and sensitivity, which are required for protein NMR. Using a motor-based shuttle system the resolution of high magnetic fields $(16.44 \mathrm{~T})$ is combined with relaxation information from the stray field $(10-0.5 \mathrm{~T})$ of the NMR magnet. The aim of this work is to study the field-dependence of longitudinal relaxation rates and the effect of local protein motions on the Lorentzian behavior of the spectral density function. Initial experiments on ubiquitin showed a deviation from the expected mono-exponential decay for a flexible loop region as well as the C-terminal residues at magnetic field strengths below $4 \mathrm{~T}$, indicating a stronger influence of local motions at these low fields. During the course of this work intrinsic problems with the shuttle setup occurred and required relaxation data could not be acquired. The effect of this intrinsic problems on the shuttle relaxometry data as well as the error diagnostics of the shuttle setup are described in detail. Additionally, relaxation data at several static magnetic fields was acquired to put the field-dependent $R_{1}$ data from shuttle relaxometry into perspective. The results indicate the necessity of low-field relaxation data for a more accurate estimation of local protein motions and an improvement of the shuttle hardware. 


\section{Contents}

1 Introduction $\quad 1$

1.1 Protein Structure and Motions . . . . . . . . . . . . . . . . . . 1

1.2 Protein NMR . . . . . . . . . . . . . . . . . . . . . . . 4

1.2.1 NMR Basic Concepts . . . . . . . . . . . . . . . . . . 4

1.2 .2 Structural Insights by NMR . . . . . . . . . . . . . . . . . . . 8

1.2 .3 The NMR Time Scale . . . . . . . . . . . . . . . . . . . 8

1.3 Thesis Outline . . . . . . . . . . . . . . . . . . . . . 11

2 High Power Relaxation Dispersion: The Extreme CPMG Experiment 13

2.1 Chemical Exchange . . . . . . . . . . . . . . . . . . . . . . . . 14

2.2 Relaxation Dispersion . . . . . . . . . . . . . . . . . . . . . . 19

2.2.1 Constant-Time CPMG . . . . . . . . . . . . . . . . . . 19

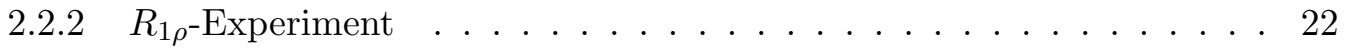

2.3 Extreme CPMG . . . . . . . . . . . . . . . . . . . 24

2.4 Conclusion . . . . . . . . . . . . . . . . . . . 30

2.5 Materials and Methods . . . . . . . . . . . . . . . . 31

2.5.1 Sample Preparation . . . . . . . . . . . . . . . . . 31

2.5.2 NMR experiments . . . . . . . . . . . . . . . . 31

2.5 .3 Data Analysis . . . . . . . . . . . . . . . . . . . . . . 31

3 Protein Folding: Application of E-CPMG to gpW Protein 33

3.1 Introduction . . . . . . . . . . . . . . . . . . . . . 33

3.2 Results and Discussion . . . . . . . . . . . . . . . 36

3.2 .1 Backbone E-CPMG Experiments . . . . . . . . . . . . . 36

3.2.2 Methyl Side-chain E-CPMG Experiments . . . . . . . . . . . . 55

3.3 Conclusion . . . . . . . . . . . . . . . . . . . 65

3.4 Materials and Methods . . . . . . . . . . . . . . . . . . 68

3.4.1 Sample preparation . . . . . . . . . . . . . 68

3.4 .2 NMR experiments . . . . . . . . . . . . . . . . . . 68

3.4 .3 Data Analysis . . . . . . . . . . . . . . . . . . . 70

4 Shuttle Relaxometry: Application to study sub- $\tau_{\mathrm{c}}$ motions $\quad 73$

4.1 Introduction . . . . . . . . . . . . . . . . . . . . 73

4.2 Results and Discussion . . . . . . . . . . . . . . . . . . . 81 
4.2 .1 Relaxometry . . . . . . . . . . . . . . . . . . 81

4.2 .2 System performance . . . . . . . . . . . . . . . . . . . . . . . . . . . .

4.3 Conclusion . . . . . . . . . . . . . . . . . . . . . . 102

4.4 Material and Methods . . . . . . . . . . . . . . . . . . . 104

4.4 .1 Sample Preparation . . . . . . . . . . . . . . . 104

4.4 .2 NMR Measurements . . . . . . . . . . . . . . . . . . . . 104

4.4 .3 Data Analysis . . . . . . . . . . . . . . . . . . . . . . . . . . . . . . . . . . . . . .

$\begin{array}{ll}\text { Appendices } & 107\end{array}$

$\begin{array}{ll}\text { A gpW Relaxation Dispersion } & 107\end{array}$

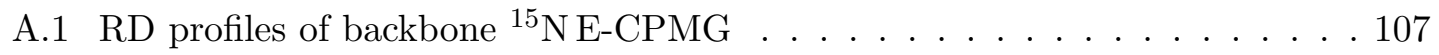

A.2 RD profiles of backbone ${ }^{1} \mathrm{HE}$ E-CPMG . . . . . . . . . . . . . . . 118

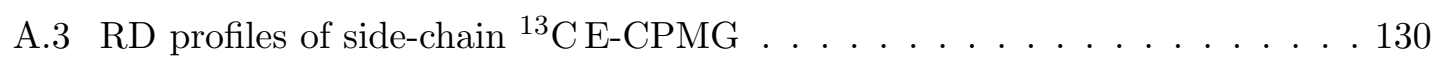

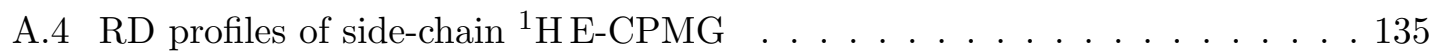

B Input for Model Free Analysis $\quad 139$

B.1 $300 \mathrm{MHz} \ldots \ldots \ldots \ldots \ldots$

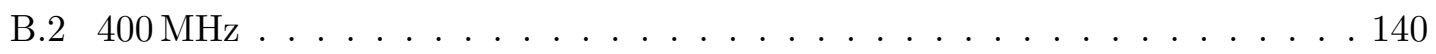

B.3 $600 \mathrm{MHz} \ldots \ldots \ldots \ldots \ldots \ldots \ldots \ldots$

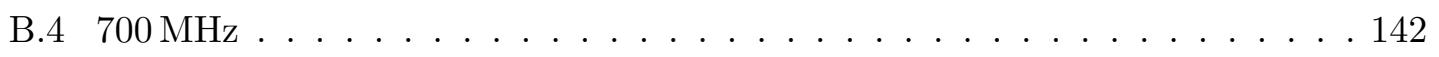

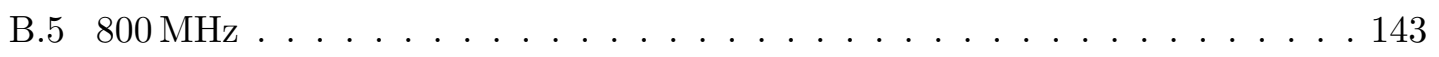

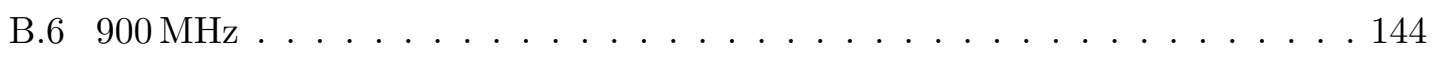

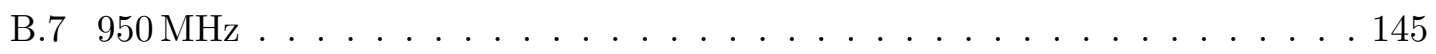

$\begin{array}{lr}\text { C Pulse Programs } & 147\end{array}$

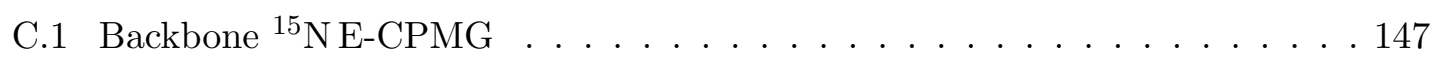

C.2 Backbone ${ }^{1} \mathrm{HE}$ E-CPMG . . . . . . . . . . . . . . . . . . . . . . . 152

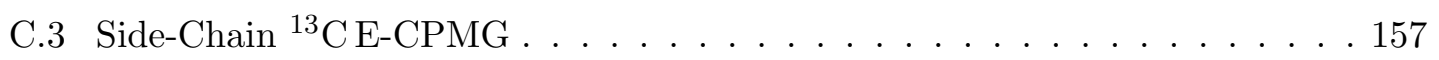

C.4 Side-Chain ${ }^{1} \mathrm{HE}$ E-CPMG . . . . . . . . . . . . . . . . . . . . . 162

C.5 Shuttle relaxometry . . . . . . . . . . . . . . . 166 


\section{List of Figures}

1.1 Scheme of the NMR time scale $\ldots \ldots \ldots \ldots \ldots$

2.1 Scheme of chemical exchange effects on the NMR signal. . . . . . . . . . 16

2.2 Simulated relaxation dispersion profile using the Luz-Meiboom model in the conventional CPMG range. . . . . . . . . . . . . . . . 21

2.3 Pulse sequence of the ${ }^{15} \mathrm{~N}$ E-CPMG experiment . . . . . . . . . . . 25

2.4 Detailed scheme of the CPMG block. . . . . . . . . . . . . . . 26

2.5 Slices from ${ }^{15} \mathrm{NE}-\mathrm{CPMG}$ experiments at different refocusing frequencies

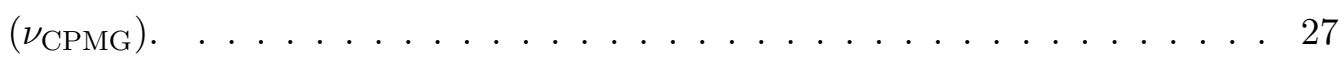

2.6 Comparison of conventional and E-CPMG fit results. . . . . . . . . . . . 28

3.1 Amino acid sequence of the 62 -residue gpW protein. . . . . . . . . . . . 33

3.2 Structure of 62 -residue gpW protein. . . . . . . . . . . . . . . . . 33

3.3 Relaxation dispersion profiles obtained from ${ }^{15} \mathrm{NE}-\mathrm{CPMG}$ with individually fitted exchange model at $275 \mathrm{~K} \ldots \ldots \ldots$. . . . . . . . . . . 36

3.4 Exchange rates of individually fitted residues from ${ }^{15} \mathrm{NE}-\mathrm{CPMG}$ at $275 \mathrm{~K} . \quad 37$

3.5 Comparison of individually and globally fitted two-state exchange model at $275 \mathrm{~K}$ for residues in the $\beta$-hairpin region. . . . . . . . . . . . . . 38

3.6 ${ }^{15} \mathrm{~N}$ E-CPMG RD profiles of backbone amide nitrogen from residues in the $\alpha$-helical region at $275 \mathrm{~K}$ indicating partial quenching of exchange contribution. . . . . . . . . . . . . . . . . . . 39

3.7 Comparison of ${ }^{15} \mathrm{NE}$-CPMG RD profiles from $\alpha$-helical residues at $263 \mathrm{~K}$

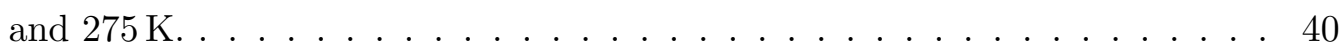

3.8 RD profiles obtained from ${ }^{15} \mathrm{NE}$-CPMG with individually fitted two and three-site exchange model at $263 \mathrm{~K}$ for residues in $\alpha$-helices. . . . . . . . . . 41

3.9 Comparison of the two- and three-site exchange model by corrected Akaike information criterion $(\mathrm{AICc}) \ldots \ldots \ldots . \ldots . \ldots 43$

3.10 Exchange rates obtained from ${ }^{15} \mathrm{~N}$ E-CPMG experiments at $263 \mathrm{~K} . \quad \ldots . . \quad .44$

3.11 Comparison of individually and globally fitted three-state exchange model

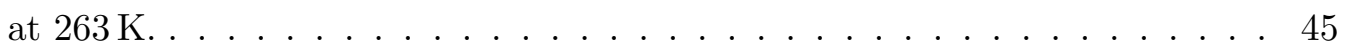

3.12 Relaxation dispersion profiles obtained from ${ }^{1} \mathrm{HE}$-CPMG with individually fitted exchange model at $263 \mathrm{~K} \ldots \ldots$. . . . . . . . . . . . 47

3.13 Exchange rates obtained from ${ }^{1} \mathrm{HE}$-CPMG experiments at $263 \mathrm{~K} . \ldots . . \quad 48$

3.14 Temperature dependent backbone E-CPMG relaxation dispersion profiles of residues in the $\beta$-hairpin region. . . . . . . . . . . . . . 50 
3.15 Temperature dependent ${ }^{1} \mathrm{HE}$-CPMG relaxation dispersion profiles of selected residues in the $\alpha$-helices. . . . . . . . . . . . . . . . . . 51

3.16 Arrhenius analysis of globally fitted exchange rates from gpW backbone E-CPMG data. . . . . . . . . . . . . . . . . . . . 5 52

3.17 Structure of gpW with highlighted backbone residues showing relaxation

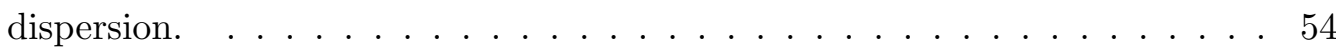

3.18 Relaxation dispersion profiles obtained from ${ }^{13} \mathrm{C}$ E-CPMG with individually fitted exchange model at $275 \mathrm{~K}$ of methyl side-chains. . . . . . . . . . . 55

3.19 Exchange rates from individually fitted ${ }^{13} \mathrm{C} \mathrm{E}-\mathrm{CPMG}$ data at $275 \mathrm{~K} . \quad \ldots .56$

3.20 Comparison of individually and globally fitted ${ }^{13} \mathrm{C}$ RD data at $275 \mathrm{~K}$. . . . 57

3.21 Comparison of ${ }^{13} \mathrm{C}$ relaxation dispersion data of methyl groups in the same residue at $275 \mathrm{~K} \ldots \ldots \ldots \ldots \ldots \ldots$

3.22 Relaxation dispersion profiles obtained from ${ }^{1} \mathrm{HE}-\mathrm{CPMG}$ with individually fitted exchange model at $275 \mathrm{~K} \ldots \ldots$. . . . . . . . . . . 6 60

3.23 Exchange rates from individual fitting of methyl ${ }^{1} \mathrm{HE}$ E-CPMG data at $275 \mathrm{~K} .61$

3.24 Comparison of individually and globally fitted ${ }^{1} \mathrm{H}$ RD profiles at $275 \mathrm{~K}$. . . 61

3.25 Temperature dependent side-chain relaxation dispersion profiles. . . . . . . 62

3.26 Arrhenius analysis of globally fitted exchange rates from gpW side-chain E-CPMG data. . . . . . . . . . . . . . . . . . . . 6 63

3.27 Structure of gpW with highlighted methyl groups showing relaxation dis-

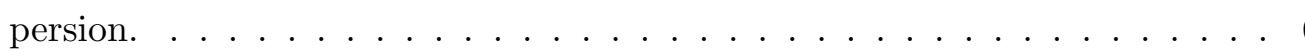

4.1 Comparison of $R_{1}$ at low $\left(\omega_{H}=21 \mathrm{MHz}, B_{0}=0.5 \mathrm{~T}\right)$ and high $\left(\omega_{H}=\right.$ $\left.950 \mathrm{MHz}, B_{0}=22.3 \mathrm{~T}\right)$ magnetic field strengths. $\ldots \ldots \ldots . \ldots 77$

4.2 Simulated frequency dependent spectral density profiles of Brownian and Fractional Brownian Dynamics. . . . . . . . . . . . . . . 79

4.3 Stray field profile of the $700 \mathrm{MHz}(16.44 \mathrm{~T})$ Oxford magnet dependent on the motor position. . . . . . . . . . . . . . . 81

4.4 Scheme of the pulse sequence for shuttle relaxometry experiments. . . . . . 82

4.5 Comparison of $2 \mathrm{D}$ planes of ubiquitin from static and shuttle pseudo-3D

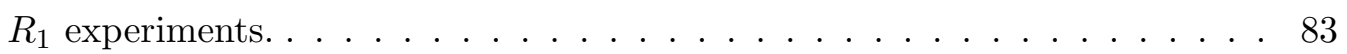

4.6 Shuttle relaxometry data of residue Isoleucine $30 \ldots \ldots$. . . . . . . . . 84

4.7 Shuttle Relaxometry data of Threonine 9 at $0.5 \mathrm{~T}$. . . . . . . . . . . 85

4.8 Field dependence of $R_{1}$ derived by shuttle relaxometry. . . . . . . . . . 86

4.9 Individual fit results of $\tau_{\mathrm{c}}$ and $S^{2}$ by shuttle relaxometry data. . . . . . . 87

4.10 Results from repeated shuttle experiments in the very low field strength

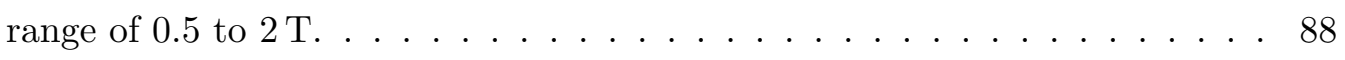

4.11 Comparison of shuttle and static relaxometry measurement. . . . . . . . . 89

4.12 Results from individual fit of $\tau_{\mathrm{c}}$ and $S^{2}$ from static relaxometry measurement. 90

4.13 Model free analysis for Lipari-Szabo Order Parameter at different static magnetic field strengths. . . . . . . . . . . . . . . . . . 91

4.14 Comparison of the intensity of the lock signal during a conventional HSQC and shuttle experiment. . . . . . . . . . . . . . . 92 
4.15 Scheme of the pulse sequence for optimization of the stabilization delay $\left(\tau_{\mathrm{st}}\right) .94$

4.16 FIDs from the $\tau_{\text {st }}$ optimization experiment. . . . . . . . . . . . . . 95

4.17 Example frames of the motor wheel motion recorded with a high-speed camera. . . . . . . . . . . . . . . . . . . . . . 98

A.1 Temperature dependent ${ }^{15} \mathrm{~N}$ relaxation dispersion profiles of gpW backbone. 112

A.2 Temperature dependent ${ }^{1} \mathrm{H}$ relaxation dispersion profiles of gpW backbone. 122

A.3 Temperature dependent ${ }^{13} \mathrm{C}$ relaxation dispersion profiles of gpW methyl side-chains. . . . . . . . . . . . . . . . . . . . . 131

A.4 Temperature dependent ${ }^{1} \mathrm{H}$ relaxation dispersion profiles of gpW methyl side-chains. . . . . . . . . . . . . . . . . . 136 


\section{List of Tables}

2.1 Comparison of individual fit results obtained from E-CPMG and conventional CPMG data sets at $275 \mathrm{~K} \ldots \ldots \ldots$. . . . . . . . . . 29

3.1 Exchange rates $k_{\text {ex }}$ obtained from globally fitted E-CPMG data. $\ldots . . .666$

4.1 Shuttle time ( $\tau_{\text {up }}$ and $\left.\tau_{\text {down }}\right)$ to different field strength position in the stray field. . . . . . . . . . . . . . . . . . . . 8 81

4.2 Model free analysis results of the correlation time at different magnetic field

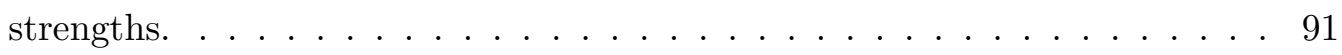

4.3 Results from the frame-wise analysis of the shuttle experiment at $0.5 \mathrm{~T}$. . . 99

4.4 Results from the frame-wise analysis of the shuttle experiment at 4.5 T. . . 100

4.5 Results from the frame-wise analysis of the shuttle experiment at $12 \mathrm{~T}$. . . 101

4.6 Field Strength and Shuttle delay for the motor positions. . . . . . . . . . . 104

A.1 Individual fit parameters of the slow exchange process from backbone ${ }^{15} \mathrm{NE}-$ CPMG data. . . . . . . . . . . . . . . . . . . . . . . 113

A.2 Individual fit parameters of the fast exchange process from backbone ${ }^{15} \mathrm{NE}-$ CPMG data. . . . . . . . . . . . . . . . . . . . . . . 115

A.3 Individual fit parameters of the slow exchange process from backbone ${ }^{1} \mathrm{HE}$ CPMG data. . . . . . . . . . . . . . . . . . . . . . . . 123

A.4 Individual fit parameters of the fast exchange process from backbone ${ }^{1} \mathrm{HE}-$ CPMG data. . . . . . . . . . . . . . . . . . . . . . 126

A.5 Individual fit parameters of the slow exchange process from side-chain ${ }^{13} \mathrm{C}$ E-CPMG data. . . . . . . . . . . . . . . . . . . . . 132

A.6 Individual fit parameters of the slow exchange process from side-chain ${ }^{1} \mathrm{HE}$ CPMG data. . . . . . . . . . . . . . . . . . . . . 137

B.1 $R_{1}, R_{2}$ and het NOE data measured on a $300 \mathrm{MHz}$ spectrometer at $294.5 \mathrm{~K} .139$

B.2 $R_{1}, R_{2}$ and het $N O E$ data measured on a $400 \mathrm{MHz}$ spectrometer at $294.5 \mathrm{~K} .140$

B.3 $R_{1}, R_{2}$ and het NOE data measured on a $600 \mathrm{MHz}$ spectrometer at $294.5 \mathrm{~K} .141$

B.4 $R_{1}, R_{2}$ and het NOE data measured on a $700 \mathrm{MHz}$ spectrometer at $294.5 \mathrm{~K} .142$

B.5 $R_{1}, R_{2}$ and het NOE data measured on a $800 \mathrm{MHz}$ spectrometer at $294.5 \mathrm{~K} .143$

B.6 $R_{1}, R_{2}$ and het NOE data measured on a $900 \mathrm{MHz}$ spectrometer at $294.5 \mathrm{~K} .144$

B.7 $R_{1}, R_{2}$ and het NOE data measured on a $950 \mathrm{MHz}$ spectrometer at $294.5 \mathrm{~K} .145$ 


\section{Chapter 1}

\section{Introduction}

\subsection{Protein Structure and Motions}

The structure and dynamics of a protein in solution are key factors in its functionality in biological processes, like molecular recognition, ligand binding or folding/unfolding. Therefore, knowledge about structure and dynamics, as well as protein kinetics, are essential to understand these complex processes, that are essential for life. Ultimately, this knowledge can be used to decipher the cause of protein malfunctioning on a molecular level and serves the development of new therapeutics and identification of drug targets.

Proteins consist of thousands of atoms and their connection as well as spatial arrangement define the protein structure. For a better description of their complex architecture, the overall structure of a protein is defined by sub-levels with increasing complexity. The first level defines the amino acid sequence (primary structure) of a protein. The amide bonds (peptide bonds) that form the backbone of a protein, create a permanent arrangement of amino acids. This connection of atomic bonds is described by the configuration of a protein, which is immutable over time (neglecting degradation), and forms the base of a protein structure.

Local structures of higher complexity can be formed due to intramolecular interactions between amino acids that are not sequential in the polypeptide chain, e.g. hydrogen bonds or disulphide bridges. The most prominent of these so-called secondary structure elements, are $\alpha$-helices and $\beta$-strands. These structural elements show characteristic dihedral angles in the backbone of a protein as well as characteristic patterns of hydrogen bonds. Thus, both can be distinguished by different experimental techniques, giving a first structural insight (Ramakrishnan and Ramachandran, 1965; Grosse et al., 1971; Billeter et al., 1984; Byler and Susi, 1986). Secondary structure elements are not limited to these two with clear characteristic properties. Any constraint that hinders the free reorientation of the amino acid chain can be considered, e.g. loop regions that connect two $\beta$-strands. In the absence of intramolecular interactions and steric effects the polypeptide chain can sample all spatial arrangements in a stochastic distribution without any preferred orientation, so-called random coil regions.

Interactions between side-chains of secondary structure elements can form global domains 
or cavities that work as motifs for ligand-binding and molecular recognition processes. These tertiary structure elements can be formed by multiple secondary structure elements of a single polypeptide chain. The formation of hydrophobic cores, which bury hydrophobic residues inside the structure to reduce energetically unfavorable interactions with aqueous solvent, can be named as an example (Efimov, 1979; Kellis et al., 1989). The highest complexity of protein structure is the interaction of multiple tertiary structures of polypeptide chains as sub-units in multimer complexes. The combination of secondary, tertiary and quaternary structure describes the conformation of a protein.

Investigating the structure of a protein is a first step in understanding its functionality but proteins are not rigid macromolecules and the spatial arrangement of its atoms can change over time. These time-dependent changes are caused by atomic motions and can play an important role in a protein's function. Atomic motions are restricted to their formed bonds, and thus cannot alter the configuration of a molecule, but allow changes in a proteins conformation. In proteins, with their highly complex structure, atomic motions can alter the structure in various ways. Empirically, these can be grouped into global and local motions and show a wide spread over the time scale of motions (Figure 1.1). Local motions only involve a few atoms, like methyl group rotations or even single atom vibrations. These are very fast and take place on a femtosecond to nanosecond time scale. Motions on these time scale usually do not affect the structural elements of a protein but can play an important role in its stability (Yang et al., 1997; Sabo et al., 2012). This range of motions is also defined as the sub- $\tau_{\mathrm{c}}$ window, since they are faster than the overall rotational correlation or tumbling time of a molecule $\left(\tau_{\mathrm{c}}\right) . \tau_{\mathrm{c}}$ is defined as the average time it takes a molecule to rotate by one radiant. For an approximation of $\tau_{\mathrm{c}}$ a spherical globular shape can be assumed, thus $\tau_{\mathrm{c}}$ is described by Stokes Law:

$$
\tau_{\mathrm{c}}=4 \pi \frac{\eta r^{3}}{3 k_{\mathrm{b}} T}
$$

in which, $\eta$ is the viscosity of the solvent, $r$ is the hydrophobic radius of a molecule, $k_{\mathrm{b}}$ is the Boltzmann constant and $T$ is the temperature. For small molecules $\tau_{\mathrm{c}}$ is in the order of picoseconds, while proteins show $\tau_{\mathrm{c}}$ values in the order of nanoseconds.

Motions slower than $\tau_{\mathrm{c}}$ commonly involve a larger number of atoms and are energetically more demanding than the previously described sub- $\tau_{\mathrm{c}}$ motions. Side-chain reorientation, aromatic ring flips and loop reorientation can take place on the nanosecond and microsecond time scale. These local changes in the structure can also cause changes in the global conformation of a protein (Smith et al., 2016). Furthermore, these dynamics also play a key role in the discussion of ligand binding mechanisms (induced fit and conformational selection) (Vogt and Di Cera, 2012). While in the induced fit theory (Koshland, 1958) the ligand induces a structural change in the receptor upon binding, the conformational selection theory (Monod et al., 1956) describes an equilibrium of conformational states of which only one binds the ligand. Both theories can be distinguished according to their underlying kinetics and their knowledge is beneficial in the development of drug target molecules (Ferruz and De Fabritiis, 2016). Ligand binding and protein folding processes 
are spread over a wide range of time scales (microseconds to seconds). This wide range of time scale is caused by the involvement of large number of atoms and steps.

Recent studies of Gleevec highlight the importance of knowledge about the underlying kinetics in binding processes (Agafonov et al., 2014). Gleevec is used in cancer therapy and works as an inhibitor of the Abl kinase. It could be shown that it also binds to Src kinase which shares $54 \%$ of sequence identity with $\mathrm{Abl}$ and forms a nearly identical binding pocket. Despite the similarity of the binding pockets, Gleevec binding shows a large difference in the binding affinities, which is 3000 times less for Src than for Abl. Agafonov and co-workers could show that the actual binding event is equally fast for both kinases but is followed by an induced conformational change. While both, Abl and Src, adapt this change they differ by its probability due to their kinetic differences. By this the divergence in the binding affinities of Gleevec to Src and Abl could be explained and highlights the key role of kinetics in binding events.

Different experimental approaches can be used to determine the dynamics and structure of a protein. X-ray crystallography (X-ray) is a well-established method for structure elucidation of proteins with atomic-resolution, since Kendrew et al. solved the first protein structure of myoglobin (Kendrew et al., 1958). The resolution of X-ray depends on the quality of the used crystals and their preparation can involve demanding optimization processes, which require large amounts of sample. Furthermore, the required crystallization can alter the native state of a protein, due to crystal packing forces. In recent years, CryoEM gained popularity as method for structure elucidation due to higher resolution, less amount of sample and lack of rigorous sample preparation compared to X-ray crystallography (Böttcher et al., 1997). The resolution of Cryo-EM depends on several factors, mainly determined by the used instrumentation and samples are prepared by directly freezing the sample from solution using liquid nitrogen. The latter not only provides a simplified sample preparation compared to X-ray crystals but also yields structural information that could be closer to the native state of a protein in solution. Nevertheless, both methods require a sample preparation that can cause deviations in the protein structure compared to its native state in solution. Furthermore, large amplitude motions could be quenched by steric hindrance in the solid phase and low populated conformational (excited) states might not be observable using these methods. While both methods can be used to report qualitative about protein dynamics (Ringe and Petsko, 1985; Bonomi et al., 2018), they still lack quantitative information about the time-dependent changes in a protein structure. As it was shown with the example of Gleevec, information about the kinetics might be crucial, to fully understand the functionality of a protein. Thus, methods to study the proteins in their natural environment are desirable. Here, Nuclear Magnetic Resonance (NMR) spectroscopy is a unique method in the study of proteins, since it provides the ability to study the structure as well as dynamic and kinetic properties of a protein, yet under physiological conditions. 


\subsection{Protein NMR}

In the following the basics of NMR will be briefly introduced following the introduction of Cavanagh et al. For a detailed description of NMR theory, the reader is referred to textbooks in the literature (Cavanagh et al., 2007).

\subsubsection{NMR Basic Concepts}

NMR spectroscopy makes use of the alignment of the spin angular momentum vector:

$$
|\vec{I}|=\hbar \sqrt{I(I+1)}
$$

in which, $I$ is the is the nuclear spin angular momentum quantum number in an external magnetic field. According to quantum mechanics only one of the vector components together with $|\vec{I}|$ can be determined. Since spins align parallel to the external field axis, the corresponding z-component of $|\vec{I}|$ is quantized by:

$$
I_{\mathrm{z}}=\hbar m_{\mathrm{I}}
$$

in which, $\hbar$ is the reduced Planck constant and $m_{\mathrm{I}}$ is the magnetic quantum number. The latter can take the values $(I, I-1, \ldots,-I+1,-I)$, where $I$ is the spin angular momentum quantum number. For nuclei commonly used in protein NMR (e.g. ${ }^{1} \mathrm{H},{ }^{13} \mathrm{C},{ }^{15} \mathrm{~N}$ and ${ }^{19} \mathrm{~F}$ ) the spin quantum number equals $1 / 2$ and the discussion will be limited to these nuclei. The secondary spin quantum number of $1 / 2$ leads to two distinct orientations of the nuclear spin, spin up $\left(\alpha\right.$-spin, $\left.\left(m_{\mathrm{I}}=+1 / 2\right)\right)$ and spin down $\left(\beta\right.$-spin $\left.\left(m_{\mathrm{I}}=-1 / 2\right)\right)$, where up and down correspond to a parallel and anti-parallel orientation to the external field, respectively. According to their spin quantum number, the two different orientations have different magnetic moments $\left(\mu_{\mathrm{z}}\right)$ :

$$
\begin{aligned}
\mu_{\mathrm{z}} & =\gamma I_{\mathrm{z}}, \\
& =\gamma \hbar m_{\mathrm{I}},
\end{aligned}
$$

in which, $\gamma$, a nucleus characteristic property, is the gyromagnetic ratio. As the secondary spin quantum number, the magnetic moment is also quantized, which results in different energies for the two distinct orientations also called Zeeman levels:

$$
E_{ \pm}= \pm \frac{1}{2} \hbar \gamma B_{0}
$$

in which, $B_{0}$ is the static magnetic field strength. In thermal equilibrium the relative population of the two states are described by the Boltzmann distribution:

$$
\frac{N_{m_{\mathrm{I}}}}{N} \approx \frac{1}{2 I+1}\left(1+\frac{m_{\mathrm{I}} \hbar \gamma B_{0}}{k_{\mathrm{b}} T}\right)
$$

in which, $N$ is the total number of spins, $N_{m_{\mathrm{I}}}$ is the number of spins in state $m_{\mathrm{I}}, k_{\mathrm{b}}$ is the Boltzmann constant and $T$ is the temperature. In thermal equilibrium the two states 
have different populations, due to the energetically preferred orientation parallel to the external field vector. This results in a net or bulk magnetization $\left(M_{0}\right)$ parallel to the z-axis (longitudinal magnetization) of the magnet field:

$$
\begin{aligned}
M_{0} & =\gamma \hbar \sum_{m_{\mathrm{I}}=-I}^{I} m_{\mathrm{I}} N_{\mathrm{m}} \\
& \approx N I(I+1) \frac{\gamma^{2} \hbar^{2} B_{0}}{3 k_{\mathrm{b}} T} .
\end{aligned}
$$

As it can be seen, $M_{0}$ depends on the gyromagnetic ratio of a nuclei $(\gamma)$, the external magnet field strength $\left(B_{0}\right)$ and the temperature $(T)$. For ${ }^{1} \mathrm{H}$ at room temperature and a magnetic field strength of $11.7 \mathrm{~T}$ (Larmor frequency of $500 \mathrm{MHz}$ ) the difference between the populations is roughly $10^{-4}$. Thus, only every 10000 th spin contributes to the detectable NMR signal, making NMR a relatively insensitive spectroscopic method.

Since the equilibrium populations are described by a Boltzmann distribution, the resulting net magnetization is also called Boltzmann or equilibrium magnetization. During an NMR experiment the equilibrium magnetization is disturbed by short radio frequency (RF) pulses that match the energy difference between the two Zeeman levels:

$$
\omega=\Delta E / \hbar=\gamma B_{0},
$$

in which, $\omega$ is the frequency of the RF pulse and $\Delta E$ is the energy difference:

$$
\Delta E=\hbar \gamma B_{0}
$$

In short, the effective field created by the RF pulses tilts the equilibrium magnetization vector towards the $\mathrm{x}-\mathrm{y}$ plane (transverse magnetization), where the magnetization starts to precess around the z-axis. The time dependent changes of the transverse magnetization induced by this precession motion can be described by the Bloch equation:

$$
\frac{d M_{\mathrm{i}}}{d t}=\gamma\left(\vec{M} \times \vec{B}_{0} \vec{e}_{\mathrm{z}}\right)_{\mathrm{i}}
$$

in which, $\vec{M}$ is the magnetization vector, $\vec{B}_{0}$ is the external field vector, $\vec{e}_{\mathrm{z}}$ is the unity vector in z-direction and i denotes the cartesian coordinates $(\mathrm{x}, \mathrm{y}, \mathrm{z})$. The solution of the Bloch equations are classic oscillator functions:

$$
\begin{aligned}
& M_{\mathrm{x}}(t)=M_{\mathrm{x}}(0) \cos \left(\omega_{0} t\right)-M_{\mathrm{y}}(0) \sin \left(\omega_{0} t\right) \\
& M_{\mathrm{y}}(t)=M_{\mathrm{x}}(0) \sin \left(\omega_{0} t\right)+M_{\mathrm{y}}(0) \cos \left(\omega_{0} t\right) .
\end{aligned}
$$

in which, $\omega_{0}$ is the angular frequency of the precession motion:

$$
\omega_{0}=-\gamma B_{0}
$$

This frequency depends on the gyromagnetic ratio $(\gamma)$ and thus is nucleus characteristic and referred to as Larmor frequency of a nucleus. Furthermore, it depends on the 
magnetic field $\left(B_{0}\right)$ that acts on the nucleus. Nuclei with the same gyromagnetic ratio still show differences in their Larmor frequency, which are caused by differences in the magnetic field that acts on a nucleus. These differences are induced by different chemical environments that influence the electronic surrounding of a nucleus. Thus, the term $B_{0}$ can be substituted by an effective magnetic field term that acts on the nucleus:

$$
\begin{gathered}
\omega=-\gamma B_{\mathrm{eff}} \\
B_{\mathrm{eff}}=B_{0}(1-\sigma),
\end{gathered}
$$

in which, $B_{0}$ is the applied external field strengths and $\sigma$ is a shielding term that either increases or decreases the effective field. This shielding term arises from the fact that electron motions in a magnetic field induce secondary magnetic fields. These fields induce a local change in the field strength and highly depend on the chemical environment of a nucleus. Thus, identical nuclei can be distinguished by changes in their Larmor frequency, according to their chemical environment. These changes are in the order of $10^{-6}$, i.e. ppm, in respect to the Larmor frequency and are the informative part in an NMR spectrum. Thus, the coordinate system of the Bloch formalism is transferred to a rotating frame system, such that the difference:

$$
\Omega=\omega-\omega_{0},
$$

describes the precession of individual nuclear spins. Since $\Omega$ depends on the chemical environment, it is referred to as chemical shift of a nucleus. Relaxation of the transverse magnetization prevents that this precession motion around the z-axis continues forever and is as crucial for NMR spectroscopy as the chemical shift.

Relaxation in general describes the transition of a system from an excited state back to its equilibrium state. Different relaxation pathways exist that cause the decay of nonequilibrium magnetization, of which two relaxation rates are generally important for NMR experiments. Longitudinal relaxation or spin-lattice relaxation $\left(R_{1}\right)$ describes the transition of non-equilibrium spin populations back to their equilibrium distribution. Thus, it determines the length of the recycle delay between the acquisition of two experiments. The transverse relaxation or spin-spin relaxation $\left(R_{2}\right)$ describes the dephasing of bulk magnetization in the $\mathrm{x}-\mathrm{y}$ plane. It directly affects the linewidth of an observed resonance and thus limits the resolution in an NMR experiments.

Relaxation in NMR bases on the coupling of nuclear spins to time-dependent local oscillating fields, which are rendered by Brownian motion of the atoms. These local fields are created by dipole-dipole (DD), chemical shift anisotropy (CSA) and quadrupole interactions. The latter only plays a role for nuclei with a spin quantum number I $>1 / 2$ and thus will be neglected in this discussion. The time-dependent changes of these fields are described by a stochastic correlation function:

$$
C(\tau)=\overline{c_{0}(t) c_{0}(t+\tau) Y_{2}^{0}[\Omega(t)] Y_{2}^{0}[\Omega(t+\tau)]}
$$


in which, $c_{0}(t)$ defines a physical interaction constant, $Y_{2}^{0}$ is a spherical harmonic function and $\Omega=(\psi(t), \phi(t))$ defines a set of polar angles that describe the orientation of a unity vector pointing in the principal direction of the interaction. Assuming a spherical molecule experiencing Brownian motion, leads to a time-independent interaction strength $\left(c_{0}(t)=c_{0}\right)$. For DD the interaction strength $\left(c_{0}=d_{\mathrm{IS}}\right)$ is defined by:

$$
d_{\mathrm{IS}}=-\sqrt{6}\left(\frac{\mu_{0}}{4 \pi}\right) \frac{\hbar \gamma_{\mathrm{I}} \gamma_{\mathrm{S}}}{r_{\mathrm{IS}}^{3}}
$$

in which, $\mu_{0}$ is the vacuum permeability, $\hbar$ is the reduced Planck constant, $r_{\text {IS }}$ is the distance between the spins and $\gamma$ is the gyromagnetic ratio of the respective spin. For $\operatorname{CSA}\left(c_{0}=c_{\mathrm{I}}\right)$ it is defined by:

$$
c_{\mathrm{I}}=\frac{\Delta \sigma \gamma_{\mathrm{I}} B_{0}}{\sqrt{3}},
$$

in which, $\Delta \sigma$ is the chemical shift anisotropy of a given nucleus.

The oscillating fields, which are created by Brownian motion, are not equally distributed over all frequency components. Their distribution is defined by the spectral density function:

$$
J(\omega)=\operatorname{Re}\left(\int_{-\infty}^{\infty} C_{00}^{2}(\tau) \exp (-i \omega \tau) d \tau\right),
$$

in which, $C_{00}^{2}$ is the orientational correlation function:

$$
C_{00}^{2}(\tau)=\frac{1}{5} \exp \left(-\tau / \tau_{\mathrm{c}}\right)
$$

following the assumption of isotropic tumbling of the molecule. The Fourier transformed of this correlation function is a Lorentzian function:

$$
J(\omega)=\frac{2}{5}\left(\frac{\tau_{\mathrm{c}}}{1+\omega^{2} \tau_{\mathrm{c}}^{2}}\right)
$$

which depends on the frequency of a motion $(\omega)$ and $\tau_{\mathrm{c}}$. Relaxation rates are described by a sum of Lorentzian functions at the respective eigenfrequencies of the spin system and the corresponding interaction strength of the relaxation mechanism. Equation (1.24) is the simplest version of the spectral density function, since it only involves the overall tumbling of a molecule. Relaxation rates are also affected by local dynamics that are faster than $\tau_{\mathrm{c}}$ and these can also be included in $J(\omega)$. The different forms of the spectral density function as well as the two mentioned relaxation rates $\left(R_{1}\right.$ and $\left.R_{2}\right)$ will be further discussed in Chapter 4.

As already mentioned, the power of NMR lies in the ability of measuring structural as well as dynamic and kinetic properties of molecules under physiological conditions. A hint of how this information is entangled in the NMR parameters was already given by a brief explanation of the chemical shift and the concept of relaxation. In the following, a few examples of structural and dynamical information obtainable by NMR parameters will be given. 


\subsubsection{Structural Insights by NMR}

Structural information of a molecule is encoded into various NMR parameters, of which chemical shift, scalar couplings and residual dipolar couplings are commonly used for structure elucidation. As described above, in the rotating frame transverse magnetization precesses around the z-axis with a nucleus characteristic frequency, the chemical shift $(\Omega$, eq. 1.18). $\Omega$ is highly determined by its close-by chemical environment and functional groups give rise to a characteristic shift in the precession frequency. Thus, it yields information about the close chemical surrounding of a nucleus. In protein NMR the secondary chemical shift (SCS) $\Delta \delta$ is defined as:

$$
\Delta \delta=\delta_{\mathrm{obs}}-\delta_{\mathrm{rc}}
$$

where $\delta_{\text {obs }}$ and $\delta_{\text {rc }}$ are the observed and random coil chemical shift of a protein. SCS allows the approximation of secondary structure elements in a protein, based on the characteristic shifts in the observed resonances. Commonly $\mathrm{C}_{\alpha}$ and $\mathrm{C}_{\beta}$ secondary shifts are compared, due to their larger sensitivity to structural changes. A positive (negative) $\mathrm{C}_{\alpha}$ and negative (positive) $\mathrm{C}_{\beta} \Delta \delta$ indicate an $\alpha$-helical ( $\beta$-strand) structure.

Direct interactions of the nuclear spins cause a splitting in the energy levels and by that also the appearance of the resonances in the spectrum. This so-called scalar coupling $\left({ }^{n} J-\right.$ coupling) is a spin-spin interaction along $n$ chemical bonds of a molecule and is usually detectable up to an $\mathrm{n}$ of 1 to 4 . In protein NMR the strength of ${ }^{3} J$-couplings is described by the empirical Karplus equation:

$$
J(\phi)=A \cos (2 \phi)+B \cos (2 \phi)+C,
$$

in which, $\phi$ is the dihedral backbone angle and A, B, C are empirically estimated parameters. Using this correlation $\phi$ can be estimated by measuring ${ }^{3} J_{\text {HAHN }}$ couplings. The secondary structure of a protein backbone can be estimated according to a Ramachandran plot, which plots the dihedral angles ( $\psi$ vs. $\phi)$ and defines characteristic areas in the plot for $\alpha$-helices and $\beta$-strands (Ramakrishnan and Ramachandran, 1965).

Distance information in proteins can be obtained by relaxation experiments utilizing the Nuclear-Overhauser-Effect (NOE) or residual dipolar couplings (RDCs). Both are based on dipole-dipole interactions between nuclear magnetic moments. The strength of this interaction is reciprocally proportional to the distance $r$ between the two dipole moments (Equation (1.20)) and declines rapidly with increasing $r$ because $d_{\mathrm{IS}}^{2} \propto r^{-6}$.

\subsubsection{The NMR Time Scale}

Since molecules are dynamic in solution, NMR structure elucidation provides an ensemble of structures, which can represent the flexibility of a molecule. In principle all NMR observables are time-averaged and include dynamic information. The chemical shift of a 
nucleus for example can be different in two exchanging conformations of a protein and depending on the time scale of the interchange only an averaged shift is observable (Section 2.1). The same holds for RDC and NOE, in which the distance between the dipoles is affected by atomic motions and therefore also is time-dependent $(r(t))$. Indeed, the observation of these dynamic processes is the unique strength of NMR spectroscopy.

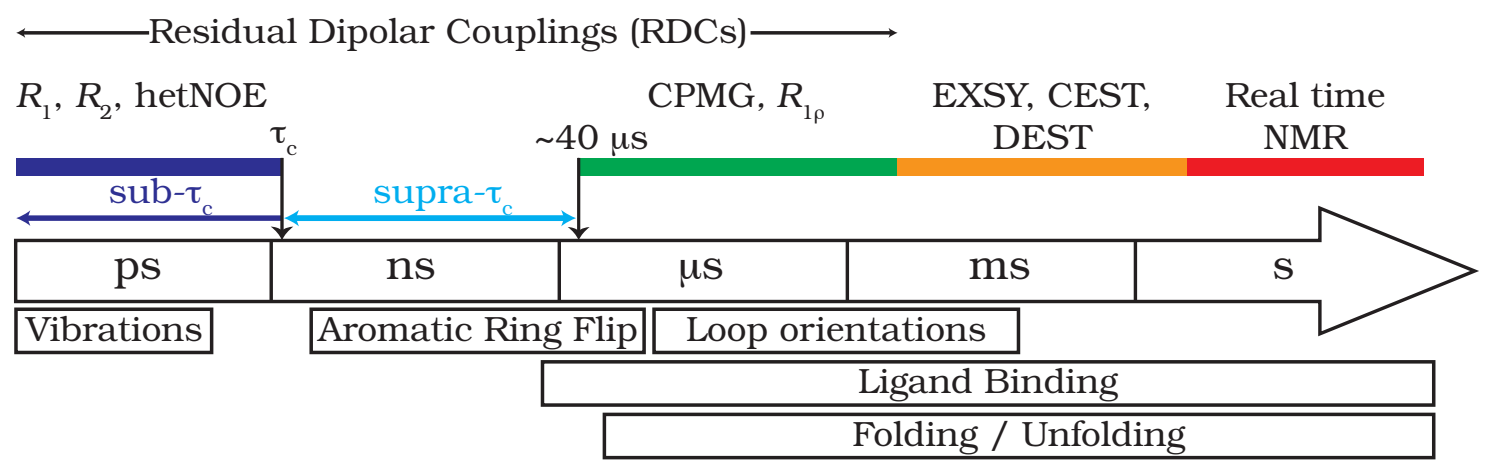

Side Chain Rotation and Reorientation

Figure 1.1: Scheme of the NMR time scale. The scheme shows the spread of protein motions and its coverage by NMR experiments. The sub- and supra- $\tau_{\mathrm{c}}$ windows are highlighted, where $\tau_{\mathrm{c}}$ is the overall rotational correlation time of a molecule. Colored boxes present the typical range of the protein motions but do not indicate their upper and lower limits. The indicated limit of the supra- $\tau_{\mathrm{c}}$ window $(\sim 40 \mu \mathrm{s})$ is connected to experimental limitations in conventional CPMG and $R_{1 \rho}$ experiments. Recent developments in these experiments lowered this limit to single-digit $\mu$ s motions and point out that it can be further reduced (Ban et al., 2012; Smith et al., 2016; Trigo-Mourino et al., 2017; Reddy et al., 2018).

Having a look at the spread of NMR experiments on the protein motion time scale (Figure 1.1), explains the popularity and power of NMR in studying protein dynamics. Similar to the large variety of protein motions, NMR experiments cover the time scale of picoseconds up to seconds.

Protein motions taking place in the sub- $\tau_{\mathrm{c}}$ window up to the correlation time $\left(\tau_{\mathrm{c}}\right)$ of a protein (fs - ns), modulate the intrinsic relaxation of a nuclear spin due to their effects on dipole-dipole interactions and perturbations in the electronic environment of a nucleus. These interactions cause local magnetic fields, which fluctuate due to Brownian motion of the atoms, as it was discussed above. The fluctuations of these fields are too fast in comparison to the chemical shift of a nucleus and thus can only be detected as a time-averaged contribution to the intrinsic relaxation rates. The study of these motions is targeted by longitudinal $\left(R_{1}\right)$ and transverse $\left(R_{2}\right)$ relaxation experiments, as well as heteronuclear NOE (hetNOE) experiments (Farrow et al., 1994). The extraction of local motion parameters of proteins by these relaxation experiments will be in focus of Chapter 4 .

Between $\tau_{\mathrm{c}}$ of a protein and the near microsecond time scale, NMR experiments cannot directly cover protein kinetics. Nevertheless, NMR can provide dynamic information in this range by residual dipolar couplings (RDCs) (Lange et al., 2008). Dipolar couplings depend on the orientation of the dipole vector in respect to the external field. This orien- 
tation is dynamically changed due to the atomic motions and thus can include dynamic information. In solution, dipolar couplings are averaged out, due to the isotropic tumbling of a molecule. Alignment media can be used to make the otherwise isotropic orientation distribution anisotropic, largely without affecting the tumbling of the molecule. This partially retrieves the dipolar coupling:

$$
D(\psi, \phi)=D_{\mathrm{a}}\left[\left(3 \cos ^{2}(\theta)-1\right)+1.5 R \sin ^{2}(\theta) \cos (2 \phi)\right],
$$

in which, $D_{\mathrm{a}}$ is the axial component of the alignment tensor, $R$ is the rhombicity and $(\theta, \phi)$ are polar angles in the alignment frame (Tjandra and Bax, 1997; Lakomek et al., 2008). Using five different alignment media, these dynamics can modeled by an order parameter $\left(S_{\mathrm{RDC}}\right)$, which describes the amplitude of motion of the dipolar vector up to the millisecond time scale (Lange et al., 2008; Lakomek et al., 2008).

Carl-Purcell-Meiboom-Gill (CPMG) (Allerhand and Gutowsky, 1964; Allerhand et al., 1965; Allerhand and Gutowsky, 1965) and transverse-rotating frame spectroscopy $\left(R_{1 \rho}\right)$ (Akke and Palmer, 1996) cover the microsecond to millisecond range of motions. The according protein motions are independent of the molecular tumbling and do not impact the intrinsic relaxation rates. Their kinetics are in the same order of magnitude as chemical shift changes and thus create time-dependent fluctuations of the latter. These so-called relaxation dispersion (RD) experiments might be one of the most informative NMR experiments, since they contain structural, thermodynamic and kinetic information. Both experiments measure the contribution of chemical exchange to the intrinsic linewidth of a resonance, dependent on an applied radio frequency pulse. The obtained data can be fitted to analytic models, which yield information about the rate of exchange (kinetics), population of the exchanging states (thermodynamics) and chemical shift difference (structure) between the states. In recent years the development of new RD experiments pushed the limits of kinetic detection further into the supra- $\tau_{\mathrm{c}}$ realm (Ban et al., 2012; Smith et al., 2016). These techniques will be a central part of this work and will be explained in more detail in Chapter 2.

Even slower motions can be targeted by chemical exchange experiments (Forsen and Hoffman, 1963). These experiments are used to observe so-called "invisible" or "dark" states, which correspond to excited (minor) conformational states of a protein. These might be so lowly populated, that they cannot be directly observed in an NMR spectrum. Chemical and dark-state exchange-mediated transfer saturation experiments (CEST and DEST, respectively) are representative experiments in this regime of protein motions. Both exploit the fact that a disturbance of an excited state by an RF pulse also affects the observable resonance. The underlying exchange process must happen in the order of ms to be targeted by these experiments.

Protein motions that occur in the order of seconds can exceed the length of conventional NMR acquisition (milliseconds to seconds). Here, so-called real time NMR techniques can be used to study such slow kinetics like for example protein folding or ligand binding. In real time NMR consecutive experiments are acquired to follow chemical shift or peak intensity changes upon binding or folding processes. The time resolution of these methods 
depends on the length of the experiment, thus 1D and more dimensional experiments are commonly used to cover motions in the order of seconds and minutes, respectively (Zeeb and Balbach, 2004; Haupt et al., 2011).

\subsection{Thesis Outline}

In this work recent developments in NMR relaxation experiments will be applied to study protein motions. The thesis is split into two parts:

The first part focuses on the study of protein motions close to the supra- $\tau_{\mathrm{c}}$ window by relaxation dispersion experiments ( $R_{1 \rho}$ and $\left.\mathrm{CPMG}\right)$.

The second part of the thesis focuses on motions faster than the overall correlation time $\left(\tau_{\mathrm{c}}\right)$, which are accessible by $R_{1}, R_{2}$ and het $N O E$ experiments.

The results and discussions of this work are presented in three chapters:

Chapter 2 introduces the differences between the conventional relaxation dispersion experiments (CPMG and $R_{1 \rho}$ ) and presents the extreme CPMG (E-CPMG) experiment. This method combines the time scales of both RD experiments and improves the accuracy of CPMG type data for the estimation of kinetic parameters of fast chemical exchange processes.

Chapter 3 shows the results of the application of E-CPMG experiments to study chemical exchange in gpW protein in solution. Backbone and side-chain E-CPMG data will be used to highlight the roles of the structural elements in the folding process and a temperature dependent study gives insight into the energy landscape of gpW.

Chapter 4 presents the use of a motor-based sample shuttle device for relaxometry experiments in the stray-field of the NMR magnet. These experiments try to answer the question if local protein motions on the picosecond time scale alter the Lorentzian behavior of the spectral density functions $(J(\omega))$ at field strengths far below conventional field strengths available in NMR spectroscopy. 


\section{Chapter 2}

\section{High Power Relaxation Dispersion: The Extreme CPMG Experiment}

The structure of a biomolecule is defined by its configuration and conformation. The configuration describes the network of atomic bonds forming the biomolecule. These bonds are stable over time and restrict the atomic motions. While the configuration is immutable, if no external forces applied, the conformation, which describes the spatial arrangement of the atoms, is dynamic and can change over time. When one hypothetical atom exists in two different conformations of a molecule, namely $\mathrm{A}$ and $\mathrm{B}$, the changes in atomic position associated with the two states can result in different chemical environments; e.g. a terminal side-chain is exposed to the solvent in state A but is buried into a hydrophobic cleft in state B. These dynamic exchange events occur at certain kinetic rates, which are determined by the population of the two states and the free energy barrier between them. Both terms, chemical and conformational exchange, describe these processes.

As already mentioned in Chapter 1, NMR offers several experiments to investigate exchange processes. The most popular ones being exchange-mediated transfer saturation (EST) (Forsen and Hoffman, 1963) and relaxation dispersion (RD) experiments (Loria et al., 1999; Akke and Palmer, 1996). Both types of experiments exploit differences in the chemical environment caused by an exchange process, which is reflected in the chemical shift of a nuclear spin and provide structural $(\Delta \omega)$, kinetic $\left(k_{\text {ex }}\right)$ and thermodynamic information (populations) of these processes.

The chemical exchange-mediated transfer saturation (CEST) experiment (Forsen and Hoffman, 1963) is used to detect exchange processes on the millisecond time scale with skewed populations. CEST relies on the perturbation of a minor populated state by weak radio frequency pulses (Vallurupalli et al., 2012). In an exchanging system the perturbation of the minor state also affects the major state. When the RF pulse is applied on-resonance on the minor state the perturbation is partially transferred to the major state and decreases the intensity of the observed major resonance. 
Relaxation dispersion experiments (CPMG and $R_{1 \rho}$ ) aim to quench the contribution of an exchange process to the transverse relaxation rate $\left(R_{2, \text { eff }}\right)$. In exchange the chemical shifts of both states stochastically interconvert, which can cause an additional dephasing of transverse magnetization. Both experiments are closely related but use different approaches to quench the dephasing caused by chemical exchange. In CPMG experiments a train of $180^{\circ}$ pulses is applied, while $R_{1 \rho}$ uses RF pulses with varying amplitude and offset. Conventional CPMG and $R_{1 \rho}$ cover different time scales, due to their different approaches for quenching the dephasing. While CPMG experiments allow the accurate detection of exchange rates in the range of $100 \mathrm{~s}^{-1}$ to $1000 \mathrm{~s}^{-1}, R_{1 \rho}$ experiments cover exchange rates of $1000 \mathrm{~s}^{-1}$ up to the supra- $\tau_{\mathrm{c}}$ window $\left(\approx 4010^{3} \mathrm{~s}^{-1}\right)$.

The improvement of conventional CPMG based relaxation dispersion experiments, accessing faster kinetics, will be discussed in this chapter.

Next, the key concept of chemical exchange and relaxation dispersion experiments will be introduced.

\subsection{Chemical Exchange}

The phenomenon of chemical exchange will be presented in this section, following the derivation from Cavanagh et al. (Cavanagh et al., 2007).

Chemical exchange describes the chemical shift modulation of a nuclear spin involved in a time dependent exchange process. The simplest exchange process imaginable is a two-state exchange, also referred to as biomolecular reaction:

$$
\mathrm{A} \underset{k_{\mathrm{BA}}}{\stackrel{k_{\mathrm{AB}}}{\rightleftharpoons}} \mathrm{B}
$$

the exchange between the discrete states $\mathrm{A}$ and $\mathrm{B}$ is characterized by the forward, $k_{\mathrm{AB}}$, and reverse, $k_{\mathrm{BA}}$, rates. The effect on transverse magnetization is described by the McConnell equation:

$$
\frac{d M^{+}(t)}{d t}=(i \Omega-R+K) M^{+}(t)
$$

where, $M^{+}(t)$ is the time-dependent transverse magnetization, $\Omega, R$ and $K$ are the chemical shift, relaxation and exchange matrices, respectively (McConnell, 1958). These three are combined as rate matrix:

$$
-i \Omega-R+K=\left(\begin{array}{cc}
-i \Omega_{\mathrm{A}}+\rho_{\mathrm{A}}+k_{\mathrm{AB}} & -k_{\mathrm{BA}} \\
-k_{\mathrm{BA}} & -i \Omega_{\mathrm{B}}+\rho_{\mathrm{B}}+k_{\mathrm{AB}}
\end{array}\right),
$$

where $\Omega$ is the chemical shift of the respective state, $\rho$ is the transverse relaxation rate, $k_{\mathrm{AB}}$ and $k_{\mathrm{BA}}$ are the forward and reverse rate, respectively. Assuming that the intrinsic transverse relaxation rates are the same in both states $\left(R_{2,0}^{\mathrm{A}}=R_{2,0}^{\mathrm{B}}=R_{2,0}\right)$, simplifies the 
eigenvalues $\lambda$ of the rate matrix to:

$$
\lambda_{ \pm}=i \Omega-R_{2, \mathrm{eff}}
$$

in which

$$
R_{2, \mathrm{eff}}=R_{2,0}+\frac{k_{\mathrm{ex}}}{2} \pm \frac{1}{\sqrt{8}}\left(k_{\mathrm{ex}}^{2}-\Delta \omega^{2}+\left[\left(k_{\mathrm{ex}}^{2}+\Delta \omega^{2}\right)^{2}-16 p_{A} p_{B} \Delta \omega^{2} k_{\mathrm{ex}}^{2}\right]^{1 / 2}\right)^{1 / 2}
$$

and

$$
\Omega=\frac{\Omega_{\mathrm{A}}+\Omega_{\mathrm{B}}}{2} \pm \frac{1}{\sqrt{8}}\left(\Delta \omega^{2}-k_{\mathrm{ex}}^{2}+\left[\left(k_{\mathrm{ex}}^{2}+\Delta \omega^{2}\right)^{2}-16 p_{\mathrm{A}} p_{\mathrm{B}} \Delta \omega^{2} k_{\mathrm{ex}}^{2}\right]^{1 / 2}\right)^{1 / 2},
$$

are the observed transverse relaxation rate and chemical shift, respectively. $p_{\mathrm{A}}$ and $p_{\mathrm{B}}$ are the populations of the two states, $k_{\mathrm{ex}}\left(\mathrm{kex}=k_{\mathrm{AB}}+k_{\mathrm{BA}}\right)$ is the rate of exchange and $\Delta \omega$ is the chemical shift difference of the two states. The time dependent magnetization (Equation (2.2)) can be calculated using analytic solutions. Their derivations rely on specific assumptions, making them only applicable to specific exchange regimes. Here, one distinguishes between slow and fast exchange regime.

In the slow exchange limit, the rate of exchange $\left(k_{\mathrm{ex}}\right)$ is smaller than the chemical shift difference $(\Delta \omega)$ of the two states. The off-diagonal terms in Equation (2.3) can be neglected and both states are independently observable in the NMR spectrum according to

$$
\begin{aligned}
& M_{\mathrm{A}}^{+}(t)=M_{\mathrm{A}}^{+}(0) \exp \left[-\left(i \Omega_{\mathrm{A}}+R_{2,0}^{\mathrm{B}}+k_{\mathrm{AB}}\right) t\right] \\
& M_{\mathrm{B}}^{+}(t)=M_{\mathrm{B}}^{+}(0) \exp \left[-\left(i \Omega_{\mathrm{B}}+R_{2,0}^{\mathrm{B}}+k_{\mathrm{BA}}\right) t\right] .
\end{aligned}
$$

In the fast exchange limit $\left(k_{\mathrm{ex}}>\Delta \omega\right)$ an average time dependent magnetization $M^{+}(t)$ is observed:

$$
M^{+}(t)=M^{+}(0) \exp \left[-\left(i \bar{\Omega}+\bar{R}_{2,0}+p_{\mathrm{A}} p_{\mathrm{B}} \Delta \omega / k_{\mathrm{ex}}\right) t\right]
$$

in which

$$
\begin{aligned}
\bar{\Omega} & =p_{\mathrm{A}} \Delta \omega_{\mathrm{A}}+p_{\mathrm{B}} \Delta \omega_{\mathrm{B}} \\
\bar{R}_{2,0} & =p_{\mathrm{A}} R_{2,0}^{\mathrm{A}}+p_{\mathrm{B}} R_{2,0}^{\mathrm{B}},
\end{aligned}
$$

are the population averaged chemical shift and transverse relaxation rate, respectively.

Figure 2.1 shows a scheme of the effect of chemical exchange on the NMR peaks. Following this scheme, the concept of chemical exchange in NMR will be explained in more detail. Here the effect of fast and slow exchange will be discussed with respect to both, equal and unequal populations.

In the absence of chemical exchange between the states $\mathrm{A}$ and $\mathrm{B}$, both equally populated $\left(p_{\mathrm{A}}=p_{\mathrm{B}}\right)$, the NMR spectra displays (Figure 2.1, a) two peaks at respective chemical shift of each state: $\omega_{\mathrm{A}}$ and $\omega_{\mathrm{B}}$ (Equation (2.6) for $k_{\mathrm{ex}}=0$ ). The linewidth of the 

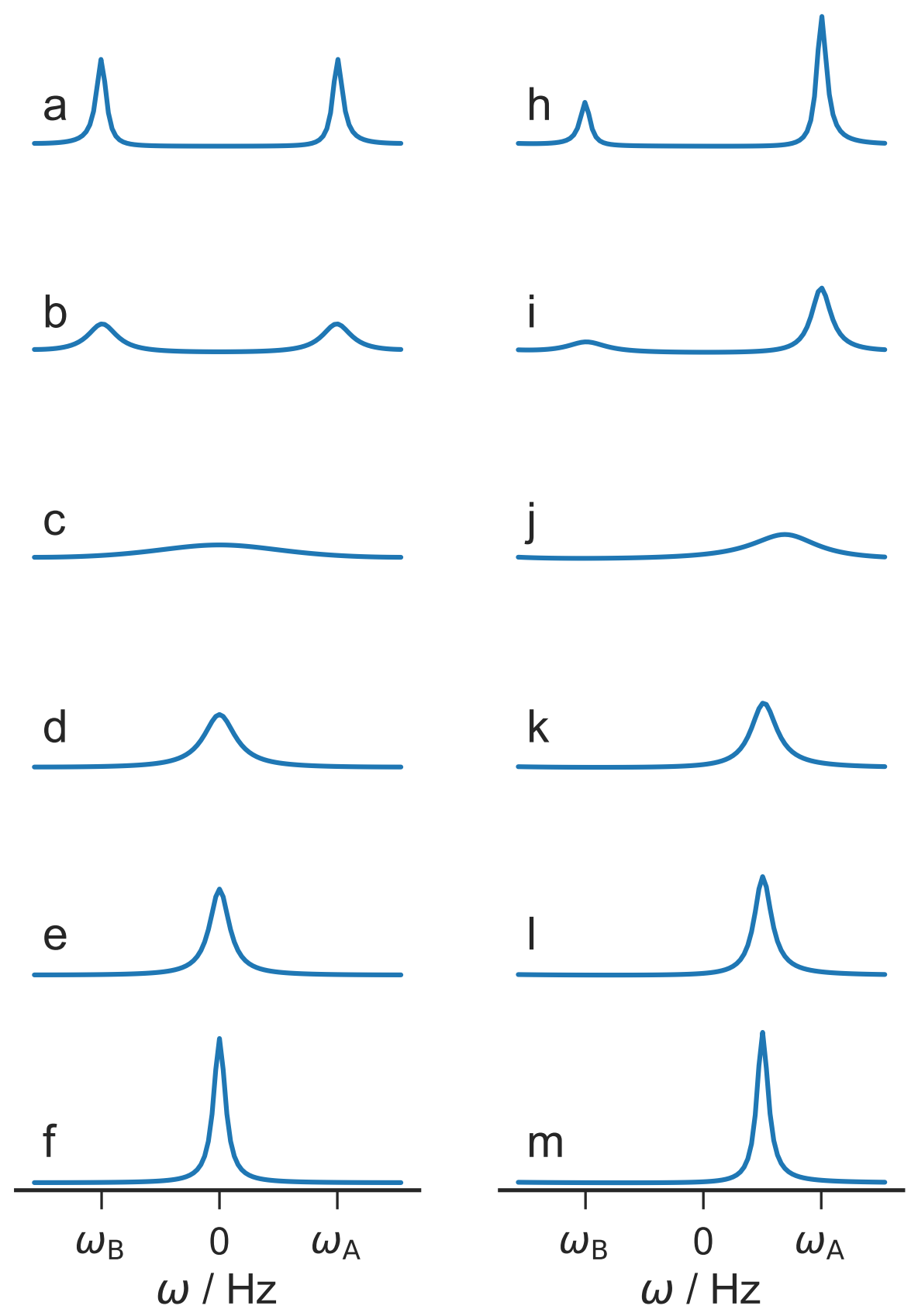

Figure 2.1: Scheme of chemical exchange effects on the NMR signal. The populations of the two states $\left(p_{\mathrm{A}}, p_{\mathrm{B}}\right)$ are equal $\left(p_{\mathrm{A}}=p_{\mathrm{B}}\right)$ in $(\mathrm{a}-\mathrm{f})$ and skewed $\left(p_{\mathrm{A}}=\right.$ $\left.4 p_{\mathrm{B}}\right)$ in $(\mathrm{g}-\mathrm{m})$. The chemical shift difference between the states was set to $\Delta \omega /(2 \pi)=$ $\left|\omega_{\mathrm{A}}-\omega_{\mathrm{B}}\right|=200 \mathrm{~Hz}$, the same intrinsic transverse relaxation rate $R_{2,0}=10 \mathrm{~s}^{-1}$ was assumed for both states and exchange rates of $0 \mathrm{~s}^{-1}$ (a, h), $20 \mathrm{~s}^{-1}$ (b, i), $200 \mathrm{~s}^{-1}$ (c, j), $900 \mathrm{~s}^{-1}(\mathrm{~d}, \mathrm{k}), 2000 \mathrm{~s}^{-1}(\mathrm{e}, \mathrm{l})$ and $10000 \mathrm{~s}^{-1}$ (f, m) were used for the simulations. 
resonances (Full Width at Half Maximum, FWHM) is defined by:

$$
F W H M=2 R_{2, \text { eff }}
$$

in which, $R_{2 \text {,eff }}$ is the effective transverse relaxation rate. Thus, the linewidth of the two peaks depends only on their intrinsic transverse relaxation rate $\left(\rho=R_{2,0}\right.$; Equation (2.5) for $\left.k_{\mathrm{ex}}=0\right)$ in the absence of chemical exchange.

Next, (Figure 2.1, b) exemplifies the spectra when the rate of exchange $\left(k_{\text {ex }}\right)$ is significantly smaller than the chemical shift difference between the states: $k_{\mathrm{ex}}<<\Delta \omega$ (slow exchange regime). Both states still evolve independently over time according to Equation (2.7) and Equation (2.8), but the exchange process causes a broadening of the peaks, due to an exchange contribution $\left(R_{\mathrm{ex}}\right)$ to the intrinsic transverse relaxation rate $\left(R_{2,0}\right)$ :

$$
R_{2, \mathrm{eff}}=R_{2,0}+R_{\mathrm{ex}}
$$

In the slow exchange regime, the exchange contribution in Equation (2.13) is defined by:

$$
R_{2, \mathrm{eff}}^{\mathrm{A}}=R_{2,0}+p_{\mathrm{B}} k_{\mathrm{ex}}
$$

Equation (2.14) shows that the linewidth of a peak is directly affected by $k_{\text {ex }}$ and the relative population of the states. Logically an increase of $k_{\text {ex }}$ leads to further broadening of the resonance. Since the integral of a peak stays the same, the peak height decreases with an increasing rate of exchange (Figure 2.1, c). This reaches its maximum at the coalescence point, the so-called intermediate exchange regime, where $k_{\mathrm{ex}} \approx \Delta \omega$. The contribution of $R_{\text {ex }}$ to the linewidth broadens the peak, such that it becomes almost invisible in the experiment (Figure 2.1, d). From this point onward the process enters the fast exchange regime, where the exchange rate becomes larger than the chemical shift difference $\left(k_{\mathrm{ex}}>\Delta \omega\right)$. In this regime the distinct information of the chemical shifts is averaged by the exchange and a single peak is observed in the spectrum, located between the chemical shifts of the two peaks (Figure 2.1, e, Equation (2.9)). Ultimately the process reaches the fast exchange limit, where $k_{\mathrm{ex}}>\Delta \omega$ (Figure 2.1, f) and the observed transverse relaxation rate is described by:

$$
R_{2, \mathrm{eff}}=p_{\mathrm{A}} R_{2,0}^{\mathrm{A}}+p_{\mathrm{B}} R_{2,0}^{\mathrm{B}}+\frac{p_{\mathrm{A}} p_{\mathrm{B}} \Delta \omega^{2}}{k_{\mathrm{ex}}}
$$

where $p_{\mathrm{A}} R_{2,0}^{\mathrm{A}}+p_{\mathrm{B}} R_{2,0}^{\mathrm{B}}$ can be simplified to $R_{2,0}$, when assuming $R_{2,0}^{\mathrm{A}}=R_{2,0}^{\mathrm{B}}$. Here the information about the distinct chemical shifts in $R_{2, \text { eff }}$ is convoluted as the population weighted chemical shift difference $\left(\phi=p_{\mathrm{A}} p_{\mathrm{B}} \Delta \omega^{2}\right)$. In the fast exchange limit, the peak becomes narrow again, due to the inverse correlation of the exchange rate with the observed transverse relaxation rate $R_{2 \text {,eff }}$ (Figure 2.1, f; Equation (2.15)).

In practice, equal populations are rare, and exchange happens between a major and minor state. Still the same concept explained so far applies. In case of no exchange the two states are separated by their chemical shifts. Due to the minor population, the respective 
peak is less intense. (Figure 2.1, h). With an increasing rate of exchange both peaks show a broadened linewidth (Figure 2.1, i). Following Equation (2.14) the linear dependency of the populations causes the minor state to broaden faster and it becomes almost invisible in the NMR spectrum (Figure $2.1, \mathrm{j}$ ). The exchange is only noticeable by changes in the linewidth of the major peak. At the coalescence point the major peak starts to shift its position (Figure 2.1, j). While in the equally populated state scenario the peak is located right between the two chemical shifts $\omega_{\mathrm{A}}$ and $\omega_{\mathrm{B}}$, here the peak is slightly shifted towards $\omega_{\mathrm{B}}$ (Equation (2.10); Figure 2.1, 1). Again, further increase of the kinetic rate causes the resonance to sharpen (Figure 2.1, m) due to the inverse correlation of the exchange rate with $R_{2, \text { eff }}$.

In case of exchange between two conformations, the major and minor populated states are synonymic for an energetically favored ground state and an excited stated, respectively. A very low population of the latter can lead to an "invisible" peak in the NMR spectrum even in the slow exchange regime. Under these conditions the exchange process can only be indirectly detected by its effects on the major peak. Since the excited state is often correlated to the biological function of a protein, the investigation of these "invisible" states is of high interest. NMR offers different experimental designs to detect these minor populated states and their corresponding exchange kinetics.

Besides exchange saturation transfer experiments (Forsen and Hoffman, 1963) like CEST (Vallurupalli et al., 2012) and DEST (Fawzi et al., 2012), relaxation dispersion is well suitable to detect these processes and works in both exchange regimes. The latter will be focus of this thesis and will be explained in more detail in the following. 


\subsection{Relaxation Dispersion}

In Section 1.2 CPMG and $R_{1 \rho}$ were already named as relaxation dispersion experiments to study protein motions on the microsecond to millisecond time scale (Vallurupalli et al., 2011; Akke and Palmer, 1996). While they follow different experimental designs for the detection of exchange kinetics, they share a basic concept. Relaxation dispersion experiments aim to quench the exchange contribution $\left(R_{\mathrm{ex}}\right)$ to the effective transverse relaxation rate $\left(R_{2, \text { eff }}\right)$. Accordingly the observed $R_{2, \text { eff }}$ decreases, which in practice leads to an increase of the peak height. While CPMG experiments uses $180^{\circ}$ pulses to quench $R_{\text {ex }}$, the $R_{1 \rho}$ experiment applies so-called spin lock pulses. Both experiments will be explained in more detail in the following.

\subsubsection{Constant-Time CPMG}

The constant-time CPMG (CT-CPMG) (Loria et al., 1999; Eichmuller and Skrynnikov, 2005 ) experiment applies a train of $180^{\circ}$ pulses during a fixed delay $T_{\mathrm{CPMG}}$ (CPMG block), which was introduced by Carl, Purcell, Meiboom and Gill (Carr and Purcell, 1954; Meiboom and Gill, 1958).

Nuclear spins precess around the z-axis with their respective chemical shifts causing a dephasing of the net magnetization. Hereby, the dephasing is directly proportional to the delay of free precession $\left(T_{\mathrm{CPMG}}\right)$. The application of a $180^{\circ}$ pulse in the center of such a delay inverts the sign of precession after $T_{\mathrm{CPMG}} / 2$ and effectively refocuses the net magnetization after $T_{\mathrm{CPMG}}$. In the presence of chemical exchange between two states, $\mathrm{A}$ and $\mathrm{B}$, the two different chemical shifts, $\omega_{\mathrm{A}}$ and $\omega_{\mathrm{B}}$ precess with their own characteristic frequency and stochastically interchange. Due to this exchange, the net magnetization is not refocused when applying a refocusing pulse in the center of $T_{\mathrm{CPMG}}$. This causes a broadening of the linewidth as it was discussed in Section 2.1. By increasing the number of refocusing pulses during $T_{\mathrm{CPMG}}$ the free delay between the pulses is reduced and thus leaves less time for the chemical shifts to interchange, ultimately improving the refocusing of the net magnetization. Eventually the frequency of refocusing pulses during $T_{\mathrm{CPMG}}$ surpasses the rate of the chemical exchange process and quenches the exchange contribution completely. At this point the transverse relaxation rate only depends on the intrinsic relaxation rate $\left(R_{2,0}\right)$.

The refocusing frequency of a CPMG pulse train is calculated by:

$$
\nu_{\mathrm{CPMG}}=\frac{1}{4 \tau_{\mathrm{cP}}},
$$

in which, $2 \tau_{\mathrm{cp}}$ corresponds to the length of a single CPMG element (Figure 2.4). In practice, the peak height of a resonance is measured in dependence of $\nu_{\mathrm{CPMG}}$. The corresponding effective transverse relaxation rates can be calculated by:

$$
R_{2, \mathrm{eff}}=-\frac{1}{T_{\mathrm{CPMG}}} \ln \left(\frac{I_{\mathrm{CPMG}}}{I_{0}}\right),
$$


in which, $T_{\mathrm{CPMG}}$ is the length of the CPMG block, $I_{\mathrm{CPMG}}$ is the intensity at the respective rotation frequency and $I_{0}$ is a reference intensity without the CPMG element (Mulder et al., 2001).

The relationship of $R_{2 \text {,eff }}$ and its corresponding $\nu_{\mathrm{CPMG}}$ yields the kinetic information of a possible exchange process. Often analytic solutions are used to describe this relationship. Their derivations rely on certain assumptions, making them applicable to only specific exchange regimes.

The Bloch-McConnell equation is usually used to describe the slow exchange regime:

$$
\frac{d}{d t}\left(\begin{array}{l}
M_{\mathrm{A}} \\
M_{\mathrm{B}}
\end{array}\right)=-\left(\begin{array}{cc}
i \Omega_{\mathrm{A}}-R_{2,0}-k_{\mathrm{AB}} & k_{\mathrm{BA}} \\
k_{\mathrm{AB}} & i \Omega_{\mathrm{B}}-R_{2,0}-k_{\mathrm{BA}}
\end{array}\right)\left(\begin{array}{l}
M_{\mathrm{A}} \\
M_{\mathrm{B}}
\end{array}\right),
$$

in which, $R_{2,0}$ is the intrinsic transverse relaxation rate, $\mathrm{M}_{\mathrm{A}}, \mathrm{M}_{\mathrm{B}}, \Omega_{\mathrm{A}}, \Omega_{\mathrm{B}}, k_{\mathrm{AB}}$ and $k_{\mathrm{BA}}$ are the intensity, chemical shift and exchange rate of state $\mathrm{A}$ and $\mathrm{B}$, respectively (McConnell, 1958).

The arising intensity of a peak in dependence of $\nu_{\mathrm{CPMG}}$ is calculated by:

$$
I\left(\tau_{\mathrm{cp}}\right)=\left(\exp \left(-R \tau_{\mathrm{cp}}\right) \exp \left(-R^{\mathrm{H}} \tau_{\mathrm{cp}}\right) \exp \left(-R^{\mathrm{H}} \tau_{\mathrm{cp}}\right) \exp \left(-R \tau_{\mathrm{cp}}\right)\right)^{n} I_{0},
$$

in which, $n=T_{\mathrm{CPMG}} \nu_{\mathrm{CPMG}}$ is the number of $360^{\circ}$ rotations in the CPMG block (two refocusing pulses), $R$ is the matrix in Equation (2.18), $R^{\mathrm{H}}$ is the Hermitian transposed of $R$ and $\tau_{\text {cp }}=\frac{1}{4 \nu_{\mathrm{CPMG}}}$ the delay between the refocusing pulses (Baldwin, 2014).

For fast exchanging sites the simplified Luz-Meiboom model (Luz and Meiboom, 1963) is applied:

$$
R_{2, \mathrm{eff}}=R_{2,0}+\frac{\Phi}{k_{\mathrm{ex}}}\left(1-\frac{4 \nu_{\mathrm{CPMG}}}{k_{\mathrm{ex}}} \tanh \left(\frac{k_{\mathrm{ex}}}{4 \nu_{\mathrm{CPMG}}}\right)\right)
$$

in which, $\Phi=4 \pi^{2} B_{0}^{2} \phi$ is the chemical shift variance with $\phi=p_{\mathrm{A}} p_{\mathrm{B}} \Delta \omega^{2}$ the population weighted chemical shift difference.

It should be noted that a flat relaxation dispersion (RD) profile not necessarily corresponds to the absence of chemical exchange. The exchange contribution $\left(R_{\mathrm{ex}}\right)$ depends also on the chemical shift difference between the two states. Thus, it must be large enough compared the exchange rate to be detectable (Equation (2.15)). Figure 2.2 shows simulated relaxation dispersion profiles using the same $k_{\mathrm{ex}}$, but different $\phi$ values. Only one of the simulated curves shows a significant exchange contribution despite assuming the same rate of exchange. In the limit where $\nu_{\mathrm{CPMG}}$ approaches 0 the maximum exchange contribution can be estimated by $\Phi / k_{\text {ex }}$ (Equation (2.20)). Thus, if $\Phi \rightarrow 0$ the chemical exchange contribution is negligible and the observed transverse relaxation rate only depends on $R_{2,0}$ as it is seen for the blue curve in Figure 2.2. RD profiles can also appear flat when the exchange kinetics are much faster than the maximum applied $\nu_{\mathrm{CPMG}}$. A comparison of $R_{2 \text {,eff }}$ and an estimated intrinsic transverse relaxation rate $\left(R_{2,0}\right)$ can be used to differentiate between flat profiles without exchange contribution and those appearing flat due to fast kinetics. 
Conventional ${ }^{15} \mathrm{~N}-\mathrm{CPMG}$ experiments reach refocusing frequencies of up to $1000 \mathrm{~s}^{-1}$ and thus cover exchange processes in the range of ms to hundreds of $\mu \mathrm{s}$. Transverse-rotating frame spectroscopy $\left(R_{1 \rho}\right)$ is the method of choice to detect faster motions than $1000 \mathrm{~s}^{-1}$ and will be briefly explained in the next section.

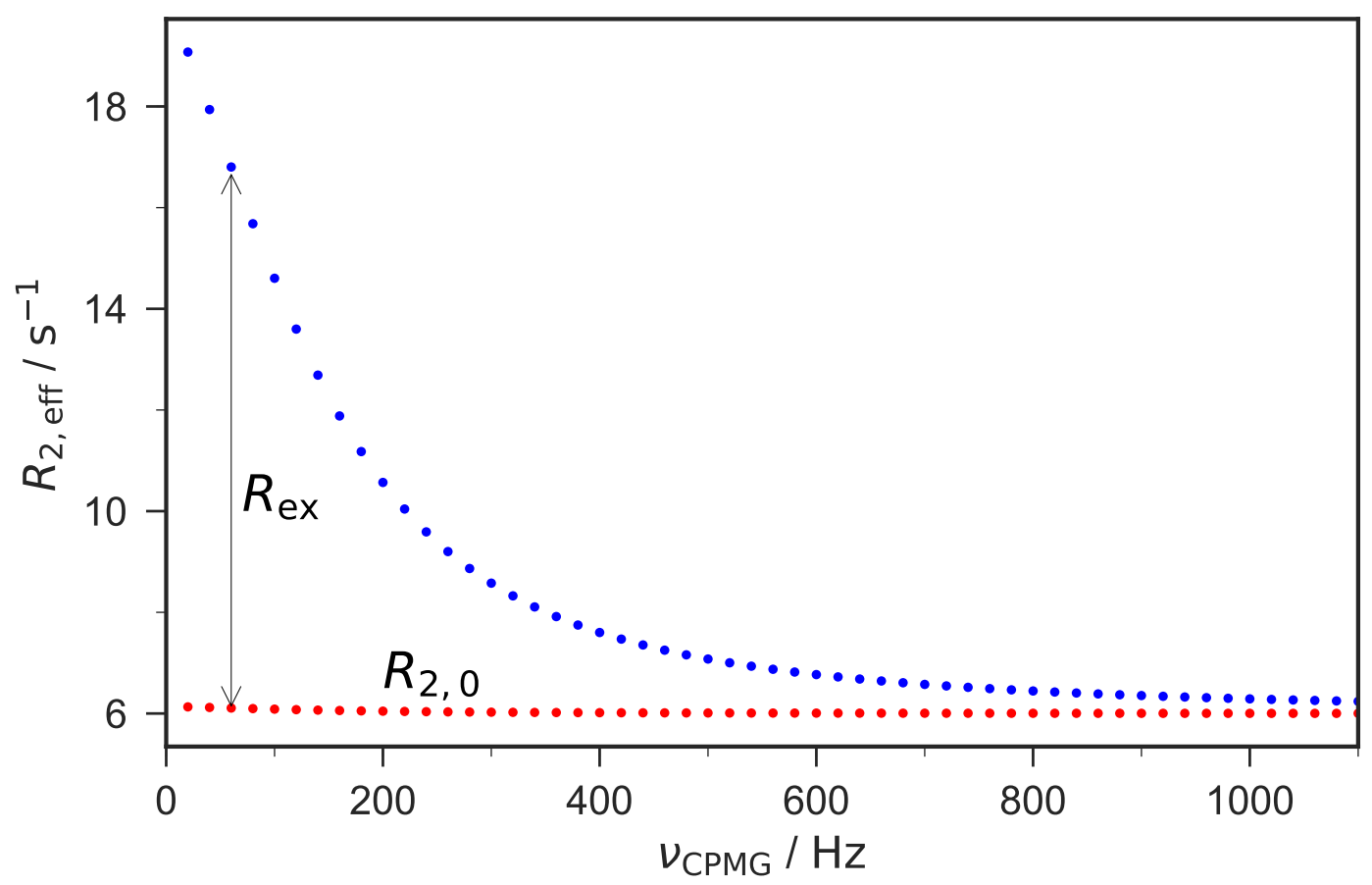

Figure 2.2: Simulated relaxation dispersion profile using the Luz-Meiboom model in the conventional CPMG range. In the presence of exchange the observed transverse relaxation rate $\left(R_{2, \mathrm{eff}}\right)$ is increased by an exchange contribution $\left(R_{\mathrm{ex}}\right)$. The application of refocusing pulses during a CPMG block of fixed length ( $\left.T_{\mathrm{CPMG}}\right)$ quenches the exchange contribution (blue). $R_{\text {ex }}$ depends on the population weighted chemical shift difference $(\phi)$ and exchange rate $\left(k_{\mathrm{ex}}\right)$. In the absence of exchange $\left(k_{\mathrm{ex}}=0\right)$ or negligible $\phi$ values, the RD profile appears flat (red). The following parameters were used for the simulation: $k_{\mathrm{ex}}=1000 \mathrm{~Hz}, B_{0}=600 \mathrm{MHz}, R_{2,0}=6 \mathrm{~Hz}, \phi=1 e^{-3} \mathrm{ppm}^{2}$ (blue) and $\phi=1 e^{-5} \mathrm{ppm}^{2}$ (red). 


\subsection{2 $\quad R_{1 \rho}$-Experiment}

The $R_{1 \rho}$-experiment utilizes a radio-frequency pulse with increasing amplitude or offset to lock the magnetization parallel to an applied effective field $B_{1}$, the so-called spin-lock. Depending on its applied power (spin lock frequency) and the offset between a resonance and the spin-lock, the magnetization is "trapped" between the z-axis and the x-y plane. Hereby $\theta$ describes the angle between the effective field and the overall field $B_{0}$ and is defined as:

$$
\theta=\tan ^{-1}\left(\nu_{\mathrm{RF}} / \Omega\right)
$$

where $\nu_{\mathrm{RF}}$ is the nutation frequency of the RF pulse and $\Omega$ the offset between a peak and the applied field of the spin-lock. Being trapped off-axis, the $\mathrm{x}$-y and z-components of the magnetization mix and an effective rate describes the relaxation. Depending on the tip angle $\theta$ the contributions of longitudinal $\left(R_{1}\right)$ and transverse relaxation rate $\left(R_{2}\right)$ add up to the rotating frame relaxation rate:

$$
R_{1 \rho}=R_{2, \mathrm{eff}} \cos \theta+R_{1} \sin \theta
$$

$R_{1 \rho}$ can be measured as off-resonance (Akke and Palmer, 1996) or on-resonance (Szyperski et al., 1993) experiment. The latter one uses a tip angle of $\theta=\pi / 2$ which eliminates the $R_{1}$ contribution according to Equation (2.22). This approach simplifies the analysis, since no further knowledge about $R_{1}$ is required. On the other hand, it requires an individual experimental setup for each resonance due to the $\theta$ dependence on $\Omega$ (Equation (2.21)). Without prior information about the active sites in a protein, this results in a large increase of measurement time.

Off-resonance $R_{1 \rho}$ targets multiple resonances, but presumes additional information about $R_{1}$ as well as transformation of the acquired data, since the effective transverse relaxation rate $\left(R_{2, \text { eff }}\right)$ still depends on $\theta$ :

$$
R_{2, \mathrm{eff}}=\frac{R_{1 \rho}}{\sin ^{2}(\theta)}-\frac{R_{1}}{\cot (\theta)}
$$

The larger the offset is $\left(\theta \rightarrow 0^{\circ}\right)$ the smaller is the contribution of transverse relaxation rate to $R_{1 \rho}$ and thus suppresses sensitivity of $R_{1 \rho}$ to the exchange contribution $\left(R_{\mathrm{ex}}\right.$, Equation (2.13)).

In general $R_{1 \rho}$ experiments require a higher amount of experimental preparation compared to CPMG experiments, as the effective field strength $\omega_{\text {eff }}$ also depends on $\nu_{\mathrm{RF}}$ and $\Omega$

$$
\omega_{\mathrm{eff}}=\left[(2 \pi \Omega)^{2}+\left(2 \pi \nu_{\mathrm{RF}}\right)^{2}\right]^{1 / 2}
$$

and the desired refocusing frequencies, as well as offsets and tip angles, need to be calculated in advance.

Though the experimental demand of $R_{1 \rho}$ experiments is higher compared to CPMG ex- 
periments, it permits access to an order of magnitude faster time scales. In recent years the detection of fast kinetics by $R_{1 \rho}$ experiments was improved (Ban et al., 2012, 2013; Smith et al., 2015; Trigo-Mourino et al., 2017). Ban et al. showed that cryogenically cooled NMR probe head allow the use of higher spin-lock frequencies, pushing the former detection limit of ${ }^{15} \mathrm{~N} R_{1 \rho}(\approx 40 \mu \mathrm{s})$ further into the supra- $\tau_{\mathrm{c}}$ window by a factor of 2 (Ban et al., 2012). Later Smith et al. used the same high-power approach to detect singledigit $\mu$ s motions using nutation frequencies up to $30010^{3} \mathrm{rad} \mathrm{s}^{-1}$ in ${ }^{1} \mathrm{H} R_{1 \rho}$ measurements (Smith et al., 2015). At low nutation frequencies $\left(\nu_{\text {eff }}<1 \mathrm{kHz}\right)$ the field strength of the spin lock is insufficient to lock the magnetization off-axis. Thus, $R_{1 \rho}$ experiments are usually used to target exchange processes on the microsecond time scale, while CPMG is used to study exchange processes near the millisecond time scale. 


\subsection{Extreme CPMG}

Due to recent work of Ban et al. the high-power relaxation dispersion (RD) approach was established for ${ }^{15} \mathrm{~N}$ RD experiments (Ban et al., 2012). Modern developments in NMR probe design allow to apply radio frequency pulses with more power than formerly believed. By that Ban and co-workers could push the accessible time scale of RD experiments further into the supra- $\tau_{\mathrm{c}}$ window, improving the detection of protein kinetics in $R_{1 \rho}$ experiments from $\approx 40 \mu$ s to $25 \mu \mathrm{s}$. Later Trigo-Mourino et al. showed that spin-lock pulses with a duration of up to $1 \mathrm{~s}$ and power comparable to the $90^{\circ}$ hard pulse can be applied without harming the hardware (Trigo-Mourino et al., 2017). Since then conventional CPMG and $R_{1 \rho}$ experiments together cover the ms to single-digit $\mu$ s time scale of protein kinetics, up to the supra- $\tau_{\mathrm{c}}$ window.

Despite their different approaches to quench the chemical exchange contribution $\left(R_{\mathrm{ex}}\right)$ to the effective transverse relaxation rate $\left(R_{2, \text { eff }}\right)$, their data can be combined for an analysis using a frequency conversion:

$$
\nu_{\mathrm{CPMG}}=\frac{\sqrt{3}}{6} \pi \nu_{\mathrm{RF}},
$$

where, $\nu_{\mathrm{CPMG}}$ and $\nu_{\mathrm{RF}}$ are the nutation frequencies of CPMG and $R_{1 \rho}$, respectively (Ishima and Torchia, 1999). Equation (2.25) is an empirical derived conversion to interchange the two experimental frequencies, using magic angle conditions in the $R_{1 \rho}$ experiment. Besides the differences in applied pulses, both methods rely on different spin states of transverse magnetization. While CPMG measures an average of in-phase and anti-phase magnetization $\left(1 / 2\left(\mathrm{~N}_{\mathrm{x}}+2 \mathrm{H}_{\mathrm{z}} \mathrm{N}_{\mathrm{x}}\right)\right)$, conventional $R_{1 \rho}$ experiments only detect in-phase magnetization to avoid cross relaxation. This causes differences in the observed $R_{2 \text {,eff }}$ and need to be considered in a combined analysis. The HEROINE experiment is a specialized $R_{1 \rho}$ experiment, in which the spin-lock is split into two blocks, centered by an INEPT transfer. This allows the use of in-phase and anti-phase during half of the effective spin-lock length and averages the relaxation contribution of both spin states, like in the CPMG experiment. By this Ban et al. already improved the accuracy of CPMG experiments, combining it with HEROINE data (Ban et al., 2013). Another challenge in combining the data of the two RD experiments lies in the difference of the applied pulses. These can cause different heating effects during the measurement, which might alter the detected $R_{2 \text {,eff }}$ differently. Here, the constant-time extreme CPMG (E-CPMG) experiment will be presented that combines the time scales of $R_{1 \rho}$ and CPMG in a single experiment using the high-power RD approach established by Ban and co-workers (Ban et al., 2012). As mentioned above, it was proven that modern NMR probes tolerate radio frequencies with hard pulse power up to a length of $1 \mathrm{~s}$ (Trigo-Mourino et al., 2017). Since CPMG experiments by design use refocusing pulses with hard pulse power to quench the exchange contribution, the high-power approach could be used to increase the accessible time scale of the conventional CPMG by far. Figure 2.3 shows the proposed pulse sequence of the ${ }^{15} \mathrm{NE}$-CPMG experiment (Reddy et al., 2018). 


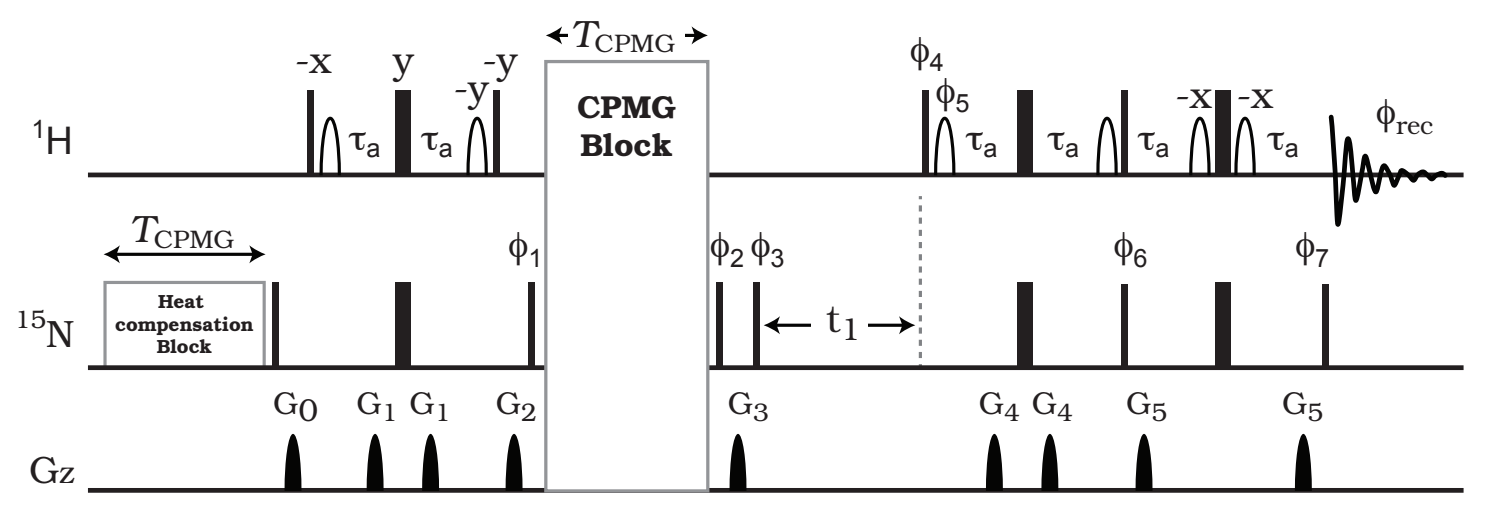

Figure 2.3: Pulse sequence of the ${ }^{15} \mathrm{~N}$ E-CPMG experiment. Narrow and wide bars depict $90^{\circ}$ and $180^{\circ}$ degree pulses. Open bars are shape pulses for water suppression. $\tau_{\mathrm{a}}$ is the INEPT delay $\left(1 /\left(4 J_{\mathrm{NH}}\right)\right), T_{\mathrm{CPMG}}$ is the CPMG block length and $t_{1}$ is the delay for chemical shift labeling of ${ }^{15} \mathrm{~N}$. At the beginning of each experiment a heat compensation block is applied, in which $180^{\circ}$ pulses were applied to achieve similar heating effects in all $\nu_{\mathrm{CPMG}}$ experiments. All pulses were applied with an x phase unless indicated differently. Details about pulse phases, delays and gradient strengths can be found in the materials and methods section of this chapter.

At first glance the pulse sequence is identical to conventional CPMG experiments. The difference lies in the CPMG block in the center of the experiment (Figure 2.4). As already mentioned, the fastest exchange process that can be quenched, depends on the maximum applied refocusing frequency or spin-lock amplitude for CPMG and $R_{1 \rho}$, respectively. In $R_{1 \rho}$ experiments higher spin-lock amplitudes can be applied than refocusing frequencies in CPMG, thus allowing $R_{1 \rho}$ to detect faster kinetics. According to Equation (2.16) the refocusing frequency of CPMG depends only on the interpulse delay $\tau_{\text {cp }}$ (Figure 2.4). Thus, decreasing $\tau_{\mathrm{cp}}$ (increasing number of refocusing pulses during $T_{\mathrm{CPMG}}$ ) pushes the limits of CPMG experiments towards the detection of kinetics in the supra- $\tau_{\mathrm{c}}$ window. In the extreme scenario when the interpulse delay becomes zero, a continuous train of $180^{\circ}$ pulses is applied. At this point the CPMG experiment is similar to an on-resonance spin-lock pulse with hard pulse power (Smith et al., 2015). Like this the E-CPMG experiment covers the range of time scales, that was formerly only accessible by combining $R_{1 \rho}$ and conventional CPMG experiments. Figure 2.4 shows the CPMG block in more detail. It is split into two halves and centered by a U-Element (Sorensen et al., 1997). The U-Element interconverts anti-phase and in-phase magnetization after half of the block length $\left(T_{\mathrm{CPMG}}\right)$. This ensures that these two spin states are equally mixed during the CPMG block. Artifacts caused by unequal contributions of in-phase and anti-phase magnetization in the different frequency experiments are reduced. This is especially important during low frequency experiments were the interpulse delay is in the order of milliseconds. On this time scale scalar coupling evolves completely and cause a larger mixing of the two spin states. While the scalar coupling is refocused at the end of $T_{\mathrm{CPMG}}$, the mixing of spin states depends on $\tau_{\mathrm{cp}}$ and an unequal contribution in different frequency experiments directly affects the 


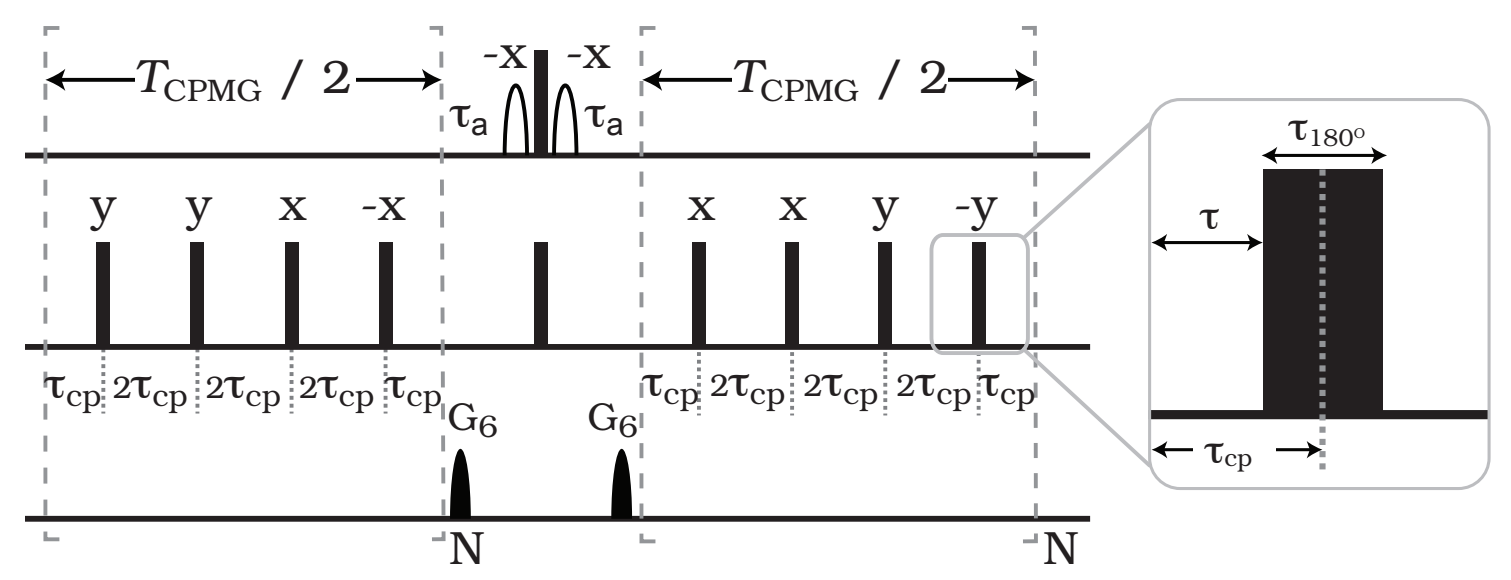

Figure 2.4: Detailed scheme of the CPMG block. Narrow and wide bars present $90^{\circ}$ and $180^{\circ}$ pulses. Open pulses depict shaped pulses for water suppression. $\tau_{\mathrm{a}}$ is the INEPT delay $\left(1 /\left(4 J_{\mathrm{NH}}\right)\right), \tau$ is the interpulse delay, $\tau_{180^{\circ}}$ is the $180^{\circ}$ pulse length and $2 \tau_{\mathrm{cp}}$ corresponds to the length of each CPMG element. The refocusing frequency $\left(\nu_{\mathrm{CPMG}}\right)$ is defined by $1 /\left(4 \tau_{\mathrm{cp}}\right)$. The CPMG block is split into two parts with a length of $T_{\mathrm{CPMG}} / 2$, centered by a U-element for interconversion of in-phase and anti-phase spin states, to reduce relaxation artifacts (Sorensen et al., 1997). A phase cycle scheme according to Yip et al. was applied to reduce artifacts caused by pulse imperfections and offset effects (Yip and Zuiderweg, 2004).

observed relaxation dispersion profile (Sorensen et al., 1997).

Furthermore, one cycle of the CPMG block contains eight refocusing pulses with phases $(\mathrm{y}, \mathrm{y}, \mathrm{x},-\mathrm{x}, \mathrm{x}, \mathrm{x}, \mathrm{y},-\mathrm{y})$ according to Yip and co-workers. This phase cycle scheme reduces artifacts, which are caused by pulse imperfections and offset effects (Yip and Zuiderweg, 2004).

The ${ }^{15} \mathrm{~N}$ E-CPMG presented here uses the Transverse Relaxation Optimized Spectroscopy (TROSY) readout to acquire the NMR signal, which comes with two benefits for the experiment (Pervushin et al., 1997). First, the TROSY sequence selectively detects the component of the ${ }^{15} \mathrm{~N}-{ }^{1} \mathrm{H}$ multiplet, which has the smallest transverse relaxation rate due to compensation of dipole-dipole and chemical shift anisotropy driven relaxation pathways. This allows the application to high molecular weight proteins which show larger intrinsic transverse relaxation rates due to their larger overall rotational correlation times $\left(\tau_{\mathrm{c}}\right)$. Second, this pulse scheme eliminates the need for a decoupling pulse sequence during acquisition and thus removes an additional heating source.

In the following the technical advantages of the E-CPMG experiment will be highlighted by using data measured on gpW protein at $275 \mathrm{~K}$ on a Bruker Avance III spectrometer operating at a ${ }^{1} \mathrm{H}$ Larmor frequency of $700 \mathrm{MHz}$. Since gpW relaxation dispersion data will be discussed in detail in Chapter 3 further introductions are skipped at this point.

The ${ }^{15} \mathrm{NE}$-CPMG experiment allows the application of RF pulses up to the nutation frequency of the hard pulse, which for the spectrometer used was $6400 \mathrm{kHz}\left(90^{\circ}\right.$ pulse 
length $39 \mu \mathrm{s})$. This permits the detection of protein kinetics up to $24 \mu \mathrm{s}$ and thus cover the same time scale that is accessible by $R_{1 \rho}$. The application of E-CPMG experiment on gpW allowed the analysis of two new resonances (V23 and D29), which were broadened beyond detection in previous CPMG experiments at $275 \mathrm{~K}$ (Sanchez-Medina et al., 2014).
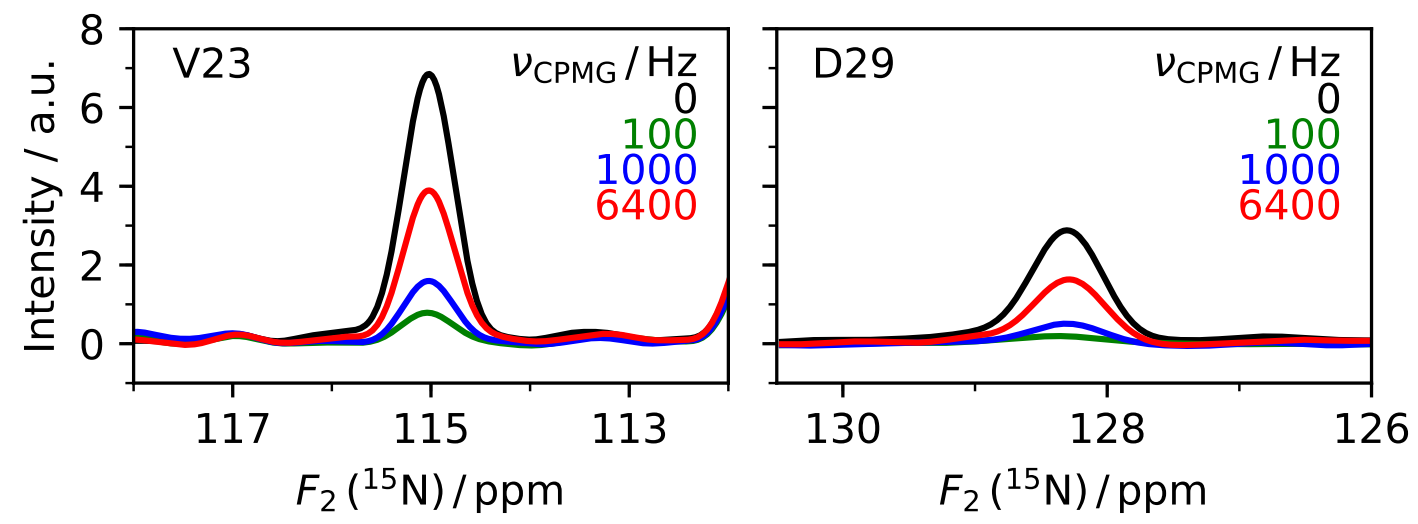

Figure 2.5: Slices from ${ }^{15} \mathrm{~N}$ E-CPMG experiments at different refocusing frequencies $\left(\nu_{\mathrm{CPMG}}\right) .1 \mathrm{D}$ slices of resonances V23 and D29 are shown as an example. E-CPMG experiments were measured with a CPMG block length $\left(T_{\mathrm{CPMG}}\right)$ of $40 \mathrm{~ms}$. Both peaks are broadened beyond detection at low refocusing frequencies $(100 \mathrm{~Hz})$. The conventional range of up to $1 \mathrm{kHz}$ is insufficient, to recover the intensity of the peaks for a feasible analysis $(1000 \mathrm{~Hz})$. The extended $\nu_{\mathrm{CPMG}}$ range of the E-CPMG further quenches the exchange contribution, allowing an analysis of these resonances under this experimental conditions $(6400 \mathrm{~Hz})$.

Figure 2.5 shows slices of the ${ }^{15} \mathrm{~N}$ E-CPMG experiment using different $\nu_{\mathrm{CPMG}}$ with resonances V23 and D29. Both were broadened close to the detection limit at low frequencies due to a large exchange contribution. Thus, their analysis is hindered in the conventional CPMG range. The use of higher $\nu_{\mathrm{CPMG}}$ (Figure 2.5 , red) quenched the large exchange contribution, raising the intensity above the detection limit.

Adding up to this, the necessity of high refocusing frequencies becomes clear while analyzing the measured RD profiles (Figure 2.6). Since the exchange contribution was not fully quenched at $\nu_{\mathrm{CPMG}}=1 \mathrm{kHz}$, information about the intrinsic relaxation rate $\left(R_{2, \mathrm{eff}}\right)$ and amplitude of $R_{\text {ex }}$ were missing. This caused an inaccurate estimation of the fit parameters during an individual analysis, using the Luz-Meiboom exchange model (Equation (2.20)). A comparison of the individual fit parameters from a cut data set, which simulates the conventional CPMG $\nu_{\mathrm{CPMG}}$ range $(1 \mathrm{kHz})$, and the full E-CPMG data set $(6.4 \mathrm{kHz})$ is shown in Table 2.1. Large differences in the intrinsic transverse relaxation rate $\left(R_{2,0}\right)$ and the chemical shift differences $(\phi)$ for the two data sets could be observed. This is expected, since the RD profiles are not completely quenched and yield no information about $R_{2,0}$. Thus, also the amplitude of $R_{\mathrm{ex}}$ is less defined and leads to inaccurate results for $\phi$. Furthermore, the individual exchange rates $\left(k_{\text {ex }}\right)$ show a large variation, when fitting the 
cut data set $\left(3-1210^{3} \mathrm{~s}^{-1}\right)$. The analysis of the full E-CPMG data set on the other hand showed a narrow distribution of the individual $k_{\mathrm{ex}}\left(7-810^{3} \mathrm{~s}^{-1}\right)$. Here, the individual fit results indicated a global exchange process that involves the residues located in the $\beta$-hairpin region. This conclusion could not be made from the individual fit results of the cut data set. Since the folding of gpW will be discussed in more detail in Chapter 3 data increases the accuracy of the individually fitted exchange parameters.
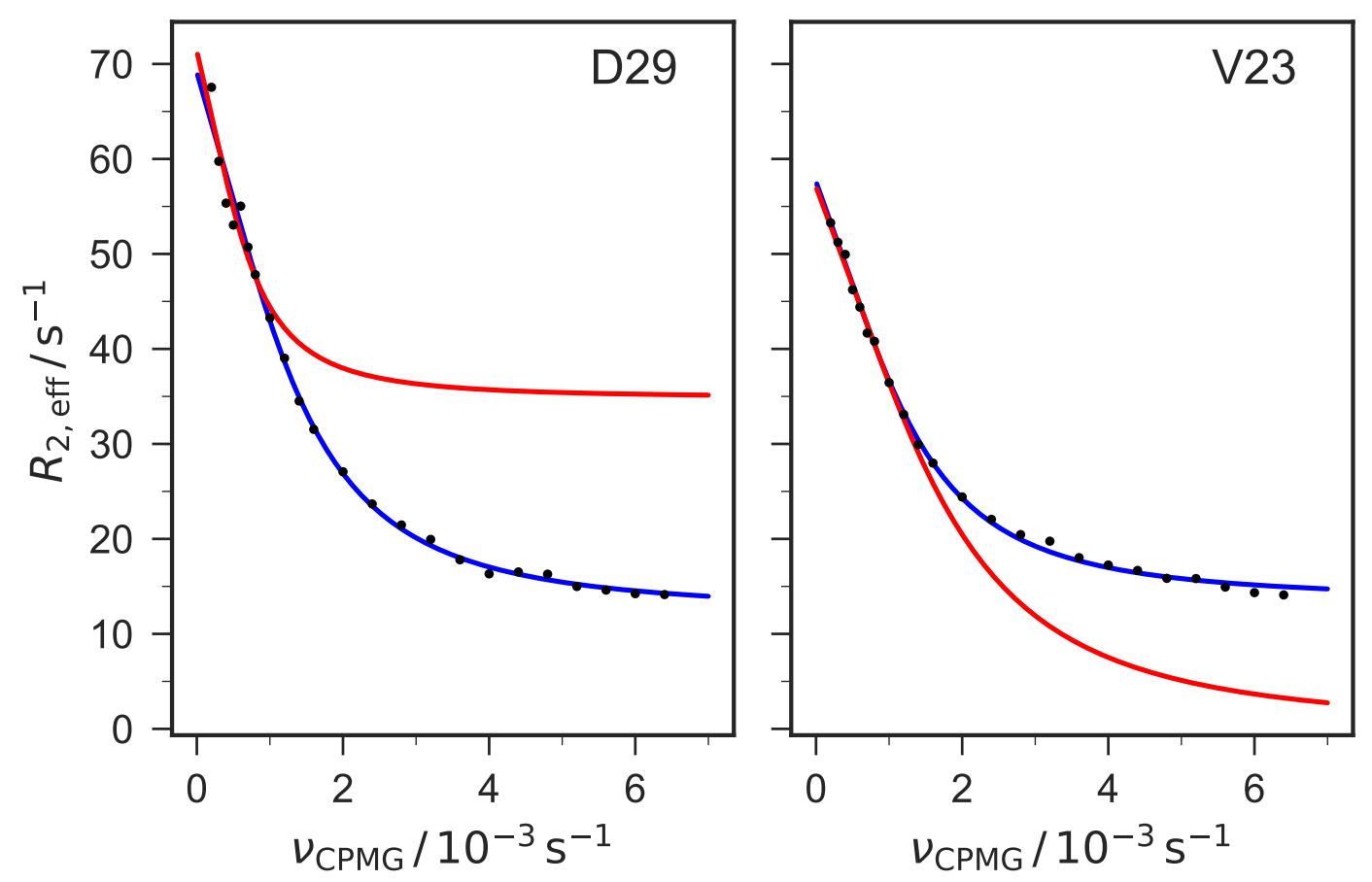

Figure 2.6: Comparison of conventional and E-CPMG fit results. E-CPMG data of residues V23 and D29 fitted to the two-state exchange Luz-Meiboom model (Equation (2.20)) using only $1 \mathrm{kHz}$ data (red), within the conventional CPMG range and the full data set acquired in E-CPMG (blue). Both RD profiles are not fully decayed within the conventional CPMG $\nu_{\mathrm{CPMG}}$ range. The missing information about the intrinsic relaxation rate $\left(R_{2,0}\right)$ and amplitude of exchange contribution $\left(R_{\mathrm{ex}}\right)$ leads to wrongly estimated kinetic parameters in the conventional data fit (red). In the E-CPMG experiment the exchange contribution can be further quenched, which allows a more accurate estimation of fast exchange processes (blue). 
Table 2.1: Comparison of individual fit results obtained from E-CPMG and conventional CPMG data sets at $275 \mathrm{~K}$. Fit results for the exchange rate $\left(k_{\mathrm{ex}}\right)$, chemical shift variance $(\phi)$ and intrinsic transverse relaxation rate $\left(R_{2,0}\right)$ are shown, using Equation (2.20). To simulate a conventional data set the E-CPMG data was cut after $\nu_{\mathrm{CPMG}}=1 \mathrm{kHz}$. The results of the $1 \mathrm{kHz}$ data set show a large fluctuation in the individually fitted $k_{\text {ex }}$ and in general larger uncertainties than the E-CPMG results. Thus information about $R_{2,0}$ are necessary for an accurate estimation of the kinetic parameters.

\begin{tabular}{llrrrcrr}
\hline \multirow{2}{*}{ Residue } & & \multicolumn{3}{c}{$6.4 \mathrm{kHz}$} & \multicolumn{3}{c}{$1.0 \mathrm{kHz}$} \\
\hline \multirow{2}{*}{ V23 } & Value & 13.44 & 1.82 & 8289 & 0 & 3.14 & 10960 \\
& Error & 0.27 & 0.04 & 188 & 0 & 0 & 0 \\
V26 & Value & 11.53 & 1.06 & 7688 & 19.26 & 0.51 & 4999 \\
& Error & 0.20 & 0.03 & 218 & 3.01 & 0.18 & 1075 \\
Q27 & Value & 10.60 & 0.47 & 7868 & 12.02 & 0.33 & 6203 \\
& Error & 0.13 & 0.02 & 327 & 1.44 & 0.10 & 1058 \\
K28 & Value & 12.39 & 0.89 & 7982 & 6.54 & 1.37 & 9627 \\
& Error & 0.19 & 0.03 & 248 & 21.34 & 2.12 & 7664 \\
D29 & Value & 12.37 & 2.39 & 8352 & 34.85 & 0.78 & 4276 \\
& Error & 0.62 & 0.10 & 334 & 8.34 & 0.44 & 1603 \\
G30 & Value & 12.50 & 0.95 & 7112 & 22.50 & 0.31 & 3341 \\
& Error & 0.28 & 0.04 & 296 & 1.90 & 0.09 & 699 \\
V33 & Value & 11.75 & 0.99 & 7398 & 0 & 2.06 & 10696 \\
& Error & 0.18 & 0.03 & 199 & 14.48 & 2.92 & 8042 \\
T36 & Value & 13.24 & 1.57 & 7648 & 0 & 2.78 & 10265 \\
& Error & 0.18 & 0.03 & 132 & 5.05 & 4.34 & 8224 \\
A37 & Value & 13.12 & 0.59 & 7770 & 13.39 & 0.55 & 7223 \\
& Error & 0.14 & 0.02 & 270 & 1.79 & 0.14 & 1000 \\
T38 & Value & 14.37 & 1.02 & 7654 & 0 & 2.43 & 11788 \\
& Error & 0.22 & 0.03 & 240 & 0 & 0 & 0 \\
S39 & Value & 13.89 & 0.51 & 7949 & 14.76 & 0.41 & 6669 \\
& Error & 0.14 & 0.02 & 321 & 1.49 & 0.11 & 990 \\
\hline
\end{tabular}




\subsection{Conclusion}

In this chapter the E-CPMG experiment was presented as fast and simple method to provide accurate exchange parameters of fast and slow exchange processes.

The discussed ${ }^{15} \mathrm{~N}$ E-CPMG experiment can be used to study kinetic processes up to $25 \mu \mathrm{s}$ and competes directly with $R_{1 \rho}$ experiments in detecting chemical exchange in the supra$\tau_{\mathrm{c}}$ range. Compared to $R_{1 \rho}$ it comes with a simplified experimental setup and covers the time scale of both conventional $\mathrm{RD}$ experiments. Thus, the detection of fast and slow kinetics is possible in a single experiment.

The larger accessible time scale and simple experimental setup of the E-CPMG experiments provides a promising tool for quick temperature dependent studies of exchange processes, like binding events and protein folding. These studies would give a more detailed insight into protein kinetics and their function in biological processes.

The fastest kinetic process that can be quenched in $\mathrm{RD}$ experiments depends on the maximum applied refocusing frequency. In E-CPMG this is equivalent to the nutation frequency $\left(\omega_{1}\right)$ of the applied effective field $\left(B_{1}\right) . \omega_{1}$ depends linearly on the gyromagnetic ratio $(\gamma)$ of a nucleus. Thus, the detection limits of the E-CPMG experiment can be further pushed into the formerly inaccessible supra- $\tau_{\mathrm{c}}$ window, by applying it to nuclei with a higher $\gamma$. The pulse sequence shown in Figure 2.3 can be adapted to measure ${ }^{13} \mathrm{C}$ and ${ }^{1} \mathrm{H}$ relaxation dispersion and their application provide access to $10 \mu \mathrm{s}$ and $4 \mu \mathrm{s}$ motions, respectively.

At this point it should be repeated that the exchange contribution to the transverse relaxation rate not only depends on the rate of exchange but also on the chemical shift variance $(\phi) .{ }^{1} \mathrm{H}$ E-CPMG gives access to the fastest kinetic processes available by RD experiments but on the other hand shows a smaller range of chemical shift dispersion compared to the low $\gamma$ nuclei ${ }^{13} \mathrm{C}$ and ${ }^{15} \mathrm{~N}$, due its rather small chemical shift range. Therefore, the exchange contribution in ${ }^{1} \mathrm{HE}-\mathrm{CPMG}$ is expected to be smaller. Anyhow, this cannot be generalized since the changes in the chemical shift upon binding largely depend on the investigated system. The use of all three nuclei as probes gives the most detailed insight into the structural changes and kinetics of exchange processes.

The next chapter will focus on the application of E-CPMG to study the kinetics and thermodynamic properties of the folding process of gpW protein. 


\subsection{Materials and Methods}

\subsubsection{Sample Preparation}

NMR experiments showed in this chapter were measured on perdeuterated, ${ }^{15} \mathrm{~N}$ labeled gpW. The protein was expressed in E. coli adapted to $100 \% \mathrm{D}_{2} \mathrm{O}$ using $\mathrm{D}_{7}$-glucose and ${ }^{15} \mathrm{NH}_{4} \mathrm{Cl}$ as carbon and nitrogen source, respectively. The protein sample was purified as described before in the literature (Gronenborn et al., 1991) and NMR samples were prepared using a concentration of $3 \mathrm{mM}$ in $20 \mathrm{mM}$ sodium phosphate buffer, $0.1 \mathrm{mM} \mathrm{NaN} 3$ at $\mathrm{pH} 6.5$, in $90 \% \mathrm{H}_{2} \mathrm{O}$ and $10 \% \mathrm{D}_{2} \mathrm{O}$ (Sborgi et al., 2011).

\subsubsection{NMR experiments}

The ${ }^{15} \mathrm{~N}$ constant-time extreme CPMG experimental data shown in this chapter was acquired at a temperature of $275 \mathrm{~K}$ using a Bruker Avance III spectrometer operating at ${ }^{1} \mathrm{H}$ Larmor frequency of $700 \mathrm{MHz}$ and equipped with a $5 \mathrm{~mm}$ triple-resonance TCI prodigy probe. Figure 2.3 displays a scheme of the pulse sequence and a transcript can be found in the Appendix. The CPMG block length $T_{\mathrm{CPMG}}$ was set to $40 \mathrm{~ms}$ and 28 different refocusing frequencies in the range of $100 \mathrm{~Hz}$ to $6400 \mathrm{~Hz}$ were acquired. Each two-dimensional experiment of the corresponding $\nu_{\mathrm{CPMG}}$ was acquired with 50 complex points $\left(t_{1, \max }=28.7 \mathrm{~ms}\right)$ in the indirect and 512 complex points in the direct dimension $\left(t_{2, \max }=56.3 \mathrm{~ms}\right)$, accumulating 16 scans. All frequencies were measured in a scan interleaved fashion and with mixed order of frequencies to average heating effects during high-power frequency experiments. The recycle delay $d_{1}$ was set to $3 \mathrm{~s}$ to further minimize heating during the measurement. The heat compensation block was set to the length of $T_{\mathrm{CPMG}}$ and missing pulses in the CPMG block were applied here to keep the overall number of pulses the same in all individual $\nu_{\mathrm{CPMG}}$ experiments. The following phase cycles were used: $\phi_{1}=4(\mathrm{x}), 4(-$ $\mathrm{x}) ; \phi_{2}=8(\mathrm{y}), 8(-\mathrm{y}) ; \phi_{3}=(\mathrm{y},-\mathrm{y},-\mathrm{x}, \mathrm{x}) ; \phi_{4}=4(-\mathrm{y}) ; \phi_{5}=(-\mathrm{y}) ; \phi_{6}=4(-\mathrm{y}) ; \phi_{7}=4(\mathrm{x}) ; \phi_{\text {rec }}=(\mathrm{y}$, $-y,-x, x), 2(-y, y, x,-x),(y,-y,-x, x)$. The following gradients were used with strength (length): $\mathrm{G}_{0}=42 \mathrm{G} \mathrm{cm}^{-1}(1 \mathrm{~ms}), \mathrm{G}_{1}=10 \mathrm{G} \mathrm{cm}^{-1}(0.5 \mathrm{~ms}), \mathrm{G}_{2}=28 \mathrm{G} \mathrm{cm}^{-1}(0.5 \mathrm{~ms}), \mathrm{G}_{3}$ $=38 \mathrm{G} \mathrm{cm}^{-1}(0.5 \mathrm{~ms}), \mathrm{G}_{4}=20 \mathrm{G} \mathrm{cm}^{-1}(0.5 \mathrm{~ms}), \mathrm{G}_{5}=37 \mathrm{G} \mathrm{cm}^{-1}(0.5 \mathrm{~ms}), \mathrm{G}_{6}=12 \mathrm{G} \mathrm{cm}^{-1}$ $(0.5 \mathrm{~ms})$.

\subsubsection{Data Analysis}

All spectra were processed using the python package NMRglue (Helmus and Jaroniec, 2013). Intensities of each individual peaks were obtained by using in-house software based on python. For the error estimation of the obtained intensities three refocusing frequencies were acquired twice and their standard deviation was used to calculate the root-meansquare-deviation (RMSD) of each individual peak.

$$
\Delta R_{2, \mathrm{eff}}=\sqrt{\sum\left(R_{2, \mathrm{eff}}^{\mathrm{exp}}-R_{2, \mathrm{eff}}^{\mathrm{repeat}}\right)^{2}}
$$


Relaxation dispersion profiles were fitted residue-wise using in-house software based on python package LMfit (Newville et al., 2014) and the Luz-Meiboom model:

$$
R_{2, \mathrm{eff}}=R_{2,0}+\frac{\Phi}{k_{\mathrm{ex}}}\left(1-\frac{4 \nu_{\mathrm{CPMG}}}{k_{\mathrm{ex}}} \tanh \left(\frac{k_{\mathrm{ex}}}{4 \nu_{\mathrm{CPMG}}}\right)\right)
$$

for fast exchange (Luz and Meiboom, 1963). An initial fit for the parameters was done using the Nelder-Mead algorithm and the so obtained fit parameters were further used as starting point for a refined fit using a least-square minimization using:

$$
\chi=\sum_{i=0}^{n} \frac{\left(\text { data }_{\mathrm{i}}-\text { model }_{\mathrm{i}}\right)}{\sigma_{\mathrm{i}}}
$$

as minimization function, in which data, model and sigma are the experimental value, calculated model and experimental uncertain of the i-th data point, respectively. For the conventional CPMG fit (Figure 2.6) the measured E-CPMG relaxation dispersion data set was cut after $\nu_{\mathrm{CPMG}}=1 \mathrm{kHz}$. 


\title{
Chapter 3
}

\section{Protein Folding: Application of E-CPMG to gpW Protein}

\subsection{Introduction}

\author{
$10 \quad 20 \quad 30 \quad 40 \quad 60$ \\ MVRQEELAAARAALHDLMTGKRVATVQKDGRRVEFTATSVSDLKKYIAELEVQTGMTQRRRG
}

Figure 3.1: Amino acid sequence of the 62-residue gpW protein. Residues that contain methyl side-chain groups are marked in red (Sborgi et al., 2011).

Proteins fold into their native structures forming secondary and tertiary structural elements, which are important for ligand binding, molecular recognition and protein interactions. In solution these structures can be found in an equilibrium with their associated unstructured form and the kinetics of these folding/unfolding processes can be the rate limiting step in protein interactions. Therefore, several NMR studies focus on the investigation of protein folding mechanisms (Farrow et al., 1995; Alexandrescu et al., 1994; Jennings and Wright, 1993). Since the formation of secondary and tertiary structure elements is likely to alter the chemical environment of a nucleus, NMR relaxation dispersion experiments offer a powerful tool to study folding processes on an atomic resolution with insights into their kinetics and structural

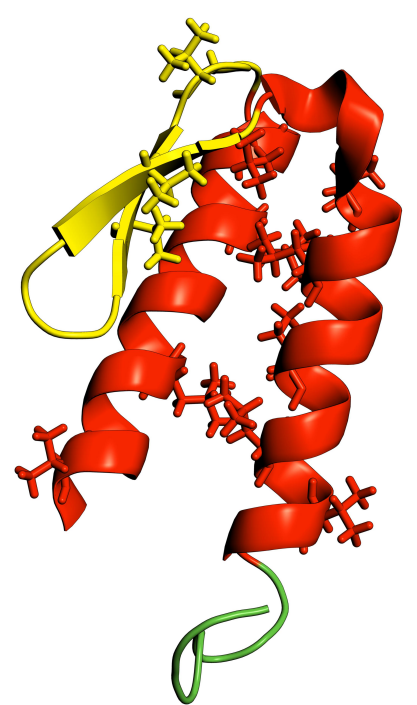

Figure 3.2: $\quad$ Structure of 62residue gpW protein. Structural elements are color-coded ( $\alpha$-helix, red; $\beta$-hairpin, yellow; disordered region, green) and Val, Leu sidechains are shown as sticks (pdb: 216r (Sanchez-Medina et al., 2014)). 
changes.

gpW protein is present in bacteriophage $\lambda$ and is involved in the last step of virus head morphogenesis, before the tail is attached to the head, forming the active virion (McClure et al., 1973). In its native form 68 residue gpW folds into a $\alpha+\beta$ topology, which is rather unusual (Figure 3.2). The structure was first described by Maxwell et al. using an automatic assignment approach (Maxwell et al., 2001) and it was revisited by Sborgi et al. ten years later (Sborgi et al., 2011). gpW forms N- and C-terminal $\alpha$-helices, which are connected via a $\beta$-hairpin region. The helices span from residue 4-19 $\left(\alpha_{1}\right)$ and 40-54 $\left(\alpha_{2}\right)$, whereas the $\beta$-strands span from residue 23-28 ( $\left.\beta_{1}\right)$ and 31-36 $\left(\beta_{2}\right)$ (Sborgi et al., 2011). The last $14 \mathrm{C}$-terminal residues of gpW are unstructured. It was shown that the last three residues of gpW play an important role for the functionality of the protein, but not for its thermal stability (Maxwell et al., 2001). Substitutions of the C-terminal residues showed no effect on the stability of the protein but largely reduced the in vitro activity of gpW. Thus, the authors hypothesized that the C-terminal region of gpW functions as binding site for another phage protein.

Due to its multiple roles in bacteriophage $\lambda$ and its novel folding topology, gpW is an attractive choice for structural and kinetic studies (Fung, 2008; Sborgi et al., 2011; SanchezMedina et al., 2014; Schonfelder et al., 2018). Thermodynamic experiments of Fung et al. showed that gpW fulfills the expected features of a so-called ultra-fast downhill folding protein (Fung et al., 2008). This class of proteins approaches the empirical folding limit of approximately $1 \mu \mathrm{s}$ for medium sized domains. They are characteristic for a maximum energy barrier smaller than the thermal energy RT $\left(\approx 2.5 \mathrm{~kJ} \mathrm{~mol}^{-1}\right.$ at $\left.298.15 \mathrm{~K}\right)$, resulting in a barrierless free energy landscape. Compared to other ultra-fast downhill folding proteins, gpW has large size but shares other attributes that seem to be common for this class. Like the downhill folding protein BBL (Naganathan and Munoz, 2005; Sadqi et al., 2006), it contains a large fraction of positive charges, a loosely packed hydrophobic core and a large contribution of local interactions (Fung et al., 2008).

Conventional CPMG experiments revealed that the folded structure of gpW in solution is in an equilibrium with a minor populated state. Medina-Sanchez and co-workers observed relaxation dispersion for residues located in the backbone of the $\beta$-hairpin region as well as methyl groups localized in the side-chains of the $\alpha$-helices. Residues in the backbone of the $\alpha$-helices on the other hand showed relatively flat dispersion profiles. According to these findings the authors hypothesized a hydrophobic collapse that drives the folding of the $\beta$-hairpin region and suggested different stability of the secondary structure elements in $\mathrm{gpW}$. This result indicates that the folding process of gpW in solution is more complex and can be interpreted in different ways (Sanchez-Medina et al., 2014).

Molecular dynamic (MD) simulation is a useful method to study the effects of atomic motions on the structure of molecules. In this theoretical approach the forces and potentials between atoms are calculated numerically using either quantum mechanical description 
or classical force fields. This method is limited by computational power and can only be applied to rather fast dynamics (up to hundreds of $\mu \mathrm{s}$ ). Since protein folding usually takes place on a slower time scale it is difficult to be targeted by MD simulation studies. Nevertheless, fast folding processes, like they were observed in gpW, can be addressed by MD simulations and their combination with NMR experiments and thermodynamic techniques provide a powerful tool for the analysis of these processes. Sborgi and coworkers analyzed a $250 \mu \mathrm{s}$ MD simulation of the folding process of gpW and combined their analysis with chemical shift information derived by NMR experiments (Sborgi et al., 2015). As the previously mentioned NMR relaxation dispersion experiments indicated, the MD simulations revealed a partially unfolded state, where the $\beta$-strands of gpW are disordered and in fast equilibrium with the folded structure (Figure 3.2). The calculated auto correlation function from the MD simulations revealed a double-exponential decay for residues in the $\alpha$-helices, which could be fitted to a fast $(1 /(0.5 \mu \mathrm{s}))$ and slow $(1 /(4.6 \mu \mathrm{s}))$ rate. According to the authors the fast rate corresponds to a helix-melting and -forming process of metastable states, while the slow rate describes an overall folding process. The latter is close to previous results $(1 /(8.8 \mu \mathrm{s}))$ obtained from IR laser induced T-jump kinetic experiments at gpW's melting temperature of $340 \mathrm{~K}$ (Fung et al., 2008). Unlike the MD simulations, experimental IR results show only one unfolding process. Furthermore, NMR experiments revealed that the second loop region (residues 35-41) and first half of the helix (residues 7-15) play an important role for the cooperative folding of gpW. Both regions show primary contacts to the hinge region (residues 19-24) and the second helix (residues 41 - 48) and thus form an interaction network that stabilize the cooperative folding process (Sborgi et al., 2015). Frank et al. used a combination NMR relaxation dispersion data and MD simulations to characterize folding intermediates of gpW (Frank et al., 2015). Using their LARMOR approach, protein backbone chemical shifts are predicted only on the bases of $\mathrm{C}_{\alpha}$ coordinates. The so obtained chemical shifts were used for coarse-grain MD modeling of an ensemble of structures of gpW. A comparison of the ensembles by LARMOR derived chemical shifts with chemical shifts from relaxation dispersion experiments (Sanchez-Medina et al., 2014) revealed a transient state in which the $\beta$-hairpin region of gpW is unfolded.

The fast folding kinetics make gpW an interesting target for the E-CPMG experiment. In this chapter the previously presented E-CPMG is used to investigate the folding kinetics of $\mathrm{gpW}$. The faster accessible timescale of this experiment might allow a deeper insight into the folding process and the atomic resolution of NMR relaxation dispersion experiments could further highlight crucial residues for the cooperative folding process. 


\subsection{Results and Discussion}

As explained in the previous section, gpW folds into its native structure, forming two terminal $\alpha$-helices that are connect via a $\beta$-hairpin structure (Figure 3.2). In this chapter the kinetic and thermodynamic properties of gpW folding will be investigated utilizing the constant-time extreme CPMG (E-CPMG) experiment, which was introduced in Chapter 2. Furthermore, its modified versions for the detection of ${ }^{13} \mathrm{C}$ and ${ }^{1} \mathrm{H}$ relaxation dispersion will be used to detect single-digit $\mu$ s kinetics.

\subsubsection{Backbone E-CPMG Experiments}

${ }^{15} \mathrm{NE}$-CPMG experiment on gpW were measured for a comparison with the results from previous relaxation dispersion experiments under conventional CPMG conditions (SanchezMedina et al., 2014). The experiments showed three different kinds of relaxation dispersion profiles and example profiles of those are shown in Figure 3.3. The last two C-terminal

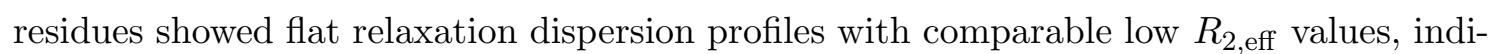
cating an exchange free effective transverse rate, as explained in Chapter 2. Relaxation
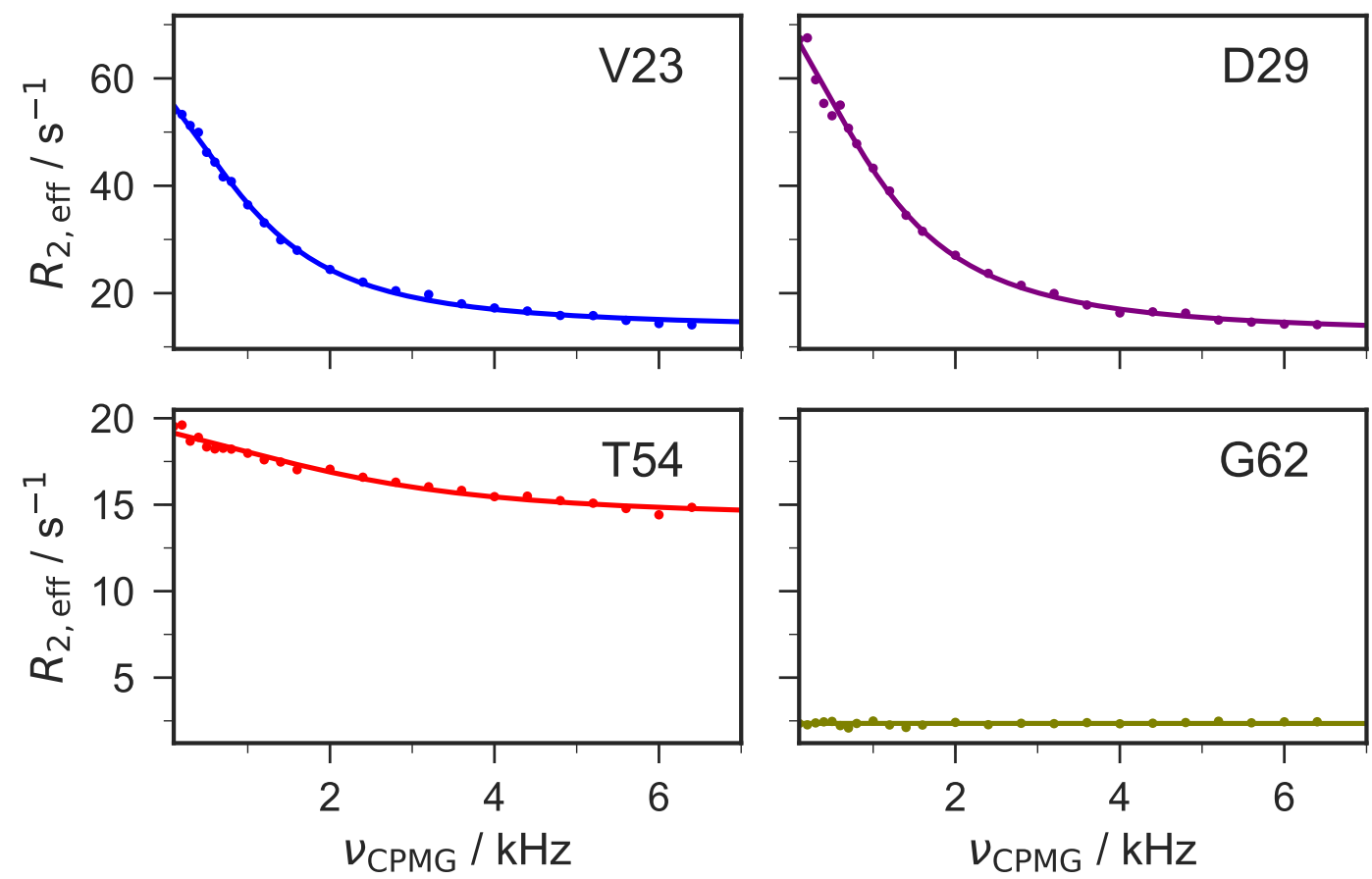

Figure 3.3: Relaxation dispersion profiles obtained from ${ }^{15} \mathrm{~N}$ E-CPMG with individually fitted exchange model at $275 \mathrm{~K}$. Displayed residues are located in different structural elements of gpW, V23 ( $\left.\beta_{1}\right)$, D29 (loop), T54 $\left(\alpha_{2}\right)$ and G62 (disordered). The structural elements can be connected to three kind of different relaxation dispersion profiles. Residues located in the $\beta$-hairpin region majorly showed RD profiles with a large exchange contribution $\left(R_{\mathrm{ex}}\right)$, that could be fully quenched within refocusing frequencies $\left(\nu_{\mathrm{CPMG}}\right)$ of up to $6400 \mathrm{~Hz}$. Residues localized in the $\alpha$-helices showed a slower decaying RD profile with a small $R_{\mathrm{ex}}$ and could not be fully quenched in the accessible $\nu_{\mathrm{CPMG}}$ range. C-terminal residues showed flat $\mathrm{RD}$ profiles, indicating no exchange contribution. 
dispersion profiles with large exchange contribution were localized in the $\beta$-hairpin region and profiles with small exchange contribution and a slower decay of $R_{2, \text { eff }}$ were found for residues in the $\alpha$-helices of gpW. In total 57 resolved peaks could be used for an individual analysis of the folding kinetic. The results of this analysis showed a clear difference between exchange rates $\left(k_{\mathrm{ex}}\right)$ fitted for residues in the $\beta$-hairpin region and $\alpha$-helices (Figure 3.4).

The $\beta$-hairpin region ranges from residue 23 to 36 , where residues 30 and 31 form a hinge that connect the two $\beta$-strands. Of these 14 residues, eight showed a distinct exchange contribution in the relaxation dispersion experiment. These included two additional resonances (V23 and D29), which were broadened beyond the detection limit in the accessible time scale of conventional CPMG experiments, as already mentioned in Chapter 2 (Sanchez-Medina et al., 2014; Reddy et al., 2018). The individually fitted exchange rates of these residues showed a narrow distribution roughly in the range of $(7-8) 10^{3} \mathrm{~s}^{-1}$, which indicated a global exchange process. In such a global exchange process all residues experience a change in their chemical environment based on the same rate of interchange of conformations. In addition, residues A37, T38 and S39, which are located in the link between the second $\beta$-strand $\left(\beta_{2}\right)$ and the C-terminal $\alpha$-helix $\left(\alpha_{2}\right)$, could also be fitted to similar exchange rates as shown in Figure 3.4.

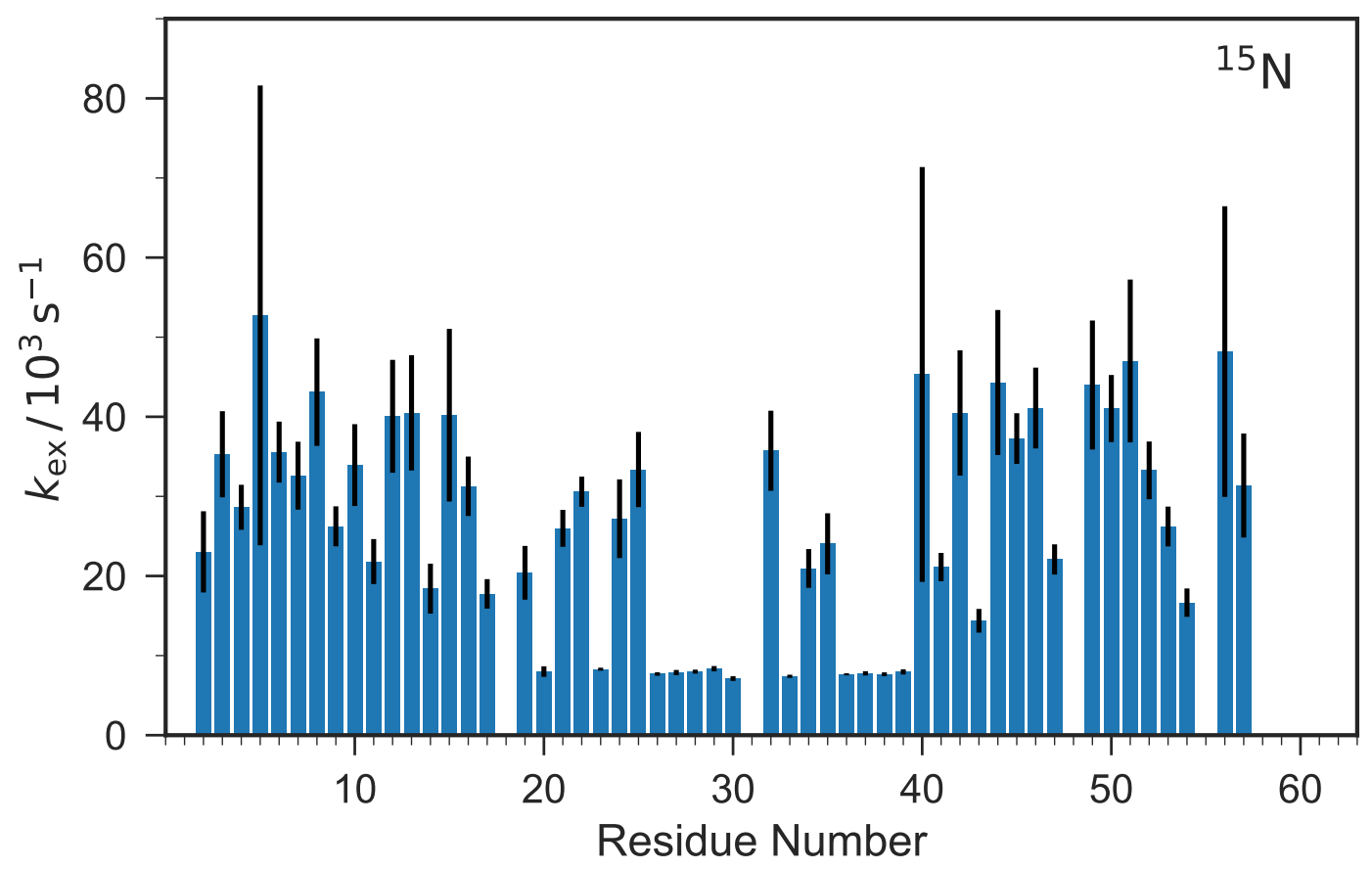

Figure 3.4: Exchange rates of individually fitted residues in gpW at $275 \mathrm{~K}$. Exchange rates were extracted by fitting the RD profiles obtained from ${ }^{15} \mathrm{NE}$-CPMG, to the Luz-Meiboom exchange model (Equation (2.20)). Residues that showed a fully quenched RD profile are located close to the $\beta$-hairpin region (residues 23 to 36 ) and were fitted to similar exchange rates $\left(k_{\mathrm{ex}}\right)$ of $\approx 8000 \mathrm{~s}^{-1}$ with small uncertainties. $k_{\mathrm{ex}}$ of $\alpha$-helical residues show a larger variation and uncertainty, which can be rationalized by the insufficiently quenched exchange contribution in the corresponding RD profiles. 
The residues V23 and D29, which could not be analysed before, fit to larger values of the population weighted chemical shift difference $\left(\phi, 1.82 \pm 0.04 \mathrm{ppm}^{2}\right.$ and $2.39 \pm 0.10 \mathrm{ppm}^{2}$, respectively) compared to other residues and thus show larger effective relaxation rates $\left(\sim 55 \mathrm{~s}^{-1}\right.$ and $\sim 70 \mathrm{~s}^{-1}$, respectively) (Table 2.1$)$. In conventional CPMG range this large exchange contribution caused V23 and D29 to be broadened beyond the detection limit and hindered their analysis.

The corresponding values for the population weighted chemical shift differences, exchange rates and intrinsic relaxation rates of the individual fit results at $275 \mathrm{~K}$ can be found in Table 2.1.

As already mentioned, the individually fitted exchange rates of the $\beta$-hairpin residues indicated a global exchange process. In total 11 residues were included in a global fit using the fast-exchange Luz-Meiboom model (Luz and Meiboom, 1963) and assuming a

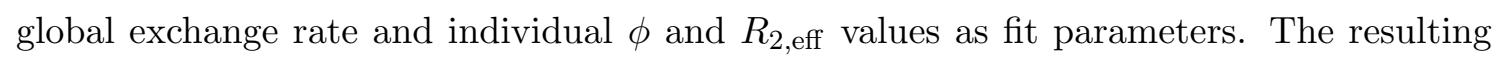
global fit was in very good agreement with the data (Figure 3.5) and the fitted exchange rate was $k_{\mathrm{ex}}=(7.95 \pm 0.79) 10^{3} \mathrm{~s}^{-1}$. Thus, the results from relaxation dispersion experiments support the assumption of a global folding process involving the $\beta$-hairpin and loop region between the second $\beta$-strand and C-terminal $\alpha$-helix.
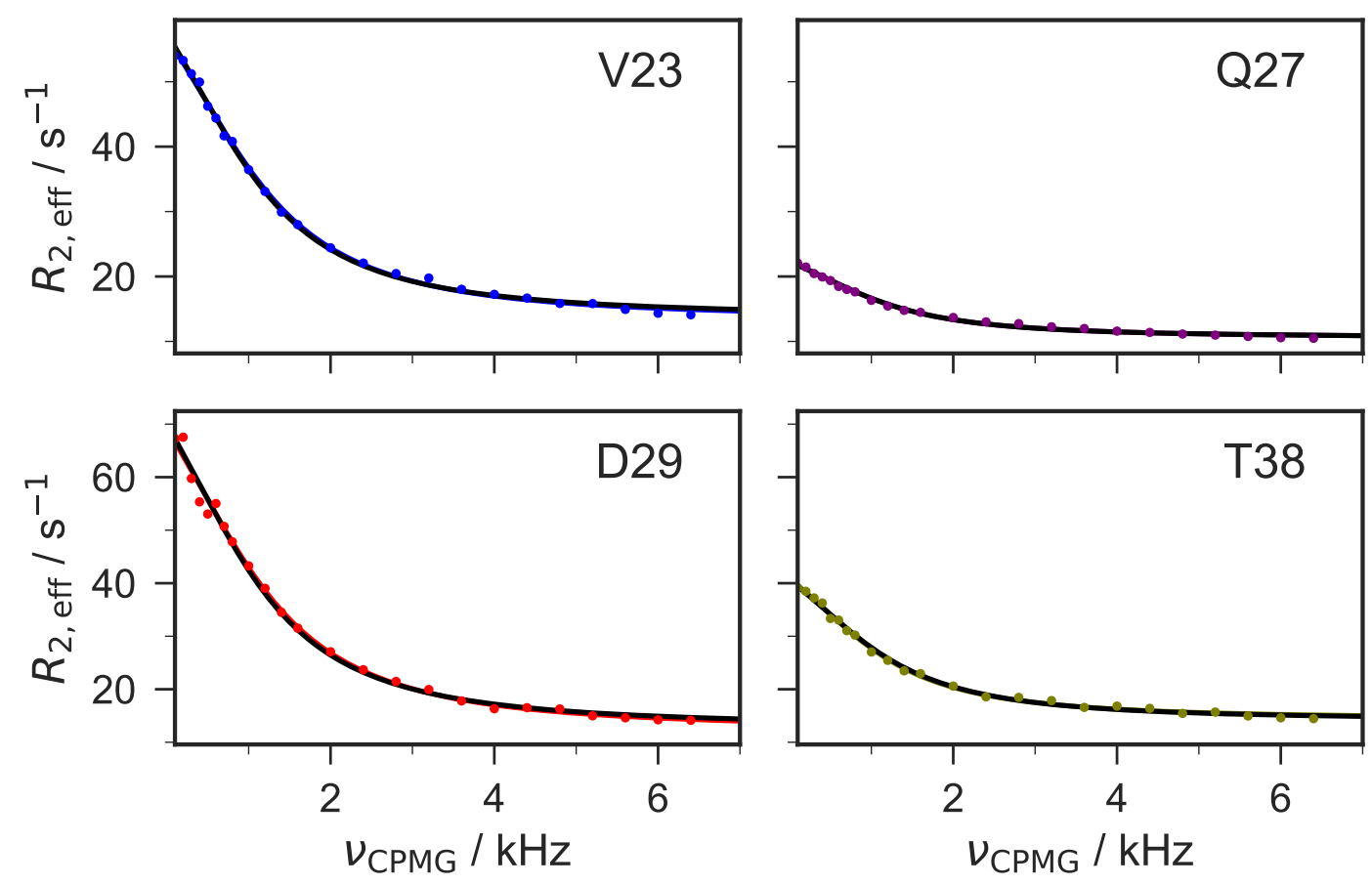

Figure 3.5: Comparison of individually and globally fitted two-state exchange model at $275 \mathrm{~K}$ for residues in the $\beta$-hairpin region. ${ }^{15} \mathrm{NE}-\mathrm{CPMG}$ obtained $\mathrm{RD}$ profiles of residues V23, Q27, D29 and T38 are shown with individually fitted (color) and globally fitted (black) Luz-Meiboom model (Equation (2.20)). The global model shows only marginal differences to the individual fit result. Thus, it can be concluded that residues in the $\beta$-hairpin region are involved in a global exchange process.

The residues in the $\alpha$-helices only showed a small exchange contribution (Figure 3.3). 
Individually fitted exchange rates of these residues are scattered over a range of (20 120) $10^{3} \mathrm{~s}^{-1}$ (Figure 3.4). Furthermore, the uncertainties of the fitted $k_{\mathrm{ex}}$ values were much larger compared to those obtained for the $\beta$-hairpin residues. Taking a closer look at the dispersion profiles of residues located in the $\alpha$-helical regions of $\mathrm{gpW}$, such as R11

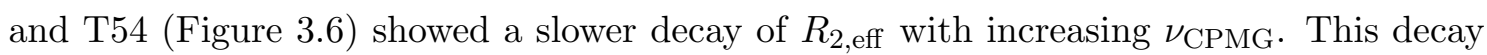
indicates the presence of an exchange event that involves the backbone amide of $\alpha$-helical residues. The exchange contribution in this relaxation dispersion profiles was rather small $\left(<5 \mathrm{~s}^{-1}\right)$ and was not fully quenched at $\nu_{\mathrm{CPMG}}=6.4 \mathrm{kHz}$. The individually fitted exchange rates shown in Figure 3.4 illustrate that a possible exchange process involving the $\alpha$-helices would take place on a much faster time scale than the observed exchange process in the $\beta$-hairpin region discussed so far. The large scattering of the fit parameters resulted from an insufficient quenching of $R_{\mathrm{ex}}$. Due to this the intrinsic relaxation rate $R_{2,0}$ is not defined and can only poorly be estimated during the fit procedure. This led to inaccurate fit results, as it was discussed in Chapter 2.

From here on the exchange process involving the $\beta$-sheet residues will be denoted as slow process and the one involving residues in the $\alpha$-helices as fast process. Still both processes take place in the fast exchange regime considering the NMR time scale.

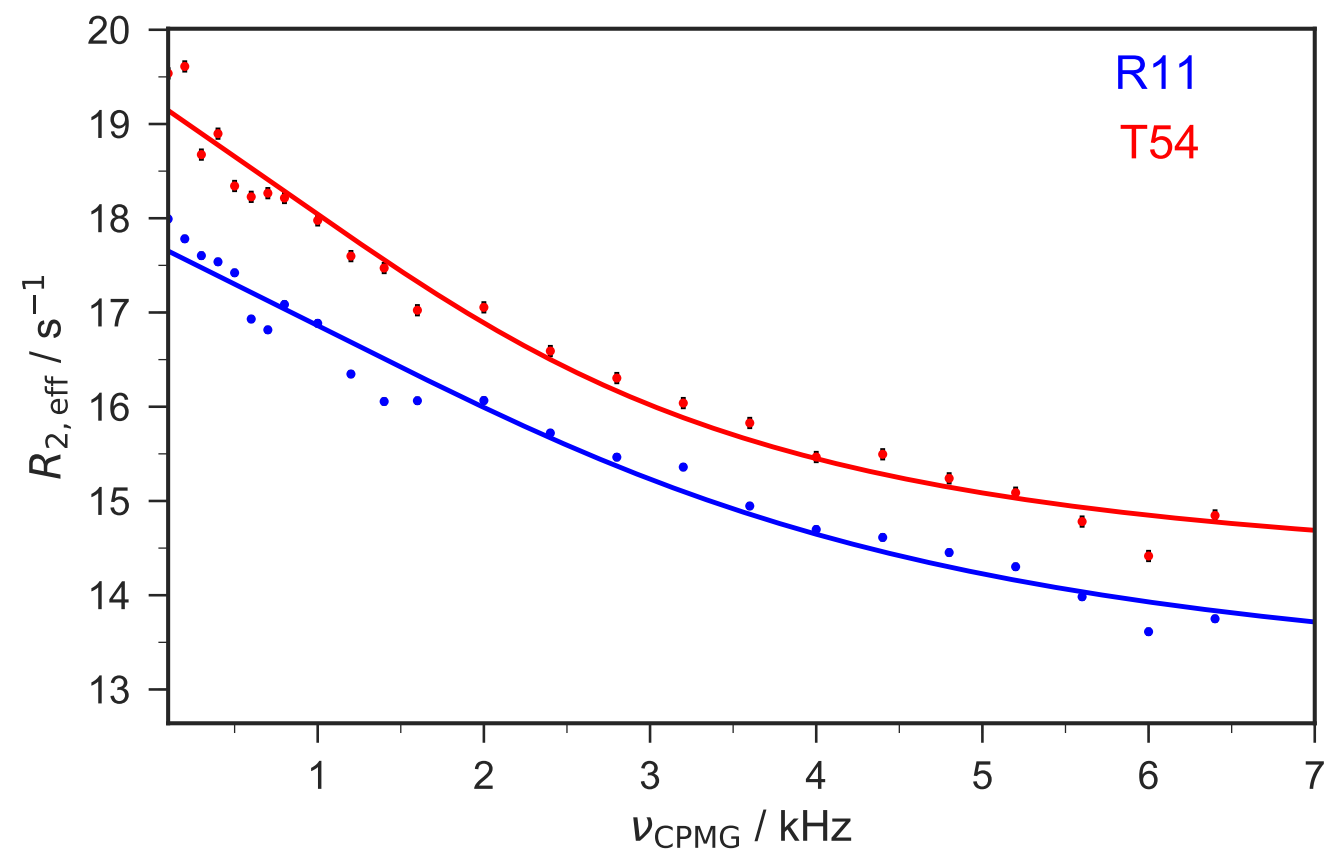

Figure 3.6: ${ }^{15} \mathrm{~N}$ E-CPMG $\mathrm{RD}$ profiles of backbone amide nitrogen from residues in the $\alpha$-helical region at $275 \mathrm{~K}$ indicating partial quenching of exchange contribution. Residues R11 (blue) and T54 (red) are shown as an example. The observed RD profiles of residues in the $\alpha$ helices show a small exchange contribution $\left(R_{\text {ex }}<5 \mathrm{~s}^{-1}\right)$ compared to residues localized in the $\beta$-hairpin $\left(R_{\mathrm{ex}}>10 \mathrm{~s}^{-1}\right) . R_{\mathrm{ex}}$ decays slowly with increasing refocusing frequency $\left(\nu_{\mathrm{CPMG}}\right)$ and could not be quenched in the accessible range of up to $\nu_{\mathrm{CPMG}}=6.4 \mathrm{kHz}$. This indicates the presence of a faster exchange process involving residues in the $\alpha$-helices. 
To further analyze the fast exchange process with ${ }^{15} \mathrm{NE}$-CPMG experiments the rate of exchange must be slowed down. This would increase the chances of quenching the process completely in the accessible time scale of the ${ }^{15} \mathrm{NE}$-CPMG experiment. At the same time the slower kinetics will cause an increase of the exchange contribution according to Equation (2.15). Thus, the measurement had to be repeated at lower temperatures. The data shown so far was already measured close to the freezing point of the used solvent. For further reduction of the temperature, the E-CPMG experiment was combined with a super-cooled sample approach (Ban et al., 2011). Thus, the sample was transferred to capillaries with an outer diameter of $1 \mathrm{~mm}$, each of which contained $25 \mu \mathrm{L}$ of gpW. Ten of such capillaries could be fitted into a conventional $5 \mathrm{~mm}$ NMR tube, which was used for further experiments at sub-zero temperatures. Due to the capillary forces the freezing point of the solvent is reduced and measurements at temperatures below $0{ }^{\circ} \mathrm{C}$ were possible. With this sample setup the ${ }^{15} \mathrm{NE}$-CPMG experiment was remeasured at $263 \mathrm{~K}$, to significantly slow down the folding kinetics of gpW.
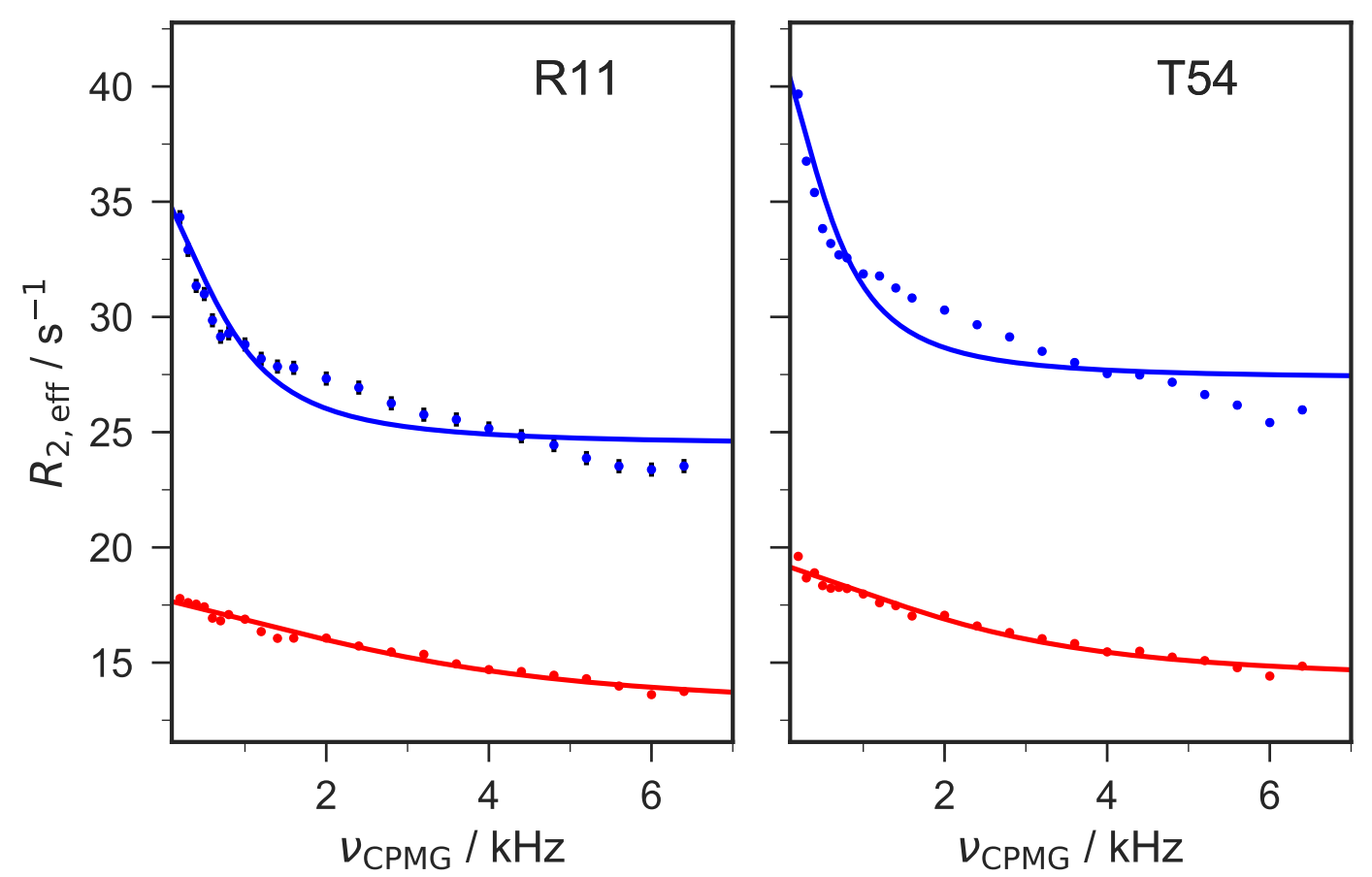

Figure 3.7: Comparison of ${ }^{15} \mathrm{~N}$ E-CPMG RD profiles from $\alpha$-helical residues at $263 \mathrm{~K}$ and $275 \mathrm{~K}$. Residues R11 and T54 are shown as an example. Relaxation dispersion data (dots) and individual fits at $263 \mathrm{~K}$ (blue) and $275 \mathrm{~K}$ (red) are shown. At $275 \mathrm{~K}$ both RD profiles show a decay in $R_{2 \text {,eff }}$ with increasing $\nu_{\text {CPMG }}$. The two-state model is in good agreement with the data but gives inaccurate fit parameters due to the insufficient quenching of $R_{\mathrm{ex}}$. At $263 \mathrm{~K}$ the $\mathrm{RD}$ profiles show a second decay at low $\nu_{\mathrm{CPMG}}$ values. This decay cannot be explained by the two-state model and indicates the presence of more than two conformational states of the $\alpha$-helices.

The observed $\beta$-hairpin exchange process was already quenched at $275 \mathrm{~K}$. Thus, the discussion of the super-cooled data will be focused on the indicated fast exchange process observed in the $\alpha$-helices. 
Figure 3.7 shows the measured relaxation dispersion profiles of residue R11 and T54, which are in the first and second $\alpha$-helix, respectively. At low temperatures all protein dynamics are slowed down. Therefore the intrinsic relaxation rate $\left(R_{2,0}\right)$ contribution to $R_{2, \text { eff }}$, caused by sub- $\tau_{\mathrm{c}}$ motions, also increased. Furthermore, the RD profiles showed a slight increase of the exchange contribution comparing data at $275 \mathrm{~K}\left(R_{\mathrm{ex}} \approx 4 \mathrm{~s}^{-1}\right)$ and $263 \mathrm{~K}\left(R_{\mathrm{ex}} \approx 6 \mathrm{~s}^{-1}\right)$. More obvious the $\mathrm{RD}$ profiles showed an initial decay that was not seen in the data collected at $275 \mathrm{~K}$. An individual analysis of the relaxation dispersion data revealed a discrepancy between the data and the two-state exchange model. The obtained model curves at $263 \mathrm{~K}$ only fitted the initial drop of $R_{2 \text {,eff }}$ and could not explain the slower decay from $\nu_{\mathrm{CPMG}} \approx 1 \mathrm{kHz}$ onward. This indicated that the observed relaxation dispersion profiles are result of more than one exchange process. At least two conformational changes, which take place on distinct time scales, seemed to involve residues of the $\alpha$-helices.
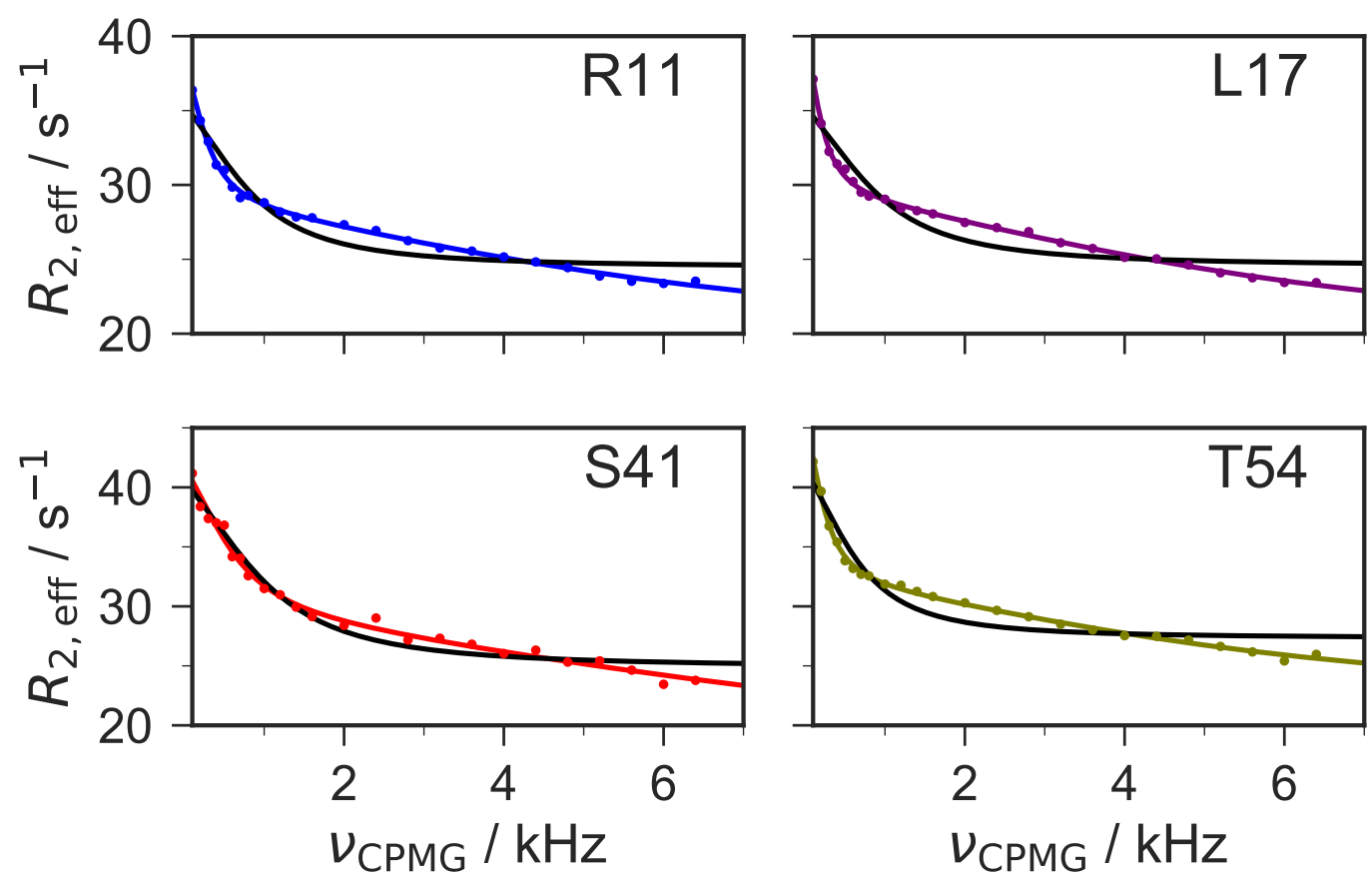

Figure 3.8: Relaxation dispersion profiles obtained from ${ }^{15}$ N E-CPMG with individually fitted two and three-site exchange model at $263 \mathrm{~K}$ for residues in $\alpha$-helices. RD profiles of residues R11, L17, S41 and T54 are shown as an example. The three-site exchange model (colored line) represents the observed relaxation dispersion better than the two-site exchange model (black line). Thus, it can be assumed that at least three conformational states are present in the exchange process of the $\alpha$-helices. A slow process that is almost quenched after $\nu_{\mathrm{CPMG}}=1 \mathrm{kHz}$ and a fast process that could not be quenched in the accessible $\nu_{\mathrm{CPMG}}$ range.

Assuming only three conformations, the simplest three-site exchange would be a linear exchange system according to:

$$
C \underset{k_{\mathrm{AC}}}{\stackrel{k_{\mathrm{CA}}}{\rightleftharpoons}} A \underset{k_{\mathrm{BA}}}{\stackrel{k_{\mathrm{AB}}}{\rightleftharpoons}} B,
$$


in which, $k_{A B}, k_{A C}, k_{B A}$ and $k_{C A}$ are the exchange rates of the corresponding states A, B and C. For this model Grey et al. provide an analytic solution in the fast exchange limit:

$$
\begin{aligned}
R_{2, e f f}=R_{2,0} & +\frac{\Phi^{F}}{k_{e x}^{F}}\left(1-\frac{4 \nu_{C P M G}}{k_{e x}^{F}} \tanh \left(\frac{k_{e x}^{F}}{4 \nu_{C P M G}}\right)\right) \\
& +\frac{\Phi^{S}}{k_{e x}}\left(1-\frac{4 \nu_{C P M G}}{k_{e x}^{S}} \tanh \left(\frac{k_{e x}^{S}}{4 \nu_{C P M G}}\right)\right),
\end{aligned}
$$

in which parameters denoted with $\mathrm{F}$ and $\mathrm{S}$ correspond to the fast and slow exchanging site, respectively (Grey et al., 2003). Equation (3.2) was used to analyze the RD profiles of residues in the $\alpha$-helices individually. The resulting model fits were in very good agreement with the relaxation dispersion data (Figure 3.8).

To compare the results of the two model fits the corrected Akaike information criterion (AICc) (Burnham and Anderson, 2002) was used. While the AICc does not certify the quality of a specific model fit, it can be used to compare models relative to each other and is defined as:

$$
A I C c=\chi^{2}+2 k+\frac{2 k(k+1)}{n-k-1},
$$

in which, $k$ is the number of fit parameters of the corresponding model, $n$ is the number of data points used and $\chi^{2}$ is a measure of the goodness of the fit. It is defined as:

$$
\chi^{2}=\sum_{i=1}^{N}\left(\frac{d a t a_{i}-\text { model }_{i}}{\sigma_{i}}\right)
$$

in which, data $a_{i}$, model $_{i}, \sigma_{i}$ are the experimental value, model value and experimental uncertainty of the i-th data point, respectively. $\chi^{2}$ is minimized during the fit procedure and describes the proximity of data and model within the experimental error. A direct comparison of the $\chi^{2}$ is insufficient for a model selection, since it does not take the number of fit parameters into account. An increase of parameters in a model tends to improve the goodness of the fit $\left(\chi^{2}\right)$ but reduces its simplicity (overfitting). Thus, a penalty for the number of fit parameters needs to be considered to avoid overfitting, during model selection. Another source for overfitting are small sample sizes, which should also be taken into account. Here, the corrected Akaike Information Criterion (AICc) is used to compare the two and three-site exchange model. 

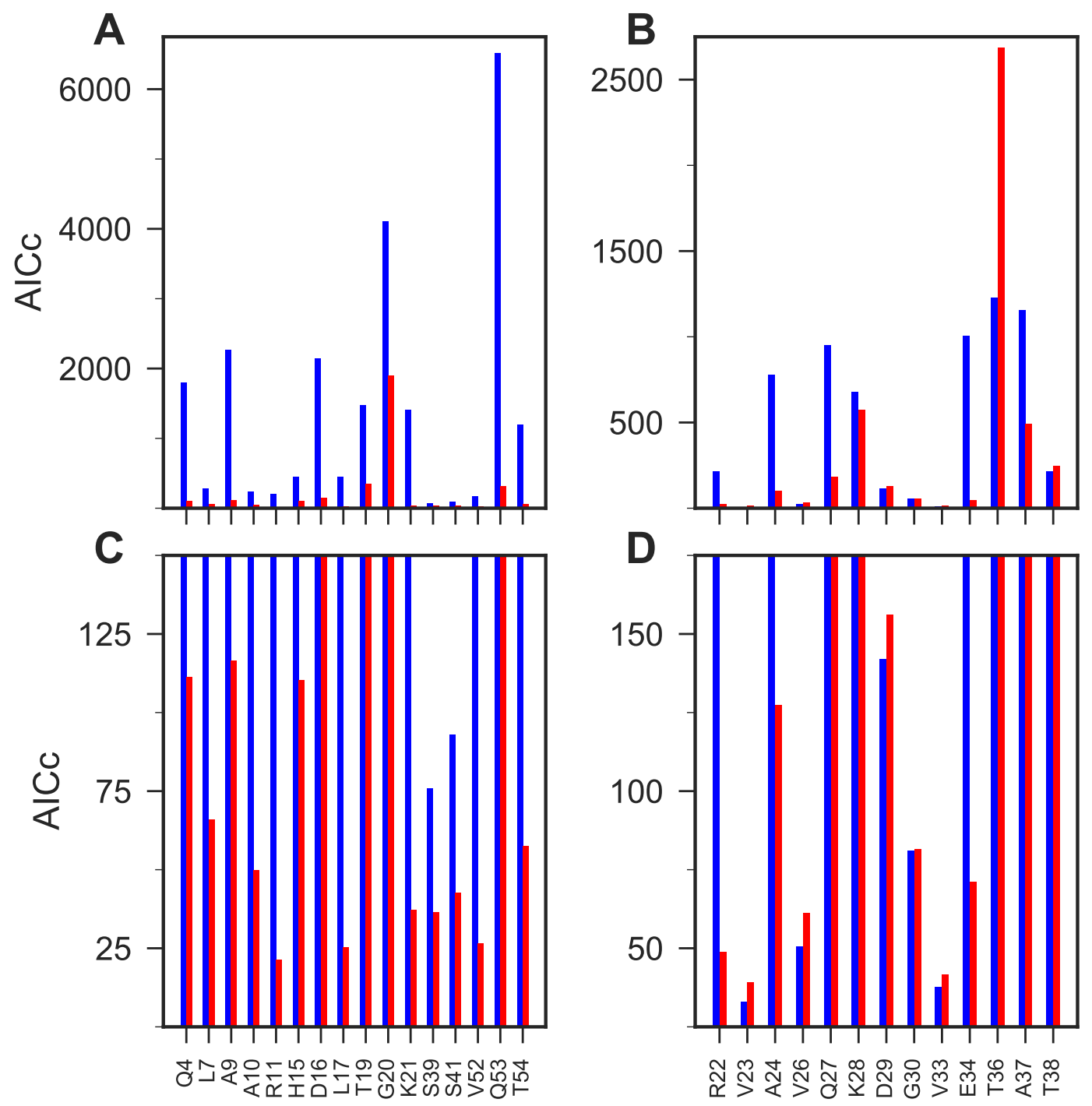

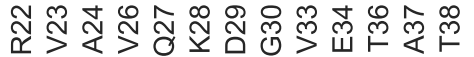

Figure 3.9: Comparison of the two- and three-site exchange model by corrected Akaike information criterion (AICc). Two-site (blue) and three-site (red) exchange are shown for selected residues. in the $\alpha$ helices $(\mathrm{A}, \mathrm{C})$ and $\beta$-hairpin region (B,D). (C,D) Zoomed in plots of the AICc values. (A,C) The AICc values for residues in the $\alpha$-helices show a large decrease when fitted to the three-site model. Thus, it is a better description of the data collected at $263 \mathrm{~K}$. (B,D) Residues in the $\beta$-hairpin region show no general tendency for one of the used exchange models. While some RD profiles (e.g. residues R22 and A37) are better described by the three-site exchange, others follow the two-site exchange model (e.g. residues V23, V33). 
A comparison of the AICc values of the two different exchange models (Figure 3.9) showed that indeed the three-site exchange model (Grey et al., 2003) provides a better explanation for the observed RD profiles for residues in the $\alpha$-helices of gpW. The AICc results for the $\beta$-strands showed an improvement especially for those residues that were fitted to a faster $k_{\text {ex }}$ in the individual analysis at $275 \mathrm{~K}$ (R22, A24 and E34) (Figure 3.4). Residues that could be fitted to a global exchange process at $275 \mathrm{~K}$ (e.g. V23, V26, G30) showed similar values in the AICc of both models. Thus, the two-state exchange model would be a better presentation of the exchange process in these residues. The residue-wise analysis also revealed that residue Q27, which could be fitted properly to a two-site exchange process at $275 \mathrm{~K}$, showed a large decrease in the AICc when fitted to the three-site exchange model (Figure 3.9, B). Differently to the $\alpha$-helical residues, the slow exchange is dominant for residue Q27 and the exchange contribution of the fast process is rather small $\left(>2 \mathrm{~s}^{-1}\right)$ and only becomes detectable at very low temperatures.

According to the model comparison using relaxation dispersion data measured $263 \mathrm{~K}$, the residues in the $\alpha$-helices in general are rather involved in a three-site exchange than twosite exchange. The residues in the $\beta$ hairpin showed no general trend, indicating that the region might also be involved in an additional exchange event but with a much smaller exchange contribution than in case of the $\alpha$ helices.
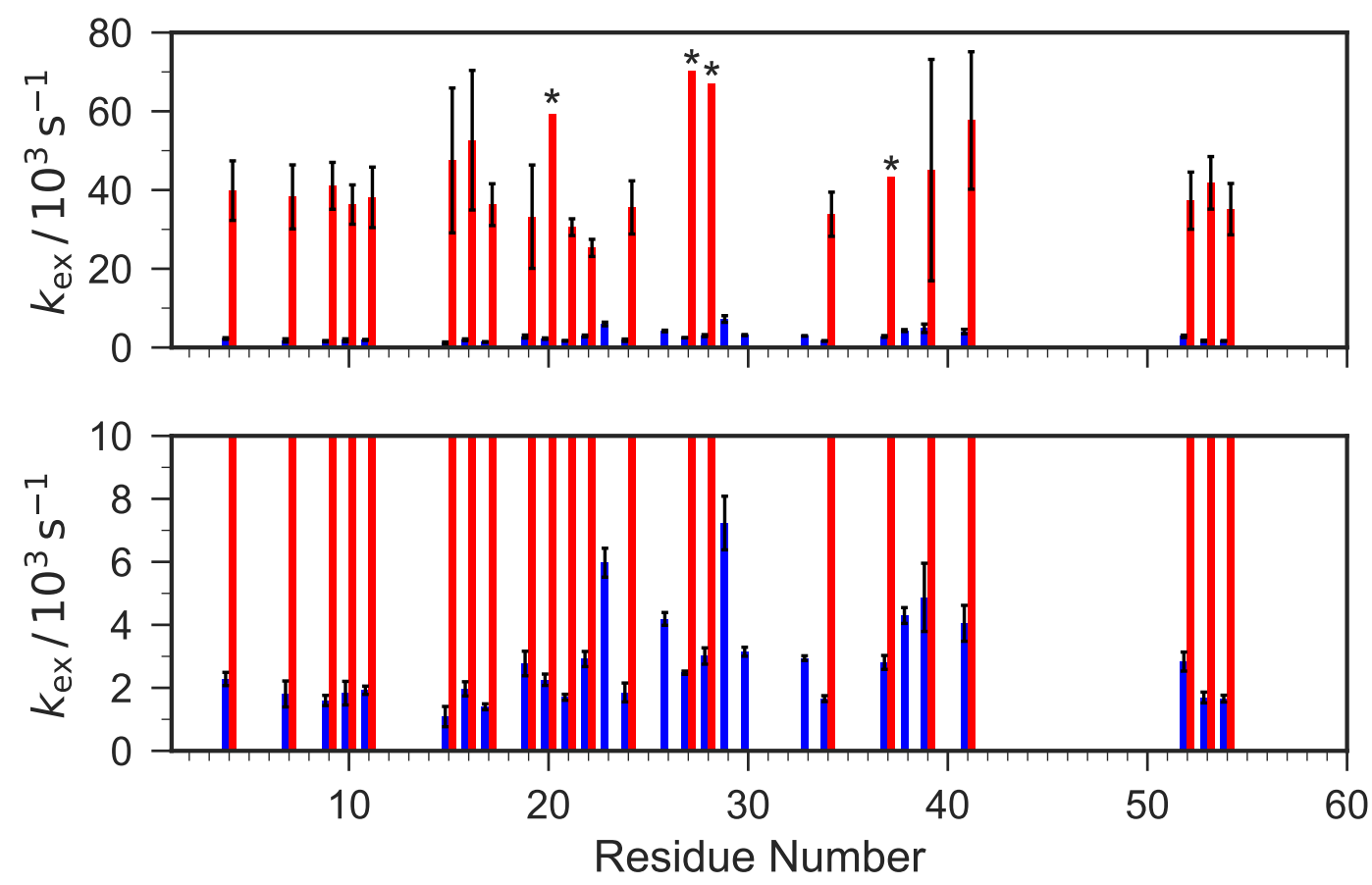

Figure 3.10: Exchange rates obtained from ${ }^{15} \mathrm{~N}$ E-CPMG experiments at $263 \mathrm{~K}$. Residues fitted to the two-state model give a single $k_{\mathrm{ex}}$ value (blue), whereas the three-state model fitting provides two different $k_{\text {ex }}$ values, $k_{\text {ex }}^{\mathrm{F}}$ (red) and $k_{\text {ex }}^{\mathrm{S}}$ (blue). The estimated values of $k_{\text {ex }}^{\mathrm{F}}$ exceed the detection limit of ${ }^{15} \mathrm{~N}$ E-CPMG experiments, thus show large uncertainties. The results of $k_{\mathrm{ex}}^{\mathrm{S}}$ are comparable with the exchange rates estimated with the two-state model for residues in the $\beta$-hairpin region. Residues marked with an asterisk $\left(^{*}\right)$ have an uncertainty larger than $100 \%$. 
The individually fitted exchange rates of residues in the $\alpha$-helices, using the three-site exchange model (Equation (3.2)), are shown together with the results of the $\beta$-hairpin residues, using the two-site exchange (Equation (2.20)), in Figure 3.10. The slow rates obtained from both models are comparable for the two secondary structure elements. At this point it is not clear if these are two distinct conformational changes that take place on the same time scale or if the detected chemical shift variances in both regions result from the same conformational change. The fast rates obtained from the three-site exchange model showed a larger uncertainty and a variation of roughly $30000 \mathrm{~s}^{-1}$. Since the RD profiles of these residues were not fully decayed, large uncertainties in the fit results of the fast process were expected (Chapter 2). Nevertheless, a global analysis for the residues in the $\alpha$-helices was performed. For this it was assumed that both, fast and slow, exchange processes were global events. Thus, the fit was performed using Equation (3.2) and $k_{\mathrm{ex}}^{\mathrm{F}}$ and $k_{\mathrm{ex}}^{\mathrm{S}}$ as global parameters. Examples of the resulting model curves are shown in Figure 3.11. The global model was in good agreement with the relaxation dispersion data, although the fast exchange process could only be inaccurately characterized.
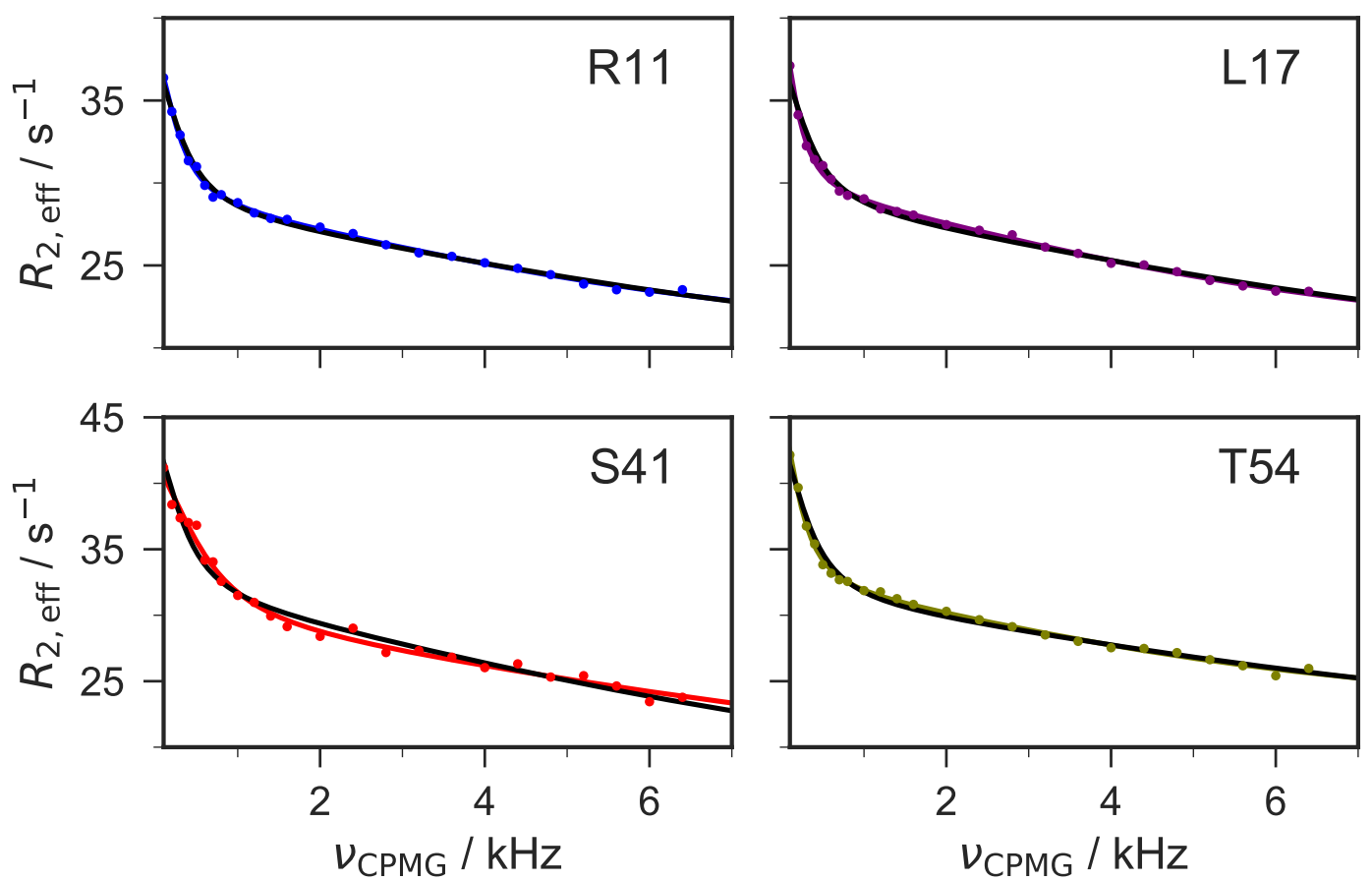

Figure 3.11: Comparison of individually and globally fitted three-state exchange model at $263 \mathrm{~K} .{ }^{15} \mathrm{~N}$ E-CPMG obtained RD profiles of residues R11, L17, S41 and T54 are shown with individually (color) and globally fitted (black) three-state exchange model (Equation (3.2)). The global model shows only marginal differences to the individual fit result. Thus, it can be concluded that residues in the $\alpha$-helices experience a global three-state exchange.

The global exchange rates were $k_{\text {ex }}^{\mathrm{S}}=(2.41 \pm 0.03) 10^{3} \mathrm{~s}^{-1}$ for the slow process and $k_{\mathrm{ex}}^{\mathrm{F}}=(48.05 \pm 5.31) 10^{3} \mathrm{~s}^{-1}$ for the fast exchange process. As already mentioned, the relaxation dispersion profiles were not fully quenched and the fitted kinetic parameters of the fast exchange process do not represent a reliable result, despite the small experimental 
error (Chapter 2). Still it can be stated that residues in the $\alpha$-helices are involved in at least two distinct conformational exchange processes. Firstly, a slow exchange process that takes place on a similar time scale as the exchange process observed for residues in the $\beta$ hairpin region. Thus, it can be hypothesized that the change in the chemical environment that causes the initial decay in the $\alpha$-helical residues is connected to the formation of the $\beta$-strand.

Secondly, a much faster process, that is dominant in the relaxation dispersion profiles of residues in the $\alpha$-helices, but too fast for an accurate characterization of the kinetic parameters using the ${ }^{15} \mathrm{~N}$ E-CPMG experiment.

The difference between the fitted $k_{\mathrm{ex}}^{\mathrm{S}}$ and $k_{\mathrm{ex}}$ of the $\beta$-strand can be rationalized by the interdependence of the fast and slow exchange rate in the three-state model. According to Grey and co-workers the rates $k_{\mathrm{ex}}^{\mathrm{S}}$ and $k_{\mathrm{ex}}^{\mathrm{F}}$ are defined as:

$$
\begin{aligned}
& k_{\mathrm{ex}}^{\mathrm{F}}=\frac{\left(k_{\mathrm{ex}}+Z\right)}{2}, \\
& k_{\mathrm{ex}}^{\mathrm{S}}=\frac{\left(k_{\mathrm{ex}}-Z\right)}{2},
\end{aligned}
$$

with:

$$
Z=\sqrt{\left(k_{\mathrm{ex}}^{2}-4 B\right)}
$$

in which, $k_{\mathrm{ex}}=k_{\mathrm{AB}}+k_{\mathrm{BA}}+k_{\mathrm{AC}}+k_{\mathrm{CA}}$ and $B=k_{\mathrm{BA}} k_{\mathrm{CA}}+k_{\mathrm{AB}} k_{\mathrm{CA}}+k_{\mathrm{BA}} k_{\mathrm{AC}}$ (Grey et al., 2003). Due to the convolution of the rates in the term $B$ and without further knowledge about these individual rates, it can only be stated that the observed exchange rate of the slow process in the three-state exchange model is expected to be smaller than the one obtained from the two-state exchange model. This convolution also prohibited a combined analysis, using both models for a global minimization of the slow exchange rate. The super-cooled sample conditions allowed measurements below the solvent freezing point but are still limited by the decreased freezing point. A further decrease in the temperature would freeze the sample and consequently making solution NMR experiments impossible. Therefore, the fast kinetics could not be slowed down further to allow a characterization using the ${ }^{15} \mathrm{NE}$-CPMG experiment. But for a reliable determination of the exchange parameter the exchange contribution had to be quenched further. In order to achieve this, higher refocusing frequencies had to be applied.

In Chapter 2 the advantages of high $\gamma$ nuclei were already discussed and enables to detect conformational exchange up to single-digit $\mu$ s lifetimes. So far, the ${ }^{15} \mathrm{NE}$-CPMG backbone experiments were residues in the $\alpha$ helices show incomplete quenching of the exchange contribution; the higher applicable $\nu_{\mathrm{CPMG}}$ of the ${ }^{1} \mathrm{HE}$-CPMG experiment was utilized in the same super-cooled conditions, striving for a complete quenching of the exchange contribution observed for residues in the $\alpha$-helices of gpW. 

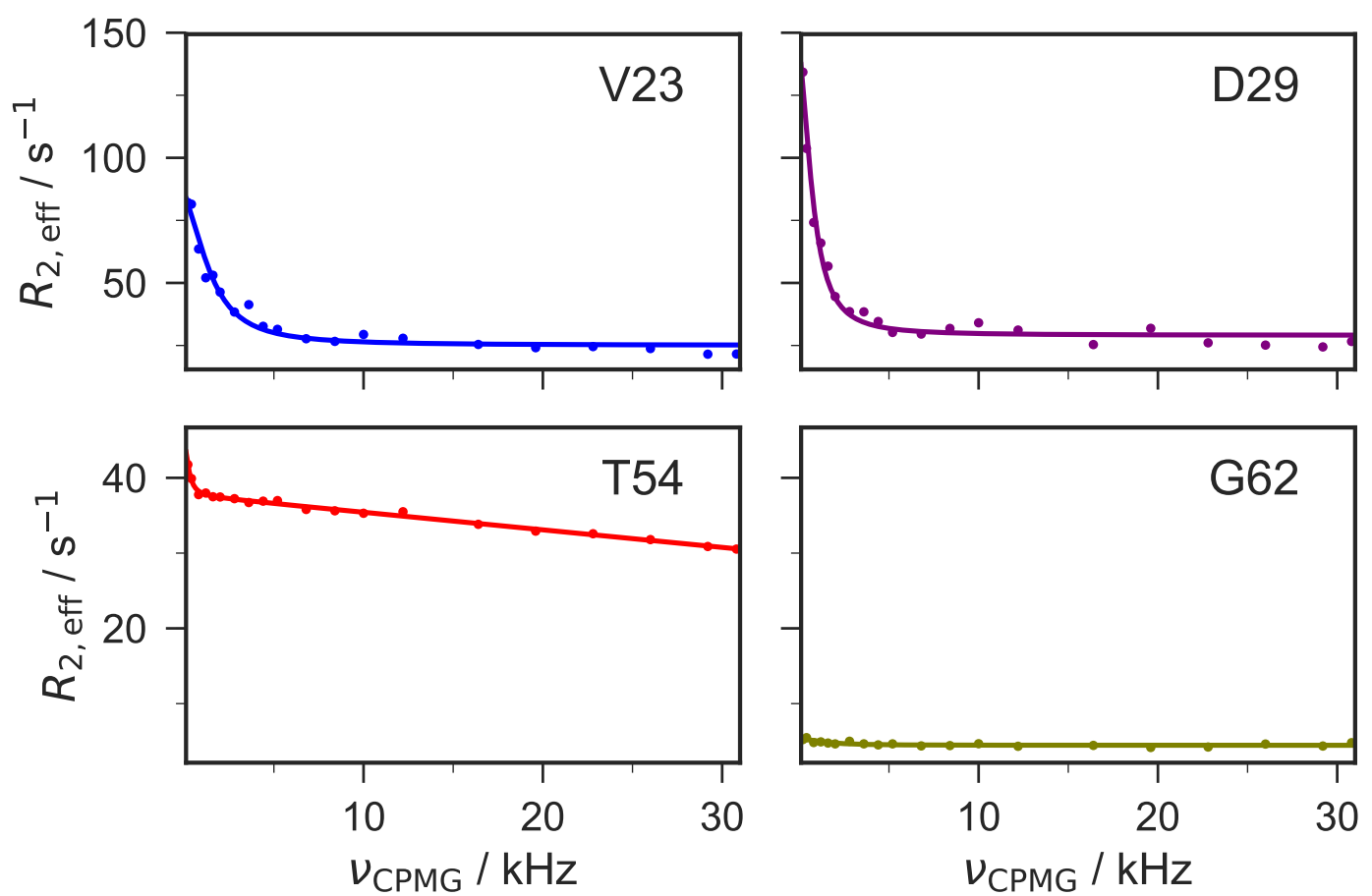

Figure 3.12: Relaxation dispersion profiles obtained from ${ }^{1} \mathrm{H}$ E-CPMG with individually fitted exchange model at $263 \mathrm{~K}$. Displayed residues are located in different structural elements of gpW; V23 ( $\left.\beta_{1}\right)$, D29 (loop), T54 $\left(\alpha_{2}\right)$ and G62 (disordered). The structural elements can be connected to three kind of different relaxation dispersion profiles. Residues located in the $\beta$-hairpin region majorly showed $\mathrm{RD}$ profiles with a large exchange contribution $\left(R_{\mathrm{ex}}\right)$, that could be fully quenched within refocusing frequencies $\left(\nu_{\mathrm{CPMG}}\right)$ of up to $32000 \mathrm{~Hz}$ and fitted to a two-state-exchange model (Equation (2.20)). Residues localized in the $\alpha$-helices showed a three-state exchange (Equation (3.2)) RD profile, that could not be fully quenched in the accessible $\nu_{\text {CPMG }}$ range. C-terminal residues showed flat RD profiles, indicating no conformational exchange.

The acquired data supported the previous findings in the ${ }^{15} \mathrm{NE}-\mathrm{CPMG}$ experiment. Relaxation dispersion profiles of residues in the $\alpha$ helices showed an initial drop in the $\mathrm{RD}$ profile followed by a slower decay of $R_{2 \text {,eff }}$. Residues in the $\beta$-hairpin majorly showed relaxation dispersion profiles of a two-site exchange (Figure 3.12). Furthermore, the flat dispersion profiles of the last two C-terminal residues could be reproduced in the refocusing range of $32 \mathrm{kHz}$. Different than expected, the increased $\nu_{\mathrm{CPMG}}$ range of the ${ }^{1} \mathrm{HE}$-CPMG experiment was still insufficient to quench the slow decay observed for $\alpha$-helical residues. Thus, the underlying exchange process for these residues is expected to be faster than single-digit microseconds.

Figure 3.13 shows the individually fitted exchange rates of residues that either showed a quenched two-state exchange relaxation dispersion profile or a three-state exchange relaxation dispersion profile. Like the previously shown results, fully quenched relaxation dispersion profiles were found in the $\beta$-hairpin region. In addition, residues S41, D42 and L43, which are located at the beginning of second helix, showed RD profiles that could 
be fitted to the two-state exchange model. These residues reported only a fast exchange process in the previous experiments. It could be hypothesized that these amide protons form polar contacts during the folding process and therefore are more sensitive to the slow process than the amide nitrogen. Previous studies showed that the second loop region (includes S41), which connects $\beta_{2}$ and the C-terminal helix, form primary contacts that stabilizes the cooperative folding process (Sborgi et al., 2015).
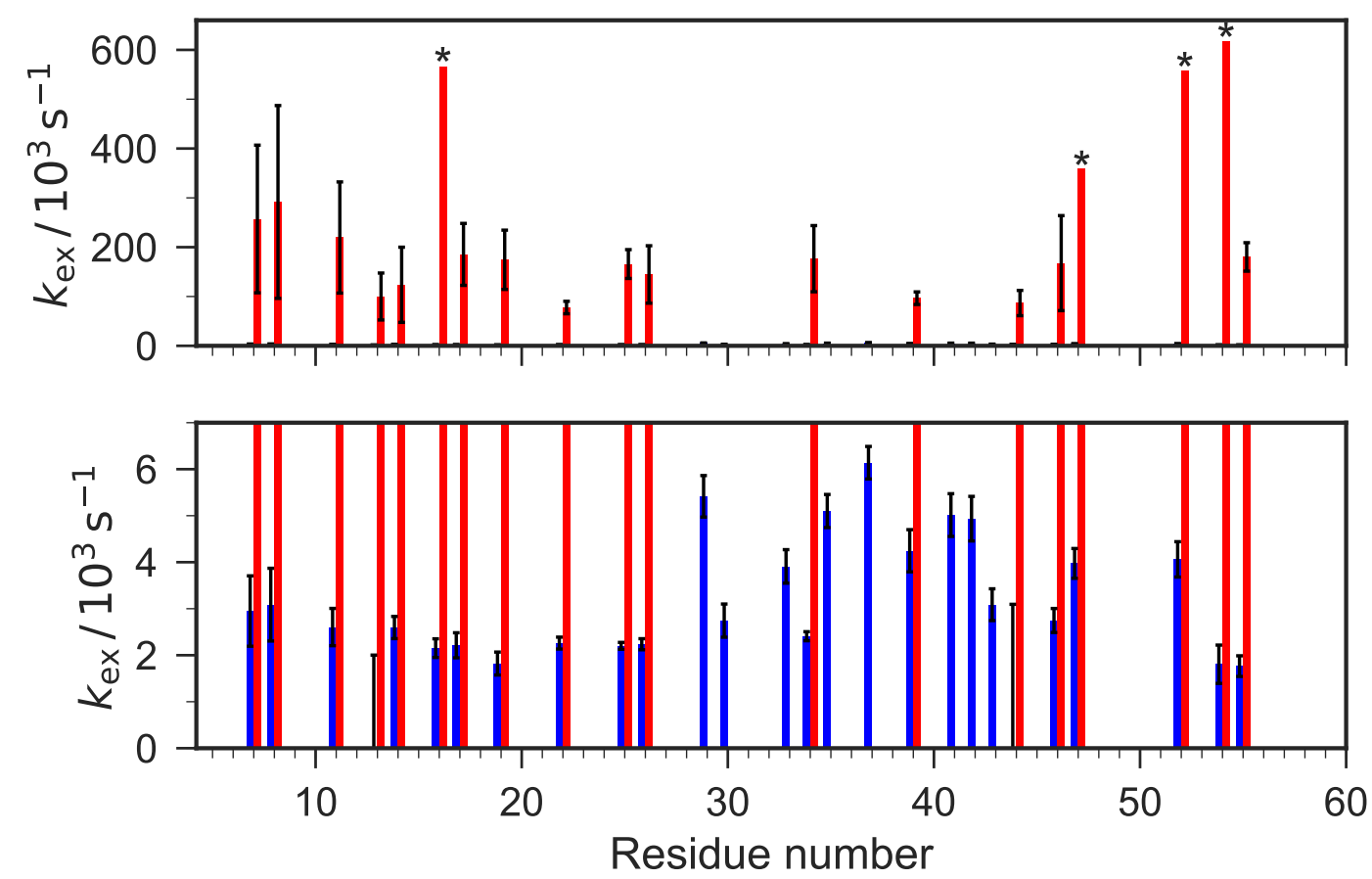

Figure 3.13: Exchange rates obtained from ${ }^{1} \mathrm{H} \mathrm{E-CPMG} \mathrm{experiments} \mathrm{at} 263 \mathrm{~K}$. Residues fitted to the two-state model give a single $k_{\text {ex }}$ value (blue), whereas the three-state model fitting provides two different $k_{\mathrm{ex}}$ values, $k_{\mathrm{ex}}^{\mathrm{F}}$ (red) and $k_{\mathrm{ex}}^{\mathrm{S}}$ (blue). The estimated values of $k_{\mathrm{ex}}^{\mathrm{F}}$ exceed the detection limit of ${ }^{1} \mathrm{HE}-\mathrm{CPMG}$ experiments, thus show large uncertainties. The results for $k_{\mathrm{ex}}^{\mathrm{S}}$ are comparable with the exchange rates estimated with the two-state model for residues in the $\beta$-hairpin region. Residues marked with an asterisk $\left(^{*}\right)$ have an uncertainty larger than $100 \%$.

As already mentioned, the relaxation dispersion profiles of residues in the $\alpha$-helix could not be quenched within the refocusing frequencies $\left(\nu_{\mathrm{CPMG}}\right)$ of up to $32 \mathrm{kHz}$. An analysis of the fast exchange process could only give a hint to the corresponding kinetic parameters, since the fitting still did not lead to accurate results (Chapter 2). This is reflected in the individual fit results that were obtained for the fast exchange rate $\left(k_{\mathrm{ex}}^{\mathrm{F}}\right)$, using Equation (3.2). These varied in a range of roughly $100-600 \mathrm{kHz}$ with uncertainties as large as the fitted parameter. In the same fit the slow exchange rate $\left(k_{\mathrm{ex}}^{\mathrm{S}}\right)$ showed more stable results ranging from $(2-4) 10^{3} \mathrm{~s}^{-1}$. These rates were comparable to the ones derived from the ${ }^{15} \mathrm{~N}$ E-CPMG analysis $\left(7-810^{3} \mathrm{~s}^{-1}\right)$.

Despite the large variation in $k_{\mathrm{ex}}^{\mathrm{F}}$, the same assumptions for a global analysis as for the ${ }^{15} \mathrm{~N}$ RD data were applied. Both processes were considered to be global conformational changes that involve all residues in the $\alpha$-helices. This assumption cannot be assured by 


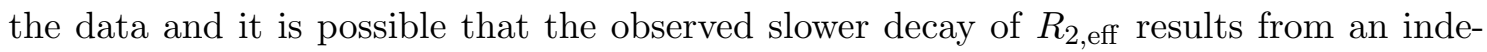
pendent process or multiple processes.

For the slow process an exchange rate of $k_{\mathrm{ex}}^{\mathrm{S}}=(3.03 \pm 0.07) 10^{3} \mathrm{~s}^{-1}$ was fitted, which is in very good agreement with results from the ${ }^{15} \mathrm{~N}$ E-CPMG experiment $\left(k_{\mathrm{ex}}^{\mathrm{S}}=(2.41 \pm\right.$ 0.03) $10^{3} \mathrm{~s}^{-1}$ ). While the global analysis of the ${ }^{15} \mathrm{NE}-\mathrm{CPMG}$ estimated a fast exchange rate of roughly $5010^{3} \mathrm{~s}^{-1}$, the ${ }^{1} \mathrm{H}$ experiment resulted in a four times faster rate $\left(k_{\text {ex }}=\right.$ $\left.(199.48 \pm 25.48) 10^{3} \mathrm{~s}^{-1}\right)$. Considering the lower maximum refocusing frequency accessible by ${ }^{15} \mathrm{~N}$, the large differences in the global fit results can be rationalized in various ways. Neither of the experiments could quantify the kinetics of the potential fast exchange process and it could only be speculated, what kind of structural changes cause the observed relaxation dispersion. Reorientation of the two $\alpha$-helices, a change in the hydrophobic packing as well as an actual unfolding of the helices could be possible.

The extended accessible time scale and simple experimental setup of the E-CPMG experiment allowed a temperature dependent analysis of the detected folding kinetics. For this analysis, experiments between $263 \mathrm{~K}$ and $285 \mathrm{~K}$ for ${ }^{15} \mathrm{~N}$ and $263 \mathrm{~K}$ and $295 \mathrm{~K}$ for ${ }^{1} \mathrm{HE}-\mathrm{CPMG}$ were measured. While conventional CPMG experiments required sample conditions in which the protein kinetics are slowed down for an accurate analysis, the ECPMG experiment can follow kinetics over a wider range of temperatures, still providing accurate kinetic parameters.

Logically, an increase of the temperature results in faster kinetics and according to Equation (2.20) also a decrease in $R_{\mathrm{ex}}$, as it is shown in Figure 3.14. The faster kinetics limit the analysis on the upper end of the temperature scale to the point, where either the $R_{\text {ex }}$ contribution is too small such that $\mathrm{RD}$ profiles become flat or the kinetics exceed the refocusing limit of the experiment and hinder a feasible analysis.

On the other end of the temperature scale the analysis is limited by the freezing point of the solvent. Commonly a mixture of $\mathrm{H}_{2} \mathrm{O}$ and $\mathrm{D}_{2} \mathrm{O}$ is used in protein NMR, leading to a lower temperature limit of around $0{ }^{\circ} \mathrm{C}$. Reducing the freezing point by super-cooled sample conditions can overcome this partially. As already discussed, low temperatures slow down all protein dynamics. Thus, the rotational correlation time $\left(\tau_{\mathrm{c}}\right)$ of a protein decreases which linearly contributes of the intrinsic relaxation rate. In addition, the slowed down kinetics increase the exchange contribution to $R_{2, \text { eff }}$. Both effects add up to broader linewidth and lower intensities at low temperatures. Especially for residues that show a large chemical shift variance, this could result in intensities beyond the detection limit at low refocusing frequencies and hinder an analysis. Thus, even in the super-cooled temperature the study of protein dynamics is limited by the lower sensitivity caused by higher $R_{2, \text { eff }}$, as exhibited by residue D29 at $263 \mathrm{~K}$ (Figure 3.14, A).

In the temperature dependent analysis of gpW kinetics only the exchange process observed in the $\beta$-hairpin region, in which the slow process is the dominant source of $R_{\mathrm{ex}}$, 

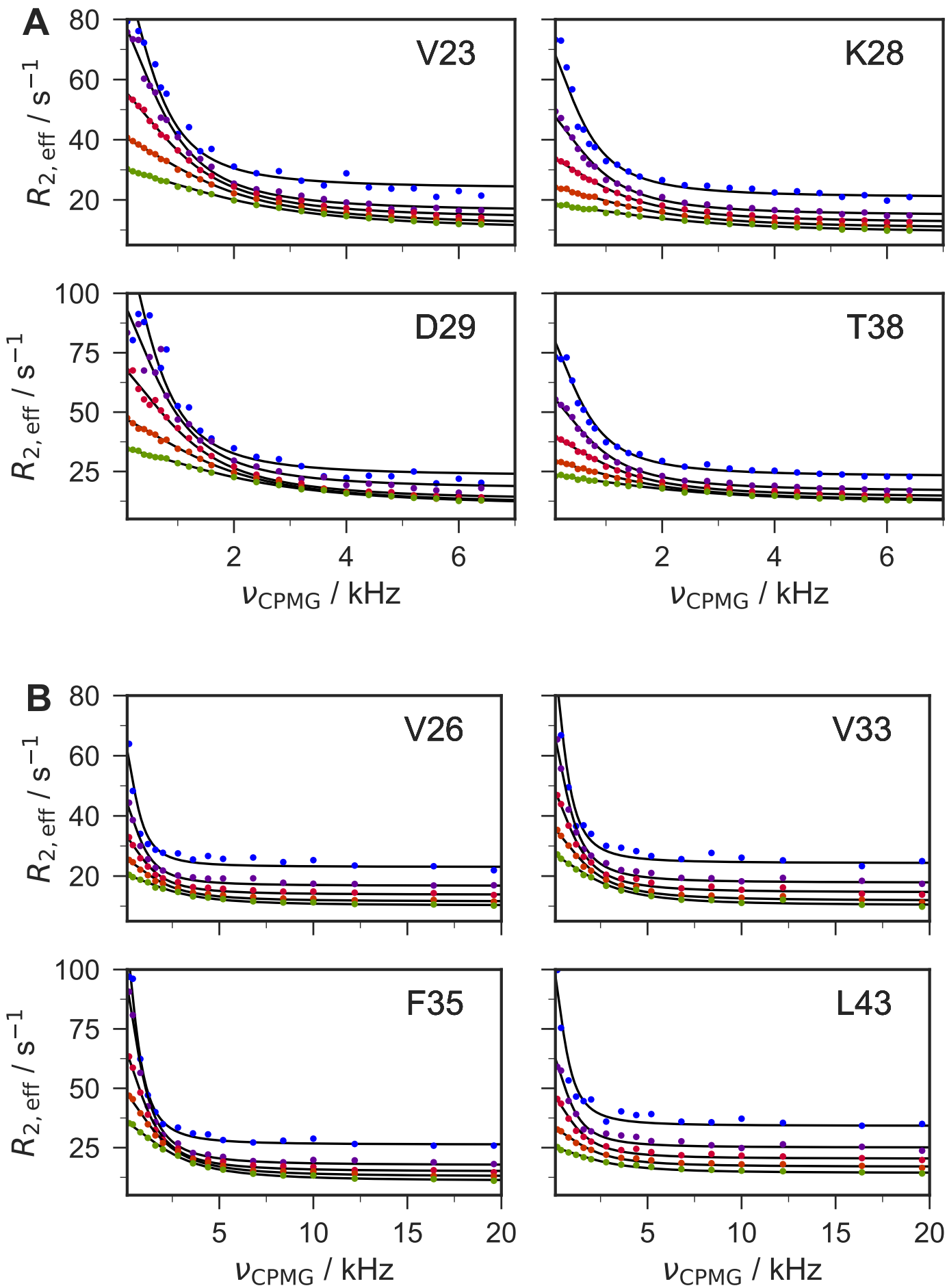

Figure 3.14: Temperature dependent backbone E-CPMG relaxation dispersion profiles of residues in the $\beta$-hairpin region. (A) ${ }^{15} \mathrm{~N}$ Relaxation dispersion data of residues V23, D29, Q27 and T38 with global fits (black line) (B) ${ }^{1} \mathrm{H}$ Relaxation dispersion data of residues V26, V33, F35 and L43 with global fits (black line). The RD data is only plotted up to $\nu_{\mathrm{CPMG}}=20 \mathrm{kHz}$ for a better demonstration. $(\mathrm{A}, \mathrm{B})$ The data was acquired at $263 \mathrm{~K}$ (blue), $270 \mathrm{~K}$ (purple), $275 \mathrm{~K}$ (red), $280 \mathrm{~K}$ (orange) and $285 \mathrm{~K}$ (green). Only residues in the $\beta$-hairpin region that showed a distinct $R_{\mathrm{ex}}$ contribution over the whole temperature range were considered for the analysis. All RD profiles are in very good agreement with the global two-state exchange model. 
could be considered.

In the $\alpha$-helices, the slow process was only visible under super-cooled conditions still showing only a small exchange contribution $\left(R_{\mathrm{ex}}<10 \mathrm{~s}\right)$. At the same temperature and with a similar exchange rate, $\beta$-strand residues showed an exchange contribution of up to $50 \mathrm{~s}^{-1}$. While the data implies a correlation between the two processes, the limited temperature window in which the slow process could be detected in the $\alpha$-helices did not allow a feasible temperature dependent analysis.

Due to the inaccuracy of the fit parameters obtained for the fast exchange process a temperature dependent analysis was also not feasible. Nevertheless the RD profiles of residues in the $\alpha$-helices showed a decreasing trend of the exchange contribution to $R_{2 \text {,eff }}$ with increasing temperature (Figure 3.15). This is expected in case of chemical exchange as it was explained above and can be used as a qualitative indication for the fast exchange process. At $275 \mathrm{~K}$ the slow exchange process is barely detectable in $\alpha$-helical residues, while the dominant $R_{\text {ex }}$ contribution of the fast process is detectable up to $295 \mathrm{~K}$.
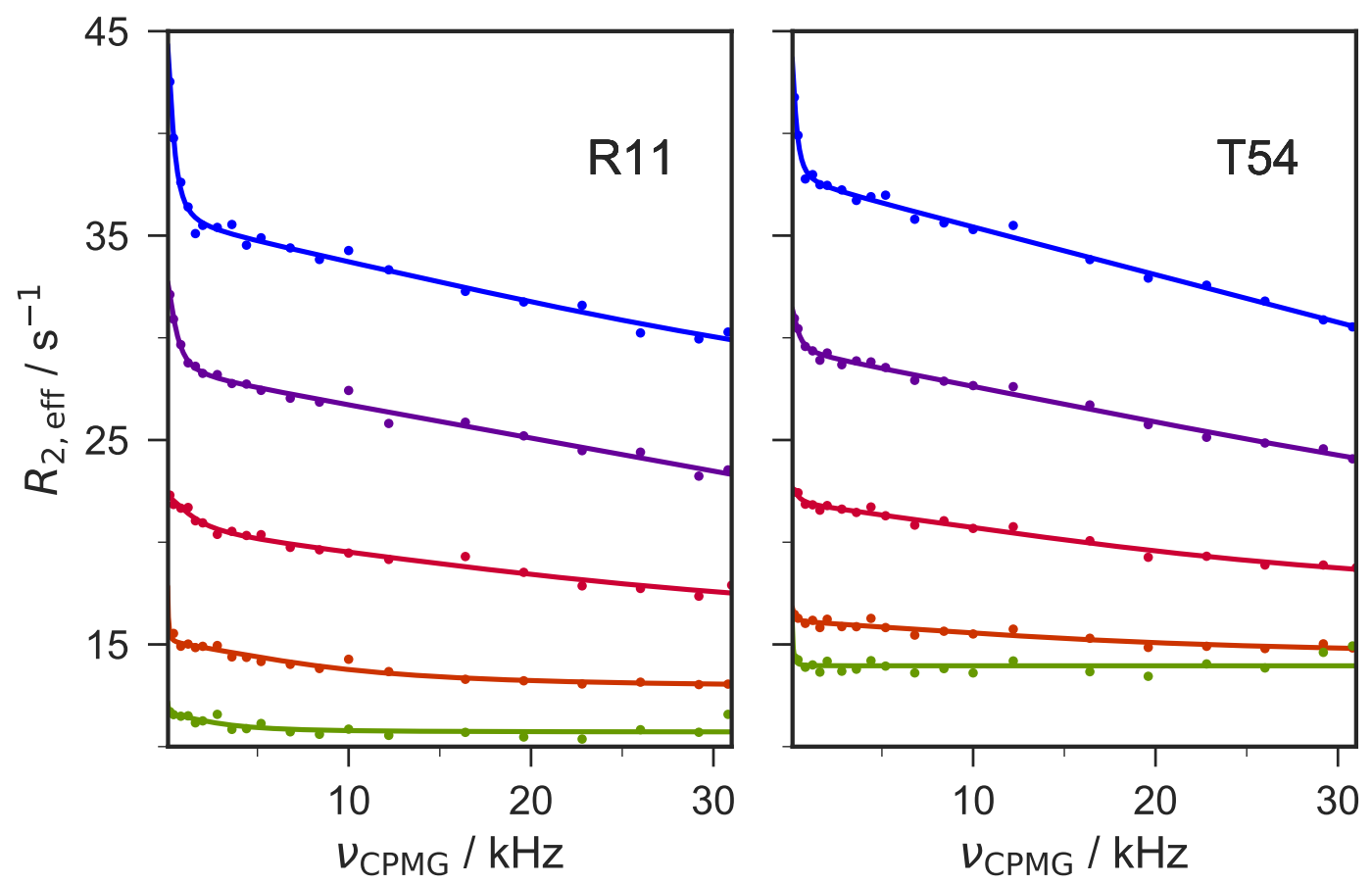

Figure 3.15: Temperature dependent ${ }^{1} \mathrm{H}$ E-CPMG relaxation dispersion profiles of selected residues in the $\alpha$-helices. The RD profiles of residues R11 $\left(\alpha_{1}\right)$ and $\mathrm{T} 54\left(\alpha_{2}\right)$ at $263 \mathrm{~K}$ (blue), $268 \mathrm{~K}$ (purple), $275 \mathrm{~K}$ (red), $285 \mathrm{~K}$ (orange) and $295 \mathrm{~K}$ (green) with best individual fit are shown. The three-state exchange is only detectable below $275 \mathrm{~K}$ and the initial decay in $R_{2 \text {,eff }}$ increases with decreasing temperature. The assumed fast exchange contribution also changes with temperature, supporting the hypothesis of two distinct exchange process involving the $\alpha$-helices. The RD profiles for residues in the $\alpha$-helices appear to be flat at $295 \mathrm{~K}$, because the exchange rate is too fast compared to the chemical shift variance.

Assuming a two-state exchange for residues in the $\beta$-hairpin region the obtained relaxation dispersion profiles were fitted with a global exchange rate using residues that showed a 
distinct conformational amplitude of $>2 \mathrm{~s}^{-1}$ in all measured temperatures. Under this assumption the globally fitted exchange rates (Table 3.1) were analyzed using the Arrhenius model:

$$
\ln \left(k_{\mathrm{ex}}\right)=\ln (A)-\frac{-E_{\mathrm{A}}}{R} \frac{1}{T},
$$

in which, $E_{\mathrm{A}}$ is the activation energy of the exchange process, $R$ is the gas constant and $A$ is a pre-exponential factor, that describes the collision frequency according to collision theory.
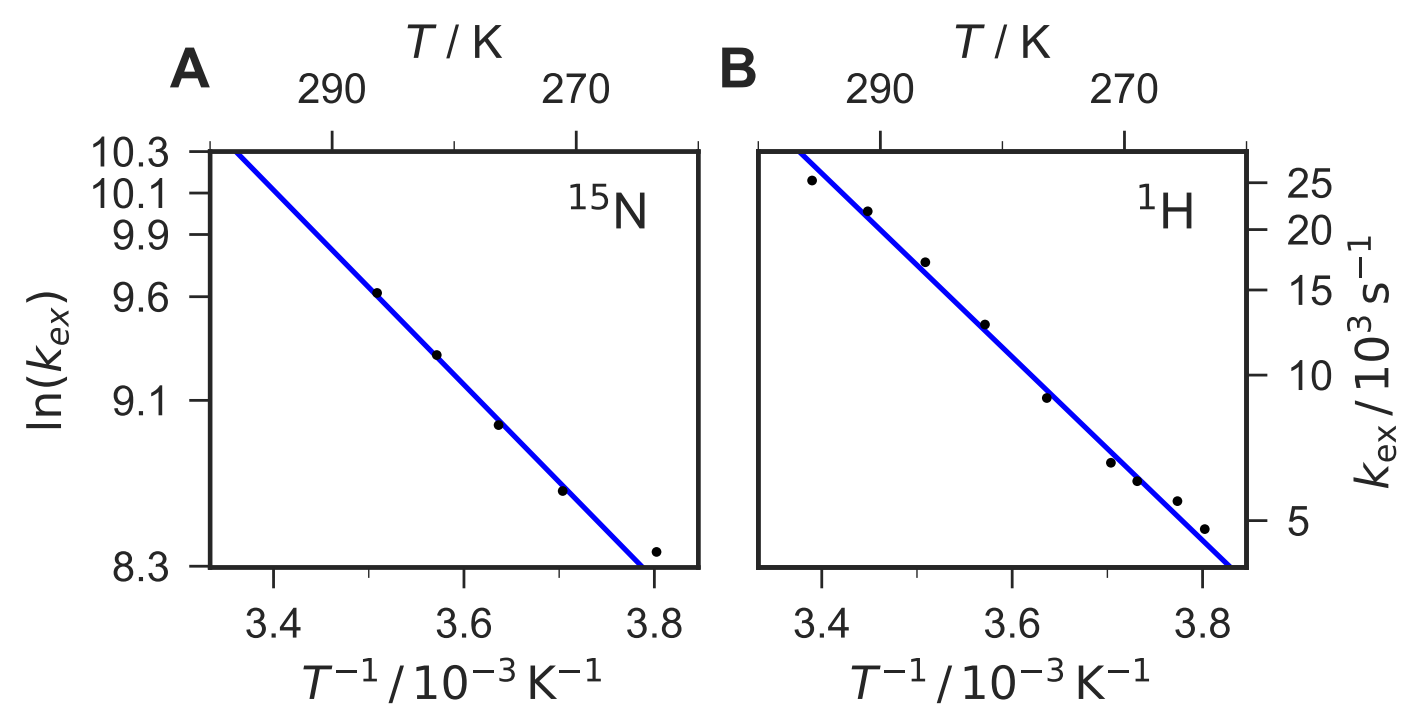

Figure 3.16: Arrhenius analysis of globally fitted exchange rates from gpW backbone E-CPMG data. (A) Arrhenius (Equation (3.8)) fitting of global exchange rates obtained from ${ }^{15} \mathrm{NE}-\mathrm{CPMG}$ experiments. The fitted Arrhenius parameters are $A=(2.11 \pm 2.05) 10^{11} \mathrm{~Hz}$ and $E_{\mathrm{A}}=39.76 \pm 2.25 \mathrm{~kJ} \mathrm{~mol}^{-1}$. An extrapolation to the melting temperature of $\mathrm{gpW}\left(T_{\mathrm{m}}=340 \mathrm{~K}\right)$ resulted in an exchange lifetime of $4.70 \pm 3.77 \mu \mathrm{s}$. (B) Arrhenius (Equation (3.8)) fitting of global exchange rates obtained from ${ }^{1} \mathrm{HE}$-CPMG. The fitted Arrhenius parameters are $A=(7.45 \pm 4.03) 10^{12} \mathrm{~Hz}$ and $E_{\mathrm{A}}=36.35 \pm 1.26 \mathrm{~kJ} \mathrm{~mol}^{-1}$. An extrapolation to $T_{\mathrm{m}}$ resulted in an exchange lifetime of $5.16 \pm 3.62 \mu \mathrm{s}$. Both extrapolated values are in good agreement with previously results from laser induced T-jump experiments $(8.8 \mu \mathrm{s})$ (Fung et al., 2008).

The resulting activation energies from the two-state exchange model fit were $39.8 \pm$ $2.2 \mathrm{~kJ} \mathrm{~mol}^{-1}$ and $36.4 \pm 1.3 \mathrm{~kJ} \mathrm{~mol}^{-1}$ for ${ }^{15} \mathrm{~N}$ and ${ }^{1} \mathrm{H}$, respectively (Figure 3.16). The ${ }^{1} \mathrm{HE}$-CPMG data results in a slightly lower energy barrier than the obtained $E_{\mathrm{A}}$ from the ${ }^{15} \mathrm{~N}$ data set. A possible explanation could be an additional contribution of solvent exchange to the observed relaxation dispersion. Such an exchange would increase the observed exchange rates and thus lead to a lower energy barrier in the temperature dependent analysis.

The fitted values of the collision number $A$ were $(2.11 \pm 2.05) 10^{11} \mathrm{~s}^{-1}$ and $(7.45 \pm$ 4.03) $10^{10} \mathrm{~s}^{-1}$ for ${ }^{15} \mathrm{~N}$ and ${ }^{1} \mathrm{HE}-\mathrm{CPMG}$, respectively. Both results showed large uncertainties, that could be rationalized as following. In a chemical reaction $A$ accounts for the 
frequency of collisions in the correct orientation that lead to a reaction. In the application to folding kinetics it would correspond to an upper limit of the exchange process at an infinite temperature. During the fit this value has to be extrapolated from the limited temperature range that is accessible. Small changes in the activation energy that describes the slope of the model curve, will have a large effect on the estimated value of $A$. While the fitted activation energies of the two data sets show similar results, the estimated $A$ value differs by a factor of roughly three. Thus, the narrow range of temperatures that is feasible for biomolecules does not allow an accurate estimation of this parameter.

Using the results of the Arrhenius analysis the kinetics at the melting temperature of gpW $\left(T_{\mathrm{m}}=340 \mathrm{~K}\right)$ could be extrapolated. This led to an exchange lifetime $\left(1 / k_{\mathrm{ex}}\right)$ of $4.70 \pm 3.77 \mu \mathrm{s}$ and $5.16 \pm 3.62 \mu \mathrm{s}$ for ${ }^{15} \mathrm{~N}$ and ${ }^{1} \mathrm{H}$ data, respectively. Both results are in very good agreement with the $8.8 \mu$ s reported by laser-induced T-jump experiments in the literature (Fung et al., 2008). These results indicate that the partial unfolding of gpW in solution can be described by a two-state exchange model with an energy barrier higher than $\approx R T$ as it would be assumed for an ultra-fast folding protein.

In summary the application of the E-CPMG experiment to the amide backbone nuclei of gpW added two new residues V23 and D29 to the analysis, which were formerly broadened beyond detection under conventional CPMG conditions. Furthermore a slow decay of $R_{2, \mathrm{eff}}$ that was primarily seen in residues located in the $\alpha$-helical region was detected (Figure 3.17). This indicates that gpW in solution is involved in an additional exchange process, which majorly involves the $\alpha$-helices (Figure 3.17). A full characterization of this second exchange process was not possible due to insufficient quenching of the exchange contribution. Nevertheless, the ${ }^{1} \mathrm{H}$ E-CPMG experiment suggested that the corresponding exchange rate is similar to or faster than $200000 \mathrm{~s}^{-1}$.

The combined use of super-cooled sample conditions and the E-CPMG approach revealed relaxation dispersion profiles that showed a discrepancy to the two-state exchange model. The use of a three-site exchange model showed a better accordance to the data. A comparison of the two model fits by the corrected Akaike information criterion supported the assumption that these residues are involved in a three-site exchange process. In this model the fitted $k_{\text {ex }}^{\mathrm{S}}$ indicated that the $\alpha$-helices are also affected by an exchange process that takes place on the same time scale as the one observed in the $\beta$-hairpin region.

These findings support the hypothesis of the hydrophobic collapse, where the preformed $\alpha$-helices form the hydrophobic core followed by the folding of the $\beta$-hairpin region as the last step of the folding process. In this scenario the large exchange contributions seen in the RD profiles of residues in the $\beta$-strands can be explained by the transition from a disordered to an ordered structure. In this conformation new intermolecular contacts and less solvent exposure cause the chemical environment of the involved nuclei to change largely. In the same folding process, the $\alpha$-helices are already formed and close contacts of the backbone amide nuclei do not change. The $\Phi$ values of these residues are expected to be smaller than for residues in the $\beta$-hairpin. Thus, a smaller exchange contribution to the transverse rate is expected and the folding process becomes visible only when the 
kinetics are slowed down at very low temperatures.
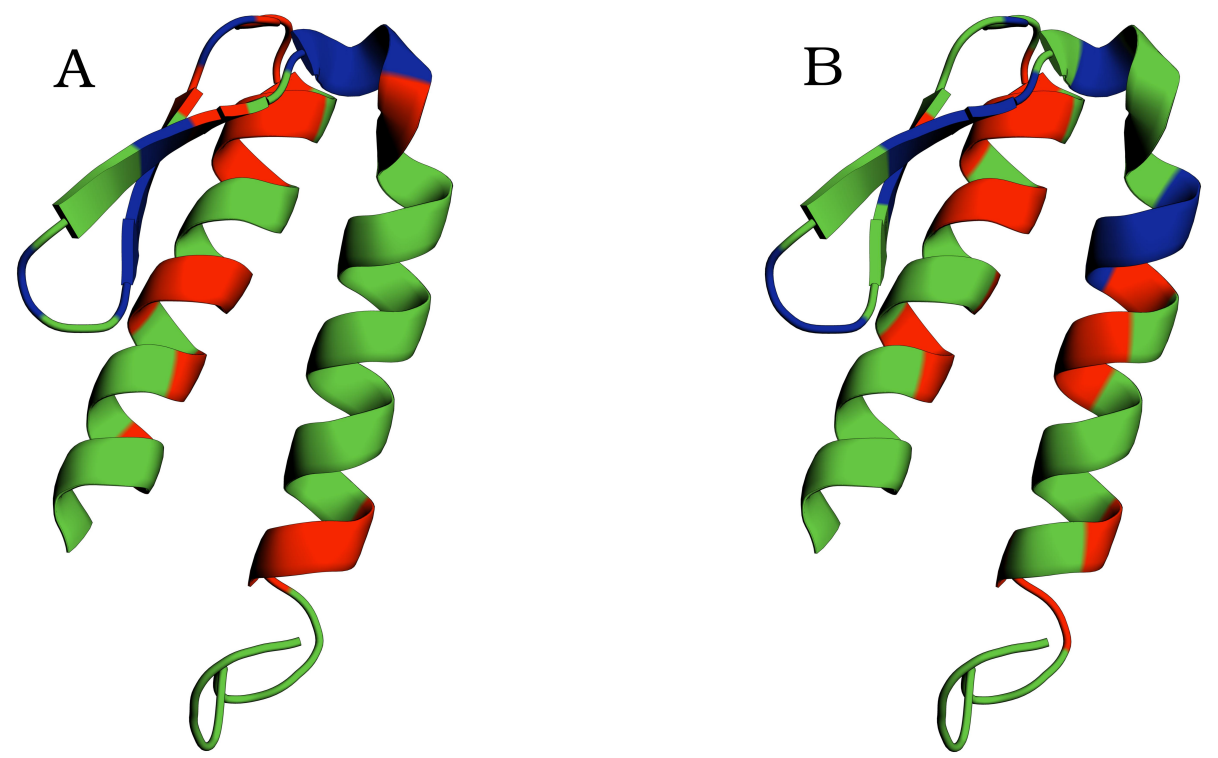

Figure 3.17: Structure of gpW with highlighted backbone residues showing relaxation dispersion. Residues used in the global analysis are highlighted in red and blue for the three and two-site exchange model, respectively (pdb: 2l6r (Sanchez-Medina et al., 2014)). (A) Global results from the ${ }^{15} \mathrm{~N}$ E-CPMG experiments and (B) ${ }^{1} \mathrm{H}$ E-CPMG experiments at $263 \mathrm{~K}$ are shown. The three-site exchange model majorly describes the relaxation dispersion profiles of residues in the $\alpha$-helices. The two-site exchange model dominates in the $\beta$-hairpin region. Residues in the loop region show no preference to one of the models.

For the slow exchange process a temperature dependent analysis of the exchange rates was done, assuming a two-state exchange. The obtained fit parameters were extrapolated to the melting temperature of gpW and were in good agreement with results reported from thermodynamic methods in the literature (Fung et al., 2008).

The results obtained from the backbone E-CPMG data support the assumption, that in solution folded gpW is in an equilibrium with a partially unfolded state of the $\beta$-hairpin region.

To further analyze the folding pathway of gpW and the role of the $\alpha$ helices in the $\beta$ hairpin formation, E-CPMG experiments on side-chain methyls were used as an additional probe. 


\subsubsection{Methyl Side-chain E-CPMG Experiments}

For studying the role of the hydrophobic core, formed by the side-chains of the residues located in the two $\alpha$-helices, a specially labeled gpW sample was used. Only methyl groups of the residues leucine, isoleucine and valine were labeled as $\mathrm{CHD}_{2}$. In total 22 resolved resonances could be analyzed, whose corresponding methyl groups were located in the $\alpha$-helical as well as in the $\beta$-hairpin region (Figure 3.2). Thus, it is expected that the side-chain experiments yield kinetic information about the $\beta$-hairpin as well as $\alpha$-helix motions.
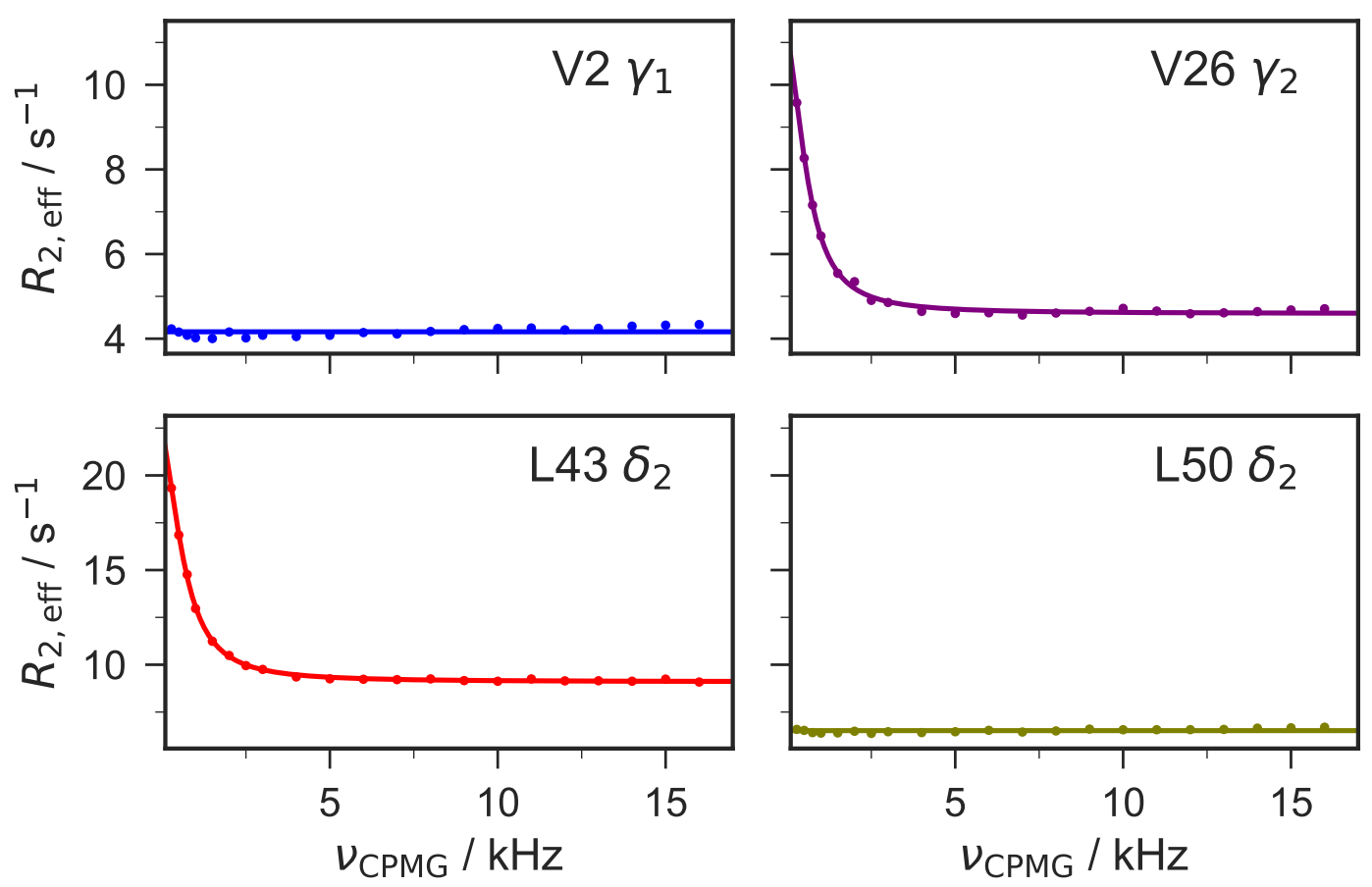

Figure 3.18: Relaxation dispersion profiles obtained from ${ }^{13}$ C E-CPMG with individually fitted exchange model at $275 \mathrm{~K}$ of methyl side-chains. Displayed residues are located in different structural elements of gpW, V2 $\gamma_{1}\left(\alpha_{1}\right)$, V26 $\gamma_{2}\left(\beta_{1}\right)$, $\mathrm{L} 43 \delta_{2}\left(\alpha_{2}\right)$ and L50 $\delta_{2}\left(\alpha_{2}\right)$. Methyl groups located in the $\beta$-helices as well as in the $\alpha$-sheets showed relaxation dispersion. The latter only reported an exchange event when pointing towards the interface of the two helices $\left(\mathrm{L} 43 \delta_{2}, \mathrm{~L} 50 \delta_{2}\right)$ and showed flat RD profiles when pointing outwards (V2 $\gamma_{1}$; Figure 3.2).

Initial ${ }^{13} \mathrm{C}$ E-CPMG experiments were measured at $275 \mathrm{~K}$ and eight methyl groups with an exchange contribution to their intrinsic relaxation rate could be identified. Hereby, only relaxation dispersion profiles with a significant exchange contribution $\left(R_{\mathrm{ex}}<2 \mathrm{~s}^{-1}\right)$ were considered. Like the previously shown experiments for the backbone amides, methyl groups that are located in the $\beta$-hairpin showed relaxation dispersion (Figure 3.18). In addition, relaxation dispersion was detected for methyl side-chains located in the $\alpha$-helices. Taking a closer look at the structure of gpW revealed, that dispersion was detectable when a methyl group points towards the interface of the two $\alpha$-helices or towards the $\beta$-hairpin. Methyl groups pointing outwards on the contrary, such as V2 and V52, which are located 
at the beginning of helix 1 and at the end of helix 2, showed flat relaxation dispersion profiles (Figure 3.18).

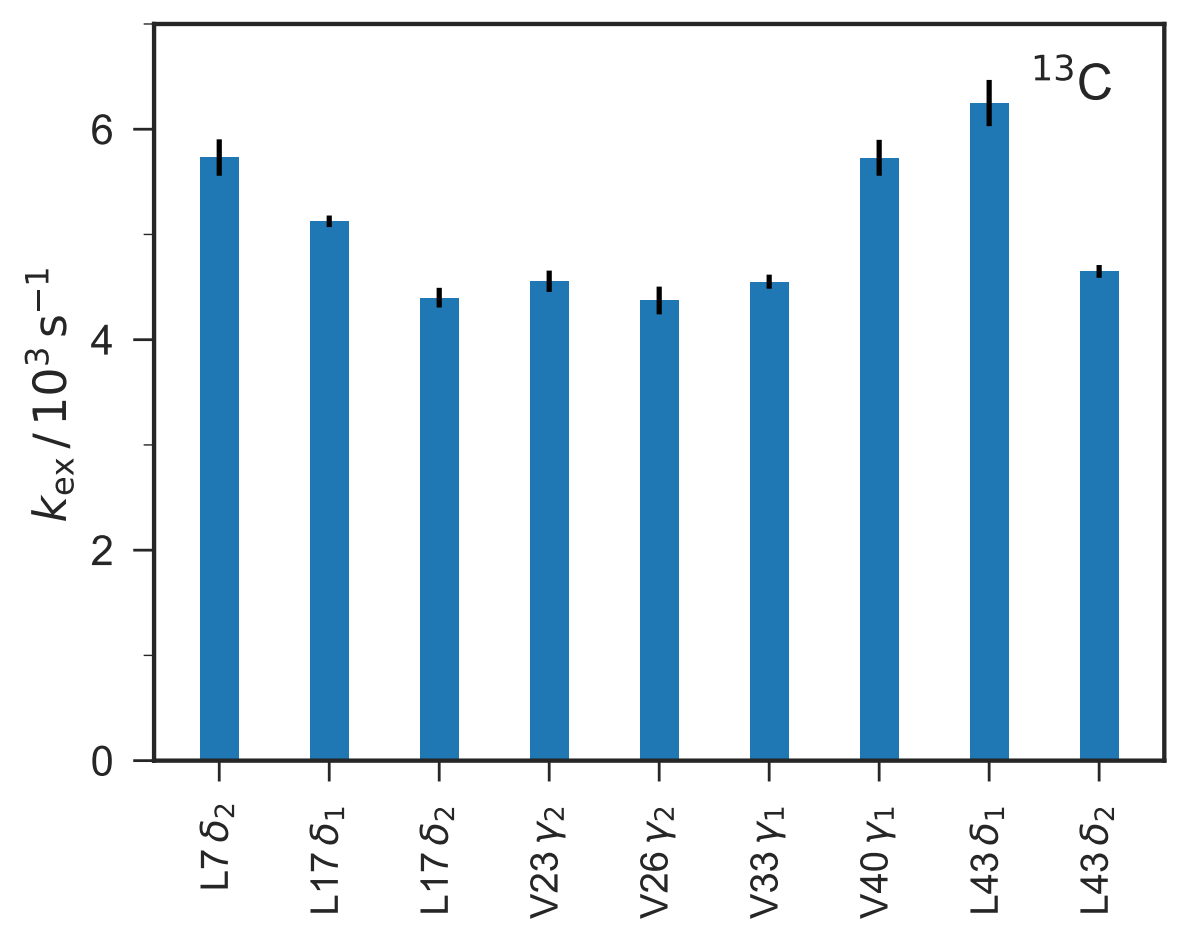

Figure 3.19: Exchange rates from individually fitted ${ }^{13} \mathrm{C}$ E-CPMG data at 275 K. Exchange rates were extracted by fitting the RD profiles obtained from ${ }^{13} \mathrm{CE}-$ CPMG to the Luz-Meiboom model (Equation (2.20)). Residues with an exchange contribution of $<2 \mathrm{~s}^{-1}$ were not considered for the analysis. All methyl groups were fitted to similar exchange rates, indicating a global exchange process. The results were also comparable to rates obtained from amide E-CPMG experiments for residues in the $\beta$-hairpin region.

A residue-wise analysis of the relaxation dispersion profiles resulted in similar exchange rates for the individual residues. The exchange rates in the range of $(4-7) 10^{3} \mathrm{~s}^{-1}$ were comparable with the results from the backbone CPMG experiments, while being generally slower than in the $\beta$-sheet amides at $275 \mathrm{~K}$. For residues V23 and V26 the kinetic rates were expected to be similar to the observed backbone $\beta$-sheet rates, assuming that they are similarly affected by the formation of the $\beta$-hairpin structure. The methyl groups located in the $\alpha$-helical residues did not report the dominating fast process that was observed in the backbone E-CPMG experiments $\left({ }^{1} \mathrm{H}\right.$ and $\left.{ }^{15} \mathrm{~N}\right)$. The fitted rates from methyl groups in the $\alpha$-helical residues were rather comparable with the slow exchange process, observed in backbone under super-cooled conditions. This supports the hypothesis that the $\alpha$-helices are involve in at least two distinct exchange processes in solution. The results also allow the hypothesis that the observed exchange processes in the methyl side-chains is correlated to the exchange process observed in the $\beta$-hairpin region of the gpW. 
The individual fitted exchange rates at $275 \mathrm{~K}$ showed a larger spread compared to the results gained from the backbone data. Nevertheless, due to the similarity to the previous results for $k_{\mathrm{ex}}$ obtained from the $\beta$-hairpin backbone analysis, a similar two-state exchange process was assumed and the relaxation dispersion profiles were fitted globally with a common exchange rate (Figure 3.20).
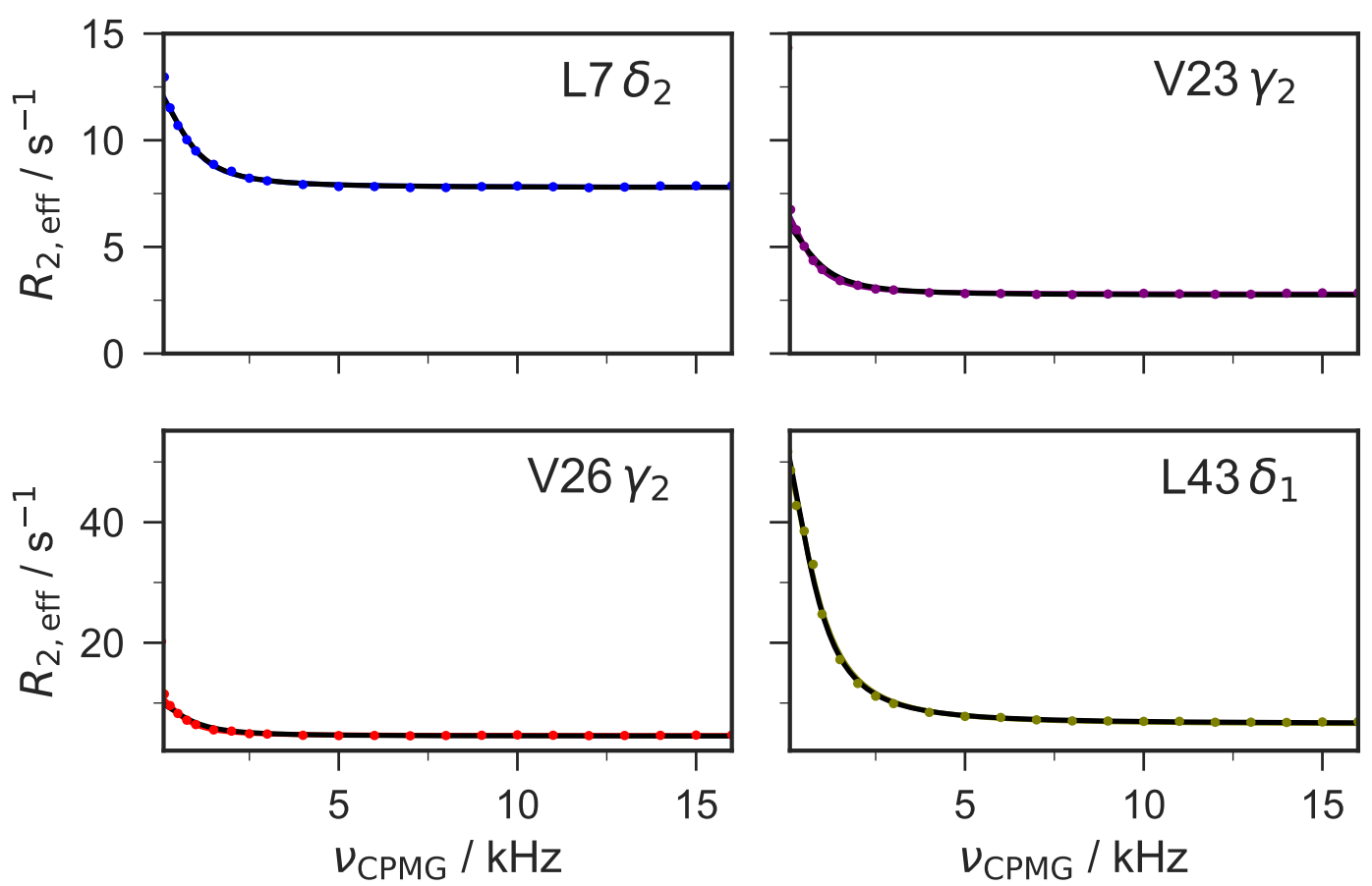

Figure 3.20: Comparison of individually and globally fitted ${ }^{13} \mathrm{C} R \mathrm{R}$ data at $275 \mathrm{~K} .{ }^{13} \mathrm{CE}$-CPMG obtained RD profiles of residues L7 $\delta_{2}, \mathrm{~V} 23 \gamma_{2}, \mathrm{~V} 26 \gamma_{2}$ and L43 $\delta_{1}$ are shown with individually (color) and globally fitted (black) Luz-Meiboom model (Equation (2.20)). The global model shows only marginal differences to the individual fit result. Thus, it can be concluded that the methyl side-chains are involved in a global exchange process.

The global fit was in very good agreement with the data and an exchange rate of $k_{\text {ex }}=$ $(4.87 \pm 0.04) 10^{3} \mathrm{~s}^{-1}$ was estimated. This estimated $k_{\mathrm{ex}}$ is smaller than the global $k_{\text {ex }}$ of the slow exchange process observed in the backbone $\beta$-hairpin at $275 \mathrm{~K}$ ( $k_{\text {ex }}$ of approximately $810^{3} \mathrm{~s}^{-1}$ and $910^{3} \mathrm{~s}^{-1}$ for ${ }^{15} \mathrm{~N}$ and ${ }^{1} \mathrm{H}$, respectively). Still it could be assumed that both, backbone and side-chain, report the same exchange process. Previous structural studies (Sborgi et al., 2015) of gpW identified the residues L7, L14, L17, V33, V40 and L43 to stabilize the hydrophobic core and assist in the hairpin formation. The results from the ${ }^{13} \mathrm{C}$ relaxation dispersion experiments correlate with these residues. Thus, it can be assumed that the formation of the hydrophobic core and the $\beta$-hairpin folding are either directly coupled or form at the same time.

Differently than in the previously presented backbone data, there was no indication of a deviation to the two-state exchange model in the methyl relaxation dispersion profiles. Neither was a fast exchange process observed as for the backbone amides in the $\alpha$-helices. 
All methyl relaxation dispersion profiles were either fully quenched in the accessible $\nu_{\text {CPMG }}$ range (up to $16000 \mathrm{~Hz}$ ) or showed no dispersion.

Interestingly, a difference in the RD profiles of $\delta_{1 / 2}$ and $\gamma_{1 / 2}$ methyl groups in the same side-chain could be observed (Figure 3.19). Only residue L17 and L43 reported similar exchange rates (Figure 3.19) in both methyl groups. For the others only one of the two methyl groups reported a notable exchange contribution. In total five methyl side-chains showed this phenomenon and the relaxation dispersion data of residue V26 and V33 are shown as an example in Figure 3.21. In both cases the intrinsic relaxation rate $R_{2,0}$ was the same, while the exchange contribution of one of the methyl groups was negligible $\left(R_{\mathrm{ex}}<1 \mathrm{~s}^{-1}\right)$. Thus, the internal dynamics, which contribute to $R_{2,0}$, of the methyl groups are the same but the exchange contribution is different. Since one of the methyl groups showed relaxation dispersion it could be assumed that the side-chain is involved in exchange process. Then the only explanation for the variation in $R_{2 \text {,eff }}$ could result from a difference in the chemical shift variation $(\phi)$ of the two methyl groups in one side-chain.
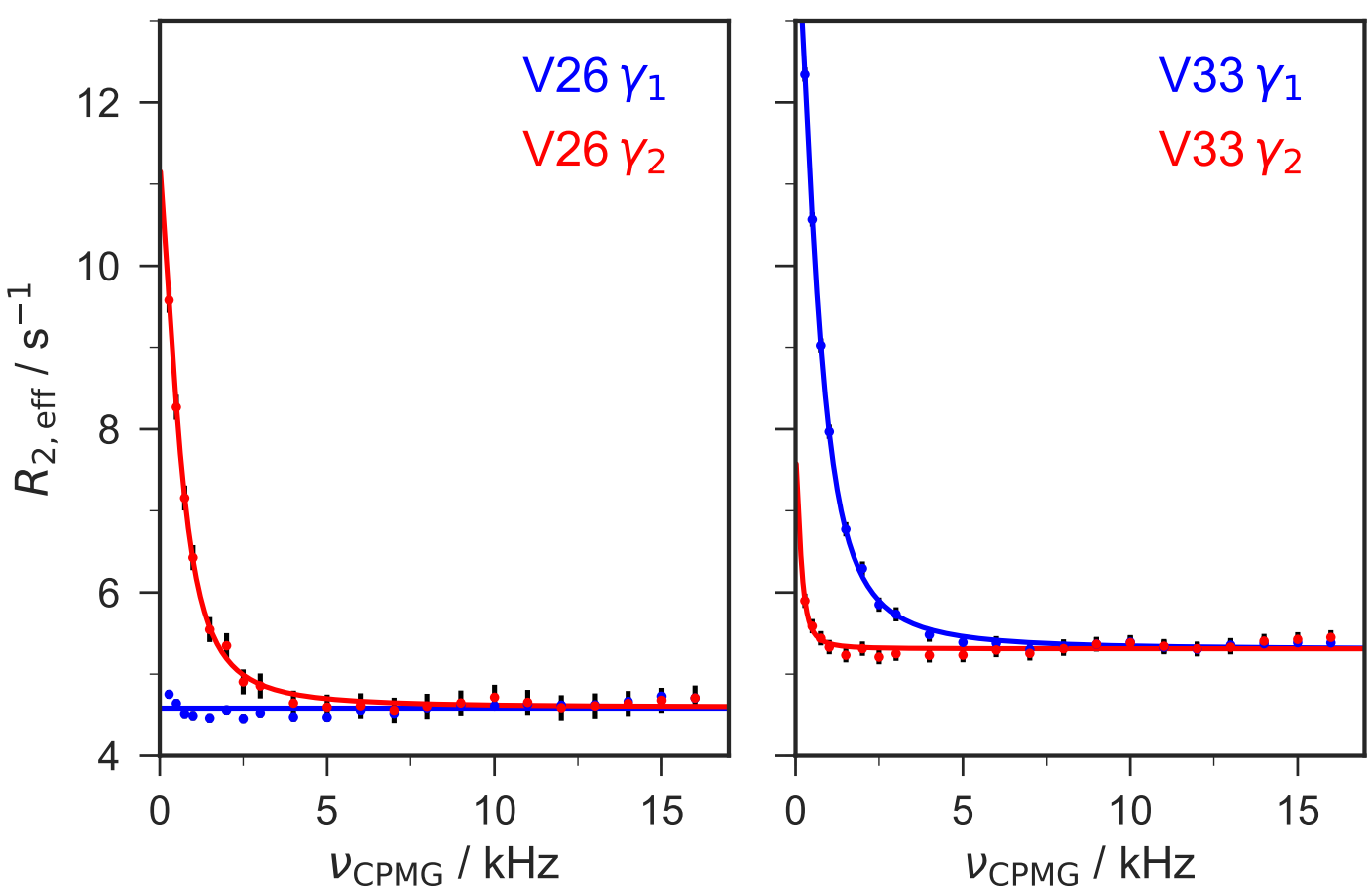

Figure 3.21: Comparison of ${ }^{13} \mathrm{C}$ relaxation dispersion data of methyl groups in the same residue at $275 \mathrm{~K}$. RD profiles of methyl groups $\gamma_{1}$ (blue) and $\gamma_{2}$ (red) of residues V26 and V33 are shown. Both show a significant difference in the exchange contribution to the intrinsic transverse relaxation rate. In case of free rotation around the C-C axis, both should report the same average kinetics. Thus, a hindrance in the free rotation that causes differences in the chemical shift variation of the two methyl groups is suspected.

In case of an equal interconversion between the rotameric states of the side-chains of leucine and valine an averaged contribution of the chemical shift changes upon chemical exchange would be expected. Consequently, the relaxation dispersion profiles should re- 
port an averaged information about the kinetics, but the shown RD profiles in Figure 3.21 are significantly different. A hindrance to the free rotation around the $\mathrm{C}-\mathrm{C}$ axis could than explain the observed deviation. In such a case one methyl group would be more affected by the chemical environment changes and thus would show a larger exchange contribution. Smith et al. (Smith et al., 2015) postulated a population shuffling model were the interconversion between rotameric states (microstates) exchanges much faster than the observed chemical exchange (macrostates) by relaxation dispersion experiments. The latter causes changes in the free energies of the rotameric states, namely trans, gauche+ and gaucheThe population changes of these microstates further reduce the chemical shift variance $\Phi_{\text {ex }}$ according to:

$$
\Phi_{\mathrm{ex}}=p_{\mathrm{A}} p_{\mathrm{B}} \delta p_{\mathrm{A}->\mathrm{B}}^{\mathrm{rot}} \Delta \omega_{\gamma}^{2},
$$

in which, $\delta p_{\mathrm{A}->\mathrm{B}}^{\mathrm{rot}}$ is the population difference between the rotameric states, $\Delta \omega_{\gamma}^{2}$ is the chemical shift difference caused by the $\gamma$ effect and $p_{\mathrm{A}}$ and $p_{\mathrm{B}}$ are the populations of the macrostates (Smith et al., 2015). The population shuffling model could explain the differences in the observed relaxation dispersion profiles of $\gamma$ and $\delta$ methyl groups of gpW but was not quantitatively proven during the course of this thesis.

The ${ }^{13} \mathrm{CE}$-CPMG experiment further indicated that the formation of $\beta$-hairpin region is connected to a hydrophobic collapse of the $\alpha$-helices. The fast exchange process that was observed for amides in the $\alpha$-helices could not be detected in the side-chains. To cross-validate these results, ${ }^{1} \mathrm{HE}$-CPMG experiments were also measured on the methyl side-chains. 

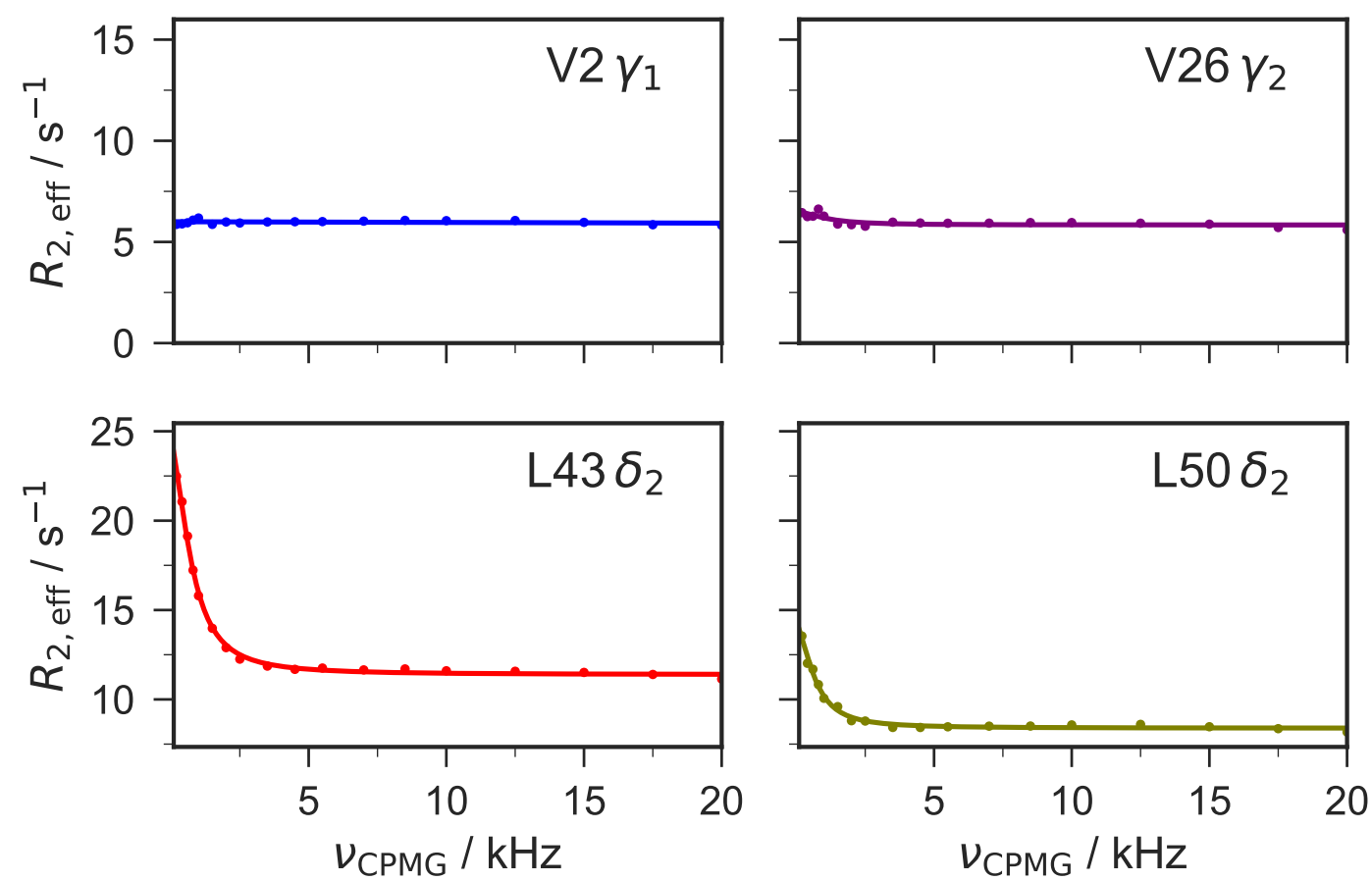

Figure 3.22: Relaxation dispersion profiles obtained from ${ }^{1} \mathrm{H}$ E-CPMG with individually fitted exchange model at $275 \mathrm{~K}$. Displayed residues are located in different structural elements of gpW, V2 $\gamma_{1}\left(\alpha_{1}\right)$, V26 $\gamma_{2}\left(\beta_{1}\right), \operatorname{L} 43 \delta_{2}\left(\alpha_{2}\right)$ and L50 $\delta_{2}\left(\alpha_{2}\right)$. The individual RD profiles showed differences to the ones obtained from ${ }^{13} \mathrm{CE}$-CPMG experiments. V26 $\gamma_{2}\left(\mathrm{~L} 50 \delta_{2}\right)$ shows a flat profile in ${ }^{1} \mathrm{H}\left({ }^{13} \mathrm{C}\right) \mathrm{E}-\mathrm{CPMG}$, while having a significant exchange contribution $\left(R_{\mathrm{ex}} \approx 6 \mathrm{~s}^{-1}\right)$ in ${ }^{13} \mathrm{C}\left({ }^{1} \mathrm{H}\right)$ E-CPMG. The terminal methyl side-chains $\left(\mathrm{V} 2 \gamma_{1}\right)$, pointing outwards, showed again flat dispersion profiles.

In the ${ }^{1} \mathrm{HE}$-CPMG experiments several methyl groups showed relaxation dispersion, which are located in both $\alpha$-helices and $\beta$-hairpin. Most of these residues already showed relaxation dispersion in ${ }^{13} \mathrm{C}$ E-CPMG experiments, except L14 $\delta_{2}$ and L50 $\delta_{2}$ (Figure 3.18 and Figure 3.22). Also, there are some methyl groups, which appear to be flat in the ${ }^{1} \mathrm{HE}-$ CPMG experiment but showed significant exchange contribution in ${ }^{13} \mathrm{CE}$-CPMG $\left(\mathrm{L} 7 \delta_{2}\right.$, V23 $\gamma_{2}$, V26 $\gamma_{2}$, V40 $\gamma_{1}$; Figure 3.19 and Figure 3.23). These observed differences in sensitivity of the two nuclei $\left({ }^{13} \mathrm{C}\right.$ and $\left.{ }^{1} \mathrm{H}\right)$ indicates the importance of chemical shift differences for the detection of conformational exchange. For this reason, the use of different nuclei in $\mathrm{RD}$ experiments is recommend for a residue-wise analysis of exchange processes.

In total seven methyl groups (with $R_{\mathrm{ex}}>2 \mathrm{~s}^{-1}$ ) were fitted individually to the two-state exchange model. The obtained exchange rates were narrowly distributed around $510^{3} \mathrm{~s}^{-1}$ and in the same order of magnitude as the $k_{\text {ex }}$ values obtained from the ${ }^{13} \mathrm{C}$ experiment (Figure 3.23). The ${ }^{1} \mathrm{HE}-\mathrm{CPMG}$ experiments neither indicated a three-state exchange or a dominating faster exchange process, as it was observed for the backbone at $275 \mathrm{~K}$. Thus, it could be concluded that the side-chain methyl groups are either not affected by this faster process or their chemical shift changes are negligible upon the conformational change. Considering, that these side-chain methyl groups are pointing outwards from the $\alpha$-helices, one could assume that they are exposed to the solvent in both folded and 


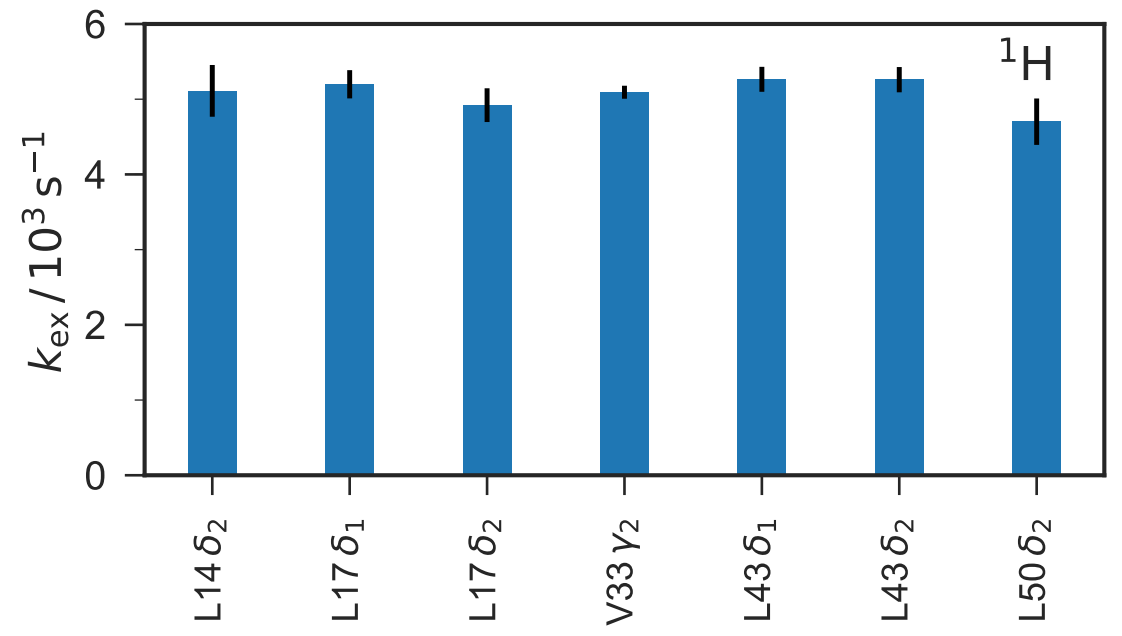

Figure 3.23: Exchange rates of individually fitted residues from methyl ${ }^{1} \mathbf{H ~ E}$ CPMG at $275 \mathrm{~K}$. Exchange rates were extracted by fitting RD profiles obtained from ${ }^{13} \mathrm{C}$ E-CPMG to the Luz-Meiboom model (Equation (2.20)). Only residues with an exchange contribution of $>2 \mathrm{~s}^{-1}$ were considered for the analysis. All methyl groups could be fitted to similar exchange rates, indicating a global exchange process.
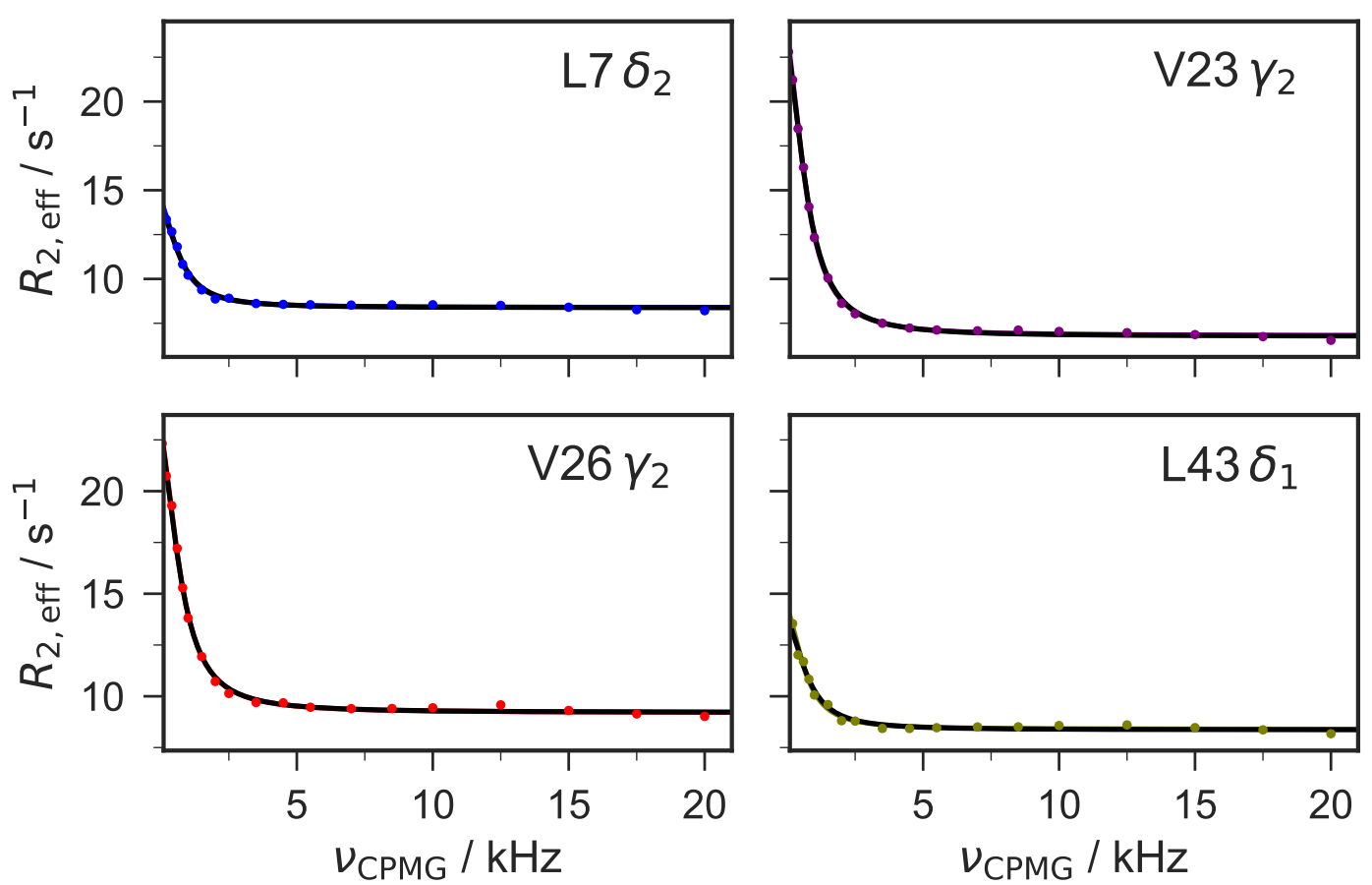

Figure 3.24: Comparison of individually and globally fitted ${ }^{1} \mathrm{H}$ RD profiles at 275 K. ${ }^{1} \mathrm{H}$ E-CPMG obtained RD profiles of residues L $7 \delta_{2}, \mathrm{~V} 23 \gamma_{2}, \mathrm{~V} 26 \gamma_{2}$ and L43 $\delta_{1}$ are shown with individually (color) and globally fitted (black) Luz-Meiboom model (Equation $(2.20))$. The global fit result $\left(k_{\mathrm{ex}}=(5.30 \pm 0.06) 10^{3} \mathrm{~s}^{-1}\right)$ is in very good agreement with the results obtained from ${ }^{13} \mathrm{CE}$-CPMG experiments $\left(k_{\mathrm{ex}}=(4.87 \pm 0.04) 10^{3} \mathrm{~s}^{-1}\right)$. Thus, both experiments report the same kinetic process. 
unfolded state. Thus, only minor changes in the chemical shifts are expected for these side-chain methyl groups upon helix folding. A dominant change in the chemical shift would be expected due to ring current effects of close-by aromatic amino acids (Perkins and Wuthrich, 1979; Kjaergaard et al., 2011). Only one of these residues is present in gpW (Y46, $\alpha_{2}$ ), which supports the hypothesis of only minor changes in the methyl chemical shifts upon helix formation. Assuming a fast exchange rate of roughly $20010^{3} \mathrm{~s}^{-1}$, as it was estimated by amide ${ }^{1} \mathrm{HE}-\mathrm{CPMG}$ experiments, this process is expected to be undetectable in the side-chain RD experiments.

The formation of the hydrophobic core on the other hand excludes solvent from the surrounding of methyl groups between the interface of the two helices. This would cause a large change in chemical environment and thus could explain the observed RD in the side-chain experiments. A global fit of the side-chain ${ }^{1} \mathrm{HE}$-CPMG resulted in a rate of
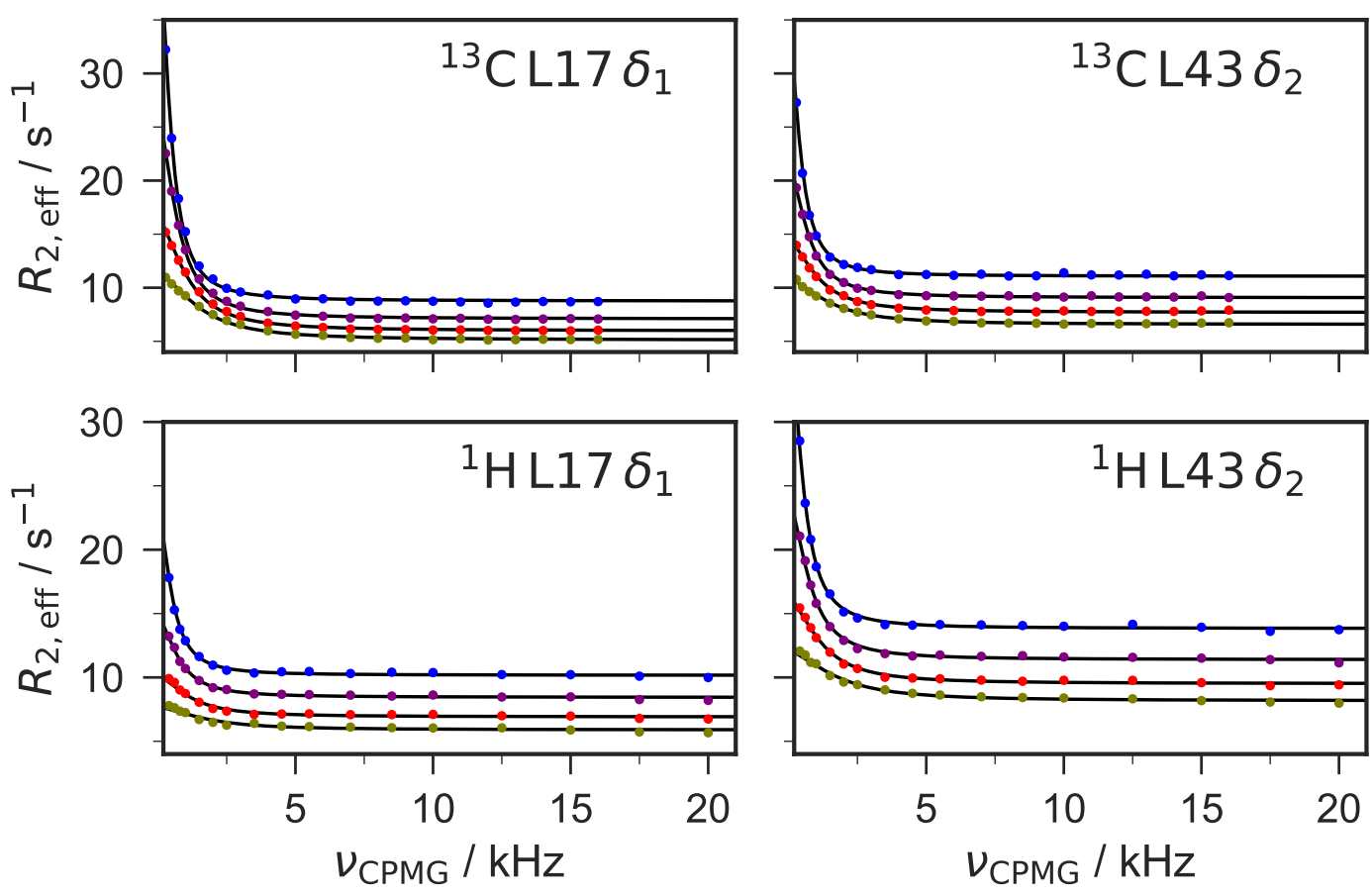

Figure 3.25: Temperature dependent side-chain relaxation dispersion profiles. Relaxation dispersion data and global fits are shown for residues $\mathrm{L} 17_{\delta_{1}}$ and $\mathrm{L} 43_{\delta_{2}}$ obtained from ${ }^{13} \mathrm{C}$ E-CPMG (top) and ${ }^{1} \mathrm{HE}$-CPMG (bottom). The data was acquired at $270 \mathrm{~K}$ blue, $275 \mathrm{~K}$ purple, $280 \mathrm{~K}$ red and $285 \mathrm{~K}$ gold. Only residues with an exchange contribution $R_{\mathrm{ex}}>2$ in the whole temperature range were considered for the analysis.

$k_{\mathrm{ex}}=(5.30 \pm 0.06) 10^{3} \mathrm{~s}^{-1}$, which is slightly faster than the rate obtained from the ${ }^{13} \mathrm{C}$ data analysis $\left(k_{\mathrm{ex}}=(4.87 \pm 0.04) 10^{3} \mathrm{~s}^{-1}\right)$. Both global models are in good agreement with the data (Figure 3.20 and Figure 3.24) and it can be assumed that they report the same kinetic process.

The side-chain relaxation dispersion experiments were repeated at several temperatures in the range of $270-290 \mathrm{~K}$ to evaluate the temperature dependence of the observed kinetics (Figure 3.25). The same procedure as the backbone temperature dependent data set was 
applied, where only residues with a significant exchange contribution in all temperatures were considered for the analysis. The resulting Arrhenius fit (Equation (3.8)) are shown in Figure 3.26. Both methyl side-chain RD data sets $\left({ }^{13} \mathrm{C}\right.$ and $\left.{ }^{1} \mathrm{H}\right)$ were fitted to similar activation energies, $E_{\mathrm{A}}=49.76 \pm 1.34 \mathrm{~kJ} \mathrm{~mol}^{-1}$ and $E_{\mathrm{A}}=52.32 \pm 0.43 \mathrm{~kJ} \mathrm{~mol}^{-1}$ for ${ }^{13} \mathrm{C}$ and ${ }^{1} \mathrm{H}$ data, respectively. The fitted collision frequency $(A)$ showed again large uncertainties of $\geq 50 \%$. An explanation for the large uncertainty of this parameter was already given during the discussion of temperature dependent analysis of the backbone data.

The fitted energy barriers of the side-chain E-CPMG data were larger compared to the results obtained from the backbone experiments. This could imply a hierarchy in the stability of the two structural elements as it was proposed before in the literature (Sborgi et al., 2015). An extrapolation of the exchange rates, as it was done for the backbone E-CPMG data using the Arrhenius model (Equation (3.8)), lead to an exchange lifetime $\left(\tau_{\mathrm{ex}}\right)$ of $3.24 \pm 1.54 \mu \mathrm{s}$ and $2.35 \pm 0.36 \mu$ s from ${ }^{13} \mathrm{C}$ and ${ }^{1} \mathrm{H}$ measurements, respectively.
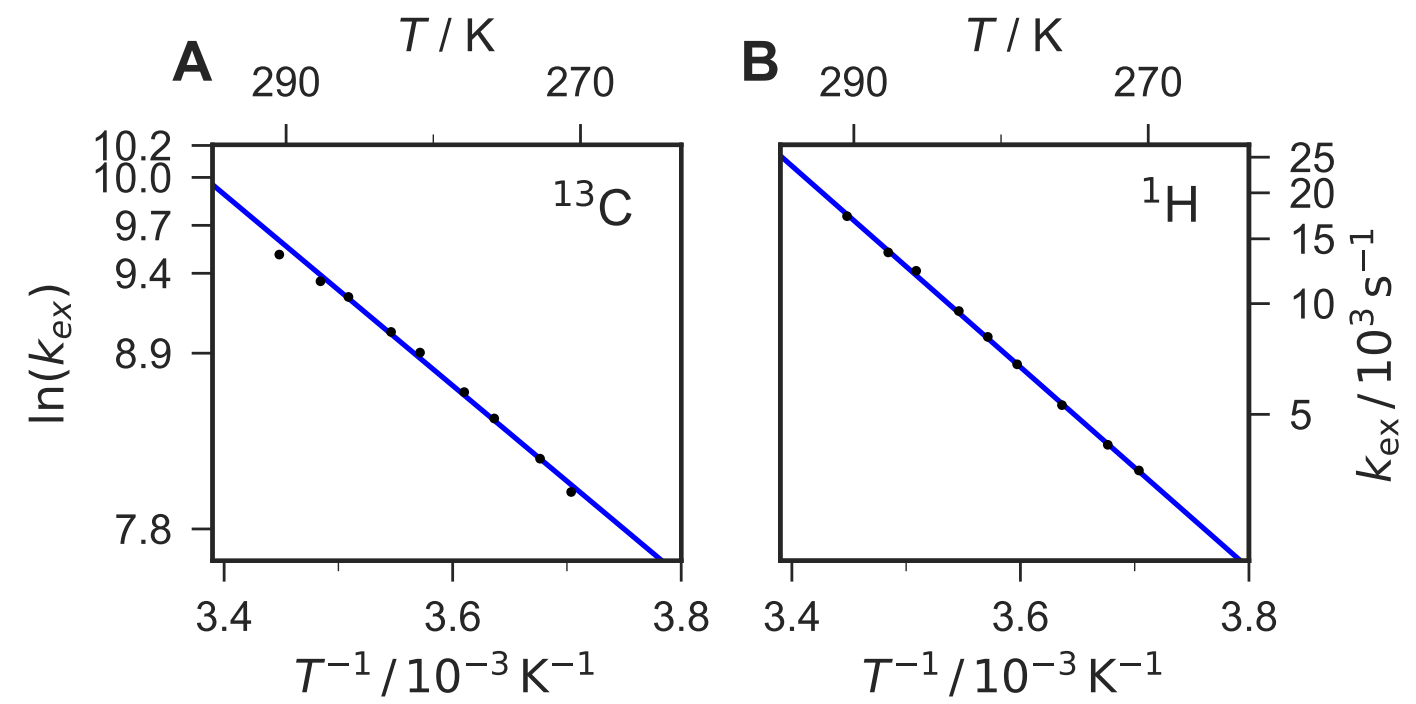

Figure 3.26: Arrhenius analysis of globally fitted exchange rates from gpW side-chain E-CPMG data. (A) Arrhenius (erefeq:arr) fitting of global exchange rates obtained from ${ }^{13} \mathrm{CE}-\mathrm{CPMG}$ experiments. The fitted Arrhenius parameters are $A=(1.36 \pm 0.79) 10^{13} \mathrm{~Hz}$ and $E_{\mathrm{A}}=49.76 \pm 1.34 \mathrm{~kJ} \mathrm{~mol}^{-1}$. An extrapolation to the melting temperature of $\mathrm{gpW}\left(T_{\mathrm{m}}=340 \mathrm{~K}\right)$ resulted in an exchange lifetime of $3.24 \pm 1.54 \mu \mathrm{s}$. (B) Arrhenius (erefeq:arr) of global exchange rates obtained from ${ }^{1} \mathrm{H}$ E-CPMG. The fitted Arrhenius parameters are $A=(4.64 \pm 8.72) 10^{12} \mathrm{~Hz}$ and $\left.E_{\mathrm{A}}=52.32 \pm 0.43 \mathrm{~kJ} \mathrm{~mol}^{-1}\right)$ An extrapolation to $T_{\mathrm{m}}$ resulted in an exchange lifetime of $2.35 \pm 0.36 \mu \mathrm{s}$. Both extrapolated values are close to the previously reported results from induced T-jump experiments $\left(\tau_{\mathrm{ex}}=8.8 \mu \mathrm{s}\right)$ (Fung et al., 2008). 
In summary the side-chain E-CPMG experiments revealed an exchange process that takes place on a similar time scale as the slow process detected in the backbone E-CPMG experiments. Methyl groups between the interface of the two helices and localized in the $\beta$-hairpin region, report this exchange event (Figure 3.27). The two methyl side-chain residues V2 and V52 that point outwards showed flat relaxation dispersion profiles in both E-CPMG experiments. Thus, it can be hypothesized that the observed chemical exchange process is connected to the formation of a hydrophobic core. The global analysis of methyl groups in both structure elements showed a good agreement to the data. This indicates that the formation of the hydrophobic core and folding of $\beta$-hairpin are the same process or at least correlated. The temperature dependent analysis of the exchange kinetics lead to a similar energy barrier in both side-chain experiments $\left(50 \mathrm{~kJ} \mathrm{~mol}^{-1}\right.$ and $\left.52 \mathrm{~kJ} \mathrm{~mol}^{-1}\right)$. These results are slightly higher than the energy barriers obtained from the backbone experiments $\left(40 \mathrm{~kJ} \mathrm{~mol}^{-1}\right.$ and $\left.36 \mathrm{~kJ} \mathrm{~mol}^{-1}\right)$. An extrapolation of the kinetics to the melting temperature of gpW resulted in an exchange lifetime $\left(\tau_{\mathrm{ex}}\right)$ of $3.24 \pm 1.54 \mu \mathrm{s}$ and $2.35 \pm 0.36 \mu \mathrm{s}$ for ${ }^{13} \mathrm{C}$ and ${ }^{1} \mathrm{H}$, respectively, which is also comparable to the previous results from backbone experiments $\left(4.7 \mu\right.$ s and $5.2 \mu \mathrm{s}$ for ${ }^{15} \mathrm{~N}$ and ${ }^{1} \mathrm{H}$, respectively).
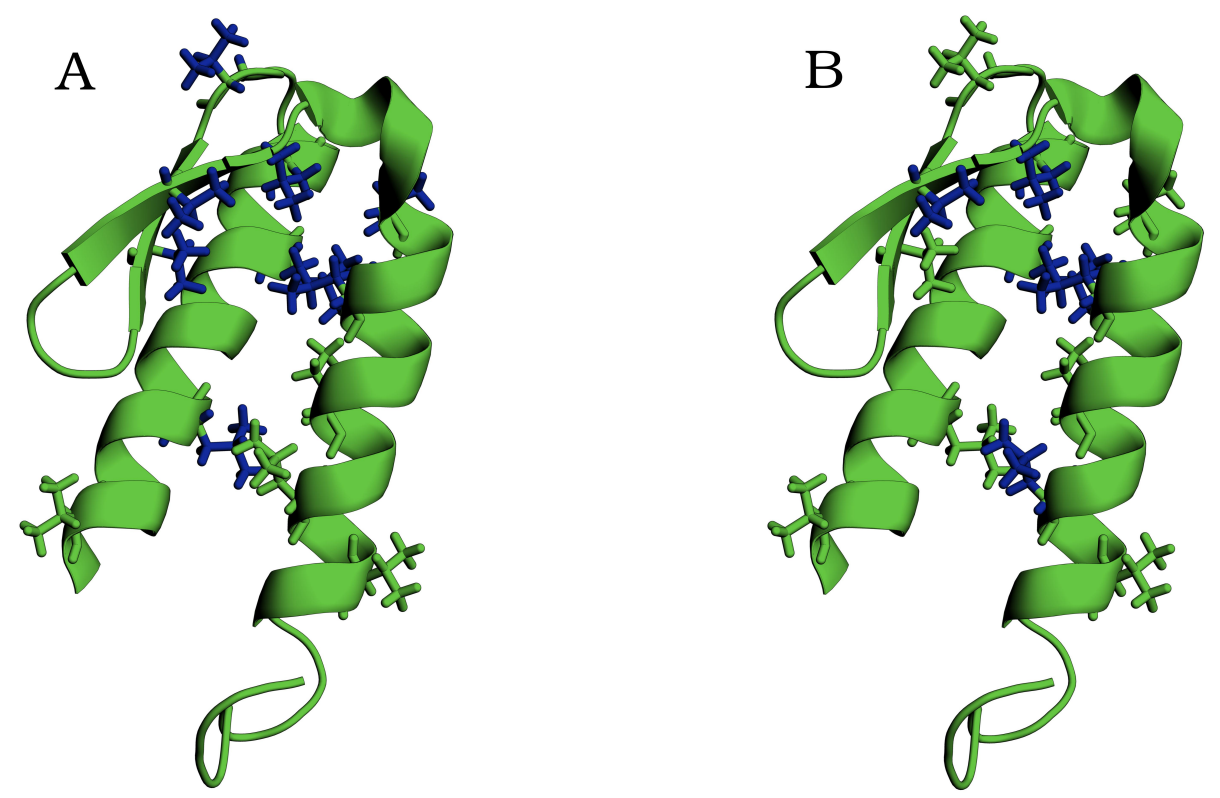

Figure 3.27: Structure of gpW with highlighted methyl groups showing relaxation dispersion. Methyl side-chains are shown as stick representation and highlighted in blue when showing dispersion (pdb: 2l6r (Sanchez-Medina et al., 2014)). (A) Methyl groups showing relaxation dispersion in ${ }^{13} \mathrm{CE}-\mathrm{CPMG}$ experiments at $275 \mathrm{~K}$. (B) Methyl groups showing relaxation dispersion in ${ }^{1} \mathrm{H} \mathrm{E-CPMG}$ experiments at $275 \mathrm{~K}$. Side-chains located in the $\beta$-hairpin region as well as between the interface of the two helices reported a chemical exchange process. The two terminal methyl side-chains (V2 and V52) point outwards the helices and showed no relaxation dispersion in neither of the experiments. These results support the hypothesis of a hydrophobic collapse that stabilizes the formation of the $\beta$-hairpin region. 


\subsection{Conclusion}

In this chapter the application of the E-CPMG experiment to the study of gpW folding kinetics was presented. ${ }^{15} \mathrm{~N}$ E-CPMG experiments added two additional residues (V23 and A29) to the analysis, which were broadened beyond detection in the conventional CPMG range (Sanchez-Medina et al., 2014). The combined use of high power relaxation dispersion and super-cooled sample conditions (Ban et al., 2011) revealed an exchange contribution to $R_{2 \text {,eff }}$ for $\alpha$-helical residues, which was not detectable in conventional CPMG experiments (Sanchez-Medina et al., 2014). In addition, these experiments showed relaxation dispersion profiles with an initial drop in $R_{2, \text { eff }}$ at low refocusing frequencies. These could not be fitted to a two-state exchange but a three-state exchange model was necessary. For the first time two distinct conformational exchange processes were detected in a single relaxation dispersion experiment. The individual analysis identified these as a slow and a fast exchange process. The latter was not fully quenched in the accessible frequency range of E-CPMG experiments hence a feasible analysis of the kinetics was not possible. Nevertheless, assuming a global three-state exchange process, the faster $k_{\text {ex }}$ was estimated to be about $200000 \mathrm{~s}^{-1}$ from ${ }^{1} \mathrm{HE}$ E-CPMG data at $263 \mathrm{~K}$. With the data collected so far it can only be speculated, which structural changes cause the exchange contribution to $R_{2, \text { eff }}$. Although a single global process was assumed for the fast rate estimation, multiple conformational changes could cause the observed RD profiles.

The slower process showed similar kinetics as they were observed for residues in the $\beta$ hairpin region. Thus, it can be hypothesized that the two structure elements are either involved in the same conformational exchange or their two processes are connected. This is supported by the results from the methyl side-chain E-CPMG experiments. Here, methyl groups that are located between the interface of the two helices showed relaxation dispersion with similar kinetics as they were observed for the slow process in the backbone experiments. A plausible explanation for these results is a hydrophobic collapse that stabilizes the formation of the $\beta$-hairpin region. In this scenario gpW exchanges between a fully folded and partially unfolded state, where only the $\beta$-hairpin region unfolds. The $\alpha$-helices are preformed and only form the hydrophobic core by translation and rotational motion. Thus, the chemical shift changes are expected to be minimal for $\alpha$-helix backbone nuclei but large for the side-chains that form the hydrophobic core. This indeed could be observed in the E-CPMG experiment, where the slow process was only visible under super-cooled conditions. The kinetic results of the backbone and side-chain measurements further indicate that the hydrophobic core and the $\beta$-hairpin formation take place on the same time scale. Thus, it can be hypothesized that the two process are correlated or are directly coupled. From previous studies it is known that the hydrophobic core stabilizes the formation of the hairpin (Maxwell et al., 2001).

Higher refocusing frequencies accessible by the E-CPMG experiment allowed a temperature dependent analysis of the folding kinetics of gpW using relaxation dispersion experiments.

An Arrhenius fit of the globally fitted exchange rates gave insights into the energy barri- 
ers of the detected conformational exchange processes. The fitted values for the backbone E-CPMG data, $39.0 \pm 2.2 \mathrm{~kJ} \mathrm{~mol}^{-1}$ for ${ }^{15} \mathrm{~N}$ and $36.3 \pm 1.3 \mathrm{~kJ} \mathrm{~mol}^{-1}$ for ${ }^{1} \mathrm{H}$, were lower than the ones obtained from the side-chain measurements, $49.8 \pm 1.3 \mathrm{~kJ} \mathrm{~mol}^{-1}$ for ${ }^{15} \mathrm{~N}$ and $52.3 \pm 0.4 \mathrm{~kJ} \mathrm{~mol}^{-1}$ for ${ }^{1} \mathrm{H}$. The difference in their energy barriers implies a hierarchy in the stability of those structural elements. According to the estimated energy barriers the hydrophobic core is more stable than the $\beta$-hairpin formation, which is in line with molecular dynamic simulations (Sborgi et al., 2015).

An extrapolation of the folding kinetics to the melting temperature of gpW at $340 \mathrm{~K}$ lead to comparable results $(4.7 \pm 3.8 \mu \mathrm{s}$ and $6.1 \pm 2.3 \mu \mathrm{s}$ from backbone, $3.2 \pm 1.5 \mu \mathrm{s}$ and $2.4 \pm 0.4 \mu$ s from side-chain measurements) with previously reported values from thermodynamic methods $\left(\tau_{\mathrm{ex}}=8.8 \mu \mathrm{s}\right)$ (Fung et al., 2008; Sborgi et al., 2015).

Table 3.1: Exchange rates $k_{\mathrm{ex}}$ obtained from globally fitted E-CPMG data. The results of the backbone amide and methyl side-chain data analysis using a two-state fast exchange model at different temperatures are shown.

\begin{tabular}{lrrrr}
\hline & ${ }^{15} \mathrm{~N}$ & ${ }^{1} \mathrm{H}^{\mathrm{N}}$ & ${ }^{13} \mathrm{C}$ & ${ }^{1} \mathrm{H}^{\mathrm{CH}_{3}}$ \\
$T / \mathrm{K}$ & $k_{\mathrm{ex}} / \mathrm{s}^{-1}$ & $k_{\mathrm{ex}} / \mathrm{s}^{-1}$ & $k_{\mathrm{ex}} / \mathrm{s}^{-1}$ & $k_{\mathrm{ex}} / \mathrm{s}^{-1}$ \\
\hline 263 & $3815 \pm 94$ & $4802 \pm 143$ & - & - \\
265 & - & $5486 \pm 151$ & - & - \\
268 & - & $6033 \pm 146$ & - & - \\
270 & $5784 \pm 111$ & $6586 \pm 110$ & $3076 \pm 34$ & $3520 \pm 28$ \\
272 & - & - & $3789 \pm 38$ & $4133 \pm 39$ \\
275 & $7949 \pm 79$ & $8966 \pm 129$ & $4873 \pm 41$ & $5297 \pm 58$ \\
278 & - & - & $5747 \pm 56$ & $6839 \pm 120$ \\
280 & $11144 \pm 109$ & $12723 \pm 175$ & $7359 \pm 83$ & $8119 \pm 148$ \\
282 & - & - & $8375 \pm 131$ & $9544 \pm 255$ \\
285 & $15034 \pm 186$ & $17118 \pm 253$ & $10426 \pm 163$ & $12276 \pm 475$ \\
287 & - & - & $11500 \pm 211$ & $13776 \pm 617$ \\
290 & - & $21818 \pm 333$ & $13591 \pm 299$ & $17282 \pm 825$ \\
295 & - & $25265 \pm 506$ & - & - \\
\hline
\end{tabular}

Previous thermodynamic studies categorize gpW as an ultrafast folding protein, whose free energy landscape follows a downhill folding model without an energy barrier (Fung et al., 2008). The temperature dependent kinetic rates obtained by the experiments presented in this work, were fitted assuming a two-state exchange model with a distinct energy barrier and could reproduce previous results for the exchange lifetime at the melting temperature. In addition, a second exchange process in the $\alpha$-helix is indicated. Thus, it can be hypothesized that the folding kinetics of gpW follow a more complex energy landscape than the downhill folding model implies.

The fast exchange process could be further analyzed, using mutations (Maxwell et al., 2000) that either stabilize or destabilize the formation of the secondary structure elements. Also, a stabilization of the $\alpha$-helix by using Tri-fluoroethanol (TFE) (Shiraki et al., 1995) could be used. Both approaches would alter the populations of the respective exchanging 
states. In case of an unfolding process of the $\alpha$-helix, stabilization of the latter would decrease the exchange contribution in the relaxation dispersion profiles and ultimately the slow decay of $R_{2 \text {,eff }}$, observed in the RD profiles, would be reduced or even disappear completely. Those approaches would give further information that could be directly connected to the structural changes involved in the folding process.

Both of these approaches do not slow down the kinetics and thus do not improve the quantitatively analysis of the fast exchange process. The latter would require a further advance of relaxation dispersion experiments into the supra- $\tau_{\mathrm{c}}$ window. Since the accessible time scale by RD only depend on the applied power of the radio frequency pulses, the E-CPMG bears the potential of reaching even deeper into the supra- $\tau_{\mathrm{c}}$ window with the development of new hardware. Assuming a rotational correlation time of 4 ns nutation, frequencies of up to $250000 \mathrm{kHz}$ would be needed to cover the complete supra- $\tau_{\mathrm{c}}$ window by relaxation dispersion experiments.

In summary, the E-CPMG relaxation dispersion data of gpW revealed a second, much faster exchange process $\left(k_{\mathrm{ex}}>20000010^{3} \mathrm{~s}^{-1}\right.$ at $\left.263 \mathrm{~K}\right)$ in the $\alpha$-helices, which so far could not be detected by conventional CPMG experiments. In addition, a much slower exchange process $\left(k_{\mathrm{ex}}<5000 k_{\mathrm{ex}}\right.$ at $\left.263 \mathrm{~K}\right)$ was detected in the relaxation dispersion profiles of residues in the $\alpha$-helices. This could only be observed under super-cooled sample conditions and an AICc based model selection showed a better consent to a three-state exchange model. The fitted exchange parameters for the slow process in the $\alpha$-helices are comparable to the ones obtained from the folding/unfolding of the $\beta$-hairpin region. This indicates that the exchange in solution is a global process, which involves large parts of the protein and thus supports the hypothesis of a hydrophobic collapse that drives the $\beta$-hairpin formation. Furthermore, the temperature dependence of the slow process could be explained by an exchange model with a distinct energy barrier $\left(40-50 \mathrm{~kJ} \mathrm{~mol}^{-1}\right)$. This leads to an exchange rate at melting temperature comparable to the previous thermodynamic experiments (Fung et al., 2008; Sborgi et al., 2015). Thus, it can be assumed that the energy landscape of $\mathrm{gpW}$ in solution is more complex than the previously proposed downhillfolding model by Fung and co-workers (Fung et al., 2008). The data presented in this chapter also showed the need of higher refocusing frequencies to fully quench the exchange contribution from the fast motion. A more accurate knowledge of the fast kinetics will help in understanding the functionality of $\mathrm{gpW}$ in an improved way. This technique can be applied to other proteins, which lack the complete characterization of motion throughout the time scale window. 


\subsection{Materials and Methods}

\subsubsection{Sample preparation}

Side-chain extreme CPMG experiments were measured on perdeuterated, ${ }^{15}$ Nlabeled gpW with selective labeling of $\delta_{1}, \delta_{2}$, respectively $\gamma_{1}, \gamma_{2}{ }^{13} \mathrm{CHD}_{2}$ methyl groups in residues Valine, Leucine and Isoleucine. The protein was expressed in E. coli adapted to $100 \% \mathrm{D}_{2} O$ using $\mathrm{D}_{7}$-glucose and ${ }^{15} \mathrm{NH}_{4} \mathrm{Cl}$ as carbon, respectively nitrogen, source (Sborgi et al., 2011; Gronenborn et al., 1991). Additional use of the precursors 2-keto-3- $\mathrm{D}_{2}-4{ }^{13} \mathrm{C}_{,} \mathrm{D}_{2^{-}}$ butyrateand 2-keto-3-methyl- $\mathrm{D}_{3}-3-\mathrm{D}_{1}-4-{ }^{13} \mathrm{C}, \mathrm{D}_{2}$-butyrate lead to the desired methyl labeling scheme as previously described in literature (Tugarinov et al., 2006).

Backbone experiments were measured on perdeuterated, ${ }^{15} \mathrm{~N}$ labeled gpW, which was prepared as explained above, while omitting the precursors for selective methyl group labeling. For experiments under super-cooled conditions the protein sample was transferred into capillaries (Wilmad, Buena, New Jersey) with an outer diameter of $0.8 \mathrm{~mm}$ and twelve of those were fitted into a conventional $5 \mathrm{~mm}$ NMR tube.

Both protein samples were purified as described before in literature (Gronenborn et al., 1991) and NMR samples were prepared using a concentration of $3 \mathrm{mM}$ in $20 \mathrm{mM}$ sodium phosphate buffer, $0.1 \mathrm{mM} \mathrm{NaN}_{3}$ at $\mathrm{pH}$ 6.5. The backbone sample and side-chain sample were solved in $90 \% \mathrm{H}_{2} \mathrm{O}$ and $10 \% \mathrm{D}_{2} \mathrm{O}$ in $100 \% \mathrm{D}_{2} \mathrm{O}$, respectively.

\subsubsection{NMR experiments}

The pulse sequences and phase cycles used for the E-CPMG experiments can be found in the Appendix.

\section{GpW Backbone Experiments}

The ${ }^{15} \mathrm{~N}$ constant-time extreme CPMG experimental data shown in this chapter was acquired at $263 \mathrm{~K}, 270 \mathrm{~K}, 275 \mathrm{~K}, 280 \mathrm{~K}$ and $285 \mathrm{~K}$. The CPMG block length $T_{\text {CPMG }}$ was set to $40 \mathrm{~ms}$ and 28 refocusing frequencies in the range of $100 \mathrm{~Hz}$ to $6400 \mathrm{~Hz}$ were acquired, including reference experiment and three repeated frequencies for an error estimation of $R_{2 \text {,eff }}$. Each two-dimensional experiment of the corresponding $\nu_{\mathrm{CPMG}}$ was acquired with 50 complex points $\left(t_{1, \max }=28.7 \mathrm{~ms}\right)$ in the indirect and 512 complex points in the direct dimension $\left(t_{2, \max }=56.3 \mathrm{~ms}\right)$, accumulating 16 scans. All frequencies were measured in a scan interleaved fashion and with mixed order of frequencies to average heating effects during high-power frequency experiments. The recycle delay $d_{1}$ was set to $3 \mathrm{~s}$ to further minimize heating during the measurement. The heat compensation block was set to the length of $T_{\mathrm{CPMG}}$ and missing pulses in the CPMG block were applied here to keep the overall number of pulses the same in all individual $\nu_{\mathrm{CPMG}}$ experiments. The following phase cycles were used: $\phi_{1}=4(\mathrm{x}), 4(-\mathrm{x}) ; \phi_{2}=8(\mathrm{y}), 8(-\mathrm{y}) ; \phi_{3}=(\mathrm{y},-\mathrm{y},-\mathrm{x}, \mathrm{x}) ; \phi_{4}=4(-\mathrm{y})$; $\phi_{5}=(-\mathrm{y}) ; \phi_{6}=4(-\mathrm{y}) ; \phi_{7}=4(\mathrm{x}) ; \phi_{\mathrm{rec}}=(\mathrm{y},-\mathrm{y},-\mathrm{x}, \mathrm{x}), 2(-\mathrm{y}, \mathrm{y}, \mathrm{x},-\mathrm{x}),(\mathrm{y},-\mathrm{y},-\mathrm{x}, \mathrm{x})$. The following gradients were used with strength given as percentage of the maximum gradient 
strength (length): $\mathrm{G}_{0}=80 \%$ ( $\left.1 \mathrm{~ms}\right), \mathrm{G}_{1}=19 \%$ (0.5 ms), $\mathrm{G}_{2}=15 \%$ (0.5 ms), $\mathrm{G}_{3}=32 \%$ $(0.5 \mathrm{~ms}), \mathrm{G}_{4}=60 \%$ (0.5 ms), $\mathrm{G}_{5}=27 \%(0.5 \mathrm{~ms}), \mathrm{G}_{6}=37 \%(0.5 \mathrm{~ms})$. A transcript of the pulse sequence can be found in the Appendix.

The ${ }^{1} \mathrm{H}^{N}$ E-CPMG data was acquired at temperature between $263 \mathrm{~K}$ and $295 \mathrm{~K}$. The CPMG block length $T_{\mathrm{CPMG}}$ was set to $20 \mathrm{~ms}$ and 24 refocusing frequencies in the range of $200 \mathrm{~Hz}$ to $30800 \mathrm{~Hz}$ were acquired, including reference experiment and three repeated frequencies for an error estimation of $R_{2 \text {,eff }}$. Each two-dimensional experiment of the corresponding $\nu_{\mathrm{CPMG}}$ was acquired with 64 complex points $\left(t_{1, \max }=36.7 \mathrm{~ms}\right)$ in the indirect and 1024 complex points in the direct dimension $\left(t_{2, \max }=112.3 \mathrm{~ms}\right)$, accumulating 8 scans for every FID. All frequencies were measured in a scan interleaved fashion and with mixed order of frequencies to average heating effects during high-power frequency experiments. The recycle delay $d_{1}$ was set to $3 \mathrm{~s}$ to further minimize heating during the measurement. The heat compensation block was set to the length of $T_{\mathrm{CPMG}}$ and missing pulses in the CPMG block were applied here to keep the overall number of pulses the same in all individual $\nu_{\mathrm{CPMG}}$ experiments. The following phase cycles were used: $\phi_{1}=\mathrm{x},-\mathrm{x}$; $\phi_{2}=2(\mathrm{x}), 2(-\mathrm{x}) ; \phi_{3}=4(\mathrm{x}), 4(-\mathrm{x}) ; \phi_{\mathrm{rec}}=-\mathrm{x}, \mathrm{x}, \mathrm{x},-\mathrm{x}, \mathrm{x},-\mathrm{x},-\mathrm{x}, \mathrm{x}$. The following gradients were used with strength given as percentage of the maximum gradient strength (length): $\mathrm{G}_{0}=80 \%$ ( $\left.1 \mathrm{~ms}\right), \mathrm{G}_{1}=19 \%$ (0.5 ms), $\mathrm{G}_{2}=15 \%$ (0.5 ms), $\mathrm{G}_{3}=32 \%$ (0.5 ms), $\mathrm{G}_{4}=60 \%$ $(0.5 \mathrm{~ms}), \mathrm{G}_{5}=27 \%(0.5 \mathrm{~ms}), \mathrm{G}_{6}=37 \%(0.5 \mathrm{~ms})$. A transcript of the pulse sequence can be found in the Appendix.

All experiments were measured on a Bruker Avance III spectrometer operating at a field strength of $16.44 \mathrm{~T}$, corresponding to a ${ }^{1} \mathrm{H}$ Larmor frequency of $700 \mathrm{MHz}$ and equipped with a $5 \mathrm{~mm}$ triple-resonance prodigy probe.

\section{GpW side-chain experiments}

The ${ }^{13} \mathrm{C}$ CT-E-CPMG experiments were acquired in the temperature range of $270 \mathrm{~K}$ to $285 \mathrm{~K}$. The CPMG block length $T_{\mathrm{CPMG}}$ was set to $100 \mathrm{~ms}$ and 22 refocusing frequencies in the range of $280 \mathrm{~Hz}$ to $16000 \mathrm{~Hz}$ were acquired, including reference experiment and three repeated frequencies for an error estimation of $R_{2, \text { eff }}$. Each $2 \mathrm{D}$ experiment of the corresponding $\nu_{\mathrm{CPMG}}$ was acquired with 50 complex points $\left(t_{1, \max }=41.4 \mathrm{~ms}\right)$ in the indirect and 1024 complex points in the direct dimension $\left(t_{2, \max }=142.5 \mathrm{~ms}\right)$, accumulating 8 scans for every FID. All frequencies were measured in a scan interleaved fashion and with mixed order of frequencies to average heating effects during high-power frequency experiments. The recycle delay $d_{1}$ was set to $3 \mathrm{~s}$ to further minimize heating during the measurement. The heat compensation block was set to the length of $T_{\mathrm{CPMG}}$ and missing pulses in the CPMG block were applied here to keep the overall number of pulses the same in all individual $\nu_{\mathrm{CPMG}}$ experiments. The following phase cycles were used: $\phi_{1}=(\mathrm{y}, \mathrm{y},-\mathrm{y},-\mathrm{y}) ; \phi_{2}=(\mathrm{x},-\mathrm{x}) ; \phi_{\mathrm{rec}}=(\mathrm{x},-\mathrm{x},-\mathrm{x}, \mathrm{x})$. The following gradients were used with strength given as percentage of the maximum gradient strength (length): $\mathrm{G}_{0}=85 \%$ (1 ms), $\mathrm{G}_{1}=19 \%$ (0.5 ms), $\mathrm{G}_{2}=13 \%$ (0.5 ms), $\mathrm{G}_{3}=31 \%$ (0.5 ms), $\mathrm{G}_{4}=71 \%(1 \mathrm{~ms}), \mathrm{G}_{5}=57 \%$ 
(1 $\mathrm{ms}), \mathrm{G}_{6}=43 \%$ (0.5 ms), $\mathrm{G}_{7}=79 \%$ (1 ms), $\mathrm{G}_{8}=65 \%$ (1 ms). A transcript of the pulse sequence can be found in the Appendix.

${ }^{1} \mathrm{HE}$-CPMG experiments were acquired in the same temperature range as the the ${ }^{13} \mathrm{C}$ experiments. The CPMG block length $\left(T_{\mathrm{CPMG}}\right)$ was set to $40 \mathrm{~ms}$ and 20 refocusing frequencies in the range of $200 \mathrm{~Hz}$ to $20000 \mathrm{~Hz}$ were acquired, including reference experiment and three repeated frequencies for an error estimation of $R_{2, \text { eff }}$. Deuterium decoupling was applied during the CPMG block to reduce HD-coupling artifacts. Each two-dimensional experiment of the corresponding $\nu_{\mathrm{CPMG}}$ was acquired with 50 complex points $\left(t_{1, \max }=41.4 \mathrm{~ms}\right)$ in the indirect and 1024 complex points in the direct dimension $\left(t_{2, \max }=142.5 \mathrm{~ms}\right)$, accumulating 16 scans for every FID. All frequencies were measured in a scan interleaved fashion and with mixed order of frequencies to average heating effects during high-power frequency experiments. The recycle delay $d_{1}$ was set to $3 \mathrm{~s}$ to further minimize heating during the measurement. The heat compensation block was set to the length of $T_{\mathrm{CPMG}}$ and missing pulses in the CPMG block were applied here to keep the overall number of pulses the same in all individual $\nu_{\mathrm{CPMG}}$ experiments. The following phase cycles were used: $\phi_{1}=(\mathrm{x},-\mathrm{x}) ; \phi_{1}=(\mathrm{x},-\mathrm{x}) ; \phi_{2}=2(\mathrm{x}), 2(-\mathrm{x}) ; \phi_{3}=4(\mathrm{y}), 4(-\mathrm{y})$; $\phi_{\text {rec }}=(-\mathrm{x}, \mathrm{x}, \mathrm{x},-\mathrm{x}, \mathrm{x},-\mathrm{x},-\mathrm{x}, \mathrm{x})$. The following gradients were used with strength given as percentage of the maximum gradient strength (length): $\mathrm{G}_{0}=85 \%$ (1 ms), $\mathrm{G}_{1}=19 \%$ ( $1 \mathrm{~ms}), \mathrm{G}_{2}=30 \%$ ( $\left.1 \mathrm{~ms}\right), \mathrm{G}_{3}=65 \%$ ( $\left.1 \mathrm{~ms}\right), \mathrm{G}_{4}=15 \%$ (1 ms). A transcript of the pulse sequence can be found in the Appendix.

All experiments were measured on a Bruker Avance III spectrometer operating at a field strength of $14.09 \mathrm{~T}$, corresponding to a ${ }^{1} \mathrm{H}$ Larmor frequency of $600 \mathrm{MHz}$, and equipped with a $5 \mathrm{~mm}$ triple-resonance room temperature probe.

\subsubsection{Data Analysis}

All two-dimensional spectra were processed using the python package NMRglue (Helmus and Jaroniec, 2013). Intensities of each individual peaks were obtained by using in-house software based on python packages (NumPy and Pandas) and Cara (Keller, 2004). For the error estimation of the obtained intensities three refocusing frequencies were acquired twice and their standard deviation was used to calculate the Root-Mean-Square-Deviation (RMSD) of each individual peak by,

$$
\Delta R_{2, \mathrm{eff}}=\sqrt{\sum\left(R_{2, \mathrm{eff}}^{e x p}-R_{2, \mathrm{eff}}^{r e p}\right)^{2}}
$$

where exp denotes value used for the experimental analysis and rep denotes the repeated point. Relaxation dispersion profiles were fitted residue-wise using in-house software based on python package LMfit (Newville et al., 2014) and the Luz-Meiboom model for two-state exchange (Equation (2.20)) (Luz and Meiboom, 1963) as well as three-state exchange 
(Equation (3.2)) (Grey et al., 2003). An initial fit for the parameters was done using the Nelder-Mead algorithm and the obtained fitted parameters were further used as a starting point for a refined fit using a least-square minimization. For all fitting procedures the target function:

$$
\chi=\sum_{i=0}^{n} \frac{\left(\text { data }_{\mathrm{i}}-\text { model }_{\mathrm{i}}\right)}{\sigma_{\mathrm{i}}},
$$

was used, in which data, model and sigma are the experimental value, calculated model and experimental uncertain of the i-th data point, respectively. 


\section{Chapter 4}

\section{Shuttle Relaxometry: Application to study sub- $\tau_{\mathrm{c}}$ motions}

\subsection{Introduction}

In the previous two chapters the discussion was limited to protein motions that take place on a time scale slower than the overall rotational correlation time $\left(\tau_{\mathrm{c}}\right)$ of a molecule. The conformational or chemical exchange between different states alter the chemical shift of a given nucleus and relaxation dispersion techniques were presented to study the kinetics of these processes. In this chapter protein motions with a time scale faster than $\tau_{\mathrm{c}}$ (sub- $\tau_{\mathrm{c}}$ window) will be discussed. The effect of sub- $\tau_{\mathrm{c}}$ motion on NMR resonances is reflected in the relaxation rates of a nuclear spin.

As briefly introduced in Chapter 1 nuclear spins align parallel to an applied magnetic field. Due to population differences in the energetic levels of the two quantized orientations $(\alpha$ and $\beta$ spin) a net magnetization parallel to the z-axis arises, the so-called Boltzmann magnetization. During an NMR experiment this equilibrium state is disturbed by radio frequency $(\mathrm{RF})$ pulses and the net magnetization vector is tilted away from the z-axis. Pulses are defined by their flip angle $\theta$, which depends on the amplitude and length of the RF pulse. Thus, a $90^{\circ}$ pulse rotates the magnetization vector into the $\mathrm{x}-\mathrm{y}$ plane. After excitation the magnetization relaxes back into its energetically favored equilibrium state and the decay of non-equilibrium magnetization is detected. The relaxation is driven by magnetic dipole, chemical shift anisotropy, quadrupole and paramagnetic interactions. Considering protein NMR spectroscopy, the latter two are mostly negligible and only need to be considered when detecting nuclei with a spin number larger than $1 / 2$ or working with para-magnetic samples. In this chapter the discussion will be limited to relaxation caused by magnetic dipole-dipole (DD) and chemical shift anisotropy (CSA) interactions. These interactions generate local oscillating fields, that fluctuate due to molecular tumbling in solution and lead to time-dependent perturbation of the chemical shift Hamiltonian. The tumbling of the molecule is caused by Brownian motions and the corresponding oscillating fields that are caused are not uniformly distributed over all rotational frequencies. The probability of a motion to occur at a given frequency is defined by the spectral density 
function:

$$
J(\omega)=\frac{2}{5} \frac{\tau_{c}}{\left(1+\omega^{2} \tau_{c}^{2}\right)},
$$

where, $\omega$ denotes the Larmor frequency and $\tau_{\mathrm{c}}$ denotes the overall rotational correlation time, which is defined as the time it takes for the product of spherical harmonics $\left(Y_{2}^{0}[\Omega(t)] Y_{2}^{0}[\Omega(t+\tau)]\right)$ to be decayed to 1/e (Equation (1.19)) (Abragam, 1961). When molecular motion is fast enough to fulfill the condition $\omega \tau_{\mathrm{c}}<<1$, the Larmor frequency dependence of the spectral density function can be neglected $(J(\omega) \simeq J(0))$. This is the so-called extreme narrowing regime and applies usually for small molecules with tumbling times in the order of ps. For very slow motions, where $\omega \tau_{\mathrm{c}}>>1$, the spectral density function is inversely proportional to $\omega^{2}$ and ultimately approaches zero at the so-called spin diffusion regime. Together with the interaction strength of underlying relaxation mechanisms the spectral density functions describe the decay rates of excited magnetization.

The magnetic dipoles of two nuclei interact through space and the dipole coupling constant in a two-spin system is defined as:

$$
d_{\mathrm{IS}}=\frac{\mu_{0}}{4 \pi} \frac{\hbar \gamma_{\mathrm{I}} \gamma_{\mathrm{S}}}{r_{\mathrm{IS}}^{3}}
$$

where $\mu_{0}$ is the magnetic constant, $\hbar$ is the reduced Planck constant, $\gamma$ is the gyromagnetic ratio of the respective nucleus and $r_{\text {IS }}$ is the distance between the two spins I and S. The interaction strength depends largely on $r_{\text {IS }}$ and thus is a sensitive measure to analyze intramolecular distance. Furthermore, the $\gamma$ of the two spins are crucial and make ${ }^{1} \mathrm{H}_{-}{ }^{1} \mathrm{H}$ relaxation a much more efficient source for relaxation than interactions with or between ${ }^{13} \mathrm{C}$ and ${ }^{15} \mathrm{~N}$ nuclei.

The chemical shift anisotropy (CSA) describes the anisotropic distribution of electrons around a nucleus. Due to the molecular tumbling the orientation of this anisotropy tensor varies and causes the local fields to change in time. The interaction strength of an axially symmetric CSA is defined as:

$$
c_{\mathrm{I}}=\frac{\Delta \sigma \gamma_{I} B_{0}}{\sqrt{3}}
$$

where $\Delta \sigma$ describes the chemical shift anisotropy of a given nucleus, $\gamma$ is the gyromagnetic ratio and $B_{0}$ is the magnetic field strength. It can be seen that the interaction strength of the CSA is directly proportional to $B_{0}$ and plays a minor role when measuring at spectrometers with low field strengths. $\Delta \sigma$ is nucleus specific and can be estimated by the order of the isotropic chemical shift range. ${ }^{1} \mathrm{H}$, compared to other nuclei used for protein NMR (e.g. ${ }^{15} \mathrm{~N}$ and ${ }^{13} \mathrm{C}$ ) have a small range of chemical shifts (-2 to $14 \mathrm{ppm}$ ) and thus have a smaller contribution of CSA to the relaxation rates. However, for relaxation the interaction strength in $\mathrm{Hz}$ counts, making the CSA of nitrogen and proton equal in size. ${ }^{13} \mathrm{C}$ or ${ }^{19} \mathrm{~F}$ CSAs are considerably larger than those of ${ }^{1} \mathrm{H}$ and ${ }^{15} \mathrm{~N}$. 
Using the spectral density function and the two above mentioned relaxation mechanisms (DD and CSA) the relaxation rates of a nuclear spin in an NMR experiment can be calculated.

The longitudinal relaxation or spin-lattice relaxation rate $R_{1}$ describes the decay of magnetization in the $\mathrm{x}-\mathrm{y}$ plane back to its Boltzmann equilibrium and is defined as:

$$
R_{1}=\left(d_{\mathrm{IS}}^{2} / 4\right)\left(J\left(\omega_{I}-\omega_{\mathrm{S}}\right)+3 J\left(\omega_{I}\right)+6 J\left(\omega_{I}+\omega_{\mathrm{S}}\right)\right)+c_{\mathrm{I}}^{2} J\left(\omega_{I}\right),
$$

where $d_{\mathrm{IS}}$ is the dipolar coupling constant (Equation (4.2)), $c_{\mathrm{I}}$ is the chemical shift anisotropy of spin I (Equation (4.3)) and $J(\omega)$ is the spectral density function at a given Larmor frequency $\omega$ (Equation (4.1)).

The transverse or spin-spin relaxation rate $R_{2}$, describes the rate of dephasing of magnetization in the $\mathrm{x}-\mathrm{y}$ plane. It directly affects the linewidth of an observed resonance and is also affected by chemical exchange as it was discussed in the previous Chapter 2 and Chapter 3. For this chapter the discussion will be limited to the intrinsic part of the transverse relaxation rate $R_{2,0}$, from now on $R_{2}$. The rate is defined by:

$$
\begin{aligned}
R_{2}= & \left(d_{\mathrm{IS}}^{2} / 8\right)\left(4 J(0)+J\left(\omega_{I}-\omega_{\mathrm{S}}\right)+3 J\left(\omega_{I}\right)+6 J\left(\omega_{\mathrm{S}}\right)+6 J\left(\omega_{I}+\omega_{\mathrm{S}}\right)\right) \\
& +c_{\mathrm{IS}} J\left(\omega_{\mathrm{I}}\right)
\end{aligned}
$$

where the terms follow the same definitions as in Equation (4.4).

The major difference between the two rates lies in the spectral density term that is independent on the Larmor frequency for $R_{2}$. This causes $R_{2}$ to be linearly dependent on $\tau_{\mathrm{c}}$. It should be noted that in a homonuclear two-spin system the zero-quantum term $J\left(\omega_{\mathrm{I}}-\omega_{\mathrm{S}}\right)$ equals $J(0)$. This makes $R_{1}$ linearly dependent on $\tau_{\mathrm{c}}$ in a homonuclear spin system.

A further important relaxation mechanism is the Nuclear Overhauser Effect (NOE), which describes the exchange of magnetization through space between two spins via dipole-dipole interaction:

$$
\delta_{\mathrm{NOE}}=d_{\mathrm{IS}}^{2}\left(-J\left(\omega_{\mathrm{I}}-\omega_{\mathrm{S}}\right)\right)+6 J\left(\omega_{\mathrm{I}}+\omega_{\mathrm{S}}\right) .
$$

Notable for the NOE rate is the change in sign with increasing field strength. At low fields the double quantum term $J\left(\omega_{I}+\omega_{\mathrm{S}}\right)$ dominates the rate by its pre-factor of six. This results in a positive NOE rate in the extreme narrowing limit. With increasing field strength, the zero quantum $J\left(\omega_{\mathrm{I}}-\omega_{\mathrm{S}}\right)$ term becomes larger. In the spin diffusion limit it dominates the rate and changes the sign. The same dependency can be seen for variations in the rotational correlation time at static fields, according to the definition of the two regimes ( $\omega \tau_{\mathrm{c}}<<1$ for extreme narrowing and $\omega \tau_{\mathrm{c}}>>1$ for spin diffusion limit).

As it can be seen from the equations above, the kinetic information lies in the spectral density functions, which reflect the molecular tumbling. Equation (4.1) is a simplified spectral density model that only takes the isotropic rotational motion into account. Local 
motions which take place on a time scale faster than $\tau_{\mathrm{c}}$ alter the spectral density function and thus the observed relaxation rates. Lipari and Szabo (Lipari and Szabo, 1982) included these motions in their model-free approach:

$$
J(\omega)=\frac{2}{5}\left(\frac{\tau_{\mathrm{c}} S^{2}}{1+\omega^{2} \tau_{\mathrm{c}}^{2}}+\frac{\tau\left(1-S^{2}\right)}{1+\omega^{2} \tau^{2}}\right),
$$

where, the additional parameters $\tau$ and $S^{2}$ are added. $\tau$ denotes a correlation time, following $\tau^{-1}=\tau_{c}^{-1}+\tau_{e}^{-1}$, where $\tau_{\mathrm{e}}^{-1}$ is the correlation time of a local motion. $S^{2}$ is the generalized Lipari-Szabo order parameter and describes the amplitude of a motion of an inter-atomic vector in a cone. $S^{2}$ ranges from 0 to 1 , where 1 represents the static case and 0 represents full coverage of the spatial area in the cone. Years later Clore and co-workers further modified the spectral density function by differentiating between fast and slow local motions:

$$
J(\omega)=\frac{2}{5}\left(\frac{\tau_{c} S^{2}}{1+\omega^{2} \tau_{c}^{2}}+\frac{\tau_{f}\left(1-S_{f}^{2}\right)}{1+\omega^{2} \tau_{f}^{2}}+\frac{\tau_{s}\left(S_{f}^{2}-S^{2}\right)}{1+\omega^{2} \tau_{s}^{2}}\right),
$$

in which, terms marked with $f$ and $s$ represent the fast and slow local motions, respectively (Clore et al., 1990). A reduced form of Equation (4.8) can be applied

$$
J(\omega)=\frac{2}{5}\left(\frac{\tau_{c} S^{2}}{1+\omega^{2} \tau_{c}^{2}}+\frac{\tau_{s}\left(S_{f}^{2}-S^{2}\right)}{1+\omega^{2} \tau_{s}^{2}}\right),
$$

when the contribution of the fast, local motion contribution $\left(\tau_{f}\right)$ is negligible.

In practice the extraction of the local kinetic information from one of these relaxation rates is not straightforward. Usually high field strengths are necessary for a sufficient resolution and sensitivity in protein NMR. These as well as large correlation times of the proteins, force the spectral density functions into the spin diffusion limit. Here $S^{2}$ and $\tau_{\mathrm{c}}$ are convoluted and cannot be determined separately by fitting spectral density models. In addition, the contribution of local motions is rather small at higher magnetic fields, considering that $\tau_{\mathrm{c}}$ is expected to be at least two orders of magnitude larger than $\tau_{\mathrm{e}}$.

Different techniques allow the estimation of the Lipari-Szabo order parameter or the overall rotational correlation time, like TRACT (Lee et al., 2006), Cross-correlated relaxation (CCR) (Sabo et al., 2012) or residual dipolar coupling (RDC) (Sabo et al., 2014) experiments. For a simultaneous determination of all local dynamics the model-free approach is well established in literature (Peng and Wagner, 1992; Korzhnev et al., 1997; Andrec et al., 1999; d'Auvergne and Gooley, 2003; Idiyatullin et al., 2003), where $R_{1}, R_{2}$ and heteronuclear NOE (hetNOE) data is combined. During the model free fitting procedure the terms $J\left(\omega_{\mathrm{I}}\right), J\left(\omega_{\mathrm{I}}+\omega_{\mathrm{S}}\right)$ and $J\left(\omega_{\mathrm{I}}-\omega_{\mathrm{S}}\right)$ are combined to a term $\alpha J\left(\beta \omega_{\mathrm{I}}\right)$, where $\alpha$ and $\beta$ are prefactors from the first-order approximation, to simplify the fit evaluation (Palmer, 2004).

Another approach to access the kinetic information embedded in the relaxation rates 
is spectral density mapping by measuring the relaxation rate at different magnetic field strengths (Kaderavek et al., 2016). Obviously, this requires the availability of several spectrometers operating at different Larmor frequencies, which is costly and rarely accessible. Furthermore, protein samples limit the use to rather high magnetic fields (> $400 \mathrm{MHz})$. Otherwise the necessary resolution for a residue-wise analysis is not ensured. Here, shuttle relaxometry provides an alternative for the measurement of relaxation rates at multiple magnetic fields.

The general concept of shuttle relaxometry is the combination of the resolution at high magnetic fields and the relaxation information at low magnetic fields. This can be achieved by shuttling the sample during the experiment to a desired position in the stray-field of the magnet bore, where magnetization relaxes during a set delay, while the signal is acquired at the high field position after the sample was shuttle back down. Since no radio frequency pulses can be applied outside the NMR probe this technique, so far, is limited to the study of longitudinal relaxation rates.
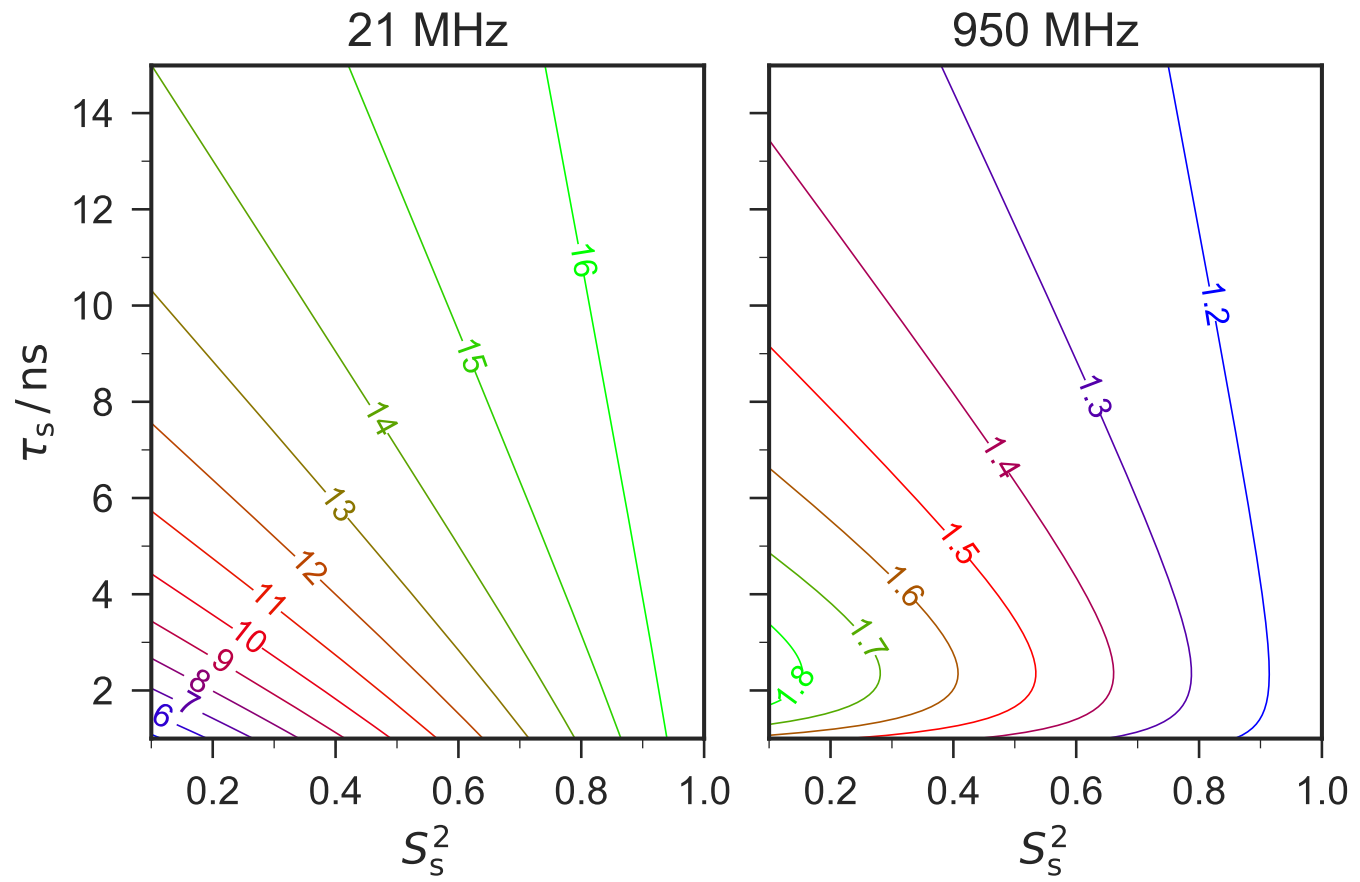

Figure 4.1: Comparison of $R_{1}$ at low $\left(\omega_{H}=21 \mathrm{MHz}, B_{0}=0.5 \mathrm{~T}\right)$ and high $\left(\omega_{H}=950 \mathrm{MHz}, B_{0}=22.3 \mathrm{~T}\right)$ magnetic field strengths. Contour plots of longitudinal relaxation rates $\left(R_{1}\right)$ estimated by the extended model free approach Equation (4.8). The following parameters were used for the calculation: $\tau_{\mathrm{c}}=5 \mathrm{~ns}, \tau_{\mathrm{f}}=5 \mathrm{ps}$ and $S_{\mathrm{f}}=0.8$. The correlation time of the slow process $\left(\tau_{\mathrm{s}}\right)$ as well as the corresponding order parameter $\left(S_{\mathrm{s}}\right)$ were varied in the range of $1-15 \mathrm{~ns}$ and $0.1-1$, respectively and contour lines represent the estimated relaxation rate. $R_{1}$ shows a much larger sensitivity to sub- $\tau_{\mathrm{c}}$ motions (especially in the low ns regime) at low magnet fields (Charlier et al., 2013). Thus, shuttle relaxometry is powerful technique to investigate local dynamics in the sub- $\tau_{\mathrm{c}}$ regime.

The use of the stray-field theoretically offers the possibility to measure relaxation rates at any magnetic field strengths between the static and earth magnetic field. Only the rela- 
tively long shuttle delays (up to $100 \mathrm{~ms}$ ) in combination with large relaxation rates at low fields limit the study to field strengths of up to $0.5 \mathrm{~T}$ (Charlier et al., 2016). By this technique, field-dependent data can be acquired over a much wider range of magnetic fields, consequently improving the spectral density mapping and accuracy of extracted dynamic parameters, while retaining the high resolution for a residue-wise analysis. Furthermore, $R_{1}$ shows a much higher sensitivity to local dynamics close to $\tau_{\mathrm{c}}$ (low nanosecond time scale) at low magnetic fields, as it is illustrated in Figure 4.1. Both highlight the power of shuttle relaxometry for studying sub- $\tau_{\mathrm{c}}$ protein motions.

Redfield and co-workers were the first to develop a pneumatic shuttle system for the measurement of protein dynamics by shuttle relaxometry (Redfield, 2003). Their combined analysis of low-field data obtained from shuttle experiments as well as high-field relaxation data was used to quantitatively measure sub- $\tau_{c}$ protein motions in SARSN (Severe Acute Respiratory Syndrome nucleocapsid protein) (Clarkson et al., 2009). It was the first highresolution relaxometry work on proteins and showed the benefits of low-field relaxation data in the estimation of sub- $\tau_{c}$ motions. In recent years another pneumatic shuttle system was developed by Bruker, which allows to use one magnet as a two-center spectrometer for liquid state DNP experiments (Lottmann et al., 2012) or using the stray-field of the magnet for relaxometry studies (Charlier et al., 2013). The pneumatic shuttle system developed by Bruker requires a specialized experimental setup, including NMR probe and sample preparation. Special sample containers need to be prepared manually, which are limited in their sample volume to $\simeq 100 \mu \mathrm{L}$ (Lottmann et al., 2012). Compared to standard $5 \mathrm{~mm}$ NMR tube this is a reduction of sample volume by roughly a factor of four. Logically this reduces the sensitivity of the experiments and leads to longer experimental times. Especially when considering the short relaxation times at low magnetic fields that already cause a reduction in the signal to noise. Furthermore, the pneumatic shuttle system cannot be combined with modern cryogenically cooled probes, which further reduces the sensitivity of these relaxometry experiments.

The discussed spectral density functions $(J(\omega))$ are the Fourier transformed stochastic correlation functions of the nucleus:

$$
J(\omega)=\operatorname{Re} \int_{-\infty}^{\infty} C_{00}^{2}(\tau) \exp (-i \omega \tau) d \tau
$$

where, $\tau_{\mathrm{c}}$ is the rotational correlation time, $i$ the imaginary number and $\omega$ is the frequency of a motion, assuming Brownian motion. Focusing on isotropic rotational diffusion, the correlation function is defined by:

$$
C_{00}^{2}(\tau)=\frac{1}{5} \exp \left(-\tau / \tau_{\mathrm{c}}\right)
$$

and its Fourier transformed results in the Lorentzian form of the spectral density function (Equation (4.1)). This form of $J(\omega)$ is used for decades to describe the relaxation behavior in NMR and deviations from this model are rarely reported in literature. The Fractional Brownian Dynamics Model is an example for such deviations (Glöckle and Nonnenmacher, 
1995; Kneller and Hinsen, 2004). Here, the spectral density function is modeled by:

$$
J_{\mathrm{FBD}}(\omega)=\frac{2 \tau_{\mathrm{c}} \sin (\beta \pi / 2)}{\left|\omega \tau_{\mathrm{c}}\right|\left(\left|\omega \tau_{\mathrm{c}}\right|^{\beta}+2 \cos (\beta \pi / 2)+\left|\omega \tau_{\mathrm{c}}\right|^{-\beta}\right)},
$$

where, $\tau_{\mathrm{c}}$ is the overall correlation time, $\omega$ is the frequency of the motion and $\beta$ represents the order of the fractional derivative $(1 \geq \beta \geq 0)$. For $\beta=1$, Equation (4.12) reduces to the Lorentzian form in Equation (4.1). The exponential character of the correlation function gets stretched with decreasing $\beta$, causing the spectral density function to decay faster with increasing frequencies (Figure 4.2). In protein NMR, field dependent studies of the spectral density function are challenging, due to the limitations in the available field range. Commercially available magnet field strengths already place a lower limit to $300 \mathrm{MHz}(\simeq 7.14 \mathrm{~T})$. In practice measurements might already be limited to $400 \mathrm{MHz}(\simeq 9.5 \mathrm{~T})$ or $500 \mathrm{MHz}(\simeq 11.9 \mathrm{~T})$ due to sensitivity and resolution of the NMR measurements. Both largely depends on the sample conditions (concentration, labeling, chemical shift dispersion) but it generally results in long measurement times at these low field strengths. Here, the discussed shuttle relaxometry approach offers the opportunity to study the field dependence of spectral density functions and the role of fast and slow local dynamics in nuclear spin relaxation.

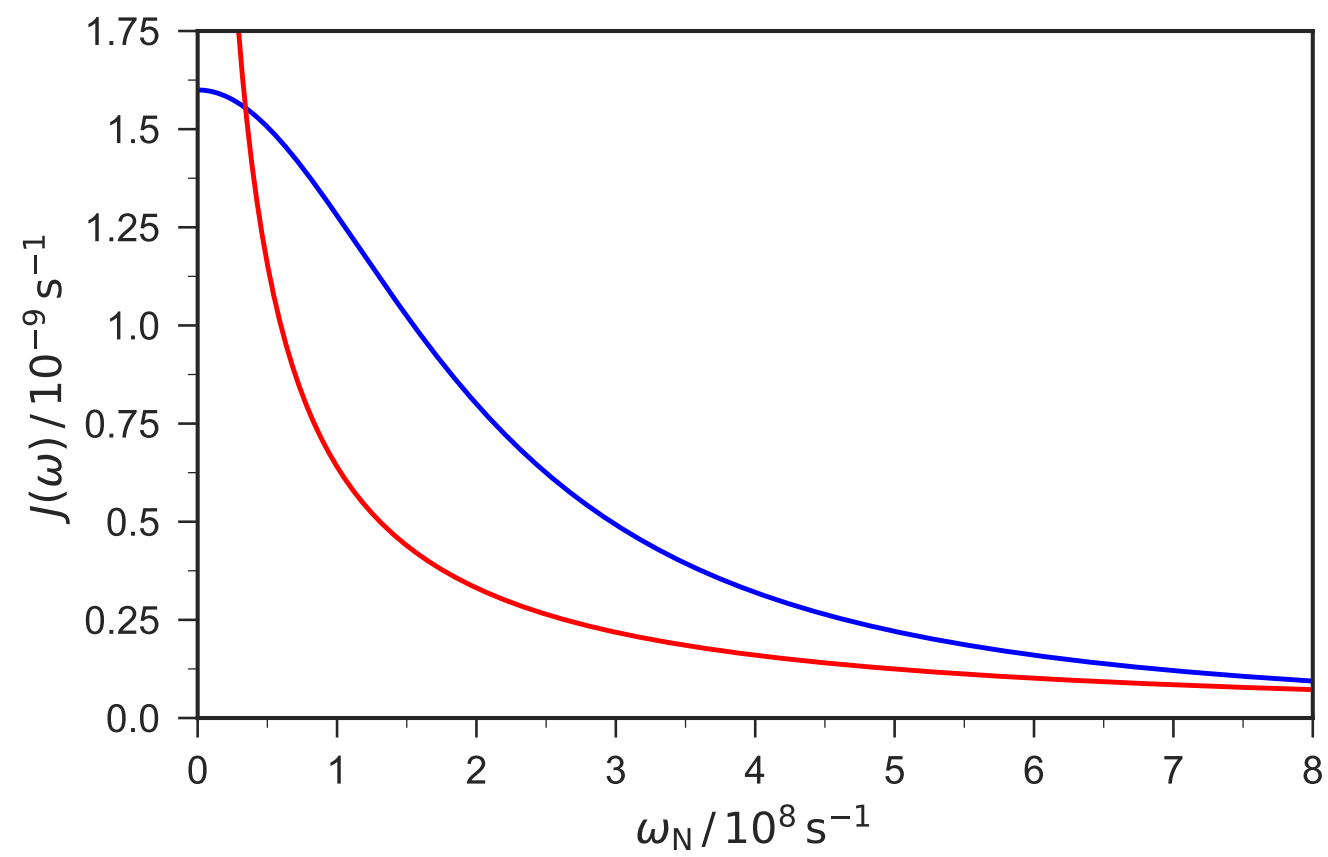

Figure 4.2: Simulated frequency dependent spectral density profiles of Brownian and Fractional Brownian Dynamics. The fractional Brownian dynamic (FBD) model describes a deviation from the classical Brownian dynamic model, in which the motion of an atomic particle at time point $t$ and $t+\tau$ are not necessarily independent anymore. Here the correlation function is "stretched" by the order of fractional derivative $(\beta)$, creating a non-Lorentzian behavior of the spectral density function $(J(\omega))$. For the simulation Equation (4.12) (red) and Equation (4.1) (blue) were used with the following parameters: $\tau_{\mathrm{c}}=5 \mathrm{~ns}, S^{2}=0.8$ and $\beta=0.5$. 
In this chapter a new, commercially available, motor-based shuttle system will be introduced to study the field-dependent Lorentzian behavior of spectral density functions at very low-fields and the effects of local motions to the longitudinal relaxation rate $R_{1}$. 


\subsection{Results and Discussion}

\subsubsection{Relaxometry}

The shuttle setup used for the relaxometry experiments in this chapter is a motor-based setup from Field Cycling Technology Ltd (Chou et al., 2012). A motor was installed on top of the NMR magnet that moves the sample-shuttle along a rail system that is inserted into the bore of the magnet with constant acceleration and deceleration. The settings of the motor are controlled by an additional software, where the desired field positions and motor parameters are defined. The motor movement is triggered by an electronic signal from the spectrometer console and thus the trigger can be directly implemented into the pulse sequence. Different to the pneumatic shuttle, mentioned in the introduction, this shuttle setup can be combined with cryogenically cooled probes. Furthermore, the setup can be used with

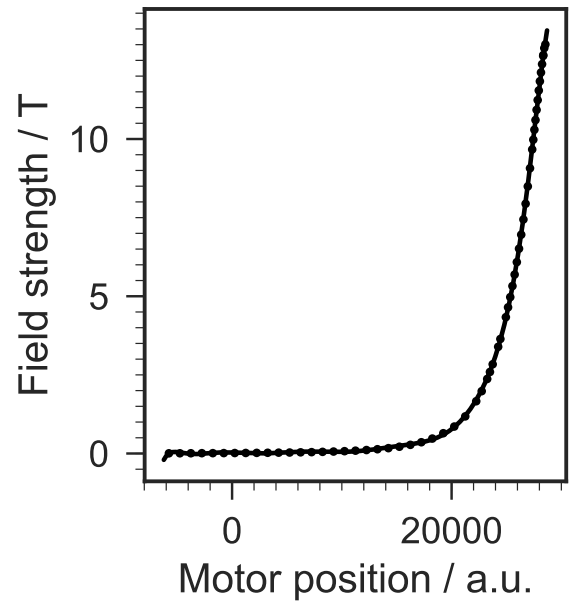

Figure 4.3: Stray field profile of the $700 \mathrm{MHz}$ oxford magnet dependent on the motor position. The field values were measured using a hall probe and the resolution of the motor positions is $0.037 \mathrm{~mm}$ per step. Values below 0 are reached outside the magnet bore. commercially available NMR tubes. For the experiments shown in this chapter, $5 \mathrm{~mm}$ NMR tubes with a medium wall thickness of $0.77 \mathrm{~mm}$ were used. Combining cryoprobes and conventional NMR tubes highly increase the sensitivity of shuttle relaxometry compared to the pneumatic shuttle approach (Chou et al., 2016).

Table 4.1: Shuttle time ( $\tau_{\text {up }}$ and $\left.\tau_{\text {down }}\right)$ to different field strength position in the stray field. The displayed delays were calculated using a sample length of $35 \mathrm{~mm}$ and motor parameters $a=350 \mathrm{rpss}$ and $v=1500 \mathrm{rpm}$ for acceleration and velocity, respectively.

\begin{tabular}{l|ccccc}
\hline Field / T & 0.50 & 0.75 & 1.00 & 1.51 & 2.01 \\
Shuttle Delay / ms & 133.5 & 128.1 & 124.2 & 118.9 & 114.8 \\
\hline \hline Field / T & 2.50 & 3.00 & 3.51 & 4.00 & 4.50 \\
Shuttle Delay / ms & 111.5 & 108.8 & 106.3 & 104.3 & 102.4 \\
\hline \hline Field / T & 5.01 & 5.51 & 6.01 & 8.02 & 10.02 \\
Shuttle Delay / ms & 100.7 & 99.1 & 97.6 & 92.6 & 88.2 \\
\hline
\end{tabular}

The motor position was calibrated, using optical sensors along the rail-system. Due to interference of the magnet field with the electronics of the optical sensors, the position of the shuttle cannot be reliably calibrated between the static field strength $(16.44 \mathrm{~T})$ and 


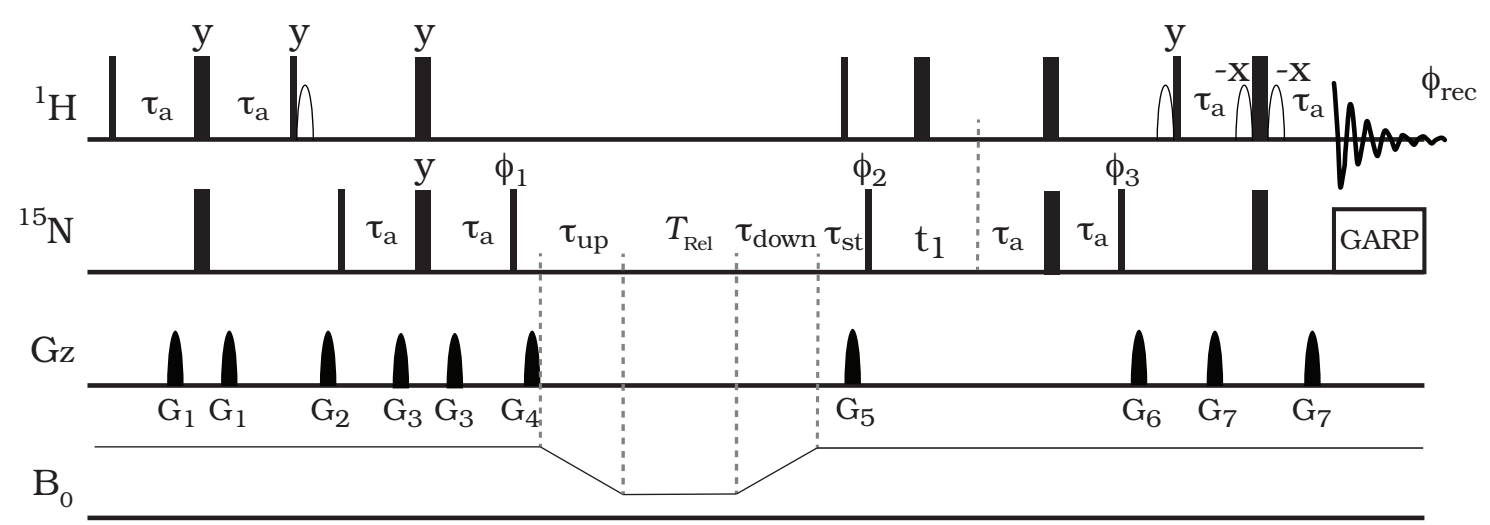

Figure 4.4: Scheme of the pulse sequence for shuttle relaxometry experiments. Narrow and wide bars denote $90^{\circ}$ and $180^{\circ}$ pulses, respectively and open bars denote selective pulses for water suppression. $\tau_{\mathrm{a}}$ is the INEPT delay $\left(1 /\left(4 J_{\mathrm{NH}}\right)\right), \tau_{\text {up }}$ and $\tau_{\text {down }}$ are the shuttle up and down times, respectively. $T_{\text {rel }}$ is the relaxation delay in the stray field and $\tau_{\text {st }}$ is the stabilization delay to avoid vibration artifacts. The pulse sequence was run as a pseudo-3D experiment were $\phi_{1}$ was incremented by $180^{\circ}$ to store negative and positive $\mathrm{N}_{Z}$ magnetization separately. Details of the phase cycle and delays can be found in the materials and methods section.

roughly $14 \mathrm{~T}$. Table 4.1 shows the calculated shuttle times for different field positions that were used during the course of this work. The pulse sequence used for the shuttle relaxometry experiments is shown in Figure 4.4 and is based on the heteronuclear $T_{1}$ experiment by Kay and co-workers (Kay et al., 1989). In the center of the pulse sequence, before the chemical shift labeling during $t_{1}$, a shuttle block is included. A $90^{\circ}$ pulse before the block controls the sign of the longitudinal magnetization and is incremented in a pseudo 3D experiment to store negative and positive magnetization separately. A gradient before the shuttle up event dephases left over water magnetization to minimize solvent exchange during the relaxation delay. The shuttle event contains four delays of which the shuttle delays $\tau_{\text {up }}, \tau_{\text {down }}$ (Table 4.1) and a stabilization delay $\tau_{\text {st }}$ are constant for a set of experiments at a given field. Thus, the intensity of a peak only depends on the variable relaxation delay $T_{\text {rel }}$. After the shuttle block, chemical shift labeling and readout were done as in an HSQC experiment.

Figure 4.5 shows spectra of ubiquitin obtained using the pulse sequence shown in Figure 4.4, under static and shuttle conditions. As it can be seen the high resolution of the static field is preserved and only the peak intensities are modulated by the relaxation rates at the low-field under shuttle conditions.

The obtained intensity profiles were fitted to an exponential decay model to estimate $R_{1}$. In case of the separated positive and negative z-magnetization experiments a monoexponential model was used:

$$
I_{\mathrm{Z}}(t)=I_{\mathrm{N}} \pm I_{\mathrm{H}} \exp \left(-R_{1} T_{\mathrm{Rel}}\right)
$$

in which, $I_{\mathrm{H}}$ is the magnetization transferred from ${ }^{1} \mathrm{H}$ to ${ }^{15} \mathrm{~N}$ during the first two INEPT 

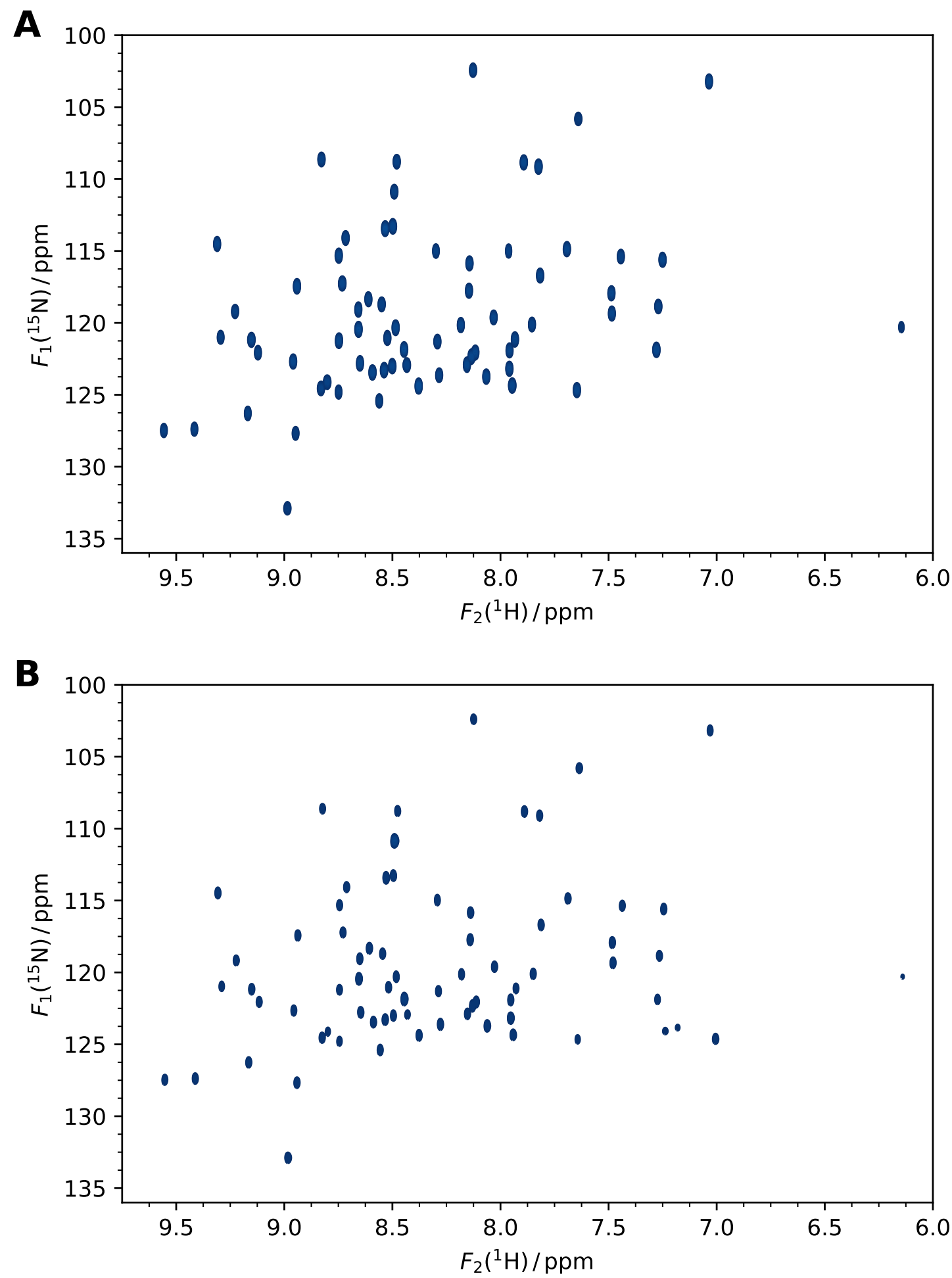

Figure 4.5: Comparison of $2 \mathrm{D}$ planes of ubiquitin from static and shuttle pseudo-3D $R_{1}$ experiments. (A) $2 \mathrm{D}$ plane of static field $R_{1}$ experiment acquired at $16.45 \mathrm{~T}$ (700 MHz Larmor frequency) using 4 scans . (B) $2 \mathrm{D}$ plane of shuttle $R_{1}$ experiment with relaxation at $0.5 \mathrm{~T}$ and signal acquisition at $16.45 \mathrm{~T}$ (700 MHz Larmor frequency). The shuttle delays and stabilization delay were set to $133.5 \mathrm{~ms}$ and $75 \mathrm{~ms}$, respectively. In both experiments the relaxation delay $\left(T_{\text {rel }}\right)$ was set to $5 \mathrm{~s}$. The high resolution of the static field is contained in both experiments, while the relaxation information of the low-field is encoded in the reduced intensity of the resonances in the shuttle experiment. 
blocks before for shuttle event, $I_{\mathrm{N}}$ is the Boltzmann magnetization of ${ }^{15} \mathrm{~N}$ in the low-field and $T_{\text {rel }}$ is the relaxation delay in the low-field. The selection of negative and positive magnetization is controlled by the phase of the $90^{\circ}$ pulse before the shuttle block. Right before this pulse the magnetization is described by the operator $I_{\mathrm{x}}$, which denotes in-phase magnetization parallel to the $\mathrm{x}$-axis. Using a pulse with $\phi=\mathrm{y}$ flips the transverse ${ }^{15} \mathrm{~N}$ magnetization anti-parallel to the z-axis $\left(I_{z}^{-}\right)$. A phase change of $\phi_{1}$ by $180^{\circ}\left(\phi_{1}=-y\right)$ also changes the orientation of the magnetization by $180^{\circ}$ and aligns it parallel to the z-axis $\left(I_{\mathrm{z}}^{+}\right)$. The absolute intensity is the same in both orientations $\left(I_{\mathrm{z}}^{+}=-I_{\mathrm{z}}^{-}\right)$. In case of the combined analysis the difference of both intensities is calculated:

$$
I_{\mathrm{z}}=I_{\mathrm{z}}^{+}-I_{\mathrm{z}}^{-}
$$

in which, + and - denote the intensities of positive and negative ${ }^{15} \mathrm{~N}$ z-magnetization, respectively. The decay of magnetization is described by the linear combination of both decay models (Equation (4.13)) and can be simplified to:

$$
I_{\mathrm{z}}(t)=I_{\mathrm{H}} \exp \left(-R_{1} T_{\mathrm{Rel}}\right)
$$

in which, the ${ }^{15} \mathrm{~N}$ Boltzmann term is eliminated and only the initially transferred magnetization from ${ }^{1} \mathrm{H}$ is fitted together with the longitudinal relaxation rate $\left(R_{1}\right)$ of ${ }^{15} \mathrm{~N}$. The
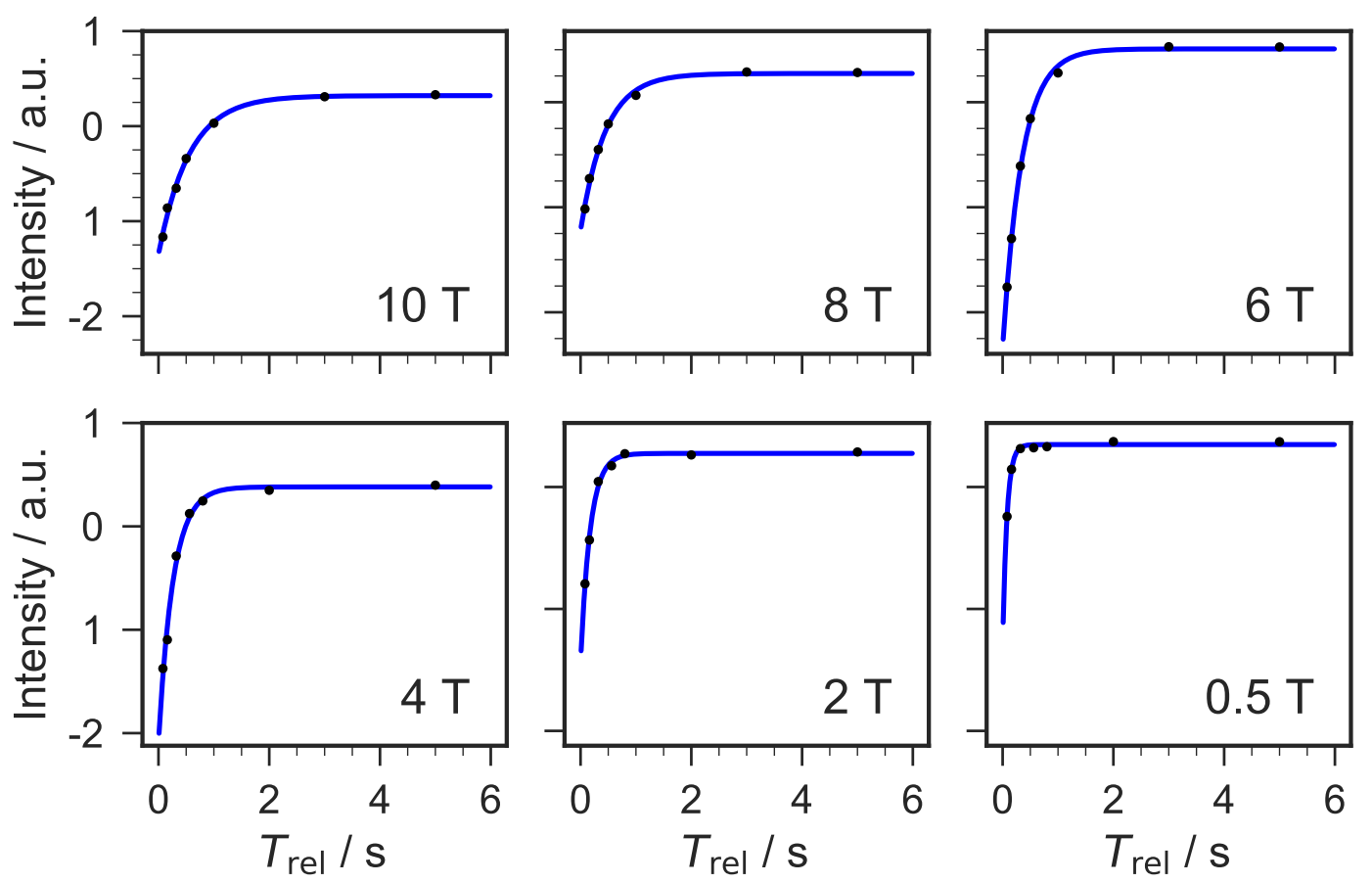

Figure 4.6: Shuttle relaxometry data of residue Isoleucine 30. The $\mathrm{N}_{\mathrm{z}}^{-}$intensity profiles of shuttle experiments at stray field strengths of $10,8,6,4,2$ and $0.5 \mathrm{~T}$ are shown together with the fitted exponential model Equation (4.13) as blue line. All experimental curves were in good agreement to the applied exponential model fit.

intensity profiles of 70 resolved peaks of ubiquitin were analyzed at different field strengths 
between 0.5 and $10 \mathrm{~T}$ using the described shuttle setup. Figure 4.6 shows the intensity profiles at selected fields of residue I30 as an example. Most of the intensity profiles followed the models of the three different analysis. Interestingly, residues between L8 and T12, as well as the last four C-terminal residues, showed a discrepancy to the expected decay model of positive and negative magnetization (Figure 4.7). For these residues Equation (4.13) only describes the intensity profiles at magnetic fields from the static field up to $4 \mathrm{~T}$. At lower fields strengths the intensities seem to "overshoot" the Boltzmann magnetization of the low-field and only reach its equilibrium state after very long relaxation delays in the low-field.
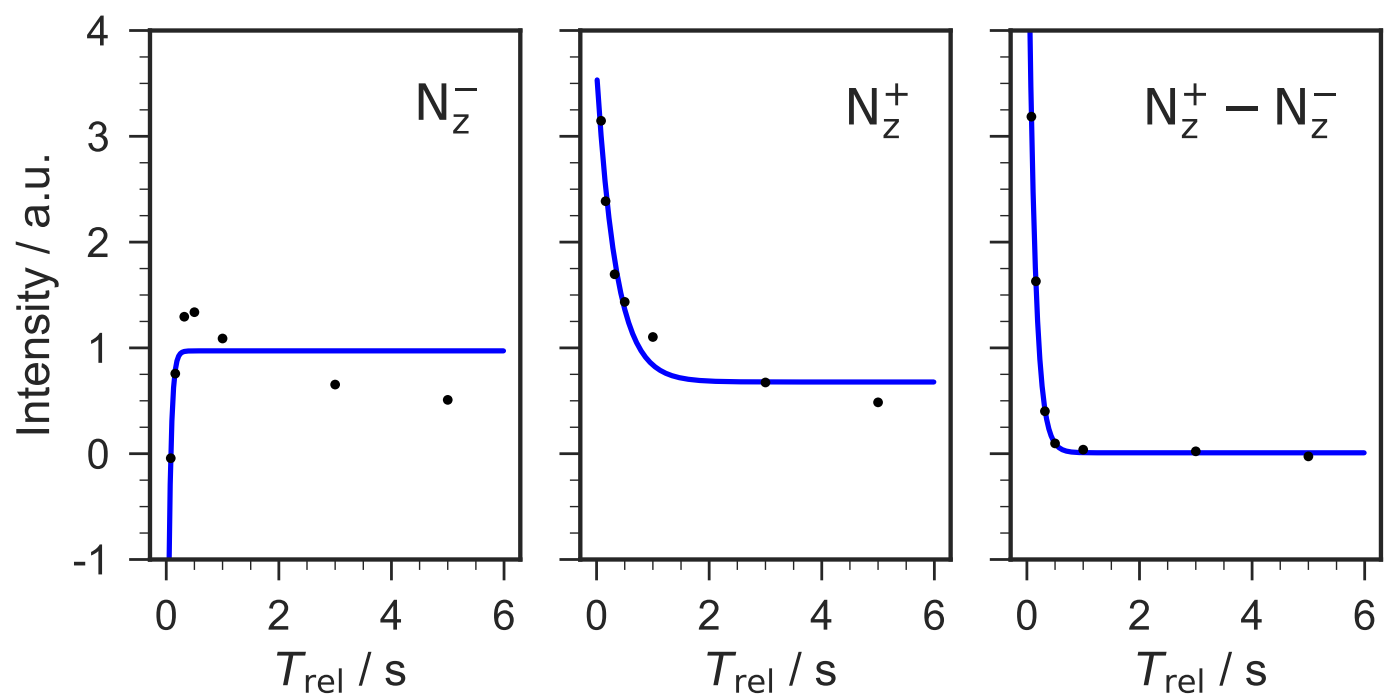

Figure 4.7: Shuttle Relaxometry data of Threonine 9 at $0.5 \mathrm{~T}$. Intensity profiles and fitted exponential decay model (blue line) of the three different analysis modes. $\mathrm{N}_{\mathrm{z}}^{-}$ (left) and $\mathrm{N}_{\mathrm{z}}^{+}$(center) derived intensities show a deviation from the exponential model, that is compensated in the $\left(\mathrm{N}_{\mathrm{z}}^{+}-\mathrm{N}_{\mathrm{Z}}^{-}\right)$derived profiles (right). Thus, it could be assumed that a relaxation mechanism, which only contributes measurably at low magnetic field strengths, caused the deviations in the individual profile analysis.

A possible explanation for this could be either an exchange with water molecules or local dynamics, which only contributes measurably at low magnetic fields. Detection of the NMR signal after gradient G4 (Figure 4.4) showed an empty spectrum, which allows the assumption that the water magnetization is suppressed as good as possible before the relaxation block. Thus, solvent exchange in the low-field can be neglected.

The residues 8-12 are located in a loop region of ubiquitin, which showed a higher flexibility in previous studies of ubiquitin backbone order parameter (Tjandra et al., 1995; Lange et al., 2008). Also, the C-terminal residues are known to show a higher mobility. Both supports the hypothesis that local dynamics are responsible for the seen deviations in the intensity profiles.

A comparison of the negative, positive and combined intensity profiles of residue T9 is shown in Figure 4.7. A similar deviation as in the negative magnetization decay was 
detected for the positive $\mathrm{N}_{\mathrm{z}}$ experiment. While the magnetization decays towards the Boltzmann equilibrium, the decay slows down in the range of delays where an "overshoot" was visible in the negative $\mathrm{N}_{\mathrm{z}}$ experiment. On the other hand, the combined analysis showed neither of these effects and could be fitted to the expected mono exponential model (Equation (4.15)). This further indicates that the cause of these deviations is a relaxation mechanism, that gets compensated when both positive and negative magnetization are combined. At this point the cause of the deviation could not be clearly characterized, but the data implies that the relaxation data from low-fields yields new insights into local dynamics on the time scale of picoseconds to nanoseconds.
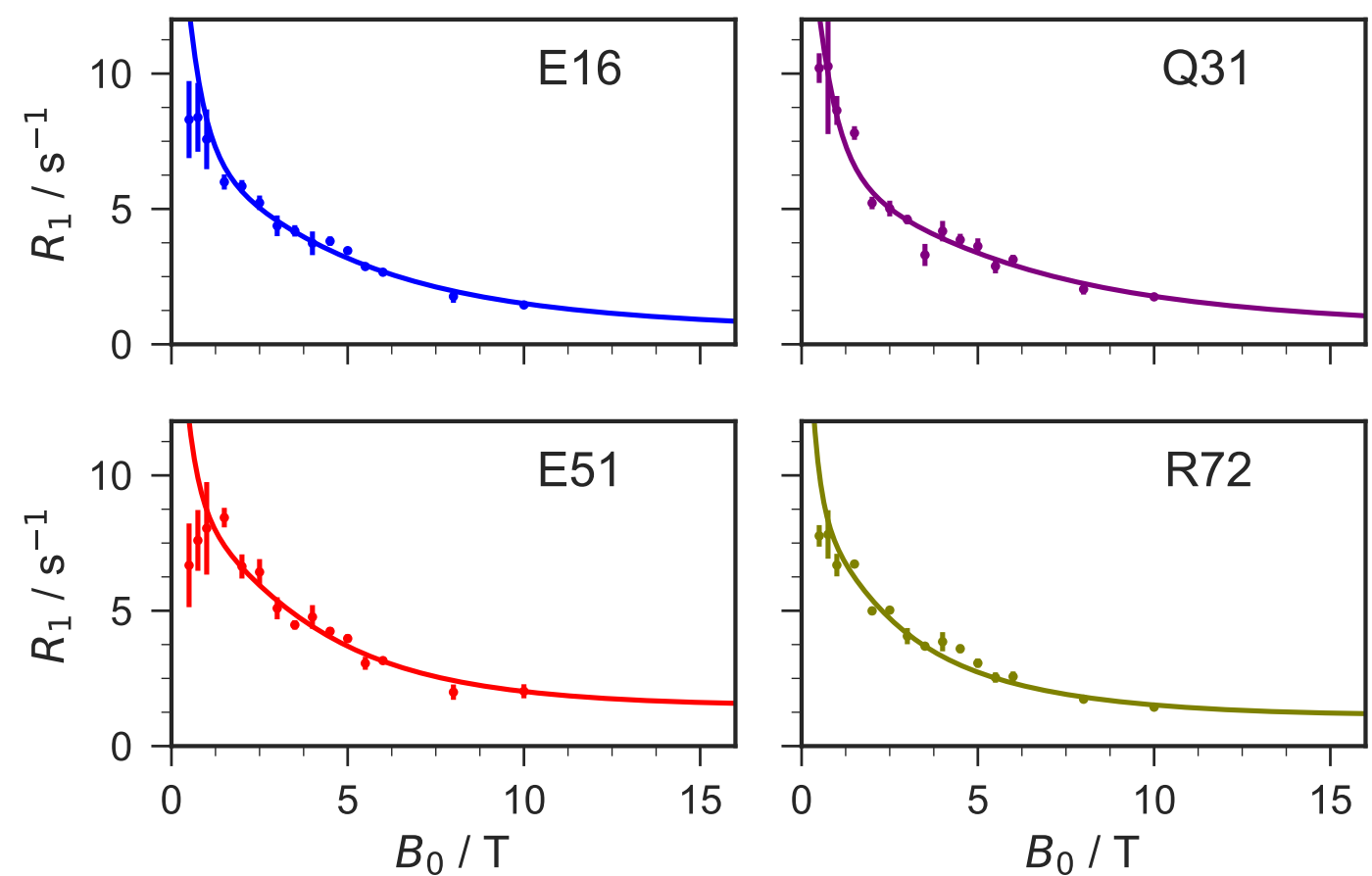

Figure 4.8: Field dependence of $R_{1}$ derived by shuttle relaxometry. $R_{1}$ values were obtained by shuttle relaxometry experiments between 0.5 and $10 \mathrm{~T}$. As an example the results for residues E16, Q31, E51 and R72 are shown. At field strengths below 2T deviations from the Lorentzian spectral density model are prominently seen (residue E16 and E51).

The fitted $R_{1}$ values derived by shuttle experiments in the range of $0.5 \mathrm{~T}$ to $10 \mathrm{~T}$ were analyzed according to the field dependent spectral density function. The rates were fitted residue-wise using Equation (4.4) and Equation (4.7) as model for the spectral density function. As it is common in spectral density mapping the zero- $\left(J\left(\omega_{\mathrm{H}}-\omega_{\mathrm{N}}\right)\right)$ and doublequantum $\left(J\left(\omega_{\mathrm{H}}+\omega_{\mathrm{N}}\right)\right)$ spectral density terms were substituted by a first order approximation of $0.92 J\left(\omega_{\mathrm{H}}\right)$ to simplify the fitting procedure (Palmer, 2004). The resulting fit curves for selected residues are shown in Figure 4.8. The obtained values for $R_{1}$ from the exponential model fits showed a scatter that was larger than the error estimated from the fit. Especially for very low-field data points a strong deviation from the expected Lorentzian profile of the spectral density function was noted (Figure 4.8, E51). Some residues showed a decreasing trend in their longitudinal relaxation rate at low fields that was larger than 
the estimated experimental error.

A comparison of the fitted local dynamic parameter showed a large variation in the results for $\tau_{\mathrm{c}}$ and $S^{2}$ of the individual residues (Figure 4.9). Firstly, the fitted $\tau_{\mathrm{c}}$ values tend to be larger than expected for ubiquitin at $21^{\circ} \mathrm{C}$ and secondly both values showed a large fluctuation in the individual results. The rotational correlation time could be estimated based on a viscosity model of the solvent and the hydrophobic radius of the protein according to Stokes law:

$$
\tau_{\mathrm{c}}=\frac{4 \pi \eta r^{3}}{3 k_{\mathrm{b}} T},
$$

in which, $\eta$ is the viscosity of the solvent, $r$ is the hydrophobic radius of a molecule, $k_{b}$ is the Boltzmann constant and $T$ is the temperature. Using the viscosity model of Cho et al. (Cho et al., 1999) and a hydrophobic radius for ubiquitin according to Thompson et al. (Thompson and Rovnyak, 2007) an overall rotational correlation time of $5.16 \mathrm{~ns}$ was estimated for ubiquitin in $100 \% \mathrm{H}_{2} \mathrm{O}$. This estimation neglected the contribution of the more viscous $\mathrm{D}_{2} \mathrm{O}$ in the actual solvent $\left(90 \% \mathrm{H}_{2} \mathrm{O}+10 \% \mathrm{D}_{2} \mathrm{O}\right)$, which would slightly increase $\tau_{\mathrm{c}}$. Compared to this theoretical value, the majority of the individual fits overestimated $\tau_{\mathrm{c}}$, even when a higher viscosity of the solvent mixture is considered. Partially this could be rationalized by the convolution of $\tau_{\mathrm{c}}$ and $S^{2}$ in the extended spectral model Equation (4.8).
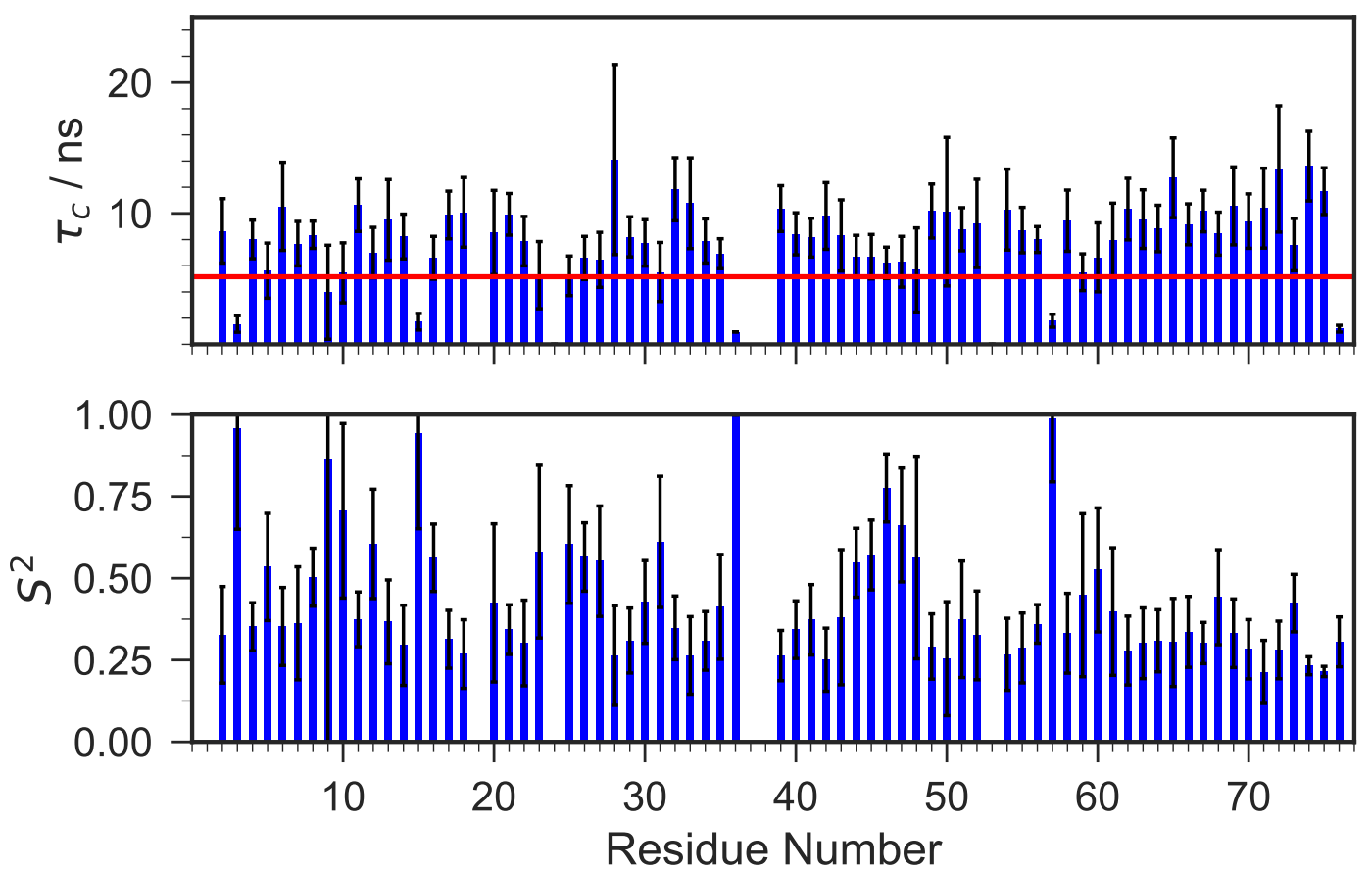

Figure 4.9: Results from individual fit for $\tau_{\mathrm{c}}$ and $S^{2}$ from shuttle relaxometry data. Values were obtained by fitting the shuttle relaxometry data to Equation (4.8). The large errors and variations in $\tau_{\mathrm{c}}$ and $S^{2}$ as well as deviations from the expected $\tau_{\mathrm{c}}$ value of $\sim 5.16 \mathrm{~ns}$ indicate an unstable fit.

The latter showed significantly smaller values than expected for the ubiquitin backbone 
order parameters. In addition, the comparison of the two bar plots showed an opposing trend for $\tau_{\mathrm{c}}$ and $S^{2}$, such that large values in $\tau_{\mathrm{c}}$ get compensated by small values of $S^{2}$ (Figure 4.9). This indicated the use of only $R_{1}$ data might not be sufficient for a deconvolution of the two parameters.

As already mentioned, a deviation from the Lorentzian behavior of the spectral density function was noted for some residues at very low field strengths. To clarify these findings the shuttle experiments were repeated for low field strengths in the range of 0.5 to $2 \mathrm{~T}$. The results of the repeated experiments are shown in Figure 4.10. The results of the fitted relaxation rates vary significantly for the individual field strengths. This variation could not be explained by the experimental error and seemed to increase with decreasing field strength.

An explanation could be found in the relaxation delay dependent intensity profiles in Figure 4.6. The increased rates at low field strengths let the magnetization decay much faster towards the ${ }^{15} \mathrm{~N}$ Boltzmann equilibrium at the low-field position. Comparing the six intensity profiles it can be seen that for $0.5 \mathrm{~T}$ only two data points characterize the exponential decay.

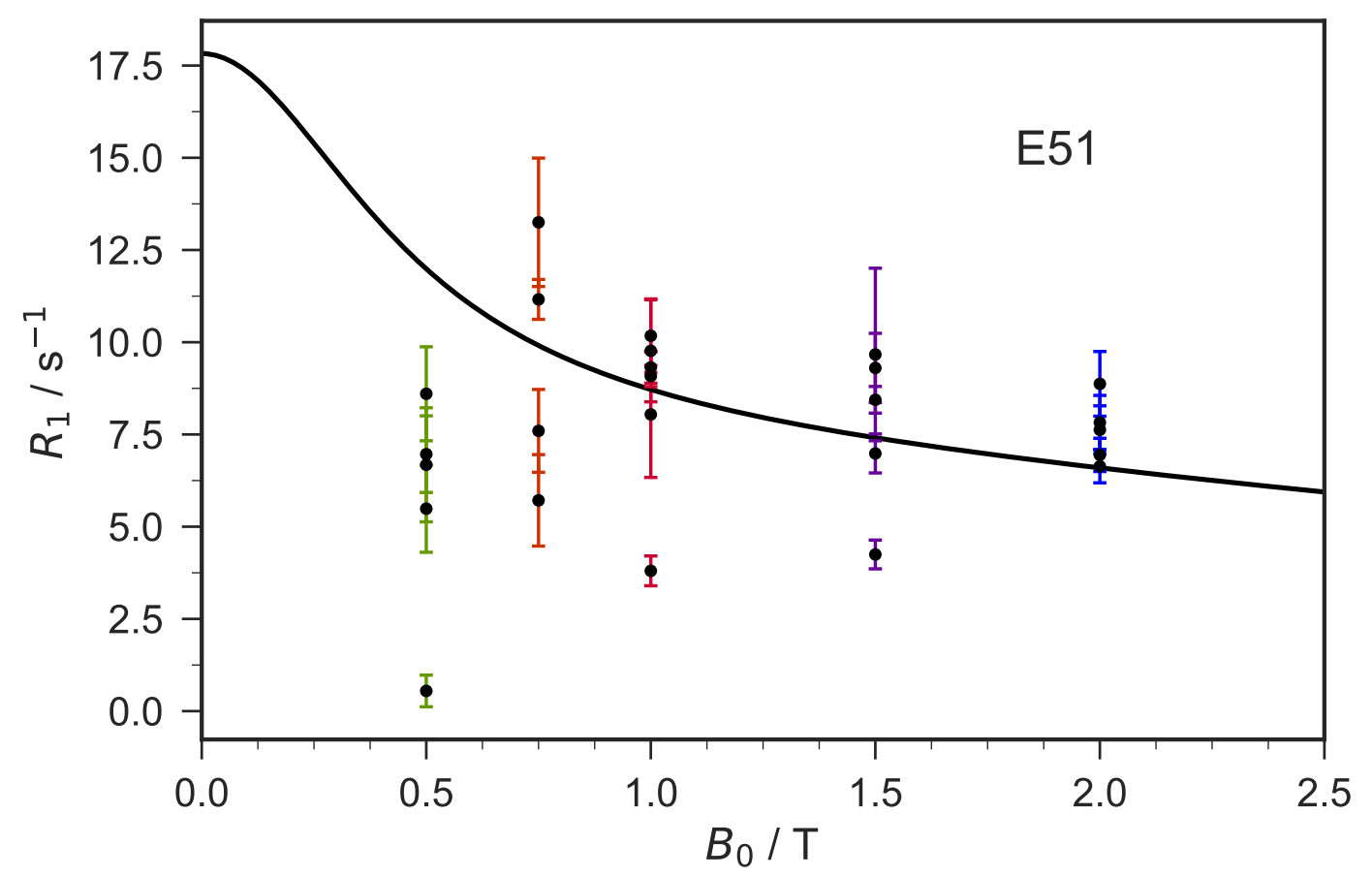

Figure 4.10: Results from repeated shuttle experiments in the very low field strength range of 0.5 to $2 \mathrm{~T}$. Distribution of fitted relaxation rates by repeated shuttle experiments in the field range of 0.5 to $2.0 \mathrm{~T}$ with Equation (4.9). The variations in $R_{1}$ values are larger than the estimated uncertainty of the fit parameters.

The used relaxation delays lead to intensities already close to the zero point of the function at $0.5 \mathrm{~T}$. Due to technical limitations a minimum relaxation delay of $80 \mathrm{~ms}$ had to be used in the shuttle experiments. Thus, the experiments could not be measured with shorter de- 
lays to improve the fitting. Assuming a relaxation rate of $12 \mathrm{~s}^{-1}$ a minimum delay of $80 \mathrm{~ms}$ causes a decay of intensity by roughly $70 \%$ without including losses during the shuttle and the stabilization delay. This results in intensity profiles at low fields that are too flat to guarantee an accurate fit of the rate using the exponential model. In addition to the shuttle experiments a set of high-field experiments under static conditions were measured to put the obtained low-field data into perspective of non-shuttle relaxation rates. A comparison of the two data sets in Figure 4.11 indicates a tendency of lower relaxation rates derived by the shuttle experiments. Due to the mentioned technical limitations shuttle experiments were only measured up to $10 \mathrm{~T}$, which corresponds to a ${ }^{1} \mathrm{H}$ Larmor frequency of $420 \mathrm{MHz}$. Thus, the best indicator for the quality of the shuttle rates are the results from data acquired at static fields operating at a Larmor frequency of $400 \mathrm{MHz}$ and $300 \mathrm{MHz}$. As Figure 4.11 shows these values do not fit into the relaxation profiles of the shuttle data, but rather are in the order of rates measured at field strengths of $5 \mathrm{~T}(\simeq 210 \mathrm{MHz})$. Both data sets were fitted residue-wise using Equation (4.7) as spectral density model. The results are plotted together with the relaxation data in Figure 4.11. A clear discrepancy between the shuttle data and conventionally acquired relaxation data could be seen. The uncertainty of the low-field fit parameters could not explain the deviation to the static field data and the measured intensity profiles (Figure 4.6) did not indicate major differences to the exponential model besides the mentioned residues (Figure 4.6). According to these findings a systematic error in the experimental setup of the shuttle experiment had to be assumed.
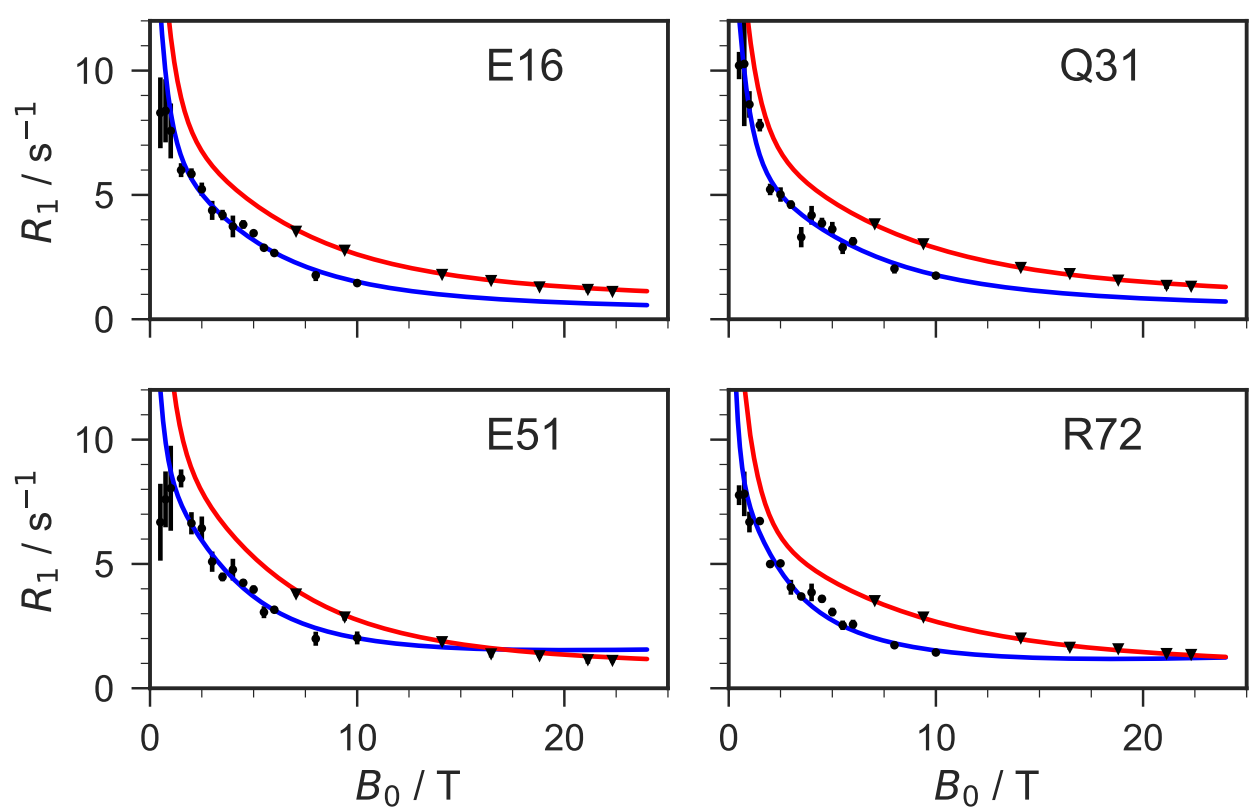

Figure 4.11: Comparison of shuttle and static relaxometry measurement. $R_{1}$ relaxation rates derived from shuttle experiments in the stray field of the magnet $(\bullet)$ and $R_{1}$ experiments at different static magnetic fields $(\boldsymbol{\nabla})$ show a strong discrepancy. The fits of field dependence of $R_{1}$ are shown in blue for shuttle and red for static field measurements.

The individually fitted longitudinal relaxation rates of the static field experiments were 
analyzed in the same way as the shuttle data and the results for $\tau_{\mathrm{c}}$ and $S^{2}$ are shown in Figure 4.12. The obtained values for $\tau_{\mathrm{c}}$ show a narrow distribution and are in good agreement with the estimated correlation time of $5.16 \mathrm{~ns}$. Only the C-terminal residues, which are very mobile and have order parameters as low as $20 \%$, showed a clear deviation, which can be rationalized by the convolution of $\tau_{\mathrm{c}}$ and $S^{2}$ in individual fits. The results for the C-terminal order parameters are higher than expected and compensate the underestimated $\tau_{\mathrm{c}}$ results. For a further evaluation of the $R_{1}$ field dependent analysis, additional
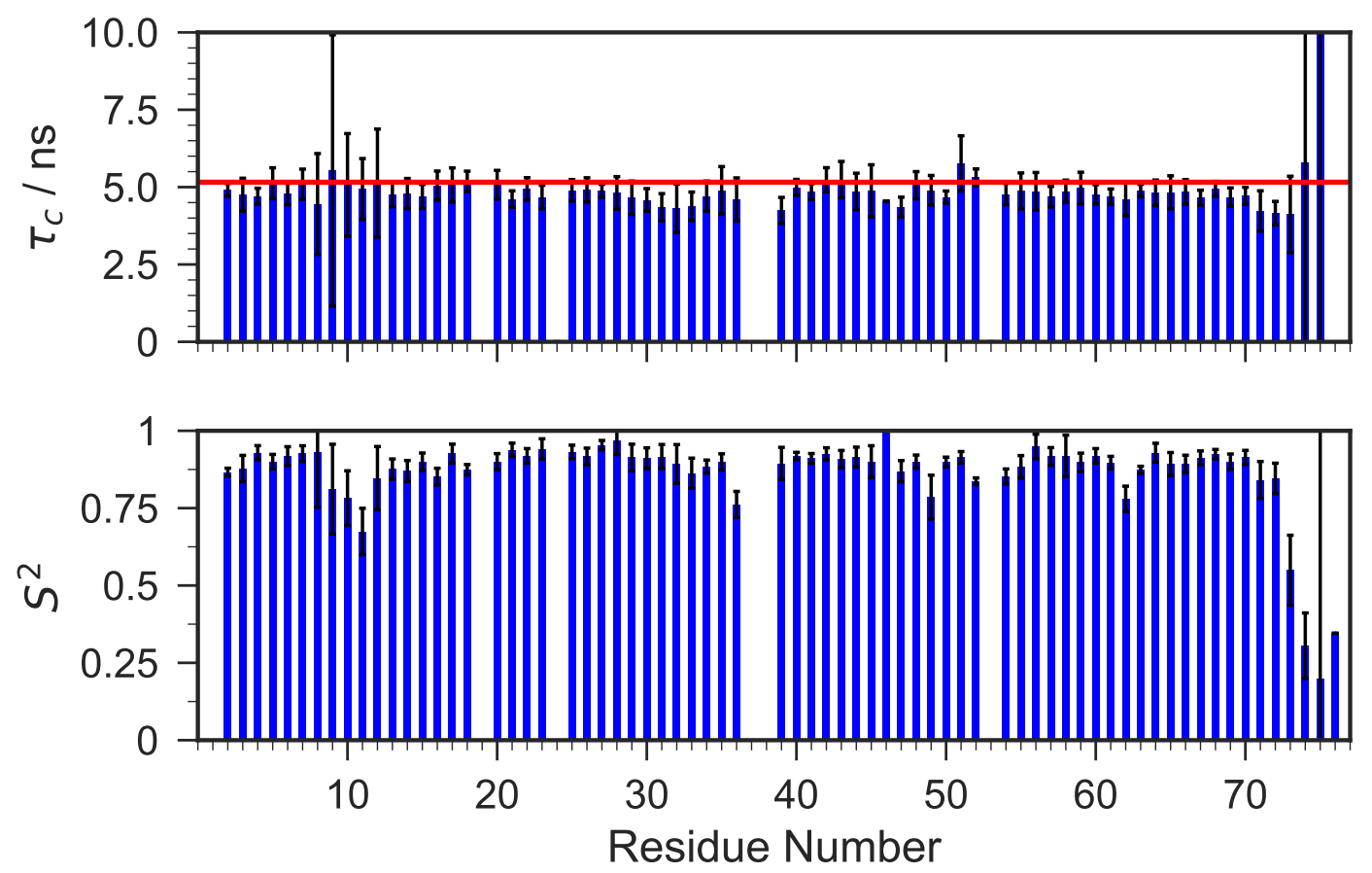

Figure 4.12: Results from individual fit of $\tau_{\mathrm{c}}$ and $S^{2}$ from static relaxometry measurement. Values were obtained by fitting $R_{1}$ data of seven different static field strengths between 7 and $22 \mathrm{~T}$ using Equation (4.8). The individual results showed a narrow distribution of $\tau_{\mathrm{c}}$ that was close to the estimated value of $\tau_{\mathrm{c}}$ (red line) assuming Stokes law (Equation (4.16)) and the $S^{2}$ values also showed reasonable results. Only residues in the C-terminal region showed large variations, which could be rationalized by the very long relaxation times due to the high flexibility of these residues. The results in general indicated that an analysis of the local motion parameter based on $R_{1}$ data is feasible.

$R_{2}$ and hetNOE experiments were acquired to compare the $R_{1}$ fit results with a model free analysis. In Table 4.2 the fitted correlation times at the measured field strengths are listed. All fitted correlation times are comparable to the estimated $\tau_{\mathrm{c}}$, while showing slightly larger values. This can be rationalized by the assumption of $100 \% \mathrm{H}_{2} \mathrm{O}$ as solvent during the estimation of $\tau_{\mathrm{c}}$. The sample contained a mixture of $90 \% \mathrm{H}_{2} \mathrm{O}$ and $10 \%$ $\mathrm{D}_{2} \mathrm{O}$ and the contribution of $\mathrm{D}_{2} \mathrm{O}$ with its higher viscosity was neglected. Therefore, the effective viscosity of the sample is underestimated during the calculation, which results in a lower estimated $\tau_{\mathrm{c}}$. 
The fitted Lipari-Szabo order parameters of the different data sets are shown in Figure 4.13. During the fitting procedure residues with large errors in the fit parameters were discarded automatically. The order parameter profiles indicate that the estimation of local dynamics at high magnetic field strengths has a larger uncertainty. With decreasing field strength more order parameters could be reliably estimated and at $300 \mathrm{MHz}$ all residues showed a decent fit result for $S^{2}$. This indicates the importance of low field data in the study of local protein dynamics by relaxation experiments.

Table 4.2: Model free analysis results of the correlation time at different magnetic field strengths. Experiments at all field strengths lead to similar results for the overall rotational correlation time $\left(\tau_{\mathrm{c}}\right)$ and are comparable to the results obtained from the field-dependent $R_{1}$ analysis.

\begin{tabular}{c|cccc}
\hline & $300 \mathrm{MHz}$ & $400 \mathrm{MHz}$ & $600 \mathrm{MHz}$ & $700 \mathrm{MHz}$ \\
\hline$\tau_{\mathrm{c}} / \mathrm{ns}$ & $6.62 \pm 0.29$ & $6.13 \pm 0.18$ & $5.69 \pm 0.29$ & $5.33 \pm 0.24$ \\
\hline \hline & $800 \mathrm{MHz}$ & $900 \mathrm{MHz}$ & $950 \mathrm{MHz}$ & estimated \\
\hline$\tau_{\mathrm{c}} / \mathrm{ns}$ & $5.58 \pm 0.30$ & $5.39 \pm 0.43$ & $5.66 \pm 0.31$ & 5.16 \\
\hline
\end{tabular}
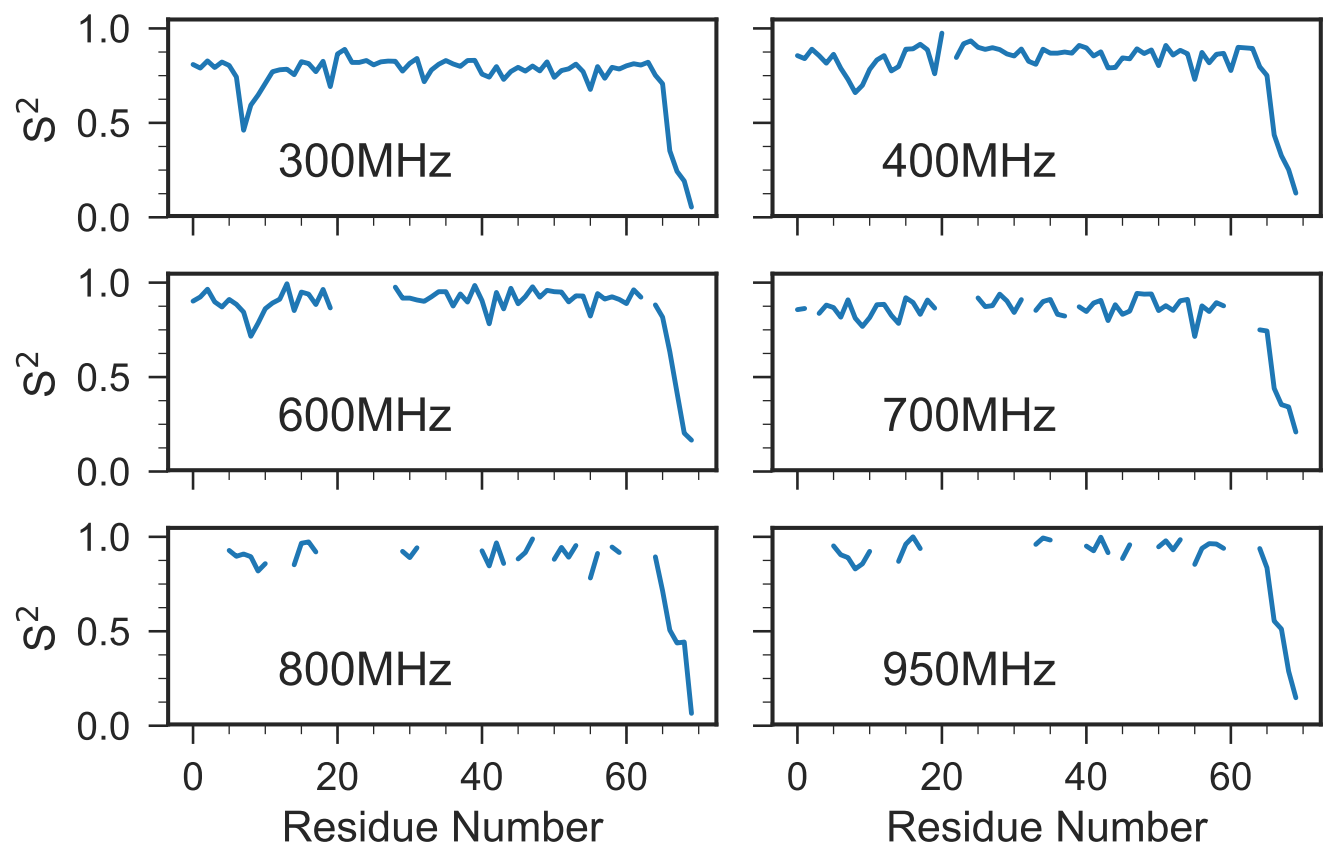

Figure 4.13: Model free analysis for Lipari-Szabo Order Parameter at different static magnetic field strengths. As model for the spectral density function, Equation (4.8) was used and residues with large uncertainties were automatically discarded during the fit procedure. While all data sets lead to similar results for fitted residues, the number of discarded residues decreased with a decreasing magnetic field strength. This indicates that the extraction of local kinetic parameters in the sub- $\tau_{\mathrm{c}}$ window from relaxation data is more feasible at low magnetic field strengths. 


\subsubsection{System performance}

During the shuttle experiment the sample leaves the probe and the usual control mechanisms for spectrum quality do not apply anymore. As already mentioned at the beginning of this chapter, the shuttle approach is limited to longitudinal relaxation measurement, since radio frequency pulses cannot be applied in the stray-field of the magnet. Phase cycle schemes for water suppression can only be applied after the shuttle block as the control over the magnetization is lost during the shuttle and relaxation delays. Furthermore, temperature stability is a problem during the shuttle experiments. In conventional NMR experiments the temperature is stable and heating effects due to pulse application is compensated by a sufficient number of dummy scans. Shuttle experiments on the other hand could be affected by temperature gradients between the low-field and static position. To handle the latter effects the temperature was set to the room temperature, which can be estimated to be also the temperature in the bore of the magnet. This minimizes the temperature differences between the two positions.
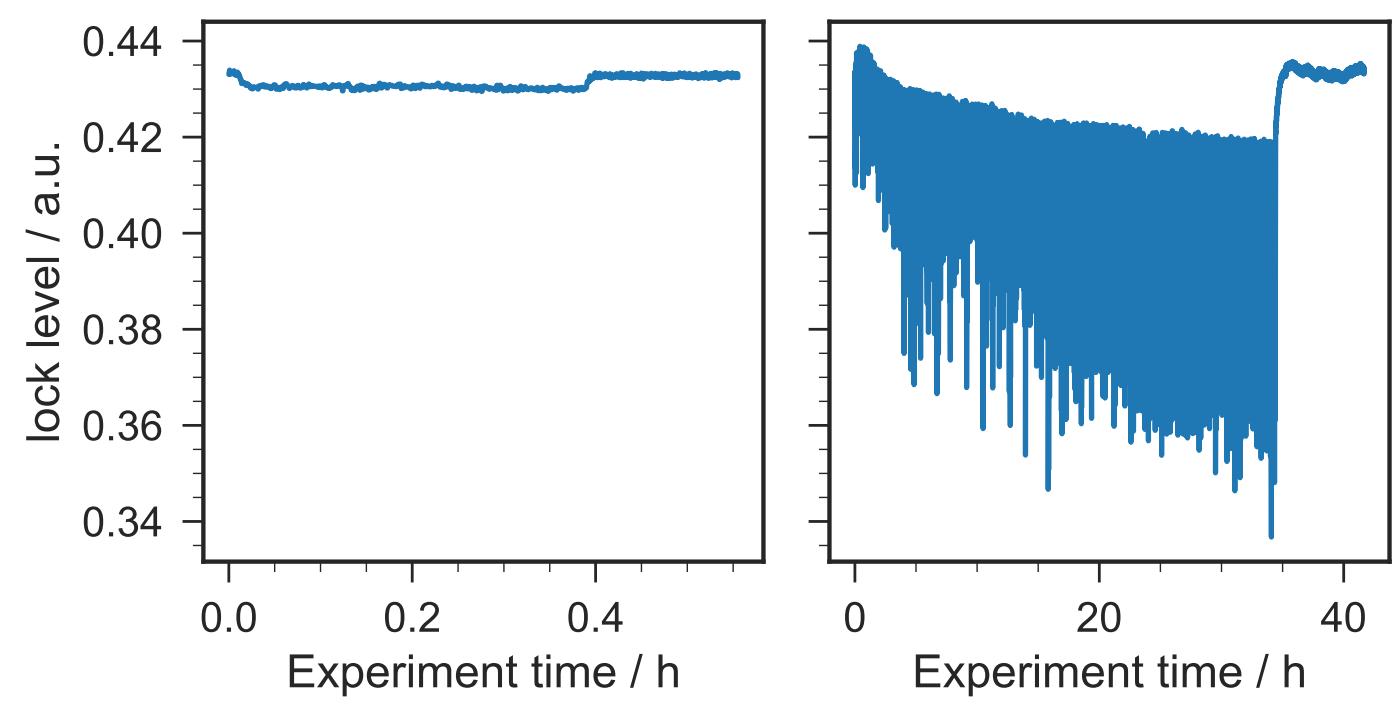

Figure 4.14: Comparison of the intensity of the lock signal during a conventional HSQC and shuttle experiment. The lock intensity was obtained using an in-house Topspin AU program, storing the lock intensity at every second. In the HSQC experiment (left) the lock level shows a small drop that results from additional heating due to pulse application. The shuttle experiment (right) shows a large fluctuation in the lock level, that increases during the course of the experiment. In addition, the maximum lock level also decreases. After both experiments the lock level reaches back its original level. This could indicate either an instability of the shuttle position or a temperature gradient in the shuttle experiments.

The reproducibility of the shuttle experiment can be monitored by the lock signal of the spectrometer. The lock signal is a reference signal measured on deuterium in the background of NMR experiments. It is used to adjust field drifts to keep the magnetic field stable between individual scans and its amplitude is used to optimize the homogeneity (shim) of the magnetic field in the probe before the experiments. The homogeneity of the 
field is disturbed by changes in the temperature as well as changes in the positioning of the sample. Thus, a change in the lock level indicates a change of either of these.

During the shuttle experiment the lock signal is lost during the shuttle block and retrieved only afterwards. Ideally the sample position in the probe as well as the sample temperature do not change during the shuttle block. Otherwise a change in the lock intensity is expected due to worse shim caused by the misplacement of the sample or a temperature gradient in the sample. For a set of shuttle experiments the lock signal was recorded at every second using an in-house Topspin AU program and for reference also during a conventional HSQC experiment. The direct comparison of the two time dependent lock profiles is shown in Figure 4.14.

In the HSQC experiment the lock signal showed a small drop at the beginning of experiment, which is caused by heating effects due to the applied radio frequency pulses that include decoupling during detection. This is a common problem and dummy scans are applied to guarantee a stabilized temperature during the actual experiment. During the experiment the lock signal is stable and reaches back to its initial level after the experiment is finished $(\mathrm{t}=\simeq 1400 \mathrm{~s})$. The lock signal of the shuttle experiment on the other hand showed a very unstable behavior. Right from the beginning the lock level fluctuated strongly and the fluctuation kept increasing while the experiment continued. At its maximum a variation of roughly $25 \%$ was seen (0.44 to 0.34, Figure 4.14). Additionally, the maximum lock level decreased during the course of the experiment. The latter could be rationalized by a temperature gradient between the two positions in the magnet bore. The fact that it did not reach an equilibrium state could be due to the long relaxation delays (up to $5 \mathrm{~s}$ ) in the low-field and long recycling delays $(5 \mathrm{~s})$ in the probe. Restoration of the lock level back to its initial position after the experiment was finished, further supports this hypothesis. The scattering of the lock level may indicate a change in the position of the sample. But it is unlikely that the positioning is back to its initial state at the end of the experiment as shown by the restoration of the lock level. A better explanation of the scattering could come from the relaxation of the lock signal $\left(\mathrm{D}_{2} \mathrm{O}\right)$, which relaxes according to its own relaxation rate at the applied field. Depending on the $R_{1}$ and the length of the relaxation delay the magnetization of the lock signal might not reach its equilibrium state during the recycling delay. To clarify this, field-dependent relaxation experiments of the deuterated solvent can be measured, by using the pulse sequence in Figure 4.4. Due to malfunctioning of the experimental setup, which will be discussed in the following, these experiments could not be measured and the relaxation data acquired so far did not allow such an analysis. Thus, this assumption could not be clarified during the course of this work.

To further check the reproducibility of the shuttle setup, a single scan experiment was established (Figure 4.15). In combination with a high-speed camera the motion of the motor wheel was monitored simultaneously while detecting the water resonance. The obtained data could be used to connect differences in the peak intensity to an erroneous performance 


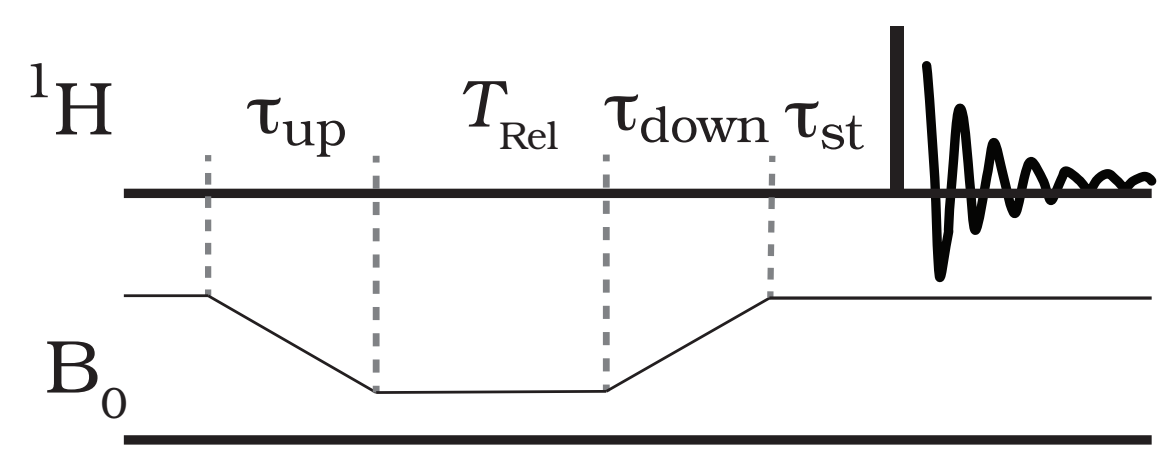

Figure 4.15: Scheme of the pulse sequence for optimization of the stabilization delay $\left(\tau_{\text {st }}\right)$. The shuttle delays $\left(\tau_{\text {up }}\right.$ and $\left.\tau_{\text {down }}\right)$ were set according to the time calculated by the shuttle software. The relaxation delay in the low-field $\left(T_{\text {rel }}\right)$ was kept constant, while the stabilization delay $\left(\tau_{\mathrm{st}}\right)$ was incremented during pseudo $2 \mathrm{D}$ acquisition. By comparison of the line shape of the water resonance the optimal delay could be estimated that compromises vibration artifacts and unwanted relaxation in the high-field position.

of the shuttle setup. In the experiment the shuttle delays $\left(\tau_{\mathrm{up}}, \tau_{\text {down }}\right)$ were fixed to the exact time calculated by the shuttle software (Table 4.6) and a variable stabilization delay $\tau_{\text {st }}$ was used. Thus, the stabilization delay could be optimized to reduce additional highfield relaxation contribution in the shuttle experiments and vibration effects. In a single scan the water signal was used to compare the intensity and the shape with increasing stabilization delay. Ideally the sample reaches the probe in the calculated time and after compensation of vibration effects, the intensity should follow an exponential increase until it reaches the Boltzmann equilibrium of the static field. In Figure 4.16 the single scan FIDs of a shuttle experiment to the $0.5 \mathrm{~T}$ position in the stray-field is shown. The single scan experiments showed "missing" FIDs that contained no signal. This phenomenon could only be explained by the fact that the $90^{\circ}$ pulse is applied before the sample reached the active volume of the probe. A partial insertion of the sample would lead to a highly disturbed signal, which could explain the shape of FID 4 in Figure 4.16. Even with an additional delay of $31 \mathrm{~ms}$ (FID 32) the sample did not reach the active volume of the probe in time to be excited by the $90^{\circ}$ pulse. In general, it is noticeable that the intensities of the FIDs do not follow the expected exponential trend but are scattered. Only from the NMR results it could be concluded that a discrepancy between the set and actually processed delays occurs with a variation of up to $33 \mathrm{~ms}$.

The video of the wheel motion that was recorded during the experiment could be used to determine at which point of the experiment the extra delay occurred. The videos with a resolution of $360 \mathrm{fps}$ (frames per second) were analyzed frame-wise (Figure 4.17). Frames in which the wheel is in motion were connected to the shuttle up and shuttle down events of the respective FIDs, according to the position of the black marks at the wheel (Figure 4.17). Table 4.3 shows the results of the frame analysis for the experiment shown in Figure 4.16. Here, the frame difference between the start and stop motion of the motor 


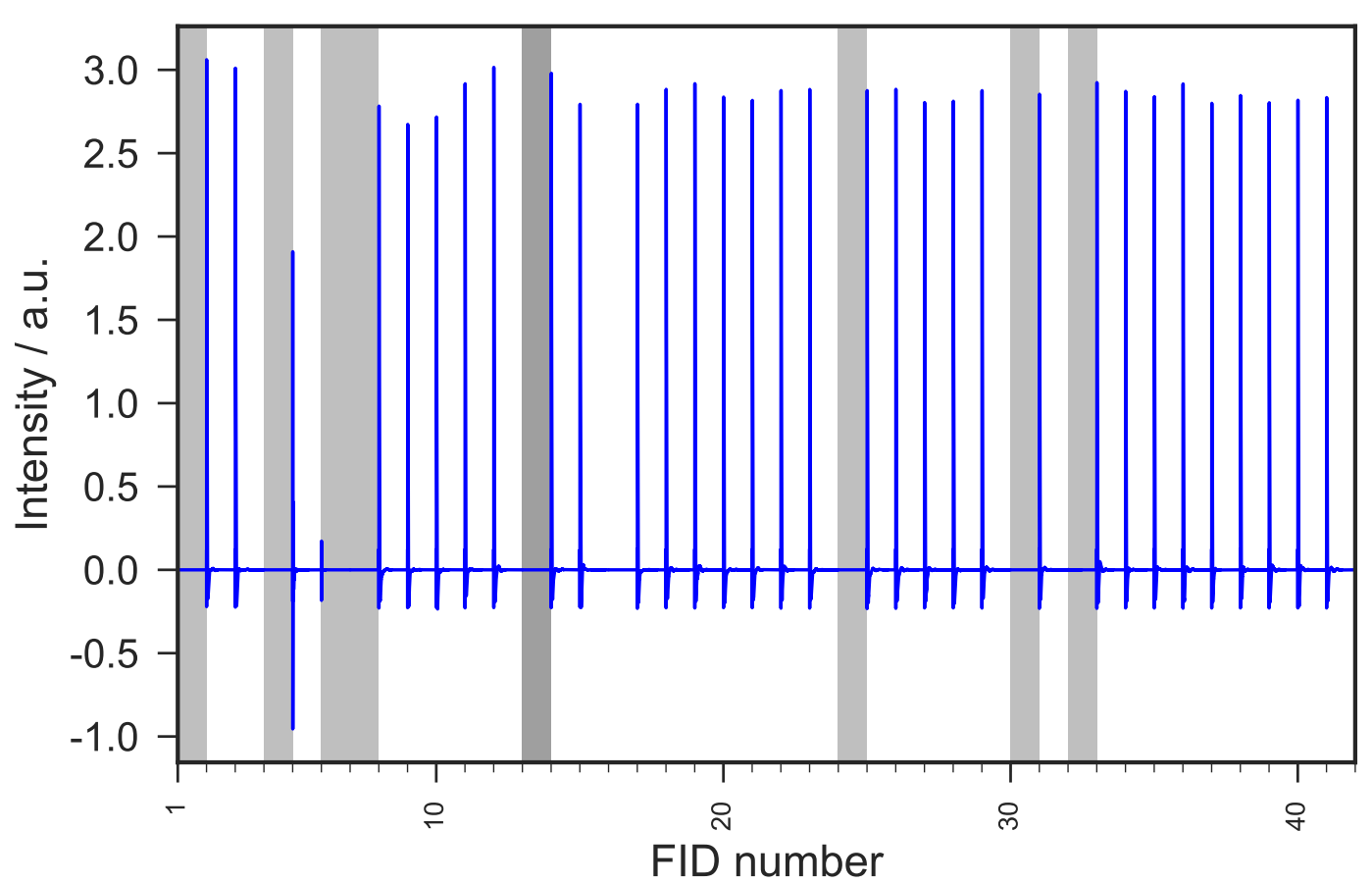

Figure 4.16: FIDs from the $\tau_{\text {st }}$ optimization experiment. During the experiment the stabilization delay was incremented by $1 \mathrm{~ms}$, so that for the $41^{\text {st }}$ FID $\tau_{\mathrm{st}}=40 \mathrm{~ms}$. Missing FIDs are highlighted by grey bars.

for each shuttle up and shuttle down event, should match the experimental $\tau_{\text {up }}$ and $\tau_{\text {down }}$ values. The difference between the stop motion of the shuttle up and start of the shuttle down event should match the residence time $T_{\text {rel }}$ at the stray-field position.

A comparison of the difference in frames of shuttle up and down events showed a stable performance of the motor. With a time resolution of $2.78 \mathrm{~ms}$ per frame $(1 / 360 \mathrm{fps})$ the estimated shuttle delays from the frame count equals $130.24 \mathrm{~ms}$. Considering the resolution of the frames and their manual selection, the result is in very good agreement with the $133 \mathrm{~ms}$ that were used in the experimental setup. This proved the reproducibility of the motor when it is in motion and excluded problems during the shuttle process.

The estimated residence times from the frame-wise analysis revealed a large variation. A deviation of up to 40 frames was observed for $T_{\text {rel }}$, which corresponds to variation of up to $111.2 \mathrm{~ms}$ in the residence times. FIDs that showed no signal in the NMR experiment have an average residence time of $389.8 \mathrm{~ms}$ (140.2 frames), which is longer than the used experimental value of $367 \mathrm{~ms}$. Here, a clear connection between the elongated residence times and missing FIDs could be made, which supports the assumption that the sample does not reach the probe in time. Further experiments at two different positions in the stray field with shorter shuttling times (motor position 25000 (102 ms) and $28000(84 \mathrm{~ms})$ ) showed the same phenomenon. In case of the experiment using motor position 25000 (Table 4.4) only one FID was missing, while the experiment using motor position 28000 (Table 4.4) showed seven missing FIDs. Thus, no correlation between the stray-field posi- 
tion or shuttle delay to the missing FIDs could be observed. However, these experiments showed correlation between the residence time and the missing signals.

The results so far lead to the conclusion that an electronic piece of the shuttle equipment creates a variable delay during the processing of the electronic signal when the shuttle is triggered during the experiment. Since the pulse program is instantaneous and continues while the sample is moved, it creates an asynchronous operation of the spectrometer. The same experiment that was presented here (Figure 4.15), was repeated in the only other laboratory that has the same shuttle, i.e. in the structural biology center in New York. These experiments showed the same phenomenon of missing FIDs as it was discussed above. Furthermore, they showed not only a deviation in $T_{\text {rel }}$, but also in the time it takes the motor to start the shuttle up event. Thus, the additional random delay appears in the pulse sequence when the shuttle is triggered, which further supports the conclusion of a malfunctioning shuttle controller.

In the presented relaxometry data of ubiquitin several scans need to be accumulated to achieve the necessary signal to noise for the intensity analysis. Random missing FIDs, as they were just discussed, cannot be traced back, since all FIDs are immediately added up. Thus, it has to be assumed that all of the measured intensity profiles have been distorted by these problems. In addition to the missing FIDs a further problem comes with the large variation in the residence time. Especially at low-fields were the relaxation rates are expected to be high, very short relaxation delays are necessary to allow an accurate characterization of the relaxation parameters. It is needless to say that a standard variation of $31 \mathrm{~ms}$ in the relaxation delay is too large for any relaxation experiment, particularly when short relaxation of the order of $100 \mathrm{~ms}$ delays are necessary. Theoretically it is possible to acquire each scan individually. The empty scans could be sorted out and the reduced number of scans in the respective FIDs could be corrected by an individual scaling factor but removing empty FIDs would not be sufficient for an accurate analysis, since one has to consider that the effective residence time $\left(T_{\text {rel }}\right)$ varies randomly also for scans with signal. This variation cannot be quantified by the individual FIDs. For a selection of scans with similar $T_{\text {rel }}$, the full NMR experiment has to be combined with a frame-wise analysis as it was presented above. The file size of the high-speed videos in these analysis was roughly 20 Gigabytes ( 4 min experiment). A single field experiment below $2 \mathrm{~T}$ using seven relaxation delays takes up to $48 \mathrm{~h}$ and high-speed footage of this experiment would range in the order of Terabytes. The amount of data and its frame-wise analysis exceeds the limitations of conventional computers.

Missing signals and longer effective residence times cause the intensities to be underestimated during the experiments and ultimately cause the relaxation rates to be underestimated during the fit. Thus, these findings are in line with the presented shuttle data and explain the deviation to the relaxation rates obtained from static relaxation experiments. While in the mentioned performance test experiments the residence time was set to a comparable large value of $500 \mathrm{~ms}$, it can only by speculated how the performance would 
be in case of more practical values like tens of milliseconds. According to the hypothesis of a delay in the processing of the electronic signal, it can be expected that the deviations will be even larger when the time between the two trigger signals is shorter. 

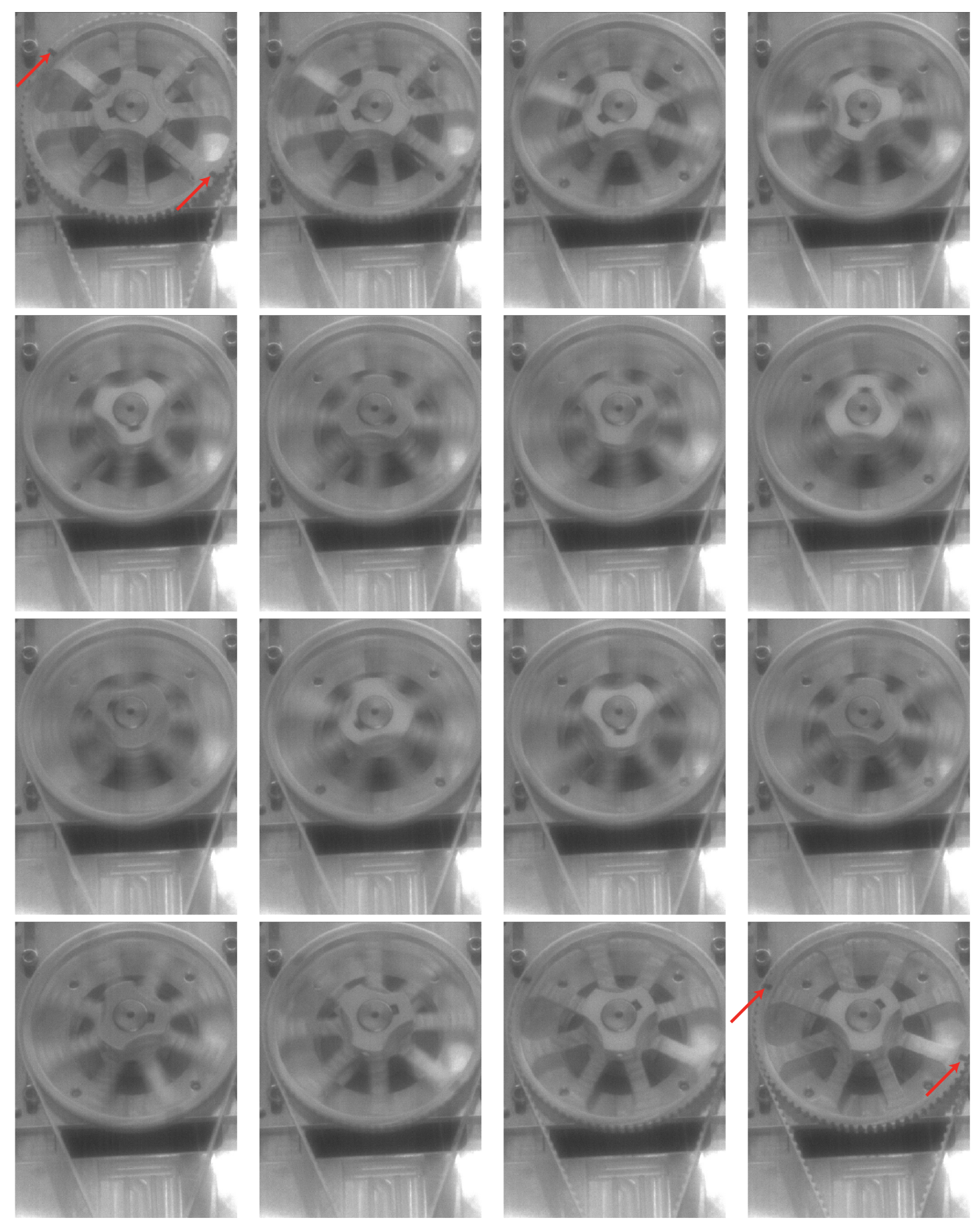

Figure 4.17: Example frames of the motor wheel motion recorded with a highspeed camera. Videos were taken with a resolution of $360 \mathrm{fps}(\approx 2.8 \mathrm{~ms})$. The first and last pictures show the wheel positions in the Down and Up state of the shuttle, respectively. The frames in between, where the wheel is in motion, were counted for a time resolved analysis of the shuttle events. 
Table 4.3: Results from the frame-wise analysis of the shuttle experiment. The table shows the frame numbers corresponding to the start and stop of the wheel motion for the shuttle up and down event. The differences should match the set delay for $\tau_{\text {up }}$ and $\tau_{\text {down }}$ (Up and Down, $133 \mathrm{~ms} \approx 48$ frames) as well as $T_{\text {rel }}$ (Residence time, $367 \mathrm{~ms} \simeq$ 132 frames). FIDs with no signal are marked with a grey background.

\begin{tabular}{|c|c|c|c|c|c|c|c|c|}
\hline \multirow[b]{2}{*}{ FID Number } & \multirow[b]{2}{*}{ Signal } & \multicolumn{2}{|c|}{ Shuttle Up } & \multicolumn{2}{|c|}{ Shuttle Down } & \multicolumn{3}{|c|}{ Difference in Frames } \\
\hline & & Start & Stop & Start & Stop & $\mathrm{Up}$ & Residence time & Down \\
\hline 1 & No & 1312 & 1360 & 1494 & 1542 & 48 & 134 & 48 \\
\hline 2 & Yes & 3081 & 3130 & 3253 & 3302 & 49 & 123 & 49 \\
\hline 3 & Yes & 4877 & 4924 & 5030 & 5077 & 47 & 106 & 47 \\
\hline 4 & No & 6617 & 6664 & 6806 & 6853 & 47 & 142 & 47 \\
\hline 5 & Yes & 8424 & 8471 & 8601 & 8648 & 47 & 130 & 47 \\
\hline 6 & No & 10218 & 10265 & 10397 & 10443 & 47 & 132 & 46 \\
\hline 7 & No & 11999 & 12046 & 12192 & 12240 & 47 & 146 & 48 \\
\hline 8 & Yes & 13781 & 13827 & 13940 & 13987 & 46 & 113 & 47 \\
\hline 9 & Yes & 15579 & 15625 & 15744 & 15791 & 46 & 119 & 47 \\
\hline 10 & Yes & 17379 & 17425 & 17552 & 17599 & 46 & 127 & 47 \\
\hline 11 & Yes & 19172 & 19219 & 19325 & 19372 & 47 & 106 & 47 \\
\hline 12 & Yes & 20932 & 20979 & 21107 & 21153 & 47 & 128 & 46 \\
\hline 13 & No & 22708 & 22756 & 22899 & 22949 & 48 & 143 & 50 \\
\hline 14 & Yes & 24486 & 24534 & 24658 & 24707 & 48 & 124 & 49 \\
\hline 15 & Yes & 26246 & 26294 & 26420 & 26470 & 48 & 126 & 50 \\
\hline 16 & No & 28025 & 28072 & 28217 & 28263 & 47 & 145 & 46 \\
\hline 17 & Yes & 29807 & 29854 & 29971 & 30017 & 47 & 117 & 46 \\
\hline 18 & Yes & 31580 & 31627 & 31752 & 31799 & 47 & 125 & 47 \\
\hline 19 & Yes & 33367 & 33414 & 33540 & 33586 & 47 & 126 & 46 \\
\hline 20 & Yes & 35165 & 35211 & 35338 & 35383 & 46 & 127 & 45 \\
\hline 21 & Yes & 36932 & 36978 & 37105 & 37152 & 46 & 127 & 47 \\
\hline 22 & Yes & 38722 & 38769 & 38879 & 38925 & 47 & 110 & 46 \\
\hline 23 & Yes & 40483 & 40529 & 40656 & 40703 & 46 & 127 & 47 \\
\hline 24 & No & 42257 & 42306 & 42450 & 42501 & 49 & 144 & 51 \\
\hline 25 & Yes & 44041 & 44087 & 44209 & 44256 & 46 & 122 & 47 \\
\hline 26 & Yes & 45839 & 45886 & 45993 & 46039 & 47 & 107 & 46 \\
\hline 27 & Yes & 47574 & 47620 & 47746 & 47793 & 46 & 126 & 47 \\
\hline 28 & Yes & 49372 & 49418 & 49545 & 49591 & 46 & 127 & 46 \\
\hline 29 & Yes & 51194 & 51241 & 51366 & 51413 & 47 & 125 & 47 \\
\hline 30 & No & 52967 & 53013 & 53158 & 53205 & 46 & 145 & 47 \\
\hline 31 & Yes & 54745 & 54791 & 54916 & 54963 & 46 & 125 & 47 \\
\hline 32 & No & 57526 & 57574 & 57715 & 57764 & 48 & 141 & 49 \\
\hline 33 & Yes & 59314 & 59359 & 59485 & 59530 & 45 & 126 & 45 \\
\hline 34 & Yes & 61103 & 61150 & 61276 & 61323 & 47 & 126 & 47 \\
\hline 35 & Yes & 62897 & 62943 & 63056 & 63103 & 46 & 113 & 47 \\
\hline 36 & Yes & 64692 & 64739 & 64845 & 64891 & 47 & 106 & 46 \\
\hline 37 & Yes & 66487 & 66533 & 66652 & 66698 & 46 & 119 & 46 \\
\hline 38 & Yes & 68283 & 68330 & 68452 & 68500 & 47 & 122 & 48 \\
\hline 39 & Yes & 70078 & 70125 & 70238 & 70284 & 47 & 113 & 46 \\
\hline 40 & Yes & 71844 & 71891 & 72017 & 72063 & 47 & 126 & 46 \\
\hline 41 & Yes & 73632 & 73679 & 73809 & 73855 & 47 & 130 & 46 \\
\hline $\bar{x} /$ frames & & & & & & 46.85 & 125.51 & 47.05 \\
\hline$\sigma /$ frames & & & & & & 0.85 & 11.07 & 1.32 \\
\hline $\bar{x} / \mathrm{ms}$ & & & & & & 130.24 & 348.92 & 130.80 \\
\hline$\sigma / \mathrm{ms}$ & & & & & & 2.37 & 30.74 & 3.67 \\
\hline
\end{tabular}


Table 4.4: Results from the frame-wise analysis of the shuttle experiment. The table shows the frame numbers of the corresponding start and stop of the wheel motion for the shuttle up and down event. The differences should match the set delay for $\tau_{\text {up }}$ and $\tau_{\text {down }}$ (Up and Down, $102 \mathrm{~ms} \approx 37$ frames) as well as $T_{\text {rel }}$ (Residence time, $398 \mathrm{~ms} \simeq$ 143 frames). FIDs with no signal are marked with a grey background.

\begin{tabular}{|c|c|c|c|c|c|c|c|c|}
\hline \multirow[b]{2}{*}{ FID } & \multirow[b]{2}{*}{ Signal } & \multicolumn{2}{|c|}{ Shuttle Up } & \multicolumn{2}{|c|}{ Shuttle Down } & \multicolumn{3}{|c|}{ Difference in Frames } \\
\hline & & Start & Stop & Start & Stop & Up & Residence time & Down \\
\hline 1 & Yes & 1506 & 1542 & 1688 & 1723 & 36 & 146 & 35 \\
\hline 2 & Yes & 3238 & 3275 & 3411 & 3448 & 37 & 136 & 37 \\
\hline 3 & Yes & 5057 & 5094 & 5224 & 5261 & 37 & 130 & 37 \\
\hline 4 & Yes & 6805 & 6841 & 6977 & 7013 & 36 & 136 & 36 \\
\hline 5 & Yes & 8562 & 8599 & 8735 & 8771 & 37 & 136 & 36 \\
\hline 6 & Yes & 10320 & 10356 & 10483 & 10521 & 36 & 127 & 38 \\
\hline 7 & No & 12069 & 12104 & 12252 & 12291 & 35 & 148 & 39 \\
\hline 8 & Yes & 13848 & 13884 & 14020 & 14057 & 36 & 136 & 37 \\
\hline 9 & Yes & 15606 & 15643 & 15779 & 15815 & 37 & 136 & 36 \\
\hline 10 & Yes & 17366 & 17402 & 17538 & 17574 & 36 & 136 & 36 \\
\hline 11 & Yes & 19124 & 19161 & 19301 & 19337 & 37 & 140 & 36 \\
\hline 12 & Yes & 20890 & 20926 & 21060 & 21096 & 36 & 134 & 36 \\
\hline 13 & No & 22646 & 22682 & 22825 & 22862 & 36 & 143 & 37 \\
\hline 14 & Yes & 24451 & 24488 & 24623 & 24660 & 37 & 135 & 37 \\
\hline 15 & Yes & 26209 & 26246 & 26368 & 26407 & 37 & 122 & 39 \\
\hline 16 & Yes & 27936 & 27973 & 28103 & 28139 & 37 & 130 & 36 \\
\hline 17 & Yes & 29691 & 29726 & 29873 & 29908 & 35 & 147 & 35 \\
\hline 18 & Yes & 31461 & 31498 & 31627 & 31664 & 37 & 129 & 37 \\
\hline 19 & Yes & 33221 & 33260 & 33395 & 33432 & 39 & 135 & 37 \\
\hline 20 & Yes & 34983 & 35020 & 35153 & 35185 & 37 & 133 & 32 \\
\hline 21 & Yes & 36780 & 36816 & 36953 & 36990 & 36 & 137 & 37 \\
\hline 22 & Yes & 38540 & 38575 & 38713 & 38749 & 35 & 138 & 36 \\
\hline 23 & Yes & 40302 & 40339 & 40475 & 40513 & 37 & 136 & 38 \\
\hline 24 & Yes & 42091 & 42127 & 42246 & 42282 & 36 & 119 & 36 \\
\hline 25 & Yes & 43843 & 43878 & 44025 & 44062 & 35 & 147 & 37 \\
\hline 26 & Yes & 45617 & 45652 & 45792 & 45828 & 35 & 140 & 36 \\
\hline 27 & Yes & 47420 & 47456 & 47592 & 47627 & 36 & 136 & 35 \\
\hline 28 & Yes & 49208 & 49244 & 49363 & 49399 & 36 & 119 & 36 \\
\hline 29 & Yes & 50964 & 50998 & 51142 & 51179 & 34 & 144 & 37 \\
\hline 30 & Yes & 52731 & 52767 & 52905 & 52943 & 36 & 138 & 38 \\
\hline 31 & Yes & 54527 & 54563 & 54685 & 54721 & 36 & 122 & 36 \\
\hline 32 & Yes & 56282 & 56320 & 56462 & 56499 & 38 & 142 & 37 \\
\hline 33 & Yes & 58048 & 58085 & 58219 & 58256 & 37 & 134 & 37 \\
\hline 34 & Yes & 59814 & 59850 & 59987 & 60024 & 36 & 137 & 37 \\
\hline 35 & Yes & 61582 & 61618 & 61754 & 61790 & 36 & 136 & 36 \\
\hline 36 & Yes & 63370 & 63406 & 63525 & 63563 & 36 & 119 & 38 \\
\hline 37 & Yes & 65118 & 65154 & 65304 & 65341 & 36 & 150 & 37 \\
\hline 38 & No & 66905 & 66942 & 67099 & 67137 & 37 & 157 & 38 \\
\hline 39 & Yes & 68715 & 68750 & 68895 & 68933 & 35 & 145 & 38 \\
\hline 40 & Yes & 70484 & 70520 & 70656 & 70693 & 36 & 136 & 37 \\
\hline 41 & Yes & 72302 & 72338 & 72489 & 72525 & 36 & 151 & 36 \\
\hline $\bar{x} /$ frames & & & & & & 36.24 & 136.54 & 36.63 \\
\hline$\sigma /$ frames & & & & & & 0.92 & 8.79 & 1.22 \\
\hline $\bar{x} / \mathrm{ms}$ & & & & & & 100.68 & 379.27 & 101.76 \\
\hline$\sigma / \mathrm{ms}$ & & & & & & 2.54 & 24.41 & 3.34 \\
\hline
\end{tabular}


Table 4.5: Results from the frame-wise analysis of the shuttle experiment. The table shows the corresponding frame numbers of the start and stop of the wheel motion for the shuttle up and down event. The differences should match the set delay for $\tau_{\text {up }}$ and $\tau_{\text {down }}$ (Up and Down, $84 \mathrm{~ms} \approx 30$ frames) as well as $T_{\text {rel }}$ (Residence time, $416 \mathrm{~ms} \simeq$ 150 frames). FIDs with no signal are marked with a grey background.

\begin{tabular}{|c|c|c|c|c|c|c|c|c|}
\hline \multirow[b]{2}{*}{ FID } & \multirow[b]{2}{*}{ Signal } & \multicolumn{2}{|c|}{ Shuttle Up } & \multicolumn{2}{|c|}{ Shuttle Down } & \multicolumn{3}{|c|}{ Difference in Frames } \\
\hline & & Start & Stop & Start & Stop & $\mathrm{Up}$ & Residence time & Down \\
\hline 1 & No & 1389 & 1417 & 1580 & 1611 & 28 & 163 & 31 \\
\hline 2 & Yes & 3145 & 3175 & 3317 & 3347 & 30 & 142 & 30 \\
\hline 3 & Yes & 4898 & 4927 & 5070 & 5100 & 29 & 143 & 30 \\
\hline 4 & No & 6653 & 6683 & 6834 & 6866 & 30 & 151 & 32 \\
\hline 5 & No & 8445 & 8476 & 8630 & 8662 & 31 & 154 & 32 \\
\hline 6 & Yes & 10219 & 10252 & 10391 & 10422 & 33 & 139 & 31 \\
\hline 7 & Yes & 11978 & 12008 & 12154 & 12184 & 30 & 146 & 30 \\
\hline 8 & Yes & 13743 & 13774 & 13903 & 13933 & 31 & 129 & 30 \\
\hline 9 & No & 15491 & 15521 & 15677 & 15707 & 30 & 156 & 30 \\
\hline 10 & Yes & 17266 & 17295 & 17428 & 17461 & 29 & 133 & 33 \\
\hline 11 & Yes & 19007 & 19036 & 19200 & 19231 & 29 & 164 & 31 \\
\hline 12 & Yes & 20757 & 20786 & 20929 & 20960 & 29 & 143 & 31 \\
\hline 13 & Yes & 22517 & 22547 & 22686 & 22718 & 30 & 139 & 32 \\
\hline 14 & Yes & 24249 & 24279 & 24430 & 24462 & 30 & 151 & 32 \\
\hline 15 & Yes & 26019 & 26050 & 26179 & 26211 & 31 & 129 & 32 \\
\hline 16 & No & 27741 & 27765 & 27917 & 27950 & 24 & 152 & 33 \\
\hline 17 & Yes & 29461 & 29491 & 29633 & 29664 & 30 & 142 & 31 \\
\hline 18 & Yes & 31220 & 31250 & 31392 & 31422 & 30 & 142 & 30 \\
\hline 19 & Yes & 32978 & 33009 & 33151 & 33181 & 31 & 142 & 30 \\
\hline 20 & No & 34750 & 34780 & 34941 & 34973 & 30 & 161 & 32 \\
\hline 21 & No & 36546 & 36577 & 36737 & 36768 & 31 & 160 & 31 \\
\hline 22 & Yes & 38305 & 38334 & 38477 & 38507 & 29 & 143 & 30 \\
\hline 23 & Yes & 40061 & 40090 & 40234 & 40264 & 29 & 144 & 30 \\
\hline 24 & Yes & 41821 & 41849 & 41994 & 42024 & 28 & 145 & 30 \\
\hline 25 & Yes & 43587 & 43618 & 43759 & 43790 & 31 & 141 & 31 \\
\hline 26 & Yes & 45345 & 45376 & 45517 & 45548 & 31 & 141 & 31 \\
\hline 27 & Yes & 47104 & 47134 & 47274 & 47304 & 30 & 140 & 30 \\
\hline 28 & Yes & 48863 & 48894 & 49038 & 49069 & 31 & 144 & 31 \\
\hline 29 & Yes & 50626 & 50657 & 50797 & 50828 & 31 & 140 & 31 \\
\hline 30 & Yes & 52386 & 52416 & 52561 & 52591 & 30 & 145 & 30 \\
\hline 31 & Yes & 54150 & 54179 & 54320 & 54351 & 29 & 141 & 31 \\
\hline 32 & Yes & 55955 & 55983 & 56129 & 56160 & 28 & 146 & 31 \\
\hline 33 & Yes & 57713 & 57741 & 57887 & 57918 & 28 & 146 & 31 \\
\hline 34 & Yes & 59472 & 59501 & 59632 & 59662 & 29 & 131 & 30 \\
\hline 35 & Yes & 61221 & 61249 & 61406 & 61436 & 28 & 157 & 30 \\
\hline 36 & Yes & 62994 & 63022 & 63156 & 63186 & 28 & 134 & 30 \\
\hline 37 & Yes & 64751 & 64781 & 64930 & 64960 & 30 & 149 & 30 \\
\hline 38 & Yes & 66519 & 66548 & 66691 & 66722 & 29 & 143 & 31 \\
\hline 39 & Yes & 68282 & 68312 & 68453 & 68483 & 30 & 141 & 30 \\
\hline 40 & Yes & 70041 & 70070 & 70215 & 70246 & 29 & 145 & 31 \\
\hline 41 & Yes & 71805 & 71835 & 71978 & 72008 & 30 & 143 & 30 \\
\hline $\bar{x} /$ frames & & & & & & 29.61 & 144.88 & 30.80 \\
\hline$\sigma /$ frames & & & & & & 1.43 & 8.47 & 0.87 \\
\hline $\bar{x} / \mathrm{ms}$ & & & & & & 82.2 & 402.4 & 85.6 \\
\hline$\sigma / \mathrm{ms}$ & & & & & & 3.9 & 23.52 & 2.42 \\
\hline
\end{tabular}




\subsection{Conclusion}

The aim of this part of the thesis was to answer the question if the relaxation behavior of proteins at very low fields follows the theoretical description of Lorentzian spectral density functions. Furthermore, the relaxation data obtained at low-field magnetic field strength should give a more accurate insight into the sub- $\tau_{\mathrm{c}}$ motions of a protein. For this a newly motor based shuttle device was used that allows an easier sample handling and pledge a high reproducibility as well as fast shuttle times and less vibration effects.

Initial experiments on ubiquitin showed a deviation from the classical exponential decay model for residues 8-12 and C-terminal residues when measuring at field strengths below $4 \mathrm{~T}(\sim 170 \mathrm{MHz})$. These low field strengths are not feasible for direct detection of protein signals, due to the low resolution and signal-to-noise. During the course of this thesis the cause of this deviations could not be clarified, while the individual analysis of $\mathrm{N}_{\mathrm{Z}}^{-}, \mathrm{N}_{\mathrm{Z}}^{+}$ and the combined analysis indicated that a relaxation mechanism that could cause this deviation.

The analysis of the field dependence of the longitudinal relaxation rates revealed a deviation to the Lorentzian spectral density function. Repetitive experiments in the range of 0.5 and $2 \mathrm{~T}$ could not reproduce the data despite a good fit of the intensity profiles. Thus, a systematic error of the shuttle setup was hypothesized and proven by a combination of shuttle experiments and associated high-speed camera footage of the motor motion. The frame-wise analysis revealed a random timing error that caused the sample to stay longer in the low-field position as set in the pulse program. This had two severe effects on the collected relaxation data. Firstly, for short relaxation delays, which are especially important at low-field experiments, it caused randomly appearing empty FIDs that get accumulated during the acquisition and could not be traced back. Secondly, it added a random deviation in the effective relaxation delays, which could not be estimated and prohibited an accurate analysis of the relaxation data. In general, these two effects caused the intensities to be lower than expected at a given relaxation delay and ultimately resulted in decreased intensities, which in turn resulted in underestimated relaxation rates.

The classical relaxometry data of ubiquitin was in good agreement while comparing $\tau_{\mathrm{c}}$ and backbone $S^{2}$ to calculated and literature values, respectively. Therefore, it could be used to validate the shuttle relaxometry data. The comparison of the classic model free and shuttle data set showed, that the shuttle experiments underestimated the relaxation rates. Thus, the results are in line with the fact that the shuttle setup had intrinsic problems.

Finally, the Lorentzian behavior of the spectral density function at low magnetic field strengths as well as the effect of local dynamics to the relaxation rate could not be studied during the course of this work, due to the discussed issues of the experimental setup. However, this work showed the importance of stability in an experimental shuttle setup as well as the need of control mechanisms of the shuttle performance, since the experimental intensity curves might not indicate experimental problems. Furthermore, only the 
use of long relaxation delays and an individual analysis of the $\mathrm{N}_{\mathrm{z}}^{+}$and $\mathrm{N}_{\mathrm{z}}^{-}$magnetization revealed deviations of the relaxation data to the exponential decay model. These findings were reproducible and indicate a relaxation mechanism or local dynamic contributions, which affects the relaxation rates only in a minor way at high magnetic field strengths. Thus, shuttle relaxometry offers a unique method to study these contributions in a residuespecific manner, ultimately giving a more detailed insight into local protein motions in the sub- $\tau_{\mathrm{c}}$ window. 


\subsection{Material and Methods}

\subsubsection{Sample Preparation}

Perdeuterated, ${ }^{15} \mathrm{~N}$-labeled ubiquitin was expressed in E. coli adapted to $100 \% \mathrm{D}_{2} \mathrm{O}$, using $\mathrm{D}_{7}$-glucose and ${ }^{15} \mathrm{NH}_{4} \mathrm{Cl}$ as carbon and nitrogen source, respectively. The protein was purified as described before in literature (Gronenborn et al., 1991) and NMR samples were prepared using a concentration of $3 \mathrm{mM}$ in $50 \mathrm{mM}$ sodium phosphate buffer of $\mathrm{pH} 6.5$ containing $100 \mathrm{mM} \mathrm{NaCl}$ and $0.05 \%$ sodium azide in $90 \% \mathrm{H}_{2} \mathrm{O} / 10 \% \mathrm{D}_{2} \mathrm{O}$.

\subsubsection{NMR Measurements}

All relaxation experiments were acquired using a perdeuterated, ${ }^{15} \mathrm{~N}$-labeled ubiquitin sample of $3 \mathrm{mM}$ at a temperature of $294.5 \mathrm{~K}$.

\section{Shuttle Relaxometry}

Shuttle relaxometry data was measured at field strengths between 0.5 and $10 \mathrm{~T}$ using the described shuttle device on a $700 \mathrm{MHz}$ Bruker AVANCE III spectrometer equipped with a $5 \mathrm{~mm}$ TCI probe. The measured field strengths and corresponding motor positions as well as shuttle delays are shown in Table 4.6. The pulse program used for the shuttle experiments is depicted in Figure 4.4 and the pulse sequence in Bruker format can be found in the appendix.

The experiment was run as a pseudo 3D-experiment were $\phi_{1}$ was incremented by $180^{\circ}$ to store negative and positive longitudinal magnetization separately. Other phases were set to $\phi_{3}=(\mathrm{x},-\mathrm{x}), \phi_{\mathrm{rec}}=(\mathrm{x},-\mathrm{x})$ and $\phi_{2}$ was incremented by $90^{\circ}$ for quadrature detection. All INEPT delays $\left(\tau_{\mathrm{a}}\right)$ were set to $2.7 \mathrm{~ms}, \tau_{\text {up }}$ and $\tau_{\text {down }}$ were set to the respective shuttle delays according to Table 4.6. To reduce vibration artifacts in the spectrum $\tau_{\text {st }}$ was set to $75 \mathrm{~ms}$. All shuttle experiments were recorded with 64 complex points and an acquisition time of $0.122 \mathrm{~s}$. The used relaxation delays $T_{\text {rel }}=[0.08,0.32,0.16,0.50,1.00,3.00,5.00] \mathrm{s}$ were measured in interleaved fashion and in a randomized order. The recycling delay was set to $5 \mathrm{~s}$ to fully recover the Boltzmann magnetization after each experiment. All experiments at field strength $\geq 2 \mathrm{~T}$ and below $2 \mathrm{~T}$ were recorded with 8 scans and 16 scans, respectively.

\begin{tabular}{l|cccccccc}
\hline Motor position / a.u. & 18660 & 19900 & 20750 & 21890 & 22720 & 23370 & 23900 & 24360 \\
Field Strength / T & 0.5 & 0.75 & 1.00 & 1.51 & 2.01 & 2.50 & 3.00 & 3.51 \\
Shuttle Delay / ms & 133.5 & 128.1 & 124.2 & 118.9 & 114.8 & 111.5 & 108.8 & 106.3 \\
\hline \hline Motor position / a.u. & 24740 & 25080 & 25385 & 25660 & 25910 & 26750 & 27450 & \\
Field Strength / T & 4.00 & 4.50 & 5.01 & 5.51 & 6.01 & 8.02 & 10.0 & \\
Shuttle Delay / ms & 104.3 & 102.4 & 100.7 & 99.1 & 97.6 & 92.6 & 88.2 & \\
\hline
\end{tabular}

Table 4.6: Field Strength and Shuttle delay for the motor positions. The sample length was set to $35 \mathrm{~mm}$ and the motor parameters for acceleration and velocity were set to $\mathrm{a}=350 \mathrm{rpss}$ and $\mathrm{v}=1500 \mathrm{rpm}$, respectively. 


\section{Static Experiments}

Static relaxation data of ubiquitin was acquired by standard pulse sequences for $R_{1}, R_{2}$ and hetNOE detection, established by Farrow and co-workers (Farrow et al., 1994). In total seven sets of statics experiments were acquired at spectrometers operating at a ${ }^{1} \mathrm{H}$ Larmor frequency of $300 \mathrm{MHz}, 400 \mathrm{MHz}, 600 \mathrm{MHz}, 700 \mathrm{MHz}, 800 \mathrm{MHz}, 900 \mathrm{MHz}$ and $950 \mathrm{MHz}$. All spectrometers were equipped with a $5 \mathrm{~mm}$ cryogenically cooled probe except the two lowfield spectrometers operating at Larmor frequencies of 300 and $400 \mathrm{MHz}$. All relaxation experiments were measured at a temperature of $294.5 \mathrm{~K}$.

$R_{1}$ relaxation data was acquired using seven relaxation delays $T_{\text {rel }}=(0.02,0.06,0.16$, $0.32,0.80,1.50,3.00) \mathrm{s}$. Spectra were recorded as pseudo-3D experiments with $64 \mathrm{com}-$ plex points and an acquisition time of $0.212 \mathrm{~s}$ using a recycle delay of $5 \mathrm{~s}$.

$R_{2}$ relaxation data was acquired using $14 \mathrm{CPMG}$ loops $n_{\text {loop }}$ in the range of 2 to 24 . A single CPMG loop length was set to $17 \mathrm{~ms}$ resulting in relaxation times of 34 to $408 \mathrm{~ms}$. Spectra were recorded as pseudo-3D experiments with 64 complex points and an acquisition time of $0.212 \mathrm{~s}$ using a recycle delay of $5 \mathrm{~s}$.

Heteronuclear NOE data was obtained measuring saturated and control experiment as interleaved 2D experiment. Spectra were recorded with 64 complex points and an acquisition time of $0.212 \mathrm{~s}$. Recycle and saturation delay were set to $5 \mathrm{~s}$.

All high field relaxation data acquired with cryogenically cooled probes was recorded with 8 scans. Experiments at $300 \mathrm{MHz}$ and $400 \mathrm{MHz}$ were recorded with 64 and 32 scans, respectively, for a better spectrum quality.

\subsubsection{Data Analysis}

\section{Shuttle Relaxometry}

The shuttle relaxometry data was processed using in-house software based on the python package NMRglue (Helmus and Jaroniec, 2013). The intensities of the individual peaks were extracted by using Cara and in-house software based on python packages (numpy, pandas). All model fits were done using the python package LMFit (Newville et al., 2014) using a least-square minimization algorithm on the target function

$$
\chi^{2}=\sum_{i}^{n}\left(\frac{\left(\exp _{i}-c a l c_{i}\right)^{2}}{\sigma_{i}^{2}}\right),
$$

where, exp is the experimental data point, calc the fitted model value and $\sigma$ the uncertainty of the i-th data point. The experimental uncertainty $\sigma$ was estimated by the noise of the individual spectra. 
The obtained intensity profiles were fitted to the following exponential decay models:

$$
\begin{gathered}
I_{z}(t)=I_{\mathrm{N}} \pm I_{\mathrm{H}} \exp \left(-R_{1} T_{\text {Rel }}\right), \\
I(t)=I_{\mathrm{H}} \exp \left(-R_{1} T_{\text {Rel }}\right),
\end{gathered}
$$

for an individual or combined analysis of $\mathrm{N}_{Z}^{+}$and $\mathrm{N}_{Z}^{-}$, respectively.

The field-dependent relaxation rates were fitted to

$$
R_{1}=\left(d_{\mathrm{IS}} / 4\right)\left(3 J\left(\omega_{I}\right)+7 J\left(0.921 \omega_{S}\right)\right)+c_{\mathrm{IS}} J\left(\omega_{I}\right)
$$

in which,

$$
J(\omega)=\frac{2}{5}\left(\frac{\tau_{\mathrm{c}} S^{2}}{1+\omega^{2} \tau_{\mathrm{c}}^{2}}+\frac{\tau\left(1-S^{2}\right)}{1+\omega^{2} \tau^{2}}\right),
$$

was used as model for the spectral density function.

\section{Model Free Experiments}

All spectra of $R_{1}, R_{2}$ and hetNOE were processed with Bruker Topspin. The relaxometry data measured at static fields was analyzed using Bruker Dynamics Center.

$R_{1}$ and $R_{2}$ data was fitted to the exponential model

$$
I_{t}=I_{0} \exp (-R t)
$$

For the model free analysis, a N-H distance of $1.02 \AA$ and a chemical shift anisotropy c c of $-160 \mathrm{ppm}$ was assumed for all residues. For an improved model fitting 1000 random selected start parameters were used.

\section{High Speed Video Analysis}

Videos of shuttle motor motion were recorded using a high-speed camera (DFK 23UP1300, The Imaging Source Europe $\mathrm{GmbH}$ ). The videos were acquired with $360 \mathrm{fps}$ using IC capture software.

For the frame-wise analysis home written python software based on the OpenCV package was used (Bradski, 2000). A frame comparison algorithm was used to preliminary select frames related to the shuttle up and down events. Start and stop frames of the motor motion were selected manually and their differences were compared with the experimental delays $\tau_{\text {up }}, \tau_{\text {down }}$ and $\tau_{\text {st }}$ (Table 4.3, Table 4.4, Table 4.5). 


\section{Appendix A}

\section{gpW Relaxation Dispersion}

A.1 RD profiles of backbone ${ }^{15} \mathrm{~N}$ E-CPMG
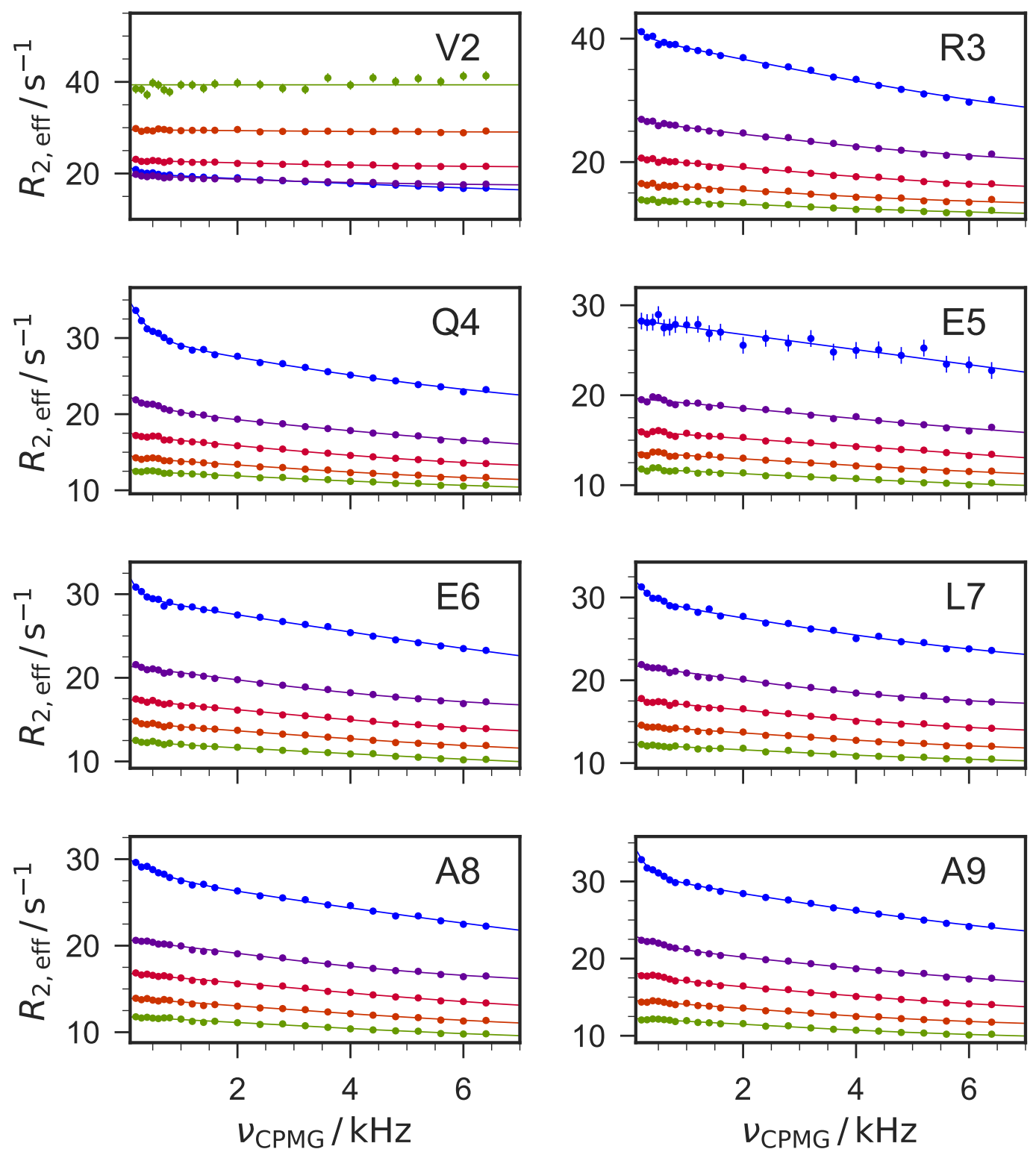

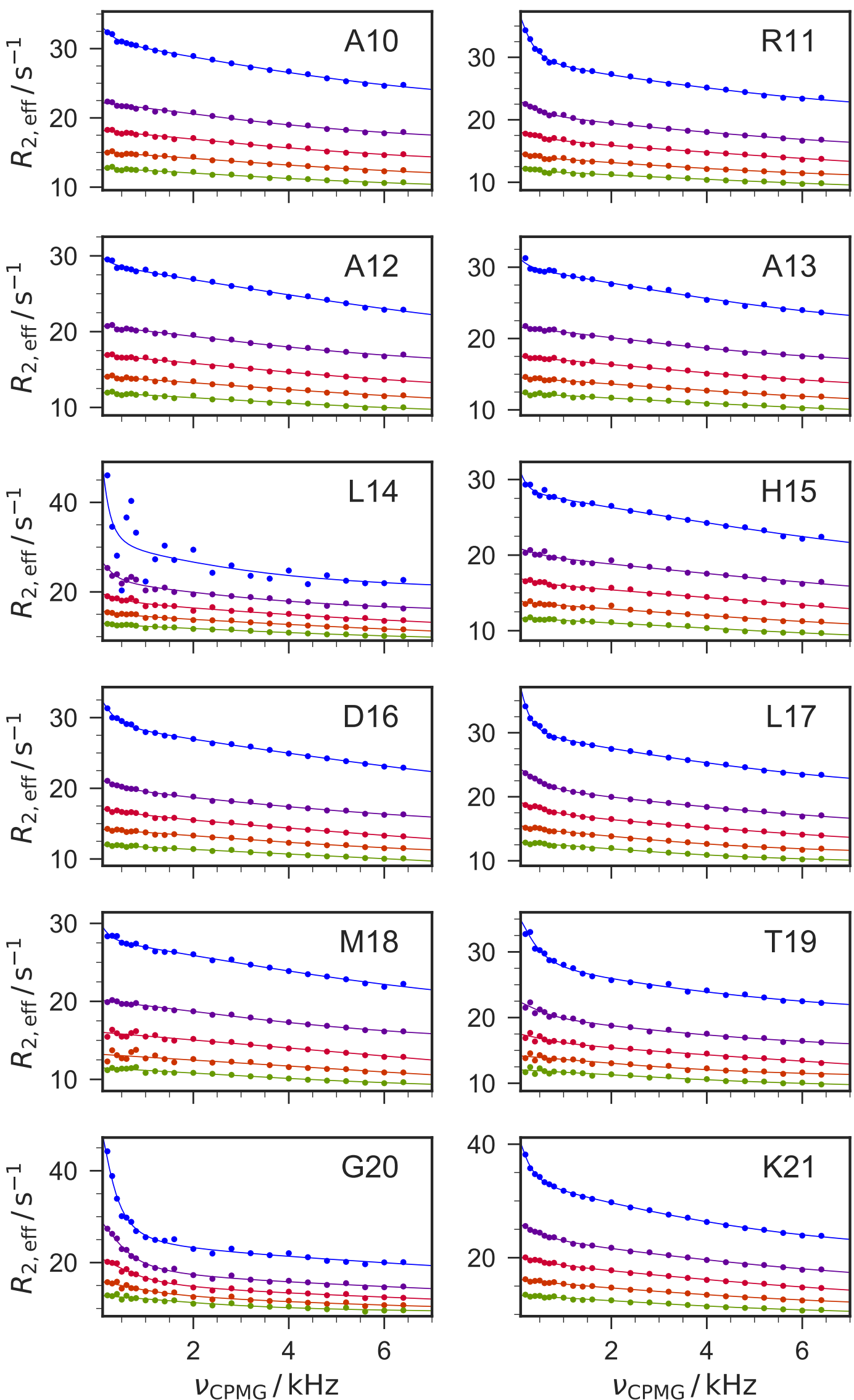

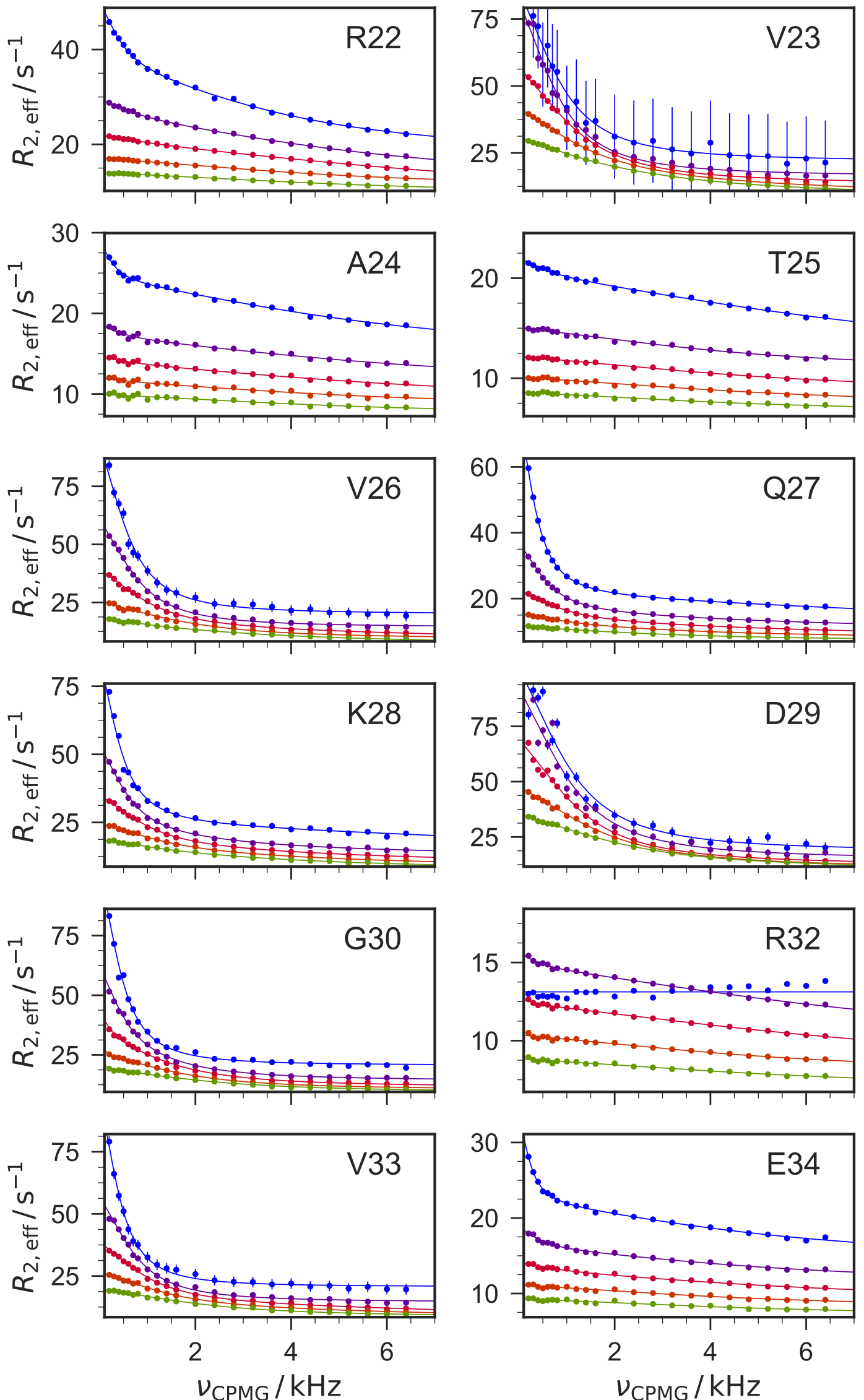

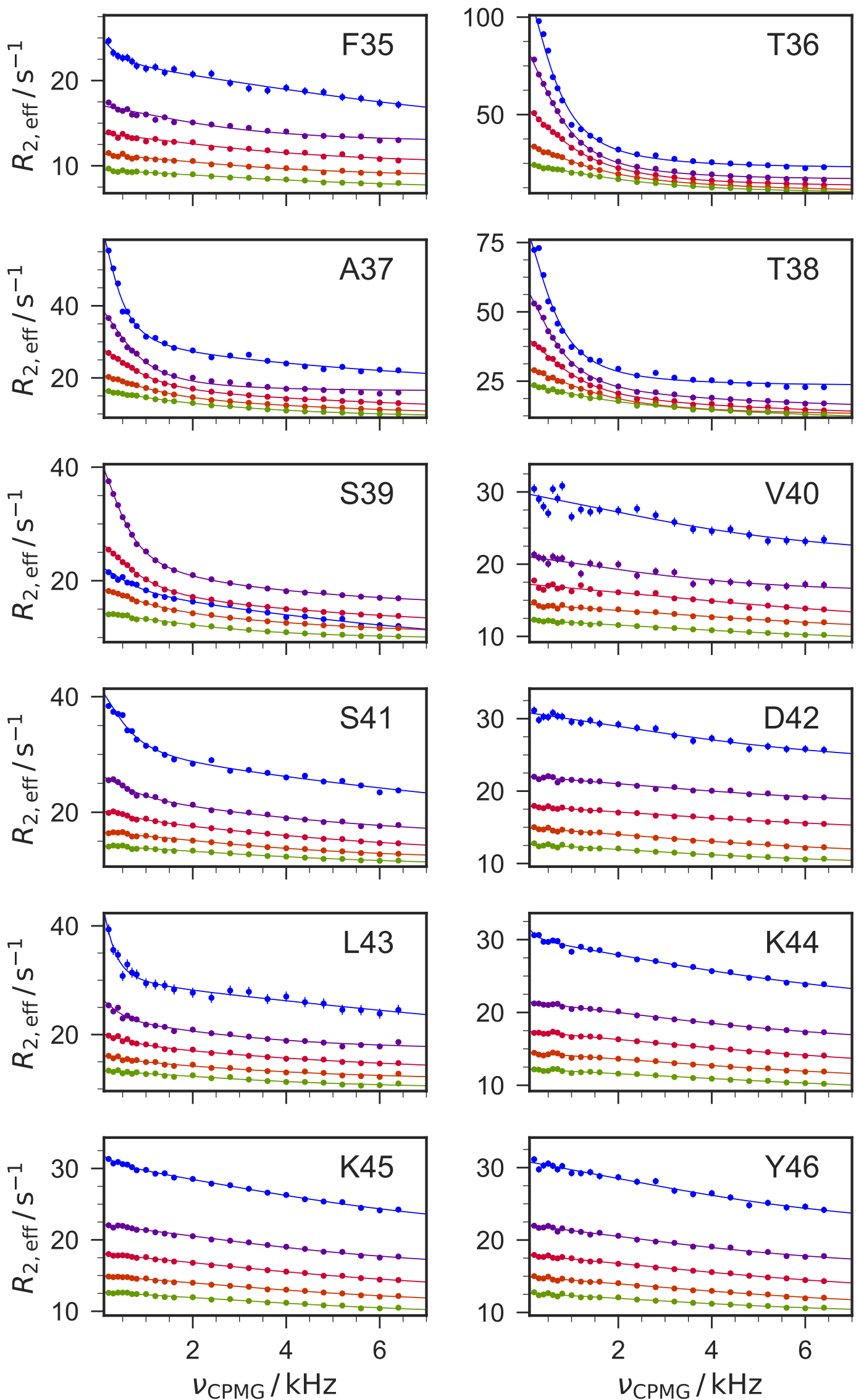

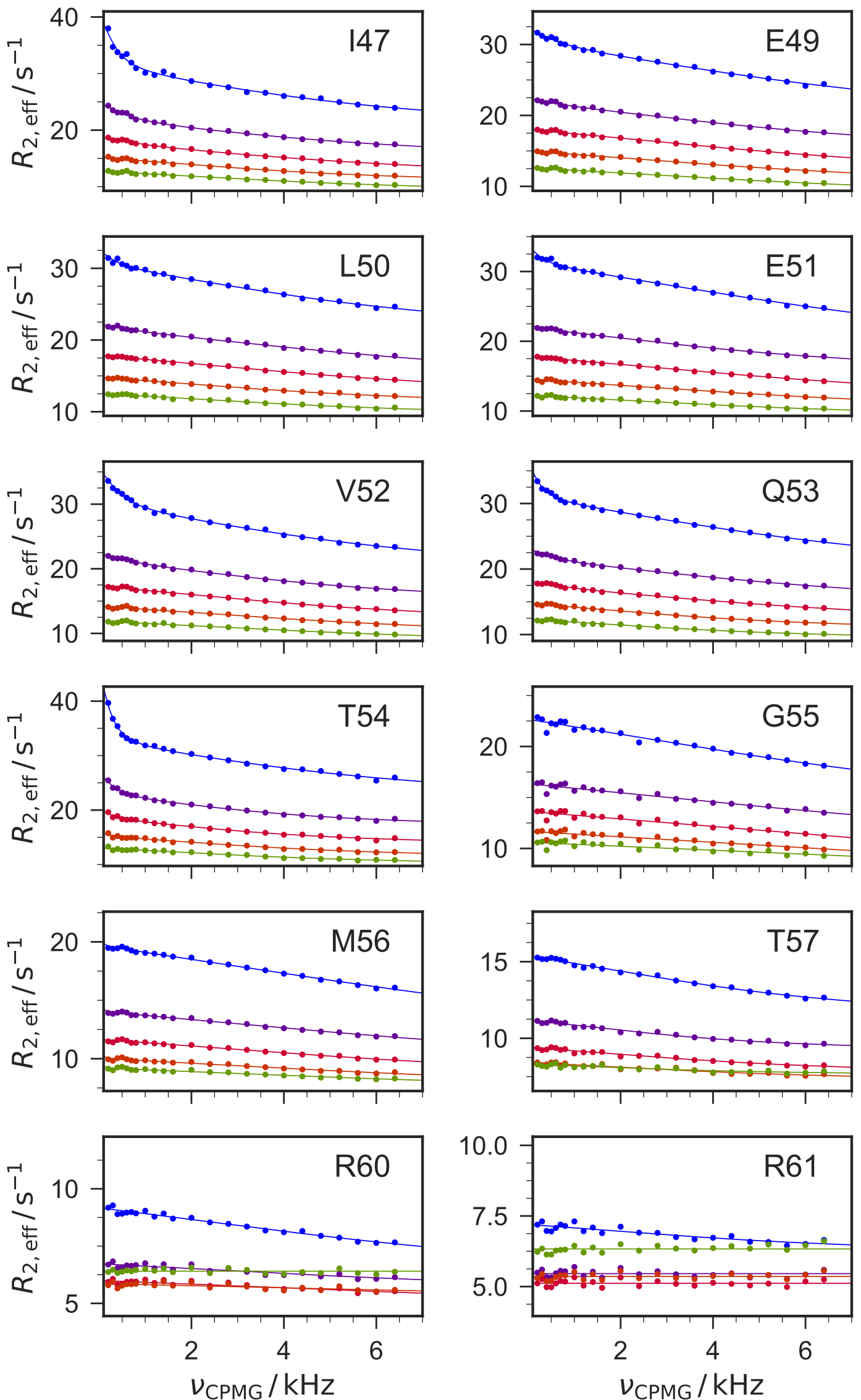


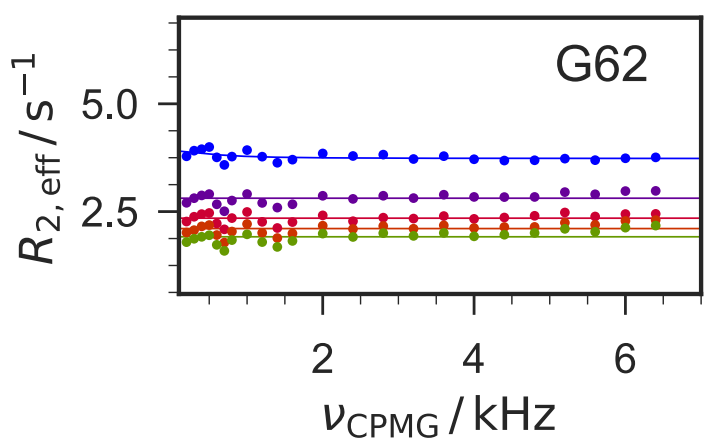

Figure A.1: Temperature dependent ${ }^{15} \mathrm{~N}$ relaxation dispersion profiles of gpW backbone. ${ }^{15} \mathrm{NE}$-CPMG experiments were measured at $263 \mathrm{~K}, 270 \mathrm{~K}, 275 \mathrm{~K}, 280 \mathrm{~K}$ and $285 \mathrm{~K}$. RD profiles are shown with the best individual fit, based on the AICc comparison described in Chapter 3. 
Table A.1: Individual fit parameters of the slow exchange process from backbone ${ }^{15} \mathbf{N}$ E-CPMG data. Fit parameters of the best individual model fit based on the AICc model selection are shown. Values in black correspond to the two-state exchange model parameters $\left(k_{\mathrm{ex}}, \phi\right.$, Equation $\left.(2.20)\right)$ and in red correspond to the three-state exchange model parameters $\left(k_{\mathrm{ex}}^{\mathrm{S}}, \phi^{\mathrm{S}}\right.$, Equation (3.2)). Flat profiles and fits with uncertainties larger than the fit parameter are marked as flat and NaN, respectively.

\begin{tabular}{|c|c|c|c|c|c|c|}
\hline Residue & Parameter & $263 \mathrm{~K}$ & $270 \mathrm{~K}$ & $275 \mathrm{~K}$ & $280 \mathrm{~K}$ & $285 \mathrm{~K}$ \\
\hline V2 & $\begin{array}{l}k_{\mathrm{ex}} / 10^{3} \mathrm{~s} \\
\phi / \mathrm{ppm}^{2}\end{array}$ & $\begin{aligned} 1.45 & \pm 0.31 \\
0.014 & \pm 0.002\end{aligned}$ & $\begin{array}{c}22.55 \pm 3.60 \\
0.284 \pm 0.066\end{array}$ & $\begin{array}{c}23.03 \pm 5.10 \\
0.196 \pm 0.064\end{array}$ & $\begin{array}{l}\mathrm{NaN} \\
\mathrm{NaN}\end{array}$ & flat \\
\hline R3 & $\begin{array}{l}k_{\mathrm{ex}} / 10^{3} \mathrm{~s} \\
\phi / \mathrm{ppm}^{2}\end{array}$ & $\begin{array}{c}1.44 \pm 0.64 \\
0.017 \pm 0.006\end{array}$ & $\begin{array}{c}1.93 \pm 1.61 \\
0.008 \pm 0.007\end{array}$ & $\begin{array}{c}35.29 \pm 5.41 \\
1.194 \pm 0.317\end{array}$ & $\begin{array}{c}30.74 \pm 6.30 \\
0.663 \pm 0.224\end{array}$ & $\begin{array}{c}33.57 \pm 9.49 \\
0.537 \pm 0.258\end{array}$ \\
\hline Q4 & $\begin{array}{l}k_{\mathrm{ex}} / 10^{3} \mathrm{~s} \\
\phi / \mathrm{ppm}^{2}\end{array}$ & $\begin{array}{c}2.28 \pm 0.21 \\
0.073 \pm 0.007\end{array}$ & $\begin{array}{c}3.88 \pm 0.67 \\
0.038 \pm 0.009\end{array}$ & $\begin{array}{c}0.75 \pm 0.73 \\
0.003 \pm 0.001\end{array}$ & $\begin{array}{l}\mathrm{NaN} \\
\mathrm{NaN}\end{array}$ & $\begin{array}{l}\mathrm{NaN} \\
\mathrm{NaN}\end{array}$ \\
\hline E5 & $\begin{array}{l}k_{\mathrm{ex}} / 10^{3} \mathrm{~s} \\
\phi / \mathrm{ppm}^{2}\end{array}$ & $\begin{array}{l}\mathrm{NaN} \\
\mathrm{NaN}\end{array}$ & $\begin{array}{l}49.23 \pm 22.46 \\
1.772 \pm 1.525\end{array}$ & $\begin{array}{l}\mathrm{NaN} \\
\mathrm{NaN}\end{array}$ & $\begin{array}{l}41.55 \pm 13.71 \\
0.825 \pm 0.494\end{array}$ & $\begin{array}{l}39.33 \pm 12.37 \\
0.591 \pm 0.333\end{array}$ \\
\hline E6 & $\begin{array}{l}k_{\mathrm{ex}} / 10^{3} \mathrm{~s} \\
\phi / \mathrm{ppm}^{2}\end{array}$ & $\begin{array}{c}1.24 \pm 0.22 \\
0.023 \pm 0.003\end{array}$ & $\begin{array}{c}29.26 \pm 2.98 \\
0.922 \pm 0.152\end{array}$ & $\begin{array}{c}35.56 \pm 3.83 \\
0.999 \pm 0.187\end{array}$ & $\begin{array}{l}\mathrm{NaN} \\
\mathrm{NaN}\end{array}$ & $\begin{array}{l}\text { NaN } \\
\text { NaN }\end{array}$ \\
\hline L7 & $\begin{array}{l}k_{\mathrm{ex}} / 10^{3} \mathrm{~s} \\
\phi / \mathrm{ppm}^{2}\end{array}$ & $\begin{array}{c}1.81 \pm 0.41 \\
0.029 \pm 0.006\end{array}$ & $\begin{array}{c}25.69 \pm 2.31 \\
0.750 \pm 0.104\end{array}$ & $\begin{array}{c}32.60 \pm 4.26 \\
0.847 \pm 0.187 \\
\end{array}$ & $\begin{array}{c}35.43 \pm 4.63 \\
0.714 \pm 0.162 \\
\end{array}$ & $\begin{array}{c}34.95 \pm 7.27 \\
0.510 \pm 0.183\end{array}$ \\
\hline A 8 & $\begin{array}{l}k_{\mathrm{ex}} / 10^{3} \mathrm{~s} \\
\phi / \mathrm{ppm}^{2}\end{array}$ & $\begin{aligned} 4.04 & \pm 0.72 \\
0.046 & \pm 0.011\end{aligned}$ & $\begin{array}{c}30.41 \pm 2.49 \\
0.940 \pm 0.127\end{array}$ & $\begin{array}{c}43.10 \pm 6.75 \\
1.352 \pm 0.388\end{array}$ & $\begin{array}{c}40.43 \pm 7.15 \\
0.944 \pm 0.301\end{array}$ & $\begin{array}{c}41.02 \pm 8.80 \\
0.739 \pm 0.287\end{array}$ \\
\hline A9 & $\begin{array}{l}k_{\mathrm{ex}} / 10^{3} \mathrm{~s} \\
\phi / \mathrm{ppm}^{2}\end{array}$ & $\begin{aligned} 1.60 & \pm 0.16 \\
0.040 & \pm 0.003\end{aligned}$ & $\begin{array}{c}3.65 \pm 0.89 \\
0.029 \pm 0.010\end{array}$ & $\begin{aligned} 4.89 & \pm 2.30 \\
0.020 & \pm 0.015\end{aligned}$ & $\begin{array}{c}30.26 \pm 3.05 \\
0.619 \pm 0.102\end{array}$ & $\begin{array}{c}32.72 \pm 4.22 \\
0.535 \pm 0.117\end{array}$ \\
\hline A 10 & $\begin{array}{l}k_{\mathrm{ex}} / 10^{3} \mathrm{~s} \\
\phi / \mathrm{ppm}^{2}\end{array}$ & $\begin{array}{c}1.83 \pm 0.37 \\
0.025 \pm 0.005\end{array}$ & $\begin{array}{c}29.90 \pm 3.83 \\
0.964 \pm 0.202\end{array}$ & $\begin{array}{c}33.93 \pm 5.14 \\
0.952 \pm 0.247\end{array}$ & $\begin{array}{c}38.75 \pm 8.38 \\
0.924 \pm 0.356\end{array}$ & $\begin{array}{c}35.97 \pm 8.19 \\
0.647 \pm 0.256\end{array}$ \\
\hline R11 & $\begin{array}{l}k_{\mathrm{ex}} / 10^{3} \mathrm{~s} \\
\phi / \mathrm{ppm}^{2}\end{array}$ & $\begin{array}{c}1.92 \pm 0.13 \\
0.091 \pm 0.006\end{array}$ & $\begin{aligned} 3.26 & \pm 0.64 \\
0.042 & \pm 0.011\end{aligned}$ & $\begin{aligned} 4.22 & \pm 1.33 \\
0.029 & \pm 0.013\end{aligned}$ & $\begin{aligned} 2.28 & \pm 1.39 \\
0.007 & \pm 0.005\end{aligned}$ & $\begin{aligned} 2.50 & \pm 2.12 \\
0.006 & \pm 0.006\end{aligned}$ \\
\hline $\mathrm{A} 12$ & $\begin{array}{l}k_{\mathrm{ex}} / 10^{3} \mathrm{~s} \\
\phi / \mathrm{ppm}^{2}\end{array}$ & $\begin{aligned} 1.73 & \pm 0.87 \\
0.011 & \pm 0.005\end{aligned}$ & $\begin{array}{c}34.13 \pm 4.92 \\
1.072 \pm 0.265\end{array}$ & $\begin{array}{c}40.06 \pm 7.09 \\
1.192 \pm 0.379\end{array}$ & $\begin{array}{l}43.25 \pm 11.75 \\
1.063 \pm 0.530\end{array}$ & $\begin{array}{l}39.38 \pm 12.04 \\
0.698 \pm 0.382\end{array}$ \\
\hline A 13 & $\begin{array}{l}k_{\mathrm{ex}} / 10^{3} \mathrm{~s} \\
\phi / \mathrm{ppm}^{2}\end{array}$ & $\begin{array}{c}1.45 \pm 0.87 \\
0.012 \pm 0.006\end{array}$ & $\begin{array}{c}32.22 \pm 3.45 \\
1.017 \pm 0.183\end{array}$ & $\begin{array}{c}40.50 \pm 7.24 \\
1.225 \pm 0.395\end{array}$ & $\begin{array}{c}42.37 \pm 9.38 \\
1.051 \pm 0.425\end{array}$ & $\begin{array}{l}41.51 \pm 11.75 \\
0.785 \pm 0.403\end{array}$ \\
\hline L14 & $\begin{array}{l}k_{\mathrm{ex}} / 10^{3} \mathrm{~s} \\
\phi / \mathrm{ppm}^{2}\end{array}$ & $\begin{array}{c}1.15 \pm 0.91 \\
0.160 \pm 0.088\end{array}$ & $\begin{array}{c}1.99 \pm 0.71 \\
0.057 \pm 0.022\end{array}$ & $\begin{array}{c}3.25 \pm 1.66 \\
0.035 \pm 0.023\end{array}$ & $\begin{array}{c}3.26 \pm 1.70 \\
0.015 \pm 0.010\end{array}$ & $\begin{array}{l}\text { NaN } \\
\text { NaN }\end{array}$ \\
\hline H15 & $\begin{array}{l}k_{\mathrm{ex}} / 10^{3} \mathrm{~s} \\
\phi / \mathrm{ppm}^{2}\end{array}$ & $\begin{aligned} 1.09 & \pm 0.32 \\
0.024 & \pm 0.004\end{aligned}$ & $\begin{aligned} 3.03 & \pm 2.10 \\
0.016 & \pm 0.013\end{aligned}$ & $\begin{aligned} 2.13 & \pm 1.49 \\
0.007 & \pm 0.005\end{aligned}$ & $\begin{array}{l}\mathrm{NaN} \\
\mathrm{NaN}\end{array}$ & $\begin{array}{l}47.42 \pm 19.10 \\
0.973 \pm 0.734\end{array}$ \\
\hline D16 & $\begin{array}{l}k_{\mathrm{ex}} / 10^{3} \mathrm{~s} \\
\phi / \mathrm{ppm}^{2}\end{array}$ & $\begin{aligned} 1.97 & \pm 0.23 \\
0.044 & \pm 0.005\end{aligned}$ & $\begin{aligned} 3.57 & \pm 0.96 \\
0.025 & \pm 0.009\end{aligned}$ & $\begin{array}{l}\mathrm{NaN} \\
\mathrm{NaN}\end{array}$ & $\begin{array}{c}38.67 \pm 6.72 \\
0.894 \pm 0.276\end{array}$ & $\begin{array}{l}\text { NaN } \\
\text { NaN }\end{array}$ \\
\hline L17 & $\begin{array}{l}k_{\mathrm{ex}} / 10^{3} \mathrm{~s} \\
\phi / \mathrm{ppm}^{2}\end{array}$ & $\begin{array}{c}1.40 \pm 0.09 \\
0.073 \pm 0.004\end{array}$ & $\begin{array}{c}3.05 \pm 0.23 \\
0.055 \pm 0.005\end{array}$ & $\begin{array}{c}4.27 \pm 0.82 \\
0.041 \pm 0.011\end{array}$ & $\begin{array}{l}\mathrm{NaN} \\
\mathrm{NaN}\end{array}$ & $\begin{array}{c}27.16 \pm 3.47 \\
0.497 \pm 0.100\end{array}$ \\
\hline M18 & $\begin{array}{l}k_{\mathrm{ex}} / 10^{3} \mathrm{~s} \\
\phi / \mathrm{ppm}^{2}\end{array}$ & $\begin{aligned} 1.26 & \pm 0.46 \\
0.016 & \pm 0.004\end{aligned}$ & $\begin{array}{c}32.92 \pm 3.97 \\
1.019 \pm 0.208\end{array}$ & $\begin{array}{l}\mathrm{NaN} \\
\mathrm{NaN}\end{array}$ & $\begin{array}{l}\mathrm{NaN} \\
\mathrm{NaN}\end{array}$ & $\begin{array}{l}36.02 \pm 10.53 \\
0.576 \pm 0.294\end{array}$ \\
\hline T19 & $\begin{array}{l}k_{\mathrm{ex}} / 10^{3} \mathrm{~s} \\
\phi / \mathrm{ppm}^{2}\end{array}$ & $\begin{array}{c}2.77 \pm 0.39 \\
0.121 \pm 0.021\end{array}$ & $\begin{array}{c}3.98 \pm 1.52 \\
0.055 \pm 0.032\end{array}$ & $\begin{array}{c}5.10 \pm 3.09 \\
0.036 \pm 0.033\end{array}$ & $\begin{array}{c}23.58 \pm 5.07 \\
0.416 \pm 0.133\end{array}$ & $\begin{array}{l}31.25 \pm 11.17 \\
0.495 \pm 0.294\end{array}$ \\
\hline G20 & $\begin{array}{l}k_{\mathrm{ex}} / 10^{3} \mathrm{~s} \\
\phi / \mathrm{ppm}^{2}\end{array}$ & $\begin{array}{c}2.26 \pm 0.18 \\
0.329 \pm 0.026\end{array}$ & $\begin{array}{c}3.89 \pm 0.33 \\
0.245 \pm 0.027\end{array}$ & $\begin{array}{c}5.50 \pm 0.84 \\
0.179 \pm 0.041\end{array}$ & $\begin{array}{c}7.46 \pm 3.38 \\
0.124 \pm 0.115\end{array}$ & $\begin{array}{c}19.05 \pm 3.54 \\
0.368 \pm 0.093\end{array}$ \\
\hline K21 & $\begin{array}{l}k_{\mathrm{ex}} / 10^{3} \mathrm{~s} \\
\phi / \mathrm{ppm}^{2}\end{array}$ & $\begin{aligned} 1.70 & \pm 0.10 \\
0.082 & \pm 0.004\end{aligned}$ & $\begin{aligned} 3.75 & \pm 0.59 \\
0.054 & \pm 0.012\end{aligned}$ & $\begin{array}{c}4.98 \pm 1.78 \\
0.028 \pm 0.016\end{array}$ & $\begin{array}{l}\mathrm{NaN} \\
\mathrm{NaN}\end{array}$ & $\begin{array}{c}31.54 \pm 4.33 \\
0.626 \pm 0.143\end{array}$ \\
\hline $\mathrm{R} 22$ & $\begin{array}{l}k_{\mathrm{ex}} / 10^{3} \mathrm{~s} \\
\phi / \mathrm{ppm}^{2}\end{array}$ & $\begin{array}{c}2.92 \pm 0.24 \\
0.184 \pm 0.020\end{array}$ & $\begin{aligned} 4.68 & \pm 0.78 \\
0.074 & \pm 0.020\end{aligned}$ & $\begin{array}{c}7.16 \pm 2.13 \\
0.042 \pm 0.024\end{array}$ & $\begin{array}{c}31.65 \pm 1.60 \\
0.995 \pm 0.084\end{array}$ & $\begin{array}{c}36.15 \pm 3.74 \\
0.845 \pm 0.152\end{array}$ \\
\hline V23 & $\begin{array}{l}k_{\mathrm{ex}} / 10^{3} \mathrm{~s} \\
\phi / \mathrm{ppm}^{2}\end{array}$ & $\begin{array}{c}5.97 \pm 0.46 \\
1.926 \pm 0.128\end{array}$ & $\begin{array}{c}5.66 \pm 0.25 \\
1.863 \pm 0.073\end{array}$ & $\begin{array}{c}8.29 \pm 0.19 \\
1.816 \pm 0.041\end{array}$ & $\begin{aligned} 8.83 & \pm 0.68 \\
1.000 & \pm 0.420\end{aligned}$ & $\begin{aligned} 9.04 & \pm 1.97 \\
0.396 & \pm 0.275\end{aligned}$ \\
\hline A 24 & $\begin{array}{l}k_{\mathrm{ex}} / 10^{3} \mathrm{~s} \\
\phi / \mathrm{ppm}^{2}\end{array}$ & $\begin{aligned} 1.85 & \pm 0.30 \\
0.042 & \pm 0.006\end{aligned}$ & $\begin{aligned} 3.22 & \pm 1.64 \\
0.023 & \pm 0.015\end{aligned}$ & $\begin{array}{l}\mathrm{NaN} \\
\mathrm{NaN}\end{array}$ & $\begin{array}{l}\mathrm{NaN} \\
\mathrm{NaN}\end{array}$ & $\begin{array}{l}32.20 \pm 11.56 \\
0.407 \pm 0.245\end{array}$ \\
\hline $\mathrm{T} 25$ & $\begin{array}{l}k_{\mathrm{ex}} / 10^{3} \mathrm{~s} \\
\phi / \mathrm{ppm}^{2}\end{array}$ & $\begin{aligned} 3.89 & \pm 1.08 \\
0.028 & \pm 0.011\end{aligned}$ & $\begin{array}{c}28.53 \pm 3.18 \\
0.613 \pm 0.110\end{array}$ & $\begin{array}{c}33.37 \pm 4.72 \\
0.609 \pm 0.146\end{array}$ & $\begin{array}{c}34.90 \pm 7.07 \\
0.500 \pm 0.175\end{array}$ & $\begin{array}{c}30.76 \pm 6.30 \\
0.306 \pm 0.104\end{array}$ \\
\hline V 26 & $\begin{array}{l}k_{\mathrm{ex}} / 10^{3} \mathrm{~s} \\
\phi / \mathrm{ppm}^{2}\end{array}$ & $\begin{array}{c}4.19 \pm 0.20 \\
1.543 \pm 0.062\end{array}$ & $\begin{array}{c}5.30 \pm 0.10 \\
1.236 \pm 0.020\end{array}$ & $\begin{aligned} 6.88 & \pm 0.45 \\
0.872 & \pm 0.107\end{aligned}$ & $\begin{array}{l}\text { NaN } \\
\text { NaN }\end{array}$ & $\begin{array}{l}\mathrm{NaN} \\
\mathrm{NaN}\end{array}$ \\
\hline Q27 & $\begin{array}{l}k_{\mathrm{ex}} / 10^{3} \mathrm{~s} \\
\phi / \mathrm{ppm}^{2}\end{array}$ & $\begin{aligned} 2.48 & \pm 0.05 \\
0.686 & \pm 0.014\end{aligned}$ & $\begin{aligned} 3.95 & \pm 0.08 \\
0.418 & \pm 0.012\end{aligned}$ & $\begin{aligned} 5.78 & \pm 0.26 \\
0.275 & \pm 0.022\end{aligned}$ & $\begin{array}{c}6.74 \pm 1.42 \\
0.109 \pm 0.054\end{array}$ & $\begin{array}{l}\mathrm{NaN} \\
\mathrm{NaN}\end{array}$ \\
\hline $\mathrm{K} 28$ & $\begin{array}{l}k_{\mathrm{ex}} / 10^{3} \mathrm{~s} \\
\phi / \mathrm{ppm}^{2}\end{array}$ & $\begin{aligned} 3.01 & \pm 0.26 \\
0.913 & \pm 0.088\end{aligned}$ & $\begin{array}{c}4.08 \pm 0.22 \\
0.674 \pm 0.060\end{array}$ & $\begin{aligned} 6.61 & \pm 0.45 \\
0.638 & \pm 0.085\end{aligned}$ & $\begin{array}{c}9.17 \pm 1.33 \\
0.506 \pm 0.179\end{array}$ & $\begin{array}{l}\mathrm{NaN} \\
\mathrm{NaN}\end{array}$ \\
\hline D29 & $\begin{array}{l}k_{\mathrm{ex}} / 10^{3} \mathrm{~s} \\
\phi / \mathrm{ppm}^{2}\end{array}$ & $\begin{array}{c}7.23 \pm 0.85 \\
3.020 \pm 0.317\end{array}$ & $\begin{array}{c}7.27 \pm 0.71 \\
2.826 \pm 0.254\end{array}$ & $\begin{array}{c}8.35 \pm 0.33 \\
2.391 \pm 0.096\end{array}$ & $\begin{array}{c}11.45 \pm 0.32 \\
2.128 \pm 0.067\end{array}$ & $\begin{array}{c}14.97 \pm 0.37 \\
1.870 \pm 0.057\end{array}$ \\
\hline G30 & $\begin{array}{l}k_{\mathrm{ex}} / 10^{3} \mathrm{~s} \\
\phi / \mathrm{ppm}^{2}\end{array}$ & $\begin{array}{c}3.15 \pm 0.14 \\
1.294 \pm 0.035\end{array}$ & $\begin{aligned} 3.47 & \pm 0.34 \\
0.597 & \pm 0.096\end{aligned}$ & $\begin{aligned} 1.62 & \pm 0.00 \\
0.076 & \pm 0.000\end{aligned}$ & $\begin{array}{l}\mathrm{NaN} \\
\mathrm{NaN}\end{array}$ & $\begin{array}{l}\mathrm{NaN} \\
\mathrm{NaN}\end{array}$ \\
\hline R32 & $\begin{array}{l}k_{\mathrm{ex}} / 10^{3} \mathrm{~s} \\
\phi / \mathrm{ppm}^{2}\end{array}$ & flat & $\begin{array}{c}2.25 \pm 0.72 \\
0.009 \pm 0.003\end{array}$ & $\begin{array}{c}1.79 \pm 1.39 \\
0.003 \pm 0.002\end{array}$ & $\begin{array}{c}33.71 \pm 5.49 \\
0.417 \pm 0.116\end{array}$ & $\begin{array}{c}37.87 \pm 9.32 \\
0.363 \pm 0.158\end{array}$ \\
\hline V33 & $\begin{array}{l}k_{\mathrm{ex}} / 10^{3} \mathrm{~s} \\
\phi / \mathrm{ppm}^{2}\end{array}$ & $\begin{array}{c}2.94 \pm 0.08 \\
1.141 \pm 0.022\end{array}$ & $\begin{array}{c}4.99 \pm 0.16 \\
1.064 \pm 0.029\end{array}$ & $\begin{array}{c}6.58 \pm 0.27 \\
0.804 \pm 0.052\end{array}$ & $\begin{array}{c}9.40 \pm 0.94 \\
0.693 \pm 0.149\end{array}$ & $\begin{array}{c}14.68 \pm 0.66 \\
0.833 \pm 0.046\end{array}$ \\
\hline E34 & $\begin{array}{l}k_{\mathrm{ex}} / 10^{3} \mathrm{~s} \\
\phi / \mathrm{ppm}^{2}\end{array}$ & $\begin{aligned} 1.66 & \pm 0.09 \\
0.093 & \pm 0.005\end{aligned}$ & $\begin{array}{c}2.94 \pm 0.52 \\
0.037 \pm 0.008\end{array}$ & $\begin{array}{c}5.05 \pm 2.97 \\
0.023 \pm 0.022\end{array}$ & $\begin{array}{c}29.94 \pm 5.48 \\
0.450 \pm 0.135\end{array}$ & $\begin{array}{c}33.34 \pm 9.74 \\
0.379 \pm 0.188\end{array}$ \\
\hline F35 & $\begin{array}{l}k_{\mathrm{ex}} / 10^{3} \mathrm{~s} \\
\phi / \mathrm{ppm}^{2}\end{array}$ & $\begin{aligned} 2.38 & \pm 0.73 \\
0.037 & \pm 0.012\end{aligned}$ & $\begin{array}{c}16.89 \pm 2.14 \\
0.381 \pm 0.063\end{array}$ & $\begin{array}{c}24.04 \pm 3.83 \\
0.467 \pm 0.112\end{array}$ & $\begin{array}{c}23.82 \pm 3.36 \\
0.344 \pm 0.073\end{array}$ & $\begin{array}{c}31.94 \pm 5.55 \\
0.399 \pm 0.116\end{array}$ \\
\hline T36 & $\begin{array}{l}k_{\mathrm{ex}} / 10^{3} \mathrm{~s} \\
\phi / \mathrm{ppm}^{2}\end{array}$ & $\begin{array}{c}4.55 \pm 0.21 \\
2.233 \pm 0.083\end{array}$ & $\begin{array}{c}5.38 \pm 0.12 \\
1.887 \pm 0.036\end{array}$ & $\begin{array}{c}6.53 \pm 1.50 \\
1.000 \pm 0.583\end{array}$ & $\begin{array}{c}9.73 \pm 1.34 \\
1.000 \pm 0.214\end{array}$ & $\begin{array}{c}15.31 \pm 0.47 \\
1.181 \pm 0.045\end{array}$ \\
\hline A 37 & $\begin{array}{l}k_{\mathrm{ex}} / 10^{3} \mathrm{~s} \\
\phi / \mathrm{ppm}^{2}\end{array}$ & $\begin{aligned} 2.81 & \pm 0.22 \\
0.522 & \pm 0.048\end{aligned}$ & $\begin{array}{c}5.14 \pm 0.22 \\
0.619 \pm 0.023\end{array}$ & $\begin{aligned} 6.22 & \pm 0.26 \\
0.403 & \pm 0.029\end{aligned}$ & $\begin{array}{c}8.47 \pm 0.58 \\
0.316 \pm 0.048\end{array}$ & $\begin{aligned} 7.83 & \pm 2.78 \\
0.089 & \pm 0.087\end{aligned}$ \\
\hline
\end{tabular}




\begin{tabular}{|c|c|c|c|c|c|c|}
\hline Residue & Parameter & $263 \mathrm{~K}$ & $270 \mathrm{~K}$ & $275 \mathrm{~K}$ & $280 \mathrm{~K}$ & $285 \mathrm{~K}$ \\
\hline \multirow{2}{*}{ T38 } & $k_{\mathrm{ex}} / 10^{3} \mathrm{~s}$ & $4.30 \pm 0.25$ & $4.93 \pm 0.21$ & $6.86 \pm 0.41$ & $10.73 \pm 0.43$ & $\mathrm{NaN}$ \\
\hline & $\phi / \mathrm{ppm}^{2}$ & $1.287 \pm 0.063$ & $1.000 \pm 0.323$ & $0.843 \pm 0.087$ & $0.928 \pm 0.040$ & $\mathrm{NaN}$ \\
\hline \multirow{2}{*}{ S39 } & $k_{\text {ex }} / 10^{3} \mathrm{~s}$ & $4.87 \pm 1.08$ & $3.95 \pm 0.10$ & $5.94 \pm 0.22$ & $7.70 \pm 1.13$ & $17.69 \pm 1.10$ \\
\hline & $\phi / \mathrm{ppm}^{2}$ & $0.120 \pm 0.042$ & $0.419 \pm 0.016$ & $0.308 \pm 0.020$ & $0.171 \pm 0.057$ & $0.423 \pm 0.035$ \\
\hline \multirow{2}{*}{ V40 } & $k_{\mathrm{ex}} / 10^{3} \mathrm{~s}$ & $29.34 \pm 13.40$ & $23.26 \pm 6.63$ & $\mathrm{NaN}$ & $43.97 \pm 15.29$ & $39.09 \pm 6.21$ \\
\hline & $\phi / \mathrm{ppm}^{2}$ & $1.414 \pm 1.049$ & $0.632 \pm 0.267$ & $\mathrm{NaN}$ & $1.066 \pm 0.683$ & $0.704 \pm 0.200$ \\
\hline \multirow{2}{*}{$\mathrm{S} 41$} & $k_{\mathrm{ex}} / 10^{3} \mathrm{~s}$ & $4.05 \pm 0.57$ & $5.22 \pm 1.29$ & $6.49 \pm 2.97$ & $27.26 \pm 2.85$ & $30.41 \pm 4.56$ \\
\hline & $\phi / \mathrm{ppm}^{2}$ & $0.245 \pm 0.040$ & $0.113 \pm 0.049$ & $0.052 \pm 0.047$ & $0.713 \pm 0.118$ & $0.587 \pm 0.145$ \\
\hline \multirow{2}{*}{ D42 } & $k_{\mathrm{ex}} / 10^{3} \mathrm{~s}$ & $33.11 \pm 7.73$ & $35.43 \pm 8.98$ & $40.48 \pm 7.87$ & $36.76 \pm 6.14$ & $35.17 \pm 7.44$ \\
\hline & $\phi / \mathrm{ppm}^{2}$ & $1.358 \pm 0.537$ & $0.828 \pm 0.364$ & $0.865 \pm 0.303$ & $0.826 \pm 0.242$ & $0.587 \pm 0.215$ \\
\hline \multirow{2}{*}{ L43 } & $k_{\mathrm{ex}} / 10^{3} \mathrm{~s}$ & $1.80 \pm 0.25$ & $2.87 \pm 0.88$ & $4.79 \pm 1.53$ & $\mathrm{NaN}$ & $25.96 \pm 4.77$ \\
\hline & $\phi / \mathrm{ppm}^{2}$ & $0.150 \pm 0.018$ & $0.065 \pm 0.028$ & $0.057 \pm 0.030$ & $\mathrm{NaN}$ & $0.462 \pm 0.132$ \\
\hline \multirow{2}{*}{ K44 } & $k_{\mathrm{ex}} / 10^{3} \mathrm{~s}$ & $1.28 \pm 0.64$ & $35.10 \pm 4.04$ & $44.31 \pm 9.11$ & $43.18 \pm 11.67$ & $50.37 \pm 21.93$ \\
\hline & $\phi / \mathrm{ppm}^{2}$ & $0.013 \pm 0.005$ & $1.186 \pm 0.236$ & $1.425 \pm 0.540$ & $1.051 \pm 0.521$ & $1.064 \pm 0.878$ \\
\hline \multirow{2}{*}{ K45 } & $k_{\mathrm{ex}} / 10^{3} \mathrm{~s}$ & $2.32 \pm 0.95$ & $34.16 \pm 3.74$ & $37.27 \pm 3.18$ & $36.23 \pm 3.94$ & $38.20 \pm 7.61$ \\
\hline & $\phi / \mathrm{ppm}^{2}$ & $0.016 \pm 0.007$ & $1.225 \pm 0.230$ & $1.145 \pm 0.172$ & $0.857 \pm 0.163$ & $0.737 \pm 0.260$ \\
\hline \multirow{2}{*}{ Y46 } & $k_{\mathrm{ex}} / 10^{3} \mathrm{~s}$ & $34.65 \pm 6.89$ & $33.63 \pm 4.87$ & $41.10 \pm 5.08$ & $39.88 \pm 7.20$ & $35.17 \pm 7.44$ \\
\hline & $\phi / \mathrm{ppm}^{2}$ & $1.831 \pm 0.626$ & $1.150 \pm 0.284$ & $1.320 \pm 0.295$ & $1.028 \pm 0.333$ & $0.587 \pm 0.215$ \\
\hline \multirow{2}{*}{ I 47} & $k_{\mathrm{ex}} / 10^{3} \mathrm{~s}$ & $2.34 \pm 0.36$ & $4.12 \pm 0.74$ & $5.63 \pm 2.29$ & $29.54 \pm 3.80$ & $34.09 \pm 5.66$ \\
\hline & $\phi / \mathrm{ppm}^{2}$ & $0.113 \pm 0.019$ & $0.064 \pm 0.017$ & $0.035 \pm 0.025$ & $0.690 \pm 0.145$ & $0.659 \pm 0.187$ \\
\hline \multirow{2}{*}{ E49 } & $k_{\mathrm{ex}} / 10^{3} \mathrm{~s}$ & $3.84 \pm 1.01$ & $\mathrm{NaN}$ & $43.99 \pm 8.11$ & $42.21 \pm 9.02$ & $45.93 \pm 15.07$ \\
\hline & $\phi / \mathrm{ppm}^{2}$ & $0.039 \pm 0.014$ & $\mathrm{NaN}$ & $1.516 \pm 0.514$ & $1.086 \pm 0.423$ & $0.990 \pm 0.604$ \\
\hline \multirow{2}{*}{ L50 } & $k_{\mathrm{ex}} / 10^{3} \mathrm{~s}$ & $2.25 \pm 0.80$ & $\mathrm{NaN}$ & $41.03 \pm 4.21$ & $32.80 \pm 3.99$ & $35.14 \pm 6.18$ \\
\hline & $\phi / \mathrm{ppm}^{2}$ & $0.022 \pm 0.008$ & $\mathrm{NaN}$ & $1.229 \pm 0.228$ & $0.655 \pm 0.135$ & $0.578 \pm 0.176$ \\
\hline \multirow{2}{*}{ E51 } & $k_{\mathrm{ex}} / 10^{3} \mathrm{~s}$ & $1.88 \pm 0.53$ & $\mathrm{NaN}$ & $47.01 \pm 10.23$ & $44.34 \pm 12.01$ & $38.41 \pm 9.44$ \\
\hline & $\phi / \mathrm{ppm}^{2}$ & $0.022 \pm 0.006$ & $\mathrm{NaN}$ & $1.613 \pm 0.656$ & $1.087 \pm 0.543$ & $0.638 \pm 0.279$ \\
\hline \multirow{2}{*}{ V52 } & $k_{\mathrm{ex}} / 10^{3} \mathrm{~s}$ & $2.83 \pm 0.30$ & $4.27 \pm 1.54$ & $\mathrm{NaN}$ & $39.35 \pm 7.87$ & $40.72 \pm 11.68$ \\
\hline & $\phi / \mathrm{ppm}^{2}$ & $0.082 \pm 0.010$ & $0.025 \pm 0.014$ & $\mathrm{NaN}$ & $0.948 \pm 0.339$ & $0.750 \pm 0.388$ \\
\hline \multirow{2}{*}{ Q53 } & $k_{\mathrm{ex}} / 10^{3} \mathrm{~s}$ & $1.69 \pm 0.17$ & $3.65 \pm 0.89$ & $4.88 \pm 2.30$ & $\mathrm{NaN}$ & $28.58 \pm 4.40$ \\
\hline & $\phi / \mathrm{ppm}^{2}$ & $0.045 \pm 0.004$ & $0.029 \pm 0.010$ & $0.020 \pm 0.015$ & $\mathrm{NaN}$ & $0.456 \pm 0.113$ \\
\hline \multirow{2}{*}{ T54 } & $k_{\mathrm{ex}} / 10^{3} \mathrm{~s}$ & $1.66 \pm 0.11$ & $2.13 \pm 0.31$ & $2.14 \pm 0.90$ & $\mathrm{NaN}$ & $28.59 \pm 5.18$ \\
\hline & $\phi / \mathrm{ppm}^{2}$ & $0.110 \pm 0.006$ & $0.044 \pm 0.007$ & $0.016 \pm 0.007$ & $\mathrm{NaN}$ & $0.459 \pm 0.134$ \\
\hline \multirow{2}{*}{ G55 } & $k_{\mathrm{ex}} / 10^{3} \mathrm{~s}$ & $\mathrm{NaN}$ & $\mathrm{NaN}$ & $\mathrm{NaN}$ & $\mathrm{NaN}$ & $\mathrm{NaN}$ \\
\hline & $\phi / \mathrm{ppm}^{2}$ & $\mathrm{NaN}$ & $\mathrm{NaN}$ & $\mathrm{NaN}$ & $\mathrm{NaN}$ & $\mathrm{NaN}$ \\
\hline \multirow{2}{*}{ M56 } & $k_{\mathrm{ex}} / 10^{3} \mathrm{~s}$ & $\mathrm{NaN}$ & $\mathrm{NaN}$ & $48.19 \pm 18.25$ & $\mathrm{NaN}$ & $\mathrm{NaN}$ \\
\hline & $\phi / \mathrm{ppm}^{2}$ & $\mathrm{NaN}$ & $\mathrm{NaN}$ & $0.854 \pm 0.608$ & $\mathrm{NaN}$ & $\mathrm{NaN}$ \\
\hline \multirow{2}{*}{ T57 } & $k_{\mathrm{ex}} / 10^{3} \mathrm{~s}$ & $33.65 \pm 3.54$ & $25.19 \pm 3.35$ & $31.37 \pm 6.54$ & $31.48 \pm 11.10$ & $24.84 \pm 10.34$ \\
\hline & $\phi / \mathrm{ppm}^{2}$ & $0.723 \pm 0.130$ & $0.263 \pm 0.054$ & $0.283 \pm 0.098$ & $0.197 \pm 0.116$ & $0.089 \pm 0.056$ \\
\hline \multirow{2}{*}{ R60 } & $k_{\mathrm{ex}} / 10^{3} \mathrm{~s}$ & $48.13 \pm 20.32$ & $\mathrm{NaN}$ & $\mathrm{NaN}$ & $\mathrm{NaN}$ & \\
\hline & $\phi / \mathrm{ppm}^{2}$ & $0.748 \pm 0.593$ & $\mathrm{NaN}$ & $\mathrm{NaN}$ & $\mathrm{NaN}$ & flat \\
\hline R61 & $\begin{array}{l}k_{\mathrm{ex}} / 10^{3} \mathrm{~s} \\
\phi / \mathrm{ppm}^{2}\end{array}$ & $\begin{array}{l}\mathrm{NaN} \\
\mathrm{NaN}\end{array}$ & flat & flat & flat & flat \\
\hline G62 & $\begin{array}{l}k_{\mathrm{ex}} / 10^{3} \mathrm{~s} \\
\phi / \mathrm{ppm}^{2}\end{array}$ & $\begin{array}{c}4.24 \pm 3.40 \\
0.004 \pm 0.003\end{array}$ & flat & flat & flat & flat \\
\hline
\end{tabular}


Table A.2: Individual fit parameters of the fast exchange process from backbone ${ }^{15} \mathrm{~N}$ E-CPMG data. Values in red correspond to the three-state exchange model parameters $\left(k_{\mathrm{ex}}^{\mathrm{F}}, \phi^{\mathrm{F}}\right.$, Equation (3.2)). Flat profiles and fits with uncertainties larger than the fit parameter are marked as flat and NaN, respectively.

\begin{tabular}{|c|c|c|c|c|c|c|}
\hline Residue & Parameter & $263 \mathrm{~K}$ & $270 \mathrm{~K}$ & $275 \mathrm{~K}$ & $280 \mathrm{~K}$ & $285 \mathrm{~K}$ \\
\hline $\mathrm{V} 2$ & $\begin{array}{l}k_{e x} / 10^{3} \mathrm{~s} \\
\phi / \mathrm{ppm}^{2}\end{array}$ & $\begin{array}{l}44.72 \pm 11.76 \\
1.358 \pm 0.639\end{array}$ & $\mathrm{LM}$ & $\mathrm{LM}$ & LM & flat \\
\hline $\mathrm{R} 3$ & $\begin{array}{l}k_{e x} / 10^{3} \mathrm{~s} \\
\phi / \mathrm{ppm}^{2}\end{array}$ & $\begin{array}{c}42.37 \pm 8.25 \\
4.000 \pm 1.774\end{array}$ & $\begin{array}{c}33.39 \pm 5.86 \\
1.459 \pm 0.398\end{array}$ & LM & LM & LM \\
\hline Q4 & $\begin{array}{l}k_{e x} / 10^{3} \mathrm{~s} \\
\phi / \mathrm{ppm}^{2}\end{array}$ & $\begin{array}{c}39.85 \pm 7.56 \\
2.262 \pm 0.719\end{array}$ & $\begin{array}{l}45.32 \pm 12.26 \\
1.770 \pm 0.817\end{array}$ & $\begin{array}{c}32.78 \pm 3.80 \\
0.910 \pm 0.171\end{array}$ & $\begin{array}{c}33.32 \pm 4.59 \\
0.693 \pm 0.158\end{array}$ & $\begin{array}{c}39.36 \pm 9.79 \\
0.673 \pm 0.294\end{array}$ \\
\hline E5 & $\begin{array}{l}k_{e x} / 10^{3} \mathrm{~s} \\
\phi / \mathrm{ppm}^{2}\end{array}$ & $\mathrm{LM}$ & LM & $\begin{array}{l}\mathrm{NaN} \\
\mathrm{NaN}\end{array}$ & LM & LM \\
\hline E6 & $\begin{array}{l}k_{e x} / 10^{3} \mathrm{~s} \\
\phi / \mathrm{ppm}^{2}\end{array}$ & $\begin{array}{l}55.76 \pm 19.58 \\
4.000 \pm 0.988\end{array}$ & LM & LM & $\begin{array}{c}40.35 \pm 8.43 \\
0.987 \pm 0.352\end{array}$ & $\begin{array}{l}\mathrm{NaN} \\
\mathrm{NaN}\end{array}$ \\
\hline L7 & $\begin{array}{l}k_{e x} / 10^{3} \mathrm{~s} \\
\phi / \mathrm{ppm}^{2}\end{array}$ & $\begin{array}{c}38.26 \pm 8.13 \\
1.929 \pm 0.684\end{array}$ & LM & $\mathrm{LM}$ & $\mathrm{LM}$ & $\mathrm{LM}$ \\
\hline A 8 & $\begin{array}{l}k_{e x} / 10^{3} \mathrm{~s} \\
\phi / \mathrm{ppm}^{2}\end{array}$ & $\begin{array}{l}59.30 \pm 29.39 \\
4.000 \pm 0.915\end{array}$ & $\mathrm{LM}$ & $\mathrm{LM}$ & LM & LM \\
\hline A9 & $\begin{array}{l}k_{e x} / 10^{3} \mathrm{~s} \\
\phi / \mathrm{ppm}^{2}\end{array}$ & $\begin{array}{c}41.07 \pm 5.95 \\
2.353 \pm 0.590\end{array}$ & $\begin{array}{l}42.56 \pm 11.29 \\
1.609 \pm 0.710\end{array}$ & $\begin{array}{l}41.90 \pm 13.29 \\
1.274 \pm 0.642\end{array}$ & LM & LM \\
\hline A10 & $\begin{array}{l}k_{e x} / 10^{3} \mathrm{~s} \\
\phi / \mathrm{ppm}^{2}\end{array}$ & $\begin{array}{c}36.30 \pm 5.00 \\
1.905 \pm 0.427\end{array}$ & LM & LM & $\mathrm{LM}$ & LM \\
\hline R11 & $\begin{array}{l}k_{e x} / 10^{3} \mathrm{~s} \\
\phi / \mathrm{ppm}^{2}\end{array}$ & $\begin{array}{c}38.14 \pm 7.69 \\
1.837 \pm 0.615\end{array}$ & $\begin{array}{l}40.00 \pm 12.37 \\
1.381 \pm 0.695\end{array}$ & $\begin{array}{l}\mathrm{NaN} \\
\mathrm{NaN}\end{array}$ & $\begin{array}{c}35.30 \pm 8.58 \\
0.760 \pm 0.291\end{array}$ & $\begin{array}{l}\mathrm{NaN} \\
\mathrm{NaN}\end{array}$ \\
\hline $\mathrm{A} 12$ & $\begin{array}{l}k_{e x} / 10^{3} \mathrm{~s} \\
\phi / \mathrm{ppm}^{2}\end{array}$ & $\begin{array}{l}48.62 \pm 14.98 \\
2.975 \pm 1.676\end{array}$ & $\mathrm{LM}$ & LM & LM & LM \\
\hline A13 & $\begin{array}{l}k_{e x} / 10^{3} \mathrm{~s} \\
\phi / \mathrm{ppm}^{2}\end{array}$ & $\begin{array}{c}36.61 \pm 8.20 \\
1.875 \pm 0.696\end{array}$ & LM & $\mathrm{LM}$ & $\mathrm{LM}$ & $\mathrm{LM}$ \\
\hline L14 & $\begin{array}{l}k_{e x} / 10^{3} \mathrm{~s} \\
\phi / \mathrm{ppm}^{2}\end{array}$ & $\begin{array}{l}\mathrm{NaN} \\
\mathrm{NaN} \\
\end{array}$ & $\begin{array}{l}25.50 \pm 10.33 \\
0.924 \pm 0.487 \\
\end{array}$ & $\begin{array}{l}\mathrm{NaN} \\
\mathrm{NaN}\end{array}$ & $\begin{array}{l}48.69 \pm 26.94 \\
1.646 \pm 1.622 \\
\end{array}$ & $\begin{array}{c}36.25 \pm 9.05 \\
0.781 \pm 0.321\end{array}$ \\
\hline H15 & $\begin{array}{l}k_{e x} / 10^{3} \mathrm{~s} \\
\phi / \mathrm{ppm}^{2}\end{array}$ & $\begin{array}{l}47.52 \pm 18.40 \\
2.871 \pm 2.039\end{array}$ & $\begin{array}{l}\mathrm{NaN} \\
\mathrm{NaN}\end{array}$ & $\begin{array}{l}\mathrm{NaN} \\
\mathrm{NaN}\end{array}$ & $\begin{array}{l}45.62 \pm 19.70 \\
1.145 \pm 0.907\end{array}$ & LM \\
\hline D16 & $\begin{array}{l}k_{e x} / 10^{3} \mathrm{~s} \\
\phi / \mathrm{ppm}^{2}\end{array}$ & $\begin{array}{l}52.66 \pm 17.73 \\
3.350 \pm 2.099\end{array}$ & $\begin{array}{c}39.25 \pm 9.78 \\
1.235 \pm 0.490\end{array}$ & $\begin{array}{l}\mathrm{NaN} \\
\mathrm{NaN}\end{array}$ & $\mathrm{LM}$ & $\mathrm{LM}$ \\
\hline L17 & $\begin{array}{l}k_{e x} / 10^{3} \mathrm{~s} \\
\phi / \mathrm{ppm}^{2}\end{array}$ & $\begin{array}{c}36.28 \pm 5.31 \\
1.874 \pm 0.454\end{array}$ & $\begin{array}{c}42.52 \pm 7.31 \\
1.645 \pm 0.478\end{array}$ & $\begin{array}{l}47.62 \pm 18.90 \\
1.637 \pm 1.125\end{array}$ & $\begin{array}{c}26.11 \pm 2.47 \\
0.598 \pm 0.083\end{array}$ & $\mathrm{LM}$ \\
\hline M18 & $\begin{array}{l}k_{e x} / 10^{3} \mathrm{~s} \\
\phi / \mathrm{ppm}^{2}\end{array}$ & $\begin{array}{l}42.09 \pm 10.97 \\
2.242 \pm 1.029\end{array}$ & $\mathrm{LM}$ & LM & LM & $\mathrm{LM}$ \\
\hline T19 & $\begin{array}{l}k_{e x} / 10^{3} \mathrm{~s} \\
\phi / \mathrm{ppm}^{2}\end{array}$ & $\begin{array}{l}33.23 \pm 13.13 \\
1.335 \pm 0.783\end{array}$ & $\begin{array}{l}33.47 \pm 18.27 \\
0.943 \pm 0.721\end{array}$ & $\begin{array}{l}\mathrm{NaN} \\
\mathrm{NaN}\end{array}$ & LM & $\mathrm{LM}$ \\
\hline G20 & $\begin{array}{l}k_{e x} / 10^{3} \mathrm{~s} \\
\phi / \mathrm{ppm}^{2}\end{array}$ & $\begin{array}{l}\mathrm{NaN} \\
\mathrm{NaN} \\
\end{array}$ & $\begin{array}{l}\mathrm{NaN} \\
\mathrm{NaN}\end{array}$ & $\begin{array}{l}\mathrm{NaN} \\
\mathrm{NaN} \\
\end{array}$ & $\begin{array}{l}\mathrm{NaN} \\
\mathrm{NaN} \\
\end{array}$ & $\mathrm{LM}$ \\
\hline K21 & $\begin{array}{l}k_{e x} / 10^{3} \mathrm{~s} \\
\phi / \mathrm{ppm}^{2}\end{array}$ & $\begin{array}{c}30.56 \pm 2.13 \\
2.062 \pm 0.214\end{array}$ & $\begin{array}{c}39.93 \pm 8.15 \\
1.906 \pm 0.623\end{array}$ & $\begin{array}{l}44.29 \pm 13.07 \\
1.828 \pm 0.885\end{array}$ & $\begin{array}{l}36.20 \pm 11.08 \\
0.993 \pm 0.426\end{array}$ & $\mathrm{LM}$ \\
\hline $\mathrm{R} 22$ & $\begin{array}{l}k_{e x} / 10^{3} \mathrm{~s} \\
\phi / \mathrm{ppm}^{2}\end{array}$ & $\begin{array}{c}25.30 \pm 2.18 \\
2.531 \pm 0.259\end{array}$ & $\begin{array}{c}35.73 \pm 4.45 \\
2.583 \pm 0.459\end{array}$ & $\begin{array}{l}49.05 \pm 11.46 \\
2.976 \pm 1.150\end{array}$ & LM & LM \\
\hline V23 & $\begin{array}{l}k_{e x} / 10^{3} \mathrm{~s} \\
\phi / \mathrm{ppm}^{2}\end{array}$ & LM & LM & LM & $\begin{array}{c}24.43 \pm 7.79 \\
1.171 \pm 0.156\end{array}$ & $\begin{array}{c}22.80 \pm 5.21 \\
1.534 \pm 0.143\end{array}$ \\
\hline A24 & $\begin{array}{l}k_{e x} / 10^{3} \mathrm{~s} \\
\phi / \mathrm{ppm}^{2}\end{array}$ & $\begin{array}{c}35.57 \pm 6.76 \\
1.704 \pm 0.521\end{array}$ & $\begin{array}{l}39.10 \pm 19.28 \\
1.162 \pm 0.922\end{array}$ & $\begin{array}{l}39.94 \pm 25.64 \\
0.971 \pm 0.968\end{array}$ & $\begin{array}{l}32.99 \pm 19.41 \\
0.546 \pm 0.458\end{array}$ & $\mathrm{LM}$ \\
\hline $\mathrm{T} 25$ & $\begin{array}{l}k_{e x} / 10^{3} \mathrm{~s} \\
\phi / \mathrm{ppm}^{2}\end{array}$ & $\begin{array}{l}46.71 \pm 14.51 \\
2.069 \pm 1.112\end{array}$ & LM & LM & LM & LM \\
\hline V26 & $\begin{array}{l}k_{e x} / 10^{3} \mathrm{~s} \\
\phi / \mathrm{ppm}^{2}\end{array}$ & LM & LM & $\begin{array}{l}\mathrm{NaN} \\
\mathrm{NaN}\end{array}$ & $\begin{array}{c}12.50 \pm 1.38 \\
0.979 \pm 0.073\end{array}$ & $\begin{array}{l}26.54 \pm 12.49 \\
1.039 \pm 0.281\end{array}$ \\
\hline Q27 & $\begin{array}{l}k_{e x} / 10^{3} \mathrm{~s} \\
\phi / \mathrm{ppm}^{2}\end{array}$ & $\begin{array}{l}\mathrm{NaN} \\
\mathrm{NaN}\end{array}$ & $\begin{array}{l}38.01 \pm 10.93 \\
1.086 \pm 0.481\end{array}$ & $\begin{array}{l}40.04 \pm 14.69 \\
1.036 \pm 0.566\end{array}$ & $\begin{array}{c}25.04 \pm 7.28 \\
0.540 \pm 0.105\end{array}$ & $\begin{array}{c}21.97 \pm 4.40 \\
0.436 \pm 0.066\end{array}$ \\
\hline $\mathrm{K} 28$ & $\begin{array}{l}k_{e x} / 10^{3} \mathrm{~s} \\
\phi / \mathrm{ppm}^{2}\end{array}$ & $\begin{array}{l}\mathrm{NaN} \\
\mathrm{NaN}\end{array}$ & $\begin{array}{c}24.24 \pm 9.65 \\
0.919 \pm 0.363\end{array}$ & $\begin{array}{l}\mathrm{NaN} \\
\mathrm{NaN}\end{array}$ & $\begin{array}{l}\mathrm{NaN} \\
\mathrm{NaN}\end{array}$ & $\begin{array}{l}29.12 \pm 25.22 \\
0.951 \pm 0.405\end{array}$ \\
\hline D29 & $\begin{array}{l}k_{e x} / 10^{3} \mathrm{~s} \\
\phi / \mathrm{ppm}^{2}\end{array}$ & $\mathrm{LM}$ & LM & LM & LM & LM \\
\hline G30 & $\begin{array}{l}k_{e x} / 10^{3} \mathrm{~s} \\
\phi / \mathrm{ppm}^{2}\end{array}$ & LM & $\begin{array}{c}10.00 \pm 0.87 \\
0.683 \pm 0.155\end{array}$ & $\begin{array}{c}10.00 \pm 0.00 \\
1.089 \pm 0.000\end{array}$ & $\begin{array}{c}11.40 \pm 0.36 \\
0.881 \pm 0.029\end{array}$ & $\begin{array}{c}15.60 \pm 0.98 \\
0.786 \pm 0.058\end{array}$ \\
\hline R32 & $\begin{array}{l}k_{e x} / 10^{3} \mathrm{~s} \\
\phi / \mathrm{ppm}^{2}\end{array}$ & flat & $\begin{array}{l}45.45 \pm 11.68 \\
1.154 \pm 0.525\end{array}$ & $\begin{array}{l}44.97 \pm 11.52 \\
0.914 \pm 0.417\end{array}$ & $\mathrm{LM}$ & $\mathrm{LM}$ \\
\hline V33 & $\begin{array}{l}k_{e x} / 10^{3} \mathrm{~s} \\
\phi / \mathrm{ppm}^{2}\end{array}$ & LM & LM & $\begin{array}{l}\mathrm{NaN} \\
\mathrm{NaN}\end{array}$ & $\begin{array}{l}\mathrm{NaN} \\
\mathrm{NaN}\end{array}$ & LM \\
\hline E34 & $\begin{array}{l}k_{e x} / 10^{3} \mathrm{~s} \\
\phi / \mathrm{ppm}^{2}\end{array}$ & $\begin{array}{c}33.85 \pm 5.61 \\
1.358 \pm 0.356\end{array}$ & $\begin{array}{c}33.51 \pm 8.24 \\
0.824 \pm 0.300\end{array}$ & $\begin{array}{l}37.15 \pm 17.79 \\
0.766 \pm 0.530\end{array}$ & $\mathrm{LM}$ & $\mathrm{LM}$ \\
\hline F35 & $\begin{array}{l}k_{e x} / 10^{3} \mathrm{~s} \\
\phi / \mathrm{ppm}^{2}\end{array}$ & $\begin{array}{l}\mathrm{NaN} \\
\mathrm{NaN}\end{array}$ & LM & LM & LM & $\mathrm{LM}$ \\
\hline T36 & $\begin{array}{l}k_{e x} / 10^{3} \mathrm{~s} \\
\phi / \mathrm{ppm}^{2}\end{array}$ & LM & LM & $\begin{array}{l}\mathrm{NaN} \\
\mathrm{NaN}\end{array}$ & $\begin{array}{l}\mathrm{NaN} \\
\mathrm{NaN}\end{array}$ & LM \\
\hline A 37 & $\begin{array}{l}k_{e x} / 10^{3} \mathrm{~s} \\
\phi / \mathrm{ppm}^{2}\end{array}$ & $\begin{array}{l}\mathrm{NaN} \\
\mathrm{NaN}\end{array}$ & $\mathrm{LM}$ & $\begin{array}{l}\mathrm{NaN} \\
\mathrm{NaN}\end{array}$ & $\begin{array}{l}45.39 \pm 27.85 \\
1.180 \pm 1.089\end{array}$ & $\begin{array}{c}23.35 \pm 5.50 \\
0.674 \pm 0.066\end{array}$ \\
\hline T38 & $\begin{array}{l}k_{e x} / 10^{3} \mathrm{~s} \\
\phi / \mathrm{ppm}^{2}\end{array}$ & LM & $\begin{array}{l}\mathrm{NaN} \\
\mathrm{NaN}\end{array}$ & $\begin{array}{l}\mathrm{NaN} \\
\mathrm{NaN}\end{array}$ & LM & $\begin{array}{l}\mathrm{NaN} \\
\mathrm{NaN}\end{array}$ \\
\hline
\end{tabular}




\begin{tabular}{|c|c|c|c|c|c|c|}
\hline Residue & Parameter & $263 \mathrm{~K}$ & $270 \mathrm{~K}$ & $275 \mathrm{~K}$ & $280 \mathrm{~K}$ & $285 \mathrm{~K}$ \\
\hline S39 & $\begin{array}{l}k_{e x} / 10^{3} \mathrm{~s} \\
\phi / \mathrm{ppm}^{2}\end{array}$ & $\begin{array}{l}\mathrm{NaN} \\
\mathrm{NaN}\end{array}$ & $\begin{array}{c}32.10 \pm 7.44 \\
0.974 \pm 0.306\end{array}$ & $\begin{array}{l}46.17 \pm 20.14 \\
1.383 \pm 0.988\end{array}$ & $\begin{array}{l}34.41 \pm 15.52 \\
0.777 \pm 0.393\end{array}$ & LM \\
\hline V40 & $\begin{array}{l}k_{e x} / 10^{3} \mathrm{~s} \\
\phi / \mathrm{ppm}^{2}\end{array}$ & LM & LM & LM & LM & LM \\
\hline $\mathrm{S} 41$ & $\begin{array}{l}k_{e x} / 10^{3} \mathrm{~s} \\
\phi / \mathrm{ppm}^{2}\end{array}$ & $\begin{array}{l}57.66 \pm 17.46 \\
4.000 \pm 1.027\end{array}$ & $\begin{array}{l}35.18 \pm 16.75 \\
1.308 \pm 0.850\end{array}$ & $\begin{array}{l}37.73 \pm 15.61 \\
1.305 \pm 0.736\end{array}$ & LM & LM \\
\hline D42 & $\begin{array}{l}k_{e x} / 10^{3} \mathrm{~s} \\
\phi / \mathrm{ppm}^{2}\end{array}$ & LM & LM & LM & LM & LM \\
\hline L43 & $\begin{array}{l}k_{e x} / 10^{3} \mathrm{~s} \\
\phi / \mathrm{ppm}^{2}\end{array}$ & $\begin{array}{l}\mathrm{NaN} \\
\mathrm{NaN} \\
\end{array}$ & $\begin{array}{c}22.48 \pm 8.03 \\
0.627 \pm 0.238\end{array}$ & $\begin{array}{l}34.70 \pm 15.78 \\
0.925 \pm 0.581\end{array}$ & $\begin{array}{l}27.72 \pm 13.71 \\
0.541 \pm 0.287\end{array}$ & LM \\
\hline K44 & $\begin{array}{l}k_{e x} / 10^{3} \mathrm{~s} \\
\phi / \mathrm{ppm}^{2}\end{array}$ & $\begin{array}{c}37.98 \pm 8.38 \\
2.015 \pm 0.752\end{array}$ & LM & LM & LM & LM \\
\hline K45 & $\begin{array}{l}k_{e x} / 10^{3} \mathrm{~s} \\
\phi / \mathrm{ppm}^{2}\end{array}$ & $\begin{array}{c}39.62 \pm 6.97 \\
2.245 \pm 0.658\end{array}$ & LM & LM & LM & LM \\
\hline Y46 & $\begin{array}{l}k_{e x} / 10^{3} \mathrm{~s} \\
\phi / \mathrm{ppm}^{2}\end{array}$ & LM & LM & LM & LM & LM \\
\hline I 47 & $\begin{array}{l}k_{e x} / 10^{3} \mathrm{~s} \\
\phi / \mathrm{ppm}^{2}\end{array}$ & $\begin{array}{l}35.11 \pm 12.97 \\
1.935 \pm 1.119\end{array}$ & $\begin{array}{c}35.33 \pm 9.26 \\
1.226 \pm 0.467\end{array}$ & $\begin{array}{l}38.42 \pm 12.74 \\
1.193 \pm 0.572\end{array}$ & LM & LM \\
\hline E49 & $\begin{array}{l}k_{e x} / 10^{3} \mathrm{~s} \\
\phi / \mathrm{ppm}^{2}\end{array}$ & $\begin{array}{l}44.75 \pm 12.45 \\
2.545 \pm 1.202 \\
\end{array}$ & $\begin{array}{l}40.87 \pm 11.24 \\
1.545 \pm 0.689 \\
\end{array}$ & LM & LM & LM \\
\hline L50 & $\begin{array}{l}k_{e x} / 10^{3} \mathrm{~s} \\
\phi / \mathrm{ppm}^{2}\end{array}$ & $\begin{array}{c}37.43 \pm 8.19 \\
1.874 \pm 0.667 \\
\end{array}$ & $\begin{array}{l}46.48 \pm 17.57 \\
1.805 \pm 1.170\end{array}$ & LM & LM & LM \\
\hline E51 & $\begin{array}{l}k_{e x} / 10^{3} \mathrm{~s} \\
\phi / \mathrm{ppm}^{2}\end{array}$ & $\begin{array}{l}52.38 \pm 19.51 \\
3.696 \pm 2.562\end{array}$ & $\begin{array}{c}34.58 \pm 4.42 \\
1.148 \pm 0.246\end{array}$ & LM & LM & LM \\
\hline V52 & $\begin{array}{l}k_{e x} / 10^{3} \mathrm{~s} \\
\phi / \mathrm{ppm}^{2}\end{array}$ & $\begin{array}{c}37.30 \pm 7.26 \\
2.004 \pm 0.621\end{array}$ & $\begin{array}{c}33.49 \pm 5.90 \\
1.152 \pm 0.280\end{array}$ & $\begin{array}{c}35.06 \pm 4.54 \\
1.031 \pm 0.225\end{array}$ & LM & LM \\
\hline Q53 & $\begin{array}{l}k_{e x} / 10^{3} \mathrm{~s} \\
\phi / \mathrm{ppm}^{2}\end{array}$ & $\begin{array}{c}41.83 \pm 6.67 \\
2.518 \pm 0.698\end{array}$ & $\begin{array}{l}42.56 \pm 11.29 \\
1.609 \pm 0.710\end{array}$ & $\begin{array}{l}41.66 \pm 13.04 \\
1.262 \pm 0.625\end{array}$ & $\begin{array}{c}29.01 \pm 3.29 \\
0.617 \pm 0.111\end{array}$ & LM \\
\hline T54 & $\begin{array}{l}k_{e x} / 10^{3} \mathrm{~s} \\
\phi / \mathrm{ppm}^{2}\end{array}$ & $\begin{array}{c}35.14 \pm 6.52 \\
1.870 \pm 0.559\end{array}$ & $\begin{array}{c}25.74 \pm 3.70 \\
0.791 \pm 0.147\end{array}$ & $\begin{array}{c}24.99 \pm 4.36 \\
0.636 \pm 0.140\end{array}$ & $\begin{array}{c}28.10 \pm 5.51 \\
0.596 \pm 0.169\end{array}$ & LM \\
\hline G55 & $\begin{array}{l}k_{e x} / 10^{3} \mathrm{~s} \\
\phi / \mathrm{ppm}^{2}\end{array}$ & LM & LM & LM & LM & LM \\
\hline M56 & $\begin{array}{l}k_{e x} / 10^{3} \mathrm{~s} \\
\phi / \mathrm{ppm}^{2}\end{array}$ & $\begin{array}{l}60.53 \pm 25.13 \\
2.751 \pm 2.217\end{array}$ & $\begin{array}{l}54.09 \pm 21.96 \\
1.315 \pm 1.020\end{array}$ & LM & $\begin{array}{l}\mathrm{NaN} \\
\mathrm{NaN}\end{array}$ & $\begin{array}{l}\mathrm{NaN} \\
\mathrm{NaN}\end{array}$ \\
\hline T57 & $\begin{array}{l}k_{e x} / 10^{3} \mathrm{~s} \\
\phi / \mathrm{ppm}^{2}\end{array}$ & LM & LM & LM & LM & LM \\
\hline R60 & $\begin{array}{l}k_{e x} / 10^{3} \mathrm{~s} \\
\phi / \mathrm{ppm}^{2}\end{array}$ & LM & LM & LM & LM & flat \\
\hline R61 & $\begin{array}{l}k_{e x} / 10^{3} \mathrm{~s} \\
\phi / \mathrm{ppm}^{2}\end{array}$ & LM & flat & flat & flat & flat \\
\hline G62 & $\begin{array}{l}k_{e x} / 10^{3} \mathrm{~s} \\
\phi / \mathrm{ppm}^{2}\end{array}$ & LM & flat & flat & flat & flat \\
\hline
\end{tabular}




\section{A.2 RD profiles of backbone ${ }^{1} \mathrm{H}$ E-CPMG}
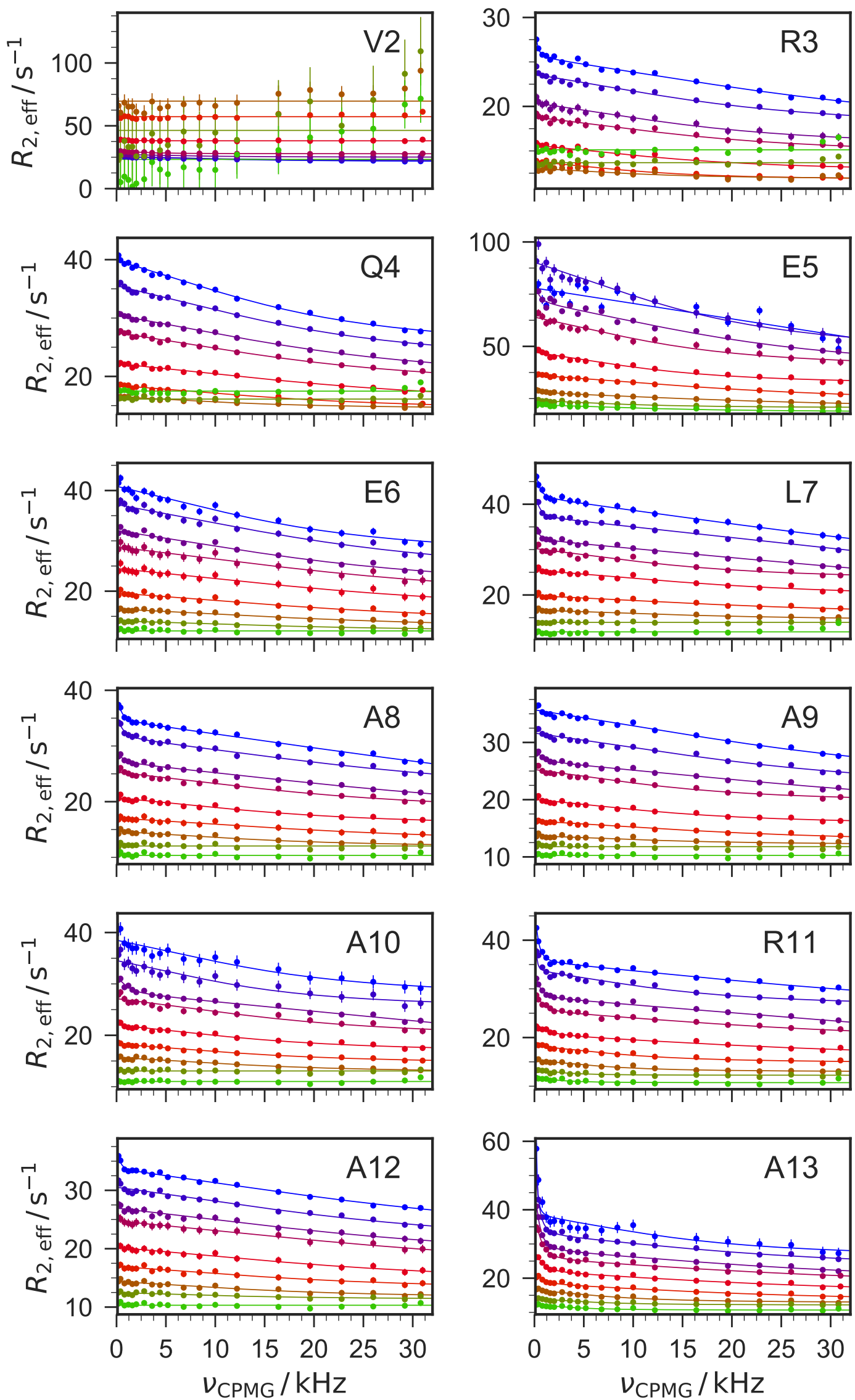

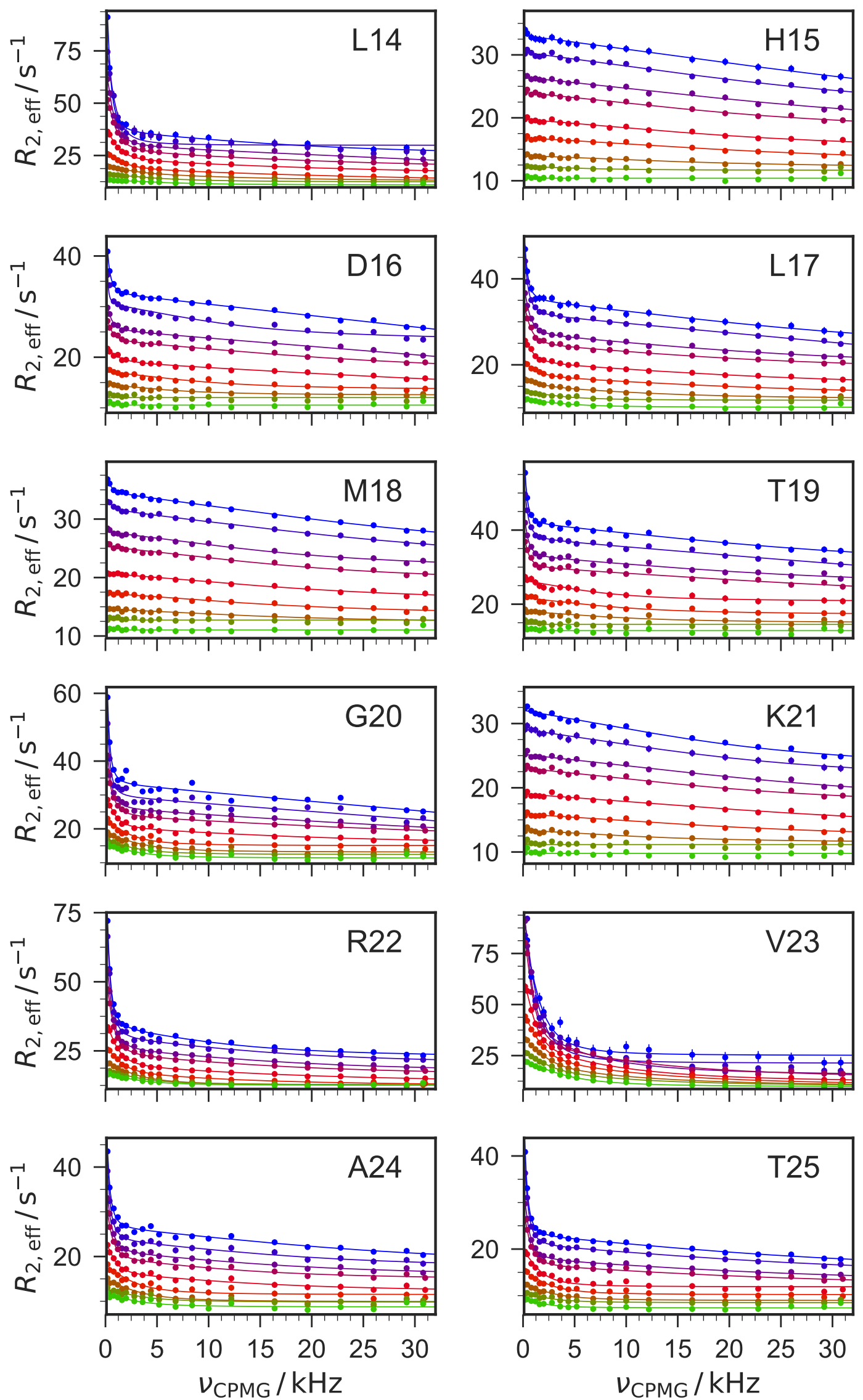

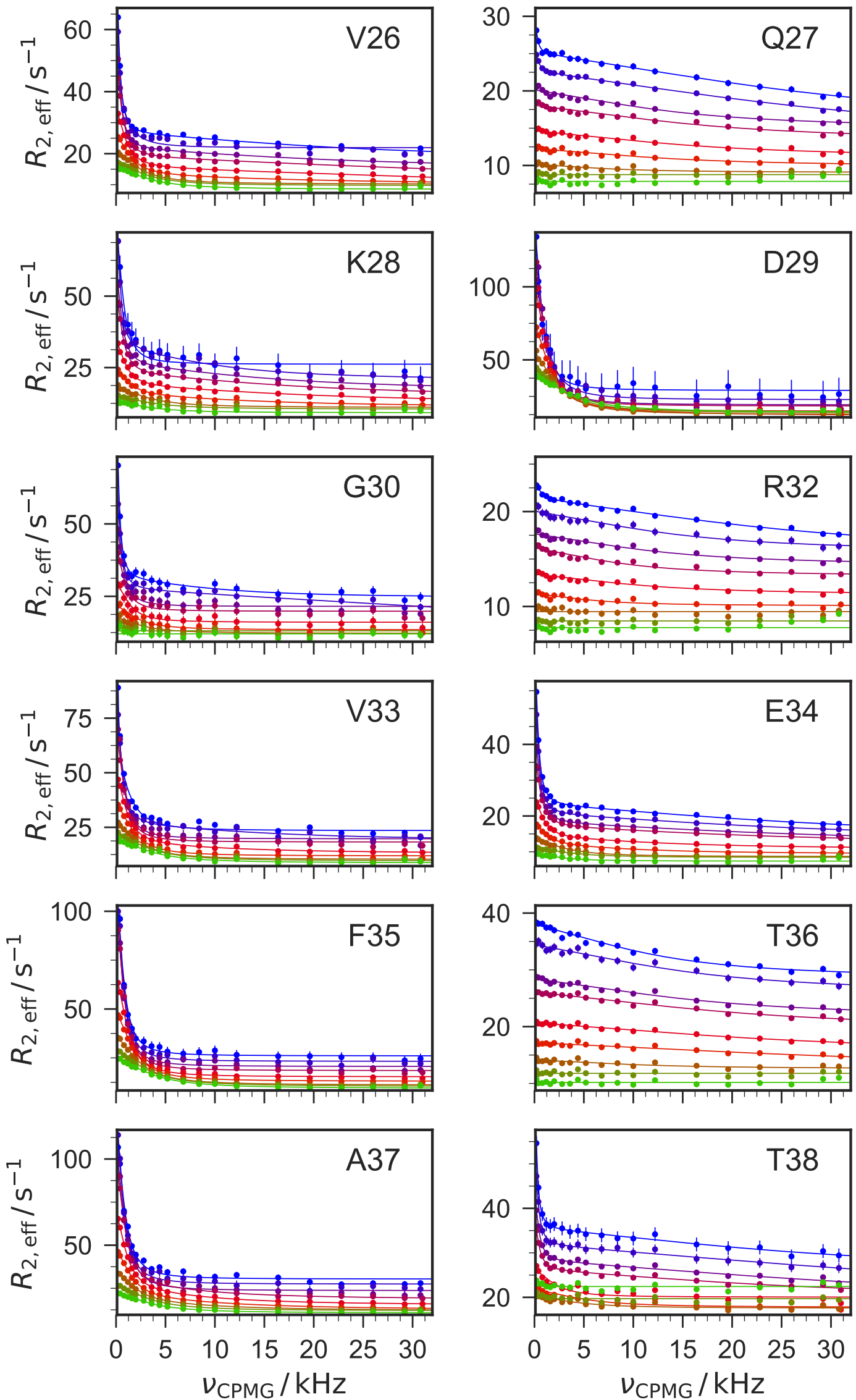

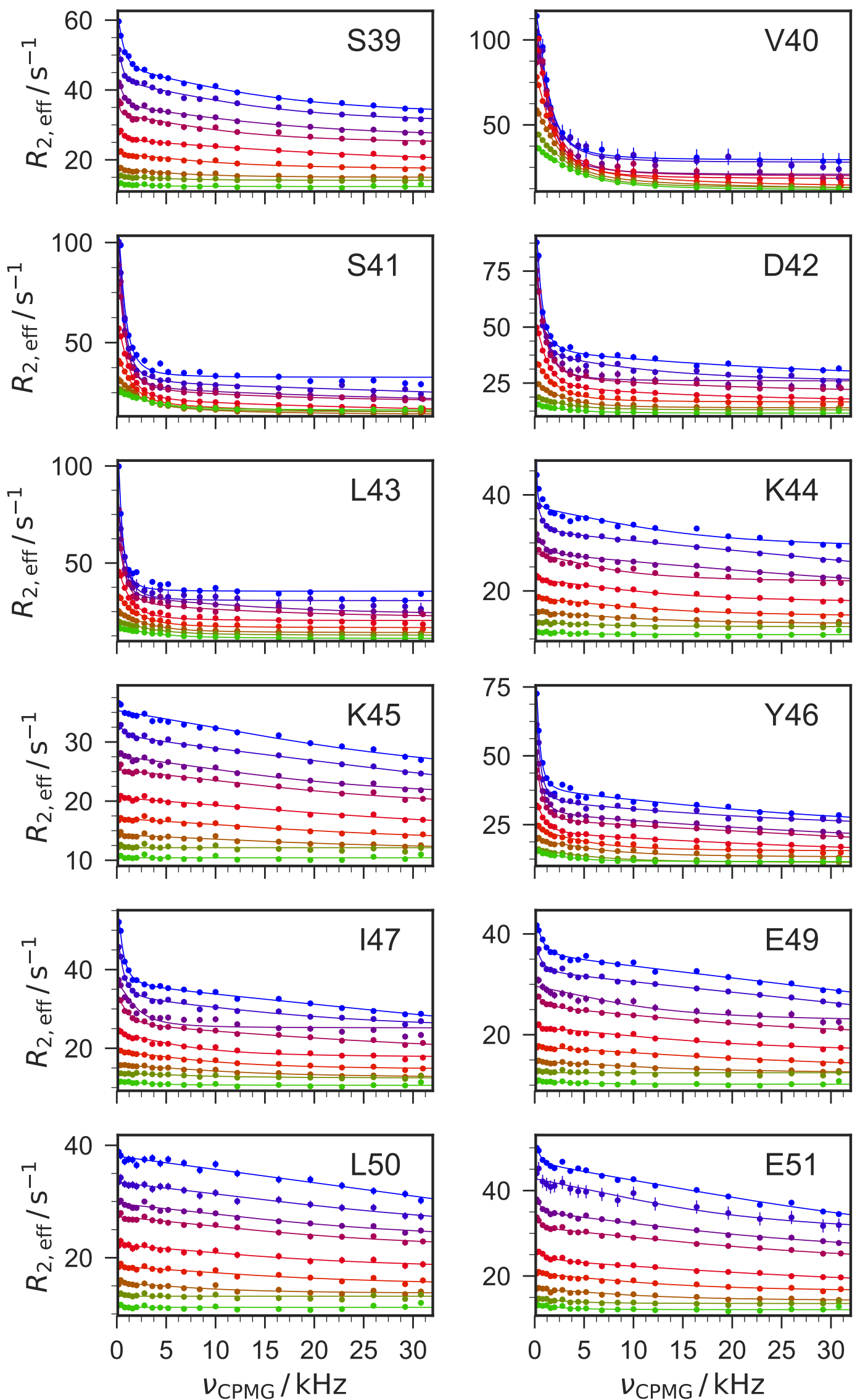

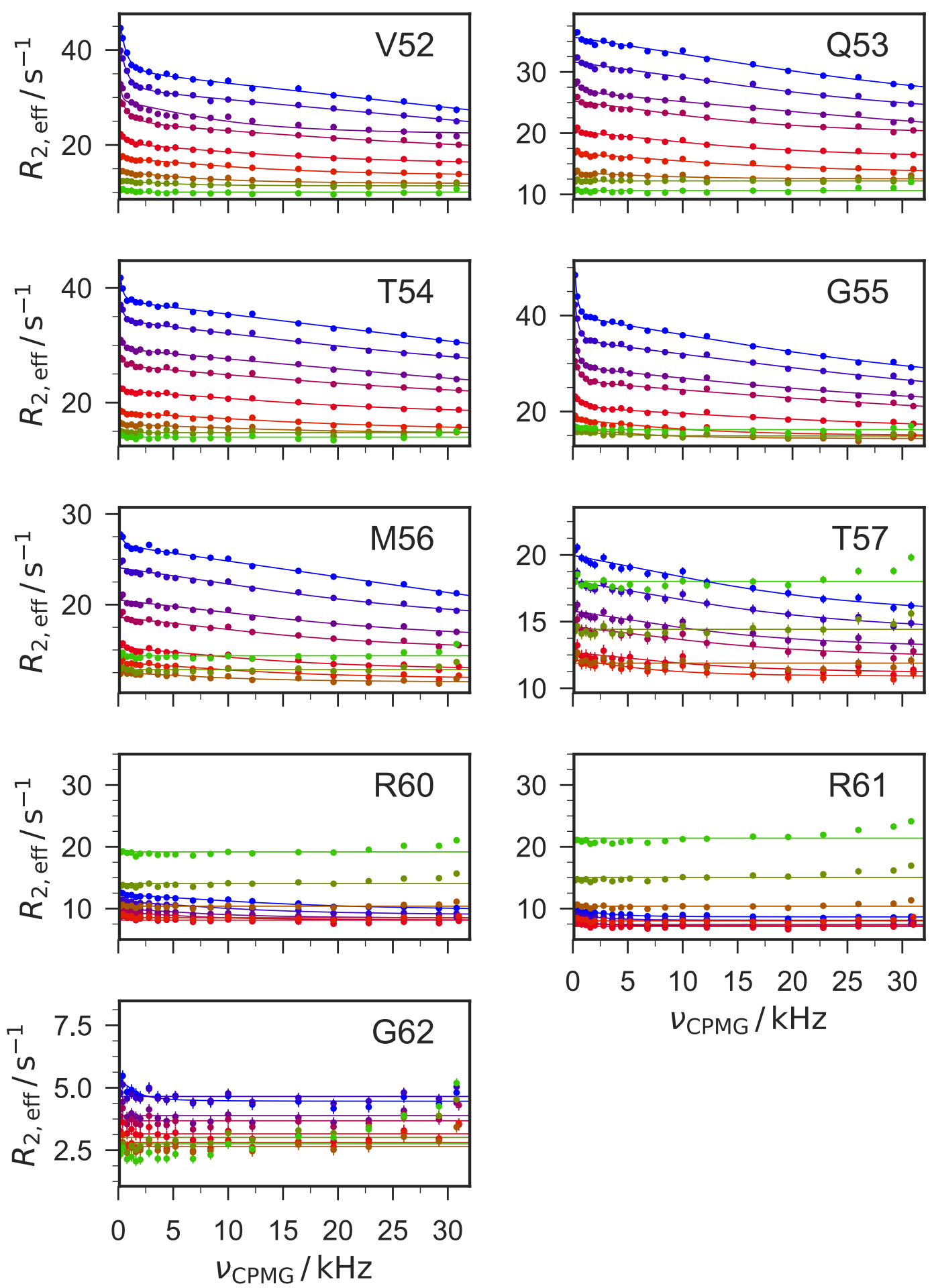

Figure A.2: Temperature dependent ${ }^{1} \mathrm{H}$ relaxation dispersion profiles of gpW backbone. ${ }^{1} \mathrm{H} \mathrm{E}$-CPMG experiments were measured at $263 \mathrm{~K}, 265 \mathrm{~K}, 268 \mathrm{~K}, 270 \mathrm{~K}, 275 \mathrm{~K}$, $280 \mathrm{~K}, 285 \mathrm{~K}, 290 \mathrm{~K}$ and $295 \mathrm{~K}$. RD profiles are shown with the best individual fit, based on the AICc comparison described in Chapter 3. 
Table A.3: Individual fit parameters of the slow exchange process from backbone ${ }^{1} \mathbf{H}$ E-CPMG data. Fit parameters of the best individual model fit based on the AICc model selection are shown. Values in black correspond to the two-state exchange model parameters $\left(k_{\text {ex }}, \phi\right.$, Equation (2.20)) and in red correspond to the three-state exchange model parameters $\left(k_{\mathrm{ex}}^{\mathrm{S}}, \phi^{\mathrm{S}}\right.$, Equation $\left.(3.2)\right)$. Flat profiles and fits with uncertainties larger than the fit parameter are marked as flat and NaN, respectively.

\begin{tabular}{|c|c|c|c|c|c|c|c|c|c|c|}
\hline Residue & Parameter & $263 \mathrm{~K}$ & $265 \mathrm{~K}$ & $268 \mathrm{~K}$ & $270 \mathrm{~K}$ & $275 \mathrm{~K}$ & $280 \mathrm{~K}$ & $285 \mathrm{~K}$ & $290 \mathrm{~K}$ & $295 \mathrm{~K}$ \\
\hline V2 & $\begin{array}{l}k_{e x} / 10^{3} \mathrm{~s} \\
\phi / \mathrm{ppm}^{2}\end{array}$ & $\begin{array}{l}77.62 \pm 13.87 \\
0.016 \pm 0.004\end{array}$ & $\begin{array}{l}65.73 \pm 12.33 \\
0.011 \pm 0.003 \\
\end{array}$ & $\begin{array}{l}53.30 \pm 12.21 \\
0.006 \pm 0.002 \\
\end{array}$ & $\begin{array}{c}22.16 \pm 4.36 \\
0.002 \pm 0.000 \\
\end{array}$ & $\begin{array}{c}5.44 \pm 4.31 \\
0.000 \pm 0.000\end{array}$ & flat & flat & flat & flat \\
\hline R3 & $\begin{array}{l}k_{e x} / 10^{3} \mathrm{~s} \\
\phi / \mathrm{ppm}^{2}\end{array}$ & $\begin{array}{c}1.92 \pm 0.80 \\
0.000 \pm 0.000\end{array}$ & $\begin{array}{c}127.43 \pm 26.45 \\
0.040 \pm 0.013\end{array}$ & $\begin{array}{c}118.64 \pm 24.19 \\
0.031 \pm 0.009\end{array}$ & $\begin{array}{c}120.61 \pm 23.78 \\
0.026 \pm 0.008\end{array}$ & $\begin{array}{c}103.84 \pm 23.16 \\
0.016 \pm 0.005\end{array}$ & $\begin{array}{l}75.60 \pm 15.94 \\
0.008 \pm 0.002\end{array}$ & $\begin{array}{l}75.35 \pm 30.91 \\
0.005 \pm 0.002 \\
\end{array}$ & flat & flat \\
\hline Q4 & $\begin{array}{l}k_{e x} / 10^{3} \mathrm{~s} \\
\phi / \mathrm{ppm}^{2}\end{array}$ & $\begin{array}{l}116.21 \pm 9.38 \\
0.092 \pm 0.011 \\
\end{array}$ & $\begin{array}{c}129.61 \pm 12.25 \\
0.090 \pm 0.013 \\
\end{array}$ & $\begin{array}{c}144.16 \pm 14.15 \\
0.084 \pm 0.014\end{array}$ & $\begin{array}{c}137.72 \pm 16.29 \\
0.066 \pm 0.013\end{array}$ & $\begin{array}{c}160.90 \pm 29.09 \\
0.059 \pm 0.018\end{array}$ & $\begin{array}{c}125.09 \pm 20.12 \\
0.028 \pm 0.007\end{array}$ & $\begin{array}{l}90.56 \pm 22.69 \\
0.010 \pm 0.003 \\
\end{array}$ & flat & flat \\
\hline E5 & $\begin{array}{l}k_{e x} / 10^{3} \mathrm{~s} \\
\phi / \mathrm{ppm}^{2}\end{array}$ & $\begin{array}{c}421.15 \pm 71.24 \\
1.692 \pm 0.467\end{array}$ & $\begin{array}{c}110.31 \pm 23.93 \\
0.252 \pm 0.081\end{array}$ & $\begin{array}{c}3.33 \pm 2.79 \\
0.001 \pm 0.001\end{array}$ & $\begin{array}{l}93.38 \pm 14.12 \\
0.117 \pm 0.025\end{array}$ & $\begin{array}{l}84.64 \pm 11.99 \\
0.068 \pm 0.013\end{array}$ & $\begin{array}{c}148.99 \pm 18.89 \\
0.101 \pm 0.021\end{array}$ & $\begin{array}{c}6.35 \pm 1.72 \\
0.000 \pm 0.000\end{array}$ & $\begin{array}{l}66.73 \pm 11.33 \\
0.012 \pm 0.003\end{array}$ & $\begin{array}{l}88.70 \pm 26.00 \\
0.016 \pm 0.006\end{array}$ \\
\hline E6 & $\begin{array}{l}k_{e x} / 10^{3} \mathrm{~s} \\
\phi / \mathrm{ppm}^{2}\end{array}$ & $\begin{array}{c}117.50 \pm 23.55 \\
0.085 \pm 0.026\end{array}$ & $\begin{array}{c}155.59 \pm 38.11 \\
0.116 \pm 0.048\end{array}$ & $\begin{array}{c}150.18 \pm 32.87 \\
0.090 \pm 0.033\end{array}$ & $\begin{array}{c}175.90 \pm 72.84 \\
0.096 \pm 0.069\end{array}$ & $\begin{array}{c}172.03 \pm 68.04 \\
0.077 \pm 0.053\end{array}$ & $\begin{array}{c}163.20 \pm 62.26 \\
0.052 \pm 0.034\end{array}$ & $\begin{array}{c}155.44 \pm 39.73 \\
0.031 \pm 0.013\end{array}$ & $\begin{array}{c}136.36 \pm 42.28 \\
0.015 \pm 0.007\end{array}$ & flat \\
\hline L7 & $\begin{array}{l}k_{e x} / 10^{3} \mathrm{~s} \\
\phi / \mathrm{ppm}^{2}\end{array}$ & $\begin{array}{c}2.95 \pm 0.76 \\
0.001 \pm 0.000\end{array}$ & 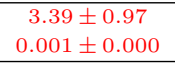 & $\begin{array}{c}2.84 \pm 1.00 \\
0.000 \pm 0.000\end{array}$ & $\begin{array}{l}99.86 \pm 21.14 \\
0.036 \pm 0.011\end{array}$ & $\begin{array}{c}135.57 \pm 35.23 \\
0.043 \pm 0.018\end{array}$ & $\begin{array}{c}158.36 \pm 62.81 \\
0.035 \pm 0.024\end{array}$ & $\begin{array}{c}120.55 \pm 45.09 \\
0.014 \pm 0.008\end{array}$ & flat & flat \\
\hline A8 & $\begin{array}{l}k_{e x} / 10^{3} \mathrm{~s} \\
\phi / \mathrm{ppm}^{2}\end{array}$ & $\begin{array}{c}3.09 \pm 0.78 \\
0.001 \pm 0.000\end{array}$ & $\begin{aligned} 4.20 & \pm 1.20 \\
0.001 & \pm 0.000\end{aligned}$ & $\begin{array}{c}4.78 \pm 2.52 \\
0.000 \pm 0.000\end{array}$ & $\begin{array}{c}140.59 \pm 31.19 \\
0.053 \pm 0.019\end{array}$ & $\begin{array}{c}115.22 \pm 21.48 \\
0.030 \pm 0.008\end{array}$ & $\begin{array}{r}158.73 \pm 56.17 \\
0.037 \pm 0.022\end{array}$ & $\begin{array}{r}104.17 \pm 27.87 \\
0.015 \pm 0.006\end{array}$ & flat & flat \\
\hline A9 & $\begin{array}{l}k_{e x} / 10^{3} \mathrm{~s} \\
\phi / \mathrm{ppm}^{2}\end{array}$ & $\begin{array}{c}191.07 \pm 55.10 \\
0.132 \pm 0.068\end{array}$ & $\begin{array}{c}162.65 \pm 40.35 \\
0.087 \pm 0.037\end{array}$ & $\begin{array}{c}5.39 \pm 2.19 \\
0.001 \pm 0.000\end{array}$ & $\begin{array}{l}96.49 \pm 16.38 \\
0.029 \pm 0.007\end{array}$ & $\begin{array}{l}98.89 \pm 17.60 \\
0.023 \pm 0.006\end{array}$ & $\begin{array}{c}139.75 \pm 35.82 \\
0.027 \pm 0.011\end{array}$ & $\begin{array}{l}93.65 \pm 36.27 \\
0.008 \pm 0.004\end{array}$ & flat & flat \\
\hline A10 & $\begin{array}{l}k_{e x} / 10^{3} \mathrm{~s} \\
\phi / \mathrm{ppm}^{2}\end{array}$ & $\begin{array}{c}106.94 \pm 30.70 \\
0.061 \pm 0.026\end{array}$ & $\begin{array}{l}90.00 \pm 23.31 \\
0.043 \pm 0.015\end{array}$ & 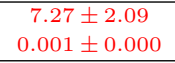 & $\begin{array}{c}122.20 \pm 33.69 \\
0.049 \pm 0.021\end{array}$ & $\begin{array}{c}102.87 \pm 17.54 \\
0.028 \pm 0.007\end{array}$ & $\begin{array}{c}103.74 \pm 12.65 \\
0.021 \pm 0.004\end{array}$ & $\begin{array}{c}103.68 \pm 22.29 \\
0.016 \pm 0.005\end{array}$ & flat & flat \\
\hline R11 & $\begin{array}{l}k_{e x} / 10^{3} \mathrm{~s} \\
\phi / \mathrm{ppm}^{2}\end{array}$ & $\begin{array}{c}2.61 \pm 0.40 \\
0.001 \pm 0.000\end{array}$ & $\begin{array}{l}\text { NaN } \\
\text { NaN }\end{array}$ & $\begin{array}{c}3.60 \pm 0.70 \\
0.001 \pm 0.000\end{array}$ & $\begin{array}{c}5.34 \pm 1.22 \\
0.001 \pm 0.000\end{array}$ & $\begin{array}{c}10.11 \pm 3.72 \\
0.001 \pm 0.000\end{array}$ & $\begin{array}{l}66.91 \pm 10.22 \\
0.012 \pm 0.002 \\
\end{array}$ & $\begin{array}{c}50.22 \pm 7.90 \\
0.006 \pm 0.001\end{array}$ & $\begin{array}{c}21.40 \pm 8.02 \\
0.001 \pm 0.000\end{array}$ & $\begin{array}{c}18.60 \pm 8.98 \\
0.001 \pm 0.000\end{array}$ \\
\hline A12 & $\begin{array}{l}k_{e x} / 10^{3} \mathrm{~s} \\
\phi / \mathrm{ppm}^{2}\end{array}$ & $\begin{array}{c}1.97 \pm 0.71 \\
0.000 \pm 0.000\end{array}$ & $\begin{array}{c}167.77 \pm 51.58 \\
0.087 \pm 0.046\end{array}$ & $\begin{array}{c}176.66 \pm 57.42 \\
0.080 \pm 0.046\end{array}$ & $\begin{array}{c}209.16 \pm 84.65 \\
0.095 \pm 0.070\end{array}$ & $\begin{array}{c}161.97 \pm 45.76 \\
0.051 \pm 0.025\end{array}$ & $\begin{array}{c}131.66 \pm 37.91 \\
0.027 \pm 0.012\end{array}$ & $\begin{array}{c}108.11 \pm 30.19 \\
0.016 \pm 0.007\end{array}$ & $\begin{array}{l}88.44 \pm 43.28 \\
0.005 \pm 0.003\end{array}$ & flat \\
\hline A 13 & $\begin{array}{l}k_{e x} / 10^{3} \mathrm{~s} \\
\phi / \mathrm{ppm}^{2}\end{array}$ & $\begin{array}{l}\text { NaN } \\
\text { NaN }\end{array}$ & $\begin{array}{c}2.70 \pm 0.26 \\
0.003 \pm 0.000\end{array}$ & $\begin{array}{c}3.44 \pm 0.26 \\
0.003 \pm 0.000\end{array}$ & $\begin{array}{c}4.87 \pm 0.59 \\
0.003 \pm 0.000\end{array}$ & $\begin{aligned} 6.28 & \pm 1.25 \\
0.002 & \pm 0.000\end{aligned}$ & $\begin{array}{c}6.74 \pm 2.48 \\
0.001 \pm 0.000\end{array}$ & $\begin{array}{c}55.30 \pm 8.78 \\
0.010 \pm 0.002\end{array}$ & $\begin{array}{c}41.09 \pm 7.46 \\
0.004 \pm 0.001\end{array}$ & $\begin{array}{c}28.12 \pm 6.55 \\
0.002 \pm 0.001\end{array}$ \\
\hline L14 & $\begin{array}{l}k_{e x} / 10^{3} \mathrm{~s} \\
\phi / \mathrm{ppm}^{2}\end{array}$ & 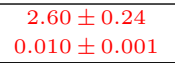 & $\begin{aligned} 5.50 & \pm 0.57 \\
0.014 & \pm 0.001\end{aligned}$ & $\begin{aligned} 4.73 & \pm 0.16 \\
0.009 & \pm 0.000\end{aligned}$ & 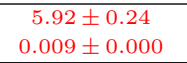 & $\begin{aligned} 7.73 & \pm 0.43 \\
0.006 & \pm 0.000\end{aligned}$ & $\begin{array}{c}11.30 \pm 0.63 \\
0.005 \pm 0.000\end{array}$ & $\begin{array}{c}18.84 \pm 3.96 \\
0.004 \pm 0.001\end{array}$ & $\begin{array}{c}36.24 \pm 2.28 \\
0.007 \pm 0.000\end{array}$ & $\begin{array}{c}41.38 \pm 5.98 \\
0.005 \pm 0.001\end{array}$ \\
\hline H15 & $\begin{array}{l}k_{e x} / 10^{3} \mathrm{~s} \\
\phi / \mathrm{ppm}^{2}\end{array}$ & $\begin{array}{l}\text { NaN } \\
\text { NaN }\end{array}$ & $\begin{array}{c}183.49 \pm 42.02 \\
0.096 \pm 0.039\end{array}$ & $\begin{array}{c}193.50 \pm 54.52 \\
0.086 \pm 0.043\end{array}$ & $\begin{array}{c}152.40 \pm 28.05 \\
0.051 \pm 0.016\end{array}$ & $\begin{array}{c}144.55 \pm 28.66 \\
0.037 \pm 0.012\end{array}$ & $\begin{array}{c}166.82 \pm 46.82 \\
0.036 \pm 0.017\end{array}$ & $\begin{array}{c}117.01 \pm 39.29 \\
0.012 \pm 0.006\end{array}$ & $\begin{array}{l}45.02 \pm 24.64 \\
0.001 \pm 0.001\end{array}$ & flat \\
\hline D16 & $\begin{array}{l}k_{e x} / 10^{3} \mathrm{~s} \\
\phi / \mathrm{ppm}^{2}\end{array}$ & $\begin{array}{c}2.15 \pm 0.20 \\
0.001 \pm 0.000\end{array}$ & $\begin{array}{l}\text { NaN } \\
\text { NaN }\end{array}$ & $\begin{array}{c}3.24 \pm 0.53 \\
0.001 \pm 0.000\end{array}$ & $\begin{array}{c}3.68 \pm 0.61 \\
0.001 \pm 0.000\end{array}$ & $\begin{array}{c}7.79 \pm 1.77 \\
0.001 \pm 0.000\end{array}$ & $\begin{array}{l}62.49 \pm 10.49 \\
0.012 \pm 0.002\end{array}$ & $\begin{array}{c}44.22 \pm 8.58 \\
0.005 \pm 0.001\end{array}$ & flat & flat \\
\hline L17 & $\begin{array}{l}k_{e x} / 10^{3} \mathrm{~s} \\
\phi / \mathrm{ppm}^{2}\end{array}$ & $\begin{array}{c}2.21 \pm 0.27 \\
0.002 \pm 0.000\end{array}$ & $\begin{array}{c}2.72 \pm 0.16 \\
0.002 \pm 0.000\end{array}$ & $\begin{array}{c}3.75 \pm 0.29 \\
0.002 \pm 0.000\end{array}$ & $\begin{array}{c}4.37 \pm 0.37 \\
0.002 \pm 0.000\end{array}$ & $\begin{array}{c}6.11 \pm 0.65 \\
0.002 \pm 0.000\end{array}$ & $\begin{array}{c}8.93 \pm 1.14 \\
0.002 \pm 0.000\end{array}$ & $\begin{array}{c}11.72 \pm 3.10 \\
0.001 \pm 0.000\end{array}$ & $\begin{array}{c}24.99 \pm 4.13 \\
0.003 \pm 0.000\end{array}$ & $\begin{array}{c}26.66 \pm 6.34 \\
0.003 \pm 0.001\end{array}$ \\
\hline M18 & $\begin{array}{l}k_{e x} / 10^{3} \mathrm{~s} \\
\phi / \mathrm{ppm}^{2}\end{array}$ & 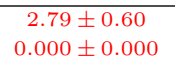 & $\begin{array}{c}4.40 \pm 1.53 \\
0.000 \pm 0.000\end{array}$ & $\begin{array}{c}117.11 \pm 16.35 \\
0.040 \pm 0.008\end{array}$ & $\begin{array}{c}128.88 \pm 22.24 \\
0.042 \pm 0.011\end{array}$ & $\begin{array}{c}138.76 \pm 21.45 \\
0.036 \pm 0.009\end{array}$ & $\begin{array}{c}116.95 \pm 22.34 \\
0.023 \pm 0.007\end{array}$ & $\begin{array}{c}115.36 \pm 26.03 \\
0.014 \pm 0.005\end{array}$ & flat & flat \\
\hline T19 & $\begin{array}{l}k_{e x} / 10^{3} \mathrm{~s} \\
\phi / \mathrm{ppm}^{2}\end{array}$ & $\begin{array}{c}1.82 \pm 0.25 \\
0.002 \pm 0.000\end{array}$ & $\begin{aligned} 2.63 & \pm 0.30 \\
0.002 & \pm 0.000\end{aligned}$ & $\begin{array}{c}2.72 \pm 0.49 \\
0.002 \pm 0.000\end{array}$ & $\begin{array}{c}3.14 \pm 0.70 \\
0.001 \pm 0.000\end{array}$ & $\begin{array}{c}46.84 \pm 9.71 \\
0.014 \pm 0.003\end{array}$ & $\begin{array}{l}59.65 \pm 12.84 \\
0.014 \pm 0.004\end{array}$ & $\begin{array}{l}63.61 \pm 18.11 \\
0.010 \pm 0.004\end{array}$ & flat & flat \\
\hline G20 & $\begin{array}{l}k_{e x} / 10^{3} \mathrm{~s} \\
\phi / \mathrm{ppm}^{2}\end{array}$ & $\begin{array}{c}1.77 \pm 0.35 \\
0.004 \pm 0.000\end{array}$ & $\begin{array}{c}2.01 \pm 0.36 \\
0.004 \pm 0.000\end{array}$ & $\begin{array}{c}3.14 \pm 0.30 \\
0.003 \pm 0.000\end{array}$ & $\begin{array}{c}4.21 \pm 0.38 \\
0.003 \pm 0.000\end{array}$ & $\begin{array}{c}5.81 \pm 0.92 \\
0.003 \pm 0.000\end{array}$ & $\begin{array}{c}15.77 \pm 2.67 \\
0.006 \pm 0.001\end{array}$ & $\begin{array}{c}22.64 \pm 3.80 \\
0.006 \pm 0.001\end{array}$ & $\begin{array}{c}19.56 \pm 3.77 \\
0.004 \pm 0.001\end{array}$ & $\begin{array}{c}20.31 \pm 4.82 \\
0.004 \pm 0.001\end{array}$ \\
\hline
\end{tabular}




\begin{tabular}{|c|c|c|c|c|c|c|c|c|c|c|}
\hline Residue & Parameter & $263 \mathrm{~K}$ & $265 \mathrm{~K}$ & $268 \mathrm{~K}$ & $270 \mathrm{~K}$ & $275 \mathrm{~K}$ & $280 \mathrm{~K}$ & $285 \mathrm{~K}$ & $290 \mathrm{~K}$ & $295 \mathrm{~K}$ \\
\hline K21 & $\begin{array}{l}k_{e x} / 10^{3} \mathrm{~s} \\
\phi / \mathrm{ppm}^{2}\end{array}$ & $\begin{array}{c}132.98 \pm 21.74 \\
0.065 \pm 0.017\end{array}$ & $\begin{array}{c}136.73 \pm 23.36 \\
0.057 \pm 0.016\end{array}$ & $\begin{array}{c}181.12 \pm 47.55 \\
0.076 \pm 0.035\end{array}$ & $\begin{array}{c}144.80 \pm 30.40 \\
0.046 \pm 0.016\end{array}$ & $\begin{array}{c}200.60 \pm 93.91 \\
0.062 \pm 0.053\end{array}$ & $\begin{array}{c}148.10 \pm 42.46 \\
0.030 \pm 0.014\end{array}$ & $\begin{array}{l}84.55 \pm 26.75 \\
0.009 \pm 0.004\end{array}$ & flat & flat \\
\hline $\mathrm{R} 22$ & $\begin{array}{l}k_{e x} / 10^{3} \mathrm{~s} \\
\phi / \mathrm{ppm}^{2}\end{array}$ & $\begin{array}{c}2.26 \pm 0.13 \\
0.007 \pm 0.000\end{array}$ & $\begin{array}{c}2.77 \pm 0.12 \\
0.007 \pm 0.000\end{array}$ & $\begin{array}{c}3.71 \pm 0.11 \\
0.007 \pm 0.000\end{array}$ & $\begin{array}{c}4.45 \pm 0.14 \\
0.007 \pm 0.000\end{array}$ & $\begin{array}{c}7.38 \pm 0.39 \\
0.006 \pm 0.000\end{array}$ & $\begin{array}{c}10.19 \pm 0.95 \\
0.005 \pm 0.001\end{array}$ & $\begin{array}{c}20.71 \pm 1.46 \\
0.009 \pm 0.001\end{array}$ & $\begin{array}{c}22.98 \pm 1.77 \\
0.006 \pm 0.000\end{array}$ & $\begin{array}{c}21.03 \pm 2.64 \\
0.005 \pm 0.001\end{array}$ \\
\hline V23 & $\begin{array}{l}k_{e x} / 10^{3} \mathrm{~s} \\
\phi / \mathrm{ppm}^{2}\end{array}$ & $\begin{array}{c}10.46 \pm 1.02 \\
0.033 \pm 0.003\end{array}$ & $\begin{aligned} 5.12 & \pm 0.00 \\
0.005 & \pm 0.000\end{aligned}$ & $\begin{aligned} 5.37 & \pm 0.49 \\
0.019 & \pm 0.002\end{aligned}$ & $\begin{aligned} 5.99 & \pm 0.37 \\
0.019 & \pm 0.001\end{aligned}$ & $\begin{aligned} 7.48 & \pm 0.40 \\
0.014 & \pm 0.001\end{aligned}$ & $\begin{array}{c}11.11 \pm 0.43 \\
0.014 \pm 0.001\end{array}$ & $\begin{array}{c}14.85 \pm 0.76 \\
0.013 \pm 0.001\end{array}$ & $\begin{array}{c}17.03 \pm 1.48 \\
0.010 \pm 0.002\end{array}$ & $\begin{array}{c}27.74 \pm 1.05 \\
0.018 \pm 0.001\end{array}$ \\
\hline A 24 & $\begin{array}{l}k_{e x} / 10^{3} \mathrm{~s} \\
\phi / \mathrm{ppm}^{2}\end{array}$ & $\begin{array}{c}2.28 \pm 0.26 \\
0.003 \pm 0.000\end{array}$ & $\begin{array}{c}2.58 \pm 0.29 \\
0.003 \pm 0.000\end{array}$ & $\begin{array}{c}3.42 \pm 0.30 \\
0.003 \pm 0.000\end{array}$ & $\begin{array}{c}3.81 \pm 0.44 \\
0.003 \pm 0.000\end{array}$ & $\begin{array}{c}5.93 \pm 1.08 \\
0.002 \pm 0.000\end{array}$ & $\begin{array}{c}18.52 \pm 3.01 \\
0.006 \pm 0.001\end{array}$ & $\begin{array}{c}21.96 \pm 3.71 \\
0.005 \pm 0.001\end{array}$ & $\begin{array}{c}22.16 \pm 5.44 \\
0.003 \pm 0.001\end{array}$ & $\begin{array}{c}21.33 \pm 6.58 \\
0.003 \pm 0.001\end{array}$ \\
\hline T25 & $\begin{array}{l}k_{e x} / 10^{3} \mathrm{~s} \\
\phi / \mathrm{ppm}^{2}\end{array}$ & $\begin{array}{c}2.20 \pm 0.07 \\
0.003 \pm 0.000\end{array}$ & $\begin{array}{c}2.80 \pm 0.14 \\
0.003 \pm 0.000\end{array}$ & $\begin{array}{c}3.60 \pm 0.20 \\
0.003 \pm 0.000\end{array}$ & $\begin{array}{c}4.29 \pm 0.30 \\
0.003 \pm 0.000\end{array}$ & $\begin{array}{c}10.89 \pm 1.45 \\
0.004 \pm 0.001\end{array}$ & $\begin{array}{c}16.17 \pm 1.96 \\
0.004 \pm 0.000\end{array}$ & $\begin{array}{c}18.84 \pm 2.15 \\
0.003 \pm 0.000\end{array}$ & $\begin{array}{c}16.23 \pm 3.40 \\
0.002 \pm 0.000\end{array}$ & $\begin{array}{c}13.07 \pm 4.20 \\
0.001 \pm 0.000\end{array}$ \\
\hline V26 & $\begin{array}{l}k_{e x} / 10^{3} \mathrm{~s} \\
\phi / \mathrm{ppm}^{2}\end{array}$ & $\begin{array}{c}2.24 \pm 0.12 \\
0.007 \pm 0.000\end{array}$ & $\begin{array}{c}1.92 \pm 0.00 \\
0.004 \pm 0.000\end{array}$ & $\begin{array}{c}3.21 \pm 0.11 \\
0.006 \pm 0.000\end{array}$ & $\begin{array}{c}4.35 \pm 0.13 \\
0.007 \pm 0.000\end{array}$ & $\begin{array}{c}6.90 \pm 0.24 \\
0.007 \pm 0.000\end{array}$ & $\begin{array}{c}10.23 \pm 0.45 \\
0.007 \pm 0.000\end{array}$ & $\begin{array}{c}17.39 \pm 0.84 \\
0.009 \pm 0.000\end{array}$ & $\begin{array}{c}20.90 \pm 0.91 \\
0.008 \pm 0.000\end{array}$ & $\begin{array}{c}23.79 \pm 1.47 \\
0.009 \pm 0.001\end{array}$ \\
\hline Q27 & $\begin{array}{l}k_{e x} / 10^{3} \mathrm{~s} \\
\phi / \mathrm{ppm}^{2}\end{array}$ & $\begin{array}{c}1.69 \pm 0.44 \\
0.000 \pm 0.000\end{array}$ & $\begin{array}{c}2.53 \pm 0.50 \\
0.000 \pm 0.000\end{array}$ & $\begin{array}{l}90.47 \pm 13.74 \\
0.024 \pm 0.005\end{array}$ & $\begin{array}{c}110.94 \pm 21.61 \\
0.027 \pm 0.008 \\
\end{array}$ & $\begin{array}{c}105.51 \pm 22.66 \\
0.019 \pm 0.006\end{array}$ & $\begin{array}{l}89.54 \pm 23.19 \\
0.011 \pm 0.004\end{array}$ & $\begin{array}{l}50.88 \pm 20.77 \\
0.003 \pm 0.001 \\
\end{array}$ & flat & flat \\
\hline K28 & $\begin{array}{l}k_{e x} / 10^{3} \mathrm{~s} \\
\phi / \mathrm{ppm}^{2}\end{array}$ & $\begin{array}{c}5.01 \pm 0.72 \\
0.013 \pm 0.001\end{array}$ & $\begin{array}{c}2.69 \pm 0.25 \\
0.007 \pm 0.000\end{array}$ & $\begin{array}{c}4.05 \pm 0.28 \\
0.007 \pm 0.000\end{array}$ & $\begin{array}{c}4.52 \pm 0.25 \\
0.007 \pm 0.000\end{array}$ & $\begin{array}{c}6.78 \pm 0.66 \\
0.006 \pm 0.001\end{array}$ & $\begin{array}{c}9.71 \pm 1.63 \\
0.005 \pm 0.001\end{array}$ & $\begin{array}{c}22.86 \pm 2.92 \\
0.008 \pm 0.001\end{array}$ & $\begin{array}{c}25.66 \pm 4.34 \\
0.006 \pm 0.001\end{array}$ & $\begin{array}{r}23.85 \pm 5.16 \\
0.005 \pm 0.001\end{array}$ \\
\hline D29 & $\begin{array}{l}k_{e x} / 10^{3} \mathrm{~s} \\
\phi / \mathrm{ppm}^{2}\end{array}$ & $\begin{array}{c}5.42 \pm 0.45 \\
0.032 \pm 0.002\end{array}$ & $\begin{array}{c}8.54 \pm 0.52 \\
0.039 \pm 0.002\end{array}$ & $\begin{array}{c}7.40 \pm 0.57 \\
0.041 \pm 0.003\end{array}$ & $\begin{array}{c}7.36 \pm 0.28 \\
0.041 \pm 0.001\end{array}$ & $\begin{array}{c}9.04 \pm 0.29 \\
0.041 \pm 0.001\end{array}$ & $\begin{array}{c}11.28 \pm 0.57 \\
0.034 \pm 0.003\end{array}$ & $\begin{array}{c}16.54 \pm 0.71 \\
0.037 \pm 0.001\end{array}$ & $\begin{array}{c}21.59 \pm 0.84 \\
0.035 \pm 0.001\end{array}$ & $\begin{array}{c}26.47 \pm 1.08 \\
0.035 \pm 0.001\end{array}$ \\
\hline G30 & $\begin{array}{l}k_{e x} / 10^{3} \mathrm{~s} \\
\phi / \mathrm{ppm}^{2}\end{array}$ & $\begin{array}{c}2.06 \pm 0.24 \\
0.007 \pm 0.000\end{array}$ & $\begin{array}{c}2.86 \pm 0.24 \\
0.006 \pm 0.000\end{array}$ & $\begin{aligned} 4.76 & \pm 0.58 \\
0.008 & \pm 0.001\end{aligned}$ & $\begin{aligned} 5.98 & \pm 0.78 \\
0.007 & \pm 0.001\end{aligned}$ & $\begin{aligned} 9.13 & \pm 1.35 \\
0.007 & \pm 0.001\end{aligned}$ & $\begin{array}{c}14.81 \pm 2.65 \\
0.007 \pm 0.001\end{array}$ & $\begin{array}{c}18.43 \pm 4.28 \\
0.005 \pm 0.001\end{array}$ & flat & flat \\
\hline R32 & $\begin{array}{l}k_{e x} / 10^{3} \mathrm{~s} \\
\phi / \mathrm{ppm}^{2}\end{array}$ & $\begin{array}{c}4.02 \pm 1.11 \\
0.000 \pm 0.000\end{array}$ & $\begin{array}{l}90.65 \pm 17.03 \\
0.020 \pm 0.005\end{array}$ & $\begin{array}{l}80.54 \pm 12.96 \\
0.014 \pm 0.003\end{array}$ & $\begin{array}{c}58.53 \pm 8.97 \\
0.009 \pm 0.002\end{array}$ & $\begin{array}{l}73.06 \pm 14.64 \\
0.008 \pm 0.002\end{array}$ & $\begin{array}{l}39.61 \pm 10.78 \\
0.003 \pm 0.001\end{array}$ & flat & flat & flat \\
\hline V33 & $\begin{array}{l}k_{e x} / 10^{3} \mathrm{~s} \\
\phi / \mathrm{ppm}^{2}\end{array}$ & $\begin{array}{l}\mathrm{NaN} \\
\mathrm{NaN}\end{array}$ & $\begin{array}{c}3.68 \pm 0.20 \\
0.012 \pm 0.001\end{array}$ & $\begin{array}{c}5.16 \pm 0.37 \\
0.016 \pm 0.001\end{array}$ & $\begin{array}{c}5.75 \pm 0.29 \\
0.016 \pm 0.001\end{array}$ & $\begin{array}{c}7.36 \pm 0.36 \\
0.013 \pm 0.001\end{array}$ & $\begin{array}{c}12.39 \pm 0.61 \\
0.016 \pm 0.001\end{array}$ & $\begin{array}{c}16.86 \pm 0.96 \\
0.015 \pm 0.001\end{array}$ & $\begin{array}{c}22.71 \pm 1.87 \\
0.015 \pm 0.001\end{array}$ & $\begin{array}{c}24.77 \pm 2.42 \\
0.014 \pm 0.001\end{array}$ \\
\hline E34 & $\begin{array}{l}k_{e x} / 10^{3} \mathrm{~s} \\
\phi / \mathrm{ppm}^{2}\end{array}$ & $\begin{array}{c}2.41 \pm 0.10 \\
0.006 \pm 0.000\end{array}$ & $\begin{array}{c}2.74 \pm 0.08 \\
0.005 \pm 0.000\end{array}$ & $\begin{array}{c}3.63 \pm 0.08 \\
0.005 \pm 0.000\end{array}$ & $\begin{array}{c}4.29 \pm 0.07 \\
0.005 \pm 0.000\end{array}$ & $\begin{array}{c}6.36 \pm 0.29 \\
0.004 \pm 0.000\end{array}$ & $\begin{array}{c}8.91 \pm 0.96 \\
0.003 \pm 0.000\end{array}$ & $\begin{array}{c}21.45 \pm 1.70 \\
0.006 \pm 0.000\end{array}$ & $\begin{array}{c}24.74 \pm 3.11 \\
0.003 \pm 0.000\end{array}$ & $\begin{array}{c}18.25 \pm 4.76 \\
0.002 \pm 0.001\end{array}$ \\
\hline F35 & $\begin{array}{l}k_{e x} / 10^{3} \mathrm{~s} \\
\phi / \mathrm{ppm}^{2}\end{array}$ & $\begin{array}{c}5.10 \pm 0.36 \\
0.024 \pm 0.001\end{array}$ & $\begin{array}{c}4.80 \pm 0.24 \\
0.024 \pm 0.001\end{array}$ & $\begin{array}{c}5.20 \pm 0.27 \\
0.023 \pm 0.001\end{array}$ & $\begin{array}{c}5.60 \pm 0.15 \\
0.025 \pm 0.001\end{array}$ & $\begin{array}{c}8.26 \pm 0.22 \\
0.023 \pm 0.001\end{array}$ & $\begin{array}{c}12.13 \pm 0.37 \\
0.023 \pm 0.001\end{array}$ & $\begin{array}{c}16.53 \pm 0.50 \\
0.022 \pm 0.001\end{array}$ & $\begin{array}{c}21.49 \pm 0.60 \\
0.021 \pm 0.001\end{array}$ & $\begin{array}{r}24.99 \pm 1.09 \\
0.020 \pm 0.001\end{array}$ \\
\hline T36 & $\begin{array}{l}k_{e x} / 10^{3} \mathrm{~s} \\
\phi / \mathrm{ppm}^{2}\end{array}$ & $\begin{array}{c}78.76 \pm 9.14 \\
0.039 \pm 0.006\end{array}$ & $\begin{array}{c}101.54 \pm 17.63 \\
0.044 \pm 0.011\end{array}$ & $\begin{array}{c}108.56 \pm 17.63 \\
0.038 \pm 0.009\end{array}$ & $\begin{array}{c}134.55 \pm 26.22 \\
0.045 \pm 0.014\end{array}$ & $\begin{array}{r}158.00 \pm 39.07 \\
0.042 \pm 0.018\end{array}$ & $\begin{array}{l}\text { NaN } \\
\text { NaN }\end{array}$ & $\begin{array}{l}71.12 \pm 34.85 \\
0.006 \pm 0.004\end{array}$ & 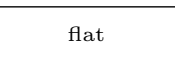 & 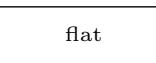 \\
\hline A37 & $\begin{array}{l}k_{e x} / 10^{3} \mathrm{~s} \\
\phi / \mathrm{ppm}^{2}\end{array}$ & $\begin{array}{c}6.14 \pm 0.35 \\
0.028 \pm 0.001\end{array}$ & $\begin{array}{c}5.29 \pm 0.26 \\
0.028 \pm 0.001\end{array}$ & $\begin{array}{c}6.38 \pm 0.34 \\
0.028 \pm 0.001\end{array}$ & $\begin{array}{c}6.14 \pm 0.32 \\
0.023 \pm 0.001\end{array}$ & $\begin{aligned} 7.53 & \pm 0.23 \\
0.018 & \pm 0.001\end{aligned}$ & $\begin{array}{c}10.65 \pm 0.32 \\
0.016 \pm 0.001\end{array}$ & $\begin{array}{c}14.35 \pm 0.96 \\
0.014 \pm 0.002\end{array}$ & $\begin{array}{c}23.12 \pm 0.83 \\
0.018 \pm 0.001\end{array}$ & $\begin{array}{c}25.08 \pm 1.08 \\
0.015 \pm 0.001\end{array}$ \\
\hline T38 & $\begin{array}{l}k_{e x} / 10^{3} \mathrm{~s} \\
\phi / \mathrm{ppm}^{2}\end{array}$ & $\begin{array}{c}1.72 \pm 0.18 \\
0.003 \pm 0.000\end{array}$ & $\begin{array}{c}2.36 \pm 0.23 \\
0.003 \pm 0.000 \\
\end{array}$ & $\begin{array}{c}3.05 \pm 0.32 \\
0.002 \pm 0.000\end{array}$ & $\begin{array}{c}3.28 \pm 0.40 \\
0.002 \pm 0.000\end{array}$ & $\begin{array}{c}14.32 \pm 3.36 \\
0.005 \pm 0.001\end{array}$ & $\begin{array}{c}33.87 \pm 8.66 \\
0.007 \pm 0.002\end{array}$ & $\begin{array}{c}30.32 \pm 8.86 \\
0.004 \pm 0.001\end{array}$ & $0.010+0.001$ & 0.0510 .001 \\
\hline S39 & $\begin{array}{l}k_{e x} / 10^{3} \mathrm{~s} \\
\phi / \mathrm{ppm}^{2}\end{array}$ & $\begin{array}{c}4.25 \pm 0.45 \\
0.004 \pm 0.000\end{array}$ & $\begin{array}{c}3.06 \pm 0.50 \\
0.002 \pm 0.000\end{array}$ & $\begin{array}{c}.09 \pm 0.55 \\
0.002 \pm 0.000\end{array}$ & $\begin{array}{l}\text { NaN } \\
\text { NaN }\end{array}$ & $\begin{array}{c}5.80 \pm 1.59 \\
0.001 \pm 0.000\end{array}$ & $\begin{array}{l}66.58 \pm 11.13 \\
0.015 \pm 0.003\end{array}$ & $\begin{array}{c}44.93 \pm 8.84 \\
0.005 \pm 0.001\end{array}$ & $\begin{array}{c}32.86 \pm 9.40 \\
0.002 \pm 0.001\end{array}$ & $\begin{array}{c}19.09 \pm 9.62 \\
0.001 \pm 0.000\end{array}$ \\
\hline V40 & $\begin{array}{l}k_{e x} / 10^{3} \mathrm{~s} \\
\phi / \mathrm{ppm}^{2}\end{array}$ & $\begin{array}{c}8.90 \pm 0.51 \\
0.043 \pm 0.002\end{array}$ & $\begin{array}{c}9.84 \pm 0.79 \\
0.042 \pm 0.003\end{array}$ & $\begin{array}{c}9.76 \pm 0.63 \\
0.043 \pm 0.002\end{array}$ & $\begin{array}{c}11.81 \pm 0.96 \\
0.049 \pm 0.004\end{array}$ & $\begin{array}{c}10.66 \pm 0.57 \\
0.049 \pm 0.002\end{array}$ & $\begin{array}{c}11.42 \pm 0.38 \\
0.036 \pm 0.002\end{array}$ & $\begin{array}{c}16.16 \pm 0.28 \\
0.037 \pm 0.001\end{array}$ & $\begin{array}{c}22.43 \pm 0.56 \\
0.038 \pm 0.001\end{array}$ & $\begin{array}{r}27.14 \pm 0.69 \\
0.035 \pm 0.001\end{array}$ \\
\hline S41 & $\begin{array}{l}k_{e x} / 10^{3} \mathrm{~s} \\
\phi / \mathrm{ppm}^{2}\end{array}$ & $\begin{array}{c}5.01 \pm 0.46 \\
0.022 \pm 0.002\end{array}$ & $\begin{array}{c}4.47 \pm 0.16 \\
0.020 \pm 0.001\end{array}$ & $\begin{array}{c}4.73 \pm 0.20 \\
0.019 \pm 0.001\end{array}$ & $\begin{array}{c}5.40 \pm 0.20 \\
0.018 \pm 0.001\end{array}$ & $\begin{array}{c}7.67 \pm 0.14 \\
0.016 \pm 0.000\end{array}$ & $\begin{array}{c}10.64 \pm 0.41 \\
0.013 \pm 0.001\end{array}$ & $\begin{array}{c}14.50 \pm 0.92 \\
0.011 \pm 0.001\end{array}$ & $\begin{array}{c}21.37 \pm 0.71 \\
0.013 \pm 0.000\end{array}$ & $\begin{array}{c}22.75 \pm 1.37 \\
0.011 \pm 0.001\end{array}$ \\
\hline D 42 & $\begin{array}{l}k_{e x} / 10^{3} \mathrm{~s} \\
\phi / \mathrm{ppm}^{2}\end{array}$ & $\begin{array}{c}4.08 \pm 0.36 \\
0.013 \pm 0.001\end{array}$ & $\begin{array}{c}3.22 \pm 0.22 \\
0.009 \pm 0.001\end{array}$ & $\begin{aligned} 6.55 & \pm 0.44 \\
0.018 & \pm 0.001\end{aligned}$ & $\begin{aligned} 6.07 & \pm 0.20 \\
0.016 & \pm 0.001\end{aligned}$ & $\begin{aligned} 7.57 & \pm 0.29 \\
0.012 & \pm 0.001\end{aligned}$ & $\begin{array}{c}14.01 \pm 0.91 \\
0.013 \pm 0.001\end{array}$ & $\begin{array}{c}18.92 \pm 1.11 \\
0.011 \pm 0.001\end{array}$ & $\begin{array}{c}20.77 \pm 1.52 \\
0.006 \pm 0.000\end{array}$ & $\begin{array}{c}20.11 \pm 2.78 \\
0.004 \pm 0.001\end{array}$ \\
\hline L43 & $\begin{array}{l}k_{e x} / 10^{3} \mathrm{~s} \\
\phi / \mathrm{ppm}^{2}\end{array}$ & $\begin{array}{c}3.09 \pm 0.34 \\
0.014 \pm 0.001\end{array}$ & $\begin{array}{c}5.15 \pm 0.48 \\
0.014 \pm 0.001\end{array}$ & $\begin{aligned} 3.22 & \pm 0.17 \\
0.010 & \pm 0.000\end{aligned}$ & $\begin{aligned} 5.30 & \pm 0.53 \\
0.010 & \pm 0.001\end{aligned}$ & $\begin{aligned} 3.76 & \pm 0.00 \\
0.001 & \pm 0.000\end{aligned}$ & $\begin{array}{l}\text { NaN } \\
\text { NaN }\end{array}$ & $\begin{array}{c}19.58 \pm 1.24 \\
0.011 \pm 0.001\end{array}$ & $\begin{array}{c}23.40 \pm 1.21 \\
0.009 \pm 0.000\end{array}$ & $\begin{array}{r}25.87 \pm 2.17 \\
0.007 \pm 0.001\end{array}$ \\
\hline K44 & $\begin{array}{l}k_{e x} / 10^{3} \mathrm{~s} \\
\phi / \mathrm{ppm}^{2}\end{array}$ & $\begin{array}{l}\text { NaN } \\
\text { NaN }\end{array}$ & $\begin{array}{c}4.38 \pm 0.50 \\
0.002 \pm 0.000\end{array}$ & $\begin{array}{c}5.55 \pm 0.94 \\
0.001 \pm 0.000\end{array}$ & $\begin{array}{c}50.91 \pm 8.36 \\
0.016 \pm 0.003\end{array}$ & $\begin{array}{l}78.80 \pm 12.10 \\
0.020 \pm 0.004\end{array}$ & $\begin{array}{l}79.18 \pm 11.48 \\
0.016 \pm 0.003\end{array}$ & $\begin{array}{l}73.56 \pm 13.03 \\
0.010 \pm 0.002\end{array}$ & $\begin{array}{l}41.29 \pm 16.17 \\
0.002 \pm 0.001\end{array}$ & $\begin{array}{l}23.30 \pm 17.54 \\
0.001 \pm 0.001\end{array}$ \\
\hline
\end{tabular}




\begin{tabular}{|c|c|c|c|c|c|c|c|c|c|c|}
\hline Residue & Parameter & $263 \mathrm{~K}$ & $265 \mathrm{~K}$ & $268 \mathrm{~K}$ & $270 \mathrm{~K}$ & $275 \mathrm{~K}$ & $280 \mathrm{~K}$ & $285 \mathrm{~K}$ & $290 \mathrm{~K}$ & $295 \mathrm{~K}$ \\
\hline K45 & $\begin{array}{l}k_{e x} / 10^{3} \mathrm{~s} \\
\phi / \mathrm{ppm}^{2}\end{array}$ & $\begin{array}{c}170.36 \pm 52.21 \\
0.110 \pm 0.058\end{array}$ & $\begin{array}{c}5.40 \pm 1.98 \\
0.001 \pm 0.000\end{array}$ & $\begin{aligned} 129.40 & \pm 24.56 \\
0.050 & \pm 0.015\end{aligned}$ & $\begin{array}{c}153.82 \pm 40.82 \\
0.057 \pm 0.025\end{array}$ & $\begin{array}{c}200.06 \pm 87.40 \\
0.068 \pm 0.054\end{array}$ & $\begin{array}{c}156.69 \pm 53.52 \\
0.035 \pm 0.020\end{array}$ & $\begin{array}{c}140.72 \pm 61.85 \\
0.019 \pm 0.014\end{array}$ & flat & flat \\
\hline Y46 & $\begin{array}{l}k_{e x} / 10^{3} \mathrm{~s} \\
\phi / \mathrm{ppm}^{2}\end{array}$ & $\begin{array}{c}2.75 \pm 0.26 \\
0.007 \pm 0.000\end{array}$ & $\begin{array}{c}3.29 \pm 0.27 \\
0.006 \pm 0.000\end{array}$ & $\begin{array}{c}4.05 \pm 0.25 \\
0.006 \pm 0.000\end{array}$ & $\begin{array}{c}4.55 \pm 0.24 \\
0.005 \pm 0.000\end{array}$ & $\begin{array}{c}6.60 \pm 0.57 \\
0.004 \pm 0.000\end{array}$ & $\begin{array}{l}\text { NaN } \\
\text { NaN }\end{array}$ & $\begin{array}{c}42.21 \pm 5.63 \\
0.014 \pm 0.002\end{array}$ & $\begin{array}{c}40.45 \pm 4.29 \\
0.010 \pm 0.001\end{array}$ & $\begin{array}{c}20.11 \pm 2.78 \\
0.004 \pm 0.001\end{array}$ \\
\hline I 47 & $\begin{array}{l}k_{e x} / 10^{3} \mathrm{~s} \\
\phi / \mathrm{ppm}^{2}\end{array}$ & 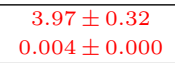 & $\begin{array}{c}4.26 \pm 0.45 \\
0.003 \pm 0.000\end{array}$ & $\begin{array}{c}12.86 \pm 2.30 \\
0.008 \pm 0.001\end{array}$ & $\begin{array}{c}7.18 \pm 0.88 \\
0.003 \pm 0.000\end{array}$ & $\begin{array}{c}47.15 \pm 6.74 \\
0.015 \pm 0.002\end{array}$ & $\begin{array}{c}64.83 \pm 7.59 \\
0.016 \pm 0.002\end{array}$ & $\begin{array}{l}82.15 \pm 10.00 \\
0.014 \pm 0.002\end{array}$ & $\begin{array}{l}55.60 \pm 16.98 \\
0.004 \pm 0.001\end{array}$ & $\begin{array}{c}22.40 \pm 9.87 \\
0.001 \pm 0.000\end{array}$ \\
\hline E49 & $\begin{array}{l}k_{e x} / 10^{3} \mathrm{~s} \\
\phi / \mathrm{ppm}^{2}\end{array}$ & $\begin{array}{c}4.89 \pm 0.79 \\
0.002 \pm 0.000\end{array}$ & $\begin{array}{c}4.48 \pm 1.13 \\
0.001 \pm 0.000\end{array}$ & $\begin{array}{l}68.86 \pm 14.68 \\
0.025 \pm 0.007\end{array}$ & $\begin{array}{c}6.45 \pm 1.67 \\
0.001 \pm 0.000\end{array}$ & $\begin{array}{c}115.35 \pm 24.15 \\
0.031 \pm 0.010\end{array}$ & $\begin{array}{c}157.18 \pm 44.27 \\
0.037 \pm 0.018\end{array}$ & $\begin{array}{l}92.02 \pm 18.38 \\
0.011 \pm 0.003\end{array}$ & flat & $\begin{array}{l}23.31 \pm 12.36 \\
0.001 \pm 0.000\end{array}$ \\
\hline L50 & $\begin{array}{l}k_{e x} / 10^{3} \mathrm{~s} \\
\phi / \mathrm{ppm}^{2}\end{array}$ & $\begin{array}{l}\mathrm{NaN} \\
\mathrm{NaN}\end{array}$ & $\begin{array}{c}190.32 \pm 75.13 \\
0.098 \pm 0.069\end{array}$ & $\begin{array}{c}145.99 \pm 38.70 \\
0.053 \pm 0.023\end{array}$ & $\begin{array}{c}139.91 \pm 33.18 \\
0.045 \pm 0.017\end{array}$ & $\begin{array}{c}123.51 \pm 33.61 \\
0.029 \pm 0.012\end{array}$ & $\begin{array}{c}122.20 \pm 42.20 \\
0.022 \pm 0.012\end{array}$ & $\begin{array}{l}62.89 \pm 19.38 \\
0.006 \pm 0.002\end{array}$ & flat & flat \\
\hline E51 & $\begin{array}{l}k_{e x} / 10^{3} \mathrm{~s} \\
\phi / \mathrm{ppm}^{2}\end{array}$ & $\begin{array}{c}3.24 \pm 1.34 \\
0.001 \pm 0.000\end{array}$ & $\begin{array}{c}110.37 \pm 26.84 \\
0.076 \pm 0.027\end{array}$ & $\begin{aligned} 3.18 & \pm 0.84 \\
0.001 & \pm 0.000\end{aligned}$ & $\begin{array}{c}3.09 \pm 0.95 \\
0.001 \pm 0.000\end{array}$ & $\begin{aligned} 8.60 \pm 2.88 \\
0.001 \pm 0.000\end{aligned}$ & $\begin{array}{l}84.54 \pm 13.67 \\
0.019 \pm 0.004\end{array}$ & $\begin{array}{l}72.79 \pm 14.26 \\
0.011 \pm 0.003\end{array}$ & $\begin{array}{c}23.44 \pm 7.71 \\
0.001 \pm 0.000\end{array}$ & $\begin{array}{c}17.88 \pm 7.39 \\
0.001 \pm 0.000\end{array}$ \\
\hline V52 & $\begin{array}{l}k_{e x} / 10^{3} \mathrm{~s} \\
\phi / \mathrm{ppm}^{2}\end{array}$ & $\begin{array}{c}4.06 \pm 0.38 \\
0.002 \pm 0.000\end{array}$ & $\begin{array}{c}4.56 \pm 0.39 \\
0.002 \pm 0.000\end{array}$ & $\begin{array}{l}\text { NaN } \\
\text { NaN }\end{array}$ & $\begin{array}{c}7.03 \pm 0.89 \\
0.002 \pm 0.000\end{array}$ & $\begin{array}{c}5.33 \pm 1.58 \\
0.001 \pm 0.000\end{array}$ & $\begin{array}{l}86.12 \pm 13.13 \\
0.017 \pm 0.004\end{array}$ & $\begin{array}{c}51.30 \pm 7.51 \\
0.007 \pm 0.001\end{array}$ & $\begin{array}{c}32.41 \pm 9.35 \\
0.002 \pm 0.001\end{array}$ & flat \\
\hline Q53 & $\begin{array}{l}k_{e x} / 10^{3} \mathrm{~s} \\
\phi / \mathrm{ppm}^{2}\end{array}$ & $\begin{array}{c}191.07 \pm 55.10 \\
0.132 \pm 0.068\end{array}$ & $\begin{array}{c}162.65 \pm 40.35 \\
0.087 \pm 0.037\end{array}$ & $\begin{array}{c}5.39 \pm 2.19 \\
0.001 \pm 0.000\end{array}$ & $\begin{array}{l}96.49 \pm 16.38 \\
0.029 \pm 0.007\end{array}$ & $\begin{array}{c}101.52 \pm 17.25 \\
0.024 \pm 0.006\end{array}$ & $\begin{array}{c}105.40 \pm 23.92 \\
0.019 \pm 0.006\end{array}$ & $\begin{array}{l}52.56 \pm 23.81 \\
0.003 \pm 0.002\end{array}$ & flat & flat \\
\hline T54 & $\begin{array}{l}k_{e x} / 10^{3} \mathrm{~s} \\
\phi / \mathrm{ppm}^{2}\end{array}$ & $\begin{array}{c}1.81 \pm 0.41 \\
0.001 \pm 0.000\end{array}$ & $\begin{array}{c}2.43 \pm 0.66 \\
0.001 \pm 0.000\end{array}$ & $\begin{array}{c}2.60 \pm 0.94 \\
0.000 \pm 0.000\end{array}$ & $\begin{array}{c}3.68 \pm 1.71 \\
0.000 \pm 0.000\end{array}$ & $\begin{array}{c}131.29 \pm 26.42 \\
0.031 \pm 0.010\end{array}$ & $\begin{array}{c}125.80 \pm 32.58 \\
0.022 \pm 0.009\end{array}$ & $\begin{array}{l}97.12 \pm 31.50 \\
0.008 \pm 0.004\end{array}$ & flat & flat \\
\hline G55 & $\begin{array}{l}k_{e x} / 10^{3} \mathrm{~s} \\
\phi / \mathrm{ppm}^{2}\end{array}$ & $\begin{array}{c}1.77 \pm 0.22 \\
0.001 \pm 0.000\end{array}$ & $\begin{array}{c}2.38 \pm 0.31 \\
0.001 \pm 0.000\end{array}$ & $\begin{array}{c}2.73 \pm 0.39 \\
0.001 \pm 0.000\end{array}$ & $\begin{array}{c}3.45 \pm 0.65 \\
0.001 \pm 0.000\end{array}$ & $\begin{array}{c}5.17 \pm 1.33 \\
0.001 \pm 0.000\end{array}$ & $\begin{array}{l}63.90 \pm 13.28 \\
0.012 \pm 0.003\end{array}$ & $\begin{array}{c}42.10 \pm 9.27 \\
0.005 \pm 0.001\end{array}$ & $\begin{array}{c}17.89 \pm 8.65 \\
0.001 \pm 0.000\end{array}$ & flat \\
\hline M56 & $\begin{array}{l}k_{e x} / 10^{3} \mathrm{~s} \\
\phi / \mathrm{ppm}^{2}\end{array}$ & $\begin{array}{c}2.21 \pm 1.20 \\
0.000 \pm 0.000\end{array}$ & $\begin{array}{c}146.22 \pm 36.25 \\
0.050 \pm 0.020\end{array}$ & $\begin{array}{c}139.93 \pm 36.08 \\
0.035 \pm 0.015\end{array}$ & $\begin{array}{c}113.85 \pm 24.70 \\
0.023 \pm 0.007\end{array}$ & $\begin{array}{l}95.00 \pm 21.65 \\
0.013 \pm 0.004\end{array}$ & $\begin{array}{l}89.47 \pm 20.95 \\
0.009 \pm 0.003\end{array}$ & $\begin{array}{l}67.48 \pm 23.02 \\
0.004 \pm 0.002\end{array}$ & flat & flat \\
\hline $\mathrm{T} 57$ & $\begin{array}{l}k_{e x} / 10^{3} \mathrm{~s} \\
\phi / \mathrm{ppm}^{2}\end{array}$ & $\begin{array}{c}118.82 \pm 26.01 \\
0.030 \pm 0.010\end{array}$ & $\begin{array}{c}115.84 \pm 27.28 \\
0.025 \pm 0.009\end{array}$ & $\begin{array}{l}90.78 \pm 21.36 \\
0.014 \pm 0.004\end{array}$ & $\begin{array}{l}98.99 \pm 31.94 \\
0.013 \pm 0.006\end{array}$ & $\begin{array}{l}74.73 \pm 28.94 \\
0.006 \pm 0.003\end{array}$ & $\begin{array}{l}46.44 \pm 16.33 \\
0.003 \pm 0.001\end{array}$ & flat & flat & flat \\
\hline R60 & $\begin{array}{l}k_{e x} / 10^{3} \mathrm{~s} \\
\phi / \mathrm{ppm}^{2}\end{array}$ & $\begin{array}{c}100.59 \pm 18.49 \\
0.014 \pm 0.004\end{array}$ & $\begin{array}{l}75.15 \pm 12.60 \\
0.009 \pm 0.002\end{array}$ & $\begin{array}{l}57.94 \pm 11.00 \\
0.005 \pm 0.001\end{array}$ & $\begin{array}{c}46.31 \pm 9.96 \\
0.003 \pm 0.001\end{array}$ & flat & flat & flat & flat & flat \\
\hline R61 & $\begin{array}{l}k_{e x} / 10^{3} \mathrm{~s} \\
\phi / \mathrm{ppm}^{2}\end{array}$ & $\begin{array}{c}20.37 \pm 4.81 \\
0.001 \pm 0.000\end{array}$ & $\begin{array}{c}16.79 \pm 5.03 \\
0.001 \pm 0.000\end{array}$ & $\begin{array}{c}10.60 \pm 2.89 \\
0.001 \pm 0.000\end{array}$ & flat & flat & flat & flat & flat & flat \\
\hline G62 & $\begin{array}{l}k_{e x} / 10^{3} \mathrm{~s} \\
\phi / \mathrm{ppm}^{2}\end{array}$ & $\begin{array}{c}10.26 \pm 4.07 \\
0.000 \pm 0.000\end{array}$ & flat & flat & flat & flat & flat & flat & flat & flat \\
\hline
\end{tabular}


Table A.4: Individual fit parameters of the fast exchange process from backbone ${ }^{1} \mathbf{H}$ E-CPMG data. Values in red correspond to the three-state exchange model parameters $\left(k_{\mathrm{ex}}^{\mathrm{F}}, \phi^{\mathrm{F}}\right)$. Flat profiles and fits with uncertainties larger than the fit parameter are marked as flat and NaN, respectively.

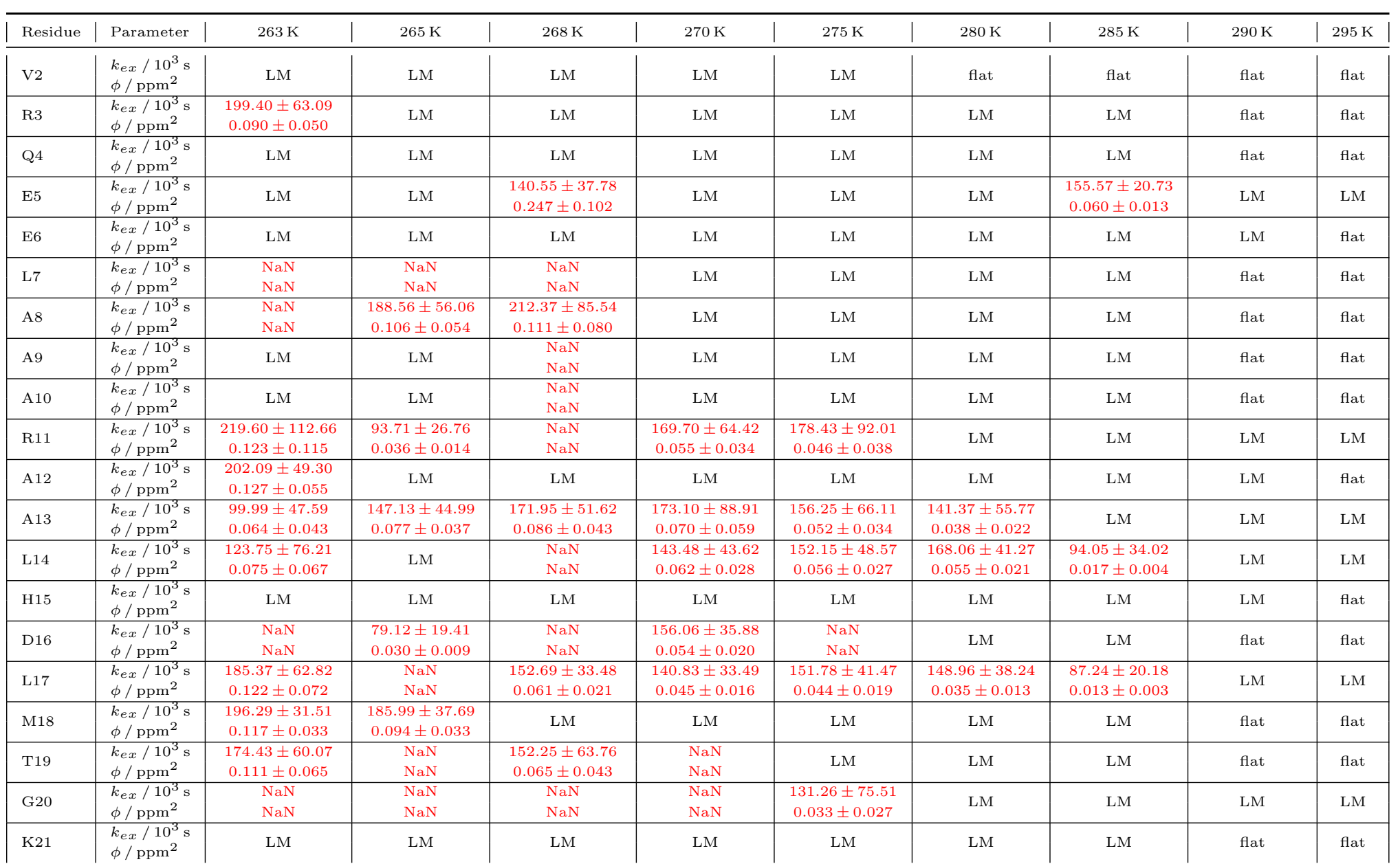




\begin{tabular}{|c|c|c|c|c|c|c|c|c|c|c|}
\hline Residue & Parameter & $263 \mathrm{~K}$ & $265 \mathrm{~K}$ & $268 \mathrm{~K}$ & $270 \mathrm{~K}$ & $275 \mathrm{~K}$ & $280 \mathrm{~K}$ & $285 \mathrm{~K}$ & $290 \mathrm{~K}$ & $295 \mathrm{~K}$ \\
\hline R22 & $\begin{array}{l}k_{e x} / 10^{3} \mathrm{~s} \\
\phi / \mathrm{ppm}^{2}\end{array}$ & $\begin{array}{l}77.61 \pm 12.62 \\
0.044 \pm 0.008\end{array}$ & $\begin{array}{c}108.07 \pm 21.78 \\
0.054 \pm 0.015\end{array}$ & $\begin{array}{c}115.54 \pm 17.71 \\
0.051 \pm 0.011\end{array}$ & $\begin{array}{c}113.94 \pm 17.88 \\
0.044 \pm 0.009\end{array}$ & $\begin{array}{c}131.75 \pm 39.26 \\
0.040 \pm 0.017\end{array}$ & $\begin{array}{l}97.12 \pm 32.34 \\
0.021 \pm 0.007\end{array}$ & LM & LM & LM \\
\hline V23 & $\begin{array}{l}k_{e x} / 10^{3} \mathrm{~s} \\
\phi / \mathrm{ppm}^{2}\end{array}$ & LM & $\begin{array}{c}10.00 \pm 0.00 \\
0.029 \pm 0.000\end{array}$ & $\begin{array}{l}51.25 \pm 13.88 \\
0.047 \pm 0.010 \\
\end{array}$ & $\begin{array}{l}87.70 \pm 24.72 \\
0.069 \pm 0.022\end{array}$ & $\begin{array}{c}67.90 \pm 9.83 \\
0.051 \pm 0.006 \\
\end{array}$ & $\begin{array}{l}93.34 \pm 12.11 \\
0.052 \pm 0.007\end{array}$ & $\begin{array}{l}89.42 \pm 14.63 \\
0.035 \pm 0.005 \\
\end{array}$ & $\begin{array}{r}80.39 \pm 19.08 \\
0.023 \pm 0.003 \\
\end{array}$ & LM \\
\hline A 24 & $\begin{array}{l}k_{e x} / 10^{3} \mathrm{~s} \\
\phi / \mathrm{ppm}^{2}\end{array}$ & $\begin{array}{c}159.97 \pm 73.61 \\
0.074 \pm 0.056\end{array}$ & $\begin{array}{c}120.39 \pm 43.94 \\
0.041 \pm 0.021\end{array}$ & $\begin{array}{c}116.74 \pm 32.92 \\
0.033 \pm 0.013\end{array}$ & $\begin{array}{l}89.17 \pm 25.03 \\
0.022 \pm 0.007\end{array}$ & $\begin{array}{c}102.32 \pm 43.67 \\
0.022 \pm 0.011\end{array}$ & LM & LM & LM & LM \\
\hline $\mathrm{T} 25$ & $\begin{array}{l}k_{e x} / 10^{3} \mathrm{~s} \\
\phi / \mathrm{ppm}^{2}\end{array}$ & $\begin{array}{c}165.81 \pm 29.22 \\
0.067 \pm 0.020\end{array}$ & $\begin{array}{c}168.52 \pm 52.97 \\
0.058 \pm 0.031\end{array}$ & $\begin{array}{c}154.01 \pm 47.65 \\
0.040 \pm 0.020\end{array}$ & $\begin{array}{c}154.58 \pm 60.44 \\
0.036 \pm 0.022\end{array}$ & LM & LM & LM & LM & LM \\
\hline V26 & $\begin{array}{l}k_{e x} / 10^{3} \mathrm{~s} \\
\phi / \mathrm{ppm}^{2}\end{array}$ & $\begin{array}{c}144.67 \pm 58.20 \\
0.065 \pm 0.041\end{array}$ & $\begin{array}{c}10.00 \pm 0.00 \\
0.006 \pm 0.000\end{array}$ & $\begin{array}{c}139.77 \pm 40.23 \\
0.047 \pm 0.021\end{array}$ & $\begin{array}{l}\text { NaN } \\
\text { NaN }\end{array}$ & $\begin{array}{l}\mathrm{NaN} \\
\mathrm{NaN}\end{array}$ & $\begin{array}{c}134.32 \pm 60.28 \\
0.021 \pm 0.013\end{array}$ & LM & LM & LM \\
\hline Q27 & $\begin{array}{l}k_{e x} / 10^{3} \mathrm{~s} \\
\phi / \mathrm{ppm}^{2}\end{array}$ & $\begin{array}{c}187.52 \pm 38.91 \\
0.095 \pm 0.035\end{array}$ & $\begin{array}{c}188.50 \pm 31.65 \\
0.086 \pm 0.025\end{array}$ & LM & LM & LM & LM & LM & flat & flat \\
\hline K28 & $\begin{array}{l}k_{e x} / 10^{3} \mathrm{~s} \\
\phi / \mathrm{ppm}^{2}\end{array}$ & LM & $\begin{array}{l}68.42 \pm 16.39 \\
0.041 \pm 0.011 \\
\end{array}$ & $\begin{array}{c}103.20 \pm 28.44 \\
0.049 \pm 0.018\end{array}$ & $\begin{array}{c}121.24 \pm 34.82 \\
0.052 \pm 0.021 \\
\end{array}$ & $\begin{array}{c}132.10 \pm 65.91 \\
0.043 \pm 0.030\end{array}$ & $\begin{array}{l}98.96 \pm 54.16 \\
0.022 \pm 0.013\end{array}$ & LM & LM & LM \\
\hline D29 & $\begin{array}{l}k_{e x} / 10^{3} \mathrm{~s} \\
\phi / \mathrm{ppm}^{2}\end{array}$ & LM & LM & LM & LM & LM & $\begin{array}{l}69.98 \pm 51.02 \\
0.018 \pm 0.009\end{array}$ & LM & LM & LM \\
\hline G30 & $\begin{array}{l}k_{e x} / 10^{3} \mathrm{~s} \\
\phi / \mathrm{ppm}^{2}\end{array}$ & $\begin{array}{l}66.52 \pm 30.65 \\
0.024 \pm 0.012 \\
\end{array}$ & $\begin{array}{l}\text { NaN } \\
\text { NaN }\end{array}$ & LM & LM & LM & LM & LM & flat & flat \\
\hline R32 & $\begin{array}{l}k_{e x} / 10^{3} \mathrm{~s} \\
\phi / \mathrm{ppm}^{2}\end{array}$ & $\begin{array}{c}162.93 \pm 30.89 \\
0.049 \pm 0.015\end{array}$ & LM & LM & LM & LM & LM & flat & flat & flat \\
\hline V33 & $\begin{array}{l}k_{e x} / 10^{3} \mathrm{~s} \\
\phi / \mathrm{ppm}^{2}\end{array}$ & $\begin{array}{c}10.00 \pm 1.33 \\
0.017 \pm 0.003\end{array}$ & $\begin{array}{c}100.88 \pm 45.30 \\
0.040 \pm 0.023\end{array}$ & LM & LM & $\begin{array}{l}82.73 \pm 36.89 \\
0.019 \pm 0.009\end{array}$ & LM & LM & LM & LM \\
\hline E34 & $\begin{array}{l}k_{e x} / 10^{3} \mathrm{~s} \\
\phi / \mathrm{ppm}^{2}\end{array}$ & $\begin{array}{c}176.66 \pm 67.11 \\
0.084 \pm 0.054\end{array}$ & $\begin{array}{c}169.58 \pm 51.09 \\
0.063 \pm 0.032\end{array}$ & $\begin{array}{c}163.44 \pm 37.27 \\
0.048 \pm 0.018\end{array}$ & $\begin{array}{c}149.36 \pm 20.86 \\
0.038 \pm 0.008\end{array}$ & $\begin{array}{l}92.73 \pm 17.46 \\
0.016 \pm 0.003\end{array}$ & $\begin{array}{l}70.16 \pm 19.61 \\
0.010 \pm 0.002\end{array}$ & LM & LM & LM \\
\hline F35 & $\begin{array}{l}k_{e x} / 10^{3} \mathrm{~s} \\
\phi / \mathrm{ppm}^{2}\end{array}$ & LM & LM & LM & LM & LM & LM & LM & LM & LM \\
\hline т36 & $\begin{array}{l}k_{e x} / 10^{3} \mathrm{~s} \\
\phi / \mathrm{ppm}^{2}\end{array}$ & LM & LM & LM & LM & LM & LM & LM & flat & flat \\
\hline A 37 & $\begin{array}{l}k_{e x} / 10^{3} \mathrm{~s} \\
\phi / \mathrm{ppm}^{2}\end{array}$ & LM & LM & LM & $\begin{array}{l}67.31 \pm 28.98 \\
0.029 \pm 0.011\end{array}$ & $\begin{array}{l}64.54 \pm 10.24 \\
0.030 \pm 0.004\end{array}$ & $\begin{array}{l}84.68 \pm 14.26 \\
0.030 \pm 0.005\end{array}$ & $\begin{array}{l}72.80 \pm 23.76 \\
0.018 \pm 0.004\end{array}$ & LM & LM \\
\hline T38 & $\begin{array}{l}k_{e x} / 10^{3} \mathrm{~s} \\
\phi / \mathrm{ppm}^{2}\end{array}$ & $\begin{array}{c}171.83 \pm 75.63 \\
0.086 \pm 0.064\end{array}$ & $\begin{array}{l}\mathrm{NaN} \\
\mathrm{NaN}\end{array}$ & $\begin{array}{l}\mathrm{NaN} \\
\mathrm{NaN}\end{array}$ & $\begin{array}{c}158.64 \pm 64.98 \\
0.052 \pm 0.034\end{array}$ & LM & LM & LM & flat & flat \\
\hline S39 & $\begin{array}{l}k_{e x} / 10^{3} \mathrm{~s} \\
\phi / \mathrm{ppm}^{2}\end{array}$ & $\begin{array}{l}96.56 \pm 12.76 \\
0.069 \pm 0.011\end{array}$ & $\begin{array}{l}91.84 \pm 12.48 \\
0.058 \pm 0.010\end{array}$ & $\begin{array}{c}104.78 \pm 14.78 \\
0.050 \pm 0.009\end{array}$ & $\begin{array}{l}71.70 \pm 14.97 \\
0.032 \pm 0.008\end{array}$ & $\begin{array}{c}146.71 \pm 37.84 \\
0.053 \pm 0.021\end{array}$ & LM & LM & LM & LM \\
\hline V40 & $\begin{array}{l}k_{e x} / 10^{3} \mathrm{~s} \\
\phi / \mathrm{ppm}^{2}\end{array}$ & LM & LM & LM & LM & $\mathrm{LM}$ & $\begin{array}{l}93.10 \pm 33.49 \\
0.039 \pm 0.014\end{array}$ & $\begin{array}{c}130.57 \pm 45.23 \\
0.034 \pm 0.014\end{array}$ & LM & LM \\
\hline S41 & $\begin{array}{l}k_{e x} / 10^{3} \mathrm{~s} \\
\phi / \mathrm{ppm}^{2}\end{array}$ & LM & $\begin{array}{l}\text { NaN } \\
\text { NaN }\end{array}$ & $\begin{array}{l}\mathrm{NaN} \\
\mathrm{NaN}\end{array}$ & $\begin{array}{c}119.47 \pm 74.44 \\
0.036 \pm 0.031\end{array}$ & $\begin{array}{c}191.01 \pm 88.63 \\
0.068 \pm 0.053\end{array}$ & $\begin{array}{c}103.32 \pm 41.84 \\
0.020 \pm 0.009\end{array}$ & $\begin{array}{l}72.58 \pm 29.24 \\
0.011 \pm 0.003\end{array}$ & LM & LM \\
\hline D42 & $\begin{array}{l}k_{e x} / 10^{3} \mathrm{~s} \\
\phi / \mathrm{ppm}^{2}\end{array}$ & $\begin{array}{l}\mathrm{NaN} \\
\mathrm{NaN}\end{array}$ & $\begin{array}{l}80.91 \pm 20.15 \\
0.049 \pm 0.014\end{array}$ & LM & $\begin{array}{c}140.37 \pm 79.69 \\
0.046 \pm 0.039\end{array}$ & $\begin{array}{c}129.72 \pm 50.19 \\
0.041 \pm 0.022\end{array}$ & LM & LM & LM & LM \\
\hline L43 & $\begin{array}{l}k_{e x} / 10^{3} \mathrm{~s} \\
\phi / \mathrm{ppm}^{2}\end{array}$ & LM & LM & $\begin{array}{c}104.69 \pm 32.34 \\
0.051 \pm 0.021\end{array}$ & $\begin{array}{c}109.80 \pm 82.71 \\
0.039 \pm 0.039\end{array}$ & $\begin{array}{c}10.00 \pm 0.00 \\
0.011 \pm 0.000\end{array}$ & $\begin{array}{c}15.19 \pm 1.48 \\
0.012 \pm 0.001\end{array}$ & LM & LM & LM \\
\hline K44 & $\begin{array}{l}k_{e x} / 10^{3} \mathrm{~s} \\
\phi / \mathrm{ppm}^{2}\end{array}$ & $\begin{array}{l}86.73 \pm 25.61 \\
0.039 \pm 0.015\end{array}$ & $\begin{array}{l}\text { NaN } \\
\text { NaN }\end{array}$ & $\begin{array}{c}221.29 \pm 105.12 \\
0.110 \pm 0.094\end{array}$ & LM & LM & LM & LM & LM & LM \\
\hline K45 & $\begin{array}{l}k_{e x} / 10^{3} \mathrm{~s} \\
\phi / \mathrm{ppm}^{2}\end{array}$ & LM & $\begin{array}{l}\mathrm{NaN} \\
\mathrm{NaN}\end{array}$ & LM & LM & LM & LM & LM & flat & flat \\
\hline
\end{tabular}




\begin{tabular}{|c|c|c|c|c|c|c|c|c|c|c|}
\hline Residue & Parameter & $263 \mathrm{~K}$ & $265 \mathrm{~K}$ & $268 \mathrm{~K}$ & $270 \mathrm{~K}$ & $275 \mathrm{~K}$ & $280 \mathrm{~K}$ & $285 \mathrm{~K}$ & $290 \mathrm{~K}$ & $295 \mathrm{~K}$ \\
\hline Y46 & $\begin{array}{l}k_{e x} / 10^{3} \mathrm{~s} \\
\phi / \mathrm{ppm}^{2}\end{array}$ & $\begin{array}{c}167.67 \pm 96.33 \\
0.128 \pm 0.123\end{array}$ & $\begin{array}{c}150.48 \pm 79.95 \\
0.078 \pm 0.065\end{array}$ & $\begin{array}{c}180.04 \pm 81.19 \\
0.104 \pm 0.080\end{array}$ & $\begin{array}{l}\text { NaN } \\
\text { NaN }\end{array}$ & $\begin{array}{c}176.82 \pm 76.59 \\
0.074 \pm 0.053\end{array}$ & $\begin{array}{c}34.32 \pm 5.99 \\
0.014 \pm 0.003\end{array}$ & LM & LM & LM \\
\hline I 47 & $\begin{array}{l}k_{e x} / 10^{3} \mathrm{~s} \\
\phi / \mathrm{ppm}^{2}\end{array}$ & $\begin{array}{l}\text { NaN } \\
\text { NaN }\end{array}$ & $\begin{array}{c}127.22 \pm 36.88 \\
0.056 \pm 0.024\end{array}$ & LM & $\begin{array}{c}188.27 \pm 86.29 \\
0.080 \pm 0.062\end{array}$ & LM & LM & LM & LM & LM \\
\hline E49 & $\begin{array}{l}k_{e x} / 10^{3} \mathrm{~s} \\
\phi / \mathrm{ppm}^{2}\end{array}$ & $\begin{array}{l}\mathrm{NaN} \\
\mathrm{NaN}\end{array}$ & $\begin{array}{l}\text { NaN } \\
\text { NaN }\end{array}$ & LM & $\begin{array}{c}147.53 \pm 32.30 \\
0.050 \pm 0.017\end{array}$ & LM & LM & LM & flat & LM \\
\hline L50 & $\begin{array}{l}k_{e x} / 10^{3} \mathrm{~s} \\
\phi / \mathrm{ppm}^{2}\end{array}$ & LM & $\mathrm{LM}$ & LM & $\mathrm{LM}$ & LM & LM & LM & flat & flat \\
\hline E51 & $\begin{array}{l}k_{e x} / 10^{3} \mathrm{~s} \\
\phi / \mathrm{ppm}^{2}\end{array}$ & $\begin{array}{c}216.68 \pm 86.70 \\
0.244 \pm 0.177\end{array}$ & LM & $\begin{array}{c}152.72 \pm 26.29 \\
0.083 \pm 0.023\end{array}$ & $\begin{array}{c}162.99 \pm 33.79 \\
0.078 \pm 0.027\end{array}$ & $\begin{array}{c}188.22 \pm 90.74 \\
0.066 \pm 0.054\end{array}$ & LM & LM & LM & LM \\
\hline V52 & $\begin{array}{l}k_{e x} / 10^{3} \mathrm{~s} \\
\phi / \mathrm{ppm}^{2}\end{array}$ & $\begin{array}{l}\text { NaN } \\
\text { NaN }\end{array}$ & $\begin{array}{l}\mathrm{NaN} \\
\mathrm{NaN}\end{array}$ & $\begin{array}{l}61.05 \pm 15.47 \\
0.023 \pm 0.007\end{array}$ & $\begin{array}{c}182.18 \pm 59.99 \\
0.070 \pm 0.038\end{array}$ & $\begin{array}{c}112.39 \pm 24.56 \\
0.027 \pm 0.008\end{array}$ & LM & LM & LM & flat \\
\hline Q53 & $\begin{array}{l}k_{e x} / 10^{3} \mathrm{~s} \\
\phi / \mathrm{ppm}^{2}\end{array}$ & $\mathrm{LM}$ & LM & $\begin{array}{l}\mathrm{NaN} \\
\mathrm{NaN}\end{array}$ & LM & LM & LM & LM & flat & flat \\
\hline T54 & $\begin{array}{l}k_{e x} / 10^{3} \mathrm{~s} \\
\phi / \mathrm{ppm}^{2}\end{array}$ & $\begin{array}{l}\text { NaN } \\
\text { NaN }\end{array}$ & $\begin{array}{c}197.26 \pm 52.30 \\
0.112 \pm 0.053\end{array}$ & $\begin{array}{c}261.54 \pm 134.42 \\
0.154 \pm 0.150\end{array}$ & $\begin{array}{c}174.88 \pm 55.89 \\
0.061 \pm 0.033\end{array}$ & LM & LM & LM & flat & flat \\
\hline G55 & $\begin{array}{l}k_{e x} / 10^{3} \mathrm{~s} \\
\phi / \mathrm{ppm}^{2}\end{array}$ & $\begin{array}{c}180.22 \pm 28.86 \\
0.157 \pm 0.043\end{array}$ & $\begin{array}{c}218.50 \pm 69.86 \\
0.176 \pm 0.102\end{array}$ & $\begin{array}{c}165.17 \pm 35.31 \\
0.079 \pm 0.028\end{array}$ & $\begin{array}{c}204.76 \pm 86.65 \\
0.094 \pm 0.071\end{array}$ & $\begin{array}{c}164.74 \pm 58.55 \\
0.044 \pm 0.026\end{array}$ & LM & LM & LM & flat \\
\hline M56 & $\begin{array}{l}k_{e x} / 10^{3} \mathrm{~s} \\
\phi / \mathrm{ppm}^{2}\end{array}$ & $\begin{array}{l}\text { NaN } \\
\text { NaN }\end{array}$ & LM & LM & $\mathrm{LM}$ & LM & LM & LM & flat & flat \\
\hline T57 & $\begin{array}{l}k_{e x} / 10^{3} \mathrm{~s} \\
\phi / \mathrm{ppm}^{2}\end{array}$ & LM & LM & LM & LM & LM & LM & flat & flat & flat \\
\hline R60 & $\begin{array}{l}k_{e x} / 10^{3} \mathrm{~s} \\
\phi / \mathrm{ppm}^{2}\end{array}$ & LM & LM & LM & LM & flat & flat & flat & flat & flat \\
\hline R61 & $\begin{array}{l}k_{e x} / 10^{3} \mathrm{~s} \\
\phi / \mathrm{ppm}^{2}\end{array}$ & LM & LM & LM & flat & flat & flat & flat & flat & flat \\
\hline G62 & $\begin{array}{l}k_{e x} / 10^{3} \mathrm{~s} \\
\phi / \mathrm{ppm}^{2}\end{array}$ & LM & flat & flat & flat & flat & flat & flat & flat & flat \\
\hline
\end{tabular}


A.3 RD profiles of side-chain ${ }^{13} \mathrm{C}$ E-CPMG
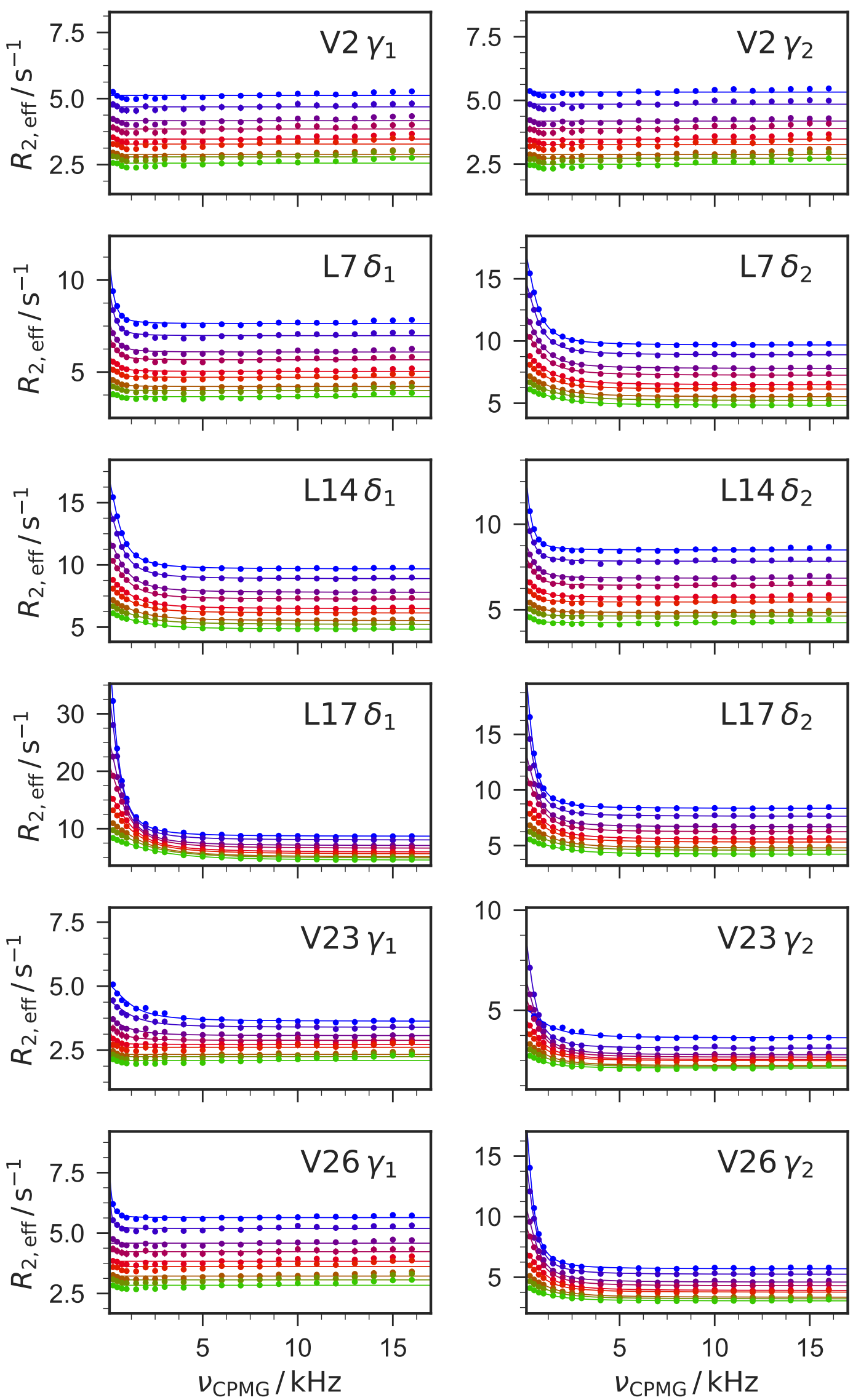

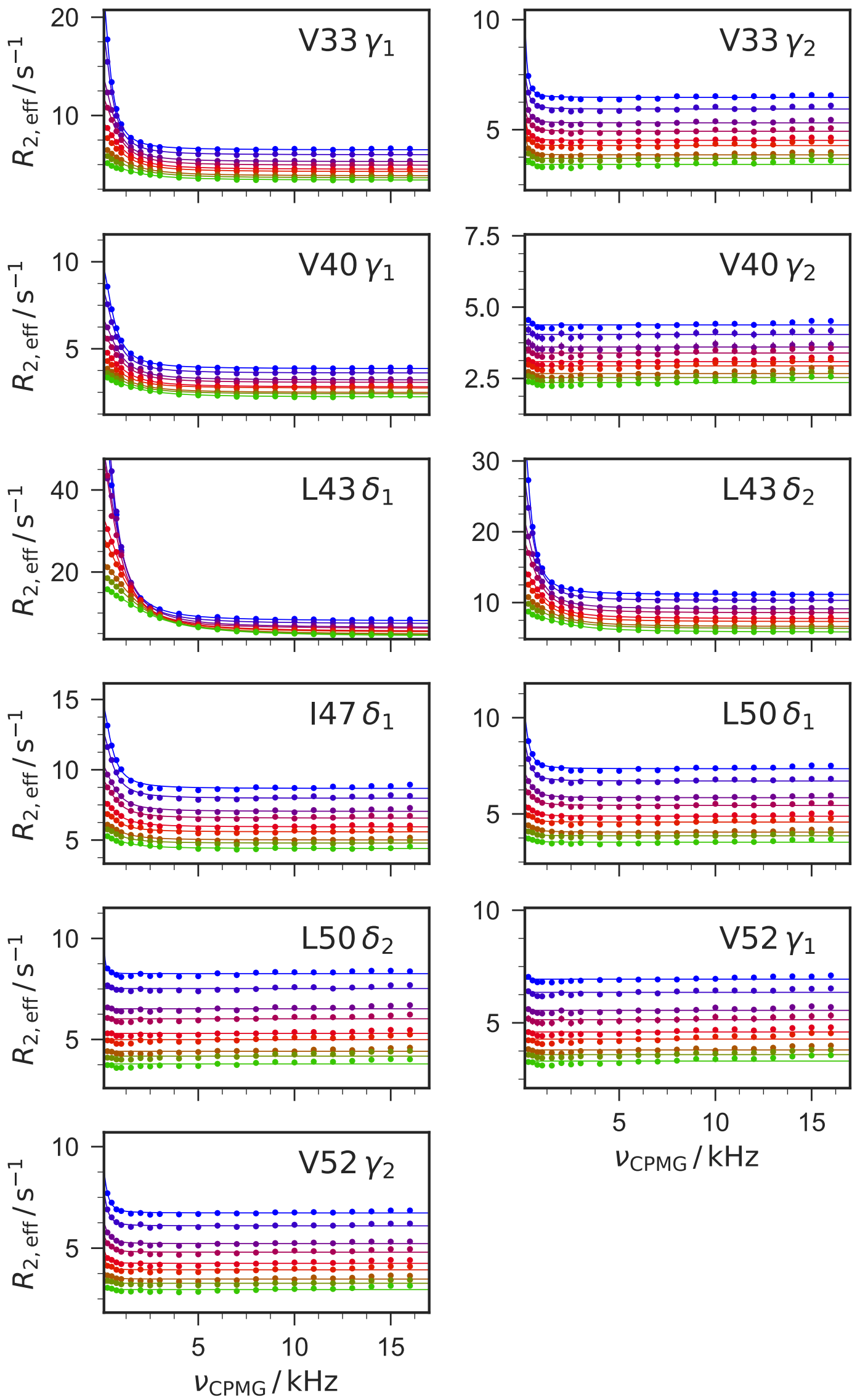

$v_{\text {CPMG }} / \mathrm{kHz}$

Figure A.3: Temperature dependent ${ }^{13} \mathrm{C}$ relaxation dispersion profiles of gpW methyl side-chains. E-CPMG experiments were measured at $270 \mathrm{~K}, 272 \mathrm{~K}, 275 \mathrm{~K}, 278 \mathrm{~K}$, $280 \mathrm{~K}, 282 \mathrm{~K}, 285 \mathrm{~K}, 287 \mathrm{~K}$ and $290 \mathrm{~K}$. 
Table A.5: Individual fit parameters of the slow exchange process from side-chain ${ }^{13} \mathrm{C} \mathrm{E-CPMG}$ data. Fit parameters of the two-state exchange model are shown. RD profiles with an exchange contribution $\left(R_{\mathrm{ex}}\right)>2 \mathrm{~s}^{-1}$ are marked as flat profiles.

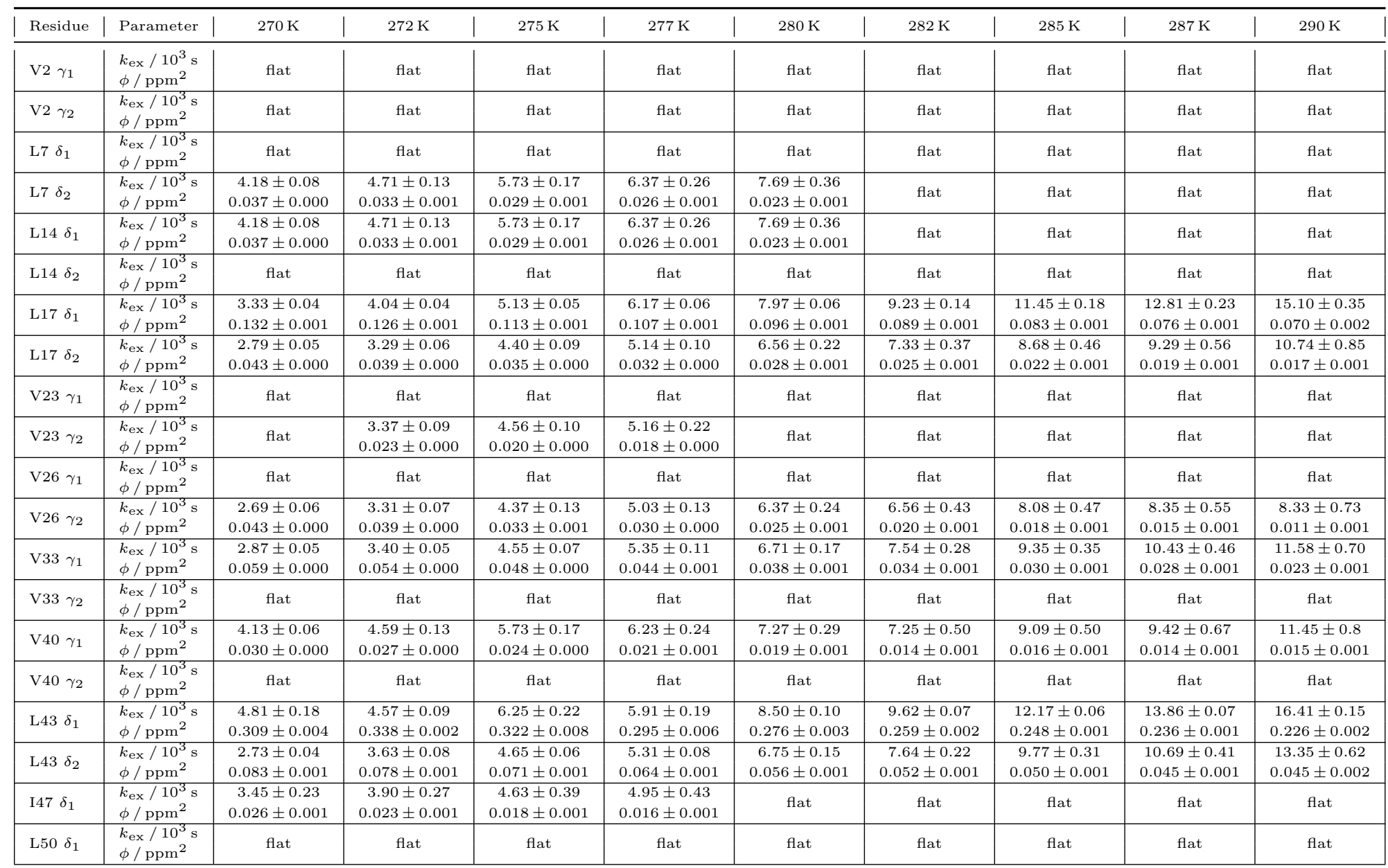




\begin{tabular}{|c|c|c|c|c|c|c|c|c|c|c|c|c|}
\hline Residue & Parameter & $270 \mathrm{~K}$ & $272 \mathrm{~K}$ & $275 \mathrm{~K}$ & $277 \mathrm{~K}$ & $280 \mathrm{~K}$ & $282 \mathrm{~K}$ & $285 \mathrm{~K}$ & $287 \mathrm{~K}$ & $290 \mathrm{~K}$ \\
\hline L50 $\delta_{2}$ & $\begin{array}{l}k_{\text {ex }} / 10^{3} \mathrm{~s} \\
\phi / \mathrm{ppm}^{2}\end{array}$ & flat & flat & flat & flat & flat & flat & flat & flat \\
\hline $\mathrm{V} 52 \gamma_{1}$ & $\begin{array}{l}k_{\text {ex }} / 10^{3} \mathrm{~s} \\
\phi / \mathrm{ppm}^{2}\end{array}$ & flat & flat & flat & flat & flat & flat & flat & flat & flat \\
\hline $\mathrm{V} 52 \gamma_{2}$ & $\begin{array}{l}k_{\mathrm{ex}} / 10^{3} \mathrm{~s} \\
\phi / \mathrm{ppm}^{2}\end{array}$ & flat & flat & flat & flat & flat & flat & flat & flat & flat \\
\hline
\end{tabular}


A.4 RD profiles of side-chain ${ }^{1} \mathrm{H}$ E-CPMG
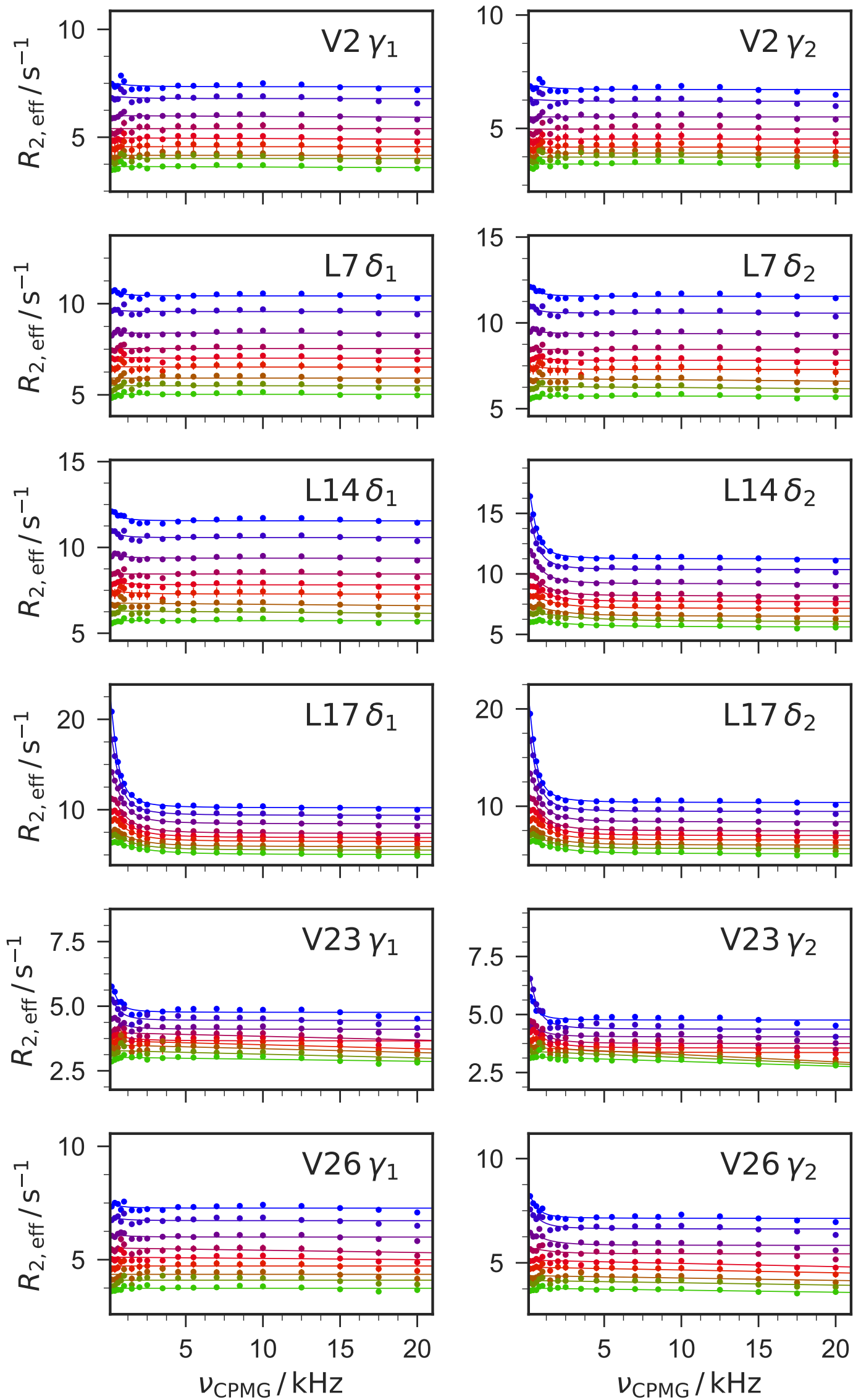

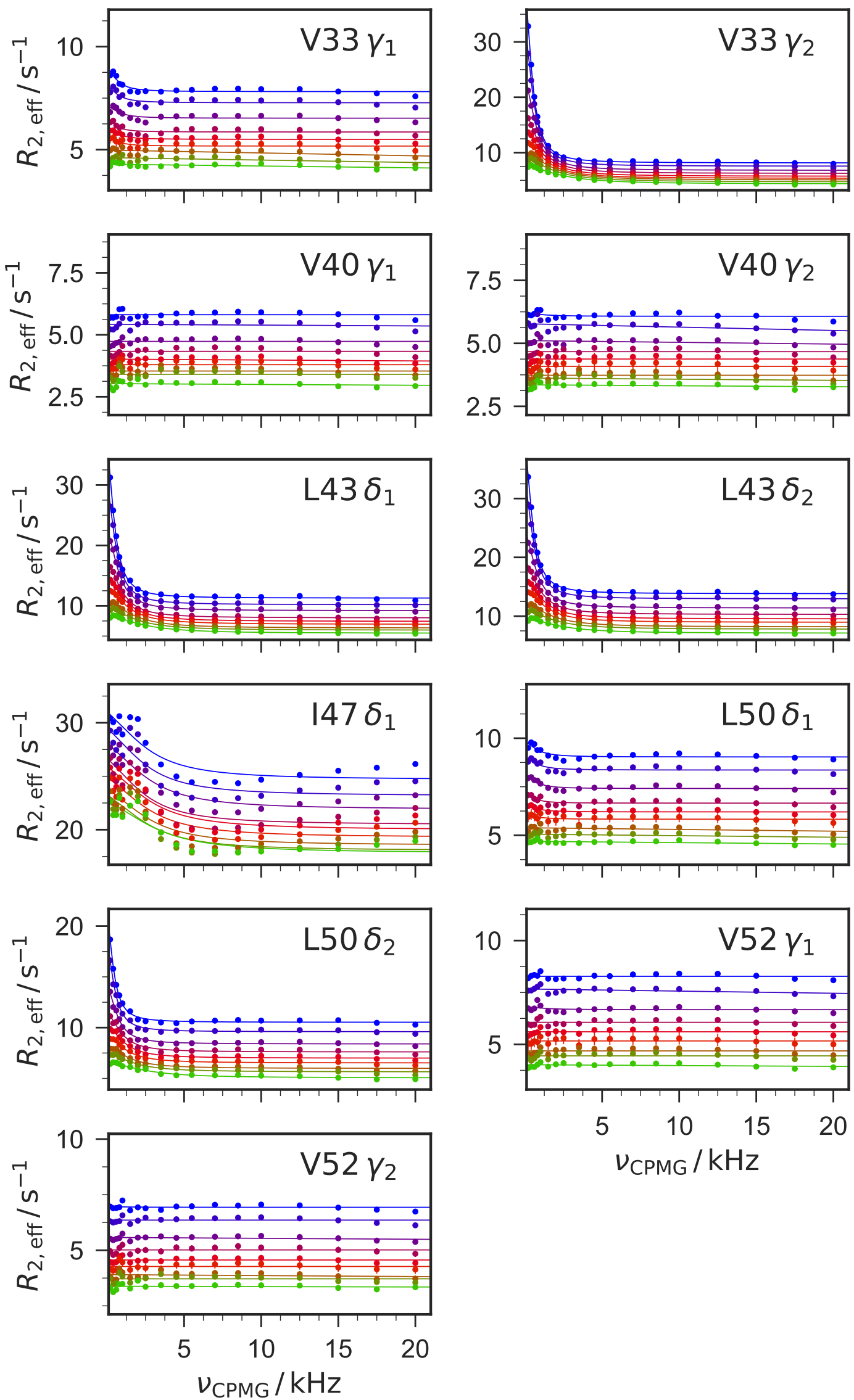

Figure A.4: Temperature dependent ${ }^{1} \mathrm{H}$ relaxation dispersion profiles of gpW methyl side-chains. E-CPMG experiments were measured at $270 \mathrm{~K}, 272 \mathrm{~K}, 275 \mathrm{~K}, 278 \mathrm{~K}$, $280 \mathrm{~K}, 282 \mathrm{~K}, 285 \mathrm{~K}, 287 \mathrm{~K}$ and $290 \mathrm{~K}$. 
Table A.6: Individual fit parameters of the slow exchange process from side-chain ${ }^{1} \mathbf{H}$ E-CPMG data. Fit parameters of the two-state exchange model are shown. RD profiles with an exchange contribution $\left(R_{\mathrm{ex}}\right)>2 \mathrm{~s}^{-1}$ are marked as flat profiles.

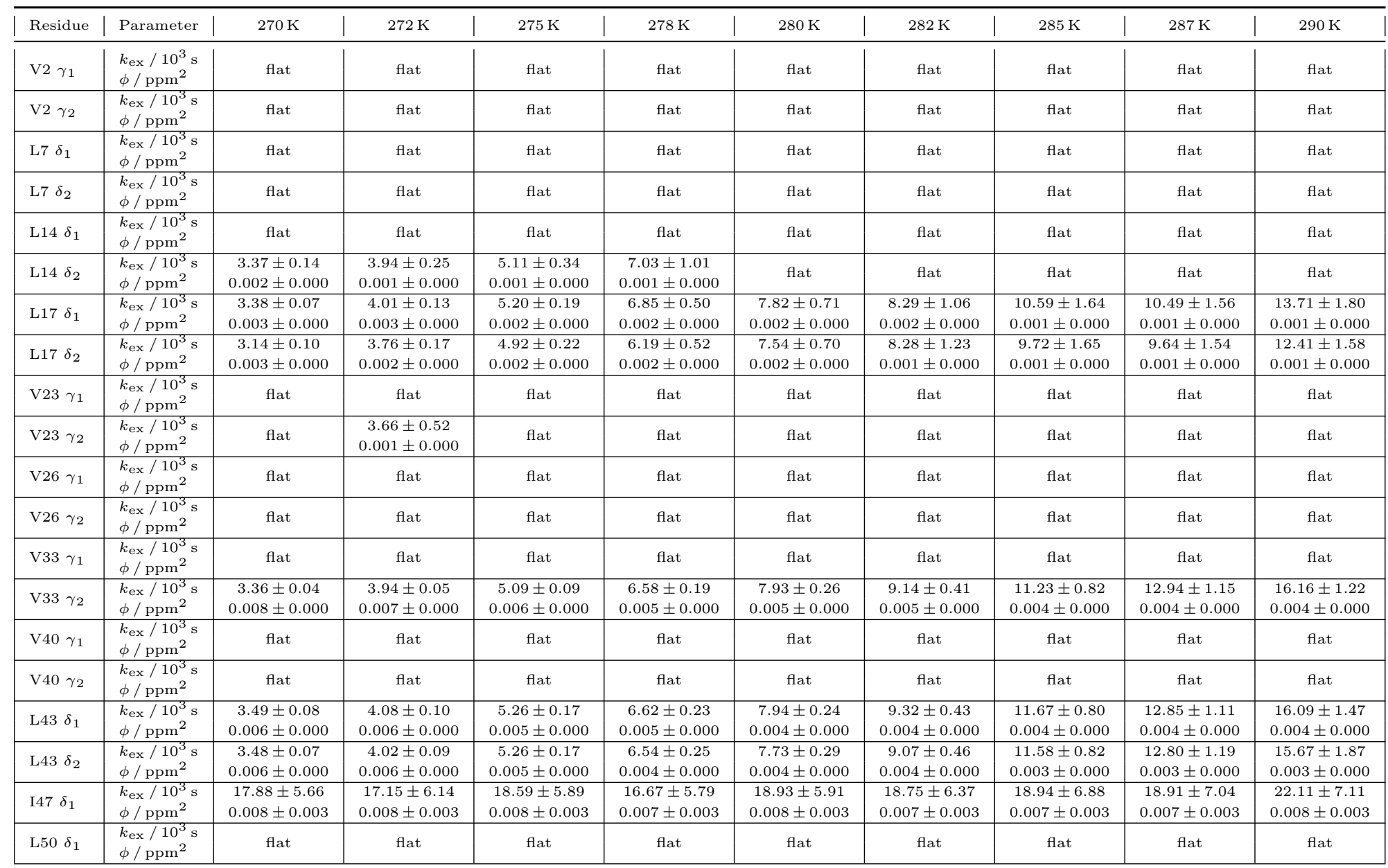




\begin{tabular}{|c|c|c|c|c|c|c|c|c|c|c|}
\hline Residue & Parameter & $270 \mathrm{~K}$ & $272 \mathrm{~K}$ & $275 \mathrm{~K}$ & $278 \mathrm{~K}$ & $280 \mathrm{~K}$ & $282 \mathrm{~K}$ & $285 \mathrm{~K}$ & $287 \mathrm{~K}$ & $290 \mathrm{~K}$ \\
\hline L50 $\delta_{2}$ & $\begin{array}{l}k_{\text {ex }} / 10^{3} \mathrm{~s} \\
\phi / \mathrm{ppm}^{2}\end{array}$ & $\begin{array}{c}3.12 \pm 0.14 \\
0.002 \pm 0.000\end{array}$ & $\begin{array}{c}3.56 \pm 0.17 \\
0.002 \pm 0.000\end{array}$ & $\begin{array}{c}4.70 \pm 0.31 \\
0.002 \pm 0.000\end{array}$ & $\begin{array}{c}6.51 \pm 0.66 \\
0.002 \pm 0.000\end{array}$ & $\begin{array}{c}7.60 \pm 0.74 \\
0.002 \pm 0.000\end{array}$ & $\begin{array}{c}8.48 \pm 0.89 \\
0.002 \pm 0.000\end{array}$ & $\begin{array}{c}9.91 \pm 1.17 \\
0.002 \pm 0.000\end{array}$ & $\begin{array}{c}11.97 \pm 1.69 \\
0.002 \pm 0.000\end{array}$ & $\begin{array}{c}17.32 \pm 2.45 \\
0.002 \pm 0.000\end{array}$ \\
\hline $\mathrm{V} 52 \gamma_{1}$ & $\begin{array}{l}k_{\text {ex }} / 10^{3} \mathrm{~s} \\
\phi / \mathrm{ppm}^{2}\end{array}$ & flat & flat & flat & flat & flat & flat & flat & flat & flat \\
\hline V52 $\gamma_{2}$ & $\begin{array}{l}k_{\text {ex }} / 10^{3} \mathrm{~s} \\
\phi / \mathrm{ppm}^{2}\end{array}$ & flat & flat & flat & flat & flat & flat & flat & flat & flat \\
\hline
\end{tabular}




\section{Appendix B}

\section{Input for Model Free Analysis}

\section{B.1 $300 \mathrm{MHz}$}

Table B.1: $R_{1}, R_{2}$ and het $N O E$ data measured on a $300 \mathrm{MHz}$ spectrometer at 294.5 K.

\begin{tabular}{|c|c|c|c|c|c|c|c|}
\hline Residue & $R_{1} / \mathrm{s}^{-1}$ & $R_{2} / \mathrm{s}^{-1}$ & het NOE & Residue & $R_{1} / \mathrm{s}^{-1}$ & $R_{2} / \mathrm{s}^{-1}$ & het NOE \\
\hline Q2 & $3.63 \pm 0.04$ & $8.08 \pm 0.06$ & $0.65 \pm 0.01$ & Q41 & $3.79 \pm 0.10$ & $8.23 \pm 0.06$ & $0.64 \pm 0.02$ \\
\hline I3 & $3.66 \pm 0.07$ & $7.80 \pm 0.08$ & $0.65 \pm 0.02$ & $\mathrm{R} 42$ & $3.84 \pm 0.06$ & $8.15 \pm 0.08$ & $0.56 \pm 0.02$ \\
\hline $\mathrm{F} 4$ & $3.87 \pm 0.04$ & $8.15 \pm 0.05$ & $0.65 \pm 0.02$ & L43 & $3.77 \pm 0.05$ & $7.92 \pm 0.07$ & $0.60 \pm 0.02$ \\
\hline V5 & $3.73 \pm 0.06$ & $7.93 \pm 0.05$ & $0.56 \pm 0.01$ & $\mathrm{I} 44$ & $3.79 \pm 0.09$ & $8.28 \pm 0.09$ & $0.62 \pm 0.02$ \\
\hline K6 & $3.83 \pm 0.01$ & $7.99 \pm 0.06$ & $0.63 \pm 0.02$ & F45 & $3.67 \pm 0.08$ & $8.22 \pm 0.04$ & $0.67 \pm 0.02$ \\
\hline $\mathrm{T} 7$ & $3.86 \pm 0.08$ & $8.08 \pm 0.05$ & $0.55 \pm 0.02$ & A46 & $4.28 \pm 0.18$ & $8.11 \pm 0.12$ & $0.62 \pm 0.03$ \\
\hline L8 & $4.03 \pm 0.19$ & $7.77 \pm 0.08$ & $0.41 \pm 0.02$ & G47 & $3.59 \pm 0.05$ & $7.30 \pm 0.06$ & $0.59 \pm 0.02$ \\
\hline $\mathrm{T} 9$ & $3.70 \pm 0.38$ & $6.91 \pm 0.12$ & $0.61 \pm 0.04$ & K48 & $3.76 \pm 0.14$ & $8.03 \pm 0.08$ & $0.54 \pm 0.02$ \\
\hline G10 & $3.70 \pm 0.14$ & $6.94 \pm 0.06$ & $0.42 \pm 0.02$ & Q49 & $3.49 \pm 0.06$ & $7.45 \pm 0.06$ & $0.44 \pm 0.01$ \\
\hline K11 & $3.33 \pm 0.11$ & $6.65 \pm 0.03$ & $0.35 \pm 0.01$ & L50 & $3.75 \pm 0.06$ & $7.70 \pm 0.05$ & $0.55 \pm 0.02$ \\
\hline $\mathrm{T} 12$ & $3.83 \pm 0.12$ & $7.63 \pm 0.08$ & $0.50 \pm 0.02$ & E51 & $3.77 \pm 0.07$ & $7.84 \pm 0.10$ & $0.60 \pm 0.02$ \\
\hline $\mathrm{I} 13$ & $3.69 \pm 0.06$ & $7.62 \pm 0.06$ & $0.60 \pm 0.02$ & D52 & $3.49 \pm 0.03$ & $7.95 \pm 0.08$ & $0.61 \pm 0.01$ \\
\hline $\mathrm{T} 14$ & $3.62 \pm 0.05$ & $7.82 \pm 0.04$ & $0.56 \pm 0.01$ & $\mathrm{R} 54$ & $3.54 \pm 0.05$ & $8.01 \pm 0.05$ & $0.63 \pm 0.02$ \\
\hline L15 & $3.71 \pm 0.07$ & $7.71 \pm 0.08$ & $0.64 \pm 0.01$ & $\mathrm{~T} 55$ & $3.66 \pm 0.09$ & $7.73 \pm 0.04$ & $0.59 \pm 0.02$ \\
\hline E16 & $3.52 \pm 0.06$ & $7.52 \pm 0.07$ & $0.59 \pm 0.01$ & L56 & $3.90 \pm 0.14$ & $8.21 \pm 0.07$ & $0.59 \pm 0.02$ \\
\hline V17 & $3.85 \pm 0.07$ & $8.20 \pm 0.05$ & $0.61 \pm 0.02$ & S57 & $3.82 \pm 0.04$ & $7.83 \pm 0.05$ & $0.52 \pm 0.01$ \\
\hline E18 & $3.64 \pm 0.05$ & $8.32 \pm 0.09$ & $0.61 \pm 0.01$ & D58 & $3.92 \pm 0.07$ & $8.16 \pm 0.05$ & $0.61 \pm 0.02$ \\
\hline $\mathrm{S} 20$ & $3.72 \pm 0.10$ & $7.63 \pm 0.09$ & $0.59 \pm 0.02$ & Y59 & $3.71 \pm 0.08$ & $7.81 \pm 0.07$ & $0.60 \pm 0.02$ \\
\hline D21 & $3.90 \pm 0.11$ & $8.19 \pm 0.08$ & $0.62 \pm 0.02$ & N60 & $3.82 \pm 0.05$ & $8.06 \pm 0.07$ & $0.59 \pm 0.02$ \\
\hline $\mathrm{T} 22$ & $3.83 \pm 0.06$ & $7.62 \pm 0.05$ & $0.54 \pm 0.01$ & I61 & $3.73 \pm 0.04$ & $7.88 \pm 0.06$ & $0.48 \pm 0.02$ \\
\hline $\mathrm{I} 23$ & $3.92 \pm 0.05$ & $8.72 \pm 0.05$ & $0.60 \pm 0.02$ & Q62 & $3.24 \pm 0.06$ & $6.85 \pm 0.05$ & $0.46 \pm 0.01$ \\
\hline $\mathrm{N} 25$ & $3.88 \pm 0.09$ & $9.06 \pm 0.09$ & $0.63 \pm 0.02$ & K63 & $3.65 \pm 0.06$ & $8.02 \pm 0.05$ & $0.57 \pm 0.01$ \\
\hline V26 & $3.82 \pm 0.04$ & $8.29 \pm 0.10$ & $0.58 \pm 0.02$ & E64 & $3.87 \pm 0.05$ & $7.92 \pm 0.06$ & $0.59 \pm 0.02$ \\
\hline $\mathrm{K} 27$ & $3.99 \pm 0.05$ & $8.41 \pm 0.05$ & $0.62 \pm 0.02$ & S65 & $3.71 \pm 0.06$ & $7.83 \pm 0.06$ & $0.64 \pm 0.02$ \\
\hline A 28 & $4.05 \pm 0.05$ & $8.49 \pm 0.11$ & $0.56 \pm 0.02$ & T66 & $3.72 \pm 0.03$ & $7.90 \pm 0.05$ & $0.53 \pm 0.02$ \\
\hline K29 & $3.79 \pm 0.07$ & $8.00 \pm 0.06$ & $0.62 \pm 0.02$ & L67 & $3.80 \pm 0.03$ & $7.89 \pm 0.06$ & $0.61 \pm 0.02$ \\
\hline $\mathrm{I} 30$ & $3.81 \pm 0.03$ & $8.06 \pm 0.08$ & $0.65 \pm 0.02$ & H68 & $3.85 \pm 0.03$ & $8.16 \pm 0.06$ & $0.56 \pm 0.02$ \\
\hline Q31 & $3.81 \pm 0.04$ & $8.20 \pm 0.08$ & $0.63 \pm 0.02$ & L69 & $3.73 \pm 0.05$ & $7.95 \pm 0.07$ & $0.65 \pm 0.02$ \\
\hline D32 & $3.78 \pm 0.05$ & $8.24 \pm 0.07$ & $0.62 \pm 0.02$ & V70 & $3.80 \pm 0.04$ & $8.25 \pm 0.05$ & $0.58 \pm 0.02$ \\
\hline K33 & $3.57 \pm 0.02$ & $7.88 \pm 0.08$ & $0.60 \pm 0.02$ & L71 & $3.53 \pm 0.06$ & $7.62 \pm 0.07$ & $0.51 \pm 0.01$ \\
\hline E34 & $3.78 \pm 0.05$ & $8.08 \pm 0.07$ & $0.63 \pm 0.02$ & $\mathrm{R} 72$ & $3.50 \pm 0.03$ & $7.25 \pm 0.02$ & $0.39 \pm 0.01$ \\
\hline G35 & $3.81 \pm 0.07$ & $8.37 \pm 0.07$ & $0.64 \pm 0.02$ & $\mathrm{~L} 73$ & $3.07 \pm 0.13$ & $5.23 \pm 0.02$ & $0.05 \pm 0.01$ \\
\hline I36 & $3.23 \pm 0.05$ & $7.46 \pm 0.19$ & $0.67 \pm 0.02$ & $\mathrm{R} 74$ & $2.56 \pm 0.24$ & $4.28 \pm 0.09$ & $-0.34 \pm 0.01$ \\
\hline D39 & $3.69 \pm 0.03$ & $7.73 \pm 0.04$ & $0.59 \pm 0.01$ & G75 & $1.68 \pm 0.24$ & $2.66 \pm 0.09$ & $-1.24 \pm 0.04$ \\
\hline $\mathrm{Q} 40$ & $3.81 \pm 0.09$ & $8.13 \pm 0.07$ & $0.56 \pm 0.02$ & G76 & $1.03 \pm 0.04$ & $1.61 \pm 0.02$ & $-2.66 \pm 0.03$ \\
\hline
\end{tabular}




\section{B.2 $400 \mathrm{MHz}$}

Table B.2: $R_{1}, R_{2}$ and het $N O E$ data measured on a $400 \mathrm{MHz}$ spectrometer at 294.5 K.

\begin{tabular}{|c|c|c|c|c|c|c|c|}
\hline Residue & $R_{1} / \mathrm{s}^{-1}$ & $R_{2} / \mathrm{s}^{-1}$ & het NOE & Residue & $R_{1} / \mathrm{s}^{-1}$ & $R_{2} / \mathrm{s}^{-1}$ & het NOE \\
\hline Q2 & $2.78 \pm 0.04$ & $7.78 \pm 0.04$ & $0.72 \pm 0.01$ & Q41 & $2.94 \pm 0.06$ & $7.93 \pm 0.05$ & $0.67 \pm 0.01$ \\
\hline I3 & $2.90 \pm 0.14$ & $7.59 \pm 0.07$ & $0.73 \pm 0.02$ & R42 & $2.96 \pm 0.06$ & $7.88 \pm 0.04$ & $0.70 \pm 0.01$ \\
\hline $\mathrm{F} 4$ & $3.06 \pm 0.06$ & $8.14 \pm 0.05$ & $0.70 \pm 0.01$ & $\mathrm{~L} 43$ & $2.93 \pm 0.07$ & $7.93 \pm 0.07$ & $0.72 \pm 0.02$ \\
\hline V5 & $2.91 \pm 0.06$ & $7.70 \pm 0.08$ & $0.74 \pm 0.02$ & I 44 & $2.96 \pm 0.04$ & $8.03 \pm 0.06$ & $0.65 \pm 0.01$ \\
\hline K6 & $2.98 \pm 0.04$ & $7.76 \pm 0.04$ & $0.72 \pm 0.02$ & F45 & $2.99 \pm 0.06$ & $8.24 \pm 0.07$ & $0.75 \pm 0.02$ \\
\hline $\mathrm{T} 7$ & $2.99 \pm 0.10$ & $8.04 \pm 0.09$ & $0.64 \pm 0.01$ & $\mathrm{~A} 46$ & $3.47 \pm 0.25$ & $10.71 \pm 0.30$ & $0.63 \pm 0.02$ \\
\hline L8 & $3.22 \pm 0.18$ & $7.43 \pm 0.10$ & $0.53 \pm 0.02$ & G47 & $2.93 \pm 0.09$ & $12.37 \pm 0.33$ & $0.60 \pm 0.01$ \\
\hline $\mathrm{T} 9$ & $3.11 \pm 0.33$ & $7.08 \pm 0.18$ & $0.55 \pm 0.02$ & K48 & $2.88 \pm 0.10$ & $7.96 \pm 0.13$ & $0.74 \pm 0.02$ \\
\hline G10 & $2.94 \pm 0.15$ & $6.74 \pm 0.10$ & $0.56 \pm 0.01$ & Q49 & $2.78 \pm 0.07$ & $7.29 \pm 0.05$ & $0.56 \pm 0.01$ \\
\hline K11 & $2.73 \pm 0.15$ & $6.84 \pm 0.07$ & $0.49 \pm 0.02$ & L50 & $2.92 \pm 0.06$ & $7.56 \pm 0.07$ & $0.66 \pm 0.01$ \\
\hline $\mathrm{T} 12$ & $3.07 \pm 0.17$ & $7.42 \pm 0.05$ & $0.59 \pm 0.01$ & $\mathrm{E} 51$ & $2.84 \pm 0.12$ & $7.63 \pm 0.10$ & $0.72 \pm 0.02$ \\
\hline $\mathrm{I} 13$ & $2.88 \pm 0.06$ & $7.59 \pm 0.07$ & $0.65 \pm 0.01$ & D52 & $2.63 \pm 0.04$ & $7.74 \pm 0.04$ & $0.64 \pm 0.01$ \\
\hline $\mathrm{T} 14$ & $2.86 \pm 0.07$ & $7.82 \pm 0.04$ & $0.66 \pm 0.01$ & $\mathrm{R} 54$ & $2.80 \pm 0.06$ & $8.07 \pm 0.05$ & $0.78 \pm 0.01$ \\
\hline L15 & $2.96 \pm 0.05$ & $7.55 \pm 0.05$ & $0.71 \pm 0.01$ & $\mathrm{~T} 55$ & $2.93 \pm 0.10$ & $7.83 \pm 0.06$ & $0.74 \pm 0.02$ \\
\hline E16 & $2.75 \pm 0.06$ & $7.29 \pm 0.08$ & $0.62 \pm 0.01$ & L56 & $3.15 \pm 0.13$ & $8.19 \pm 0.05$ & $0.75 \pm 0.02$ \\
\hline V17 & $3.01 \pm 0.11$ & $8.06 \pm 0.07$ & $0.72 \pm 0.01$ & S57 & $3.01 \pm 0.09$ & $7.68 \pm 0.07$ & $0.70 \pm 0.01$ \\
\hline E18 & $2.80 \pm 0.05$ & $8.27 \pm 0.08$ & $0.71 \pm 0.02$ & D58 & $3.09 \pm 0.11$ & $8.55 \pm 0.12$ & $0.69 \pm 0.02$ \\
\hline $\mathrm{S} 20$ & $2.96 \pm 0.13$ & $13.52 \pm 0.53$ & $0.71 \pm 0.02$ & Y59 & $2.97 \pm 0.08$ & $7.79 \pm 0.07$ & $0.69 \pm 0.01$ \\
\hline D21 & $3.08 \pm 0.08$ & $8.15 \pm 0.07$ & $0.72 \pm 0.01$ & N60 & $3.00 \pm 0.06$ & $8.09 \pm 0.05$ & $0.68 \pm 0.02$ \\
\hline $\mathrm{T} 22$ & $2.99 \pm 0.08$ & $7.53 \pm 0.06$ & $0.72 \pm 0.01$ & $\mathrm{I} 61$ & $2.95 \pm 0.05$ & $7.84 \pm 0.06$ & $0.70 \pm 0.01$ \\
\hline $\mathrm{I} 23$ & $3.10 \pm 0.08$ & $9.09 \pm 0.07$ & $0.72 \pm 0.02$ & Q62 & $2.62 \pm 0.09$ & $6.72 \pm 0.04$ & $0.53 \pm 0.02$ \\
\hline $\mathrm{N} 25$ & $3.03 \pm 0.09$ & $9.58 \pm 0.08$ & $0.78 \pm 0.02$ & K63 & $2.83 \pm 0.05$ & $7.93 \pm 0.02$ & $0.69 \pm 0.01$ \\
\hline $\mathrm{V} 26$ & $2.97 \pm 0.05$ & $7.82 \pm 0.07$ & $0.63 \pm 0.01$ & E64 & $3.06 \pm 0.08$ & $7.80 \pm 0.05$ & $0.72 \pm 0.01$ \\
\hline $\mathrm{K} 27$ & $3.07 \pm 0.06$ & $8.39 \pm 0.04$ & $0.70 \pm 0.02$ & S65 & $2.95 \pm 0.10$ & $7.88 \pm 0.07$ & $0.66 \pm 0.01$ \\
\hline A 28 & $3.08 \pm 0.08$ & $8.35 \pm 0.11$ & $0.84 \pm 0.03$ & T66 & $2.95 \pm 0.06$ & $7.91 \pm 0.05$ & $0.67 \pm 0.02$ \\
\hline K29 & $2.98 \pm 0.06$ & $8.13 \pm 0.04$ & $0.76 \pm 0.02$ & L67 & $3.00 \pm 0.05$ & $7.67 \pm 0.03$ & $0.77 \pm 0.02$ \\
\hline $\mathrm{I} 30$ & $2.99 \pm 0.02$ & $8.03 \pm 0.03$ & $0.73 \pm 0.02$ & H68 & $2.98 \pm 0.03$ & $8.19 \pm 0.07$ & $0.74 \pm 0.02$ \\
\hline Q31 & $3.01 \pm 0.02$ & $8.16 \pm 0.04$ & $0.72 \pm 0.02$ & L69 & $2.99 \pm 0.07$ & $8.12 \pm 0.06$ & $0.73 \pm 0.02$ \\
\hline D32 & $2.93 \pm 0.01$ & $8.22 \pm 0.04$ & $0.72 \pm 0.01$ & V70 & $2.99 \pm 0.06$ & $8.25 \pm 0.07$ & $0.68 \pm 0.02$ \\
\hline K33 & $2.88 \pm 0.06$ & $7.82 \pm 0.08$ & $0.75 \pm 0.02$ & $\mathrm{~L} 71$ & $2.76 \pm 0.02$ & $7.44 \pm 0.05$ & $0.56 \pm 0.01$ \\
\hline E34 & $2.87 \pm 0.00$ & $7.84 \pm 0.03$ & $0.67 \pm 0.02$ & $\mathrm{R} 72$ & $2.84 \pm 0.07$ & $7.19 \pm 0.04$ & $0.55 \pm 0.01$ \\
\hline G35 & $2.89 \pm 0.01$ & $8.31 \pm 0.05$ & $0.77 \pm 0.02$ & L73 & $2.61 \pm 0.13$ & $5.27 \pm 0.07$ & $0.32 \pm 0.01$ \\
\hline I36 & $2.52 \pm 0.06$ & $7.44 \pm 0.05$ & $0.81 \pm 0.02$ & $\mathrm{R} 74$ & $2.33 \pm 0.24$ & $4.34 \pm 0.08$ & $-0.02 \pm 0.01$ \\
\hline D39 & $3.03 \pm 0.07$ & $7.68 \pm 0.08$ & $0.68 \pm 0.01$ & G75 & $1.51 \pm 0.24$ & $2.67 \pm 0.10$ & $-0.67 \pm 0.02$ \\
\hline $\mathrm{Q} 40$ & $2.96 \pm 0.04$ & $8.10 \pm 0.05$ & $0.71 \pm 0.01$ & G76 & $0.97 \pm 0.04$ & $1.60 \pm 0.02$ & $-1.48 \pm 0.01$ \\
\hline
\end{tabular}




\section{B.3 $600 \mathrm{MHz}$}

Table B.3: $R_{1}, R_{2}$ and het $N O E$ data measured on a $600 \mathrm{MHz}$ spectrometer at 294.5 K.

\begin{tabular}{|c|c|c|c|c|c|c|c|}
\hline Residue & $R_{1} / \mathrm{s}^{-1}$ & $R_{2} / \mathrm{s}^{-1}$ & hetNOE & Residue & $R_{1} / \mathrm{s}^{-1}$ & $R_{2} / \mathrm{s}^{-1}$ & hetNOE \\
\hline Q2 & $1.87 \pm 0.06$ & $8.18 \pm 0.08$ & $0.74 \pm 0.00$ & Q41 & $1.99 \pm 0.08$ & $8.47 \pm 0.05$ & $0.84 \pm 0.00$ \\
\hline I3 & $1.97 \pm 0.09$ & $8.21 \pm 0.04$ & $0.85 \pm 0.00$ & $\mathrm{R} 42$ & $1.87 \pm 0.09$ & $7.76 \pm 0.12$ & $0.88 \pm 0.00$ \\
\hline F4 & $2.03 \pm 0.08$ & $8.57 \pm 0.02$ & $0.88 \pm 0.00$ & $\mathrm{~L} 43$ & $1.93 \pm 0.09$ & $8.38 \pm 0.08$ & $0.82 \pm 0.00$ \\
\hline V5 & $1.91 \pm 0.08$ & $7.99 \pm 0.04$ & $0.83 \pm 0.00$ & I44 & $1.90 \pm 0.08$ & $7.96 \pm 0.11$ & $0.86 \pm 0.01$ \\
\hline K6 & $1.90 \pm 0.09$ & $7.67 \pm 0.18$ & $0.86 \pm 0.00$ & F45 & $1.99 \pm 0.09$ & $9.35 \pm 0.11$ & $0.86 \pm 0.00$ \\
\hline $\mathrm{T} 7$ & $1.99 \pm 0.12$ & $8.34 \pm 0.04$ & $0.75 \pm 0.00$ & A 46 & $2.32 \pm 0.24$ & $8.43 \pm 0.17$ & $0.75 \pm 0.01$ \\
\hline L8 & $2.35 \pm 0.19$ & $7.99 \pm 0.06$ & $0.73 \pm 0.01$ & G47 & $2.00 \pm 0.08$ & $7.40 \pm 0.21$ & $0.78 \pm 0.00$ \\
\hline $\mathrm{T} 9$ & $2.16 \pm 0.31$ & $7.68 \pm 0.23$ & $0.66 \pm 0.01$ & K48 & $1.90 \pm 0.09$ & $8.58 \pm 0.09$ & $0.81 \pm 0.00$ \\
\hline G10 & $2.10 \pm 0.15$ & $6.96 \pm 0.14$ & $0.70 \pm 0.00$ & Q49 & $1.91 \pm 0.08$ & $7.83 \pm 0.09$ & $0.68 \pm 0.00$ \\
\hline K11 & $1.92 \pm 0.12$ & $7.29 \pm 0.05$ & $0.65 \pm 0.00$ & $\mathrm{~L} 50$ & $1.99 \pm 0.10$ & $10.00 \pm 0.14$ & $0.84 \pm 0.00$ \\
\hline $\mathrm{T} 12$ & $2.14 \pm 0.18$ & $7.81 \pm 0.03$ & $0.70 \pm 0.00$ & E51 & $1.85 \pm 0.13$ & $7.93 \pm 0.11$ & $0.79 \pm 0.00$ \\
\hline $\mathrm{I} 13$ & $1.85 \pm 0.08$ & $7.95 \pm 0.16$ & $0.85 \pm 0.00$ & D52 & $1.74 \pm 0.06$ & $8.27 \pm 0.03$ & $0.81 \pm 0.00$ \\
\hline $\mathrm{T} 14$ & $1.94 \pm 0.09$ & $8.38 \pm 0.06$ & $0.76 \pm 0.00$ & $\mathrm{R} 54$ & $1.88 \pm 0.08$ & $8.73 \pm 0.04$ & $0.86 \pm 0.00$ \\
\hline L15 & $2.01 \pm 0.07$ & $9.21 \pm 0.07$ & $0.87 \pm 0.00$ & $\mathrm{~T} 55$ & $1.88 \pm 0.12$ & $8.27 \pm 0.18$ & $0.81 \pm 0.00$ \\
\hline E16 & $1.78 \pm 0.03$ & $7.62 \pm 0.06$ & $0.82 \pm 0.00$ & $\mathrm{~L} 56$ & $2.10 \pm 0.13$ & $8.51 \pm 0.03$ & $0.89 \pm 0.00$ \\
\hline V17 & $1.98 \pm 0.12$ & $8.60 \pm 0.04$ & $0.81 \pm 0.00$ & $\mathrm{~S} 57$ & $2.04 \pm 0.13$ & $8.51 \pm 0.11$ & $0.83 \pm 0.00$ \\
\hline E18 & $1.73 \pm 0.09$ & $8.63 \pm 0.04$ & $0.79 \pm 0.00$ & D58 & $2.10 \pm 0.13$ & $9.12 \pm 0.12$ & $0.81 \pm 0.00$ \\
\hline $\mathrm{S} 20$ & $1.92 \pm 0.11$ & $7.87 \pm 0.19$ & $0.78 \pm 0.00$ & Y59 & $1.89 \pm 0.11$ & $7.98 \pm 0.04$ & $0.84 \pm 0.00$ \\
\hline D21 & $2.11 \pm 0.12$ & $8.88 \pm 0.10$ & $0.83 \pm 0.00$ & N60 & $2.05 \pm 0.09$ & $8.41 \pm 0.04$ & $0.85 \pm 0.00$ \\
\hline $\mathrm{T} 22$ & $1.99 \pm 0.10$ & $7.63 \pm 0.14$ & $0.83 \pm 0.00$ & $\mathrm{I} 61$ & $1.91 \pm 0.09$ & $8.26 \pm 0.04$ & $0.86 \pm 0.00$ \\
\hline $\mathrm{I} 23$ & $2.13 \pm 0.11$ & $10.35 \pm 0.07$ & $0.88 \pm 0.00$ & Q62 & $1.73 \pm 0.09$ & $8.04 \pm 0.08$ & $0.61 \pm 0.00$ \\
\hline $\mathrm{N} 25$ & $2.01 \pm 0.10$ & $11.78 \pm 0.05$ & $0.82 \pm 0.01$ & K63 & $1.88 \pm 0.07$ & $8.40 \pm 0.02$ & $0.81 \pm 0.00$ \\
\hline V26 & $2.02 \pm 0.08$ & $8.09 \pm 0.09$ & $0.85 \pm 0.00$ & E64 & $2.07 \pm 0.11$ & $8.26 \pm 0.08$ & $0.82 \pm 0.00$ \\
\hline $\mathrm{K} 27$ & $2.00 \pm 0.08$ & $8.94 \pm 0.03$ & $0.91 \pm 0.01$ & S65 & $1.90 \pm 0.12$ & $8.31 \pm 0.04$ & $0.78 \pm 0.00$ \\
\hline A 28 & $2.07 \pm 0.04$ & $8.77 \pm 0.12$ & $0.89 \pm 0.00$ & T66 & $1.95 \pm 0.08$ & $8.16 \pm 0.03$ & $0.76 \pm 0.00$ \\
\hline K29 & $1.98 \pm 0.03$ & $8.55 \pm 0.01$ & $0.91 \pm 0.00$ & $\mathrm{~L} 67$ & $2.02 \pm 0.07$ & $7.79 \pm 0.12$ & $0.86 \pm 0.00$ \\
\hline $\mathrm{I} 30$ & $1.94 \pm 0.04$ & $8.39 \pm 0.06$ & $0.87 \pm 0.00$ & H68 & $1.98 \pm 0.05$ & $8.57 \pm 0.04$ & $0.84 \pm 0.01$ \\
\hline Q31 & $2.06 \pm 0.03$ & $8.36 \pm 0.09$ & $0.90 \pm 0.01$ & L69 & $2.00 \pm 0.06$ & $8.19 \pm 0.08$ & $0.84 \pm 0.00$ \\
\hline D32 & $2.00 \pm 0.02$ & $8.74 \pm 0.02$ & $0.84 \pm 0.00$ & V70 & $2.02 \pm 0.07$ & $8.76 \pm 0.11$ & $0.89 \pm 0.01$ \\
\hline K33 & $1.93 \pm 0.01$ & $8.13 \pm 0.03$ & $0.87 \pm 0.00$ & L71 & $1.92 \pm 0.04$ & $7.83 \pm 0.08$ & $0.79 \pm 0.00$ \\
\hline E34 & $1.92 \pm 0.02$ & $8.23 \pm 0.07$ & $0.83 \pm 0.00$ & $\mathrm{R} 72$ & $2.00 \pm 0.10$ & $7.59 \pm 0.06$ & $0.74 \pm 0.00$ \\
\hline G35 & $1.89 \pm 0.02$ & $8.33 \pm 0.18$ & $0.86 \pm 0.00$ & $\mathrm{~L} 73$ & $2.06 \pm 0.14$ & $6.16 \pm 0.10$ & $0.44 \pm 0.00$ \\
\hline I36 & $1.64 \pm 0.01$ & $7.94 \pm 0.02$ & $0.95 \pm 0.00$ & $\mathrm{R} 74$ & $1.85 \pm 0.23$ & $4.69 \pm 0.05$ & $0.27 \pm 0.00$ \\
\hline D39 & $2.07 \pm 0.09$ & $8.36 \pm 0.10$ & $0.84 \pm 0.00$ & G75 & $1.29 \pm 0.22$ & $3.07 \pm 0.12$ & $-0.15 \pm 0.00$ \\
\hline Q40 & $1.91 \pm 0.08$ & $8.47 \pm 0.03$ & $0.85 \pm 0.00$ & G76 & $0.87 \pm 0.05$ & $1.75 \pm 0.02$ & $-1.06 \pm 0.00$ \\
\hline
\end{tabular}




\section{B.4 $700 \mathrm{MHz}$}

Table B.4: $R_{1}, R_{2}$ and het $N O E$ data measured on a $700 \mathrm{MHz}$ spectrometer at 294.5 K.

\begin{tabular}{|c|c|c|c|c|c|c|c|}
\hline Residue & $R_{1} / \mathrm{s}^{-1}$ & $R_{2} / \mathrm{s}^{-1}$ & het NOE & Residue & $R_{1} / \mathrm{s}^{-1}$ & $R_{2} / \mathrm{s}^{-1}$ & hetNOE \\
\hline Q2 & $1.59 \pm 0.04$ & $7.74 \pm 0.05$ & $0.70 \pm 0.00$ & Q41 & $1.66 \pm 0.05$ & $8.12 \pm 0.07$ & $0.86 \pm 0.00$ \\
\hline I3 & $1.59 \pm 0.05$ & $7.66 \pm 0.10$ & $0.90 \pm 0.00$ & R42 & $1.62 \pm 0.06$ & $7.36 \pm 0.07$ & $0.88 \pm 0.00$ \\
\hline $\mathrm{F} 4$ & $1.73 \pm 0.05$ & $8.28 \pm 0.07$ & $0.92 \pm 0.00$ & L43 & $1.50 \pm 0.09$ & $7.35 \pm 0.15$ & $0.84 \pm 0.00$ \\
\hline V5 & $1.59 \pm 0.04$ & $7.42 \pm 0.08$ & $0.86 \pm 0.00$ & I44 & $1.70 \pm 0.03$ & $7.43 \pm 0.06$ & $0.91 \pm 0.00$ \\
\hline K6 & $1.72 \pm 0.03$ & $7.33 \pm 0.15$ & $0.88 \pm 0.00$ & F45 & $1.63 \pm 0.05$ & $7.68 \pm 0.18$ & $0.88 \pm 0.00$ \\
\hline $\mathrm{T} 7$ & $1.55 \pm 0.11$ & $7.80 \pm 0.14$ & $0.78 \pm 0.00$ & A 46 & $1.71 \pm 0.14$ & $7.72 \pm 0.50$ & $0.73 \pm 0.01$ \\
\hline L8 & $1.65 \pm 0.14$ & $7.31 \pm 0.09$ & $0.74 \pm 0.00$ & G47 & $1.66 \pm 0.07$ & $8.57 \pm 0.06$ & $0.73 \pm 0.00$ \\
\hline T9 & $1.46 \pm 0.15$ & $8.87 \pm 0.03$ & $0.66 \pm 0.01$ & K48 & $1.61 \pm 0.05$ & $8.10 \pm 0.08$ & $0.86 \pm 0.00$ \\
\hline G10 & $1.51 \pm 0.13$ & $7.37 \pm 0.16$ & $0.64 \pm 0.00$ & Q49 & $1.53 \pm 0.07$ & $7.18 \pm 0.06$ & $0.69 \pm 0.00$ \\
\hline K11 & $1.41 \pm 0.12$ & $6.97 \pm 0.11$ & $0.59 \pm 0.00$ & L50 & $1.67 \pm 0.07$ & $7.72 \pm 0.22$ & $0.90 \pm 0.00$ \\
\hline $\mathrm{T} 12$ & $1.49 \pm 0.13$ & $7.35 \pm 0.13$ & $0.69 \pm 0.00$ & E51 & $1.36 \pm 0.12$ & $7.47 \pm 0.10$ & $0.77 \pm 0.00$ \\
\hline I13 & $1.68 \pm 0.05$ & $7.80 \pm 0.14$ & $0.84 \pm 0.01$ & D52 & $1.44 \pm 0.03$ & $7.77 \pm 0.08$ & $0.76 \pm 0.00$ \\
\hline T14 & $1.53 \pm 0.07$ & $7.97 \pm 0.08$ & $0.76 \pm 0.00$ & R54 & $1.58 \pm 0.05$ & $8.50 \pm 0.10$ & $0.88 \pm 0.00$ \\
\hline L15 & $1.65 \pm 0.04$ & $7.08 \pm 0.14$ & $0.89 \pm 0.00$ & T55 & $1.56 \pm 0.10$ & $8.60 \pm 0.24$ & $0.84 \pm 0.00$ \\
\hline E16 & $1.53 \pm 0.01$ & $7.03 \pm 0.02$ & $0.71 \pm 0.00$ & L56 & $1.70 \pm 0.09$ & $8.38 \pm 0.14$ & $0.87 \pm 0.00$ \\
\hline V17 & $1.55 \pm 0.11$ & $8.42 \pm 0.11$ & $0.79 \pm 0.00$ & S57 & $1.65 \pm 0.10$ & $7.56 \pm 0.13$ & $0.85 \pm 0.00$ \\
\hline E18 & $1.53 \pm 0.04$ & $8.35 \pm 0.13$ & $0.79 \pm 0.00$ & D58 & $1.67 \pm 0.11$ & $7.80 \pm 0.16$ & $0.85 \pm 0.00$ \\
\hline $\mathrm{S} 20$ & $1.60 \pm 0.08$ & $7.04 \pm 0.52$ & $0.79 \pm 0.00$ & Y59 & $1.58 \pm 0.07$ & $7.60 \pm 0.12$ & $0.84 \pm 0.00$ \\
\hline D21 & $1.74 \pm 0.07$ & $8.06 \pm 0.11$ & $0.86 \pm 0.00$ & N60 & $1.69 \pm 0.06$ & $8.06 \pm 0.11$ & $0.82 \pm 0.00$ \\
\hline $\mathrm{T} 22$ & $1.62 \pm 0.07$ & $7.73 \pm 0.13$ & $0.80 \pm 0.00$ & I61 & $1.68 \pm 0.05$ & $8.11 \pm 0.09$ & $0.86 \pm 0.00$ \\
\hline I 23 & $1.75 \pm 0.07$ & $10.60 \pm 0.11$ & $0.91 \pm 0.00$ & Q62 & $1.41 \pm 0.07$ & $6.39 \pm 0.17$ & $0.68 \pm 0.00$ \\
\hline $\mathrm{N} 25$ & $1.66 \pm 0.09$ & $12.43 \pm 0.09$ & $0.86 \pm 0.00$ & K63 & $1.56 \pm 0.05$ & $7.91 \pm 0.10$ & $0.78 \pm 0.00$ \\
\hline V26 & $1.65 \pm 0.06$ & $7.68 \pm 0.09$ & $0.86 \pm 0.00$ & E64 & $1.66 \pm 0.08$ & $7.54 \pm 0.07$ & $0.83 \pm 0.00$ \\
\hline $\mathrm{K} 27$ & $1.75 \pm 0.04$ & $8.56 \pm 0.08$ & $0.92 \pm 0.00$ & S65 & $1.58 \pm 0.11$ & $7.98 \pm 0.10$ & $0.84 \pm 0.00$ \\
\hline $\mathrm{A} 28$ & $1.80 \pm 0.02$ & $8.03 \pm 0.05$ & $0.92 \pm 0.00$ & T66 & $1.58 \pm 0.06$ & $7.88 \pm 0.08$ & $0.76 \pm 0.00$ \\
\hline K29 & $1.73 \pm 0.01$ & $7.92 \pm 0.06$ & $0.88 \pm 0.00$ & L67 & $1.70 \pm 0.03$ & $7.48 \pm 0.15$ & $0.92 \pm 0.00$ \\
\hline I30 & $1.75 \pm 0.01$ & $7.66 \pm 0.05$ & $0.91 \pm 0.00$ & $\mathrm{H} 68$ & $1.68 \pm 0.03$ & $8.21 \pm 0.06$ & $0.85 \pm 0.00$ \\
\hline Q31 & $1.81 \pm 0.01$ & $7.60 \pm 0.07$ & $0.89 \pm 0.00$ & L69 & $1.67 \pm 0.03$ & $7.30 \pm 0.07$ & $0.92 \pm 0.00$ \\
\hline D32 & $1.79 \pm 0.01$ & $8.12 \pm 0.03$ & $0.90 \pm 0.00$ & V70 & $1.71 \pm 0.05$ & $11.54 \pm 0.12$ & $0.92 \pm 0.00$ \\
\hline K33 & $1.71 \pm 0.01$ & $7.56 \pm 0.05$ & $0.92 \pm 0.00$ & L71 & $1.71 \pm 0.02$ & $7.07 \pm 0.04$ & $0.69 \pm 0.00$ \\
\hline E34 & $1.66 \pm 0.01$ & $7.17 \pm 0.02$ & $0.85 \pm 0.00$ & $\mathrm{R} 72$ & $1.63 \pm 0.08$ & $6.59 \pm 0.09$ & $0.78 \pm 0.00$ \\
\hline G35 & $1.67 \pm 0.01$ & $8.13 \pm 0.33$ & $0.87 \pm 0.00$ & L73 & $1.58 \pm 0.11$ & $4.91 \pm 0.15$ & $0.46 \pm 0.00$ \\
\hline I36 & $1.46 \pm 0.00$ & $7.36 \pm 0.04$ & $0.94 \pm 0.00$ & R74 & $1.38 \pm 0.12$ & $4.17 \pm 0.09$ & $0.25 \pm 0.00$ \\
\hline D39 & $1.70 \pm 0.07$ & $7.59 \pm 0.05$ & $0.86 \pm 0.00$ & G75 & $1.07 \pm 0.12$ & $2.97 \pm 0.24$ & $-0.09 \pm 0.00$ \\
\hline Q40 & $1.62 \pm 0.05$ & $8.05 \pm 0.08$ & $0.83 \pm 0.00$ & G76 & $0.73 \pm 0.05$ & $2.00 \pm 0.07$ & $-0.61 \pm 0.00$ \\
\hline
\end{tabular}




\section{B.5 $800 \mathrm{MHz}$}

Table B.5: $R_{1}, R_{2}$ and het $N O E$ data measured on a $800 \mathrm{MHz}$ spectrometer at 294.5 K.

\begin{tabular}{|c|c|c|c|c|c|c|c|}
\hline Residue & $R_{1} / \mathrm{s}^{-1}$ & $R_{2} / \mathrm{s}^{-1}$ & hetNOE & Residue & $R_{1} / \mathrm{s}^{-1}$ & $R_{2} / \mathrm{s}^{-1}$ & hetNOE \\
\hline Q2 & $1.41 \pm 0.05$ & $9.22 \pm 0.05$ & $0.75 \pm 0.0$ & Q41 & $1.44 \pm 0.05$ & $9.45 \pm 0.07$ & $0.90 \pm 0.0$ \\
\hline I3 & $1.43 \pm 0.07$ & $8.83 \pm 0.07$ & $0.97 \pm 0.0$ & $\mathrm{R} 42$ & $1.30 \pm 0.11$ & $8.71 \pm 0.08$ & $0.91 \pm 0.0$ \\
\hline $\mathrm{F} 4$ & $1.52 \pm 0.07$ & $9.63 \pm 0.06$ & $0.99 \pm 0.0$ & $\mathrm{~L} 43$ & $1.35 \pm 0.06$ & $8.89 \pm 0.10$ & $0.88 \pm 0.0$ \\
\hline V5 & $1.35 \pm 0.05$ & $8.77 \pm 0.06$ & $0.95 \pm 0.0$ & I 44 & $1.41 \pm 0.05$ & $8.80 \pm 0.04$ & $0.96 \pm 0.0$ \\
\hline K6 & $1.37 \pm 0.12$ & $9.29 \pm 0.11$ & $0.94 \pm 0.0$ & F45 & $1.38 \pm 0.05$ & $9.43 \pm 0.11$ & $0.96 \pm 0.0$ \\
\hline $\mathrm{T} 7$ & $1.44 \pm 0.08$ & $9.22 \pm 0.12$ & $0.82 \pm 0.0$ & A 46 & $1.82 \pm 0.19$ & $10.08 \pm 0.19$ & $0.80 \pm 0.0$ \\
\hline L8 & $1.82 \pm 0.14$ & $8.98 \pm 0.08$ & $0.78 \pm 0.0$ & G47 & $1.49 \pm 0.05$ & $8.28 \pm 0.09$ & $0.86 \pm 0.0$ \\
\hline $\mathrm{T} 9$ & $1.70 \pm 0.23$ & $9.30 \pm 0.14$ & $0.78 \pm 0.0$ & K48 & $1.37 \pm 0.06$ & $9.90 \pm 0.07$ & $0.86 \pm 0.0$ \\
\hline G10 & $1.67 \pm 0.10$ & $10.85 \pm 0.15$ & $0.75 \pm 0.0$ & Q49 & $1.46 \pm 0.06$ & $8.70 \pm 0.06$ & $0.80 \pm 0.0$ \\
\hline K11 & $1.53 \pm 0.08$ & $8.31 \pm 0.07$ & $0.70 \pm 0.0$ & L50 & $1.51 \pm 0.08$ & $9.40 \pm 0.08$ & $0.96 \pm 0.0$ \\
\hline $\mathrm{T} 12$ & $1.65 \pm 0.12$ & $8.70 \pm 0.08$ & $0.79 \pm 0.0$ & E51 & $1.29 \pm 0.07$ & $8.75 \pm 0.07$ & $0.84 \pm 0.0$ \\
\hline $\mathrm{I} 13$ & $1.49 \pm 0.06$ & $9.92 \pm 0.12$ & $0.95 \pm 0.0$ & D52 & $1.23 \pm 0.03$ & $9.30 \pm 0.05$ & $0.79 \pm 0.0$ \\
\hline $\mathrm{T} 14$ & $1.42 \pm 0.06$ & $9.60 \pm 0.09$ & $0.80 \pm 0.0$ & $\mathrm{R} 54$ & $1.34 \pm 0.05$ & $9.94 \pm 0.08$ & $0.89 \pm 0.0$ \\
\hline L15 & $1.47 \pm 0.06$ & $8.71 \pm 0.05$ & $0.96 \pm 0.0$ & $\mathrm{~T} 55$ & $1.38 \pm 0.07$ & $11.84 \pm 0.20$ & $0.92 \pm 0.0$ \\
\hline E16 & $1.28 \pm 0.03$ & $8.44 \pm 0.05$ & $0.87 \pm 0.0$ & $\mathrm{~L} 56$ & $1.51 \pm 0.09$ & $9.60 \pm 0.10$ & $0.95 \pm 0.0$ \\
\hline V17 & $1.47 \pm 0.08$ & $9.64 \pm 0.08$ & $0.86 \pm 0.0$ & $\mathrm{~S} 57$ & $1.54 \pm 0.09$ & $9.04 \pm 0.12$ & $0.88 \pm 0.0$ \\
\hline E18 & $1.30 \pm 0.12$ & $9.63 \pm 0.07$ & $0.88 \pm 0.0$ & D58 & $1.53 \pm 0.08$ & $9.41 \pm 0.13$ & $0.87 \pm 0.0$ \\
\hline $\mathrm{S} 20$ & $1.37 \pm 0.07$ & $9.12 \pm 0.11$ & $0.87 \pm 0.0$ & Y59 & $1.41 \pm 0.07$ & $8.80 \pm 0.09$ & $0.89 \pm 0.0$ \\
\hline D21 & $1.56 \pm 0.08$ & $9.73 \pm 0.09$ & $0.95 \pm 0.0$ & N60 & $1.46 \pm 0.06$ & $9.42 \pm 0.08$ & $0.90 \pm 0.0$ \\
\hline $\mathrm{T} 22$ & $1.43 \pm 0.05$ & $10.86 \pm 0.19$ & $0.88 \pm 0.0$ & $\mathrm{I} 61$ & $1.45 \pm 0.06$ & $9.28 \pm 0.07$ & $0.95 \pm 0.0$ \\
\hline $\mathrm{I} 23$ & $1.55 \pm 0.08$ & $12.93 \pm 0.15$ & $0.93 \pm 0.0$ & Q62 & $1.32 \pm 0.06$ & $7.76 \pm 0.07$ & $0.74 \pm 0.0$ \\
\hline $\mathrm{N} 25$ & $1.46 \pm 0.06$ & $15.47 \pm 0.14$ & $0.88 \pm 0.0$ & K63 & $1.41 \pm 0.05$ & $9.50 \pm 0.06$ & $0.78 \pm 0.0$ \\
\hline $\mathrm{V} 26$ & $1.41 \pm 0.05$ & $9.14 \pm 0.07$ & $0.94 \pm 0.0$ & E64 & $1.50 \pm 0.07$ & $8.81 \pm 0.09$ & $0.93 \pm 0.0$ \\
\hline $\mathrm{K} 27$ & $1.54 \pm 0.06$ & $10.12 \pm 0.08$ & $0.95 \pm 0.0$ & S65 & $1.37 \pm 0.10$ & $9.37 \pm 0.09$ & $0.86 \pm 0.0$ \\
\hline $\mathrm{A} 28$ & $1.49 \pm 0.04$ & $9.67 \pm 0.03$ & $0.92 \pm 0.0$ & T66 & $1.44 \pm 0.05$ & $9.11 \pm 0.07$ & $0.80 \pm 0.0$ \\
\hline K29 & $1.45 \pm 0.03$ & $9.51 \pm 0.05$ & $0.97 \pm 0.0$ & $\mathrm{~L} 67$ & $1.52 \pm 0.05$ & $9.48 \pm 0.10$ & $0.97 \pm 0.0$ \\
\hline $\mathrm{I} 30$ & $1.52 \pm 0.03$ & $9.42 \pm 0.05$ & $0.97 \pm 0.0$ & H68 & $1.43 \pm 0.04$ & $9.64 \pm 0.06$ & $0.89 \pm 0.0$ \\
\hline Q31 & $1.55 \pm 0.02$ & $9.51 \pm 0.04$ & $0.94 \pm 0.0$ & L69 & $1.47 \pm 0.05$ & $8.83 \pm 0.05$ & $0.93 \pm 0.0$ \\
\hline D32 & $1.50 \pm 0.01$ & $9.86 \pm 0.03$ & $0.94 \pm 0.0$ & V70 & $1.48 \pm 0.06$ & $11.73 \pm 0.09$ & $0.90 \pm 0.0$ \\
\hline K33 & $1.43 \pm 0.01$ & $9.11 \pm 0.03$ & $0.90 \pm 0.0$ & $\mathrm{~L} 71$ & $1.42 \pm 0.03$ & $8.84 \pm 0.05$ & $0.83 \pm 0.0$ \\
\hline E34 & $1.42 \pm 0.01$ & $8.73 \pm 0.03$ & $0.88 \pm 0.0$ & $\mathrm{R} 72$ & $1.56 \pm 0.07$ & $8.12 \pm 0.08$ & $0.88 \pm 0.0$ \\
\hline G35 & $1.41 \pm 0.01$ & $11.65 \pm 0.18$ & $0.90 \pm 0.0$ & $\mathrm{~L} 73$ & $1.72 \pm 0.09$ & $6.10 \pm 0.07$ & $0.68 \pm 0.0$ \\
\hline I36 & $1.23 \pm 0.01$ & $9.17 \pm 0.03$ & $1.03 \pm 0.0$ & $\mathrm{R} 74$ & $1.70 \pm 0.18$ & $5.25 \pm 0.07$ & $0.44 \pm 0.0$ \\
\hline D39 & $1.61 \pm 0.07$ & $9.04 \pm 0.09$ & $0.88 \pm 0.0$ & G75 & $1.37 \pm 0.20$ & $5.06 \pm 0.13$ & $0.17 \pm 0.0$ \\
\hline Q40 & $1.46 \pm 0.05$ & $9.54 \pm 0.05$ & $0.93 \pm 0.0$ & G76 & $0.85 \pm 0.03$ & $1.74 \pm 0.03$ & $-0.61 \pm 0.0$ \\
\hline
\end{tabular}




\section{B.6 $900 \mathrm{MHz}$}

Table B.6: $R_{1}, R_{2}$ and het $N O E$ data measured on a $900 \mathrm{MHz}$ spectrometer at 294.5 K.

\begin{tabular}{|c|c|c|c|c|c|c|c|}
\hline Residue & $R_{1} / \mathrm{s}^{-1}$ & $R_{2} / \mathrm{s}^{-1}$ & het NOE & Residue & $R_{1} / \mathrm{s}^{-1}$ & $R_{2} / \mathrm{s}^{-1}$ & het NOE \\
\hline Q2 & $1.22 \pm 0.12$ & $9.00 \pm 0.32$ & $0.80 \pm 0.0$ & Q41 & $1.31 \pm 0.15$ & $9.69 \pm 0.30$ & $0.95 \pm 0.0$ \\
\hline I3 & $1.27 \pm 0.16$ & $9.20 \pm 0.37$ & $0.99 \pm 0.0$ & $\mathrm{R} 42$ & $1.25 \pm 0.16$ & $8.48 \pm 0.36$ & $0.94 \pm 0.0$ \\
\hline F4 & $1.29 \pm 0.15$ & $9.68 \pm 0.38$ & $0.99 \pm 0.0$ & L43 & $1.20 \pm 0.15$ & $12.03 \pm 0.47$ & $0.92 \pm 0.0$ \\
\hline V5 & $1.20 \pm 0.13$ & $8.44 \pm 0.27$ & $0.98 \pm 0.0$ & I44 & $1.26 \pm 0.14$ & $8.28 \pm 0.32$ & $0.98 \pm 0.0$ \\
\hline K6 & $1.33 \pm 0.15$ & $8.97 \pm 0.39$ & $0.98 \pm 0.0$ & F45 & $1.22 \pm 0.14$ & $12.11 \pm 0.47$ & $0.98 \pm 0.0$ \\
\hline $\mathrm{T} 7$ & $1.28 \pm 0.16$ & $9.68 \pm 0.36$ & $0.86 \pm 0.0$ & A 46 & $1.62 \pm 0.29$ & $9.13 \pm 0.37$ & $0.83 \pm 0.0$ \\
\hline L8 & $1.57 \pm 0.23$ & $9.15 \pm 0.36$ & $0.77 \pm 0.0$ & G47 & $1.34 \pm 0.15$ & $8.64 \pm 0.30$ & $0.85 \pm 0.0$ \\
\hline T9 & $1.45 \pm 0.28$ & $9.61 \pm 0.36$ & $0.74 \pm 0.0$ & K48 & $1.23 \pm 0.13$ & $9.74 \pm 0.36$ & $0.94 \pm 0.0$ \\
\hline G10 & $1.48 \pm 0.20$ & $9.88 \pm 0.44$ & $0.73 \pm 0.0$ & Q49 & $1.31 \pm 0.15$ & $8.68 \pm 0.27$ & $0.83 \pm 0.0$ \\
\hline K11 & $1.38 \pm 0.17$ & $8.39 \pm 0.28$ & $0.74 \pm 0.0$ & L50 & $1.30 \pm 0.17$ & $11.40 \pm 0.44$ & $0.96 \pm 0.0$ \\
\hline $\mathrm{T} 12$ & $1.45 \pm 0.20$ & $8.77 \pm 0.32$ & $0.80 \pm 0.0$ & E51 & $1.12 \pm 0.13$ & $9.05 \pm 0.33$ & $0.87 \pm 0.0$ \\
\hline $\mathrm{I} 13$ & $1.25 \pm 0.14$ & $9.85 \pm 0.36$ & $0.93 \pm 0.0$ & D52 & $1.14 \pm 0.12$ & $9.15 \pm 0.26$ & $0.90 \pm 0.0$ \\
\hline $\mathrm{T} 14$ & $1.22 \pm 0.13$ & $9.88 \pm 0.30$ & $0.84 \pm 0.0$ & R54 & $1.23 \pm 0.15$ & $9.92 \pm 0.38$ & $0.96 \pm 0.0$ \\
\hline L15 & $1.29 \pm 0.15$ & $10.71 \pm 0.38$ & $1.01 \pm 0.0$ & T55 & $1.25 \pm 0.16$ & $12.84 \pm 0.53$ & $0.92 \pm 0.0$ \\
\hline E16 & $1.18 \pm 0.10$ & $8.28 \pm 0.15$ & $0.92 \pm 0.0$ & L56 & $1.32 \pm 0.19$ & $9.86 \pm 0.45$ & $0.97 \pm 0.0$ \\
\hline V17 & $1.28 \pm 0.17$ & $9.90 \pm 0.40$ & $0.86 \pm 0.0$ & S57 & $1.31 \pm 0.18$ & $9.04 \pm 0.37$ & $0.90 \pm 0.0$ \\
\hline E18 & $1.16 \pm 0.14$ & $9.44 \pm 0.46$ & $0.93 \pm 0.0$ & D58 & $1.39 \pm 0.20$ & $12.18 \pm 0.55$ & $0.93 \pm 0.0$ \\
\hline $\mathrm{S} 20$ & $1.23 \pm 0.15$ & $8.57 \pm 0.31$ & $0.90 \pm 0.0$ & Y59 & $1.24 \pm 0.16$ & $9.00 \pm 0.37$ & $0.93 \pm 0.0$ \\
\hline D21 & $1.37 \pm 0.18$ & $13.20 \pm 0.62$ & $0.92 \pm 0.0$ & N60 & $1.31 \pm 0.15$ & $9.59 \pm 0.35$ & $0.96 \pm 0.0$ \\
\hline $\mathrm{T} 22$ & $1.30 \pm 0.15$ & $11.09 \pm 0.42$ & $0.90 \pm 0.0$ & I61 & $1.31 \pm 0.16$ & $9.23 \pm 0.37$ & $0.95 \pm 0.0$ \\
\hline I 23 & $1.31 \pm 0.16$ & $13.48 \pm 0.53$ & $0.96 \pm 0.0$ & Q62 & $1.15 \pm 0.12$ & $10.17 \pm 0.39$ & $0.70 \pm 0.0$ \\
\hline N25 & $1.32 \pm 0.17$ & $16.67 \pm 0.44$ & $0.91 \pm 0.0$ & K63 & $1.23 \pm 0.13$ & $9.30 \pm 0.29$ & $0.81 \pm 0.0$ \\
\hline V26 & $1.30 \pm 0.16$ & $9.02 \pm 0.33$ & $0.96 \pm 0.0$ & E64 & $1.32 \pm 0.16$ & $8.85 \pm 0.36$ & $0.93 \pm 0.0$ \\
\hline $\mathrm{K} 27$ & $1.34 \pm 0.16$ & $10.20 \pm 0.35$ & $0.97 \pm 0.0$ & S65 & $1.31 \pm 0.17$ & $9.40 \pm 0.38$ & $0.91 \pm 0.0$ \\
\hline $\mathrm{A} 28$ & $1.37 \pm 0.14$ & $11.13 \pm 0.26$ & $0.98 \pm 0.0$ & T66 & $1.26 \pm 0.14$ & $10.07 \pm 0.24$ & $0.84 \pm 0.0$ \\
\hline K29 & $1.32 \pm 0.12$ & $9.49 \pm 0.20$ & $0.96 \pm 0.0$ & L67 & $1.28 \pm 0.13$ & $9.27 \pm 0.29$ & $0.99 \pm 0.0$ \\
\hline I30 & $1.32 \pm 0.11$ & $9.17 \pm 0.20$ & $0.97 \pm 0.0$ & $\mathrm{H} 68$ & $1.27 \pm 0.13$ & $9.63 \pm 0.29$ & $0.95 \pm 0.0$ \\
\hline Q31 & $1.34 \pm 0.12$ & $11.25 \pm 0.20$ & $0.98 \pm 0.0$ & L69 & $1.30 \pm 0.13$ & $12.46 \pm 0.42$ & $0.93 \pm 0.0$ \\
\hline D32 & $1.32 \pm 0.10$ & $9.94 \pm 0.12$ & $0.95 \pm 0.0$ & V70 & $1.28 \pm 0.15$ & $12.76 \pm 0.42$ & $0.94 \pm 0.0$ \\
\hline K33 & $1.31 \pm 0.10$ & $9.71 \pm 0.12$ & $0.95 \pm 0.0$ & $\mathrm{~L} 71$ & $1.33 \pm 0.12$ & $8.87 \pm 0.18$ & $0.92 \pm 0.0$ \\
\hline E34 & $1.26 \pm 0.09$ & $8.96 \pm 0.14$ & $0.91 \pm 0.0$ & $\mathrm{R} 72$ & $1.37 \pm 0.18$ & $10.81 \pm 0.44$ & $0.88 \pm 0.0$ \\
\hline G35 & $1.25 \pm 0.09$ & $12.48 \pm 0.25$ & $0.92 \pm 0.0$ & $\mathrm{~L} 73$ & $1.64 \pm 0.23$ & $7.40 \pm 0.37$ & $0.73 \pm 0.0$ \\
\hline I36 & $1.13 \pm 0.08$ & $8.92 \pm 0.21$ & $1.05 \pm 0.0$ & R74 & $1.65 \pm 0.28$ & $4.96 \pm 0.23$ & $0.52 \pm 0.0$ \\
\hline D39 & $1.41 \pm 0.17$ & $9.41 \pm 0.27$ & $0.86 \pm 0.0$ & G75 & $1.35 \pm 0.27$ & $4.05 \pm 0.27$ & $0.28 \pm 0.0$ \\
\hline $\mathrm{Q} 40$ & $1.30 \pm 0.15$ & $9.93 \pm 0.32$ & $0.91 \pm 0.0$ & G76 & $0.91 \pm 0.08$ & $1.52 \pm 0.13$ & $-0.06 \pm 0.0$ \\
\hline
\end{tabular}




\section{B.7 $950 \mathrm{MHz}$}

Table B.7: $R_{1}, R_{2}$ and het $N O E$ data measured on a $950 \mathrm{MHz}$ spectrometer at 294.5 K.

\begin{tabular}{|c|c|c|c|c|c|c|c|}
\hline Residue & $R_{1} / \mathrm{s}^{-1}$ & $R_{2} / \mathrm{s}^{-1}$ & hetNOE & Residue & $R_{1} / \mathrm{s}^{-1}$ & $R_{2} / \mathrm{s}^{-1}$ & hetNOE \\
\hline Q2 & $1.17 \pm 0.05$ & $10.92 \pm 0.19$ & $0.82 \pm 0.00$ & Q41 & $1.28 \pm 0.07$ & $11.19 \pm 0.20$ & $0.90 \pm 0.00$ \\
\hline I3 & $1.27 \pm 0.09$ & $10.27 \pm 0.29$ & $0.94 \pm 0.00$ & $\mathrm{R} 42$ & $1.19 \pm 0.08$ & $10.14 \pm 0.30$ & $0.96 \pm 0.00$ \\
\hline F4 & $1.28 \pm 0.08$ & $11.22 \pm 0.22$ & $1.01 \pm 0.00$ & $\mathrm{~L} 43$ & $1.19 \pm 0.08$ & $10.47 \pm 0.42$ & $0.92 \pm 0.00$ \\
\hline V5 & $1.14 \pm 0.06$ & $10.46 \pm 0.20$ & $0.98 \pm 0.00$ & I 44 & $1.20 \pm 0.06$ & $10.25 \pm 0.16$ & $0.98 \pm 0.00$ \\
\hline K6 & $1.24 \pm 0.06$ & $10.17 \pm 0.16$ & $0.99 \pm 0.00$ & F45 & $1.23 \pm 0.09$ & $11.34 \pm 0.29$ & $0.98 \pm 0.00$ \\
\hline $\mathrm{T} 7$ & $1.23 \pm 0.08$ & $11.10 \pm 0.24$ & $0.85 \pm 0.00$ & A 46 & $1.56 \pm 0.18$ & $11.66 \pm 0.44$ & $0.85 \pm 0.01$ \\
\hline L8 & $1.55 \pm 0.12$ & $10.55 \pm 0.18$ & $0.78 \pm 0.00$ & G47 & $1.31 \pm 0.08$ & $11.48 \pm 0.74$ & $0.82 \pm 0.00$ \\
\hline $\mathrm{T} 9$ & $1.46 \pm 0.20$ & $11.62 \pm 0.29$ & $0.76 \pm 0.01$ & K48 & $1.25 \pm 0.08$ & $12.09 \pm 0.28$ & $0.92 \pm 0.00$ \\
\hline G10 & $1.46 \pm 0.10$ & $9.64 \pm 0.20$ & $0.76 \pm 0.00$ & Q49 & $1.28 \pm 0.06$ & $10.43 \pm 0.19$ & $0.84 \pm 0.00$ \\
\hline K11 & $1.38 \pm 0.09$ & $10.53 \pm 0.25$ & $0.70 \pm 0.00$ & $\mathrm{~L} 50$ & $1.25 \pm 0.08$ & $10.98 \pm 0.20$ & $0.95 \pm 0.00$ \\
\hline $\mathrm{T} 12$ & $1.44 \pm 0.11$ & $10.50 \pm 0.20$ & $0.82 \pm 0.00$ & E51 & $1.10 \pm 0.07$ & $10.05 \pm 0.29$ & $0.87 \pm 0.00$ \\
\hline $\mathrm{I} 13$ & $1.25 \pm 0.07$ & $11.07 \pm 0.23$ & $0.93 \pm 0.00$ & D52 & $1.07 \pm 0.04$ & $11.06 \pm 0.17$ & $0.91 \pm 0.00$ \\
\hline $\mathrm{T} 14$ & $1.21 \pm 0.07$ & $11.73 \pm 0.16$ & $0.84 \pm 0.00$ & $\mathrm{R} 54$ & $1.19 \pm 0.07$ & $11.66 \pm 0.25$ & $0.93 \pm 0.00$ \\
\hline L15 & $1.26 \pm 0.07$ & $10.46 \pm 0.23$ & $0.99 \pm 0.00$ & $\mathrm{~T} 55$ & $1.26 \pm 0.09$ & $11.73 \pm 0.34$ & $0.91 \pm 0.00$ \\
\hline E16 & $1.09 \pm 0.03$ & $9.84 \pm 0.12$ & $0.86 \pm 0.00$ & $\mathrm{~L} 56$ & $1.28 \pm 0.11$ & $11.42 \pm 0.31$ & $0.95 \pm 0.00$ \\
\hline V17 & $1.21 \pm 0.08$ & $11.47 \pm 0.29$ & $0.87 \pm 0.00$ & $\mathrm{~S} 57$ & $1.29 \pm 0.09$ & $10.75 \pm 0.28$ & $0.88 \pm 0.00$ \\
\hline E18 & $1.14 \pm 0.07$ & $11.44 \pm 0.27$ & $0.93 \pm 0.00$ & D58 & $1.37 \pm 0.10$ & $11.28 \pm 0.29$ & $0.90 \pm 0.00$ \\
\hline $\mathrm{S} 20$ & $1.18 \pm 0.07$ & $10.96 \pm 0.34$ & $0.83 \pm 0.00$ & Y59 & $1.22 \pm 0.08$ & $10.46 \pm 0.26$ & $0.88 \pm 0.00$ \\
\hline D21 & $1.36 \pm 0.09$ & $11.65 \pm 0.20$ & $0.92 \pm 0.00$ & N60 & $1.28 \pm 0.07$ & $11.14 \pm 0.26$ & $0.92 \pm 0.00$ \\
\hline $\mathrm{T} 22$ & $1.27 \pm 0.07$ & $10.43 \pm 0.18$ & $0.85 \pm 0.00$ & $\mathrm{I} 61$ & $1.26 \pm 0.08$ & $12.56 \pm 0.59$ & $0.93 \pm 0.00$ \\
\hline $\mathrm{I} 23$ & $1.29 \pm 0.08$ & $16.35 \pm 0.36$ & $0.95 \pm 0.00$ & Q62 & $1.11 \pm 0.10$ & $9.84 \pm 0.19$ & $0.70 \pm 0.00$ \\
\hline $\mathrm{N} 25$ & $1.32 \pm 0.09$ & $19.40 \pm 0.57$ & $0.88 \pm 0.00$ & K63 & $1.21 \pm 0.05$ & $11.44 \pm 0.20$ & $0.83 \pm 0.00$ \\
\hline V26 & $1.28 \pm 0.07$ & $10.44 \pm 0.18$ & $0.93 \pm 0.00$ & E64 & $1.26 \pm 0.08$ & $10.82 \pm 0.22$ & $0.92 \pm 0.00$ \\
\hline $\mathrm{K} 27$ & $1.28 \pm 0.06$ & $11.94 \pm 0.23$ & $0.95 \pm 0.00$ & S65 & $1.28 \pm 0.09$ & $14.25 \pm 1.02$ & $0.87 \pm 0.00$ \\
\hline A 28 & $1.35 \pm 0.05$ & $11.74 \pm 0.23$ & $0.96 \pm 0.00$ & T66 & $1.22 \pm 0.06$ & $11.51 \pm 0.20$ & $0.83 \pm 0.00$ \\
\hline K29 & $1.27 \pm 0.04$ & $11.41 \pm 0.12$ & $0.94 \pm 0.00$ & $\mathrm{~L} 67$ & $1.27 \pm 0.06$ & $10.31 \pm 0.23$ & $1.01 \pm 0.00$ \\
\hline $\mathrm{I} 30$ & $1.32 \pm 0.04$ & $11.46 \pm 0.14$ & $0.97 \pm 0.00$ & H68 & $1.22 \pm 0.05$ & $11.32 \pm 0.22$ & $0.96 \pm 0.00$ \\
\hline Q31 & $1.30 \pm 0.03$ & $11.57 \pm 0.18$ & $0.94 \pm 0.00$ & L69 & $1.26 \pm 0.06$ & $10.27 \pm 0.15$ & $0.94 \pm 0.00$ \\
\hline D32 & $1.32 \pm 0.02$ & $11.78 \pm 0.10$ & $0.97 \pm 0.00$ & V70 & $1.24 \pm 0.06$ & $13.18 \pm 0.22$ & $0.97 \pm 0.00$ \\
\hline K33 & $1.25 \pm 0.02$ & $11.35 \pm 0.13$ & $0.92 \pm 0.00$ & L71 & $1.24 \pm 0.03$ & $10.63 \pm 0.09$ & $0.88 \pm 0.00$ \\
\hline E34 & $1.21 \pm 0.02$ & $10.99 \pm 0.11$ & $0.93 \pm 0.00$ & $\mathrm{R} 72$ & $1.34 \pm 0.08$ & $9.80 \pm 0.16$ & $0.87 \pm 0.00$ \\
\hline G35 & $1.19 \pm 0.01$ & $11.87 \pm 0.15$ & $0.94 \pm 0.00$ & $\mathrm{~L} 73$ & $1.62 \pm 0.10$ & $7.29 \pm 0.24$ & $0.72 \pm 0.00$ \\
\hline I36 & $1.10 \pm 0.01$ & $11.30 \pm 0.11$ & $0.99 \pm 0.00$ & $\mathrm{R} 74$ & $1.68 \pm 0.19$ & $6.60 \pm 0.16$ & $0.59 \pm 0.00$ \\
\hline D39 & $1.35 \pm 0.07$ & $11.12 \pm 0.21$ & $0.87 \pm 0.00$ & G75 & $1.39 \pm 0.21$ & $4.35 \pm 0.35$ & $0.35 \pm 0.00$ \\
\hline Q40 & $1.24 \pm 0.06$ & $11.33 \pm 0.20$ & $0.92 \pm 0.00$ & G76 & $0.93 \pm 0.04$ & $2.74 \pm 0.09$ & $-0.00 \pm 0.00$ \\
\hline
\end{tabular}




\section{Appendix C}

\section{Pulse Programs}

The pulse programs used to acquire the E-CPMG data shown in Chapter 2 and Chapter 3 as well as the used shuttle experiments to acquire the relaxation data in Chapter 4 are presented here in Bruker notation.

\section{C.1 Backbone ${ }^{15}$ N E-CPMG}

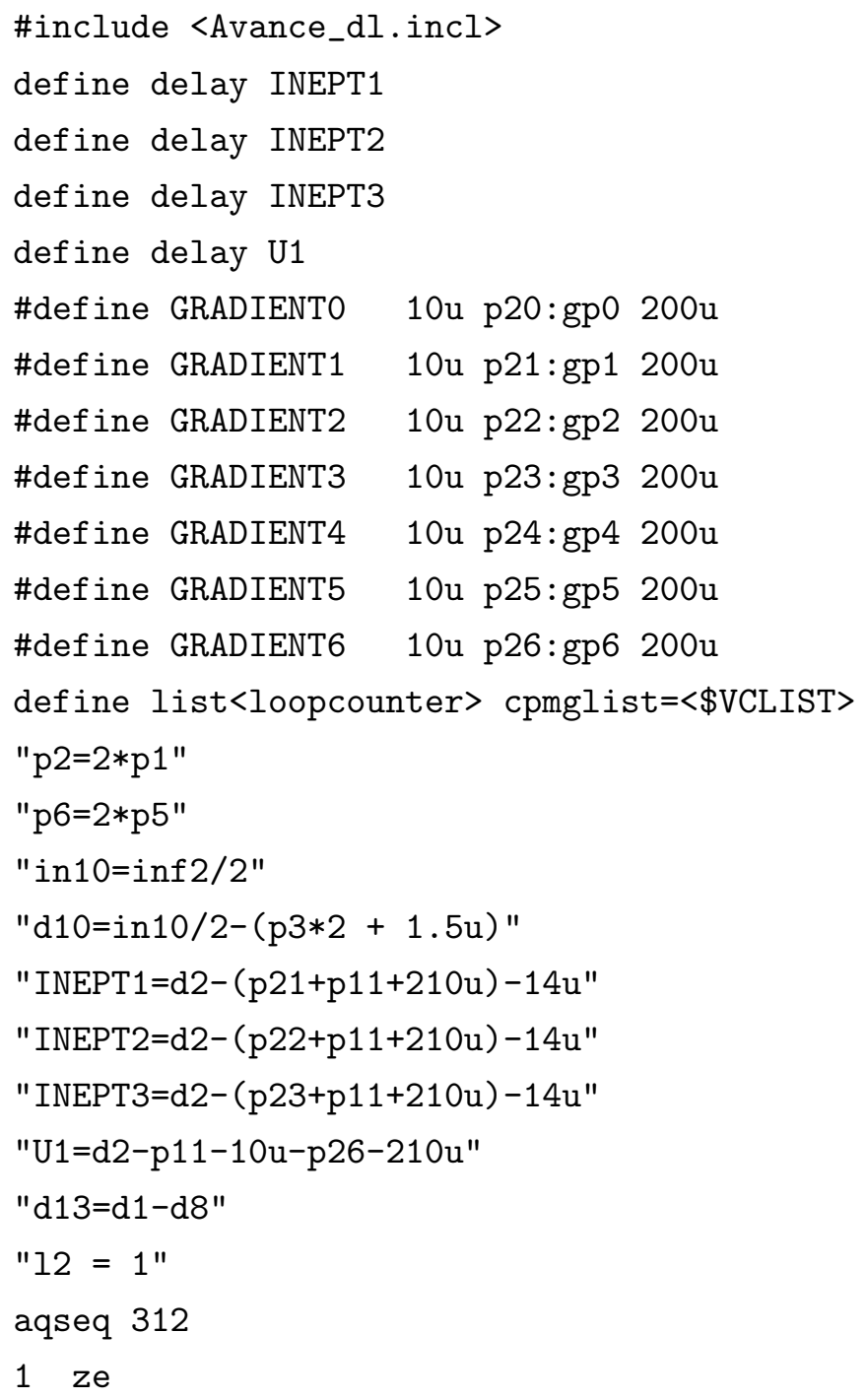


$20 \mathrm{~m}$ sto

$26 \mathrm{~m}$

$33 \mathrm{~m}$

$43 \mathrm{~m}$

20u pl1:f1

20u pl2:f2

20u pl3:f3

if "cpmglist < 113"

\{

if "cpmglist > 0"

\{

"13 = cpmglist"

"14=113-13"

"d14=d8/(16*13)-p5"

"d16=d8/(16*14)-p5"

\}

else

\{

"13=0"

"14=113"

"d14=p5"

"d16=p5"

\}

\}

else

\{

"13=113"

"14=0"

"d14=p5"

"d16=p5"

\}

d13*0. 5

$5 \mathrm{~d} 16$

(p6 ph21):f3

d16

d16

(p6 ph21):f3

d16

d16

(p6 ph20):f3

d16 
d16

(p6 ph22):f3

d16

d16

(p6 ph20):f3

d16

d16

(p6 ph20):f3

d16

d16

(p6 ph21):f3

d16

d16

(p6 ph23):f3

d16

lo to 5 times 14

$810 \mathrm{u}$

$\mathrm{d} 13 * 0.5$

$20 \mathrm{u} \mathrm{pl1:f1}$

20u pl2:f2

20u pl3:f3

20u LOCKH_ON

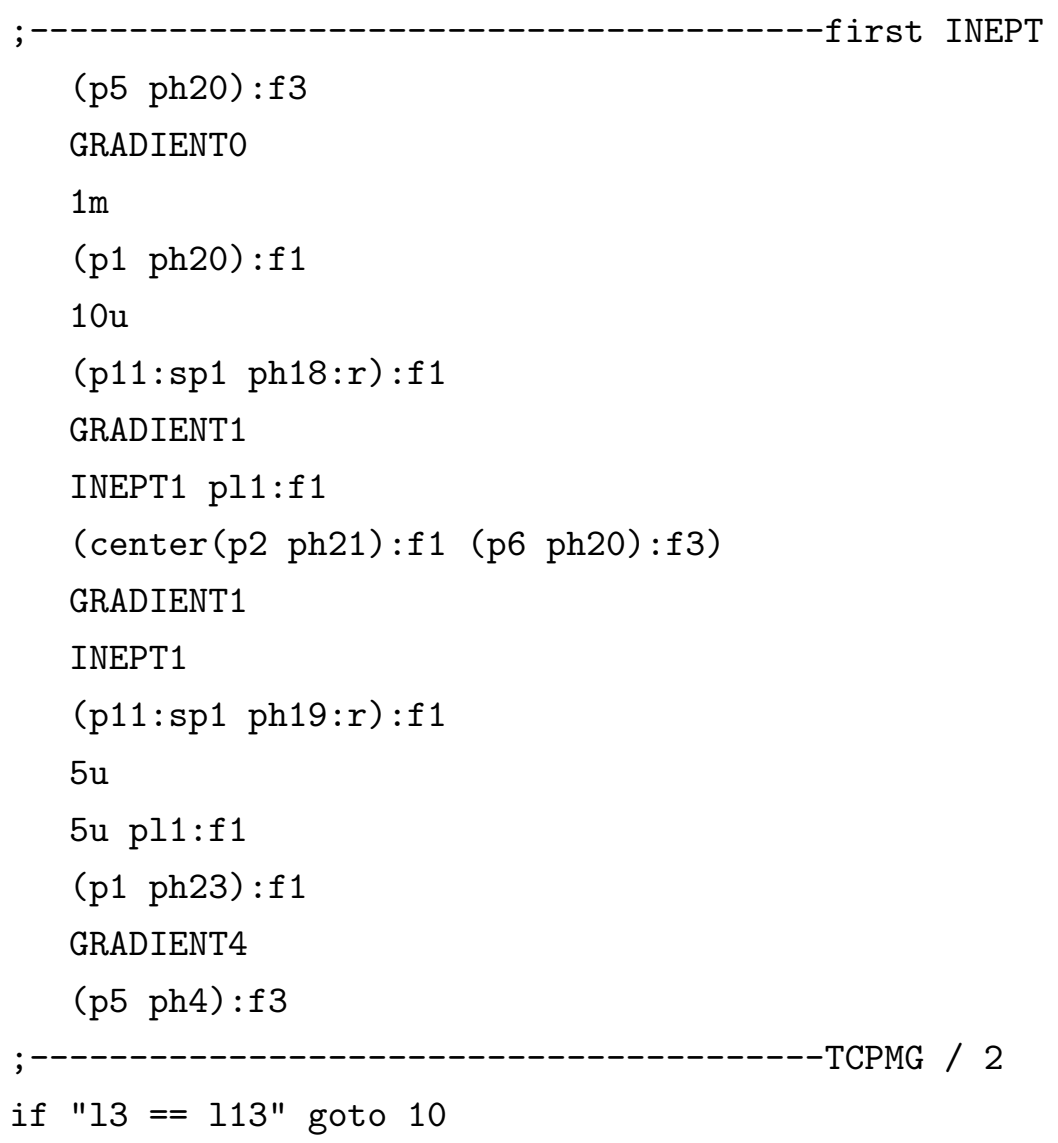




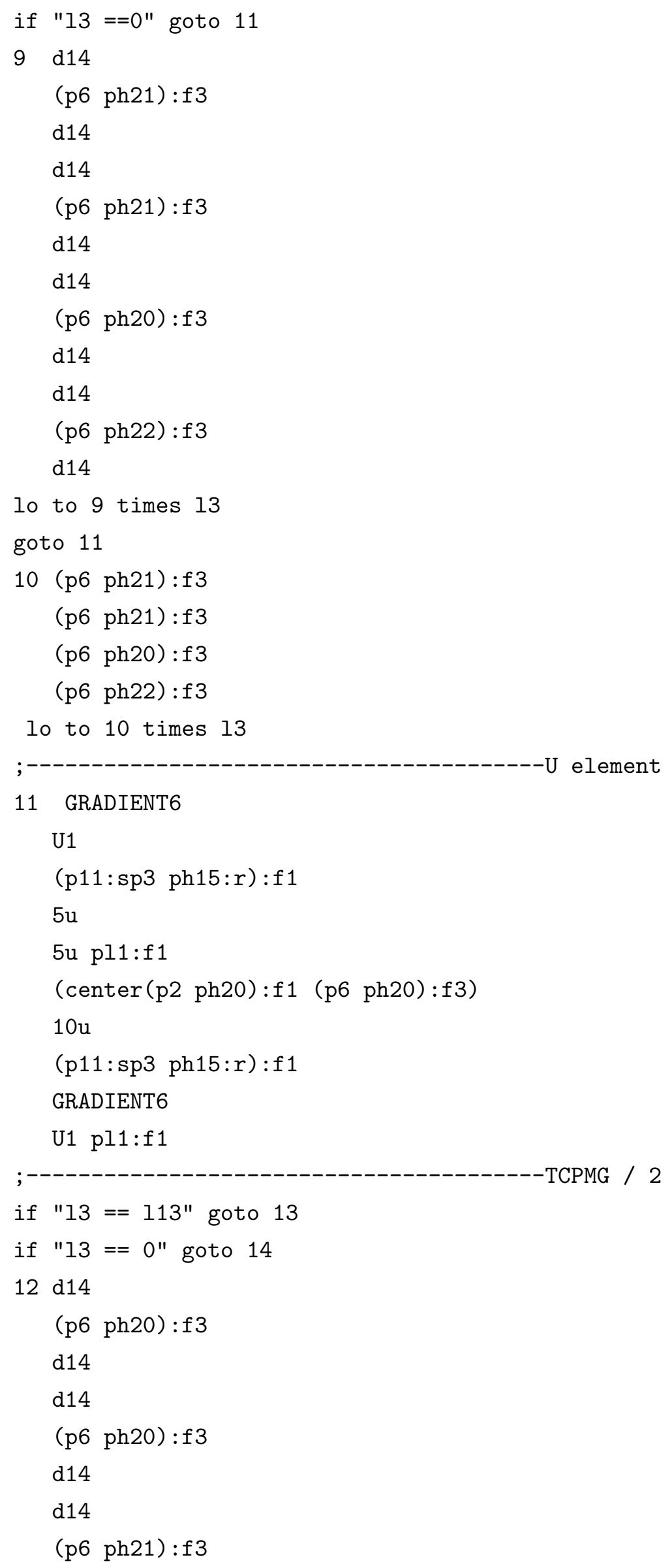




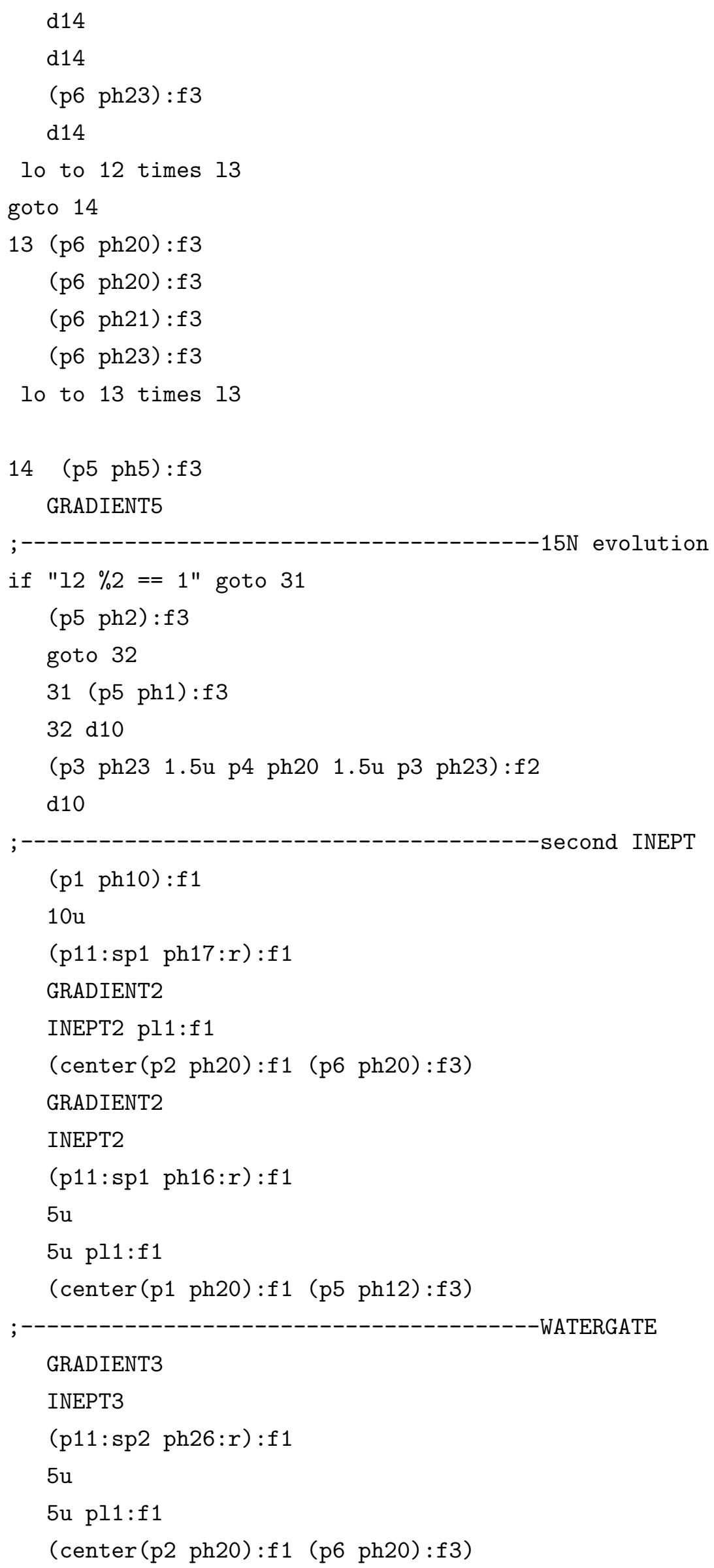


$10 \mathrm{u}$

(p11:sp2 ph26:r):f1

GRADIENT3

INEPT3 LOCKH_OFF

(p5 ph11):f3

;----------------------------------acquisition

goscnp ph31

$3 \mathrm{~m}$ st cpmglist.inc

lo to 3 times $\mathrm{nbl}$

3m ipp1 ipp2 ipp4 ipp5 ipp10 ipp11 ipp12 ipp31

lo to 4 times ns

$1 \mathrm{~m} \mathrm{mc} \# 0$ to 4

$\mathrm{F} 1 \mathrm{QF}()$

F2EA(ip10*2 \& ip12*2 \& ip17*2 \& rpp1 \& rpp2 \& rpp4

\& rpp5 \& rpp10 \& rpp11 \& rpp12 \& rpp31 \& iu2, $i p 1 * 2$ \& ip $2 * 2$ \& ip31*2 \& id10)

$10 \mathrm{u}$ do:f 1

$10 \mathrm{u}$ do:f2

$10 \mathrm{u}$ do:f3

$10 \mathrm{u}$ LOCKH_OFF

exit

ph1 =1 $3 \begin{array}{lll}2 & 0\end{array}$

ph2 $=1 \quad 3 \quad 0 \quad 2$

ph4 $=\begin{array}{llllllll}0 & 0 & 0 & 0 & 2 & 2 & 2 & 2\end{array}$

ph5 $=1 \begin{array}{lllllllllllllllllllll}1 & 1 & 1 & 1 & 1 & 1 & 1 & 3 & 3 & 3 & 3 & 3 & 3 & 3 & 3\end{array}$

ph31=1 $3 \begin{array}{llllllllllllllll} & 0 & 3 & 1 & 0 & 2 & 3 & 1 & 0 & 2 & 1 & 3 & 2 & 0\end{array}$

ph10=3 $3 \begin{array}{lll}3 & 3\end{array}$

ph11=0 0000

ph12=3 $3 \begin{array}{llll}3 & 3\end{array}$

ph15 $=2$

ph16 $=0$

$\operatorname{ph} 17=1$

$\operatorname{ph} 18=2$

ph19=3

$\operatorname{ph} 20=0$

$\operatorname{ph} 21=1$

$\operatorname{ph} 22=2$

$\mathrm{ph} 23=3$

ph26 $=2$

\section{C.2 Backbone ${ }^{1}$ H E-CPMG}

\#include <Avance_dl.incl> 


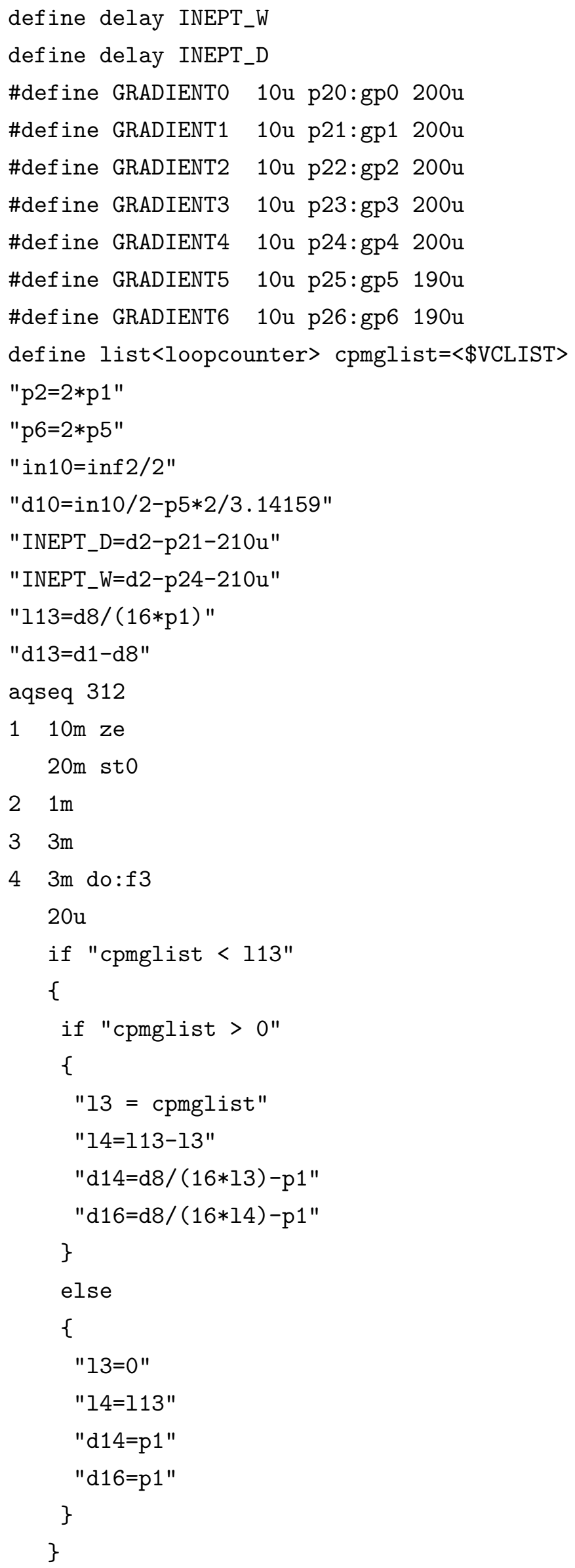




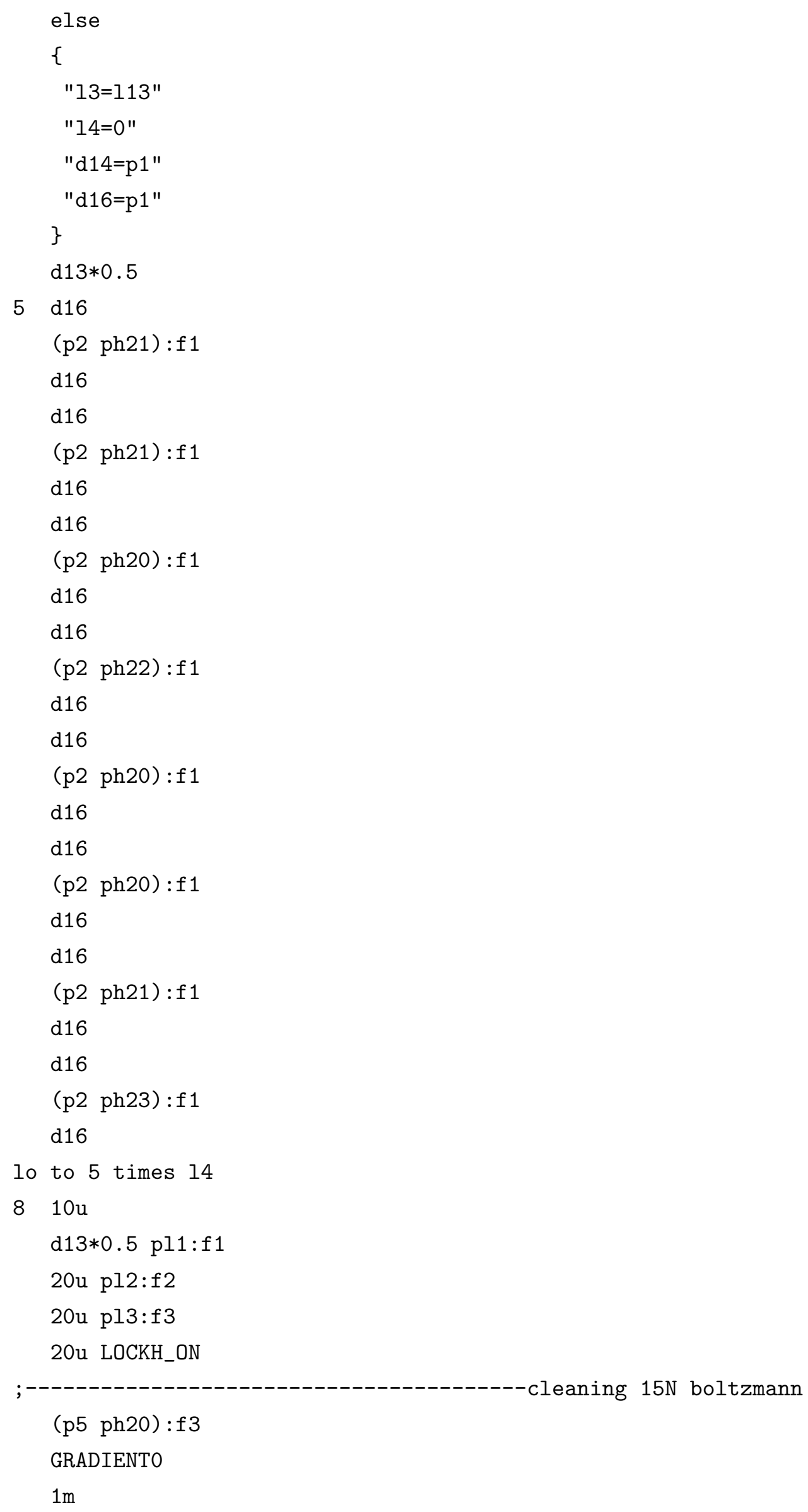




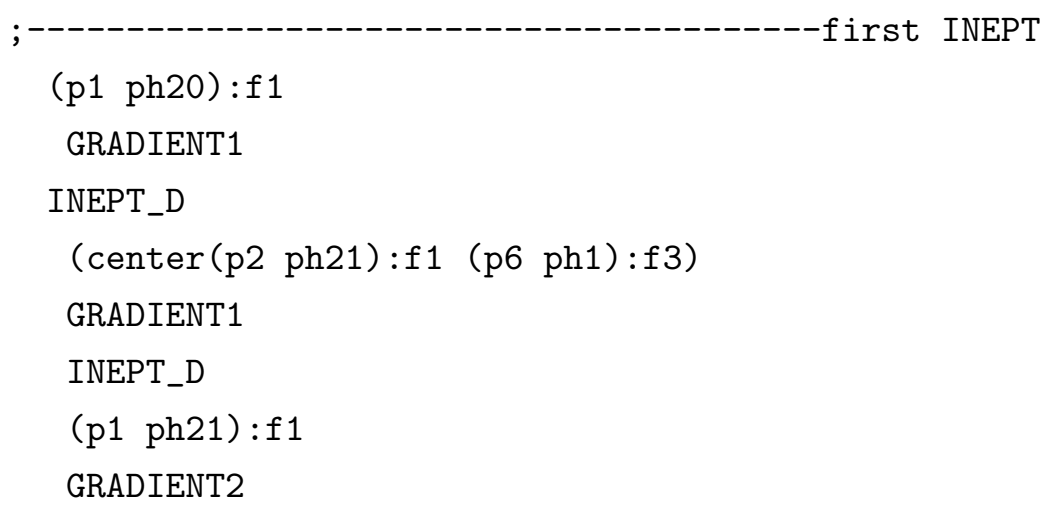


INEPT_W

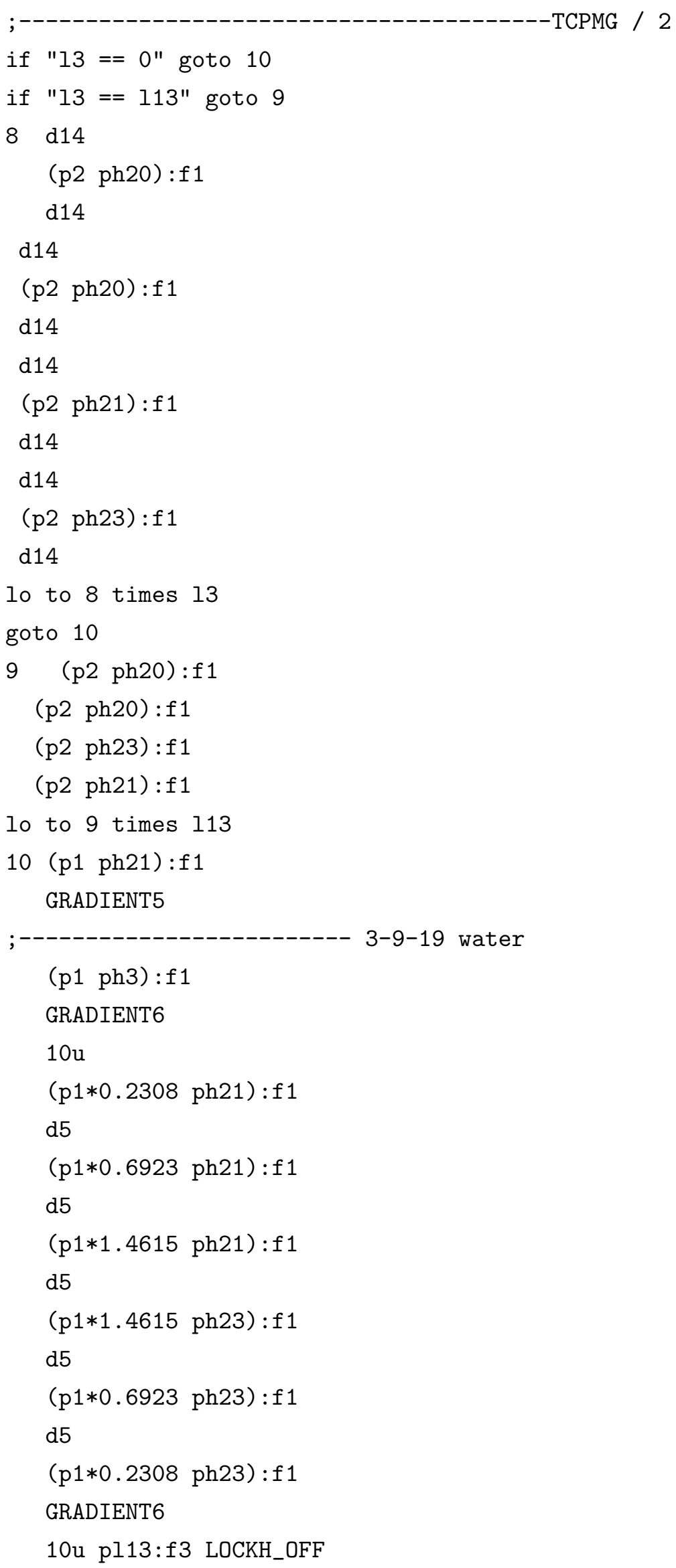




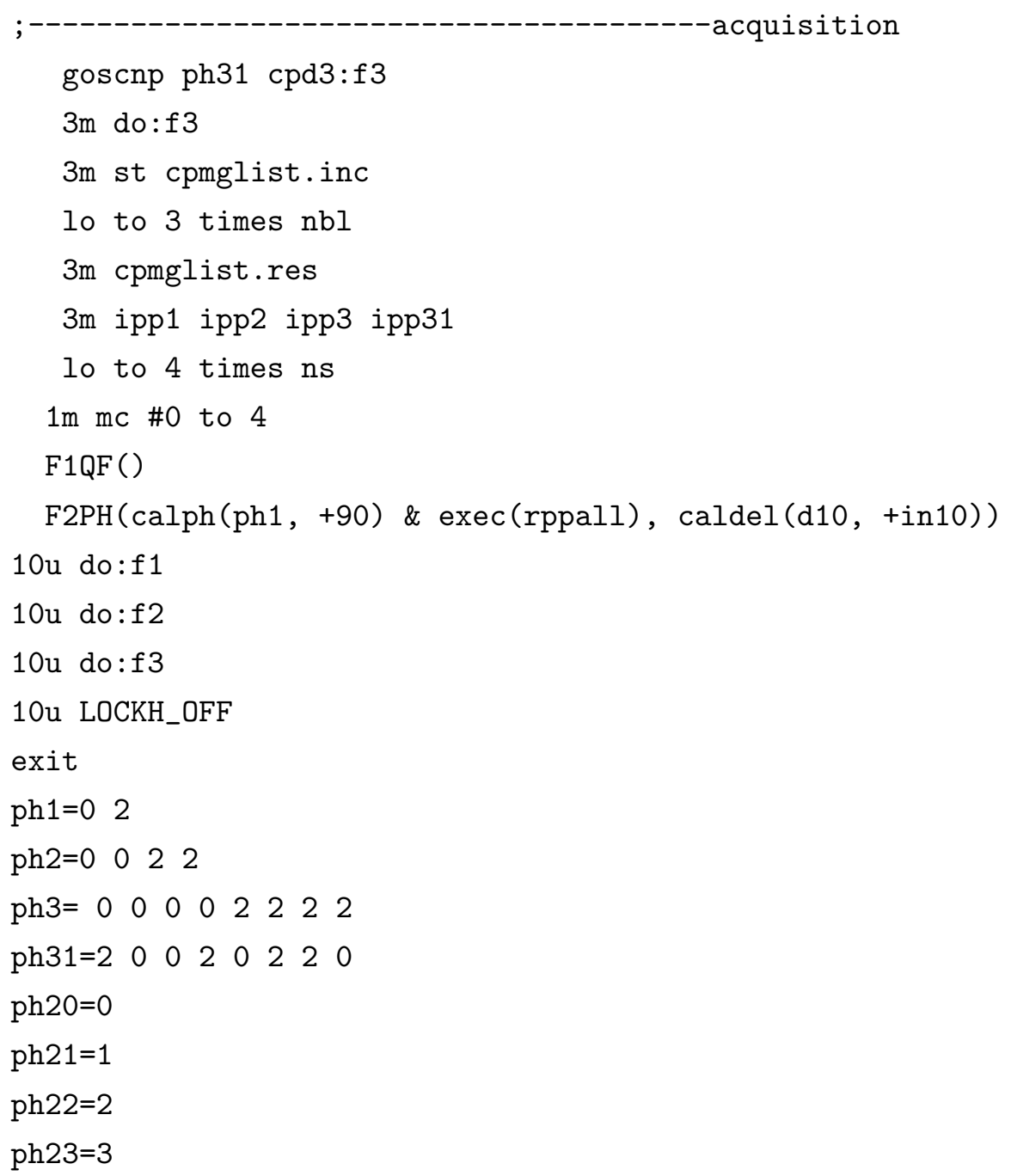

\section{C.3 Side-Chain ${ }^{13}$ C E-CPMG}

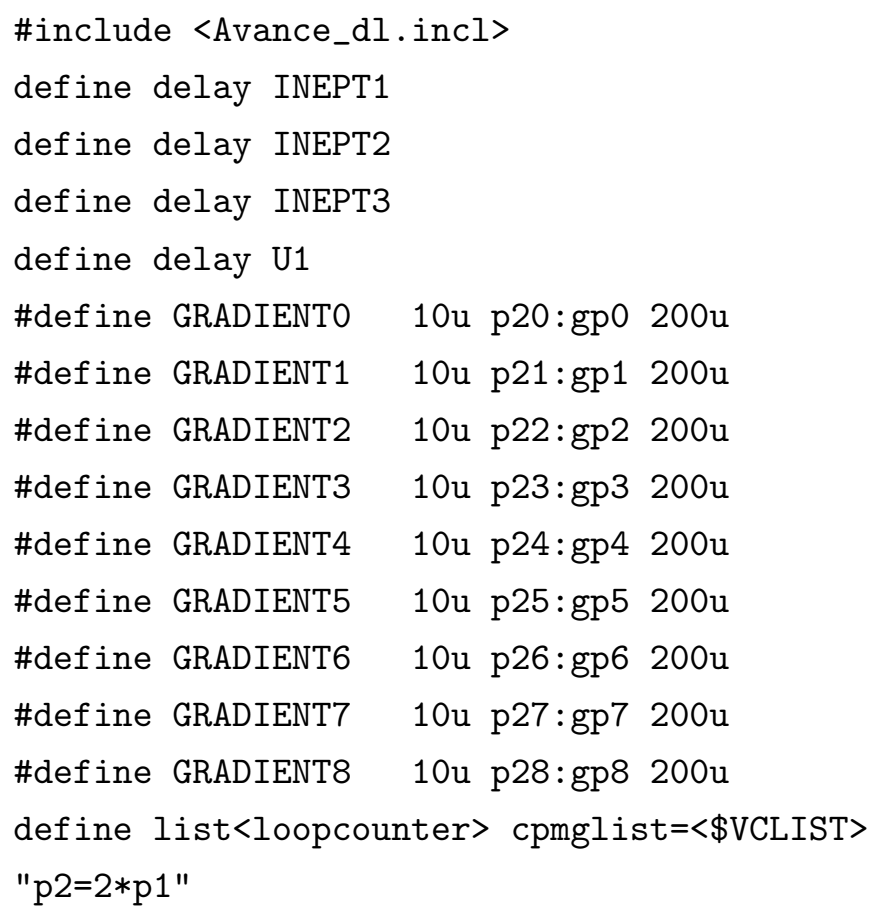




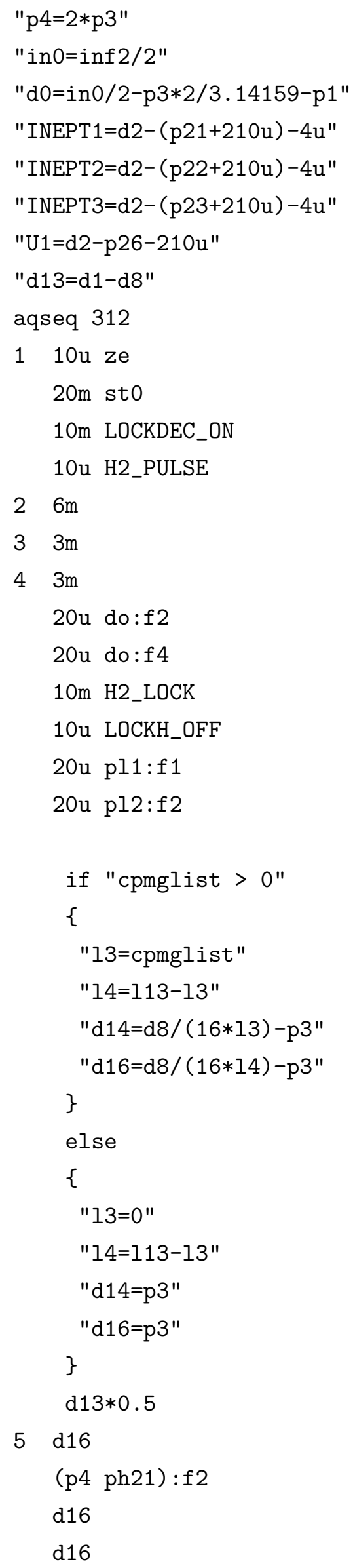




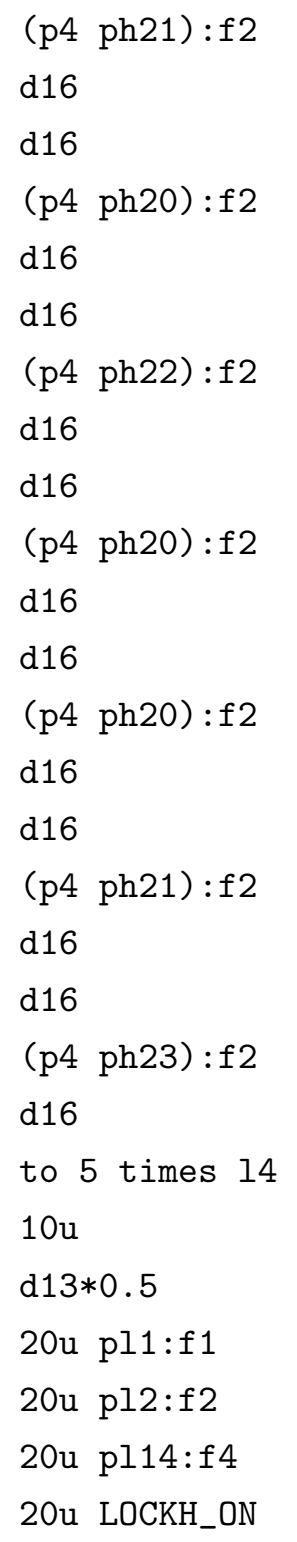

;---------------------------------------first INEPT

(p3 ph20):f2

GRADIENTO

$1 \mathrm{~m}$

10u H2_PULSE

$(\mathrm{p} 1 \mathrm{ph} 20): \mathrm{f} 1$

GRADIENT1

INEPT1

(center(p2 ph21):f1 (p3 ph20 2u p4 ph21 2u p3 ph20):f2)

GRADIENT1

INEPT1

(p1 ph21):f1

GRADIENT4

(p3 ph4):f2 


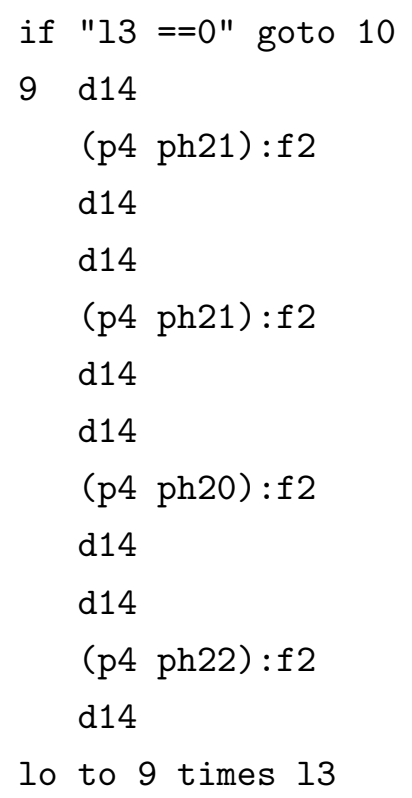

U1

(center(p2 ph21):f1 (p3 ph20 2u p4 ph21 2u p3 ph20):f2)

GRADIENT6

U1

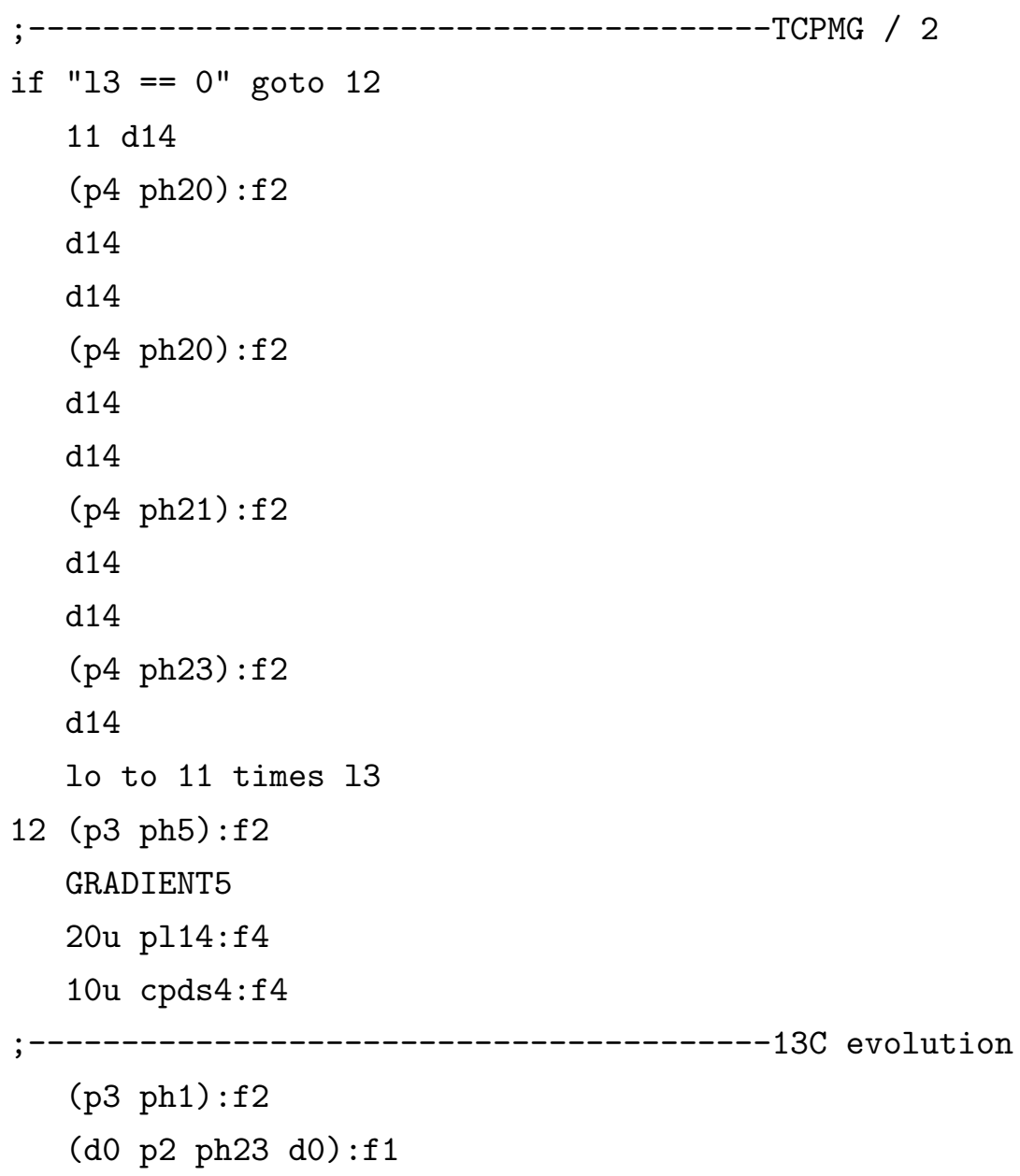




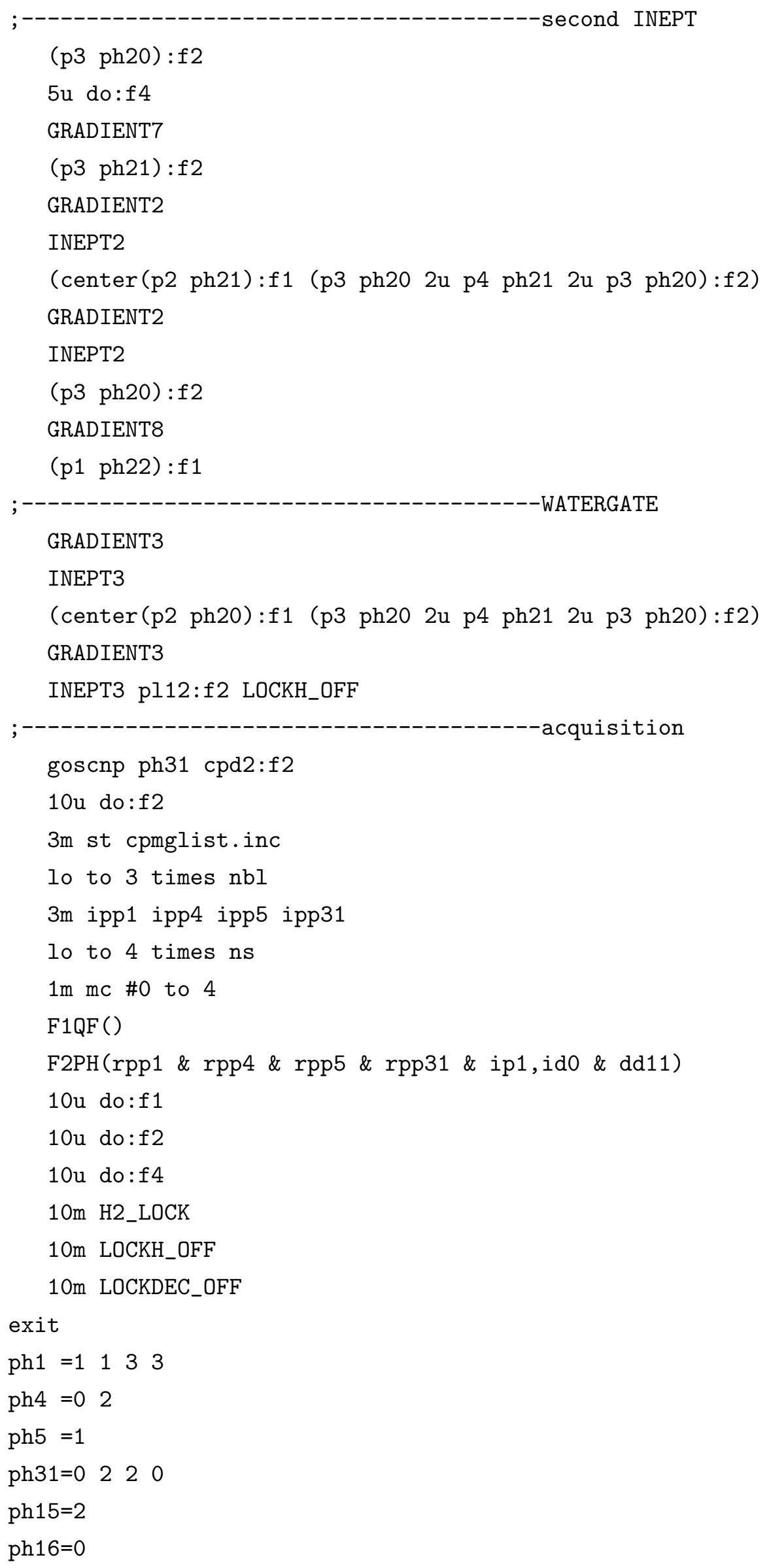


$\operatorname{ph} 17=1$
$\operatorname{ph} 18=2$
$\operatorname{ph} 19=3$
$\operatorname{ph} 20=0$
$\operatorname{ph} 21=1$
$\operatorname{ph} 22=2$
$\operatorname{ph} 23=3$
$\operatorname{ph} 26=2$

\section{C.4 Side-Chain ${ }^{1}$ H E-CPMG}

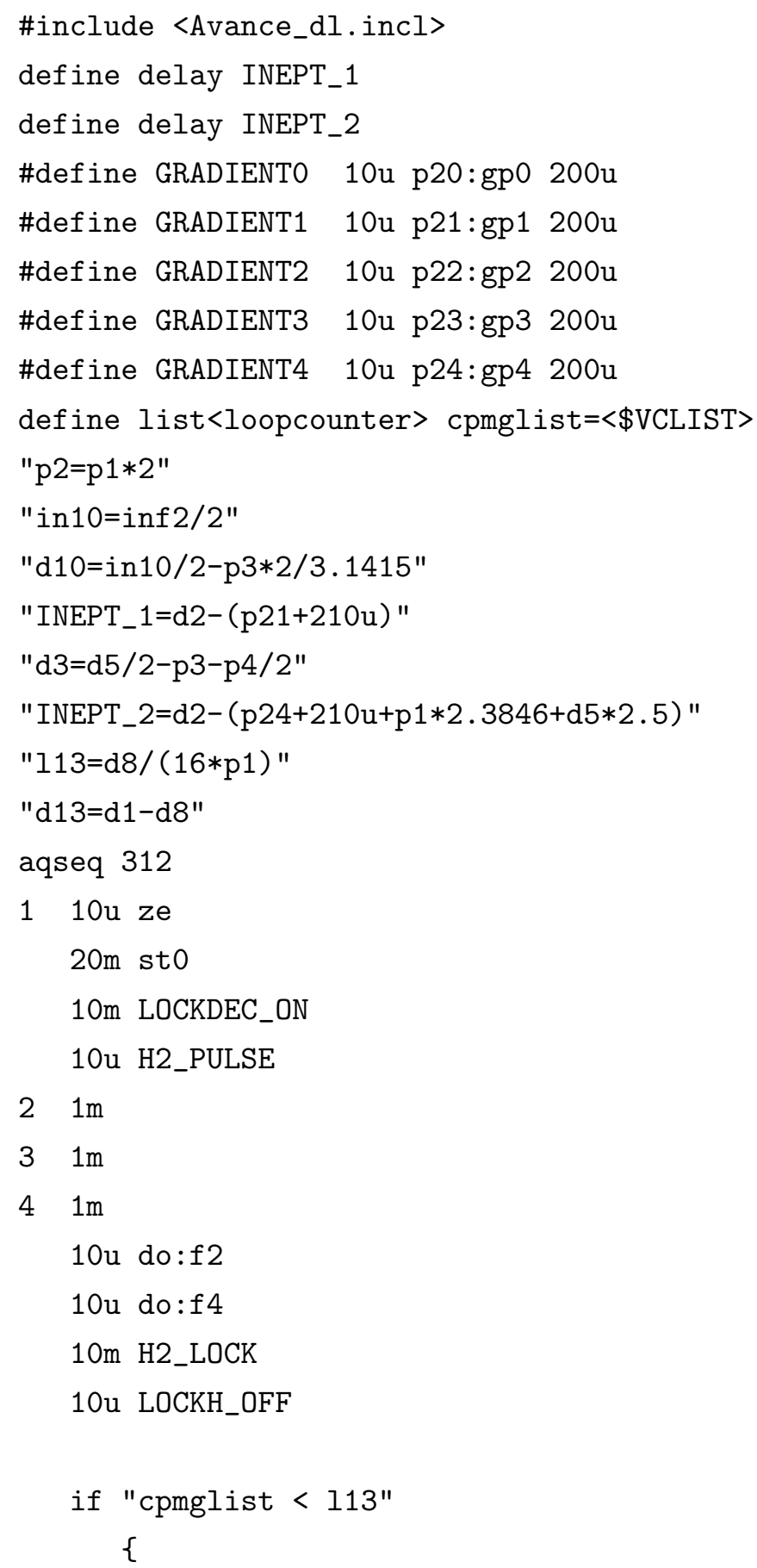




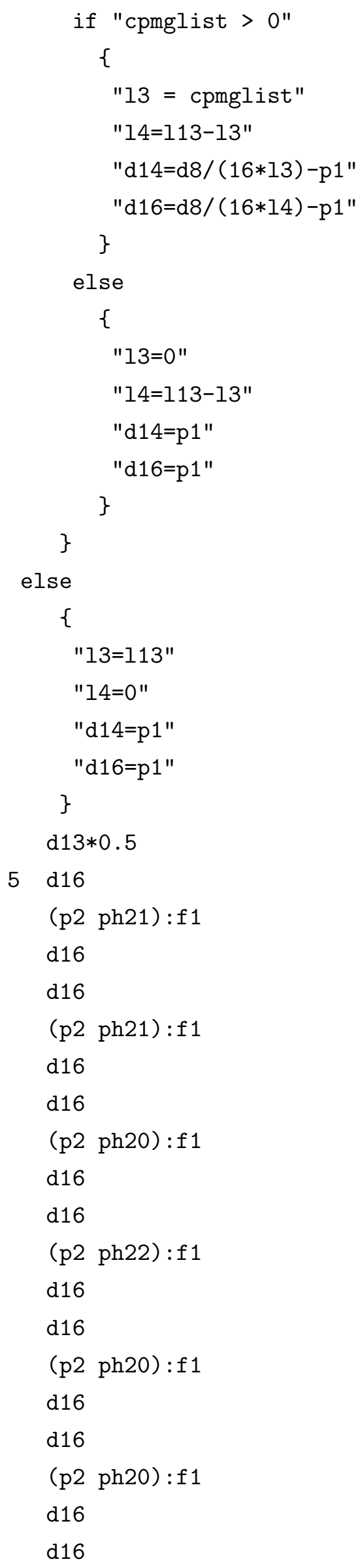




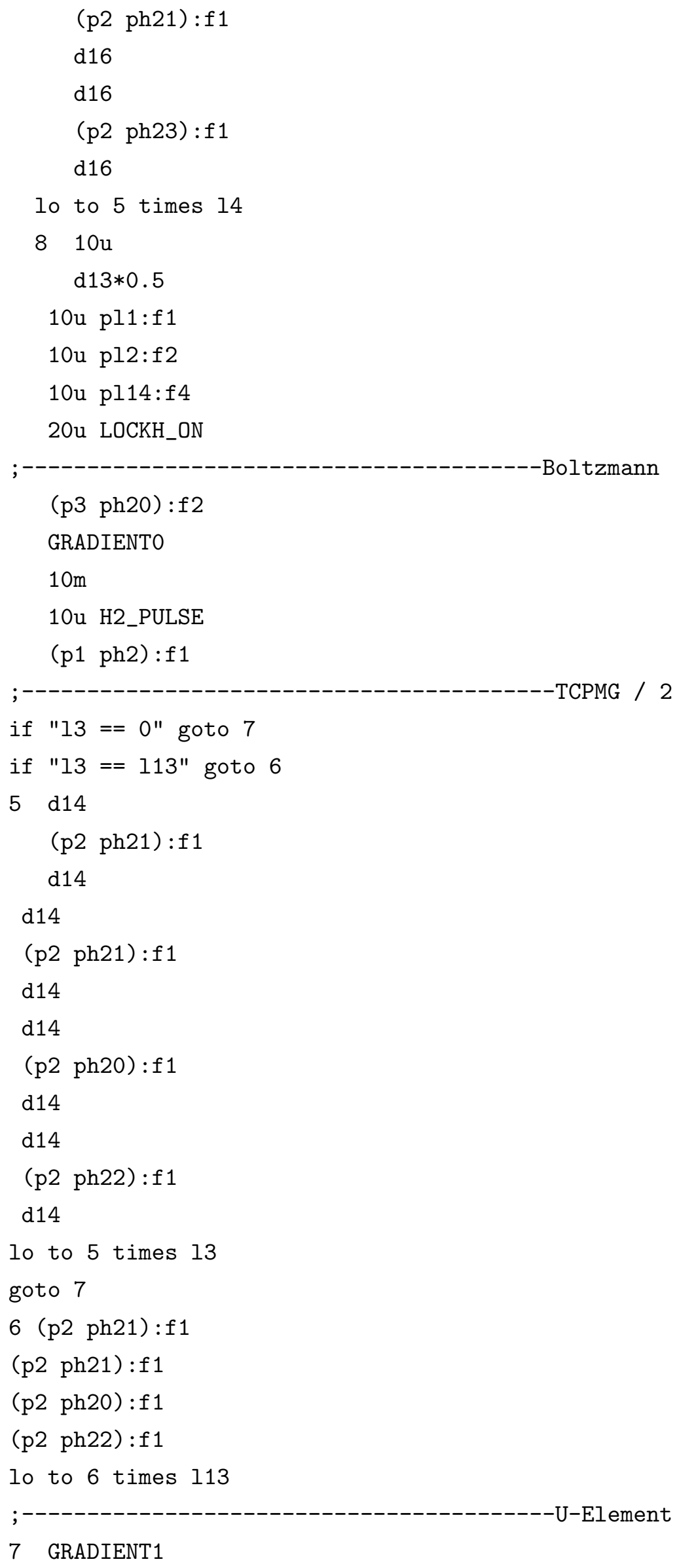

7 GRADIENT1 
INEPT_1

(center ( $\mathrm{p} 2 \mathrm{ph} 20): \mathrm{f} 1$ (p3 ph20 2u p4 ph21 2u p3 ph20):f2)

GRADIENT1

INEPT_1

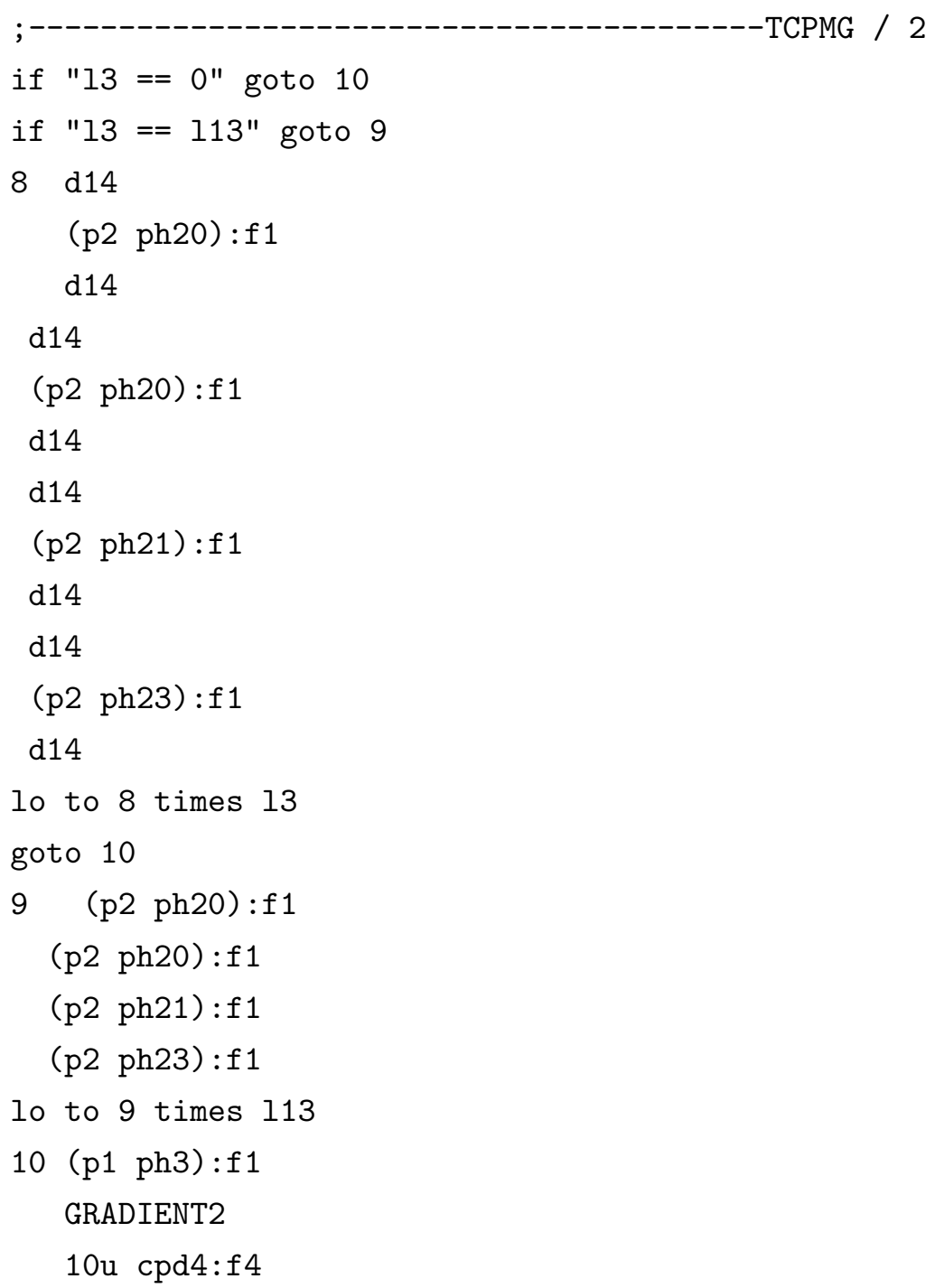




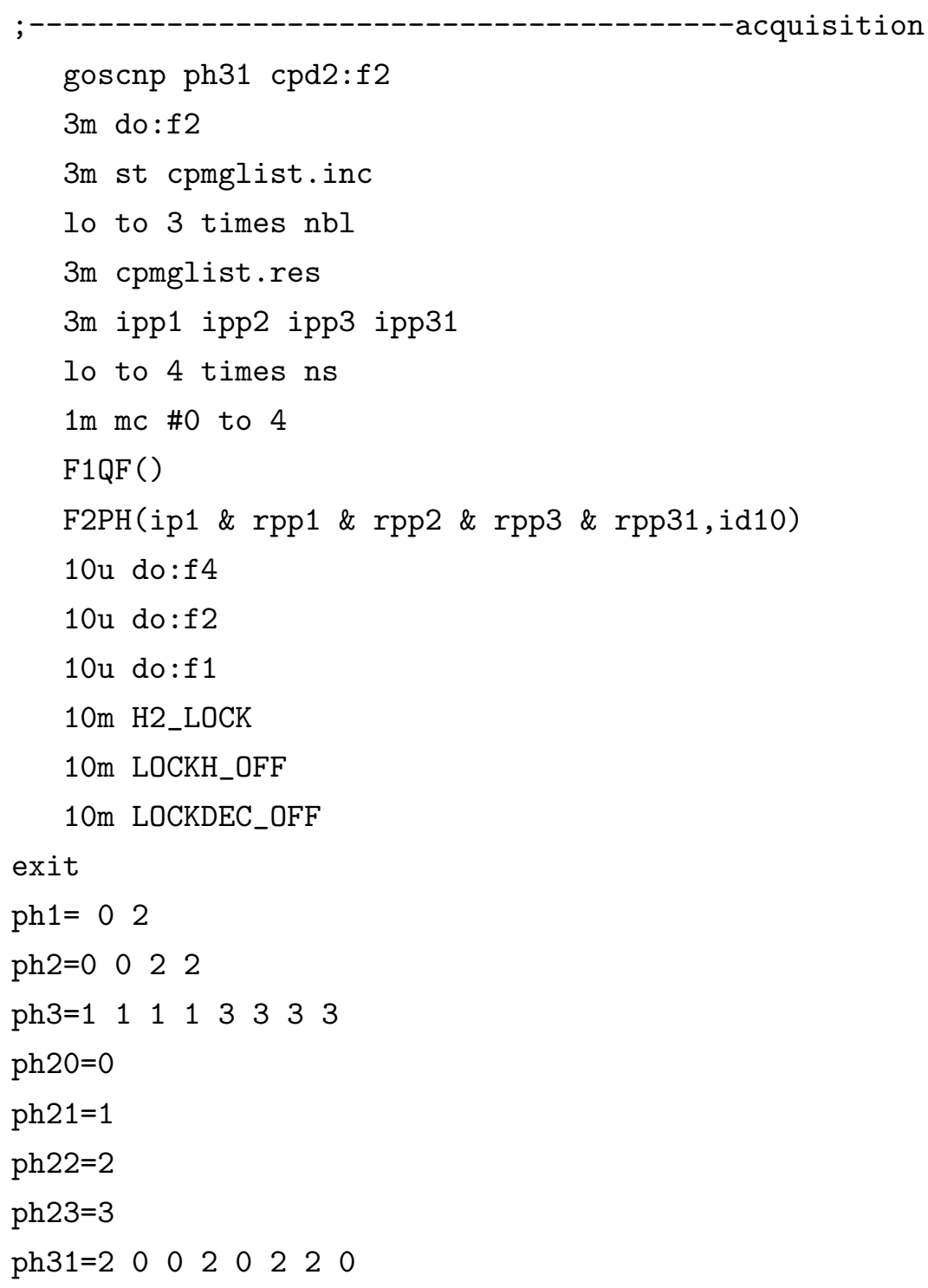

\section{C.5 Shuttle relaxometry}

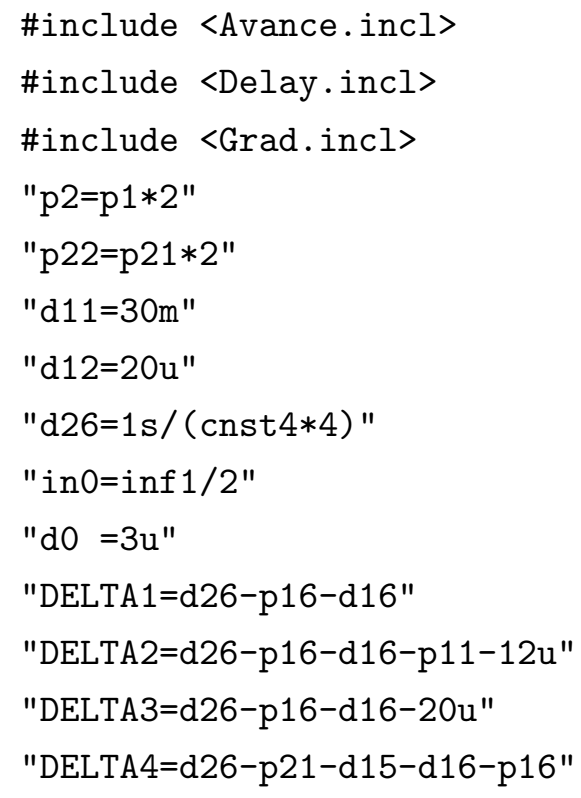


$1 \mathrm{ze}$

$20 \mathrm{~m}$

d11 pl16:f3

50u TTL4_HIGH

2 d1 do:f3

3 d12 pl1:f1 pl3:f3

50u UNBLKGRAD

(p1 ph20)

p16:gp3

d16

DELTA1

(center (p2 ph21) (p22 ph5):f3)

DELTA1

p16:gp3

d16

(p1 ph21)

$4 \mathrm{u}$ pl0:f1

(p11:sp1 ph20:r):f1

$4 \mathrm{u}$

p16:gp4

d16 pl1:f1

(p21 ph20):f3

$20 \mathrm{u}$

p16:gp6

d16

DELTA3

(center (p2 ph21) (p22 ph21):f3)

DELTA3

$20 \mathrm{u}$

p16:gp6

d16

(p21 ph1):f3

$50 \mathrm{u}$

p16:gp7

d16

; relaxation

50u TTL4_LOW ; shuttle-up

d21 ; shuttle delay

vd

50u TTL4_HIGH ; shuttle-down

d25 ; shuttle delay + stabilization delay

(p1 ph20):f1 


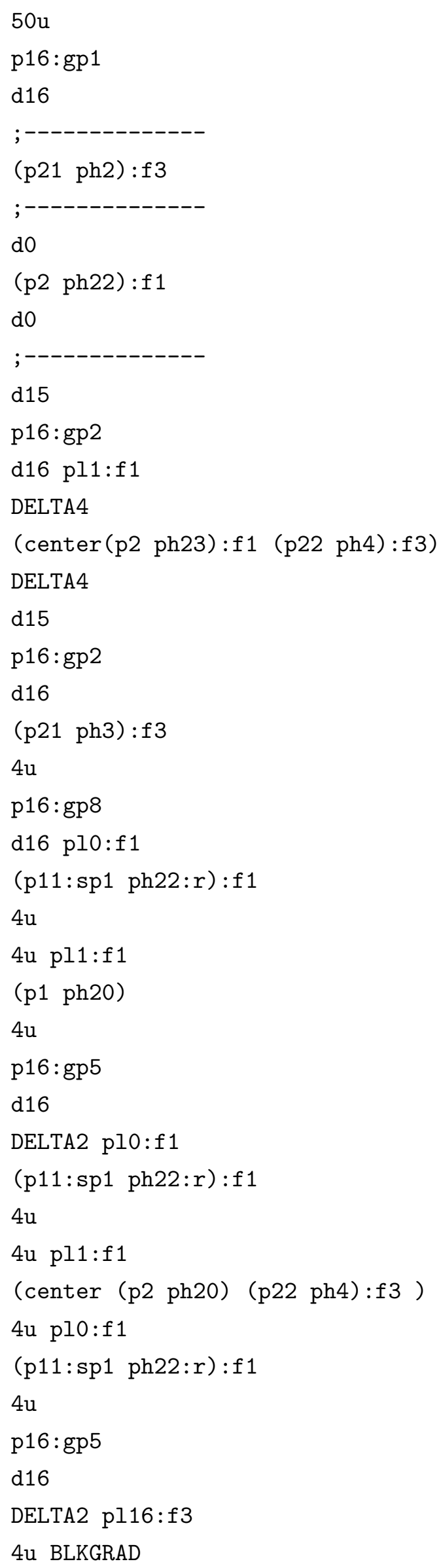


go=2 ph31 cpd3:f3

$1 \mathrm{~m}$ do:f3 mc \#0 to 2

F1I (ivd, 113)

F1PH(ip2 \& ip5, id0)

F2QF $(i p 1 * 2)$

exit

$\operatorname{ph} 20=0$

ph21 $=1$

$\operatorname{ph} 22=2$

$\mathrm{ph} 23=3$

ph1 1

$\mathrm{ph} 2=1$

ph $3=02$

ph5 $=0$

ph31= 02 


\section{Bibliography}

A. Abragam. The Principles of Nuclear Magnetism. Clarendon Press, 1961.

R. V. Agafonov, C. Wilson, R. Otten, V. Buosi, and D. Kern. Energetic dissection of gleevec's selectivity toward human tyrosine kinases. Nature Structural \& Molecular Biology, 21(10):848-853, 2014.

M. Akke and A. G. Palmer. Monitoring macromolecular motions on microsecond to millisecond time scales by $\mathrm{r}(1)$ rho-r(1) constant relaxation time nmr spectroscopy. Journal of the American Chemical Society, 118(4):911-912, 1996.

A. T. Alexandrescu, C. Abeygunawardana, and D. Shortle. Structure and dynamics of a denatured 131-residue fragment of staphylococcal nuclease - a heteronuclear nmr-study. Biochemistry, 33(5):1063-1072, 1994.

A. Allerhand and H. S. Gutowsky. Spin-echo nmr studies of chemical exchange .1. some general aspects. Journal of Chemical Physics, 41(7):2115-+, 1964.

A. Allerhand and H. S. Gutowsky. Spin-echo nmr studies of chemical exchange .4. intramolecular exchange of a coupled ab system. Journal of Chemical Physics, 42(12): $4203-+, 1965$.

A. Allerhand, F. M. Chen, and H. S. Gutowsky. Spin-echo nmr studies of chemical exchange .3. conformational isomerization of cyclohexane and d11-cyclohexane. Journal of Chemical Physics, 42(9):3040-+, 1965.

M. Andrec, G. T. Montelione, and R. M. Levy. Estimation of dynamic parameters from $\mathrm{nmr}$ relaxation data using the lipari-szabo model-free approach and bayesian statistical methods. Journal of Magnetic Resonance, 139(2):408-421, 1999.

A. J. Baldwin. An exact solution for r-2,(eff) in cpmg experiments in the case of two site chemical exchange. Journal of Magnetic Resonance, 244:114-124, 2014.

D. Ban, M. Funk, R. Gulich, D. Egger, T. M. Sabo, K. F. A. Walter, R. B. Fenwick, K. Giller, F. Pichierri, B. L. de Groot, O. F. Lange, H. Grubmuller, X. Salvatella, M. Wolf, A. Loidl, R. Kree, S. Becker, N. A. Lakomek, D. Lee, P. Lunkenheimer, and C. Griesinger. Kinetics of conformational sampling in ubiquitin. Angewandte ChemieInternational Edition, 50(48):11437-11440, 2011. 
D. Ban, A. D. Gossert, K. Giller, S. Becker, C. Griesinger, and D. Lee. Exceeding the limit of dynamics studies on biomolecules using high spin-lock field strengths with a cryogenically cooled probehead. Journal of Magnetic Resonance, 221:1-4, 2012.

D. Ban, A. Mazur, M. G. Carneiro, T. M. Sabo, K. Giller, L. M. I. Koharudin, S. Becker, A. M. Gronenborn, C. Griesinger, and D. Lee. Enhanced accuracy of kinetic information from ct-cpmg experiments by transverse rotating-frame spectroscopy. Journal of Biomolecular NMR, 57(1):73-82, 2013.

M. Billeter, P. Schultze, and K. Wuthrich. Determination of protein secondary structure from patterns of distance constraints observed by nmr in solution. Experientia, 40(6): 603-603, 1984.

M. Bonomi, R. Pellarin, and M. Vendruscolo. Simultaneous determination of protein structure and dynamics using cryo-electron microscopy. Biophysical Journal, 114(7): 1604-1613, 2018.

B. Böttcher, S. A. Wynne, and R. A. Crowther. Determination of the fold of the core protein of hepatitis b virus ky electron cryomicroscopy. Nature, 386(6620):88-91, 1997.

G. Bradski. The OpenCV Library. Dr. Dobb's Journal of Software Tools, 2000.

K. Burnham and D. Anderson. Model selection and multimodel inference: a practical information-theoretic approach. Springer, 2002.

D. M. Byler and H. Susi. Examination of the secondary structure of proteins by deconvolved ftir spectra. Biopolymers, 25(3):469-487, 1986.

H. Y. Carr and E. M. Purcell. Effects of diffusion on free precession in nuclear magnetic resonance experiments. Phys. Rev., 94:630-638, 1954.

J. Cavanagh, W. J. Fairbrother, A. G. Palmer, M. Rance, and N. J. Skelton. Protein NMR Spectroscopy: Principles and Practice, 2nd Edition. Elsevier Academic Press, 2007.

C. Charlier, S. N. Khan, T. Marquardsen, P. Pelupessy, V. Reiss, D. Sakellariou, G. Bodenhausen, F. Engelke, and F. Ferrage. Nanosecond time scale motions in proteins revealed by high-resolution nmr relaxometry. J Am Chem Soc, 135(49):18665-72, 2013.

C. Charlier, S. F. Cousin, and F. Ferrage. Protein dynamics from nuclear magnetic relaxation. Chemical Society Reviews, 45(9):2410-2422, 2016.

C. H. Cho, J. Urquidi, S. Singh, and G. Wilse Robinson. Thermal offset viscosities of liquid h2o, d2o, and t2o. The Journal of Physical Chemistry B, 103(11):1991-1994, 1999.

C. Y. Chou, M. L. Chu, C. F. Chang, and T. H. Huang. A compact high-speed mechanical sample shuttle for field-dependent high-resolution solution nmr. Journal of Magnetic Resonance, 214:302-308, 2012. 
C. Y. Chou, M. Chu, C. F. Chang, T. N. Yu, T. H. Huang, and D. Sakellariou. High sensitivity high-resolution full range relaxometry using a fast mechanical sample shuttling device and a cryo-probe. Journal of Biomolecular Nmr, 66(3):187-194, 2016.

M. W. Clarkson, M. Lei, E. Z. Eisenmesser, W. Labeikovsky, A. Redfield, and D. Kern. Mesodynamics in the sars nucleocapsid measured by $\mathrm{nmr}$ field cycling. Journal of Biomolecular Nmr, 45(1-2):217-225, 2009.

G. Marius Clore, Attila Szabo, Ad Bax, Lewis E. Kay, Paul C. Driscoll, and Angela M. Gronenborn. Deviations from the simple two-parameter model-free approach to the interpretation of nitrogen-15 nuclear magnetic relaxation of proteins. Journal of the American Chemical Society, 112(12):4989-4991, 1990.

E. J. d'Auvergne and P. R. Gooley. The use of model selection in the model-free analysis of protein dynamics. Journal of Biomolecular Nmr, 25(1):25-39, 2003.

A. V. Efimov. Packing of alpha-helices in globular-proteins - layer-structure of globin hydrophobic cores. Journal of Molecular Biology, 134(1):23-40, 1979.

C. Eichmuller and N. R. Skrynnikov. A new amide proton r-1 rho experiment permits accurate characterization of microsecond time-scale conformational exchange. Journal of Biomolecular Nmr, 32(4):281-293, 2005.

N. A. Farrow, R. Muhandiram, A. U. Singer, S. M. Pascal, C. M. Kay, G. Gish, S. E. Shoelson, T. Pawson, J. D. Formankay, and L. E. Kay. Backbone dynamics of a free and a phosphopeptide-complexed src homology-2 domain studied by n-15 nmr relaxation. Biochemistry, 33(19):5984-6003, 1994.

N. A. Farrow, O. W. Zhang, J. D. Formankay, and L. E. Kay. Comparison of the backbone dynamics of a folded and an unfolded sh3 domain existing in equilibrium in aqueous buffer. Biochemistry, 34(3):868-878, 1995.

N. L. Fawzi, J. F. Ying, D. A. Torchia, and G. M. Clore. Probing exchange kinetics and atomic resolution dynamics in high-molecular-weight complexes using dark-state exchange saturation transfer nmr spectroscopy. Nature Protocols, 7(8):1523-1533, 2012.

N. Ferruz and G. De Fabritiis. Binding kinetics in drug discovery. Molecular Informatics, 35(6-7):216-226, 2016.

S. Forsen and R. A. Hoffman. Study of moderately rapid chemical exchange reactions by means of nuclear magnetic double resonance. Journal of Chemical Physics, 39(11):2892, 1963.

A. Frank, S. Law, L. Ahlstrom, and C. Brooks III. Predicting protein backbone chemical shifts from $c_{\alpha}$ coordinates: Extracting high resolution experimental observables from low resolution models. J. Chem. Theory Comput., 11:325-331, 2015. 
A. Fung, P. Li, R. Godoy-Ruiz, J. M. Sanchez-Ruiz, and V. Munoz. Expanding the realm of ultrafast protein folding: gpw, a midsize natural single-domain with alpha+beta topology that folds downhill. Journal of the American Chemical Society, 130(23):7489$7495,2008$.

Adam Fung. Mutational Analysis of the Downhill Folding Protein GPW: Towards Tuning Stability of a Molecular Rheostat Candidate. Thesis, 2008.

W. G. Glöckle and T. F. Nonnenmacher. A fractional calculus approach to self-similar protein dynamics. Biophysical Journal, 68(1):46-53, 1995.

M. J. Grey, C. Y. Wang, and A. G. Palmer. Disulfide bond isomerization in basic pancreatic trypsin inhibitor: Multisite chemical exchange quantified by cpmg relaxation dispersion and chemical shift modeling. Journal of the American Chemical Society, 125(47):14324$14335,2003$.

A. M. Gronenborn, D. R. Filpula, N. Z. Essig, A. Achari, M. Whitlow, P. T. Wingfield, and G. M. Clore. A novel, highly stable fold of the immunoglobulin binding domain of streptococcal protein-g. Science, 253(5020):657-661, 1991.

R. Grosse, K. R. H. Repke, and J. Malur. Determination of secondary structure of proteins in biomembranes by means of circular-dichroism. Acta Biologica Et Medica Germanica, 27(5-6):K25-\&, 1971.

C. Haupt, R. Patzschke, U. Weininger, S. Groger, M. Kovermann, and J. Balbach. Transient enzyme-substrate recognition monitored by real-time nmr. Journal of the American Chemical Society, 133(29):11154-11162, 2011.

J. J. Helmus and C. P. Jaroniec. Nmrglue: an open source python package for the analysis of multidimensional nmr data. Journal of Biomolecular Nmr, 55(4):355-367, 2013.

D. Idiyatullin, V. A. Daragan, and K. H. Mayo. (nh)-n-15 backbone dynamics of protein gb1: Comparison of order parameters and correlation times derived using various "model-free" approaches. Journal of Physical Chemistry B, 107(11):2602-2609, 2003.

R. Ishima and D. A. Torchia. Estimating the time scale of chemical exchange of proteins from measurements of transverse relaxation rates in solution. Journal of Biomolecular Nmr, 14(4):369-372, 1999.

P. A. Jennings and P. E. Wright. Formation of a molten globule intermediate early in the kinetic folding pathway of apomyoglobin. Science, 262(5135):892-896, 1993.

P. Kaderavek, V. Zapletal, R. Fiala, P. Srb, P. Padrta, J. P. Precechtelova, M. Soltesova, J. Kowalewski, G. Widmalm, J. Chmelik, V. Sklenar, and L. Zidek. Spectral density mapping at multiple magnetic fields suitable for c-13 nmr relaxation studies. Journal of Magnetic Resonance, 266:23-40, 2016. 
L. E. Kay, D. A. Torchia, and A. Bax. Backbone dynamics of proteins as studied by n-15 inverse detected heteronuclear nmr-spectroscopy - application to staphylococcal nuclease. Biochemistry, 28(23):8972-8979, 1989.

Rochus L.J. Keller. The Computer Aided Resonance Assignment Tutorial. Cantina Verlag, 2004.

J. T. Kellis, K. Nyberg, and A. R. Fersht. Energetics of complementary side-chain packing in a protein hydrophobic core. Biochemistry, 28(11):4914-4922, 1989.

J. C. Kendrew, G. Bodo, H. M. Dintzis, R. G. Parrish, H. Wyckoff, and D. C. Phillips. 3-dimensional model of the myoglobin molecule obtained by x-ray analysis. Nature, 181 (4610):662-666, 1958.

M. Kjaergaard, V. Iesmantavicius, and F. M. Poulsen. The interplay between transient alpha-helix formation and side chain rotamer distributions in disordered proteins probed by methyl chemical shifts. Protein Science, 20(12):2023-2034, 2011.

G. R. Kneller and K. Hinsen. Fractional brownian dynamics in proteins. J Chem Phys, 121(20):10278-83, 2004.

D. M. Korzhnev, V. Y. Orekhov, and A. S. Arseniev. Model-free approach beyond the borders of its applicability. J Magn Reson, 127(2):184-91, 1997.

D. E. Koshland. Application of a theory of enzyme specificity to protein synthesis. Proceedings of the National Academy of Sciences of the United States of America, 44(2): 98-104, 1958.

N. A. Lakomek, O. F. Lange, K. F. A. Walter, C. Fares, D. Egger, P. Lunkenheimer, J. Meiler, H. Grubmuller, S. Becker, B. L. de Groot, and C. Griesinger. Residual dipolar couplings as a tool to study molecular recognition of ubiquitin. Biochemical Society Transactions, 36:1433-1437, 2008.

O. F. Lange, N. A. Lakomek, C. Fares, G. F. Schroder, K. F. Walter, S. Becker, J. Meiler, H. Grubmuller, C. Griesinger, and B. L. de Groot. Recognition dynamics up to microseconds revealed from an rdc-derived ubiquitin ensemble in solution. Science, 320 (5882):1471-5, 2008.

D. Lee, C. Hilty, G. Wider, and K. Wuthrich. Effective rotational correlation times of proteins from nmr relaxation interference. J Magn Reson, 178(1):72-6, 2006.

G. Lipari and A. Szabo. Model-free approach to the interpretation of nuclear magneticresonance relaxation in macromolecules .1. theory and range of validity. Journal of the American Chemical Society, 104(17):4546-4559, 1982.

J. P. Loria, M. Rance, and A. G. Palmer. A trosy cpmg sequence for characterizing chemical exchange in large proteins. Journal of Biomolecular Nmr, 15(2):151-155, 1999. 
Philip Lottmann, Thorsten Marquardsen, Alexander Krahn, Andreas Tavernier, Peter Höfer, Marina Bennati, Frank Engelke, and Christian Griesinger. Evaluation of a shuttle dnp spectrometer by calculating the coupling and global enhancement factors of l-tryptophan. Applied Magnetic Resonance, 43(1-2):207-221, 2012.

Z. Luz and S. Meiboom. Nuclear magnetic resonance study of protolysis of trimethylammonium ion in aqueous solution - order of reaction with respect to solvent. Journal of Chemical Physics, 39(2):366-370, 1963.

K. L. Maxwell, A. R. Davidson, H. Murialdo, and M. Gold. Thermodynamic and functional characterization of protein $\mathrm{w}$ from bacteriophage lambda - the three c-terminal residues are critical for activity. Journal of Biological Chemistry, 275(25):18879-18886, 2000.

K. L. Maxwell, A. A. Yee, V. Booth, C. H. Arrowsmith, M. Gold, and A. R. Davidson. The solution structure of bacteriophage lambda protein $\mathrm{w}$, a small morphogenetic protein possessing a novel fold. Journal of Molecular Biology, 308(1):9-14, 2001.

S. McClure, L. MacHattie, and M. Gold. A sedimentation analysis of dna found escherichia coli infected with phage $\lambda$ mutants. Virology, 54:1-18, 1973.

H. M. McConnell. Reaction rates by nuclear magnetic resonance. Journal of Chemical Physics, 28(3):430-431, 1958.

S. Meiboom and D. Gill. Modified spin-echo method for measuring nuclear relaxation times. Rev. Sci. Instrum., 29:688-691, 1958.

J. Monod, J. Wyman, and J.-P. Changeux. On the nature of allosteric transitions: A plausible model. Journal of Molecular Biology, 12:88-118, 1956.

F. A. A. Mulder, N. R. Skrynnikov, B. Hon, F. W. Dahlquist, and L. E. Kay. Measurement of slow (mu s-ms) time scale dynamics in protein side chains by n-15 relaxation dispersion nmr spectroscopy: Application to asn and gln residues in a cavity mutant of t4 lysozyme. Journal of the American Chemical Society, 123(5):967-975, 2001.

A. N. Naganathan and V. Munoz. Scaling of folding times with protein size. Journal of the American Chemical Society, 127(2):480-481, 2005.

Matthew Newville, Till Stensitzki, Daniel B. Allen, and Antonino Ingargiola. LMFIT: Non-Linear Least-Square Minimization and Curve-Fitting for Python, September 2014.

A. G. Palmer. Nmr characterization of the dynamics of biomacromolecules. Chemical Reviews, 104(8):3623-3640, 2004.

J. W. Peng and G. Wagner. Mapping of the spectral densities of $\mathrm{n}-\mathrm{h}$ bond motions in eglin-c using heteronuclear relaxation experiments. Biochemistry, 31(36):8571-8586, 1992.

S. J. Perkins and K. Wuthrich. Ring current effects in the conformation dependent nmr chemical-shifts of aliphatic protons in the basic pancreatic trypsin-inhibitor. Biochimica Et Biophysica Acta, 576(2):409-423, 1979. 
K. Pervushin, R. Riek, G. Wider, and K. Wuthrich. Attenuated t-2 relaxation by mutual cancellation of dipole-dipole coupling and chemical shift anisotropy indicates an avenue to $\mathrm{nmr}$ structures of very large biological macromolecules in solution. Proceedings of the National Academy of Sciences of the United States of America, 94(23):12366-12371, 1997.

C. Ramakrishnan and G. N. Ramachandran. Stereochemical criteria for polypeptide and protein chain conformations .2. allowed conformations for a pair of peptide units. Biophysical Journal, 5(6):909-+, 1965.

J. Reddy, S. Pratihar, D. Ban, S. Frischkorn, S. Becker, C. Griesinger, and D. Lee. Simultaneous determination of fast and slow dynamics in molecules using extreme cpmg relaxation dispersion experiments. Journal of Biomolecular NMR, 70(1):1-9, 2018.

A. G. Redfield. Shuttling device for high-resolution measurements of relaxation and related phenomena in solution at low field, using a shared commercial $500 \mathrm{mhz} \mathrm{nmr}$ instrument. Magnetic Resonance in Chemistry, 41(10):753-768, 2003.

D. Ringe and G. A. Petsko. Mapping protein dynamics by x-ray-diffraction. Progress in Biophysics \& Molecular Biology, 45(3):197-235, 1985.

T. M. Sabo, D. Bakhtiari, K. F. Walter, R. L. McFeeters, K. Giller, S. Becker, C. Griesinger, and D. Lee. Thermal coefficients of the methyl groups within ubiquitin. Protein Sci, 21(4):562-70, 2012.

T. M. Sabo, C. A. Smith, D. Ban, A. Mazur, D. Lee, and C. Griesinger. Orium: Optimized rdc-based iterative and unified model-free analysis. Journal of Biomolecular Nmr, 58 (4):287-301, 2014.

M. Sadqi, D. Fushman, and V. Munoz. Atom-by-atom analysis of global downhill protein folding. Nature, 442(7100):317-321, 2006.

C. Sanchez-Medina, A. Sekhar, P. Vallurupalli, M. Cerminara, V. Munoz, and L. E. Kay. Probing the free energy landscape of the fast-folding gpw protein by relaxation dispersion nmr. J Am Chem Soc, 136(20):7444-51, 2014.

L. Sborgi, A. Verma, V. Munoz, and E. de Alba. Revisiting the nmr structure of the ultrafast downhill folding protein gpw from bacteriophage lambda. PLoS One, 6(11): e26409, 2011.

L. Sborgi, A. Verma, S. Piana, K. Lindorff-Larsen, M. Cerminara, C. M. Santiveri, D. E. Shaw, E. de Alba, and V. Munoz. Interaction networks in protein folding via atomicresolution experiments and long-time-scale molecular dynamics simulations. J Am Chem Soc, 137(20):6506-16, 2015.

J. Schonfelder, D. De Sancho, R. Berkovich, R. B. Best, V. Munoz, and R. Perez-Jimenez. Reversible two-state folding of the ultrafast protein gpw under mechanical force. Communications Chemistry, 1, 2018. 
K. Shiraki, K. Nishikawa, and Y. Goto. Trifluoroethanol-induced stabilization of the alphahelical structure of beta-lactoglobulin - implication for non-hierarchical protein-folding. Journal of Molecular Biology, 245(2):180-194, 1995.

C. A. Smith, D. Ban, S. Pratihar, K. Giller, C. Schwiegk, B. L. de Groot, S. Becker, C. Griesinger, and D. Lee. Population shuffling of protein conformations. Angew Chem Int Ed Engl, 54(1):207-10, 2015.

C. A. Smith, D. Ban, S. Pratihar, K. Giller, M. Paulat, S. Becker, C. Griesinger, D. Lee, and B. L. de Groot. Allosteric switch regulates protein-protein binding through collective motion. Proc Natl Acad Sci U S A, 113(12):3269-74, 2016.

M. D. Sorensen, A. Meissner, and O. W. Sorensen. Spin-state-selective coherence transfer via intermediate states of two-spin coherence in is spin systems: Application to e.cosytype measurement of j coupling constants. Journal of Biomolecular Nmr, 10(2):181-186, 1997.

T. Szyperski, P. Luginbuhl, G. Otting, P. Guntert, and K. Wuthrich. Protein dynamics studied by rotating frame n-15 spin relaxation-times. Journal of Biomolecular Nmr, 3 (2):151-164, 1993.

L. E. Thompson and D. Rovnyak. Accessible nmr experiments studying the hydrodynamics of (15) n-enriched ubiquitin at low fields. Biochem Mol Biol Educ, 35(1):49-56, 2007.

N. Tjandra and A. Bax. Direct measurement of distances and angles in biomolecules by $\mathrm{nmr}$ in a dilute liquid crystalline medium (vol 278, pg 1111, 1997). Science, 278(5344): 1697-1697, 1997.

N. Tjandra, S. E. Feller, R. W. Pastor, and A. Bax. Rotational diffusion anisotropy of human ubiquitin from n-15 nmr relaxation. Journal of the American Chemical Society, 117(50):12562-12566, 1995.

P. Trigo-Mourino, C. Griesinger, and D. Lee. Label-free nmr-based dissociation kinetics determination. Journal of Biomolecular Nmr, 69(4):229-235, 2017.

V. Tugarinov, V. Kanelis, and L. E. Kay. Isotope labeling strategies for the study of high-molecular-weight proteins by solution nmr spectroscopy. Nature Protocols, 1(2): 749-754, 2006.

P. Vallurupalli, G. Bouvignies, and L. E. Kay. Increasing the exchange time-scale that can be probed by cpmg relaxation dispersion nmr. Journal of Physical Chemistry B, 115 (49):14891-14900, 2011.

P. Vallurupalli, G. Bouvignies, and L. E. Kay. Studying "invisible" excited protein states in slow exchange with a major state conformation. J Am Chem Soc, 134(19):8148-61, 2012 .

A. D. Vogt and E. Di Cera. Conformational selection or induced fit? a critical appraisal of the kinetic mechanism. Biochemistry, 51(30):5894-5902, 2012. 
D. W. Yang, Y. K. Mok, J. D. FormanKay, N. A. Farrow, and L. E. Kay. Contributions to protein entropy and heat capacity from bond vector motions measured by nmr spin relaxation. Journal of Molecular Biology, 272(5):790-804, 1997.

G. N. B. Yip and E. R. P. Zuiderweg. A phase cycle scheme that significantly suppresses offset-dependent artifacts in the r-2-cpmg n-15 relaxation experiment. Journal of Magnetic Resonance, 171(1):25-36, 2004.

M. Zeeb and J. Balbach. Protein folding studied by real-time nmr spectroscopy. Methods, 34(1):65-74, 2004. 


\section{Curriculum Vitae}

\section{$\underline{\text { Personal Data }}$}

Name: $\quad$ Sebastian Frischkorn

Date of Birth: 01.08.1990

Place of Birth: Bremerhaven, Germany

\section{$\underline{\text { Education }}$}

2015 - Present Ph.D. Student, Physics of Complex and Biological Systems, MaxPlanck Institute for Biophysical Chemistry, Göttingen, Germany Supervisor: Prof. Dr. Christian Griesinger

2012 - 2015 M. Sc., Department of Chemistry, Georg-August University, Göttingen, Germany, thesis: "Determination of thermal coefficients in large biomolecules via spin dynamics"

2009 - 2012 B. Sc., Department of Chemistry, Georg-August University, Göttingen, Germany, thesis: "Anisotrope NMR-Parameter zur Analyse organometallischer Verbindungen"

\section{$\underline{\text { Scientific Communications }}$}

$\underline{\text { Poster presentations }}$

2018 Protein folding and binding kinetics from NMR Relaxation experiments, Sebastian Frischkorn, Jithender Reddy Gurrala, Stefan Becker, Donghan Lee, Christian Griesinger and Pablo Trigo-Mourino, Humboldt Kolleg: The Impact of Bioimaging and Structural Biology in the Field of Life Science, Rio de Janeiro, Brasil

2018 Field-dependent high-resolution spectral density mapping using a fast, mechanical based shuttle system, Sebastian Frischkorn, Ching-Yu Chou, Stefan Becker and Christian Griesinger, $58^{\text {th }}$ ENC, Orlando, United States

2017 Windowless ${ }^{13}$ C-CPMG Experiment for Accurate Estimation of Faster Dynamics, Jithender Reddy Gurrala, Supriya Pratihar, Stefan Becker, Donghan Lee, Christian Griesinger, $57^{\text {th }}$ ENC, Asilomar, United States

\section{$\underline{\text { Publications }}$}

AC. Pöppler, S. Frischkorn, D. Stalke, M. John, Toluene and lithium amide diffusion into polystyrene: a slice-selective NMR-spectroscopic study. ChemPhysChem, 14, 3103-7, 2013

J. Reddy, S. Pratihar, D. Ban, S. Frischkorn, S. Becker, C. Griesinger and D. Lee, Simultaneous determination of fast and slow dynamics in molecules using extreme CPMG relaxation dispersion experiments. Journal of Biomolecular NMR, 70(1):1-9, 2018 